\title{
INFLUENCES ON THE OCEANIC BIOGEOCHEMICAL CYCLING OF THE HYBRID-TYPE METALS: COBALT, IRON, AND MANGANESE
}

By

Abigail Emery Noble

B.S., Haverford College, 2004

Submitted in partial fulfillment of the requirements for the degree of

Doctor of Philosophy at the MASSACHUSETTS INSTITUTE OF TECHNOLOGY and the WOODS HOLE OCEANOGRAPHIC INSTITUTION

February 2012

(C) 2012 Abigail E. Noble

All rights reserved.

The author hereby grants to MIT and WHOI permission to reproduce and to distribute publicly paper and electronic copies of this thesis document in whole or in part in any medium now known or hereafter created.

Signature of Author

Certified by

Joint Program in Oceanography Massachusetts Institute of Technology and Woods Hole Oceanographic Institution October 6, 2011

\begin{tabular}{lr}
\hline & Dr. Mak A. Saito \\
Accepted by & Thesis Supervisor
\end{tabular}

Accepted by

Dr. Roger Summons Chair, Joint Committee for Chemical Oceanography Woods Hole Oceanographic Institution 


\title{
INFLUENCES ON THE OCEANIC BIOGEOCHEMICAL CYCLING OF THE HYBRID-TYPE METALS: COBALT, IRON, AND MANGANESE By
}

\author{
Abigail Emery Noble
}

Submitted to the MIT/WHOI Joint Program in Oceanography October 6, 2011, in partial fulfillment of the requirements for the degree of Doctor of Philosophy in the field of Chemical Oceanography

\section{Thesis Abstract}

Trace metal cycling is one of many processes that influence ocean ecosystem dynamics. Cobalt, iron, and manganese are redox active trace metal micronutrients with oceanic distributions that are influenced by both biological and abiotic sources and sinks. Their open ocean concentrations range from picomolar to nanomolar, and their bioavailabilities can impact primary production. Understanding the biogeochemical cycling of these hybrid-type metals with an emphasis on cobalt was the focus of this thesis. This was accomplished by determining the dissolved distributions of these metals in oceanic regions that were characterized by different dominant biogeochemistries.

A large subsurface plume of dissolved cobalt, iron, and manganese was found in the Eastern South Atlantic. The cause of this plume is a combination of reductive dissolution in coastal sediments, wind-driven upwelling, advection, biological uptake, and remineralization. Additional processes that are discussed as sources of metals to the regions studied during this thesis include isopycnal uplift within cold-core eddies (Hawaii), ice melt (McMurdo Sound, Antarctica), riverine input (Arctic Ocean), and winter mixing (McMurdo Sound). The biological influence on surface ocean distributions of cobalt was apparent by the observation of linear relationships between cobalt and phosphate in mid to low latitudes. The cobalt:phosphate ratios derived from these correlations changed over orders of magnitude, revealing dynamic variability in the utilization, demand, and sources of this micronutrient. Speciation studies suggest that there may be two classes of cobalt binding ligands, and that organic complexation plays an important role in preventing scavenging of cobalt in the ocean.

These datasets provided a basis for comparing the biogeochemical cycles of cobalt, iron, and manganese in three oceanic regimes (Hawaii, South Atlantic, McMurdo Sound). The relative rates of scavenging for these metals show environmental variability: in the South Atlantic, cobalt, iron, and manganese were scavenged at very different rates, but in the Ross Sea, mixing and circulation over the shallow sea was fast, scavenging played a minor role, and the cycles of all three metals were coupled. Studying the distributions of these metals in biogeochemically distinct regions is a step toward a better understanding of their oceanic cycles.

Thesis supervisor: Mak Saito

Title: Associate Scientist 


\section{Acknowledgements}

I'd like to thank my advisor, Mak, for his support throughout my time in Woods Hole, for getting me to try my hand at jamming, for torturing me with his deadpan deliveries, for teaching me the paranoia that accompanies trace metal clean techniques, for introducing me to sukiyaki, for his understanding and flexibility, for somehow making me laugh whenever I think the world is ending, for having faith in me when I didn't, and lastly for the freedom I was given, whether I thought I wanted it or not. I'm sorry, that was a horrible run-on sentence. I've learned a lot during my time in this crazy enchanted place that will serve me well moving forward and I'm grateful to have had the opportunity to be a part of this great community. I'd like to thank my committee: Ed Boyle, Carl Lamborg, Phoebe Lam, and Bill Landing. The interest that they expressed in my data during committee meetings despite their 1 million other commitments was inspiring. I always left with a long laundry list of interesting calculations or experiments to try, different ways to plot the data, and new questions to ask. My only regret is that I didn't take full advantage of their input and resources during my time here.

Thanks of course to the academic programs office. I'd be confused, unregistered, generally lost, and paying late fees up the wazoo without you. Thank you for taking care of me!

Erin, Tyler, Dawn, and Matt - thanks for your support and friendship both in and outside the lab. I feel lucky to have been a part of a team that has ears and arms to listen, support, and encourage each other through anything.

I'd like to thank my friends who made me laugh, helped me gain new perspectives on life, taught me how to grow, and supported me through some of my hardest years adjusting to living with chronic back pain, particularly Mr. Willis Montgomery and 1 Wilson Rd.

I also want to thank the captain and crew of the R/V Knorr for their awesomeness. Whenever I had questions or problems, someone was able to answer or fix the problem right away, or moments later. Their genuine willingness to help, support, and work with the science party is unparalleled. I've never had such a pleasant time working long long days for weeks on end over and over again. Participating in expeditions on the Knorr has had a tremendously positive influence on my love for oceanographic work.

Lastly, I'd like to thank Mum and Dad, Whit and Liz, Grandmom and Grandpop, and Andrew. I never imagined that the biggest challenge I would face during my $\mathrm{PhD}$ would be to learn pain management. You are the reason that I have been able to persevere. Thank you for your love and infinite support. Thank you for trying to teach me to be kind to myself, to look for the bright side of every situation, and to breathe.

Funding for this research was provided by the the National Science Foundation Chemical Oceanography (Division of Ocean Sciences OCE-0452883, OCE-0752291, OCE-0928414, OCE-0732665, OCE-0440840, OCE-0327225), the Center for Microbial Research and Education, the WHOI Coastal Ocean Institute, and the WHOI Ocean Life Institute, WHOI Academic Programs Office, and a Fye Teaching Fellowship. 


\section{Table of Contents}

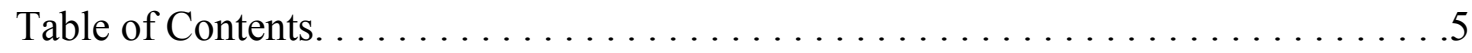

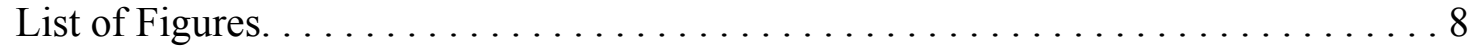

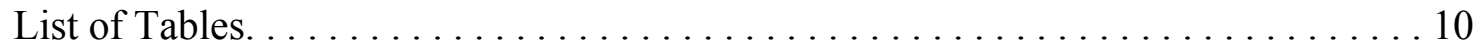

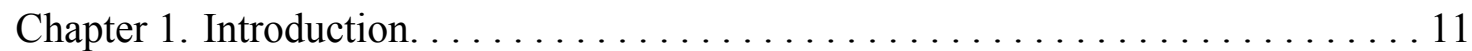

$1.1 \mathrm{Co}, \mathrm{Fe}$, and $\mathrm{Mn}$ biogeochemistry in the ocean: micronutritive importance

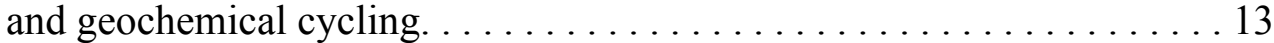

1.2 Data Chapters. . . . . . . . . . . . . . . . . . . . . . . 16

Chapter 2. Cobalt, manganese, and iron near the Hawaiian Islands: A potential concentrating mechanism for cobalt within a cyclonic eddy and implications for the

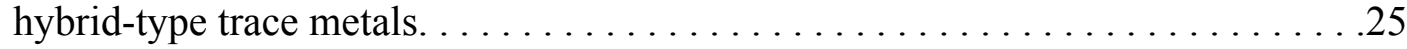

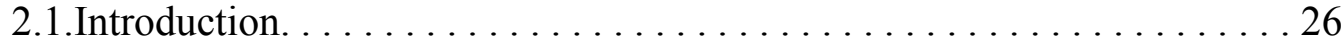

2.1.1. Potential influences on trace metal distributions among the Hawaiian Islands. . . . . . . . . . . . . . . . . 26

2.1.2. Influences on Cobalt, manganese, and iron marine

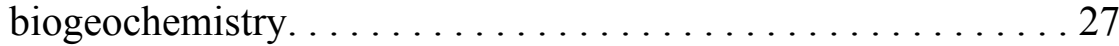

2.1.3. Mesoscale eddies near the Hawaiian Islands. . . . . . . . . . . . . . 27

2.1.4. The influence of eddies on trace metal biogeochemistry. . . . . 28

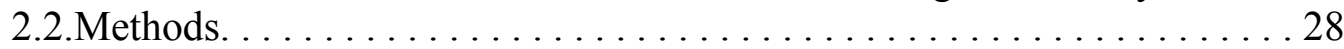

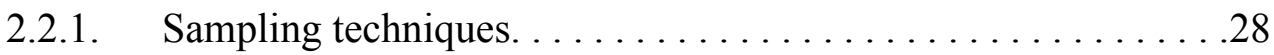

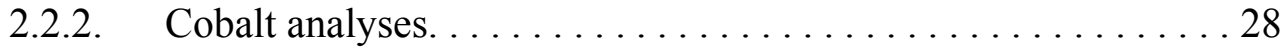

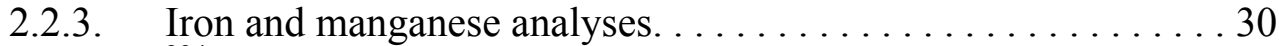

2.2.4. ${ }^{234} \mathrm{Th}$ analyses. . . . . . . . . . . . . . . . . . . . 30

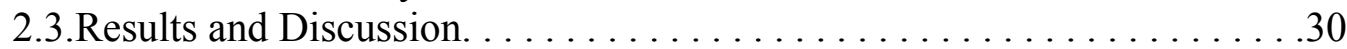

2.3.1. Oceanographic Properties. . . . . . . . . . . . . . . 30

2.3.2. General upper water column metal concentrations among the Hawaiian Islands. . . . . . . . . . . . . . . . . . . 30

2.3.3. An eddy influence on cobalt: biological entrainment and concentrating mechanism. .................. 33

2.3.4. The upper water column cobalt-phosphate correlation: a mechanistic explanation and a minimum productivity

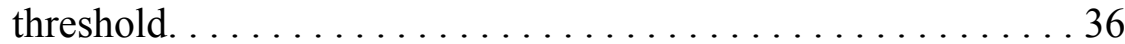

2.3.5. The implications of a biological concentrating mechanism for the hybrid-type metals. . . . . . . . . . . . . . . . . . 39

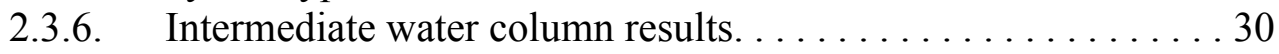

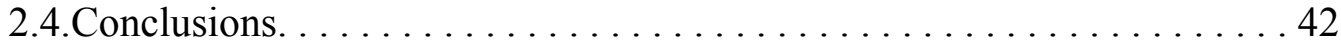

Chapter 3. Basin-scale inputs of cobalt, iron, and manganese from the BenguelaAngola Front to the South Atlantic Ocean. . . . . . . . . . . . . . . . 45

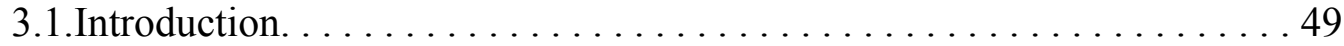

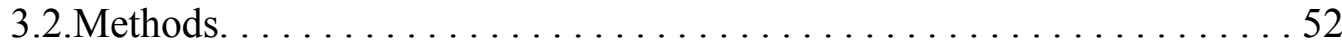

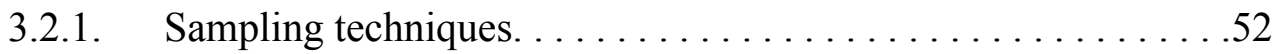

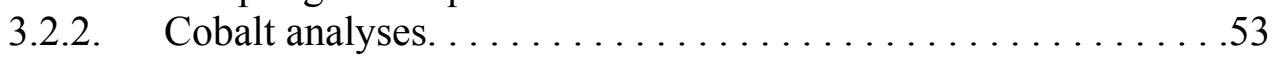

3.2.3. Iron and manganese analyses. . . . . . . . . . . . 56 
3.2.4. Sampling and analysis of $\mathrm{N}_{2} \mathrm{O} \ldots \ldots \ldots \ldots \ldots \ldots \ldots \ldots \ldots \ldots$

3.2.5. Nutrient and oxygen analyses. . . . . . . . . . . . . . . . . . . . . .

3.2.6. Particle trap collection and analysis. . . . . . . . . . . . 59

3.2.7. Suspended particle collection and analysis . . . . . . . . . . 60

3.2.8. Data analysis and repository. .................61

3.3. Results and Discussion. . . . . . . . . . . . . . . . . . . . .62

3.3.1. Spatial distribution observations. . . . . . . . . . . .63

3.3.2. Cobalt, iron, and manganese plumes across the South Atlantic

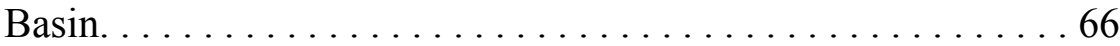

3.3.3. Implications for the hybrid-type metals with ocean deoxygenation. ........................ . . . .

3.3.4. A sedimentary metal source. . . . . . . . . . . . . . 72

3.3.5. Consideration of aeolian and riverine sources. . . . . . . . . 76

3.3.6. Vertical and lateral metal fluxes to the South Atlantic Basin . . . 79

3.3.7. Shallow coastal cobalt plume. .............. 83

Chapter 4. Dissolved and particulate cobalt, iron, and manganese under the McMurdo

Sound seasonal sea ice. . . . . . . . . . . . . . . . . . . . . . . . 109

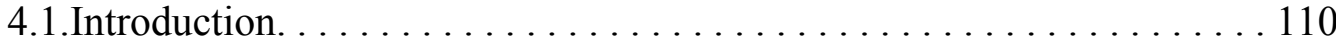

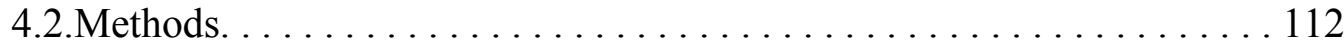

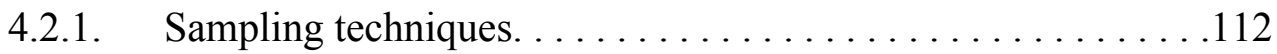

4.2.2. Cobalt analyses. . . . . . . . . . . . . . . . . . 113

4.2.3. Total dissolved and total dissolvable iron and manganese

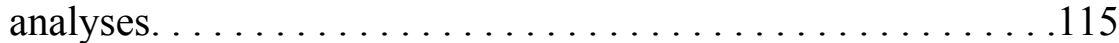

4.2.4. Particulate metal analyses. . . . . . . . . . . . . . . 115

4.2.5. Nutrient analyses. . . . . . . . . . . . . . . . . . . 117

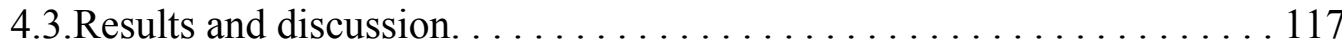

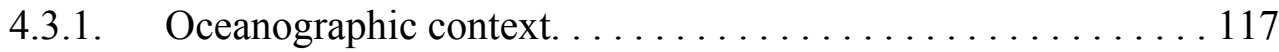

4.3.2. Total dissolved and labile cobalt distributions. . . . . . . . . 118

4.3.3. Total dissolved manganese and iron distributions. . . . . . . 120

4.3.4. Particulate metal distributions and a comparison of acid leachable particulate iron to particulate iron. . . . . . . . . . . 122

4.3.5. Consideration of island effects, terrestrial input, and/or sedimentary influences. . . . . . . . . . . . . . . 125

4.3.6. Particulate and dissolved cobalt and manganese distributions: evidence of manganese-oxidizing bacteria and fast mixing. . . .127

4.3.7. Sea ice sources. . . . . . . . . . . . . . . . . . . . . . 129

4.4.Conclusions. . . . . . . . . . . . . . . . . . . . . . . . . 130

Chapter 5. Distributions of dissolved cobalt from the US North Atlantic GEOTRACES cruise: relationships with phosphate and oxygen, and a comparison to the South

Atlantic Ocean. . . . . . . . . . . . . . . . . . . . . . . . . . . 149

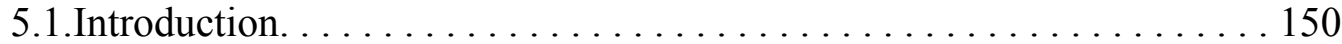

5.2.Methods. . . . . . . . . . . . . . . . . . . . . . 152 
5.2.1. NAGZT cruise sampling techniques. . . . . . . . . . . . . . 152

5.2.2. CoFeMUG cruise sampling techniques. . . . . . . . . . 153

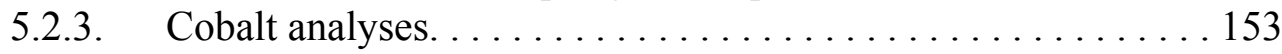

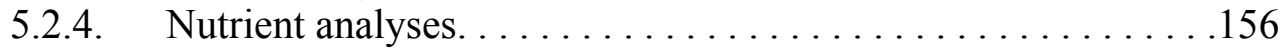

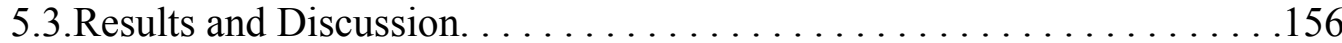

5.3.1. Description of the oceanographic regions studied . . . . . . . . 157

5.3.2. Sources of cobalt to the N. E. Atlantic. . . . . . . . . . . . . 158

5.3.3. $\mathrm{Co}: \mathrm{PO}_{4}{ }^{3-}$ relationships in the Atlantic. . . . . . . . . . . 160

5.3.4. Complexation and speciation. . . . . . . . . . . . . 164

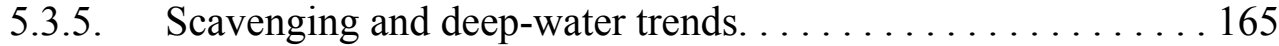

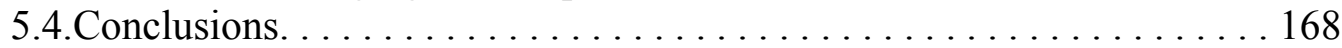

Chapter 6. Identification of a novel redox sensitive strong cobalt ligand class in oxygen minimum zones and a simple total dissolved cobalt preservation technique by the

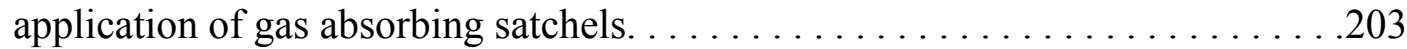

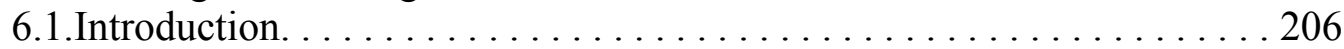

6.2.Methods. . . . . . . . . . . . . . . . . . . . . . . . . 210

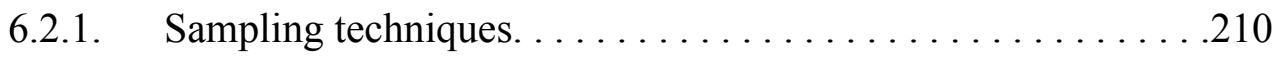

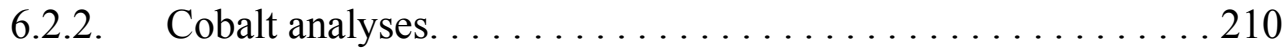

6.2.3. Preservation technique. ................... 212

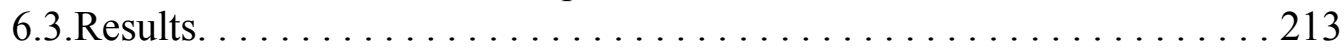

6.3.1. Comparison of at-sea to stored analyses. . . . . . . . . 213

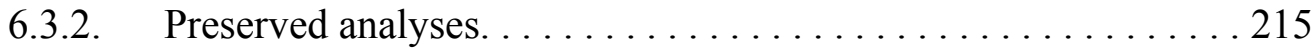

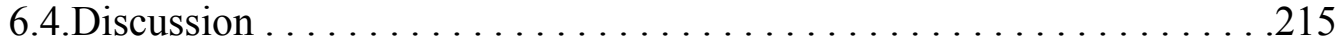

6.4.1. A method of preservation for total cobalt analysis by ACSV . . . 216

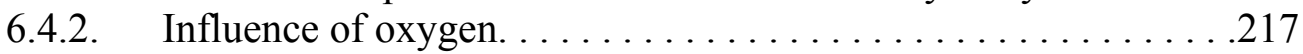

6.4.3. Potential influence of particle load. . . . . . . . . . . . . . . 219

6.4.4. The role of complexation and a new avenue for the exploration of

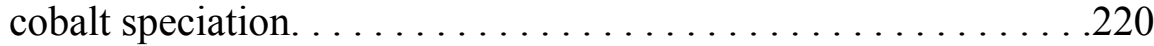

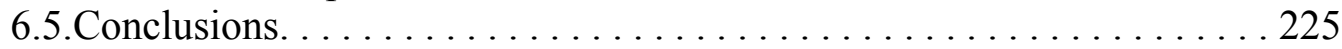

Appendix I: Prospects for a multi-element method to measure cobalt, iron, and

manganese concentrations in seawater via inductively-coupled plasma mass

spectrometry. . . . . . . . . . . . . . . . . . . . . . 239

Appendix II: Photodegradation experiments with Vitamin $B_{12} \ldots \ldots \ldots \ldots \ldots \ldots . \ldots 265$

Appendix III: Dissolved cobalt in the Beaufort Sea from the Canadian Arctic

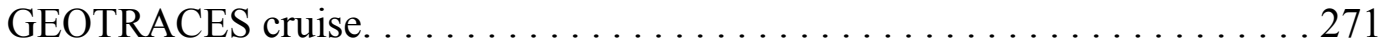

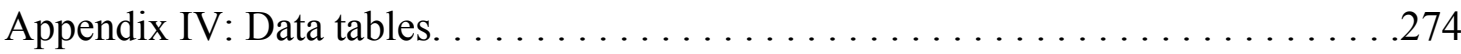

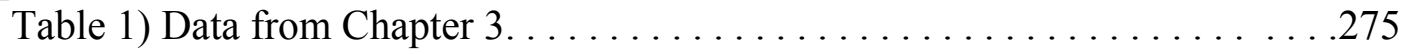

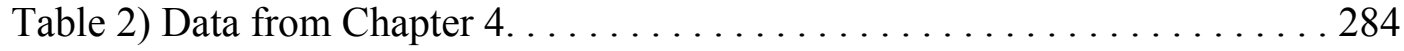

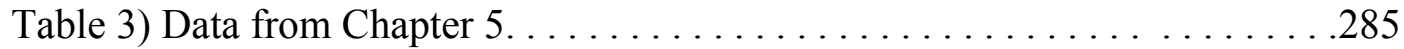

Table 4) Data from Chapter 6. . . . . . . . . . . . . . . . . . . . . . 290

Table 5) Data from Appendix III. . . . . . . . . . . . . . . . . . . . . . . . 294 


\section{List of Figures \\ Chapter 2}

Figure 1) Sampling sites, map and bathymetry around the Hawaiian Islands. . . .28

Figure 2) E-Flux II cobalt profile overlay and Transect 6 contour plot . . . . . . 34

Figure 3) Vertical profiles of $\mathrm{Mn}, \mathrm{Fe}, \mathrm{Co}, \mathrm{PO}_{4}{ }^{3-}$, and hydrographic data at Sta.

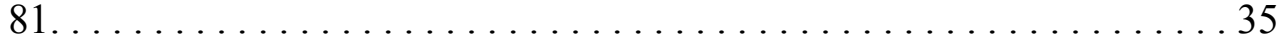

Figure 4) Surface profiles of $\mathrm{Co}, \mathrm{NO}_{2}{ }^{-}+\mathrm{NO}_{3}{ }^{-}$, and $\sigma$ from E-Flux III. . . . . . . .35

Figure 5) Contour plot of ${ }^{234} \mathrm{Th} /{ }^{238} \mathrm{U}$ for E-Flux III. . . . . . . . . . . . . . . . 36

Figure 6) Illustration of cobalt concentrating mechanism. . . . . . . . . . 36

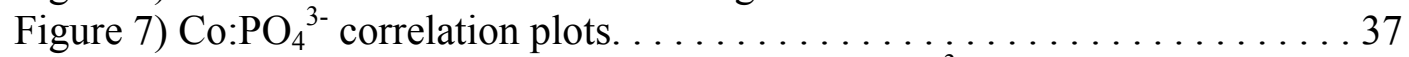

Figure 8) Schematic of processes that influence $\mathrm{Co}: \mathrm{PO}_{4}{ }^{3-}$ relationships in hybrid-

type metal-phosphate space $\left(\mathrm{HTM}: \mathrm{PO}_{4}{ }^{3-}\right) \ldots \ldots \ldots \ldots \ldots \ldots \ldots \ldots$

Figure 9) $\mathrm{Chl}$ a profiles for E-Flux II and III . . . . . . . . . . . . . . 39

Figure 10) Profiles of $\mathrm{Mn}, \mathrm{Co}, \mathrm{PO}_{4}{ }^{3-}$, and hydrographic data to $1000 \mathrm{~m}$ for E-Flux

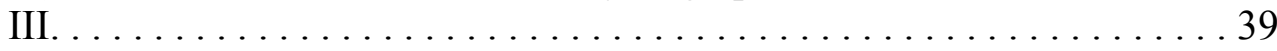

\section{Chapter 3}

Figure 1) Cruise track and ocean sections of total Co, Fe, Mn, and labile Co . . .95

Figure 2) Ocean sections of $\mathrm{PO}_{4}{ }^{3-}, \mathrm{NO}_{2}{ }^{-}+\mathrm{NO}_{3}{ }^{-}$, silicate, and $\mathrm{O}_{2} \ldots \ldots \ldots \ldots 6$

Figure 3) Example profiles of total $\mathrm{Co}, \mathrm{Fe}$, and $\mathrm{Mn} . \ldots \ldots \ldots \ldots \ldots$. . . . . . 97

Figure 4) Estimated dust deposition to the Atlantic and surface transect of Co, Al,

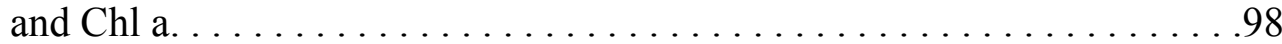

Figure 5) Upper water column sections of $\mathrm{Co}, \mathrm{Fe}, \mathrm{Mn}$ and $\mathrm{O} 2$ for the main transect. . . . . . . . . . . . . . . . . . . . . . . .99

Figure 6) Upper $800 \mathrm{~m}$ hydrography for near-coastal and coastal stations. . . . . 100

Figure 7) $\mathrm{Co}: \mathrm{O}_{2}$ and $\mathrm{Co}: \mathrm{N}_{2} \mathrm{O}$ scatter plots for data between 300 and $800 \mathrm{~m} \ldots \ldots 101$

Figure 8) Scatter plots of $\mathrm{Co}, \mathrm{Fe}, \mathrm{Mn}$ and $\mathrm{s}$ at 400, 600, and $800 \mathrm{~m}$ along the main

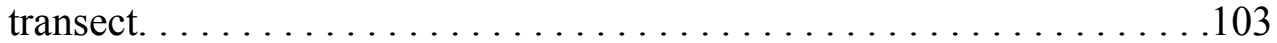

Figure 9) Comparison of particulate and dissolved profiles at Sta. 13 and Sta.

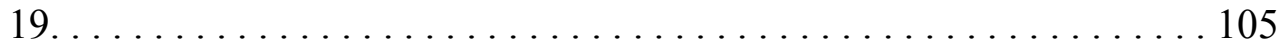

Figure 10) Vertical profiles of $\mathrm{Co}, \mathrm{Fe}, \mathrm{Mn}, \mathrm{BAT}$, and $\mathrm{O}_{2}$ at coastal stations . . . .106

Figure 11) Sediment trap data at Sta. 13 and 19 for total, P, Co, Mn, and Fe

fluxes. . . . . . . . . . . . . . . . . . . . . . . . . . . . . . . 107

\section{Chapter 4}

Figure 1) Map of Antarctica, Ross Sea, McMurdo Sound, and sampling stations. . . . . . . . . . . . . . . . . . . . . . . . . . . . . . . 137

Figure 2) Profiles of dissolved cobalt, iron, manganese, and phosphate. . . . . . 139

Figure 3) Overlay of averaged profiles from this study and CORSACS study. . .141

Figure 4) $\mathrm{Co}: \mathrm{PO}_{4}{ }^{3-}$ scatter plot of data from this study, and CORSACS study. . .142

Figure 5) Profiles of particulate cobalt, iron, manganese, aluminum, and

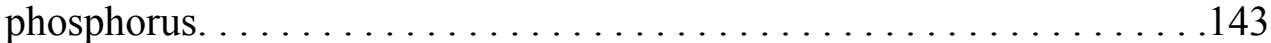

Figure 6) Scatter plots of particulate bioactive trace metals. . . . . . . . . . . . 144

Figure 7) Comparison of dissolved and total dissolvable iron and manganese . 145

Figure 8) Comparison of particulate iron to ALP iron. . . . . . . . . . . . . 146 
Figure 9) Comparison of average ALP Fe distributions from CORSACS and this study......................................

Figure 10) Profiles of metal:Al ratios in suspended particulate material compared to nearby sediment composition . . . . . . . . . . . . . . . . . . . . . . 148

\section{Chapter 5}

Figure 1) Map and cruise track for CoFeMUG and NAGZT expeditions. ......181

Figure 2) Ocean sections of $\mathrm{TCo}, \mathrm{Co}^{\prime}, \mathrm{PO}_{4}{ }^{3-}, \mathrm{NO}_{2}{ }^{-}+\mathrm{NO}_{3}{ }^{-}, \mathrm{O}_{2}, \mathrm{AOU}$, salinity, and temperature for NAGZT. ........................... 183

Figure 3) OMZ sections for CoFeMUG and NAGZT expeditions and corresponding scatterplot showing the linear covariance with $\mathrm{O}_{2} \ldots \ldots 185$

Figure 4) Profiles of TCo and Co' from the NAGZT expedition. .......... 187

Figure 5) Surface transects of cobalt for CoFeMUG and NAGZT expeditions, and surface Co:Al scatter plot showing a linear relationship within the Saharan dust plume.................................... 189

Figure 6) $\mathrm{Co}: \mathrm{PO}_{4}{ }^{3-}$ and $\mathrm{PO}_{4}{ }^{3-}: \mathrm{NO}_{2}{ }^{-}+\mathrm{NO}_{3}{ }^{-}$scatter plots, illustrating basin offsets and ecological stoichiometries in the upper $300 \mathrm{~m} \ldots \ldots \ldots \ldots \ldots \ldots 1$

Figure 7) $\mathrm{TCo}: \mathrm{PO}_{4}{ }^{3-}$ and $\mathrm{Co}^{\prime}: \mathrm{PO}_{4}{ }^{3-}$ scatter plots illustrating the relationship between complexation and the observation of a kink in the $\mathrm{TCo}^{\mathrm{PO}} \mathrm{P}_{4}{ }^{3-}$ relationship.................................193

Figure 8) TCo: $\mathrm{PO}_{4}{ }^{3-}$ scatter plots illustrating the range in depth to which a TCo and $\mathrm{PO}_{4}{ }^{3-}$ linear relationship is observed, depth of the observed kink, and overall linearity in $\mathrm{TCo}_{\mathrm{PO}}{ }_{4}{ }^{3-}$ relationships among these four regions. . 195

Figure 9) Regional global TCo: $\mathrm{PO}_{4}{ }^{3-}$ comparisons, illustrating the range in $\mathrm{PO}_{4}{ }^{3-}$ inventories and range in slopes. . . . . . . . . . . . . . . . 196

Figure 10) Upper water column profiles of $\mathrm{TCo}, \mathrm{Co}^{\prime}, \mathrm{PO}_{4}{ }^{3-}$, fluorescence, and density for select NAGZT stations. . . . . . . . . . . . . . . 197

Figure 11) Regional comparison of full depth $\mathrm{TCo} \mathrm{PO}_{4}{ }^{3-}$ relationships, illustrating the shape of the scavenging curls and the different depths at which scavenging becomes apparent. ......................... 199

Figure 12) Comparison of the shape of scavenging curls in different regions . . .200

Figure 13) Temperature:salinity scatter plot illustrating the location and depths at which different water masses sampled plot in the corresponding TCo: $\mathrm{PO}_{4}{ }^{3-}$

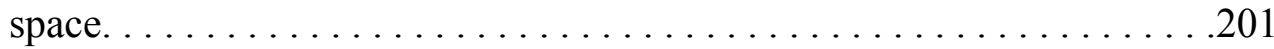

\section{Chapter 6}

Figure 1) Profiles and scatter plots comparing $\mathrm{TCo}_{a t-s e a}$ to $\mathrm{TCo}_{\text {stored }}$ for 6 stations across 3 different oceanic regimes. .....................233

Figure 2) Profile and scatter plot comparison of Sta. 9 NAGZT TCo ${ }_{a t-s e a}$ to

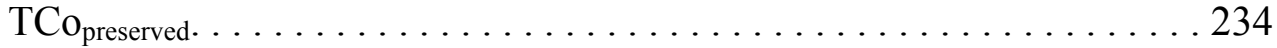

Figure 3) Percent of TCo lost during storage compared to $\mathrm{O}_{2}, \%$ complexation, and

$\mathrm{Co}^{\prime}{ }_{\text {at-sea }}$ for NAGZT analyses......................... 235

Figure 4) Profiles of $\mathrm{Co}_{\text {at-sea }}$ and $\mathrm{Co}_{\text {stored }}$ for Sta. 5 and 9 from NAGZT. . . . . 236

Figure 5) Profiles of all cobalt analyses for Sta. 9 from NAGZT and scatter plots illustrating that $\mathrm{Co}^{\prime}$ and $\mathrm{CoL}_{\mathrm{Omz}}$ are lost during storage. ........... 237

Figure 6) Schematic for possible mechanisms for cobalt loss............238 


\section{List of Tables}

\section{Chapter 2}

Table 1) Data from E-Flux II and III expeditions.................... 31

\section{Chapter 3}

Table 2) Upper water column species inventories from E-Flux III. . . . . . . . . .36

Table 1) SAFe and GEOTRACES standard seawater analyses for dissolved Co.....................................92

Table 2) Comparison of shelf-width and iron concentrations in upwelling zones. ................................... 92

Table 3) Comparison of chemical properties for stations in and near the BenguelaAngola Frontal Zone............................... 93

\section{Chapter 5}

Table 1) Blank analyses for NAGZT. .........................173

Table 2) Standard seawater analyses run during NAGZT. .............175

Table 3) Comparison of North and South Atlantic basin environmental characteristics...................................177

Table 4) Global published Co: $\mathrm{PO}_{4}{ }^{3-}$ ratios. . . . . . . . . . . . . . . . . . 179

Table 5) North and South Atlantic $\mathrm{Co}: \mathrm{O}_{2}$ linear relationships...............180

\section{Chapter 6}

Table 1) Sample inventory for cobalt analyses. ................... 231 Appendix IV

Table 2) Cobalt speciation analyses on a Ross Sea sample..............231

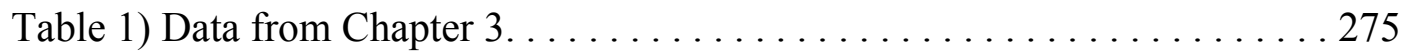

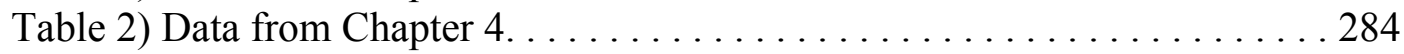

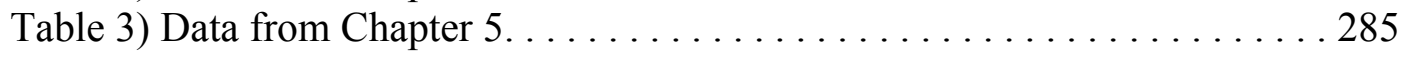

Table 4) Data from Chapter 6................................ 290

Table 5) Data from Appendix III. . . . . . . . . . . . . . . . . . . . . . . . 294 


\section{Chapter 1: Introduction}

Bioactive trace metal cycling is one of many processes that regulate ocean ecosystem dynamics. The concentrations of many bioactive metals are vanishingly low in seawater, and the role these metals play varies in importance from vital, to a useful substitute, to toxic, depending on the concentration and the organism of interest. Bioactive metals like iron, zinc, and cobalt for example, are present in picomolar to nanomolar concentrations, and can limit or co-limit the growth of primary producers in the ocean (Martin et al. 1989, Fitzwater 1996, Saito and Moffett 2002, Wu and Boyle 2002, Moore et al. 2004). These metals are incorporated into proteins and enzymes that participate in important biological processes, including light, carbon, and nitrogen acquisition, among others (Morel et al. 2003 and references therein). The diversity in metal requirements among ocean biota coupled with the selection pressure that low metal abundances exert are often not as simple as the concept of one ultimately limiting nutrient (Sunda and Huntsman 1995, Saito et al 2002, Morel et al. 2003, Saito et al. 2008). For example, zinc, cadmium and cobalt interreplacement has been demonstrated in the diatom Thalassiosira weissflogii (Lane and Morel 2000), and the abundant cyanobacteria, Prochlorococcus and Synechococcus, have an absolute requirement for cobalt that cannot be met by zinc or cadmium (Sunda and Huntsman 1995, Saito et al. 2002). These complexities highlight the need to simultaneously study multiple chemical parameters in order to understand these ecological systems.

The study of bioactive trace metal cycling is challenging due to the difficulties involved in accurately measuring the oceanic concentrations which are orders of 
magnitude lower than concentrations found on land and in man-made products. Advances in clean sampling and analysis techniques have made these measurements possible, though considerable care is required to prevent sample contamination. Among the biologically active trace elements, cobalt, iron, and manganese are of particular interest to me because they have closely linked, complex, geochemical characteristics. They are neighboring transition metals with water column distributions that are affected by biological utilization, redox chemistry, and scavenging. Their low solubilities in oxygenated seawater cause them to have small oceanic inventories. As a result, changes in the sources and sinks of these elements can dramatically affect the regional ecology. Geochemical influences on the distributions of these elements in the ocean include aeolian input, hydrothermal input, riverine discharge, sediment resuspension/adsorption, horizontal advection of dissolved and particulate metals, and loss through scavenging on particles (Landing and Bruland 1987, Sedwick et al. 1992, Lam et al. 2008, Lamborg et al. 2008, Shelley et al. 2010, Thuroczy et al. 2010, Saito et al. 2004, Boyle et al. 2005, Bruland et al. 2005, Sedwick et al. 2005). The fieldwork in this thesis sampled environments influenced by varying extents of these processes. An important goal of this thesis was to attempt to derive a broader understanding of cobalt, iron, and manganese biogeochemical behaviors by investigating their relative distributions in environments with these differing dominant geochemistries. The study sites covered oligotrophic waters (South Atlantic Gyre), an upwelling system (Benguela Upwelling), strongly aeolian influenced waters (W. African N. E. Atlantic), eddy-influenced entrainment (off the Hawaiian Islands), fluvial discharge (Beaufort Sea), and an isolated shelf system (beneath the McMurdo Sound sea ice). 


\subsection{Cobalt, iron, and manganese biogeochemistry in the ocean: micronutritive importance and geochemical cycling}

Cobalt has multiple known uses in the cell. From co-limitation culture studies, it has been observed that cobalt can satisfy some of the zinc requirements in the coccolithophore Emiliania huxleyi and the eukaryotic algae Phaeocystis antarctica, and largely satisfies zinc requirements in the diatoms Thalassiosira pseudonana and Thalassiosira oceanica (Sunda and Huntsman 1995, Saito and Goepfert 2008). This likely occurs via substitution of cobalt for zinc in the active sites of metalloenzymes like carbonic anhydrase. Prochlorococcus and Synechococcus, however, have an absolute requirement for cobalt that cannot be met by zinc or cadmium (Sunda and Huntsman 1995, Saito et al. 2002). Cobalt is also found at the metal center of vitamin $B_{12}$, a biomolecule that is thought to be produced solely by some bacteria and archaea. This means that all eukaryotes must have a method to acquire $\mathrm{B}_{12}$ from the environment, or function under a biochemistry that does not require it (Bertrand et al. 2007). In the Ross Sea (where temperatures are typically too cold to support cyanobacteria that are thought to be a significant source of $\left.\mathrm{B}_{12}\right), \mathrm{B}_{12}$ is thought to be present in particularly low concentrations or is drawn down very quickly, and iron and $\mathrm{B}_{12}$ colimitation has been observed (Bertrand et al. 2007).

Manganese is used to oxidize water to $\mathrm{O}_{2}$ in Photosystem II, to detoxify cells from harmful $\mathrm{O}_{2}^{-}$radicals via its incorporation into superoxide dismutase, and is oxidized by manganese-oxidizing bacteria (Tebo et al. 1984; Moffett and Ho 1996; Morel et al. 2003; Tebo et al. 2004). Manganese has often been observed in elevated concentrations in the upper ocean of tropical and subtropical waters, but is scavenged at intermediate depths due 
to the presence of manganese-oxidizing bacteria (Cowen and Bruland 1985). Manganese is relevant to cobalt biogeochemistry because manganese-oxidizing bacteria have been observed to co-oxidize cobalt by competitive inhibition via the same oxidative pathway (Moffett and Ho 1996, Tebo et al. 1984), and is hypothesized to be a major removal mechanism for cobalt in the ocean. Cobalt may also be precipitated with manganese oxide production via adsorption and subsequently released when it dissolves. Dissolved manganese is often elevated within oxygen minimum zones in the open ocean (Martin and Knauer 1985; Johnson et al. 1992), and this has recently been observed for cobalt as well (Saito et al. 2004, 2005; Noble et al. in review). In contrast, the cycling of cobalt and manganese was observed to be decoupled in the Sargasso Sea because cobalt was dominated by uptake and remineralization by phytoplankton whereas manganese was dominated by bacterial redox processes (Moffett and Ho 1996). This plus the knowledge that low concentrations of oxygen are thought to slow manganese oxidation rates (Tebo 1991), contribute to the observed oxygen minimum zone dissolved manganese maximum due to input from remineralized sinking organic matter (Johnson et al. 1992, 1996). Photoreduction reactions in the euphotic zone have been studied for both manganese and iron (Sunda and Huntsman 1988, Barbeau et al. 2003), but to my knowledge, few studies have investigated the photoreduction of Co(III) in the ocean (Moffett and Ho 1996).

Iron is required in photosystems I and II, and iron is important in many enzymatic pathways of the microbial nitrogen cycle, including nitrogen fixation (Morel et al. 2003; Saito et al. 2011). Due to the intense biological iron requirements of many biota and the low surface concentrations observed in the gyres, iron is thought to limit approximately 
$40 \%$ of ocean primary production (Moore et al. 2004). Cobalt can behave like iron, displaying depletion in surface waters with a subsurface remineralization signal, and scavenging at depth (Fig. 3, Noble and Saito et al. 2008). The distinctions in their biogeochemistries are highlighted in situations in which these two elements display different distributions. Iron is delivered to the ocean primarily via dust deposition and sediment interactions (Moore and Braucher 2008), though increasing evidence suggests that hydrothermal inputs may also be significant (Saito et al. in prep, Yucel et al. in press). Manganese also has a significant aeolian source, can be released from sediments, and has been observed to have hydrothermal sources (Middag et al. 2010, Saito et al. in prep). Cobalt, however, comprises a much smaller fraction of crustal material than iron $(\mathrm{Co}: \mathrm{Fe}$ in crust $\sim 1: 2600$ ) and dust is not often considered to be a primary source of cobalt to the open ocean (Taylor and McLennan 1985, Lamborg et al. 2008). Additionally, in the same study in which both iron and manganese hydrothermal plumes were observed, no plume of cobalt was observed (Chapter 3 ). In the open and coastal oceans, different scavenging rates of iron, cobalt, and manganese have also been observed (Noble et al. in review). Cobalt is more slowly scavenged than iron and manganese, likely due to its slower oxidation kinetics, and without a significant aeolian source, cobalt distributions may help identify subtle sedimentary sources of iron (Saito et al. 2004, Saito and Schneider 2006, Noble et al. in review).

The chemical speciation of these three metals is also important to their nutritive utilization in the ocean and biogeochemical cycling. As all three metals are redox active under seawater conditions, processes that have the potential to stabilize either the oxidized 
or reduced forms in seawater are of interest as they can increase the solubility of the element. Complexation by organic ligands is thought to protect iron and cobalt from scavenging to some degree (Johnson et al. 1997; Saito and Moffett 2001). While two classes of ligands, one weaker and one stronger, have been observed for iron speciation (Rue and Bruland 1994), cobalt speciation appears to be dominated by a single class of very strong ligands with conditional stability constants greater than $10^{16.8}$ (Saito et al. 2005), though a second class of oxygen minimum zone (OMZ) stabilized, strong inorganic ligands may also exist (Chapter 6). The structure of these ligands is largely unknown, and changes in speciation caused by chemical perturbation of the redox state of these ligands may reveal important clues regarding their structure. Manganese may also be complexed by some marine siderophores (Parker et at. 2007; Homann et al. 2009), though the influence of complexation on manganese scavenging is still largely unknown. Speciation also likely influences the bioavailability of the metal to microorganisms. For example, cellularly-bound reductases may reduce iron at the cell surface in order to bring the iron across the cellular membrane (Shaked et al. 2005). Presumably, if a cell possesses more diverse metal-acquiring machinery, it will be better suited to outcompete other organisms for available metals.

\subsection{Data Chapters}

Due to the complexity of the aforementioned influences on these metals, they are categorized as "hybrid-type," defined by the simultaneous influence of biological and abiotic processes on their oceanic distributions (Bruland and Lohan 2003, Noble and Saito et al. 2008). The data chapters of this thesis were designed to improve our understanding 
of hybrid-type metal cycling in the ocean, with an emphasis on cobalt. In Chapter 2, we proposed a simplified schematic of these different environmental factors where the biotic and abiotic influences can be visualized as vectors in space defined by hybrid-type metal concentration and a macronutrient like soluble reactive phosphate (Chapter 2, Fig. 8: Noble and Saito et al. 2008). This simplified schematic is derived from a Redfieldian approach, where we suggest that if the processes of biological uptake and remineralization are the primary influences on the distributions of the metal in a given environment, the metal and phosphate will tend to be linearly correlated (Noble and Saito et al. 2008). For instance, a strong cobalt-phosphate correlation found in the Peru Upwelling system $\left(\mathrm{Co}: \mathrm{PO}_{4}{ }^{3-}, \mathrm{r}^{2}=\right.$ 0.96) implied that biological processes controlled the surface water distribution of cobalt (Saito et al. 2004). With changes in phytoplankton community structure, the slope of the line may change, due to varying cellular quotas for different organisms. Conversely, abiotic processes such as scavenging, atmospheric input, or sediment resuspension tend to decouple macronutrients and the hybrid-type metal. This type of effect has been observed in the North Atlantic off the coast of New England, where a strong cobalt-salinity correlation $\left(\mathrm{r}^{2}=0.93\right)$ was observed in the absence of a Co: $\mathrm{PO}_{4}{ }^{3-}$ correlation, indicative of a coastal cobalt input obscuring the influence of biological utilization (Saito and Moffett 2002).

The field data for this thesis are concentrated in Chapters 2-5 and Appendix III, which explore the distributions of cobalt, iron, and manganese in oceanic regions that are dominated by different physical, chemical, and biological environments. Chapter 2 explores eddies off the coast of Hawaii as a mechanism for bringing metals to the surface 
ocean and concentrating them there via a cycle of shallow remineralization and reentrainment (Noble and Saito et al. 2008).

Chapter 3 presents evidence of a basin-scale subsurface plume of hybrid-type metals coincident with the oxygen minimum in the South East Atlantic, and we attempt to gain some perspective on the relative scavenging rates of these metals. The source of the metals is likely reductive dissolution from coastal sediments and these metals reach the basin via advection, upwelling, biological uptake, and remineralization. In this complicated system, we also make an effort to incorporate both particulate and particle flux data to better understand the cycling of these metals. This study also identified a plume of iron and manganese concentrations above the Southern Mid-Atlantic Ridge. These results are compiled in a separate manuscript that discusses the geochemical implications of finding a hydrothermal iron plume in a slow spreading ridge system such as the South Atlantic (Saito et al. in prep).

Chapter 4 looks at metal distributions beneath the McMurdo Sound seasonal sea ice as a potential source of metals via sediment resuspension and ice melt. This dataset is an important end member for a number of reasons. In this cold climate, cyanobacteria are virtually non-existent. This is useful because cyanobacteria are often the dominant organisms in the oligotrophic gyres, they have an absolute requirement for cobalt, and some produce cobalt binding ligands in seawater, possessing the capability to change the chemical speciation of cobalt (Saito et al. 2005, Partensky et al. 1999, Sunda and Huntsman 1995). The Ross Sea and McMurdo Sound are relatively well-mixed, and 
oxygen concentrations are generally quite high which reduces the potential for complex redox cycling having a significant impact on the dissolved profiles.

The data in Chapter 5 presents evidence of aeolian input to the North Atlantic, as well as a likely sedimentary source associated with high productivity and upwelling, similar to what was identified in the South Atlantic system. The juxtaposition of the North and South Atlantic studies allows for a regional comparison of cobalt distributions: one region is influenced by weak upwelling and strong aeolian input (North), and the other is influenced by strong upwelling and weak aeolian input (South). Chapter 5 also returns to the schematic representation of biologic and abiotic influences on the distributions of hybrid-type metals (Chapter 2, Noble and Saito et al. 2008). Here, we compare the linear relationships between cobalt and phosphate across different regions of the Atlantic Ocean and attempt to gain some understanding of what influences the large dynamic range of ecological stoichiometries observed.

Chapter 6 and Appendices I and II shift the focus to methods for cobalt analysis, sample preservation, sample shelf-life, and influences on chemical speciation. In Chapter 6 , total cobalt and labile cobalt measurements were determined in samples that were stored for differing amounts of time as well as under different conditions. These results suggest that a second class of redox sensitive strong ligands may exist for cobalt in oxygen minimum zones, and that the addition of gas absorbing satchels can be applied to explore cobalt chemical speciation. The application of these gas absorbing satchels was also determined to be successful as a short term storage alternative to making immediate analyses at sea for total cobalt. Appendix I expanded upon previous method work focused 
on the determination of iron, manganese, and cobalt by magnesium hydroxide coprecipitation techniques (Saito and Schneider 2006). Previous work had determined that cobalt was inefficiently scavenged by this technique and the work in Appendix I demonstrates that the nature of cobalt scavenging requires a relatively large precipitate, making the matrix of the analyte difficult for analysis by inductively-coupled plasma mass spectrometry (ICP-MS). This was followed by investigations of different chelating resins to remove salts. Appendix II investigated the effect of photoreduction on the lability of cobalt within the cobalt-containing compounds cyanocobalamin and coenzyme $\mathrm{B}_{12}$. This was achieved by combining UV-Vis spectroscopy with competitive ligand adsorptive cathodic stripping voltammetry. Exposure to ultraviolet light quickly destroyed these compounds and effectively release cobalt within seconds to minutes, however; exposure to natural light degraded cyanocobalamin over the course of hours, and the degraded product was observed to still bind cobalt at conditional stability constants in excess of $10^{16.8}$.

Finally, in the last of the field observations, Appendix III presents results of cobalt analyses from the GEOTRACES Canadian Arctic expedition. These samples came from the Beaufort Sea and show intense surface maxima at all stations for dissolved cobalt. As the Arctic Ocean is known to have a strong fluvial influence, these samples provide a good boundary condition for potential riverine input of cobalt. 


\section{References for Chapter 1:}

Barbeau, K. B., E. L. Rue, C. G. Trick, K. W. Bruland, and A. Butler. 2003. Photochemical reactivity of siderophores produced by marine heterotrophic bacteria and cyanobacteria based on characteristic Fe(III) binding groups. Limnol. Oceanogr. 48: 1069-1078.

Bertrand, E. M. and others 2007. Vitamin $\mathrm{B}_{12}$ and iron co-limitation of phytoplankton growth in the Ross Sea. Limnol. Oceanogr. 52: 1079-1093.

Boyle, E. A., B. A. Bergquist, R. A. Kayser, and N. Mahowald. 2005. Iron, manganese, and lead at Hawaii Ocean Time-series station ALOHA: Temporal variability and an intermediate water hydrothermal plume. Geochim. Cosmo. Acta 69: 933-952.

Bruland, K. W., and M. C. Lohan. 2003. Controls of Trace Metals in Seawater. In H. D. H. K.K. Turekian [ed.], Treatise on Geochemistry. Elsevier Science Ltd.

Bruland, K. W., E. L. Rue, G. J. Smith and G. R. DiTullio. 2005. Iron, macronutrients and diatom blooms in the Peru Upwelling regime: Brown and blue waters of Peru. Mar. Chem. 93: 81-103

Cowen, J. P., and K. W. Bruland. 1985. Metal deposits associated with bacteria: implications for Fe and Mn marine biogeochemistry. Deep-Sea Res 32: 253-272.

Fitzwater, S. E., K. H. Coale, R. M. Gordon, K. S. Johnson, and M. E. Ondrusek. 1996. Iron deficiency and phytoplankton growth in the equatorial Pacific. Deep Sea Research Part II: Topical Studies in Oceanography 43: 995-1015.

Homann, V. V., M. Sandy, J. A. Tincu, A. S. Templeton, B. M. Tebo, and A. Butler. 2009. Loihichelins A-F, a Suite of Amphiphilic Siderophores Produced by the Marine Bacterium Halomonas LOB-5. Journal of Natural Products 72: 884-888.

Johnson, K. S. and others 1992. Manganese Flux from Continental Margin Sediments in a Transect Through the Oxygen Minimum. Science 257: 1242-1245.

Johnson, K. S., K. H. Coale, W. M. Berelson, and R. M. Gordon. 1996. On the formation of the manganese in the oxygen minimum. Geochim. Cosmochim. Acta 60: 12911299.

Johnson, K. S., R. M. Gordon, and K. H. Coale. 1997. What controls dissolved iron in the world ocean? Mar. Chem 57: 137-161.

Lam, P. J., and J. K. B. Bishop. 2008. The continental margin is a key source of iron to the HNLC North Pacific Ocean Geophys. Res. Lett. 35. doi:10.1029/2008GL033294

Lamborg, C. H. and others 2008. The flux of bio- and lithogenic material associated with sinking particles in the mesopelagic "twilight zone" of the northwest and North Central Pacific Ocean. Deep Sea Research Part II: Topical Studies in Oceanography 55: 1540-1563.

Landing, W. M., and K. W. Bruland. 1987. The contrasting biogeochemistry of iron and manganese in the Pacific Ocean. Geochimica et Cosmochimica Acta 51 : 29-43.

Lane, T. W., and F. M. M. Morel. 2000. Regulation of carbonic anhydrase expression by zinc, cobalt, and carbon dioxide in the marine diatom Thalassiosira weissflogii. Plant Physiol. 123: 1-8.

Martin, J. H., and G. A. Knauer. 1985. Lateral transport of Mn in the north-east Pacific Gyre oxygen minimum. Nature 314: 524-526. 
Martin, J. H., R. M. Gordon, S. Fitzwater, and W. W. Broenkow. 1989. VERTEX: phytoplankton/iron studies in the Gulf of Alaska. Deep-Sea Res. 36: 649-680.

Middag, R., H. J. W. De Baar, P. Laan, P. H. Cai, and J. C. Van Ooijen. Dissolved manganese in the Atlantic sector of the Southern Ocean. Deep Sea Research Part II: Topical Studies in Oceanography In press.

Moffett, J. W., and J. Ho. 1996. Oxidation of cobalt and manganese in seawater via a common microbially catalyzed pathway. Geochim. Cosmo. Acta 60: 3415-3424.

Moore, J. K., and O. Braucher. 2008. Sedimentary and mineral dust sources of dissolved iron to the World Ocean. Biogeosciences 4: 1279-1327.

Moore, J. K., S. C. Doney, and K. Lindsay. 2004. Upper ocean ecosystem dynamics and iron cycling in a global three-dimensional model. Global Biogeochem. Cycles 18: GB4028. doi:4010.1029/2004GB002220. .

Morel, F. M. M., A. J. Milligan, and M. A. Saito. 2003. Marine Bioinorganic Chemistry: The Role of Trace of Metals in the Oceanic Cycles of Major Nutrients, p. 113-143. In H. D. H. K.K. Turekian [ed.], Treatise on Geochemistry. Elsevier Science Ltd.

Noble, A. E., M. A. Saito, K. Maiti, and C. Benitez-Nelson. 2008. Cobalt, manganese, and iron near the Hawaiian Islands: A potential concentrating mechanism for cobalt within a cyclonic eddy and implications for the hybrid-type trace metals. Deep Sea Res II 55: 1473-1490.

Parker, D. L., T. Morita, M. L. Mozafarzadeh, R. Verity, J. K. McCarthy, and B. M. Tebo. 2007. Inter-relationships of $\mathrm{MnO} 2$ precipitation, siderophore-Mn(III) complex formation, siderophore degradation, and iron limitation in $\mathrm{Mn}(\mathrm{II})$-oxidizing bacterial cultures. Geochimica et Cosmochimica Acta 71: 5672-5683.

Partensky, F., W. R. Hess, and D. Vaulot. 1999. Prochlorococcus, a Marine Photosynthetic Prokaryote of Global Significance. Microb. Molec. Biology Rev. 63: 106-127.

Rue, E. L., and K. W. Bruland. 1995. Complexation of iron(III) by natural organic ligands in the Central North Pacific as determined by a new competitive ligand equilibration/adsorptive cathodic stripping voltammetric method. Marine Chemistry 50: 117-138.

Saito, M. A. and others. Iron conservation by reduction of metalloenzyme inventories in the marine diazotroph Crocosphaera watsonii. 2011. Proceedings of the National Academy of Sciences. doi/10.1073/pnas.1006943108.

Saito, M. A., and D. L. Schneider. 2006. Examination of precipitation chemistry and improvements in precision using the $\mathrm{Mg}(\mathrm{OH})_{2}$ preconcentration ICP-MS method for high-throughput analysis of open-ocean Fe and Mn in seawater. Anal. Chim. Acta 565: 222-233.

Saito, M. A., and J. W. Moffett. 2001. Complexation of cobalt by natural organic ligands in the Sargasso Sea as determined by a new high-sensitivity electrochemical cobalt speciation method suitable for open ocean work. Mar. Chem. 75: 49-68.

Saito, M. A., and T. J. Goepfert. 2008. Zinc-cobalt colimitation in Phaeocystis antarctica. Limnol. Oceanogr. 53: 266-275.

Saito, M. A., G. Rocap, and J. W. Moffett. 2005. Production of cobalt binding ligands in a Synechococcus feature at the Costa Rica Upwelling Dome. Limnol. Oceanogr. 50: 279-290. 
Saito, M. A., J. W. Moffett, and G. Ditullio. 2004. Cobalt and Nickel in the Peru Upwelling Region: a Major Flux of Cobalt Utilized as a Micronutrient. Global Biogeochem. Cycles 18: doi:10.1029/2003GB002216

Saito, M. A., and J. W. Moffett. 2002. Temporal and spatial variability of cobalt in the Atlantic Ocean. Geochim. Cosmochim. Acta 66: 1943-1953.

Saito, M. A., J. W. Moffett, S. W. Chisholm, and J. B. Waterbury. 2002. Cobalt limitation and uptake in Prochlorococcus. Limnol. Oceanogr. 47: 1629-1636.

Saito, M. A., T. J. Goepfert, and J. T. Ritt. 2008. Some thoughts on the concept of colimitation: three definitions and the importance of bioavailability. Limnol. Oceanogr. 53: 276-290.

Sedwick, P. N., G. M. McMurtry, and J. D. Macdougall. 1992. Chemistry of hydrothermal solutions from Pele's Vents, Loihi Seamount, Hawaii. Geochimica et Cosmochimica Acta 56: 3643-3667.

Sedwick, P. N. and others 2005. Iron in the Sargasso Sea (Bermuda Atlantic Time-series Study region) during summer: Eolian imprint, spatiotemporal variability, and ecological implications. Global Biogeochem. Cycles 19. doi:10.1029/2004GB002445

Shaked, Y., A. B. Kustka, and F. M. M. Morel. 2005. A general kinetic model for iron acquisition by eukaryotic phytoplankton. Limnol. Oceanogr. 50: 872-882.

Shelley, R. U., B. Zachhuber, P. N. Sedwick, P. J. Worsfold, and M. C. Lohan. 2010. Determination of total dissolved cobalt in UV-irradiated seawater using flow injection with chemiluminescence detection. Limnol. Oceanogr. Meth. 8: 352-362.

Sunda, W. G., and S. A. Huntsman. 1988. Effect of sunlight on redox cycles of manganese in the southwestern Sargasso Sea. Deep-Sea Research 35: 1297-1317.

Sunda, W. G., and S. A. Huntsman. 1995. Cobalt and Zinc interreplacement in marine phytoplankton: biological and geochemical implications. Limnol. Oceanogr. 40 : 1404-1417.

Taylor, S. R., and S. M. McLennan. 1985. The Continental Crust: its Composition and Evolution. Blackwell Scientific Publications.

Tebo, B., K. Nealson, S. Emerson, and L. Jacobs. 1984. Microbial meditation of Mn(II) and $\mathrm{Co}(\mathrm{II})$ precipitation at the $\mathrm{O}_{2} / \mathrm{H}_{2}$ interfaces in two anoxic fjords. Limnol. Oceanogr. 29: 1247-1258.

Tebo, B. 1991. Manganese(II) oxidation in the suboxic zone of the Black Sea. Deep-Sea Res. A 38 (2) S883-S905.

Tebo, B. M. and others 2004. BIOGENIC MANGANESE OXIDES: Properties and Mechanisms of Formation. Annual Review of Earth and Planet. Sci. 32 : 287-328.

Thuróczy, C.-E., M. Boye, and R. Losno. 2010. Dissolution of atmospheric cobalt and zinc in seawater. Biogeosci. 7. doi:10.5194/bg-7-1927-2010

$\mathrm{Wu}$, J., and E. A. Boyle. 2002. Iron in the Sargasso Sea: Implications for the processes controlling dissolved Fe distribution in the ocean. Global Biogeochem. Cycles 16: 331-337.

Yucel, M., A. Gartman, C. S. Chan, and G. W. Luther. Hydrothermal vents as a kinetically stable source of iron-sulphide-bearing nanoparticles to the ocean. Nature Geosci 4: In press. 


\title{
Chapter 2: Cobalt, Manganese, and iron near the Hawaiian Islands: A potential concentrating mechanism for cobalt within a cyclonic eddy and implications for the hybrid-type metals
}

Reprinted with permission of Deep-Sea Research II

Noble, A. E., M. A. Saito, K. Maiti, and C. Benitez-Nelson. 2008. Cobalt, manganese, and iron near the Hawaiian Islands: A potential concentrating mechanism for cobalt within a cyclonic eddy and implications for the hybrid-type trace metals. Deep Sea Res II 55: 1473-1490.

\begin{abstract}
The vertical distributions of cobalt, iron, and manganese in the water column were studied during the E-Flux Program (E-Flux II and III), which focused on the biogeochemistry of cold-core cyclonic eddies that form in the lee of the Hawaiian Islands. During E-Flux II (January 2005) and E-Flux III (March 2005), 17 stations were sampled for cobalt $(n=147)$, all of which demonstrated nutrient-like depletion in surface waters. During E-Flux III, two depth profiles collected from within mesoscale cold-core eddy, Cyclone Opal, revealed small distinct maxima in cobalt at $\sim 100 \mathrm{~m}$ depth and a larger inventory of cobalt within the eddy. We hypothesize that this was due to a cobalt concentrating effect within the eddy, where upwelled cobalt was subsequently associated with sinking particulate organic carbon (POC) via biological activity and was released at a depth coincident with nearly complete POC remineralization [Benitez-Nelson, C., Bidigare, R.R., Dickey, T.D., Landry, M.R., Leonard, C.L., Brown, S.L., Nencioli, F., Rii, Y.M., Maiti, K., Becker, J.W., Bibby, T.S., Black, W., Cai, W.J., Carlson, C.A. Chen, F., Kuwahara, V.S., Mahaffey, C., McAndrew, P.M., Quay, P.D., Rappe, M.S., Selph, K.E., Simmons, M.P., Yang, E.J., 2007. Mesoscale eddies drive increased silica export in the subtropical Pacific Ocean. Science $316,1017-1020]$. There is also evidence for the formation of a correlation between cobalt and soluble reactive phosphorus during E-Flux III relative to the E-Flux II cruise that we suggest is due to increased productivity, implying a minimum threshold of primary production below which cobalt-phosphate coupling does not occur. Dissolved iron was measured in E-Flux II and found in somewhat elevated concentrations $(\sim 0.5 \mathrm{nM})$ in surface waters relative to the iron depleted waters of the surrounding Pacific [Fitzwater, S.E., Coale, K.H., Gordon, M.R., Johnson, K.S., Ondrusek, M.E., 1996. Iron deficiency and phytoplankton growth in the equatorial Pacific. Deep-Sea Research II 43 (4-6), 995-1015], possibly due to island effects associated with the iron-rich volcanic soil from the Hawaiian Islands and/or anthropogenic inputs. Distinct depth maxima in total dissolved cobalt were observed at 400-600 m depth, suggestive of the release of metals from the shelf area of comparable depth that surrounds these islands.
\end{abstract}




\title{
Cobalt, manganese, and iron near the Hawaiian Islands: A potential concentrating mechanism for cobalt within a cyclonic eddy and implications for the hybrid-type trace metals
}

\author{
Abigail E. Noble a,b,1, Mak A. Saito ${ }^{\mathrm{a}, *, 1}$, Kanchan Maiti $^{\mathrm{c}}$, Claudia R. Benitez-Nelson ${ }^{\mathrm{c}}$ \\ a Marine Chemistry and Geochemistry Department, Woods Hole Oceanographic Institution, 266 Woods Hole Road, MS\#51, Woods Hole, MA 02543, USA \\ ${ }^{\mathrm{b}}$ MIT-WHOI Joint Program in Chemical Oceanography, Massachusetts Institute of Technology, 77 Massachusetts Avenue, Cambridge, MA 02139, USA \\ c Department of Geological Sciences, University of South Carolina, Columbia, SC 29208, USA
}

\section{A R T I C L E I N F O}

\section{Article history:}

Accepted 6 February 2008

Available online 22 May 2008

Keywords:

Hybrid-type metals

Cobalt

Iron

Manganese

Trace metal biogeochemistry

Lee eddies

\begin{abstract}
A B S T R A C T
The vertical distributions of cobalt, iron, and manganese in the water column were studied during the E-Flux Program (E-Flux II and III), which focused on the biogeochemistry of cold-core cyclonic eddies that form in the lee of the Hawaiian Islands. During E-Flux II (January 2005) and E-Flux III (March 2005), 17 stations were sampled for cobalt $(n=147)$, all of which demonstrated nutrient-like depletion in surface waters. During E-Flux III, two depth profiles collected from within a mesoscale cold-core eddy, Cyclone Opal, revealed small distinct maxima in cobalt at $\sim 100 \mathrm{~m}$ depth and a larger inventory of cobalt within the eddy. We hypothesize that this was due to a cobalt concentrating effect within the eddy, where upwelled cobalt was subsequently associated with sinking particulate organic carbon (POC) via biological activity and was released at a depth coincident with nearly complete POC remineralization [Benitez-Nelson, C., Bidigare, R.R., Dickey, T.D., Landry, M.R., Leonard, C.L., Brown, S.L., Nencioli, F., Rii, Y.M., Maiti, K., Becker, J.W., Bibby, T.S., Black, W., Cai, W.J., Carlson, C.A., Chen, F., Kuwahara, V.S., Mahaffey, C., McAndrew, P.M., Quay, P.D., Rappe, M.S., Selph, K.E., Simmons, M.P., Yang, E.J., 2007. Mesoscale eddies drive increased silica export in the subtropical Pacific Ocean. Science 316, 1017-1020]. There is also evidence for the formation of a correlation between cobalt and soluble reactive phosphorus during E-Flux III relative to the E-Flux II cruise that we suggest is due to increased productivity, implying a minimum threshold of primary production below which cobalt-phosphate coupling does not occur. Dissolved iron was measured in E-Flux II and found in somewhat elevated concentrations $(\sim 0.5 \mathrm{nM})$ in surface waters relative to the iron depleted waters of the surrounding Pacific [Fitzwater, S.E., Coale, K.H., Gordon, M.R., Johnson, K.S., Ondrusek, M.E., 1996. Iron deficiency and phytoplankton growth in the equatorial Pacific. Deep-Sea Research II 43 (4-6), 995-1015], possibly due to island effects associated with the iron-rich volcanic soil from the Hawaiian Islands and/or anthropogenic inputs. Distinct depth maxima in total dissolved cobalt were observed at $400-600 \mathrm{~m}$ depth, suggestive of the release of metals from the shelf area of comparable depth that surrounds these islands.
\end{abstract}

(c) 2008 Elsevier Ltd. All rights reserved.

\section{Introduction}

In recent years, the importance of bioactive trace elements for marine phytoplankton nutrition has become evident. In particular, iron is now believed to limit primary productivity in major regions of the oceans, and other bioactive trace elements are known to be important micronutrients (Martin et al., 1991; Sunda and Huntsman, 1995; Saito et al., 2002, 2004; Morel et al., 2003; and references therein, Bruland et al., 2005). The processes that control the distributions of trace elements in seawater have been generally outlined in recent years (e.g., Edmond et al., 1979; Martin et al., 1991; Johnson et al., 1997; Gordon et al., 1998; Boyle

\footnotetext{
* Corresponding author. Tel.: +1508 2892393; fax: +1508 4572193 .

E-mail address: mak@whoi.edu (M.A. Saito).

${ }^{1}$ Co-authors.
}

0967-0645/\$ - see front matter (c) 2008 Elsevier Ltd. All rights reserved. doi:10.1016/j.dsr2.2008.02.010 et al., 2005), but much of the specific nature of what controls their distributions remains to be understood. In particular, the hybridtype trace metals, which are simultaneously influenced by both micronutrient and scavenging processes, can show significant variability in seawater (Bruland and Lohan, 2004, and references therein). There is much to be learned with regard to how these competing processes influence the distributions of hybrid-type metals like iron and cobalt, and how that in turn interacts with marine ecosystem processes.

\subsection{Potential influences on trace metal distributions among the Hawaiian Islands}

There are a number of potential controls on trace meta distribution in the marine water column, including: (1) aeolian deposition, (2) hydrothermal inputs, (3) riverine inputs, (4) 
exports to and imports from sediments, (5) horizontal advection, and (6) loss by export of metals associated with organic and mineral particles (Sedwick et al., 1992; Wong et al., 1995; DeCarlo et al., 2004, 2005; Saito et al., 2004; Boyle et al., 2005; Bruland et al., 2005; Sedwick et al., 2005). Recent studies in the vicinity of the Hawaiian Islands have found evidence that all of these processes are influencing trace metal distributions. Temporal variability in surface iron at station ALOHA $\left(22^{\circ} 45^{\prime} \mathrm{N}, 158^{\circ} 00^{\prime} \mathrm{W}\right)$, located north of the Hawaiian Islands in the Subtropical North Pacific Gyre, has been attributed to both aeolian input and biological uptake (Boyle et al., 2005). A study by Brown et al (2005) revealed highly variable surface dissolved iron concentrations from a transect across the North Pacific Subtrobical Gyre that ended at station ALOHA. While surface concentrations near the Hawaiian Islands were notably higher than those at more open-ocean stations within the gyre, deep concentrations were comparable, and variability was argued to be due to both aeolian deposition and fluvial input (Brown et al., 2005).

Hydrothermal studies in this area have demonstrated that seamounts and vents provide input of metals to deep waters Dissolved ferrous iron concentrations reached almost $1 \mathrm{mmol} \mathrm{kg}$ in the fluids of Pele's Vents, an area of the Loihi Seamount, and the plume from Loihi is believed to influence iron concentrations at depth at station ALOHA based on high $\delta^{3} \mathrm{He}$ values indicative of a hydrothermal signal (Sedwick et al., 1992; Boyle et al., 2005). Four years after this vent study, a volcanic event destroyed the sampling area and gave way to several new venting sites. Later studies conducted in this area by a remote sampler observed similar high Fe concentrations (Wheat et al., 2000).

Rivers have been identified as a source of trace metals to Hawaiian watersheds and surrounding waters. Two studies, conducted by DeCarlo et al. (2004, 2005), examined concentrations of lead, zinc, copper, barium, cobalt, arsenic, nickel, vanadium, and chromium in streambeds on the island of Oahu. They found that most metals are supplied to the ocean via the particulate fraction during storm events, and that the dissolved fraction comprises only $1 \%$ of the total metal contribution (DeCarlo et al., 2004, 2005). The dissolved fraction of metals showed a combination of sources including anthropogenic contributions from urban areas (vehicular traffic and industria operations) and agriculture (fertilizers) (DeCarlo et al., 2004). Finally, release from shelf sediments around the islands is also likely source of metals as the islands of Maui, Kahoolawe, Molokai, and Lana'i are surrounded by an expansive shelf ( $\sim 500 \mathrm{~m}$ depth; Fig. 1B) comprised of mafic and felsic minerals (Chase et al., 1980).

\subsection{Influences on cobalt, manganese, and iron marine} biogeochemistry

Although trace elements are now known to be essential components for biology, there are still relatively few studies of trace element concentrations in marine seawater environments. As such, we have only a cursory knowledge of what controls their distribution with depth in the water column. A brief summary of the biogeochemistry of cobalt, manganese, and iron is presented here.

Cobalt is now believed to exhibit micronutrient behavior (Saito and Moffett, 2002; Saito et al., 2004) as well as characteristics associated with scavenged elements in the water column (Johnson et al., 1988). This combined imprint of biological and scavenging processes on the vertical profile is characteristic of hybrid-type metals (Bruland and Lohan, 2004). Nutrient-like depletion of cobalt has been observed in the upper water column of several environments including the Sargasso Sea, North Pacific, Costa Rica upwelling dome, and Peru upwelling region (Martin et al., 1989; Saito and Moffett, 2002; Saito et al., 2004, 2005). While cobalt generally behaves similarly to iron in the upper water column, it has been observed to be co-oxidized by manganese oxidizing bacteria (Tebo et al., 1984; Moffett and Ho, 1996). A manganese oxidizing marine Bacillus was demonstrated to oxidize cobalt in the absence of manganese, leading to further work that suggests manganese and cobalt may be oxidized via the same biological pathway (Tebo et al., 1984; Moffett and Ho, 1996). Competitive inhibition experiments confirm this theory, though it was determined that these processes occur primarily in coastal environments such as Waquoit Bay, MA, and not in the euphotic zone of the oligotrophic Sargasso Sea where biological uptake by phytoplankton dominates cobalt distribution (Moffett and Ho, 1996).

Iron also behaves as a hybrid-type metal. An earlier synthesis of data noted that surface concentrations of dissolved iron are generally below $0.2 \mathrm{nM}$ with an average deep concentration of $0.76 \mathrm{nM}$ in the open ocean (Johnson et al., 1997). However significant variability has been observed in depth profile shape and absolute concentration. For example, Sedwick et al. (2005) observed high aerosol deposition concurrent with dissolved iron concentrations reaching $1.8 \mathrm{nM}$ within a cyclonic eddy in the Sargasso Sea. These findings confirm that iron distribution in surface waters should be significantly affected by dust deposition, primary productivity, and transport processes such as advection and mixing (Sedwick et al., 2005; Boyle et al., 2005). As these factors are all known to change seasonally, there is the potential for spatial and temporal variability in dissolved iron concentrations.

Manganese is considered a scavenged element, showing elevated concentrations at the surface due to aeolian deposition and near surface redox cycling including reductive dissolution of manganese oxides (Sunda and Huntsman, 1985, 1988). Rapid depletion with depth is due to sorption to sinking particles and/or oxidation by manganese-oxidizing bacteria (Emerson et al., 1982; Tebo et al., 1984). Secondary maxima in manganese found at intermediate depths are likely due to redox reactions occurring near oxygen minimum zones (Johnson et al., 1996). Diffusion out of sediments in regions of low oxygen bottom water also can be a source of manganese to deep water (Thamdrup et al., 1994).

\subsection{Mesoscale eddies near the Hawaiian Islands}

Mesoscale eddies are ubiquitous features in the world's oceans and are hypothesized to play an essential role in processes ranging from heat transport to upper ocean ecology and biogeochemistry (e.g., Cheney and Richardson, 1976; McGillicuddy et al., 1998; Oschlies and Garcon, 1998; Fischer et al., 2002; Sakamoto et al., 2004). Here, we focus on the role of eddies as a mechanism by which nutrient-enriched deep waters are upwelled into lit surface waters, thereby increasing biological community production and potentially enhancing particle export to deeper waters (e.g., Falkowski et al., 1991; McGillicuddy et al., 1998, 2007; BenitezNelson et al., 2007). Mesoscale eddies are produced by a variety of physical interactions, such as wind and baroclinic instabilities, but the inability to routinely predict eddy formation has made the study of these mesoscale features logistically difficult.

Wind-induced mesoscale eddies regularly occur off the leeward shores of the Hawaiian Islands throughout the year, increasing in frequency during periods of high trade wind intensity (OctoberMarch) (Patzert, 1969; Lumpkin, 1998; Chavanne et al., 2002; Dickey et al., 2008). These northeasterly winds are funneled through the volcanic peaks of the Hawaiian Islands, mainly Maui and Hawaii, causing divergent and convergent flow patterns at the surface that induce localized upwelling (cold-core cyclonic) and downwelling (warm-core anticyclonic) mesoscale eddies. The eddies tend to be of significant size ( $\sim 180 \mathrm{~km}$ in diameter) and have a typical life-span of 3-8 months (Patzert, 1969; Lumpkin, 1998; Chavanne et al., 2002; 


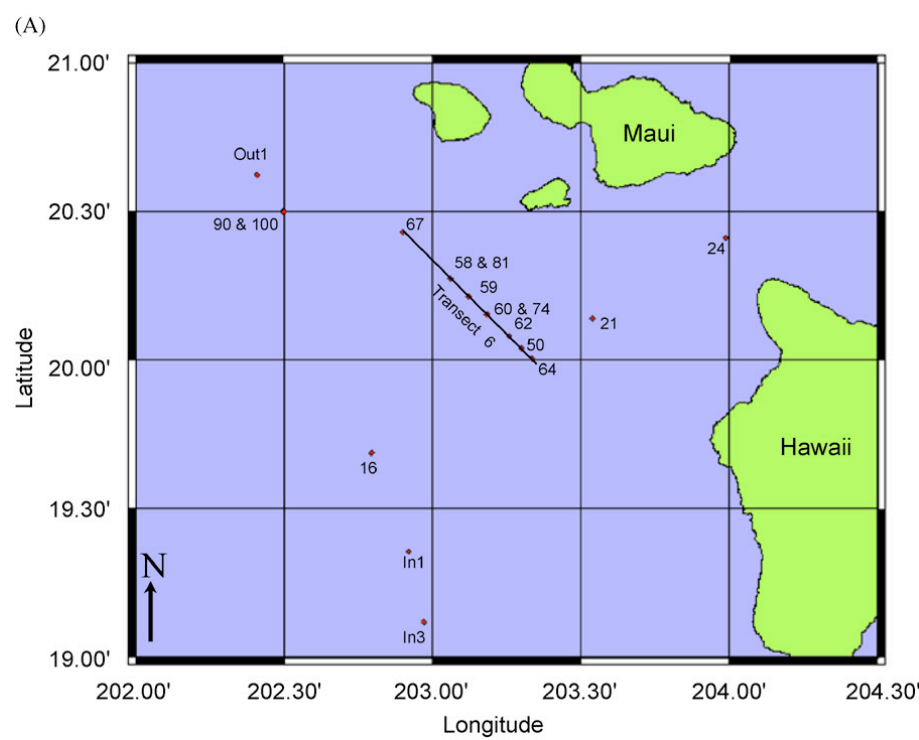

(B)

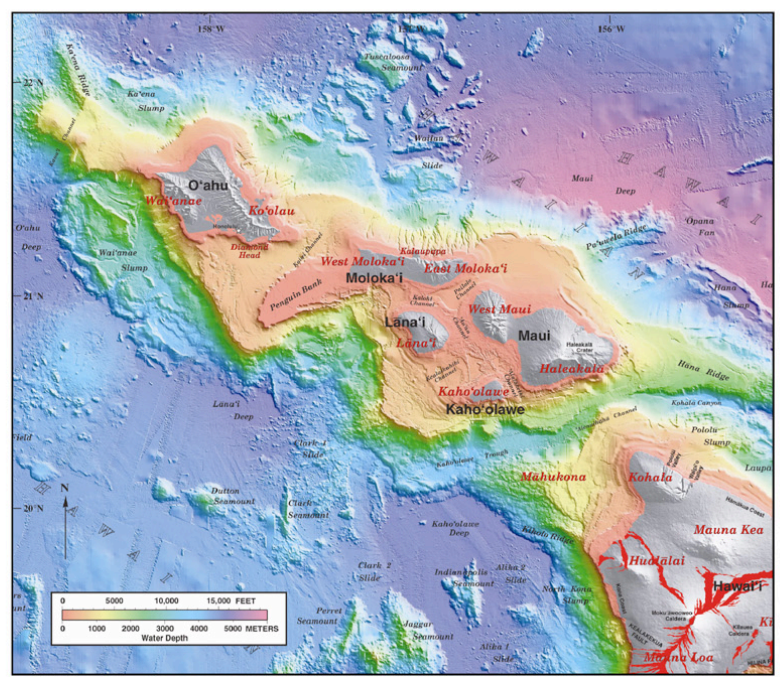

Fig. 1. (A) Sampling area for E-Flux II in January 2005 and E-Flux III in March 2005. (B) Bathymetry around the Hawaiian Islands. There is a prominent shelf at $\sim 500 \mathrm{~m}$ surrounding the islands in proximity to the study site (modified from USGS; Eakins et al., 2003).

Calil et al., 2008). A number of studies have demonstrated that Hawaiian lee eddies are highly productive features, resulting in large increases in primary production, plankton biomass, and even larger organisms, such as the Pacific Blue Marlin (Falkowski et al., 1991; Olaizola et al., 1993; Allen et al., 1996; Seki et al., 2001, 2002; Bidigare et al., 2003; Vaillancourt et al., 2003; Benitez-Nelson et al., 2007; Brown et al., 2008, Rii et al., 2008). Lumpkin (1998) determined that on average, nine cyclonic eddies occur each year in the lee of the Hawaiian Islands, with one eddy per month generated during the winter from 1993 to 1995. Their regular formation therefore provides an ideal natural laboratory for investigating eddy-enhanced biological production and carbon export in an accessible subtropical oligotrophic setting.

\subsection{The influence of eddies on trace metal biogeochemistry}

The logistical difficulties in locating eddies in combination with the potential for contamination of trace metal samples without specialized equipment aboard has led to relatively few studies on the influence of eddies on trace metal biogeochemistry. Macronutrient upwelling has been invoked as an important influence on primary productivity in the mesoscale eddies found in the Sargasso Sea (McGillicuddy et al., 1998). Cobalt depletion has been observed in the photic zone of the same region and is hypothesized to be due to the enhanced productivity associated with eddy-induced upwelling (Saito and Moffett, 2002). A time-series analysis of cobalt over 1 year showed sharp depletions of cobalt concurrent 
with decreases in water temperature at $\sim 44 \mathrm{~m}$, consistent with the passing of cyclonic mesoscale eddies over the region and/or mixing events. Because hybrid-type trace elements are not greatly enriched in deep waters relative to surface waters as macronutrients are, upwelling cannot resupply the euphotic zone with these hybrid-type elements to the same extent as it can with macronutrients. Thus, enhanced productivity and export flux could explain the depletion of the small reservoir of cobalt observed with mixing and/or upwelling events (Saito and Moffett, 2002).

The anticyclonic eddies off the western coast of Canada have lifespans of more than a year, and were recently examined for iron biogeochemistry (Johnson et al., 2005). These Haida eddies typically form in the high-nutrient low-chlorophyll (HNLC) region of the Gulf of Alaska, and Haida-2000 was found to entrain a large amount of iron as it moved westward from the coastal region. Total dissolved iron reached $5 \mathrm{nM}$ at $25 \mathrm{~m}$ depth and $3 \mathrm{nM}$ at $100 \mathrm{~m}$ depth during eddy formation, and was found to be $1.5-2$ times higher than background concentrations even 16 months later (Johnson et al., 2005). These iron concentrations are $\sim 3$ orders of magnitude above what is generally found in this HNLC area (less than $0.05 \mathrm{nM}$ ). In contrast, the downwelling and isolating effects of the anticyclonic eddy Haida-2000 resulted in rapid macronutrient utilization and depletion within the eddy (Peterson et al., 2005).

Here, we present detailed analyses of cobalt, manganese and iron from the E-Flux sampling program to investigate trace metal dynamics in the presence and absence of a cyclonic eddy in the lee of the island of Hawaii. Mechanisms that may control the distribution of these trace metals are discussed as well as implications regarding their control in oligotrophic waters. Three themes are presented: (1) a potential concentrating mechanism for cobalt within cyclonic eddies, (2) the possible existence of a minimum biological productivity threshold for the formation of a cobalt and soluble reactive phosphorus correlation, and (3) the influence of island effects on trace metal concentrations in this region.

\section{Methods}

\subsection{Sampling techniques}

During the E-Flux II cruise (January 10-27, 2005), a full tracemetal sampling program was employed in the vicinity of the Hawaiian Islands within a grid of $-157.5^{\circ} \mathrm{W}$ to $-156.0^{\circ} \mathrm{W}$ and $19.7^{\circ} \mathrm{N}$ to $20.5^{\circ} \mathrm{N}$. Acid-cleaned, teflon-coated Go-Flo sampling bottles of 10 and $2.5 \mathrm{~L}$ capacity (General Oceanics Inc.) were deployed on Kevlar wire aboard the R/V Wecoma at 14 stations. The 10 - $\mathrm{L}$ bottles were tripped with epoxy-coated lead messengers and reported sampling depths were based on the wire payout. The 2.5-L bottles were deployed on an epoxy-coated Go-Flo rosette and programmed to trip sequentially using a timer-controlled mechanism. Exact sampling depths for the trace metal rosette were recorded by a pressure sensor attached to the rosette.

During the E-Flux III cruise (March 10-28, 2005), a smaller set of trace metal samples were collected from the CTD rosette aboard the R/V Wecoma. The CTD utilized 10-L X-Niskin sampling bottles on a Seabird Rosette. During this cruise, only unfiltered trace metal samples were collected because our trace-metal sampling program was not aboard. Samples were collected in trace metal cleaned bottles (see below) directly from the CTD while wearing clean gloves and minimizing time that each bottle was open. The $\mathrm{X}$-Niskin bottles on the rosette were equipped with silicone rubber O-rings. The samples were subsampled for cobalt, then acidified upon return to the laboratory in a cleanroom environment. It should be pointed out that 10 -L X-Niskins, like those found on the R/V Wecoma CTD, also are used by some laboratories for trace metal sampling on Kevlar line, suggesting those bottles may not necessarily be contaminated. However, the use of a metal conducting hydrowire and lack of HEPA filtered-air environment for sampling are potential sources of contamination in our E-Flux III samples. The fact that the samples appear to be uncontaminated could be related to the high frequency of CTD casts during E-Flux III ( $>100$ CTD casts), and the continual seawater rinsing of the rosette and hydrowire associated with each deployment. Due to the high iron content typically found in natural particulate material relative to dissolved iron and the lack of filtration of these samples, iron data for E-Flux III are not reported.

All sample bottles for storing seawater until analysis were soaked overnight in the acidic detergent Citranox, rinsed thoroughly with Milli-Q water (Millipore), filled with $10 \% \mathrm{HCl}$ to soak for 10 days, and rinsed thoroughly with Milli-Q water adjusted to $\mathrm{pH} 2$ prior to use. Immediately following collection during E-Flux II, Go-Flo bottles were pressurized with $99.999 \% \mathrm{~N}_{2}$ at $\sim 5 \mathrm{psi}$, and seawater was filtered through teflon tubing and a $147-\mathrm{mm}, 0.4-\mu \mathrm{m}$ polycarbonate sandwich filter into acid washed polyethylene and teflon sample bottles in a positive pressure class-100 clean environment. All tubing and filters were detergent and acid-soaked prior to use. During E-Flux II, samples intended for total dissolved cobalt and cobalt speciation measurements were refrigerated in the dark until analysis and were not acidified. Samples intended for dissolved Fe and Mn analyses were acidified to $\mathrm{pH} 1.7$ with high purity $\mathrm{HCl}$ (Seastar Inc.) and stored at room temperature.

Samples from E-Flux III were not filtered. Manganese analyses from those samples are described as total dissolvable manganese following previously reported sample collection techniques (samples were acidified to $<\mathrm{pH} 2$ and allowed to sit for several months prior to analysis, similar to Sedwick et al., 2005). Cobalt analyses from those samples are described as total cobalt because the particulate fraction in seawater is negligible and samples were UV irradiated and not acidified. E-Flux II samples, however, were filtered and iron and manganese are described as "dissolved" concentrations following previously reported sample collection techniques (filtered samples were also acidified to $<\mathrm{pH} 2$ and allowed to sit for several months prior to analysis, similar to Sedwick et al., 2005) and cobalt concentrations are described as total dissolved cobalt and labile cobalt following previously reported data using identical sampling and processing methods (Saito and Moffett, 2001, 2002; Saito et al., 2002, 2004, 2005).

During E-Flux II, Go-Flo sampling bottles on the rosette occasionally tripped at incorrect depths. To determine when this occurred, filtered nutrient samples collected from all GoFlo bottles were compared with nutrient sample data from corresponding CTD rosette casts. When nutrient values from GoFlo bottles were not oceanographically consistent with the CTD nutrient data, the trace metal sample was not used. In addition, triplicate trace metal measurements were run on samples that did not produce oceanographically consistent results to assay for processing contamination. If subsequent repeated analyses revealed a lower, more reasonable concentration, the lower number was used, assuming that the previous sample aliquot had been contaminated during analysis preparation.

\subsection{Cobalt analyses}

Concentrations of total, total dissolved, and labile cobalt were determined using a previously described cathodic stripping voltammetry (CSV) method (Saito and Moffett, 2001) both during the cruise and immediately upon returning to the laboratory. Measurements were made using an Eco-Chemie $\mu$ Autolab system connected to a Metrohm 663 VA Stand equipped with a hanging mercury drop electrode and a teflon sampling cup. 
For total and total dissolved cobalt analyses, samples were UVirradiated for $1 \mathrm{~h}$ prior to analysis using a Metrohm UV digester to degrade the organic ligands that bind the cobalt and allow complete binding by the added electroactive cobalt ligand dimethylglyoxime. Samples were analyzed in $8.5-\mathrm{mL}$ aliquots with the addition of $30 \mu \mathrm{L}$ recrystalized dimethylglyoxime (DMG, $0.1 \mathrm{M}$ in methanol), $1.5 \mathrm{~mL}$ purified nitrite (1.5 M in Milli-Q water), and $50 \mu \mathrm{L}$ purified EPPS buffer ( $0.5 \mathrm{M}$ in Milli-Q water). Reagent purification protocols were identical to those previously published (Saito and Moffett, 2001). Analysis began with a $180 \mathrm{~s}$ purge with $99.999 \% \mathrm{~N}_{2}$. Each sample was conditioned at $-0.6 \mathrm{~V}$ for $90 \mathrm{~s}$ at a stir-rate of $2500 \mathrm{rpm}$ followed by a $10 \mathrm{~s}$ equilibration step and a linear sweep from -0.6 to $-1.4 \mathrm{~V}$ at a rate of $10 \mathrm{~V} \mathrm{~s}^{-1}$ (Saito and Moffett, 2001). Cobalt concentrations were determined by the standard additions technique, with initial concentrations measured in triplicate followed by four 25-pM additions. The analytical blank was determined by analyzing seawater that had been UV-irradiated for $1 \mathrm{~h}$, equilibrated overnight with prepared Chelex ${ }^{\mathbb{R}} 100$ resin beads (Bio-Rad), and UV-irradiated a second time to degrade any leached synthetic ligands (Saito and Moffett, 2001). Blanks were run at the beginning and end of the sample analyses, averaged, and used to adjust reported concentrations. Averaged blank concentrations for this dataset were $3.4 \pm 2.0 \mathrm{pM}$ $(n=5)$ and $5.6 \pm 1.4 \mathrm{pM}(n=8)$ depending on the reagent batch used. Two suites of reagents were used during the analysis process, and the reagent batch was noted and the concentrations were adjusted according to the blank calculated for that suite of reagents. Selected samples were run in triplicate to verify their concentrations. The precision of this method is excellent. For example, mean and standard deviation of triplicate analyses of a near surface water sample gave a concentration of $32 \pm 0.7 \mathrm{pM}$

For labile cobalt analyses, $8.5 \mathrm{~mL}$ of sample were pipetted into an acid-washed teflon vial that was preconditioned with a small aliquot of the sample water. Thirty microliters of DMG were added to the vial and allowed to equilibrate overnight in the dark prior to analysis (Saito et al., 2004). Analyses were then performed as described for total concentrations with the addition of the remaining two reagents and use of the standard addition technique. Previously, we determined that natural cobalt ligands in seawater have a conditional stability constant of $>10^{16.8}$ (Saito et al., 2005). This suggests that the cobalt is bound very tightly to the cobalt ligands. Therefore we can define labile cobalt as the fraction of total dissolved cobalt that is either bound to weak organic and inorganic ligands in seawater or present as free $\mathrm{Co}(\mathrm{II})$, and is thus exchangeable with the complexing agent (DMG) used for analysis (Saito et al., 2004, 2005). The difference between the total dissolved cobalt and the labile cobalt can then be used as an estimation of the strong cobalt ligand concentration. All samples measured for labile cobalt were filtered. Analytical blanks fo labile measurements were calculated by the same method used for total and total dissolved measurements.

\subsection{Iron and manganese analyses}

Total dissolvable and dissolved iron and manganese were measured using inductively coupled plasma mass spectrometry (ICP-MS) as described in detail (Saito and Schneider, 2006). Briefly, 13.0-mL aliquots of acidified seawater were weighed into acidleached polypropylene centrifuge tubes, to which $100 \mu \mathrm{L}$ of an ${ }^{57} \mathrm{Fe}$ solution was added and allowed to equilibrate overnight. Concentrated ammonia (Seastar) was added to induce $\mathrm{Mg}(\mathrm{OH})_{2}$ and trace metal co-precipitation into a pellet. This was accomplished by allowing a precipitate to form for $3 \mathrm{~min}$, followed by centrifugation for $3 \mathrm{~min}$ at $3000 \mathrm{rpm}(1460 \times \mathrm{g})$ using an Eppendorf Centrifuge 5810R. The supernatant was decanted, the sample was centrifuged a second time to further remove residual seawater, and the pellet was re-dissolved in $500 \mu \mathrm{L}$ of $5 \%$ nitric acid (Seastar) with trace amounts of indium. The $5 \%$ nitric acid resuspension solution was used as a blank, and signal suppression due to matrix effects was adjusted for by using a ratio of the indium signals in the blank and sample to calibrate the effective blank of each isotope for each sample. All samples were run in duplicate. SAFe seawater intercalibration standards were analyzed for iron with the surface (S1) and deep samples (D2) yielding values of 0.10 and $0.86 \mathrm{nM}$ respectively, which are within the reported ranges of $0.097 \pm 0.043$ and $0.91 \pm 0.17 \mathrm{nM}$ (Johnson et al., 2007).

\section{4. ${ }^{234}$ Th analyses}

Profiles of total ${ }^{234} \mathrm{Th}$ were collected from the center of Cyclone Opal (E-Flux III) from six separate casts using a CTD rosette with 10-L X-Niskin sampling bottles. Sample depths ranged from 0 to $400 \mathrm{~m}$ at each IN station. ${ }^{234} \mathrm{Th}$ samples were processed using the 4-L manganese oxide co-precipitation technique described in detail by Pike et al. (2005) and Rutgers van der Loeff et al. (2006). ${ }^{234} \mathrm{Th}$ samples were counted directly on a 5-sample gas-flow proportional low-level RIS $\emptyset$ beta counter for at least $12 \mathrm{~h}$ or until counting errors were $<3 \%$. Samples were then recounted after $>150$ days ( $\sim 6$ halflives) since collection to precisely determine background count rates, which averaged $0.51 \pm 0.05 \mathrm{cpm}$. After background recounting, total ${ }^{234} \mathrm{Th}$ samples were purified using ion exchange chemistry. Recovery of the added ${ }^{230} \mathrm{Th}$ yield monitor was quantified by inductively coupled plasma-mass spectroscopy with addition of a ${ }^{229}$ Th internal standard (Pike et al., 2005; Rutgers van der Loeff et al., 2006). Corrections were applied to ${ }^{234} \mathrm{Th}$ activities based on the ${ }^{230}$ Th recovery for each sample, which averaged $0.89 \pm 0.06$. All data are decay corrected to the time of collection and reported with a propagated error that includes uncertainties associated with sampling uncertainty, counting, and other calibration errors. Water column activity of the parent ${ }^{238} \mathrm{U}$ was calculated from salinity data $\left({ }^{238} \mathrm{Udpm} \mathrm{L}^{-1}=0.0686 \times\right.$ salinity; Chen et al., 1986).

\section{Results and discussion}

\subsection{Oceanographic properties}

In 2004-2005, the E-Flux program conducted three cruises to study eddies that form regularly in the lee of the Hawaiian Islands. We present trace metal data from the second and third of these cruises, focusing on data collected from E-Flux II (W0501B), and three unfiltered vertical profiles from E-Flux III (W0503B). E-Flux III sampled a large mesoscale eddy, Cyclone Opal, while E-Flux Il focused on the eddy generation region, but had no clear eddy signals. Shallow stations were sampled to $\sim 400 \mathrm{~m}$, while the stations discussed in detail here (E-Flux II: 50, 81 and E-Flux III: In 1 , In 3, Out 1) were all sampled to between 1200 and $1500 \mathrm{~m}$. Station locations and regional bathymetry are shown in Fig. $1 \mathrm{~A}$ and B. A total of 112 cobalt samples were analyzed for total dissolved cobalt and labile cobalt from E-Flux II with selected dissolved iron and dissolved manganese measurements, and three depth profiles were analyzed for total cobalt and total dissolvable manganese collected from E-Flux III. The chemistry of this area is characterized by oligotrophic waters uniquely affected by both island effects and hydrothermal activity that are likely to contribute to trace metal concentrations (Chase et al., 1980; Boyle et al., 2005).

3.2. General upper water column metal concentrations among the Hawaiian Islands

Total dissolved cobalt, dissolved iron, and dissolved manganese were measured throughout the water column during E-Flux II 
with a focus on cobalt biogeochemistry. A compilation of all cobalt samples demonstrates depletion in surface waters $(\sim 0-200 \mathrm{~m})$ likely due to biological utilization in the vicinity of the Hawaiian Islands along Transect 6 (Table 1; Fig. 2A and B). Cobalt gradually increases with depth in all profiles until $400-600 \mathrm{~m}$, consistent with our previous reports of a nutrient-like profile for this element (Saito and Moffett, 2001; Saito et al., 2002, 2004, 2005).

Surface-water cobalt appears to have significant temporal variability. During the E-Flux II cruise there were a few station locations that were revisited (Fig. $1 \mathrm{~A}$ ), including: $20.5^{\circ} \mathrm{N}$,

Table 1

Collected data from E-Flux II and III

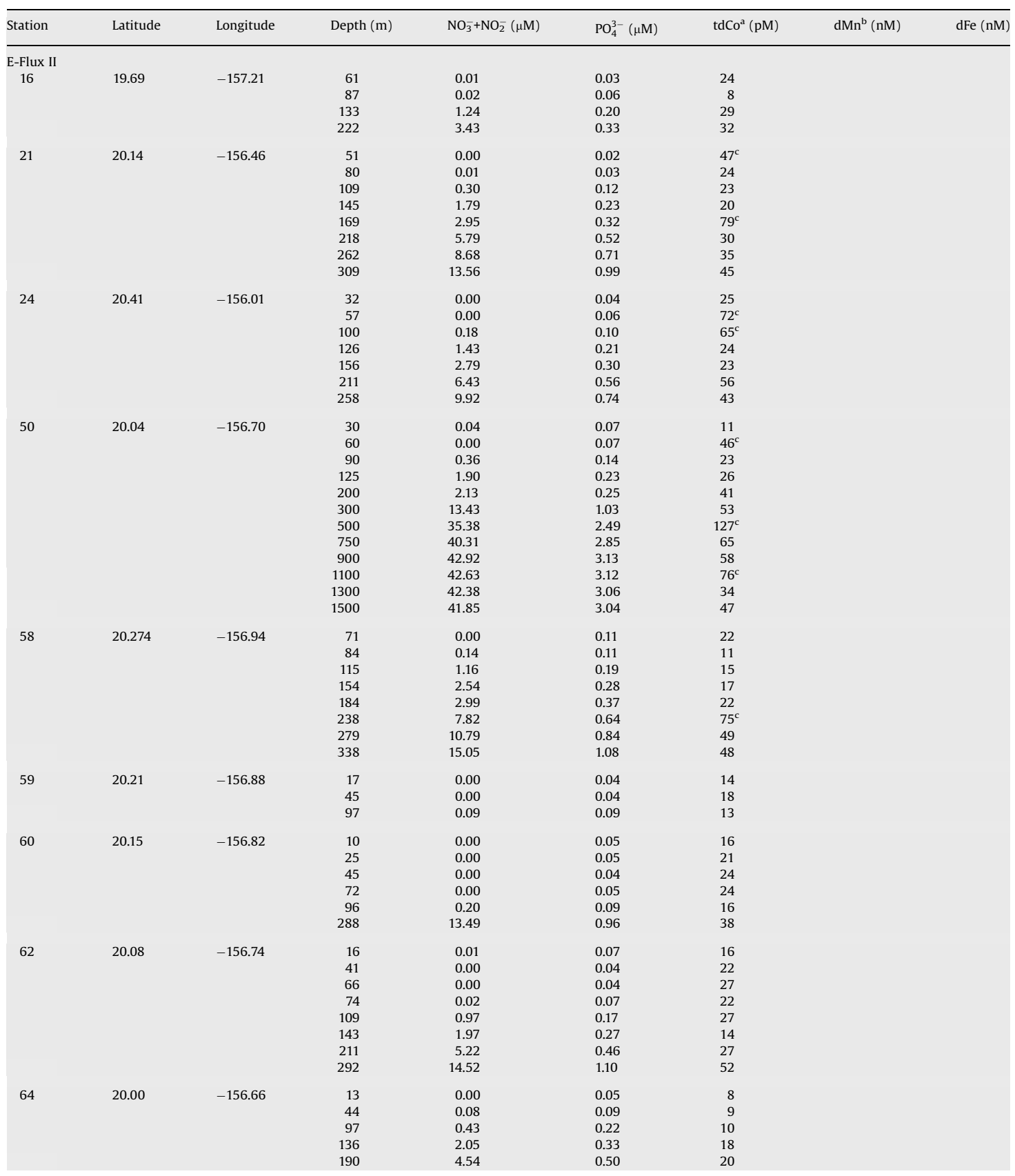




\begin{tabular}{|c|c|c|c|c|c|c|c|c|}
\hline Station & Latitude & Longitude & Depth (m) & $\mathrm{NO}_{3}^{-}+\mathrm{NO}_{2}^{-}(\mu \mathrm{M})$ & $\mathrm{PO}_{4}^{3-}(\mu \mathrm{M})$ & $\mathrm{tdCo}^{\mathrm{a}}(\mathrm{pM})$ & $\mathrm{dMn}^{\mathrm{b}}(\mathrm{nM})$ & $\mathrm{dFe}(\mathrm{nM})$ \\
\hline \multirow[t]{7}{*}{67} & \multirow[t]{7}{*}{20.43} & \multirow[t]{7}{*}{-157.10} & 49 & 0.00 & 0.03 & 3 & & \\
\hline & & & 65 & 0.00 & 0.05 & 24 & & \\
\hline & & & 89 & 0.06 & 0.08 & $73^{c}$ & & \\
\hline & & & 115 & 1.63 & 0.19 & 19 & & \\
\hline & & & 145 & 2.76 & 0.29 & 17 & & \\
\hline & & & 193 & 5.22 & 0.45 & 21 & & \\
\hline & & & 281 & 11.43 & 0.83 & $97^{c}$ & & \\
\hline \multirow[t]{3}{*}{74} & \multirow[t]{3}{*}{20.15} & \multirow[t]{3}{*}{-156.82} & 10 & 0.02 & 0.08 & 30 & & \\
\hline & & & 20 & 0.05 & 0.09 & 0 & & \\
\hline & & & 80 & 0.38 & 0.13 & 11 & & \\
\hline \multirow[t]{23}{*}{81} & \multirow[t]{23}{*}{20.27} & \multirow{23}{*}{-156.94} & 10 & 0.02 & 0.08 & 13 & 1.53 & 0.18 \\
\hline & & & 35 & 0.04 & 0.07 & 12 & 1.56 & \\
\hline & & & 45 & 0.01 & 0.05 & 11 & 1.51 & 0.43 \\
\hline & & & 57 & 0.00 & 0.06 & 14 & 1.44 & \\
\hline & & & 68 & 0.01 & 0.04 & 18 & 1.58 & 0.67 \\
\hline & & & 80 & 0.00 & 0.05 & 16 & 1.41 & 0.84 \\
\hline & & & 120 & 2.16 & 0.29 & 25 & 0.81 & 0.54 \\
\hline & & & 160 & 2.20 & 0.22 & 27 & 0.56 & 0.47 \\
\hline & & & 265 & 8.70 & 0.71 & 33 & 0.30 & \\
\hline & & & 318 & 17.00 & 1.43 & 59 & 0.21 & 0.48 \\
\hline & & & 363 & 19.42 & 1.08 & 67 & 0.22 & \\
\hline & & & 465 & 27.96 & 2.12 & 75 & 0.30 & 0.68 \\
\hline & & & 514 & 36.35 & 2.69 & 77 & 0.37 & \\
\hline & & & 551 & 37.91 & 2.80 & 77 & 0.42 & 0.76 \\
\hline & & & 600 & 30.37 & 2.66 & 64 & 0.45 & 0.97 \\
\hline & & & 662 & 41.52 & 3.10 & 61 & 0.50 & 0.97 \\
\hline & & & 720 & 43.05 & 3.32 & 59 & 0.43 & 0.95 \\
\hline & & & 789 & 37.56 & 2.93 & 52 & 0.38 & 0.79 \\
\hline & & & 913 & 38.02 & 2.83 & 48 & 0.38 & 1.22 \\
\hline & & & 1054 & 42.01 & 3.22 & 39 & 0.40 & 1.25 \\
\hline & & & 1214 & 38.38 & 3.02 & 38 & 0.35 & 1.43 \\
\hline & & & 1317 & 39.17 & 3.10 & 42 & 0.37 & 1.39 \\
\hline & & & 1417 & 41.09 & 3.16 & 36 & 0.40 & 1.48 \\
\hline 90 & 20.50 & -157.50 & 15 & 0.11 & 0.03 & 15 & & \\
\hline & & & 30 & 0.00 & 0.08 & 6 & & \\
\hline & & & 60 & 0.00 & 0.06 & 13 & & \\
\hline & & & 90 & 0.02 & 0.07 & 10 & & \\
\hline & & & 110 & 1.17 & 0.20 & 11 & & \\
\hline & & & 200 & 5.05 & 0.43 & 22 & & \\
\hline & & & 300 & 13.00 & 0.91 & 41 & & \\
\hline & & & 531 & 35.30 & 2.56 & 66 & & \\
\hline & & & 609 & 38.92 & 2.82 & 59 & & \\
\hline & & & 879 & 41.97 & 3.00 & 48 & & \\
\hline & & & 955 & 42.22 & 3.00 & 54 & & \\
\hline 100 & 20.50 & -157.50 & 24 & 1.16 & 0.12 & 5 & & \\
\hline & & & 36 & 0.01 & 0.06 & 4 & & \\
\hline & & & 92 & 0.00 & No data & 16 & & \\
\hline & & & 134 & 3.35 & 0.30 & 18 & & \\
\hline & & & 261 & 2.15 & 1.49 & 38 & & \\
\hline & & & 360 & 20.25 & 1.49 & 61 & & \\
\hline & & & 455 & 31.67 & 2.47 & 72 & & \\
\hline Station & Latitude & & Longitude & Depth (m) & $\mathrm{NO}_{3}^{-}+\mathrm{NO}_{2}^{-}(\mu \mathrm{M})$ & & & Total Co (pM) \\
\hline E-Flux III & & & & & & & & \\
\hline In 1 & 19.36 & & -157.08 & 5 & 0.09 & & & 18 \\
\hline & & & & 25 & 0.02 & & & 18 \\
\hline & & & & 45 & 0.14 & & & 26 \\
\hline & & & & 75 & 1.10 & & & 30 \\
\hline & & & & 100 & 2.18 & & & 37 \\
\hline & & & & 125 & 2.94 & & & 24 \\
\hline & & & & 175 & 3.39 & & & 26 \\
\hline & & & & 250 & 4.41 & & & 39 \\
\hline & & & & 500 & 11.20 & & & 65 \\
\hline & & & & 750 & 35.62 & & & 53 \\
\hline & & & & 1000 & 41.01 & & & 47 \\
\hline In 3 & 19.12 & & -157.03 & 5 & 0.06 & 0. & & 12 \\
\hline & & & & 25 & 0.20 & 0. & & 14 \\
\hline & & & & 45 & 0.14 & 0. & & 21 \\
\hline & & & & 75 & 1.59 & 0. & & 24 \\
\hline & & & & 100 & 2.25 & 0. & & 29 \\
\hline & & & & 125 & 3.04 & 0. & & 22 \\
\hline & & & & 175 & 4.63 & 0. & & 27 \\
\hline
\end{tabular}




\begin{tabular}{|c|c|c|c|c|c|c|}
\hline Station & Latitude & Longitude & Depth (m) & $\mathrm{NO}_{3}^{-}+\mathrm{NO}_{2}^{-}(\mu \mathrm{M})$ & $\mathrm{PO}_{4}^{3-}(\mu \mathrm{M})$ & Total Co (pM) \\
\hline & & & 250 & 12.43 & 1.02 & 47 \\
\hline & & & 500 & 35.09 & 2.65 & 82 \\
\hline & & & 750 & 41.25 & 3.04 & 51 \\
\hline & & & 1000 & 42.37 & 3.08 & 49 \\
\hline \multirow[t]{12}{*}{ Out 1} & 20.62 & -157.59 & 5 & 0.37 & 0.09 & 11 \\
\hline & & & 25 & 0.31 & 0.09 & 12 \\
\hline & & & 45 & 0.32 & 0.09 & 13 \\
\hline & & & 75 & 0.21 & 0.10 & 12 \\
\hline & & & 100 & 0.15 & 0.12 & 17 \\
\hline & & & 125 & 0.16 & 0.13 & 20 \\
\hline & & & 150 & 1.80 & 0.28 & 23 \\
\hline & & & 175 & 3.06 & 0.39 & 27 \\
\hline & & & 250 & 7.84 & 0.70 & 37 \\
\hline & & & 500 & 32.46 & 2.43 & 80 \\
\hline & & & 750 & 41.44 & 3.05 & 54 \\
\hline & & & 1000 & 42.26 & 3.10 & 55 \\
\hline
\end{tabular}

$-157.5^{\circ} \mathrm{W}$ (90 and 100 : sampled 1 day apart) $20.27^{\circ} \mathrm{N},-156.8^{\circ} \mathrm{W}$ (81 and 58: sampled 4 days apart), and $20.15^{\circ} \mathrm{N},-156.82^{\circ} \mathrm{W}$ (60 and 74: sampled 3 days apart). All three sites showed different surface cobalt concentrations from one sampling date to another, indicative of temporal variability on time scales as short as a few days. The replicate stations were sampled within 1-4 days of each other, and surface cobalt concentrations vary by up to $\sim 20 \mathrm{pM}$. This variability is likely related to changes in physical processes such as water mass movement, as well as changes in the distribution of cobalt due to biological uptake. This variability may also be due to aeolian deposition as cobalt has been observed to be affected by aeolian input in some regions such as the Mediterranean and off the coast of North Africa in the Atlantic (Herrera-Melian et al., 1994). However, the abundance of cobalt in crustal material is significantly lower than that of iron (Co:Fe ratio in crustal composition is 1:2600; Taylor and McLennan, 1985), which suggests that much larger fluxes of dust and/or higher dust solubility may be necessary to create a significant cobalt source to the upper water column relative to iron.

The abundance of manganese and iron in the upper water column near Hawaii is believed to be seasonally affected by high dust input in the spring (March-June) with lower temporal aerosol concentrations observed between July and January (Parrington et al., 1983; Perry et al., 1999; Johnson et al., 2003) During the E-Flux II cruise, dissolved manganese in the upper water column followed the expected trend of previous studies (Johnson et al., 1996): it is present in higher concentrations $(\sim 2 \mathrm{nM})$ in the surface waters presumably due to aeolian deposition, and is depleted quickly with depth due to sorption to sinking particles and/or manganese oxidizing bacteria below the euphotic zone (Fig. 3A). Our data are generally oceanographically consistent with previously reported dissolved manganese concentrations that have ranged from 1.75 to $<0.3 \mathrm{nM}$ at Station ALOHA (Boyle et al., 2005).

We observed significant iron depletion in the upper tens of meters, consistent with the lower relative dust input in January and biological utilization of iron by phytoplankton (Fig. 3B). The range of dissolved iron concentrations during the E-Flux II cruise are somewhat higher $(0.18-1.5 \mathrm{nM}$; Fig. $3 \mathrm{~B})$ than observed at Station ALOHA (below detection limit to $\sim 1 \mathrm{n} \mathrm{M}$; Boyle et al., 2005). We believe this is likely due to the closer proximity to the Hawaiian Islands and associated island effects. Comparisons with studies done during the PlumEx experiment near the Galapagos Islands are consistent with this reasoning, where surface iron was generally found to be low in surface waters except near the islands (Gordon et al.. 1998). In that study, transects identified the source of iron to be upwelling from the Equatorial Undercurrent. In addition, some of the increase, though a small component of the upwelled iron, was attributed to interaction of the Equatorial Undercurrent with shallow island topography resulting in higher particulate iron concentrations (Gordon et al., 1998). In contrast to iron, surface-water cobalt did not appear to be influenced by island effects and did not show any significant increases near the Galapagos Islands (Saito et al., 2004) or near the Hawaiian Islands (this study; Fig. 3C). This difference between iron and cobalt in surface waters may be due to the much lower abundance of cobalt in crustal material than that of iron mentioned above, hence requiring much more particulate resuspension or aeolian deposition from island material to noticeably elevate surface cobalt concentrations (Saito and Moffett, 2002). This also has implications for time-series studies near volcanic islands suggesting island proximity effects may have a particular influence on surface water iron.

\subsection{An eddy influence on cobalt: biological entrainment and concentrating mechanism}

There have only been a few studies that focus on the potential influence of eddies on trace metal distributions (Saito et al., 2002; Johnson et al., 2005; Sedwick et al., 2005). It is now becoming clear that there are multiple possibilities for trace metal distributions depending upon the specific nature of the eddy. As mentioned earlier, we observed depletion of cobalt concurrently with decreases in water temperature that we interpreted as being associated with the passing of a cyclonic eddy or mixing events in the Sargasso Sea during a 1999 time-series (Saito and Moffett, 2002). This depletion likely occurs due to a combination of macronutrient upwelling and the resulting increase in phytoplankton growth (McGillicuddy et al., 1998). In this case, upwelling of macronutrient rich waters does not replenish the depleted surface-water cobalt because cobalt's hybrid-type vertical profile has so little cobalt to be upwelled from depth due to its continual scavenging in intermediate and deep waters. 
(A)

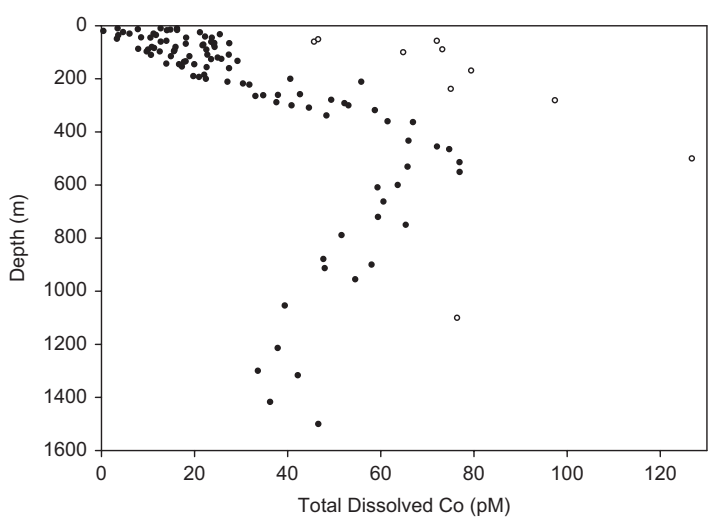

(B)

Station Number

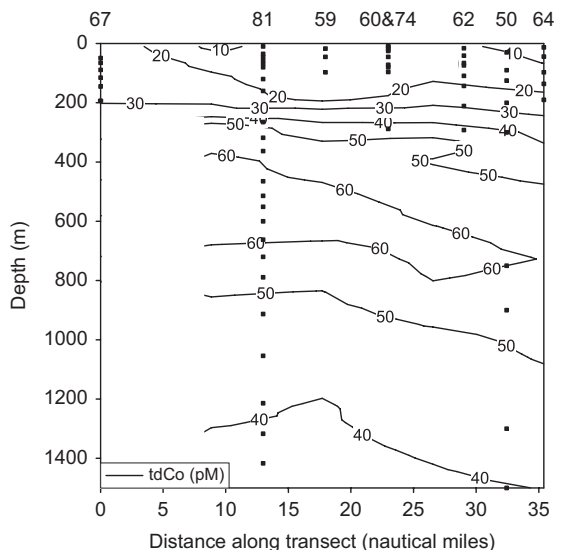

Fig. 2. (A) Sample overlay for E-Flux II total dissolved cobalt data. Closed circles represent the vast majority of samples analyzed, while the open circles represent high cobalt concentrations outside the generally observed trend. These points are denoted in Table 1 with superscript "c". With these high-throughput analyses, occasional samples were measured that were not oceanographically consistent with the bulk of the data. Note the depletion of total dissolved cobalt in the surface waters as a result of biological uptake. (B) E-Flux II Transect 6 (see Fig. 1A) contour plot showing cobalt concentration trends with depth (tdCo denotes "total dissolved cobalt"). The data from which the contour plot was ma de are overlain as black squares.

A coastal anticyclonic eddy in the North Pacific was observed to show a very different mechanism of eddy influence on trace metal distributions (Johnson et al., 2005). The coastal formation of this eddy entrained significant dissolved and particulate iron, transporting it into the iron-limited region of the North Pacific while macronutrients were drawn down due to eddy isolation from surrounding waters (Peterson et al., 2005). In addition to these eddy-related mechanisms controlling trace metal distributions (a macronutrient upwelling-associated depletion process, and a transport and containment process), we now propose a third type suggested by this study: a mechanism for concentrating cobalt from intermediate waters into the upper water column.

Three vertical cobalt profiles taken during examination of Cyclone Opal from E-Flux III show an increase in cobalt concentrations inside the eddy relative to outside the eddy (Fig. 4). The integrated inventories of cobalt between 5 and $100 \mathrm{~m}$ also show higher cobalt within the eddy with values of 2.5 and $2.0 \mu \mathrm{mol} \mathrm{Co} \mathrm{m}{ }^{-2}$, relative to $1.2 \mu \mathrm{mol} \mathrm{Co} \mathrm{m}^{-2}$ in surrounding waters (Table 2). The two profiles from within the eddy also show small maxima at $100 \mathrm{~m}$ in both profiles, whereas no such maximum is present in the Out station. These differences in cobalt abundances are resolvable since the detection limit for this voltammetry method is $\leqslant 3 \mathrm{pM}$ (Vega et al., 1997; Saito and Moffett, 2002). We attribute the maxima to shallow and extensive remineralization occurring at that depth. This is supported by three datasets: (1) ${ }^{234} \mathrm{Th}$ flux data (Fig. 5; Maiti et al., 2008), (2) microscopy observations of remineralization (Benitez-Nelson et al., 2007), and (3) absence of a significant organic matter export flux (Benitez-Nelson et al., 2007). Despite a significant increase in primary productivity, Cyclone Opal did not produce a large organic export flux; on the contrary, near complete remineralization of sinking organic matter was observed at $\sim 100 \mathrm{~m}$, with sinking diatom frustules appearing to have little remaining organic matter associated with them, thus preventing significant particle export of organic matter $\left(1.1 \mathrm{mmolC} \mathrm{m}{ }^{-2} \mathrm{~d}^{-1}\right.$ outside Opal versus $1.8 \mathrm{mmol} \mathrm{C} \mathrm{m}^{-2} \mathrm{~d}^{-1}$ inside Opal; Benitez-Nelson et al., 2007). This is consistent with the accumulation of dissolved organic carbon which accounted for $>85 \%$ of the measured primary production within Cyclone Opal. Similar results were obtained using mass balance estimates of inorganic carbon (Chen et al., 2008). The nitrate+nitrite and phosphate values measured within the upper $110 \mathrm{~m}$ of Cyclone Opal also showed accumulation with remineralization within the eddy, with an integrated inventory of $91 \mathrm{mmol} \mathrm{N} \mathrm{m}^{-2}$ and $17.1 \mathrm{mmol} \mathrm{P} \mathrm{m}^{-2}$ within Opal relative to $24 \mathrm{mmol} \mathrm{N} \mathrm{m}^{-2}$ and $9.8 \mathrm{mmol} \mathrm{P} \mathrm{m}^{-2}$ in surrounding waters (Benitez-Nelson et al., 2007). We propose that sinking particulate cobalt is being similarly remineralized before export.

These observations from Cyclone Opal suggest that there is a mechanism at work, where cobalt is concentrated within the eddy by a succession of processes: supply of cobalt to the upper euphotic zone, biological utilization of cobalt and its export in particles, and shallow and significant remineralization of cobalt from organic matter, as illustrated in Fig. 6. The likely source of cobalt to the euphotic zone is upwelling, however, lateral advection and/or aeolian deposition are also plausible sources. We can calculate the amount of cobalt delivered to the upper water column by upwelling within Cyclone Opal and compare it with the changes in inventory we have observed. Examination of isopyncal surfaces on a transect across the Cyclone Opal with the stations we have focused on (Nencioli et al., 2008; Benitez-Nelson et al., 2007; and Fig. 4) showed isopyncal uplift of $\sim 75 \mathrm{~m}$ at In Station 1. Integrating the cobalt inventory from 75 to $175 \mathrm{~m}$ at Out Station 1 yielded $2.0 \mu \mathrm{M} \mathrm{m}^{-2}$, compared to $2.5 \mu \mathrm{M} \mathrm{m}^{-2}$ found in the top $100 \mathrm{~m}$ of In Station 1 (see integrated inventory calculation above). These calculations are thus consistent with upwelling as the primary source of cobalt to the euphotic zone. However, the upper $100 \mathrm{~m}$ inside Cyclone Opal has $\sim 25 \%$ more cobalt than can be explained by one-time eddy-induced upwelling, suggesting three possibilities: (1) that the excess cobalt may have been concentrated from the displaced pre-existing water mass and then added to the sampled water mass by the proposed biological concentrating mechanism, (2) additional eddy-induced upwelling may have occurred and the cobalt was similarly concentrated, or (3) other sources such as lateral advection and aeolian deposition contributed to the cobalt inventory. The presence of the described small remineralization peaks in the two cobalt profiles from within Cyclone Opal (Fig. 4) are consistent with the first and second interpretations, although we cannot discount the third possibility of alternate sources.

This concentrating mechanism could be especially relevant to hybrid-type elements because their oceanic reservoirs are quite small (due to low deepwater concentrations and lack of intermediate and deepwater accumulation), and hence the 


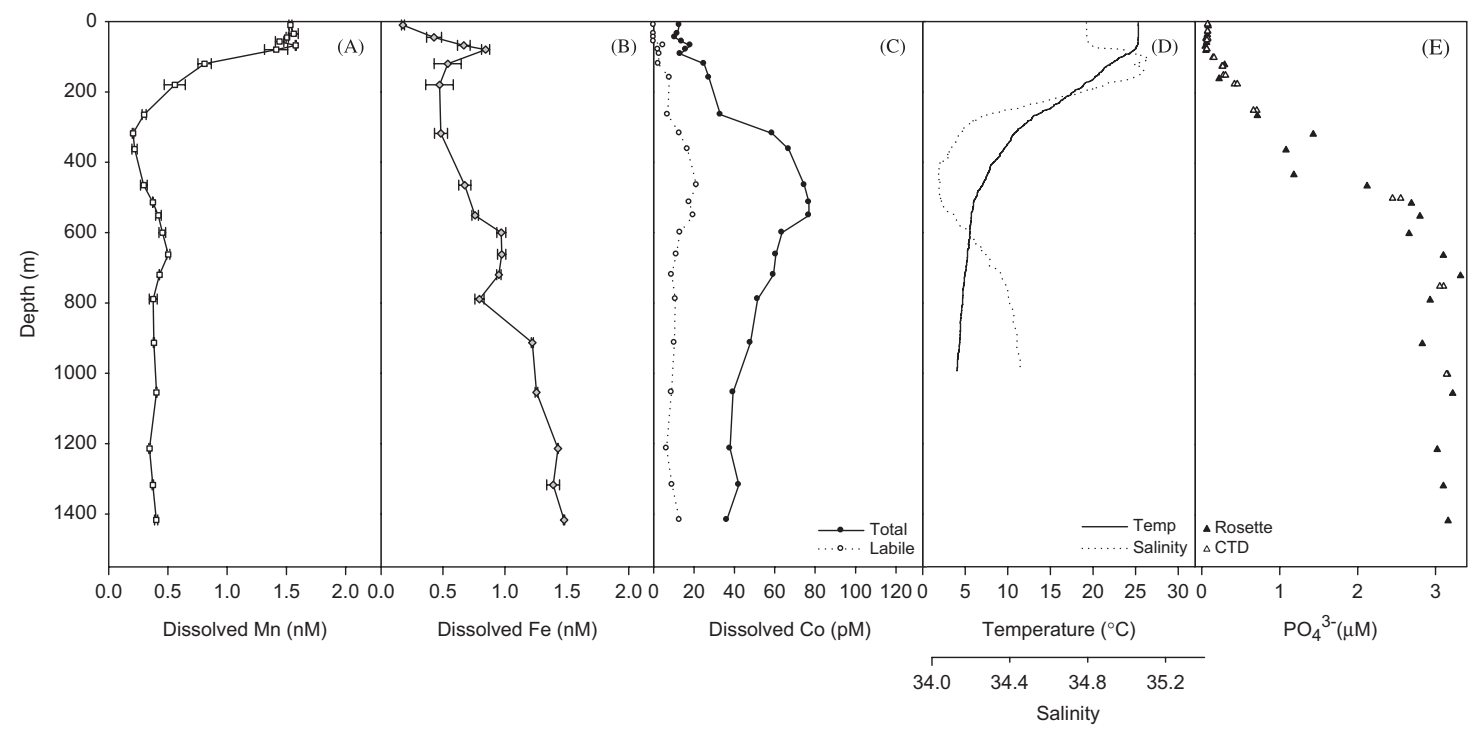

Fig. 3. Vertical profiles for dissolved manganese, iron, cobalt, and phosphate and hydrographic data for Station 81 from E-Flux II. High concentrations of cobalt are present at $400-600 \mathrm{~m}$ in all stations and suggest an intermediate depth source for cobalt. Phosphate data from the CTD bottle cast are plotted with Go-Flo rosette cast data to confirm sampling accuracy. Labile cobalt is defined as the fraction of the total dissolved cobalt that is not bound by strong organic ligands (Saito and Moffett, 2002).

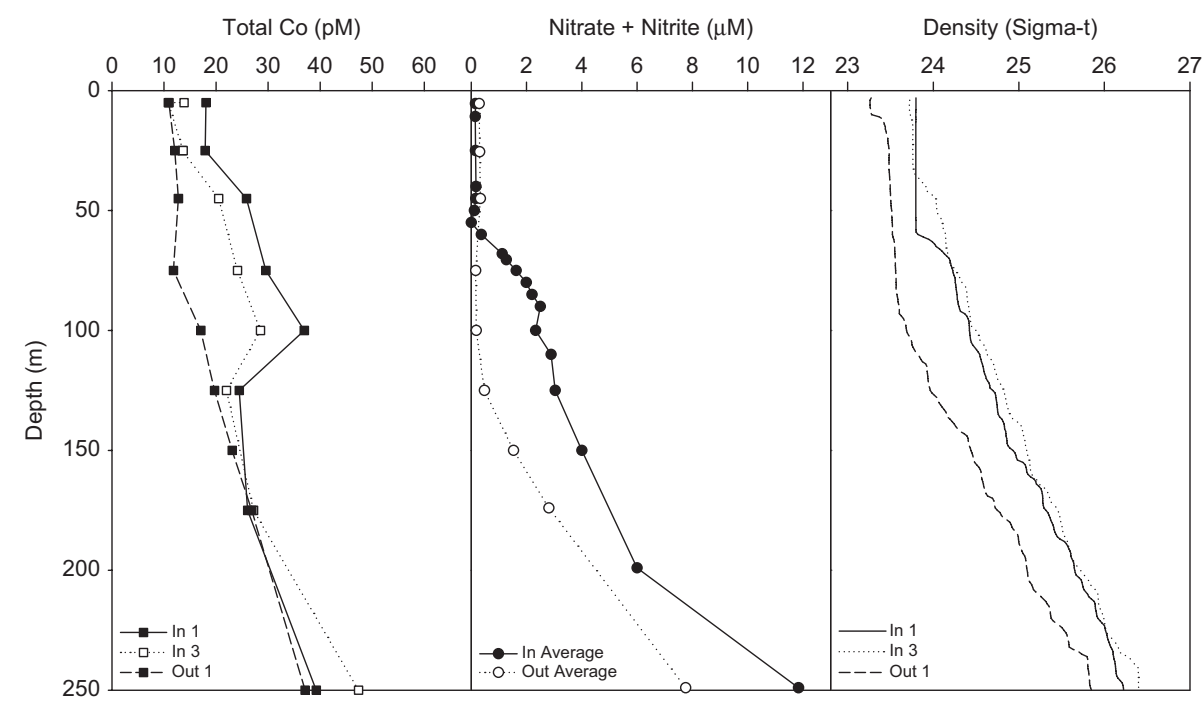

Fig. 4. Surface total cobalt profiles for E-Flux III from inside Cyclone Opal (In 1 and 3 ) and outside (Out 1). Shallow cobalt maxima are observed in two profiles within Cyclone Opal, but not in surrounding waters. These maxima occur at the same depth that the ${ }^{234} \mathrm{Th}$ disequilibria ends $(\sim 100 \mathrm{~m}$; see Fig. 5), suggesting that these shallow cobalt maxima are related to export by particle flux and subsequent shallow remineralization. Averaged nitrate+nitrite ( $N+N)$ values from In and Out stations $(n=3$ and 5 , respectively) in E-Flux III also show a slight bulge between $\sim 75$ and $100 \mathrm{~m}$ and an increase in upper column $\mathrm{N}+\mathrm{N}$ inventory, likely due to a combination of the shoaling isopycnals and a remineralization signal (data from Nencioli et al., 2008).

remineralization signature can make a significant impression on the vertical profile of the trace element. While this process is certainly not exclusive to hybrid-type elements, the impact of these signatures on the overall distributions of macronutrients and nutrient-type trace metals is relegated to a subtle one because of their much larger oceanic inventories with accumula- tion during thermohaline circulation. In addition, if the remineralization were somewhat deeper, as is often expected outside of eddy environments, any small maxima would be masked by the higher concentrations associated with the much larger marine inventories of those elements. It is this combination of eddyinduced upwelling, shallow and efficient remineralization, and the 
Table 2

Inventories in the upper $100 \mathrm{~m}$

\begin{tabular}{llllll}
\hline Station & $\begin{array}{l}\text { Range of total } \\
\text { cobalt }(\mathrm{pM})\end{array}$ & $\begin{array}{l}\text { Co inventory } \\
\left(\mu \mathrm{mol} \mathrm{m}^{-2}\right)\end{array}$ & $\begin{array}{l}\mathrm{PC} \text { inventory } \\
\left(\mathrm{g} \mathrm{m}^{-2}\right)\end{array}$ & $\begin{array}{l}\text { Total Chl } a \\
\text { inventory } \\
\left(\mathrm{g} \mathrm{m}^{-2}\right)\end{array}$ & $\begin{array}{l}\text { Nitrate+nitrite inventory } \\
\left(\mathrm{mmol} \mathrm{N} \mathrm{m}^{-2}\right)\end{array}$ \\
\hline In 1 E-Flux III & $18-37$ & 2.5 & 2.9 & 31 & $\begin{array}{l}\text { Primary productivity } \\
\left(\mathrm{mmol} \mathrm{C} \mathrm{m}^{-2} \mathrm{~d}^{-1}\right)\end{array}$ \\
In 3 E-Flux III & $11-29$ & 2.0 & 1.8 & 25 & 91 (average In) \\
Out 1 E-Flux III & $11-23$ & 1.2 & 1.8 & 9.9 & 24 (average Out) \\
\hline
\end{tabular}

a Benitez-Nelson et al. (2007)

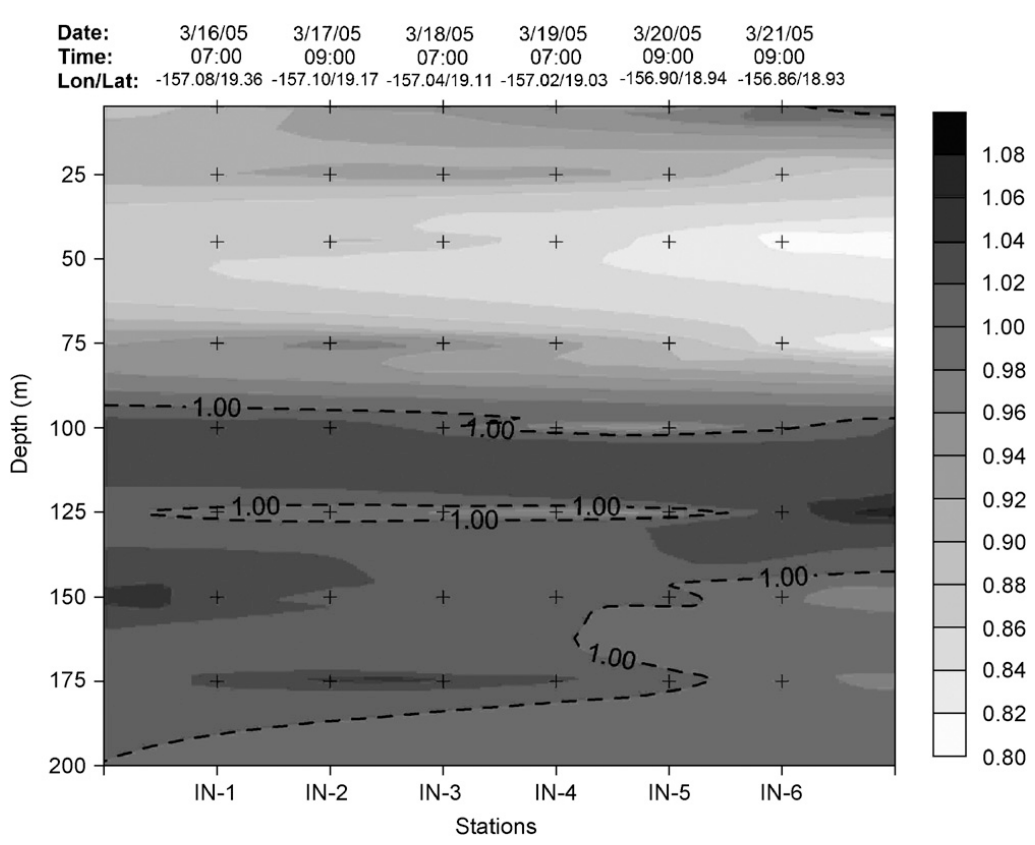

Fig. 5. Contour plot of the ${ }^{234} \mathrm{Th} /{ }^{238} \mathrm{U}$ ratio for the six IN stations at the center of E-Flux III Cyclone Opal. ${ }^{234} \mathrm{Th} /{ }^{238} \mathrm{U}$ ratio equal to 1 represents that the daughter $\left({ }^{234} \mathrm{Th}\right)$ and the parent $\left({ }^{238} \mathrm{U}\right)$ isotope are in equilibrium. ${ }^{234} \mathrm{Th} /{ }^{238} \mathrm{U}$ ratio of less than 1 represents particle scavenging whereas ${ }^{234} \mathrm{Th} /{ }^{238} \mathrm{U}$ ratio greater than 1 represents particle remineralization. A remineralization feature is clearly observed at $\sim 100 \mathrm{~m}$, coinciding with the peak observed in cobalt at this depth (see Fig. 4). Adapted from Maiti et al.

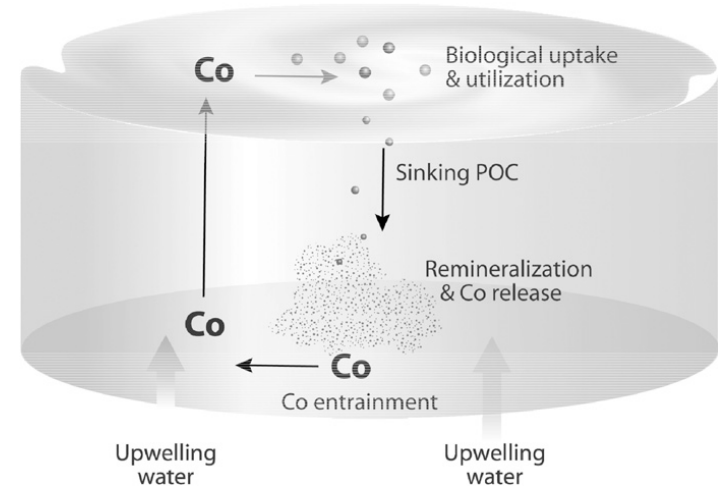

Fig. 6. A proposed cobalt concentrating mechanism within Cyclone Opal, where the cobalt is concentrated by a succession of events: upwelling, biological utilization, and subsequent export associated with POC, shallow and significant remineralization of cobalt from organic matter. hybrid-type metal scarcity in intermediate waters that creates and allows this hybrid-type metal concentrating effect.

\subsection{The upper water column cobalt-phosphate correlation: a mechanistic explanation and a minimum productivity threshold}

The nutrient-like process influencing cobalt distributions in the upper water column is likely related to the role of cobalt as a micronutrient for phytoplankton and the microbial communities. We and others have previously described correlations of total dissolved cobalt and soluble reactive phosphorus (described as phosphate or P herein) in the Gulf of Alaska, Peru upwelling region, and Equatorial Atlantic (Sunda and Huntsman, 1995; Saito and Moffett, 2002; Saito et al., 2004). Recent results also show this cobalt and phosphate correlation in the Ross Sea and throughout the North Pacific and Bering Sea (Saito and Noble, in preparation; Saito et al., in preparation). These findings are similar to the correlations seen between other trace metals and macronutrients, such as cadmium with phosphate and zinc with silica (e.g., Boyle et al., 1976; Bruland, 1980; Cullen et al., 2003), with the important exception that those micro- and macro-nutrient pairs correlate 
throughout the water column, whereas cobalt and phosphate only correlate in the upper ocean. Cobalt and phosphate appear to be decoupled, however, in the Sargasso Sea and the Western Atlantic shelf/slope area (Saito and Moffett, 2002), presumably due to a combination of extremely oligotrophic conditions in the Sargasso Sea and coastal inputs in the shelf/slope area, which result in a cobalt-salinity correlation instead. In addition, the slopes of these cobalt-phosphate relationships, which imply their relative utilization rates $(\Delta \mathrm{Co} / \Delta \mathrm{P}$; Redfield, 1958; Sunda and Huntsman, 1995$)$ vary significantly from region to region from $27 \mu \mathrm{mol} \mathrm{Co} \mathrm{mol}^{-1} \mathrm{P}$ $\left(r^{2}=0.83\right)$ in the Ross Sea (Saito and Noble, unpublished results) to $560 \mu \mathrm{mol} \mathrm{Co} \mathrm{mol}{ }^{-1} \mathrm{P}\left(r^{2}=0.63\right)$ in the Equatorial Atlantic (Saito and Moffett, 2002), with the Peru upwelling region $(248 \mu \mathrm{mol} \mathrm{Co}$ $\left.\mathrm{mol}^{-1} \mathrm{P}\right)$, Northeast Pacific (36-40 $\mu \mathrm{mol} \mathrm{Co} \mathrm{mol}^{-1} \mathrm{P}$ ) and this study (see below) falling within this range (Saito et al., 2004; Sunda and Huntsman, 1995).

In E-Flux II, there is a weak relationship between Co and $\mathrm{P}$ in the upper water column (shallower than $300 \mathrm{~m})\left(r^{2}=0.63, \Delta \mathrm{Co} / \Delta \mathrm{P}=\right.$ $29 \mu \mathrm{mol} \mathrm{Co} \mathrm{mol}^{-1} \mathrm{P}, n=35$; Fig. 7A). Note that this does not take DOP into account due to limited data and information on bioavailability at this time (Karl and Yanagi, 1997). In contrast, the E-Flux III cobalt dataset above $250 \mathrm{~m}$ produces a strong correlation $\left(r^{2}=0.91, n=17\right)$ between cobalt and phosphate with a $\Delta \mathrm{Co} / \Delta \mathrm{P}$ of $37 \mu \mathrm{mol} \mathrm{Co} \mathrm{mol}{ }^{-1} \mathrm{P}$ (Fig. 7B). The variability in Co:P data from E-Flux II does not appear to be due to analytical or sampling errors, phosphate analyses were compared between discrete samples taken at comparable depths from both the trace metal sampler and CTD and showed excellent agreement (Fig. 3E) In addition, since samples from E-Flux III were unfiltered, there is the possibility that the particulate fraction caused the Co:P correlation. However, we and others have previously observed Co:P correlations in the dissolved fraction (Martin et al., 1989; Saito and Moffett, 2002; Saito et al., 2004), and the component of cobalt associated with particles is generally quite small when compared to typical dissolved concentrations (Sherrell and Boyle, 1992). For example, particulate cobalt is estimated to be only $\sim 2.6 \%$ of total dissolved cobalt at $100 \mathrm{~m}$ and $\sim 1.6 \%$ of total dissolved cobalt at $1500 \mathrm{~m}$ near Bermuda (data from Saito 2002 in combination with Sherrell and Boyle, 1992). The cobalt concentrations measured in E-
Flux III were comparable to those of E-Flux II, which is also consistent with a relatively small particulate fraction of cobalt.

This Co:P correlation in surface waters has only recently been recognized (Sunda and Huntsman, 1995; Saito and Moffett, 2002; Saito et al., 2004) and the question of why cobalt and phosphate are coupled as nutrients in some areas and decoupled in others has yet to be directly addressed. The geochemistry of phosphate is typically dominated by biological processes in the upper water column (e.g., Redfield, 1958; Bronk et al., 1994; Tyrell, 1999; Benitez-Nelson, 2000). As mentioned earlier, cobalt is affected both by abiotic and biological processes, and it is logical that the extent to which biology controls cobalt should control the correlation between cobalt and phosphate. The small inventory of cobalt (less than $0.1 \mathrm{nM}$ in most deep waters and even less in surface waters) is likely critical to its high variability, and hence biological processes of even moderate amplitude could have a major influence on cobalt geochemistry. The size of this inventory is evident when compared to other elements: cobalt concentrations in deep waters are only 2-3 times higher than in surface waters, where phosphate concentrations are 25-30 times higher and zinc and cadmium have an even greater discrepancy ( $>100$-fold).

The influence of each biotic and abiotic source and sink of cobalt can be considered as a vector in cobalt-phosphate space to demonstrate the tug-of-war between processes on cobalt's geochemistry (Fig. 8A). This decomposition of processes into vectors should also apply to other hybrid-type trace metals (HTMs), in particular iron. The aeolian deposition of cobalt that has been reported in some locations (Wong et al., 1995; HerreraMelian et al., 1994) would result in a noticeable increase in cobalt with no observable increase in phosphate (shown as an upwards vector in Fig. 8A). There are also microbial oxidation pathways for manganese and cobalt that may remove cobalt from coastal waters independently of phosphate, resulting in a downward biotic scavenging vector, where in this case biotic scavenging refers to microbial cobalt oxidation and not utilization as a micronutrient (Tebo et al., 1984; Moffett and Ho, 1996; Saito et al., 2004). Other abiotic scavenging processes would also likely result in the preferential removal of hybrid-type metals such as cobalt relative to phosphate in the water column and a downward vector.
(A)

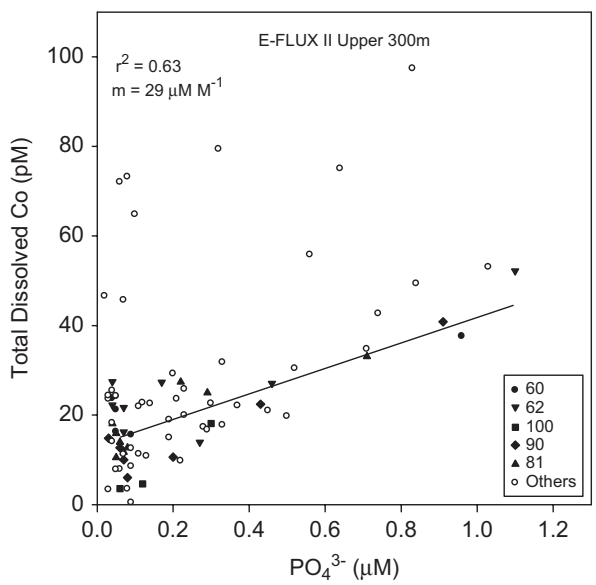

(B)

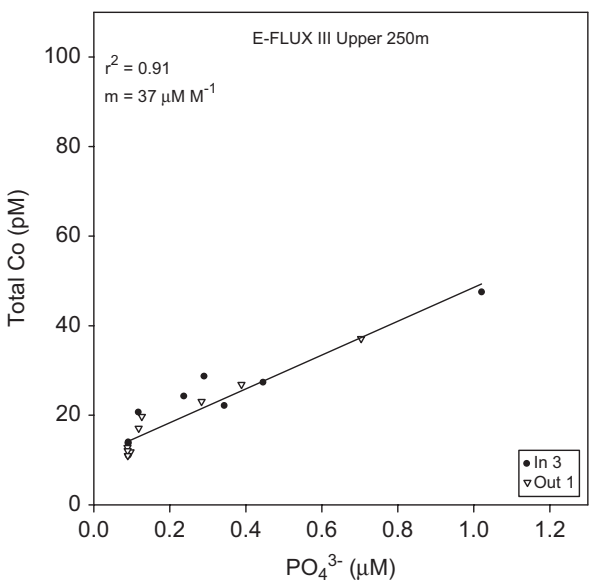

Fig. 7. Co:P correlation from E-Flux II and III surface waters. There is a strong correlation between Co and P during E-Flux III $\left(r^{2}=0.91\right)$ with a slope of $37 \mu \mathrm{mol} \mathrm{Co} \mathrm{mol}^{-1} \mathrm{P}$, and a weaker correlation during E-Flux II. A slope of $29 \mu \mathrm{mol} \mathrm{Co} \mathrm{mol}^{-1} \mathrm{P}\left(r^{2}=0.63\right)$ was calculated from the aggregate of all profiles for E-Flux II that had enough data points $(\geqslant 4)$ to identify oceanographically consistent features and did not have concentrations identified as outside the generally observed trend for the upper water column (refer to Fig. 2(A)). These points are plotted with open circles and labeled "others." 
Interestingly, because scavenging depletes cobalt relative to phosphate in intermediate and deep waters, the upwelling of water masses results in a range of vectors (depending on the extent of scavenging), with a lower Co:P slope than found in the upper water column (Fig. 8A).

Biological activity in the upper water column can be broadly described by a diagonal line in this vector diagram, similar to the correlation of $\mathrm{Co}: \mathrm{P}$ that we have observed. This line encompasses two diagonal vectors, biological utilization of cobalt as a micronutrient (downwards), and remineralization (upwards). Both of these vectors also involve uptake and release of phosphate. The slope of the biological utilization vector can be considered as the composite cellular quota of the biological community for the hybrid-type metal and phosphate in the surrounding waters (Fig. 8B). This range of biological cellular

(A)

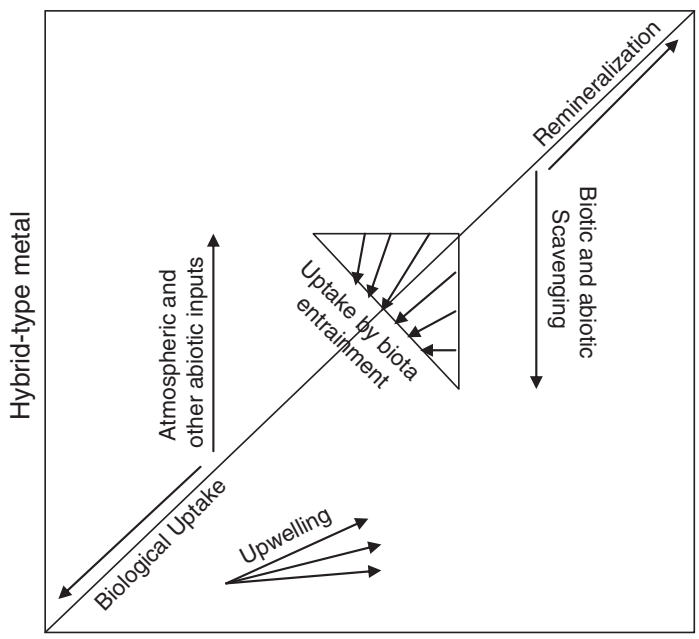

Phosphate

(B)

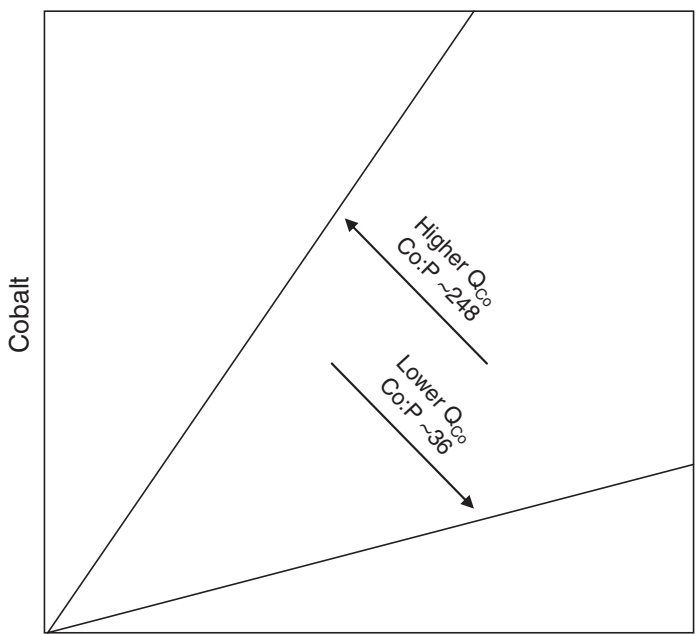

Phosphate quotas is visualized by a group of arrows each symbolizing an important phytoplankton group in the community (Fig. 8A). Together this phytoplankton community acts to entrain cobalt into a micronutrient geochemical role and creates the composite $\mathrm{Co}: \mathrm{P}$ of the structure we observe. Little is known about hybridtrace metal remineralization relative to phosphorus at this point, but presumably hybrid-type metals and phosphate are both remineralized relatively efficiently in order to support the observed upper water column correlations (Strzepek et al., 2005). Alternatively, incorporation of trace elements into biogenic minerals such as opal is believed to be a relatively small reservoir at this time (e.g., zinc in diatom frustules; Ellwood and Hunter, 1999). Presumably, if the phytoplankton community structure remains consistent (as well as the associated average cellular quota), uptake and remineralization of cobalt and phosphate should not affect the HTM:P slope, only the movement up or down the diagonal HTM:P line.

The Co:P correlation in the upper water column is beginning to appear to as a common oceanographic feature with the notable exception of oligotrophic regions such as the Sargasso Sea and coastal environments (Saito and Moffett, 2002). The site of this study occurs in the North Pacific oligotrophic gyre, yet is clearly influenced by island and eddy effects, and hence could be considered a transition from an oligotrophic system to a moderately more productive one. When one compares the various vectors in cobalt-phosphate space described above and in Fig. 8A, it is clear that a correlation between these two nutrients would form when the two biological processes of uptake and remineralization dominate over the remaining processes of aeolian deposition, upwelling, and scavenging ([uptake+remineralization] $\gg$ [dust+upwelling+scavenging]). Uptake and remineralization work along the HTM:P line, whereas dust and scavenging work along the vertical axis. The former tend to enhance correlations, whereas the latter tend to destroy them. Upwelling ranges from nearly horizontal (upwelling deep water with high phosphate and low cobalt), to slightly inclined (upwelling from shallower depths where there is some Co:P correlation). We believe the waters sampled during our E-Flux II and III cruises may have been transitioning to such a system where biological uptake and remineralization began to dominate over those other

Fig. 8. Schematic of the various processes influencing the Co:P correlation in hybrid-type metal-phosphate (HTM:P) space. (A) The diagonal HTM:P line represents the aggregate biological cellular quotas for the hybrid-type trace metal and P. Remineralization and biological uptake should change the placement of a given sample along that line, but not deviate from it. Deviation from this line is caused by processes affecting the metal concentration but not phosphate cons bic concentic scavenging refers to co-oxidation by manganese oxidizers and is distinct from biological utilization as a micronutrient), aeolian deposition, or release from sediments. The process of "uptake by biota/entrainment" should manipulate both the hybrid-trace metal and phosphate simultaneously, first through biological uptake then through remineralization of particulate organic matter. Each phytoplankton species within the natural phytoplankton assemblage likely has a unique macronutrient and micronutrient cellular stoichiometry based on the specific environmental concentrations of those nutrients (e. Sunda and Hunts(1995). Thus each arrow in the "uptake by biota/ents man, 1995). Thus each aroup represents a potential species and its stoichiometry, and the summation of these species create the aggregate community ratio of HTM:P. Upwelling will affect both elements, however due to the depletion of HTM in intermediate waters by scavenging, the ratio of HTM:P in upwelled waters will be lower than in surface waters, with the range of arrows representing variability in the extent of HTM scavenging. (B) Variations in aggregate biological cellular quotas for the hybridtype trace metal and P can influence the slope of the HTM:P line significantly. A range of Co:P correlations that have been observed previously are shown range of Co:P conn $Q_{\mathrm{Co}}=248 \mu: \mathrm{C}$ Martin et al., 1989). Factors that could affect $Q_{\text {co }}$ include biological community composition, community shift, biochemical substitution of zinc for cobalt in eukaryotic phytoplankton, and changes in the bioavailability of cobalt. 


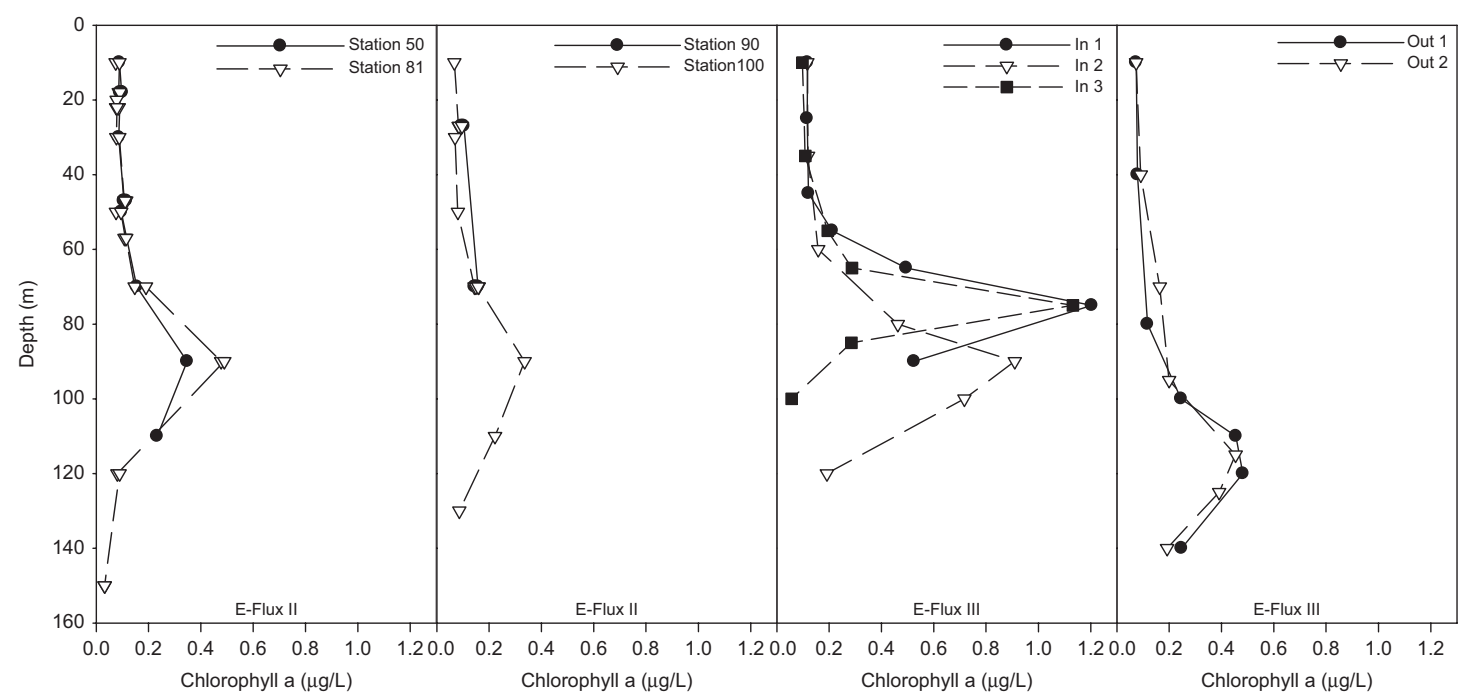

Fig. 9. Total Chlorophyll $a$ data for select E-Flux II and III In and Out stations. There is a noticeable increase in Chlorophyll $a$ concentration at the E-Flux III In stations in comparison to E-Flux II and the Out stations in E-Flux III.

processes, perhaps due to the combination of eddy and island induced increases in productivity. This interpretation suggests that there is a minimum productivity threshold above which the formation of a Co:P correlation occurs in the upper water column. While it is tempting to try to assign a specific productivity value for this correlation formation, it is clearly dependent on the relative magnitude of uptake and remineralization versus the aeolian deposition, upwelling, and scavenging, all of which can vary significantly between environments.

There is evidence for this increase in biological processes controlling uptake and remineralization by Cyclone Opal. The chlorophyll concentrations in the photic zone during E-Flux III are considerably higher than those during E-Flux II (Fig. 9; Rii et al., 2008). Primary productivity and particulate organic carbon (POC) data were not available for E-Flux II; however, comparison of primary productivity and biomass for In and Out stations during the study of Cyclone Opal provides a range for the proposed threshold. Primary productivity inside Opal was determined to be $\sim 128 \mathrm{mmol} \mathrm{C} \mathrm{m}^{-2} \mathrm{~d}^{-1}$ based on phytoplankton growth and $\Delta^{18} \mathrm{O}$ data, compared to $\sim 46 \mathrm{mmol} \mathrm{C} \mathrm{m}^{-2} \mathrm{~d}^{-1}$ determined for outside Opal (Benitez-Nelson et al., 2007). Estimated phytoplankton biomass revealed an inventory of $220 \mathrm{mmol} \mathrm{C} \mathrm{m}^{-2}$ within Opal compared to $114 \mathrm{mmol} \mathrm{C} \mathrm{m}^{-2}$ in surrounding waters (BenitezNelson et al., 2007). Fucoxanthin, an accessory pigment of diatoms, had large inventory changes from outside to inside Opal, highlighting the impact of a strong diatom bloom on aggregate biomass estimates $\left(5.5 \mathrm{mg} \mathrm{m}^{-2} \mathrm{In}, 1.0 \mathrm{mg} \mathrm{m}^{-2}\right.$ Out) (BenitezNelson et al., 2007). In general, Cyclone Opal resulted in greater primary productivity and a shift in species composition, including the significant presence of diatoms, and both findings could have contributed to the concentrating mechanism as well as the entrainment of cobalt as a micronutrient.

There is likely a temporal component to the formation of the Co:P correlation that could be occurring on a timescale of weeks to months. This is consistent with our data where a weak correlation is observed during the earlier cruise (E-Flux II). However, the higher cobalt values from the Out station of the
Cyclone Opal E-Flux III cruise still fall on the Co:P line (Fig. 7B), suggesting the upper water column in the region may have been sufficiently "biologically entrained" since the previous cruise to allow formation of the Co:P correlation.

As mentioned above, large variations of $\mathrm{Co:P}$ slopes have been observed in different environments (e.g., Saito et al., 2004). In the Peru upwelling region, a large change in Co:P slope was observed when the complexation of cobalt changed significantly, with much higher slope when significant labile cobalt was present (Saito et al., 2004). This suggests that the bioavailability of hybridtype trace metals, in addition to the average community cellular quota described above, may also be important in controlling the HTM:P slope. The relative abundance of cobalt and zinc, via biological substitution in metalloenzymes, could also influence this slope (Sunda and Huntsman, 1995; Saito et al., 2002). In summary, small changes in biological productivity, biological diversity, bioavailability, and scavenging components in the water column may have large effects on the distribution of HTMs and their distribution relative to phosphate.

\subsection{The implications of a biological concentrating mechanism for hybrid-type metals}

The small marine inventories of hybrid-type metals results not only in their tendency to limit productivity (e.g., iron limitation), but they also leave the distributions of those elements relatively "exposed" to the influences of individual processes. In contrast, an element or nutrient with a much larger inventory (e.g., $\mathrm{Zn}, \mathrm{Cd}, \mathrm{Ni}$ nitrate, phosphate) is "buffered" against observable effects of such processes by the larger seawater reservoirs. As a result, any biotic or abiotic processes could presumably have a significant influence on the distribution because the change in cobalt concentration is significant relative to the cobalt already present. For example, $0.3 \mathrm{pmol} \mathrm{L}^{-1} \mathrm{~d}^{-1}$ of biological uptake (Saito et al., 2004) relative to a typical surface water $20 \mathrm{pM}$ dissolved cobalt concentration is significant fraction of the total cobalt. If that cobalt is taken up by 
the biota and remineralized at the $100-300 \mathrm{~m}$ depth, it will be evident as a peak since the concentrations at this depth are only $50 \mathrm{pM}$. Cd and Zn, however, would not show a peak since their concentrations at those depths are in the nanomolar range for $\mathrm{Cd}$ and $\mathrm{Zn}(\sim 1$ and $\sim 8 \mathrm{nM}$, respectively). The concentrating process we propose here is likely not specific to eddies, but the combination of upwelling, shallow remineralization, isolation from surrounding waters, and a large number of analyses has helped to identify it.

One potential criticism of this concentrating mechanism is that if cobalt and phosphate are correlated, why would cobalt be concentrated in the upper water column while macronutrients or non-hybrid-type micronutrients are not? The answer to this critique is that those elements must also be affected similarly, but their much larger marine inventories make the resulting effects relatively small and hence they are likely obscured by the much higher concentrations at depth. For the hybrid-type elements such as cobalt and iron; however, this concentrating effect is likely significant and may be important for overall oceanic distributions. While we do not have iron data from E-Flux III, there does appear to be a shallow maxima in our iron data from the E-Flux II cruise, suggesting that such processes may be important for iron as well, even at lower productivities (Fig. 3B). In the coming years, improving our understanding of the competing controls on hybrid-type trace metals will continue to be a high priority.

\subsection{Intermediate water column results}

A maximum in cobalt concentration was observed at 400-600 $\mathrm{m}$ depth during both E-Flux II and E-Flux III (Figs. 2A and $10 \mathrm{~B}$ ). To a lesser extent, a deeper maximum was also present for manganese and perhaps iron at $\sim 600-700 \mathrm{~m}$ depth (Fig. $3 \mathrm{~A}$ and $B$ ). Two plausible sources for these maxima are hydrotherma input and/or island effects. The influence of hydrothermal sources on intermediate depth trace metal concentrations has been reported for the nearby Hawaiian Ocean Time-series station ALOHA (Boyle et al., 2005). In that study, helium $\left(\delta^{3} \mathrm{He}\right)$ served as a proxy for a hydrothermal signal, and enhanced dissolved iron concentrations were attributed to hydrothermal activity at Loih Seamount. It does not appear that the cobalt signal observed in the lee of the Hawaiian Islands was from a hydrothermal source due to the large difference in depth of signals: the iron concentration feature found at Station ALOHA was identified a approximately $1000-1500 \mathrm{~m}$ depth, similar to the $\left(\delta^{3} \mathrm{He}\right)$ signal and depth of Loihi Seamount (Boyle et al., 2005). While the smal manganese and iron maxima may be associated with hydrothermal activity, the cobalt feature we observed between Mau and Hawaii was significantly shallower $(400-600 \mathrm{~m})$ than the summits of Loihi and the nearby Mahukona Seamount, which is likely extinct (Garcia et al., 1990; Moore and Clague, 2004). It is improbable that the cobalt feature has a hydrothermal source without an explanation for transport across isopycnals.

Given the proximity of the E-Flux study sites to Hawaii and Maui, the islands and their associated shelf areas are the most likely sources of trace metals to the water column. As shown in the bathymetry data in Fig. 1B, there is a substantial shelf at $\sim 400-600 \mathrm{~m}$ depth particularly around the islands of Maui, Kahoolawe, Molokai, and Lana'i. Circulation of water masses between the islands may entrain significant concentrations of metals at the depth of the observed cobalt maxima. Recent results have demonstrated significant thermally driven water-rock circulation through porous basalt using excess ${ }^{223} \mathrm{Ra}$ off the lee side of Hawaii at depths coincident with and in very close geographic proximity to our observed cobalt peak (Moore et al., 2008). Moore et al. suggest that this ${ }^{223} \mathrm{Ra}$ signal was likely not a sediment resuspension signal as visual observation of the relatively young Puna Ridge on the other side of the island (where significant ${ }^{223} \mathrm{Ra}$ was also detected) revealed very little sediment cover (Moore et al., 2008). Given the similarities in depth and location of the ${ }^{223} \mathrm{Ra}$ and cobalt signatures, this thermally driven water-rock circulation may contribute significant cobalt from basaltic rock at intermediate depths between the Hawaiian Islands.

Island run-off and shelf submarine sediment resuspension are also possible sources of metals to the water column (Charette et al., 2007), although these phenomenon likely occur at shallower depths than intermediate cobalt depth peak observed here. In addition, it is possible that the Hawaiian eddies themselves contribute lateral transport of an intermediate depth source. The cyclonic circulation of Cyclone Opal penetrated to greater than $250 \mathrm{~m}$ depth, with water masses observed to maintain some cyclonic circulation to the $400 \mathrm{~m}$ maximum depth of ADCP measurements (Nencioli et al., 2008). Cyclone Opal also had a symmetrical shape with a diameter of $\sim 150 \mathrm{~km}$, large enough for its edges to come into contact with the shelves associated with Maui and Hawaii.

Manganese has previously been invoked as a tracer of sedimentary input and lateral advection (Martin et al., 1985), although secondary maxima in manganese have been convincingly argued to be the result of in situ redox processes in oxygen minimum zones (Johnson et al., 1996). Since the water column is well oxygenated here, a water-rock/sedimentary source seems more likely.

Interestingly, the chemical speciation of cobalt in this intermediate depth maximum shows only a small increase in labile cobalt relative to the total dissolved fraction, implying that this putative island source is releasing a non-labile form (Fig. 3C). This is in contrast to our previous studies in the Peru upwelling system, where we observed labile cobalt and proposed that it was being released from a sedimentary source through microbial reduction of manganese oxide and simultaneous low bottom water oxygen concentrations that prevented microbial reoxidation of cobalt and manganese before sedimentary escape (Saito et al. 2004 and references therein). Given that the intermediate depth waters near the Hawaiian Islands are well-oxygenated and the seafloor likely has a significantly different mineralogy than near Peru, it seems likely that the mechanism for cobalt release from the shelf environment may be quite distinct from that of the Peru upwelling region.

Manganese in the deeper waters typically displays depletion due to scavenging by sinking particles and/or microbial oxidizing activity. Our manganese depth profiles are consistent with previously reported concentrations by Boyle et al. (2005) with the exception of the above mentioned small maximum. In addition to the iron maxima mentioned above, iron concentrations we measure in the intermediate waters are somewhat higher $(0.68-1.48 \mathrm{nM})$ than those observed by Boyle et al. $(0.50-1.00 \mathrm{nM} ; 2005)$, perhaps due to closer proximity to either island/sedimentary/groundwater sources or hydrothermal sources. We are not aware of $\delta^{3} \mathrm{He}$ data from between the Hawaiian Islands that would allow us to confirm any hydrothermal signatures found from this region. These higher iron values appear to be real, given our successful intercalibration with the surface and deepwater SAFe standards (Johnson et al., 2007). We did not observe a peak in iron at $400-600 \mathrm{~m}$ depth as we did for cobalt; however, this may be due the rapid scavenging of iron in seawater (Wu and Luther, 1995) relative to cobalt, which might prevent an iron signal from appearing beyond immediate proximity with the shelf. We believe that this is a reasonable hypothesis given previous evidence suggesting the mechanisms for sedimentary release of cobalt and iron are different (Saito et al., 2004; Johnson et al., 1988). 


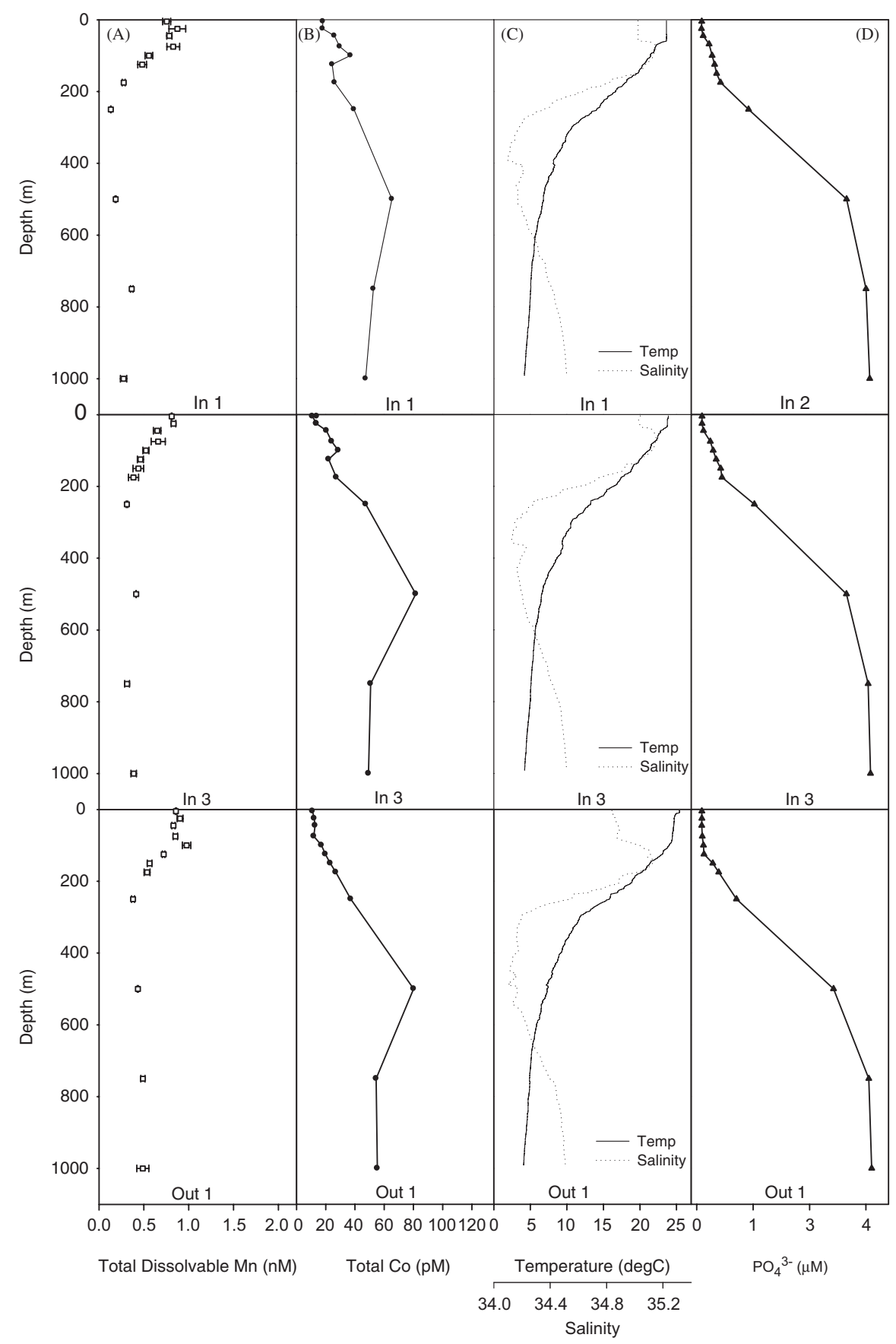

Fig. 10. Vertical profiles for total dissolvable manganese, total cobalt, phosphate, and hydrographic data for select stations in E-Flux III. Note that phosphate data were unavailable for In 1. Phosphate data for In 2 were less than one nautical mile from In 1, and are presented in place of In 1. High concentrations of cobalt are present at $400-600 \mathrm{~m}$ in all stations. The peaks at $400-600 \mathrm{~m}$ suggest that there is an intermediate depth source for influx of Co. The peaks are supported by replicates analyses, and by the supporting adjacent points in the profile. 


\section{Conclusions}

We have identified processes that affect the biogeochemistry of cobalt, manganese, and iron in both the surface and intermediate waters surrounding the Hawaiian Islands. We suggest that the shallow and near complete remineralization of sinking POC within Cyclone Opal resulted in a Co:P correlation and an accumulation of cobalt in the upper water column. This potential concentrating mechanism may be a general process important to the hybrid-type metals throughout the oceans. A change in Co:P correlation from January to March was observed, which we suggest may be due to a minimum primary productivity threshold. In addition, a cobalt maximum at $400-600 \mathrm{~m}$ was found for all stations studied. This was attributed to a source of cobalt and potentially manganese from the shelf that surrounds Maui, Kahoolawe, Molokai, and Lana'i islands. This sharp increase was not found for iron, and iron concentrations were somewhat higher than reported at the nearby station ALOHA.

\section{Acknowledgments}

We thank the Captain and crew of the R/V Wecoma, Marc Willis, Shimi Rii and Bob Bidigare for Chlorophyll data and supportive nutrient data, Ed Boyle for his suggestion of comparing cobalt and thorium data, E. Paul Oberlander for his help with graphics design of Fig. 6, and the WHOI ICP-MS facility and Dave Schneider. We thank two anonymous reviewers and editor Dennis McGillicuddy for helpful comments on this manuscript. This research was supported by NSF Grants OCE-0327225, OCE0452883, OPP-0440840, the Office of Naval Research, the Center for Environmental Bioinorganic Chemistry at Princeton, and the Center for Microbial Oceanography and Education.

\section{References}

Allen, C.B., Kanda, J., Laws, E.A., 1996. New production and photosynthetic rates within and outside a cyclonic mesoscale eddy in the North Pacific subtropical gyre. Deep-Sea Research I 43, 917-936.

Benitez-Nelson, C.R., 2000. The biogeochemical cycling of phosphorus in marine systems. Earth-Science Reviews 51, 109-135.

Benitez-Nelson, C., Bidigare, R.R., Dickey, T.D., Landry, M.R., Leonard, C.L., Brown, S.L., Nencioli, F., Rii, Y.M., Maiti, K., Becker, J.W., Bibby, T.S., Black, W., Cai, W.J., Carlson, C.A., Chen, F., Kuwahara, V.S., Mahaffey, C., McAndrew, P.M., Quay, P.D., Carlson, C.A., Chen, F., Kuwahara, V.S., Mahaffey, C., McAndrew, P.M., Quay, P.D.,
Rappe, M.S., Selph, K.E., Simmons, M.P., Yang, E.J., 2007. Mesoscale eddies drive Rappe, M.S., Selph, K.E., Simmons, M.P., Yang, E.J., 2007. Mesoscale eddies drive
increased silica export in the subtropical Pacific Ocean. Science 316, increased $1017-1020$.

Bidigare, R.R., Benitez-Nelson, C., Leonard, C.L., Quay, P.D., Parsons, M.L., Foley, D.G. Seki, M.P., 2003. Influence of a cyclonic eddy on microheterotroph biomass and carbon export in the lee of Hawaii. Geophysical Research Letters 30 (6), 1318 carbon export in the lee of Hawaii. Geophysical Research Letters 30 (6), 1318.
Boyle, E.A., Sclater, F., Edmond, J.M., 1976. Marine geochemistry of cadmium. Nature 263 (5572), 42-44.

Boyle, E.A., Berquist, B.A., Kayser, R.A., Mahowald, N., 2005. Iron, manganese, and lead at Hawaii Ocean Time-series station ALOHA: temporal variability and an intermediate water hydrothermal plume. Geochimica et Cosmochimica Acta $69,933-952$

Bronk, D.A., Gilbert, P.M., Ward, B.B., 1994. Nitrogen uptake, dissolved organic nitrogen release, and new production. Science 265, 1843-1852.

Brown, M.T., Landing, W.M., Measures, C.I., 2005. Dissolved and particulate Fe in the western and central North Pacific: results from the 2002 IOC cruise. Geochemistry Geophysics Geosystems 6 (10).

Brown, S.L., Landry, M.R., Selph, K.E., Yang, E.J., Rii, Y.M., Bidigare, R.R., 2008 Diatoms in the desert: Plankton community response to a mesoscale eddy in the subtropical North Pacific. Deep-Sea Research II, this issue [doi:10.1016 j.dsr2.2008.02.012].

Bruland, K., 1980. Oceanographic distributions of cadmium, zinc, nickel, and copper in the North Pacific. Earth and Planetary Science Letters 47 (2), 176-198.

Bruland, K.W., Lohan, M.C., 2004. Controls of trace metals in seawater. In: Elderfield, H., Holland, H.D., Turekian, K.K. (Eds.), Treatise on Geochemistry, vol. 6, The Oceans and Marine Geochemistry. 6.02, pp. 23-47.

Bruland, K.W., Rue, E.L., Smith, G.J., DiTullio, G.R., 2005. Iron, macronutrients, and diatom blooms in the Peru upwelling regime: brown and blue waters of Peru. Marine Chemistry 93, 81-103.
Calil, P.H.R., Richards, K.J., Jia, Y., Bidigare, R.R., 2008. Eddy activity in the lee of the Hawaiian Islands. Deep-Sea Research II, this issue [doi:10.1016] j.dsr2.2008.01.008].

Charette, M.A., Gonneeaa, M.E., Morris, P.J., Statham, P., Fones, G., Planquette, H., Salter, I., Garabatob, A.N., 2007. Radium isotopes as tracers of iron sources fueling a Southern Ocean phytoplankton bloom. Deep-Sea Research II 54, 1989-1998.

Chase, T.E., Miller, C.P., Seekins, B.A., Normark, W.B., Gutmacher, C.E., Wilde, P., Young, J.D., 1980. Topography of the Southern Hawaiian Islands. United States Geological Survey Open File Map 81-120, Plates 1-3.

Chavanne, C., Flament, P., Lumpkin, R., Dousset, B., Bentamy, A., 2002. Scatterometer observations of wind variations induced by oceanic islands: implications or wind-driven ocean circulation. Canadian Journal of Remote Sensing 28 (3), 466-474.

Chen, J.H., Edwards, R.L., Wasserburg, G.J., 1986. ${ }^{238} \mathrm{U},{ }^{234} \mathrm{U}$ and ${ }^{232} \mathrm{Th}$ in seawater. Earth and Planetary Science Letters 80, 241-251

Chen, F., Cai, W.-J., Wang, Y., Rii, Y.M., Bidigare, R.R., Benitez-Nelson, C.R., 2008. The carbon dioxide system and net community production within a cyclonic eddy in the lee of Hawaii. Deep-Sea Research II, this issue [doi:10.1016] j.dsr2.2008.01.011].

Cheney, R.E., Richardson, P.L., 1976. Observed decay of a cyclonic Gulf Stream ring. Deep-Sea Research 23 (2), 143-155.

Cullen, J., Chase, Z., Coale, K., Fitzwater, S., Sherrell, R., 2003. Effect of iron limitation on the cadmium to phosphorus ratio of natural phytoplankton assemblages from the Southern Ocean. Limnology and Oceanography 48 (3). 1079-1087.

DeCarlo, E.H., Beltran, V.L., Tomlinson, M.S., 2004. Composition of water and suspended sediment in streams of urbanized subtropical watersheds in Hawaii. Applied Geochemistry 19, 1011-1037.

DeCarlo, E.H., Tomlinson, M.S., Anthony, S.S., 2005. Trace elements in streambed sediments of small subtropical streams on O'ahu, Hawai'i: results from the USGS NAWQA Program. Applied Geochemistry 20, 2157-2188.

Dickey, T.D., Nencioli, F., Kuwahara, V.S., Leonard, C.L., Black, W., Rii, Y.M., Bidigare, R.R., Zhang, Q., 2008. Physical and bio-optical observations of oceanic cyclones
w. R.R., Zhang, Q., 2008. Physical and bio-optical observations of oceanic cyclones
west of the island of Hawai'i. Deep-Sea Research II, this issue [doi:10.1016/ j.dsr2.2008.01.006].

Eakins, B.W., Robinson, J.E., Kanamatsu, T., Naka, J., Smith, J.R., Takahashi, E., Clague, D.A., 2003. Hawaii's Volcanoes Revealed. United States Geological Survey Geologic Investigations Series I-2809.

Edmond, J.M., Measures, C., McDuff, R.E., Chan, L.H., Collier, R., Grant, B., Gordon, L.I., Corliss, J.B., 1979. Ridge crest hydrothermal activity and the balances of the major and minor elements in the ocean: the Galapagos data. Earth and Planetary Science Letters 46, 1-18.

Ellwood, M.J., Hunter, K.A., 1999. Determination of the Zn/Si ratio in diatom opal: a method for the separation, cleaning and dissolution of diatoms. Marine Chemistry 66, 149-160

Emerson, S., Kalhorn, S., Jacobs, L., Tebo, B.M., Nealson, K.H., Rosson., R.A., 1982. Environmental oxidation rate of manganese(II): bacterial catalysis. Geochimica et Cosmochima Acta 46 (6), 1073-1079.

Falkowski, P.G., Ziemann, D., Kolber, Z., Bienfang, P.K., 1991. Role of eddy pumping in enhancing primary production in the ocean. Nature $352,55-58$.

Fischer, A.S., Weller, R.A., Rudnick, D.L., Eriksen, C.C., Lee, C.M., Brink, K.H., Fox, C.A., Leben, R.R., 2002. Mesoscale eddies, coastal upwelling, and the upper-ocean heat budget in the Arabian Sea. Deep-Sea Research II 49 (12), 2231-2264.

Garcia, M.O., Kurz, M.D., Muenow, D.W., 1990. Mahukona: the missing Hawaiian volcano. Geology 18, 1111-1114.

Gordon, R.M., Johnson, K.S., Coale, K.H., 1998. The behaviour of iron and other trace elements during the IronEx-I and PlumEx experiments in the Equatorial Pacific. Deep-Sea Research II 45, 995-1041.

Herrera-Melian, J., Hernandez-Brito, J., Gelado-Caballero, M., Perez-Pena, J., 1994. Direct determination of cobalt in unpurged oceanic seawater by high speed Direct determination of cobalt in unpurged oceanic seawater by high speed
adsorptive cathodic stripping voltammetry. Analytica Chimica Acta 299, adsorptive

Johnson, K.S., Stout, P.M., Berelson, W.M., Sakamoto-Arnold, C.M., 1988. Cobalt and copper distributions in the waters of Santa Monica Basin, California. Nature 332, 527-530.

Johnson, K.S., Coale, K.H., Berelson, W.M., Gordon, R.M., 1996. On the formation of the manganese maximum in the oxygen minimum. Geochimica et Cosmochimica Acta 60 (8), 1291-1299.

Johnson, K.S., Gordon, R.M., Coale, K.H., 1997. What controls dissolved iron concentrations in the world ocean? Marine Chemistry 57, 137-161.

Johnson, K.S., Elrod, V.A., Fitzwater, S.E., Plant, J.N., Chavez, F.P., Tanner, S.J., Gordon, R.M., Westphal, D.L., Perry, K.D., Wu, J., Karl, D.M., 2003. Surface ocean-lower atmosphere interactions in the Northeast Pacific Ocean gyre: aerosols, iron and the ecosystem response. Global Biogeochemical Cycles 17. doi:10.1029/ 2002GB002004

Johnson, W.K., Miller, L.A., Sutherland, N.E., Wong, C.S., 2005. Iron transport by mesoscale Haida eddies in the Gulf of Alaska. Deep-Sea Research II 52, 933-953.

Johnson, K.S., Boyle, E., Bruland, K., Coale, K., Measures, C., Moffett, J., Aguilar-Islas, A., Barbeau, K., Bergquist, B., Bowie, A., Buck, K., Cai, Y., Chase, Z., Cullen, J., Doi, T., Elrod, V., Fitzwater, S., Gordon, M., King, A., Laan, P., Laglera-Baquer, P., Landing, W., Lohan, M., Mendez, J., Milne, A., Obata, H., Ossiander, L., Plant, J., Sarthou, G., Sedwick, P., Smith, G.J., Sohst, B., Tanner, S., van den Berg, S., Wu, J., 2007. Developing standards for dissolved iron in seawater. EOS 88 (11), $131-132$. 
Karl, D.M., Yanagi, K., 1997. Partial characterization of the dissolved organic phosphorus pool in the Oligotrophic North Pacific Ocean. Limnology and Oceanography 42 (6), 1398-1405.

Lumpkin, C.F. 1998. Eddies and currents in the Hawaii islands. Ph.D. Thesis, University of Hawaii.

Maiti, K., Benitez-Nelson, C.R., Rii, Y.M., Bidigare, R.R., 2008. The influence of mature cyclonic eddy on particle export in the lee of Hawaii. Deep-Sea Research II, this issue [doi: 10.1016/j.dsr2.2008.02.008]

Martin, J.H. Knauer, G.A., Broenkow, W.W., 1985. VERTEX: the lateral transport of $\mathrm{Mn}$ in the northeast Pacific. Deep-Sea Research I 32 (11), 1405-1427.

Martin, J.H., Gordon, R.M., Fitzwater, S., Broenkow, W.W., 1989. VERTEX: phytoplankton/iron studies in the Gulf of Alaska. Deep-Sea Research I 36 (5) $649-680$

Martin, J.H., Gordon, R.M., Fitzwater, S.E., 1991. The case for iron. Limnology and Oceanography $36(8), 1793-1802$.

McGillicuddy, D.J., Robinson, A.R., Siegel, D.A., Jannasch, H.W., Johnson, R., Dickey, T.D., McNeil, J., Michaels, A.F., Knap, A.H., 1998. Influence of mesoscale eddies on new production in the Sargasso Sea. Nature 394, 263-266.

McGillicuddy, D.J., Anderson, L.A., Bates, N.R., Bibby, T., Buesseler, K.O., Carlson, C.A., Davis, C.S., Ewart, C., Falkowski, P.G., Goldthwait, S.A., Hansell, D.A., Jenkins, W.J., Johnson, R., Kosnyrev, V.K., Ledwell, J.R., Li, Q.P., Siegel, D.A., Steinberg, D.K., 2007. Eddy/wind interactions stimulate extraordinary midocean plankton blooms. Science 316, 1021-1026.

Moffett, J.W., Ho, J., 1996. Oxidation of cobalt and manganese in seawater via common microbially catalyzed pathway. Geochimica et Cosmochimica Acta 60 (18), 3415-3424

Moore, J.G., Clague, D.A., 2004. Hawaiian submarine manganese-iron oxide crusts - a dating tool? GSA Bulletin 116, 337-347.

Moore, W.S., Ussler, W., Paull, C.K., 2008. Short-lived radium isotopes in the Hawaiian margin: evidence for large fluid fluxes through the Puna Ridge. Marine Chemistry 109, 421-430.

Morel, F.M.M., Milligan, A.J., Saito, M.A., 2003. Marine bioinorganic chemistry: the role of trace metals in the oceanic cycles of major nutrients. In: Elderfield, $H$., Holland, H.D. Turekian, KK. (Eds), Treatise on Geochemistry, vol. 6, Oceans and Marine Geochemistry. 6.05, pp. 113-143.

Nencioli, F., Kuwahara, V.S., Dickey, T.D., Rii, Y.M., Bidigare, R.R., 2008. Physical dynamics and biological implications of a mesoscale eddy in the lee of Hawai'i: Cyclone Opal observations during E-Flux III. Deep-Sea Research II, this issue Cyclone Opal observations durin
[doi:10.1016/j.dsr2.2008.02.003].

Olaizola, M., Ziemann, D.A., Bienfang, P.K., Walsh, W.A., Conquest, L.D., 1993. Eddyinduced oscillations of the pycnocline affect the floristic composition and depth distribution of phytoplankton in the subtropical Pacific. Marine Biology 116, 533-542.

Oschlies, A., Garcon, V., 1998. Eddy-induced enhancement of primary production in a model of the North Atlantic Ocean. Nature 394, 266-269.

Parrington, J.R., Zoller, W.H., Aras, N.K., 1983. Asian dust: seasonal transport to the Hawaiian Islands. Science 220, 195-198.

Patzert, W.C., 1969. Eddies in Hawaiian Islands. Technical Report. HIG-69-8, Hawail Institute of Geophysics, University of Hawail.

Perry, K.D., Cahill, T.A., Schnell, R.C., Harris, J.M., 1999. Long-range transport of anthropogenic aerosols to the National Oceanic and Atmospheric Administration baseline station at Mauna Loa Observatory, Hawaii. Journal of Geophysica Research 104, 18521-18533.

Peterson, T.D., Frank, A.W., Harrison, P.J., 2005. Macronutrient dynamics in an anticyclonic mesoscale eddy in the Gulf of Alaska. Deep-Sea Research II 52 909-932.

Pike, S.M., Buesseler, K.O., Andrews, J.A., Savoye, N., 2005. Quantification of ${ }^{234} \mathrm{Th}$ recovery in small volume sea water samples by inductively coupled plasma mass spectrometry. Journal of Radioanalytical and Nuclear Chemistry 263 (2) $355-360$

Redfield, S.C., 1958. The biological control of chemical factors in the environment American Scientist 46, 205-222.

Rii, Y.M., Brown, S.L., Nencioli, F., Kuwahara, V.S., Dickey, T.D., Karl, D.M., Bidigare, R.R., 2008. The transient oasis: Nutrient-phytoplankton dynamics and particle export in Hawaiian lee cyclones. Deep-Sea Research II, this issue [doi:10.1016/ j.dsr2.2008.01.013

Rutgers van der Loeff, M.R., Sarin, M.M., Baskaran, M., Benitez-Nelson, C., Buessele K.O., Charette, M., Dai, M., Gustafsson, O., Masque, P., Morris, P.J., 2006. A review of present techniques and methodological advances in analyzing ${ }^{234} \mathrm{~A}$ in aquatic systems. Marine Chemistry $100(3 / 4), 190-212$

Saito, M.A., Moffett, J.W., 2001. Complexation of cobalt by natural organic ligands in the Sargasso Sea as determined by a new high-sensitivity electrochemica cobalt speciation method suitable for open ocean work. Marine Chemistry 75 , $49-68$.
Saito, M.A., Moffett, J.W., 2002. Temporal and spatial variability of cobalt in the Athicic Ocean Geochimica et Cosmochimica Acta 66, 1943-1953.

Saito, MA Schneider D. 2006. Examination of precipitation chemistry and improvements in precision using the $\mathrm{Mg}(\mathrm{OH})_{2}$ preconcentration inductively coupled plasma mass spectrometry (ICP-MS) methods for high-throughput analysis of open-ocean Fe and Mn in seawater. Analytica Chimica Acta 565, $222-233$

Saito, M.A., Moffett, J.W., Chisholm, S.W., Waterbury, J.B., 2002. Cobalt limitation and uptake in Prochlorococcus. Limnology and Oceanography 47 (6) 1629-1636.

Saito, M.A., Moffett, J.W., DiTullio, G.R., 2004. Cobalt and nickel in the Peru upwelling region: a major flux of labile cobalt utilized as a micronutrient. Cycles 18. doi:10.1029/2003GB002216.

Saito, M.A., Rocap, G., Moffett, J.W., 2005. Production of cobalt binding ligands in a Synechococcus feature at the Costa Rica upwelling dome. Limnology and Oceanography 50 (1), 279-290.

Sakamoto, C., Yuji, O., Kanazawa, K., Ayano, E., Nishimura, T., Ando, M., Kikuchi, A., Okano, T., 2004. Temperature- and pH-responsive aminopropyl-silica ionexchange columns grafted with copolymers of $\mathrm{N}$-isopropylacrylamide. Journal of Chromatography A 1030, 247-253.

Sedwick, P.N., McMurty, G.M., Macdougall, J.D., 1992. Chemistry of hydrotherma solutions from Pele's Vents, Loihi Seamount, Hawaii. Geochimica et Cosmochimica Acta 56, 3643-3667.

Sedwick, P.N., Church, T.M., Bowie, A.R., Marsay, C.M., Ussher, S.J., Achilles, K.M., Lethaby, P.J., Johnson, R.J., Sarin, M.M, McGillicuddy, D.J., 2005. Iron in the Sargasso Sea (Bermuda Atlantic Time-Series Study Region) during summer Eolian imprint, spatiotemporal variability, and ecological implications. Global Biogeochemical Cycles 19. doi:10.1029/2004GB002445.

Seki, M.P., Polovina, J.J., Brainard, R.E., Bidigare, R.R., Leonard, C.L., Foley, D.G., 2001 Biological enhancement at cyclonic eddies tracked with GOES thermal imagery in Hawaiian waters. Geophysical Research Letters 28 (8), 1583-1586.

Seki, M.P., Lumpkin, R., Flament, P., 2002. Hawaii cyclonic eddies and blue marlin catches: the case study of the 1995 Hawaiian International Billfish Tournament. Journal of Oceanography 58 (5) 739-745.

Sherrell, R.M., Boyle, E.A., 1992. The trace metal composition of suspended particles in the oceanic water column near Bermuda. Earth and Planetary Science Letters $111,155-174$.

Strzepek, R.F., Maldonado, M.T., Higgins, J.L., Hall, J., Safi, K., Wilhelm, S.W., Boyd, P.W., 2005. Spinning the "Ferrous Wheel": the importance of the microbial P.W., 2005. Spinning the "Ferrous Wheel": the importance of the microbial
community in an iron budget during the FeCycle experiment. Global community in an iron budg

Sunda, W.G., Huntsman, S.A., 1985. Regulation of cellular manganese and manganese transport rates in the unicellular alga Chlamydomonas. Limnology manganese transport rates in
and Oceanography $30,71-80$.

Sunda, W.G.. Huntsman, S.A., 1988. Effect of sunlight on redox cycles of manganese in the southwestern Sargasso Sea. Deep-Sea Research 35, 1297-1317.

Sunda, W.G., Huntsman, S.A., 1995. Cobalt and zinc interreplacement in marine phytoplankton: biological and geochemical implications. Limnology an

Taylor, S.R., McLennan, S.M., 1985. The Continental Crust: Its Composition and Evolution. Blackwell Scientific Publications.

Tebo, B., Nealson, K., Emerson, S., Jacobs, L., 1984. Microbial mediation of Mn(II) and $\mathrm{Co}(\mathrm{II})$ precipitation at the $\mathrm{O}_{2} / \mathrm{H}_{2}$ interfaces in two anoxic fjords. Limnology and Oceanography $29,1247-1258$.

Thamdrup, B., Glud, R.N., Hansen, J.W., 1994. Manganese oxidation and in situ manganese fluxes from a coastal sediment. Geochimica et Cosmochimica Acta 58 (11), 2563-2570.

Tyrell, T., 1999. The relative influence of nitrogen relative to phosphorus on oceanic primary production. Nature $400,525-531$

Vaillancourt, R.D., J, M., Seki, M.P., Parsons, M.L., Bidigare, R.R., 2003. Impact of a cyclonic eddy on phytoplankton community structure and photosynthetic competency in the subtropical North Pacific Ocean. Deep-Sea Research I 50 829-847.

Vega, M., van den, B., Constant, M.G., 1997. Determination of cobalt in seawater by catalytic adsorptive cathodic stripping voltammetry. Analytical Chemistry 69 874-881.

Wheat, C.G., Jannasch, H.W., Plant, J.N., Moyer, C.L., Sansone, F.J., McMurty, G.M., 2000. Continuous sampling of hydrothermal fluids from Loihi Seamount afte the 1996 event. Journal of Geophysical Research 105 (B8), 19,353-19,367.

Wong, G.T., Pai, S., Chung, S., 1995. Cobalt in the Western Philippine Sea Oceanologica Acta 18 (6), 631-638.

$\mathrm{Wu}$, J., Luther, G.W., 1995. Complexation of Fe(III) by natural organic ligands in the Northwest Atlantic Ocean by a competitive ligand equilibration method and a kinetic approach. Marine Chemistry 50, 159-177. 


\title{
Chapter 3: Basin-scale inputs of cobalt, iron, and manganese from the Benguela-Angola Front into the South Atlantic Ocean
}

\author{
Revisions submitted to Limnology and Oceanography
}

\begin{abstract}
We present full-depth zonal sections of total dissolved iron, cobalt, manganese, and labile cobalt from the South Atlantic Ocean. A basin-scale plume from the African coast appears to be a major source of dissolved metals to this region, with high cobalt concentrations in the oxygen minimum zone of the Angola Dome and extending $2500 \mathrm{~km}$ into the subtropical gyre. Metal concentrations are elevated along the coastal shelf, likely due to reductive dissolution and resuspension of particulate matter. Lateral advection coupled with upwelling, biological uptake, and remineralization deliver these elevated coastal concentrations to the basin, as evident in differences between the two zonal transects with distinct physical processes. Scavenging rates within the coastal plume differ for the three metals; iron is removed fastest, manganese removal is 2.5 times slower, and cobalt scavenging cannot be discerned from water mass mixing, persisting in the water column. Because scavenging, biological utilization, and export constantly deplete the oceanic inventories of these three hybrid-type metals, point sources of the scale observed here likely serve as vital drivers of their oceanic cycles. Manganese concentrations are elevated in surface waters across the basin, likely due to coupled redox processes acting to concentrate the dissolved species there. These observations of basin-scale hybrid metal plumes combined with the recent projections of expanding oxygen minimum zones suggest a potential mechanism for effects on ocean primary production and nitrogen fixation via increases in trace metal source inputs.
\end{abstract}




\section{Basin-scale inputs of cobalt, iron, and manganese from the Benguela-Angola front to the South Atlantic Ocean}

Abigail E. Noble, ${ }^{\mathrm{a}, \mathrm{b}}$ Carl H. Lamborg, ${ }^{\mathrm{a}}$ Dan C. Ohnemus, ${ }^{\mathrm{a}, \mathrm{b}}$ Phoebe J. Lam, ${ }^{\mathrm{a}}$ Tyler J. Goepfert, ${ }^{\mathrm{a}, \mathrm{b}}$ Chris I. Measures, ${ }^{\mathrm{c}}$ Caitlin H. Frame, ${ }^{\mathrm{a}, \mathrm{b}}$ Karen L. Casciotti, ${ }^{\mathrm{a}}$ Giacomo R. DiTullio, ${ }^{\mathrm{d}}$ Joe Jennings, ${ }^{\mathrm{e}}$ Mak A. Saito, ${ }^{\mathrm{a}}$

a) Woods Hole Oceanographic Institution (WHOI), Woods Hole, Massachusetts

b) Massachusetts Institute of Technology (MIT) - WHOI Joint Program in Chemical Oceanography Massachusetts Institute of Technology, Cambridge, Massachusetts

c) University of Hawaii, Honolulu Hawaii

d) College of Charleston, Charleston, South Carolina

e) College of Oceanic \& Atmospheric Sciences, Oregon State University, Corvallis, Oregon.

* Corresponding author:msaito@whoi.edu

Running head: Basin-scale inputs of $\mathrm{Co}, \mathrm{Fe}$, and $\mathrm{Mn}$

Revisions for Limnology and Oceanography

July 27, 2011 
Acknowledgements: We thank Gabrielle Rocap, Chad Hammerschmidt, Alysia Cox, Marian Westley, and Mike Sieracki for assistance during the cruise, Erin Bertrand and Ed Sholkovitz, for helpful conversations, and Ken Johnson and an anonymous reviewer for useful comments. We are particularly indebted to the Captain and Crew of the R/V Knorr: without their exceptional support, this ocean section would not have been possible. We also thank Scot Birdwhistell in the Woods Hole Oceanographic Institution (WHOI) inductively coupled plasma mass spectrometry (ICP-MS) facility. This research was supported US National Science Foundation Chemical Oceanography (Division of Ocean Sciences OCE-0452883, OCE-0752291, OCE-0928414, OCE-1031271), the Center for Microbial Research and Education, the WHOI Coastal Ocean Institute, and the WHOI Ocean Life Institute. 


\begin{abstract}
We present full-depth zonal sections of total dissolved iron, cobalt, manganese, and labile cobalt from the South Atlantic Ocean. A basin-scale plume from the African coast appears to be a major source of dissolved metals to this region, with high cobalt concentrations in the oxygen minimum zone of the Angola Dome and extending $2500 \mathrm{~km}$ into the subtropical gyre. Metal concentrations are elevated along the coastal shelf, likely due to reductive dissolution and resuspension of particulate matter. Lateral advection coupled with upwelling, biological uptake, and remineralization deliver these elevated coastal concentrations to the basin, as evident in two zonal transects with distinct physical processes that exhibit different metal distributions. Scavenging rates within the coastal plume differ for the three metals; iron is removed fastest, manganese removal is 2.5 times slower, and cobalt scavenging cannot be discerned from water mass mixing, persisting in the water column. Because scavenging, biological utilization, and export constantly deplete the oceanic inventories of these three hybrid-type metals, point sources of the scale observed here likely serve as vital drivers of their oceanic cycles. Manganese concentrations are elevated in surface waters across the basin, likely due to coupled redox processes acting to concentrate the dissolved species there. These observations of basinscale hybrid metal plumes combined with the recent projections of expanding oxygen minimum zones suggest a potential mechanism for effects on ocean primary production and nitrogen fixation via increases in trace metal source inputs.
\end{abstract}




\subsection{Introduction}

Iron, manganese, and cobalt play essential roles as micronutrients in ocean ecosystems. These metals are involved in photosynthesis, nitrogen fixation, and many other cellular processes (Morel et al. 2003), but can exist in such low concentrations in seawater that they may limit or colimit the growth of primary producers in the open ocean (Moore et al. 2004; Saito et al. 2008). Iron is predicted to limit 40\% of ocean productivity and much of the marine nitrogen fixation capacity (Moore et al. 2009). Photosystems I and II (PSI and PSII) are iron intensive, and iron is required in many enzymatic pathways of the microbial nitrogen loop, including nitrogen fixation (Morel et al. 2003; Saito et al. 2011). Manganese, while typically abundant in the upper ocean of tropical and subtropical waters, is used to oxidize water to $\mathrm{O}_{2}$ in PSII, to detoxify cells from harmful $\mathrm{O}_{2}{ }^{-}$radicals via its incorporation into superoxide dismutase, and it is an electron acceptor for manganese-oxidizing bacteria (Tebo et al. 1984; Moffett and Ho 1995; Morel et al. 2003). Cobalt has been demonstrated to co-limit the growth of some phytoplankton in culture studies (Saito and Goepfert 2008 and references therein), and some field studies either as inorganic cobalt (Saito et al. 2005) or as the metallocenter of vitamin $\mathrm{B}_{12}$ in high latitude environments (Bertrand et al. 2007; Panzeca et al. 2006). Cobalt can also be incorporated into the active site of carbonic anhydrase in diatoms (Roberts et al. 1997), and is required by the abundant marine cyanobacteria, Prochlorococcus and Synechococcus (Saito et al. 2002).

Recent decades of field sampling and careful low-level analyses have identified sources and sinks to the water column trace metal inventory that include aeolian deposition, hydrothermal venting, riverine discharge, anthropogenic-derived input, 
reductive dissolution, sediment resuspension, biological uptake and remineralization, and scavenging (Bruland and Lohan, 2003). With these observations, dissolved metal profiles have been categorized into conservative, nutrient-like, scavenged, and hybrid-type (Bruland et al. 2003). Iron, cobalt, and manganese fall into the hybrid-type category since their distributions are controlled by a combination of phytoplankton uptake, which creates nutrient-like distributions in the photic zone, and scavenging, which creates scavenged-like distributions in intermediate and deep waters. These three metals are distinct from other trace element micronutrients, such as zinc and nickel, because they are redox active, and have small oceanic inventories and whole ocean residence times on the order of 40-150 years due to scavenging (Bruland et al. 1994; Saito and Moffett 2002). Scavenging plays an important role in preventing accumulation of hybrid-type metals with thermohaline circulation, which is seen in the accumulation of macronutrients like phosphate and nitrate, and nutrient-type trace metals like cadmium and zinc between ocean basins. The reduced forms of these hybrid-type metals can be introduced to oxygenated seawater by reductive dissolution within anoxic pore waters (Heggie and Lewis 1984), exchange with particles (Lam et al. 2006; Lohan and Bruland 2008), organic matter decomposition, and photoreduction (Sunda and Huntsman 1988), while scavenging removes them from the water column. The biotic oxidation of manganese and cobalt by manganese-oxidizing bacteria is thought to be an important removal mechanism for these two metals (Tebo 1984; Moffett and Ho 1996). Complexation by organic ligands is thought to protect iron and cobalt from scavenging to some degree (Johnson et al. 1997; Saito and Moffett 2001), and recent work has suggested that manganese may also be complexed by some marine 
siderophores (Parker et at. 2007; Homann et al. 2009), although the influence of complexation on manganese scavenging is still largely unknown.

A primary goal of this study was to investigate how the relative distributions of cobalt, iron, and manganese change across a strong biogeochemical gradient found in the oxygen minimum zone (OMZ) of the South Atlantic Ocean. This was accomplished by collection and analysis of a full-depth zonal cross-section of these dissolved metals during the CoFeMUG cruise (Cobalt, Iron, and Microorganisms from the Upwelling to the Gyre) in November, 2007. The South Atlantic Ocean is understudied with respect to trace metals (Moore et al. 2009), and understanding the biogeochemical cycling of metals near the Angola Dome and Benguela Upwelling is of economic interest to due to the presence of a major fishery. An oxygen minimum zone extends from the coast, generated by a combination of high productivity and poor ventilation (Lass and Mohrholz 2008; Mohrholz et al. 2008), and this redox gradient likely affects hybrid-type metal distributions. The South Atlantic has also been presumed to not be subject to iron limitation due to its low macronutrient abundances and aeolian input; however, the lower limit of iron concentrations during the low dust season had not previously been investigated in detail. The cruise track was chosen to cross the oligotrophic gyre and penetrate the Benguela Upwelling during a period of high upwelling and low dust deposition. We present evidence of significant sources of metals to the South Atlantic Basin from the African coast and discuss their biological interactions and relative removal rates. 


\subsection{Methods}

3.2.1 Sampling Techniques - The CoFeMUG cruise aboard the R/V Knorr (16 November - 13 December 2007, Chief Scientist, Saito) employed a trace metal sampling program for three transects in the South Atlantic Ocean. The main transect crossed the basin from west to east, beginning at $330^{\circ} \mathrm{E}, 11^{\circ} \mathrm{S}$ and ending at $12.2^{\circ} \mathrm{E}, 14.75^{\circ} \mathrm{S}$ (Fig. 1, 2). This transect consisted of 19 stations that were sampled to $800 \mathrm{~m}$ or to near bottom in profiles of 11 or 22 depths, respectively. Shallow and deep casts alternated at a 2.5 degree longitudinal spacing. This was followed by a short, north to south, coastal transect (Sta. 20-23) and a short, east to west, southern transect at $25^{\circ} \mathrm{S}$ (Sta. 23-27, Figs. 1, 2). Acidcleaned, teflon-coated X-Niskin sampling bottles (OceanTest Equipment) of $8 \mathrm{~L}$ capacity were deployed on an epoxy-coated rosette (Sea-Bird Electronics) attached to a nonmetallic line and were programmed to trip at predetermined depths and record sampling depths.

All bottles used to store seawater prior to analysis were soaked overnight in the acidic detergent, Citranox, rinsed thoroughly with Milli-Q water (Millipore), filled with $10 \% \mathrm{HCl}$ to soak for 10 days, rinsed thoroughly with Milli-Q water adjusted to $\mathrm{pH} 2$, and double-bagged. Low-density polyethylene bottles were used for trace metal sample storage and high-density polyethylene bottles were used for nutrient sample storage. Immediately following collection, the X-Niskins were pressurized with $99.999 \% \mathrm{~N}_{2}$, and seawater was filtered through teflon tubing and a $142 \mathrm{~mm}, 0.4 \mu \mathrm{m}$ polycarbonate plastic sandwich filter (Geotech Environmental Equipment) into sample storage bottles in a positive pressure class-100 clean environment. All tubing and filters were acid-washed prior to use. 
Samples intended for dissolved iron and manganese analyses were acidified to $\mathrm{pH} 1.7$ with high purity $\mathrm{HCl}$ (Seastar) within 6 months of sampling, and stored acidified at room temperature for at least 8 months prior to analysis. Samples intended for all cobalt analyses were not acidified and were kept at $4^{\circ} \mathrm{C}$ in darkness until analysis.

3.2.2 Cobalt Analyses - Concentrations of total dissolved and labile cobalt were determined using a previously described cathodic stripping voltammetry (CSV) method (Saito and Moffett 2001; Saito et al. 2004) both during the cruise and upon returning to the laboratory. Measurements were made using the Eco-Chemie $\mu$ Autolab-III systems connected to Metrohm 663 VA Stands equipped with hanging mercury drop electrodes and Teflon sampling cups. Standard additions were carried out with Metrohm 765 Dosimats using a programmed dosing procedure (Noble and Saito et al. 2008).

For total dissolved cobalt analyses, samples were ultraviolet (UV)-irradiated for $1 \mathrm{~h}$ prior to analysis using a Metrohm 705 UV digester to degrade the organic ligands that bind cobalt and allow binding by the added electroactive cobalt ligand, dimethylglyoxime. Samples were analyzed in $8.5 \mathrm{~mL}$ aliquots with the addition of $30 \mu \mathrm{L}$ recrystalized dimethylglyoxime (DMG, $0.1 \mathrm{~mol} \mathrm{~L}^{-1}$ in methanol), $1.5 \mathrm{~mL}$ purified sodium nitrite (1.5 mol L ${ }^{-1}$ in Milli-Q water), and $50 \mu \mathrm{L}$ purified N-(2-hydroxyethyl)piperazine-N-(3propanesulfonic acid) (EPPS) buffer $\left(0.5 \mathrm{~mol} \mathrm{~L}^{-1}\right.$ in Milli-Q water) (see Saito and Moffett 2001 for cleanup protocols). Analysis began with a 180 s purge with $99.999 \% \mathrm{~N}_{2}$. Each sample was conditioned at $-0.6 \mathrm{~V}$ for $90 \mathrm{~s}$ at a stir-rate of 2500 revolutions per minute (rpm) followed by a $10 \mathrm{~s}$ equilibration step and a linear sweep from $-0.6 \mathrm{~V}$ to $-1.4 \mathrm{~V}$ at a rate of $10 \mathrm{~V} \mathrm{~s}^{-1}$. Cobalt concentrations were determined by the standard additions 
technique, with initial concentrations measured in triplicate followed by four $25 \mathrm{pmol} \mathrm{L}^{-1}$ cobalt additions.

While the precision of this method using a single set of reagents is excellent (e.g. mean and standard deviation of triplicate analyses of a surface water sample $=32 \pm 0.7$ pmol L ${ }^{-1}$ ), careful analysis of reagent blanks is a crucial component of the cobalt method, and presented a new challenge for application to the analysis of an ocean section. The analytical blank was determined by analyzing seawater that had been UV-irradiated for $1 \mathrm{~h}$, equilibrated overnight with prepared Chelex-100 resin beads (Bio-Rad), and UV-irradiated a second time to degrade any leached synthetic ligands. Blanks for each batch of reagent (nitrite, DMG, EPPS) were subtracted from the initial sample concentration. During the cruise, two batches of reagents were contaminated as observed by both an abrupt and systematic increase in sample concentrations at Sta. 13 and 15 relative to adjacent profiles and by confirmation by reanalysis of the reagent blank while at sea. Upon returning to the lab, the systematic offsets for these samples were again confirmed using two independent techniques: reanalysis in the lab by CSV, and analysis by inductively coupled plasma mass spectrometry (ICP-MS). This in-lab CSV reanalysis of 7 profiles (114 samples), performed with one set of reagents with a blank of 6 pmol L $\mathrm{L}^{-1} \pm 1.7(n=19)$, quantified the contamination of the reagents as an average offset of 28 and $47 \mathrm{pmol} \mathrm{L}^{-1}$ relative to the pre-contaminated blanks (both $6 \mathrm{pmol} \mathrm{L}^{-1}$ ), and was consistent with the at-sea reanalysis of the blank (where the offset was determined by the average difference between the at-sea and in-lab analyses for a given reagent batch). However, an additional artifact was uncovered by this comparison of at-sea to in-lab analyses: datapoints in the $100-500 \mathrm{~m}$ 
OMZ range (5 datapoints per profile) were found to have a signal loss of cobalt during reanalysis, potentially due to oxidation or precipitation in the sample bottles with time and were excluded from the offset calculation (Noble and Saito in prep.). The averaged blank concentrations for the seven reagent batches used in this dataset were $4 \mathrm{pmol} \mathrm{L}^{-1} \pm 1.9(n=$ 4), 6 pmol L ${ }^{-1} \pm 1.7(n=19), 7$ pmol L-1 $\pm 1.7(n=4), 7$ pmol L-1 $\pm 1.7(n=4), 8$ pmol L-1 $\pm 0.9(n=3), 28 \mathrm{pmol} \mathrm{L}^{-1} \pm 1.4(n=4)$, and $49 \mathrm{pmol} \mathrm{L}^{-1} \pm 6.6(n=2) .12$ stations were analyzed 15 months after sampling $(4,6,8,10,12,14,16,23,24,25,26,27)$. Of those stations, 10,12,14, and 16 have been excluded from the dataset presented here because samples appeared to have experienced some loss of cobalt with time within the OMZ described above (less than $100 \mu \mathrm{mol} \mathrm{O}_{2} \mathrm{~kg}^{-1}$ ), which was apparent by comparison to adjacent profiles that were measured at sea. In contrast, Sta. 4, 6, and 8 (found outside the OMZ) were oceanographically consistent with adjacent profiles that were analyzed at sea, and have been included, as well as Sta. 23-27. With the exception of 2 datapoints from Sta. 23 and 4 datapoints from Sta. 24, the in-lab analyses from these stations were from waters

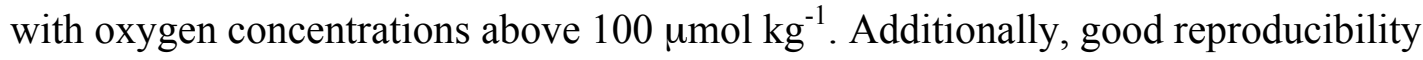
between shipboard and shore-based determinations was observed previously for samples from the well-oxygenated Ross Sea $>17$ months after sampling. In this case the shorebased recovery was $97 \% \pm 12 \%$ relative to the at sea values $(n=9$, Noble and Saito unpubl.). This suggests that non-OMZ samples can be successfully preserved by this storage technique. In future studies, care should be taken to minimize the number of reagent batches used to avoid systematic errors, to carefully constrain and minimize the 
cobalt reagent blank, and to continually monitor for small but important systematic error caused by contamination.

For labile cobalt analyses, $8.5 \mathrm{~mL}$ of filtered sample was added to clean Teflon vials preconditioned with a small aliquot of sample water. $30 \mu \mathrm{L}$ of DMG was added to each vial and allowed to equilibrate overnight in the dark (Saito et al. 2004). Analyses were then performed as described for total concentrations with the addition of the remaining two reagents and use of the standard addition technique. Previously, we determined that natural cobalt ligands in seawater have conditional stability constants of $>10^{16.8}$ (Saito et al. 2005), suggesting that the cobalt is bound very tightly to the cobalt ligands. Thus, we define labile cobalt as the fraction of total dissolved cobalt that is either bound to weak organic and inorganic ligands in seawater or present as free $\mathrm{Co}(\mathrm{II})$, and is then exchangeable with the complexing agent (DMG) used for analysis (Saito et al. 2004; Saito et al. 2005). The difference between the total dissolved cobalt and the labile cobalt can then be used as an estimation of the strong cobalt ligand concentration. Three sets of reagents were used for these analyses, with blanks of $4 \mathrm{pmol} \mathrm{L}^{-1} \pm 1.9(n=4), 5 \mathrm{pmol} \mathrm{L}^{-1} \pm$ $0.7(n=3)$, and $7 \mathrm{pmol} \mathrm{L}^{-1} \pm 1.7(n=4)$.

At the time of this expedition, low level total seawater cobalt intercalibration data were not yet available. In recent years, our lab has participated in the GEOTRACES Intercalibration effort (www.geotraces.org), which has documented the importance of UV oxidation for total cobalt analysis, and our results are presented in Table 1 (Bruland 2010).

3.2.3 Iron and Manganese Analyses - Total dissolved iron and manganese were measured using inductively coupled plasma mass spectrometry (ICP-MS), as described by 
Saito and Schneider (2006). Briefly, $13.0 \mathrm{~mL}$ aliquots of acidified seawater were weighed into cleaned polypropylene centrifuge tubes and ${ }^{57} \mathrm{Fe}$ was added for isotope dilution analysis and equilibrated overnight. Concentrated ammonium hydroxide (Seastar) was added to induce $\mathrm{Mg}(\mathrm{OH})_{2}$ and trace metal co-precipitation for 3 minutes, followed by centrifugation for 3 minutes at $3000 \mathrm{rpm}(1460 \mathrm{xg})$ using an Eppendorf Centrifuge 5810R. The supernatant was decanted, the sample was centrifuged and decanted again, and the pellet was dissolved in 5\% nitric acid (Seastar) containing $1 \mathrm{ppb}$ indium. The 5\% nitric acid resuspension solution was used to estimate the ${ }^{56} \mathrm{Fe},{ }^{57} \mathrm{Fe}$, and ${ }^{55} \mathrm{Mn}$ reagent and instrument blank, and signal suppression due to matrix effects was accounted for by using a ratio of the indium in the blank to the indium in the resuspended sample solution, as described in Saito and Schneider (2006). SAFe seawater intercalibration standards were analyzed at the beginning of each analysis day with iron concentrations of $0.96 \pm 0.095$ $\mathrm{nmol} \mathrm{L} \mathrm{L}^{-1}$ for $\mathrm{D} 2$ and $0.125 \pm 0.046 \mathrm{nmol} \mathrm{L}^{-1}$ for $\mathrm{S} 1(n=10)$, which are within the reported ranges of $0.91 \pm 0.17 \mathrm{nmol} \mathrm{L}^{-1}$ and $0.097 \pm 0.043 \mathrm{nmol} \mathrm{L}^{-1}$ (Johnson et al. 2007).

3.2.4 Sampling and analysis of $\mathrm{N}_{2} \mathrm{O}$ - Nitrous oxide $\left(\mathrm{N}_{2} \mathrm{O}\right)$ samples were collected from the trace metal rosette by twice overfilling glass $165 \mathrm{~mL}$ serum bottles (Wheaton) using tygon tubing. They were poisoned with $100 \mu \mathrm{L}$ of saturated $\mathrm{HgCl}_{2}$ solution and then sealed with butyl stoppers (MicroLiter Analytics) and aluminum crimps. Poisoned samples were stored for at least 2 months before analysis, however; previous research has demonstrated the integrity of this storage method for up to six months (McIlvin and Casciotti, in press). Isotopic analyses of $\mathrm{N}_{2} \mathrm{O}$ were made using a Finnigan Delta ${ }^{\mathrm{PLUS}} \mathrm{XP}$ isotope ratio mass spectrometer (McIlvin and Casciotti, 2010). 
3.2.5 Nutrient and oxygen analyses - Nutrient samples were filtered and frozen in acid-washed $60 \mathrm{~mL}$ high-density polyethylene bottles until analysis. The frozen samples were thawed in a warm water bath and stored in the dark for $20-24 \mathrm{~h}$ prior to analyses. This protocol has been found to increase the recovery efficiency of silicic acid in frozen samples, and has no observed adverse effects on the other nutrients. Immediately before analysis, aliquots of the samples were transferred to $15 \mathrm{~mL}$ polypropylene cups and an Alpkem autosampler. Technicon AutoAnalyzer II ${ }^{\mathrm{TM}}$ components were used to measure phosphate and ammonium; and Alpkem RFA $300^{\mathrm{TM}}$ components were used for silicic acid, nitrate plus nitrite, and nitrite. All five of the macronutrients were analyzed simultaneously. The nutrient methods were essentially those employed by the Oregon State University lab during the World Ocean Circulation Experiment (WOCE) and Southern Ocean Joint Global Ocean Flux Study (JGOFS) cruises. The phosphate method was a modification of the molybdenum blue procedure of Bernhardt and Wilhelms (1967), in which phosphate was determined as reduced phosphomolybdic acid employing hydrazine as the reductant. The nitrate + nitrite analysis used the basic method of Armstrong et al. (1967). Sulfanilamide and N-(1-Napthyl) ethylenediamine dihydrochloride react with nitrite to form a colored diazo compound. For the nitrate + nitrite analysis, nitrate is first reduced to nitrite using an open tubular cadmium reductor and imidazole buffer as described by Patton (1983). Nitrite analysis was performed on a separate channel, omitting the cadmium reductor and the buffer. The determination of silicic acid was based on that of Armstrong et al. (1967) as adapted by Atlas et al. (1971). Addition of an acidic molybdate reagent forms silicomolybdic acid which is then reduced by stannous chloride. An indophenol blue 
ammonium method was modified from ALPKEM RFA methodology, which references Methods for Chemical Analysis of Water and Wastes, March 1984, EPA-600/4-79-020, "Nitrogen Ammonia", Method 350.1 (Colorimetric, Automated Phenate). A detailed description of the continuous segmented flow procedures used can be found in Gordon et al. (1994).

Oxygen sensor data were calibrated by 5 Winkler titrations performed at Sta. 1, 2, 13, 22, and 24. Oxygen data were obtained from the CTD rosette Sea-Bird sensor (SBE$43)$ at every station $(0-800 \mathrm{~m})$ and at every deep station (full depth) from the trace metal rosette Sea-Bird SEACAT SBE-19 sensor. Titration water samples were taken immediately after rosette recovery by overfilling volume-calibrated flasks by one flask volume and then sealing with ground-glass stoppers. Standard Winkler reactions with iodate standards were performed and used to calibrate the Sea-Bird sensor and SEACAT sensor data. In cases where data was available from both sensors, the SBE-43 sensor data was used rather than the SEACAT sensor data due to its closer relationship to the calibration titrations $\left(r^{2}=0.9995\right.$ and 0.995 for Sta. 1 and 13 , respectively).

\subsubsection{Particle trap collection and analysis - Sinking particulate matter was}

collected using a surface-tethered, drogued-at-depth system and acid-cleaned polycarbonate particle interceptor tubes, similar to that used during JGOFS and at Hawaii Ocean Time Series (HOTS) and Bermuda Atlantic Time Series (BATS) (Steinberg et al. 2001). Collection tubes were held in PVC frames at depths of 60, 150 and $500 \mathrm{~m}$ at Sta. 13, and only the shallower two depths at Sta. 19. Six to eight tubes were deployed at each depth, and held $250 \mathrm{~mL}$ of a double strength seawater brine (made by freezing filtered 
uncontaminated local seawater) at the bottom of each tube below a layer of filtered seawater that was buffered with borate to $\mathrm{pH} 8.2$ and that doubled the alkalinity. No additional preservative was used, and we have found that for short duration deployments, brine without preservative gives comparable fluxes of carbon, nitrogen and thorium as did brines preserved with formaldehyde (Lamborg et al., 2008). The system was deployed for 3 days at Sta. 13 and 4 at Sta. 19. Two capped tubes, loaded with filtered water and brine, were on-board the array and used as a process blanks, and subjected to all the same processing protocols as the samples. After recovery, the tubes were allowed to stand for $>1$ $\mathrm{h}$ and brine/ particle mixture screened $(350 \mu \mathrm{m})$ to remove zooplankton swimmers. The remaining particles were collected onto acid-cleaned and pre-weighed $25 \mathrm{~mm}$ diameter, 1 $\mu \mathrm{m}$ pore sized polycarbonate Nuclepore filters. Following the filtering, the membranes were rinsed with a small amount of $\mathrm{pH} 8.2$, borate-buffered Milli-Q water. Prior to analysis, samples were desiccated and weighed cleanly in a laminar flow bench. Minor and trace elements were determined by ICP-MS as described in Lamborg et al. (2008).

\subsubsection{Suspended particle collection and analysis - Size-fractionated particles} were collected via in situ pumps (McLane Research Laboratories, Inc.) on McLane-style filter holders. The $>51 \mu \mathrm{m}$ size-fraction was collected on acid-cleaned polyester pre-filters (Sefar), followed in-line by collection of the $1-51 \mu \mathrm{m}$ size-fraction on combusted, acidcleaned quartz microfiber filters (Whatman QMA). Process blank filters were a complete filter set that was loaded in a plastic housing, attached to a pump, and deployed. These “dipped blank" filters were exposed to seawater but did not have water actively pumped through them, and were otherwise processed identically. QMA subsamples were digested 
in teflon bombs (Savillex) in 50\% v/v nitric acid (Seastar Baseline) for 4 hours at $135^{\circ} \mathrm{C}$, centrifuged to remove filter slurry, and diluted to $5 \% \mathrm{v} / \mathrm{v} \mathrm{HNO}_{3}$ for analysis by ICP-MS (Thermo Element2). Indium was used as an internal drift monitor, and concentrations were determined using multi-element external standard curves. The recovery of $\mathrm{Mn}, \mathrm{Co}$, and Fe from the MESS-3 sediment standard using this digestion procedure was $97 \%, 94 \%$, and $84 \%$, respectively, of certified values. All reported concentrations have had the process blanks removed. Sample handing and analyses were all conducted in HEPAfiltered clean environments following trace-metal procedures.

3.2.8 Data analysis and repository - Linear relationships between cobalt and oxygen, $\mathrm{N}_{2} \mathrm{O}$ and cobalt, and cobalt and $\delta^{15} \mathrm{~N}$ were determined by applying a two-way linear regression least squares fit in MATLAB using the script lsqfitma.m. The cobalt plume size calculations were determined by creating a grid of the South Atlantic basin, where each sampling point represented the corner of an enclosed area (either trapezoidal or triangular in shape). The area of each box was determined by depth and distance between data points, and the total cobalt in each box was determined by calculating trapezoid areas to interpolate between sampling points across the basin. The plume was defined by the 100 $\mu \mathrm{mol} \mathrm{kg}{ }^{-1} \mathrm{O}_{2}$ contour where the elevated cobalt was found. The cobalt within the plume was divided by the sum of the cobalt within the upper $1000 \mathrm{~m}$ to estimate relative inventories. The trace metal data presented in this manuscript (429 total dissolved iron values, 429 total dissolved manganese values, 346 total cobalt values, and 290 labile cobalt values, with fewer than 7 outliers for each species analyzed) have been deposited into the Biological and Chemical Oceanography Data Management Office database under the 
CoFeMUG program (BCO-DMO http://bcodmo.org/). Ocean sections were created in Ocean Data View with the exception of these few outliers (Schlitzer 2009).

\subsection{Results and Discussion}

The overarching scientific objective of this research cruise was to examine the distributions of cobalt, iron, and manganese in the oxygen minimum zone of the South Atlantic and Benguela Upwelling Region, motivated by previous observations of elevated cobalt in the OMZs of both the Costa Rica Dome and the Peru Upwelling Region (Saito et al. 2004, 2005). The expedition consisted of three transects: the main zonal transect (Sta. 1-19, main transect hereon), a short meridional transect along the coast within the Benguela Upwelling (Sta. 19-23), and a short transect south of the main transect extending from the shelf back into the gyre (Sta. 23-27, southern transect hereon). The cruise proceeded from west to east across the basin, from regions of low productivity in the oligotrophic subtropical gyre to higher productivity in the Angola Gyre and finally to the highest productivity regions associated with coastal upwelling of macronutrients (Figs. 1, 2). The high productivity coastal regions were further distinguished by water masses of the Angola Dome (the southern edge of which is observed in Sta. 17 and 18), the Benguela Upwelling region that covers much of the Namibian and South African Atlantic coast (Weeks and Shillington, 1994), and the dynamic Angola-Benguela Frontal Zone (ABFZ) that lies between them (roughly between $14.5^{\circ} \mathrm{S}$ to $18.5^{\circ} \mathrm{S}$ for the northern and southern extents varying by season and climatology; Lass and Morholz 2008; Kostianoy and Lutjeharms, 1999). The metal distributions within three broad geographic distinctions are presented in this manuscript: the low surface concentrations in the oligotrophic gyre, the 
large plumes observed within the subsurface OMZ of the South Atlantic, and the localized, elevated concentrations along the coast. The salient, large basin-scale features apparent in the ocean section are presented first, followed by the coastal observations and discussion of potential supply mechanisms. A discussion of the hydrothermal contributions of iron and manganese plumes found above the Mid-Atlantic Ridge (Saito et al., unpubl.), and a discussion of the ecological stoichiometry of cobalt in this region relative to the North Atlantic (Noble et al, unpubl.) will be presented elsewhere.

3.3.1 Spatial distribution observations - The western and central region of the main transect sampled the oligotrophic subtropical gyre and was characterized by surface drawdown of total cobalt to $6 \mathrm{pmol} \mathrm{L}^{-1}$, labile cobalt to below detection, and iron to 0.05 nmol L ${ }^{-1}$, due to biological utilization (Figs. 1, 3). These low surface iron and cobalt concentrations were within a range that can be limiting to some phytoplankton growth (Sunda and Huntsmann 1995), although a few stations may reflect some aeolian deposition that could relieve micronutrient stress (Sta. $9,10 \mathrm{~m} ; 0.34 \mathrm{nmol} \mathrm{L}^{-1} \mathrm{Fe}, 6 \mathrm{pmol} \mathrm{L}^{-1} \mathrm{M}$ labile Co). These low surface concentrations are consistent with modeling studies by Mahowald et al. (2005) that predict low dust input to the South Atlantic (Fig. 4a), relative to large inputs like the Sahara Desert to the north (Vink and Measures 2001).

In contrast to cobalt and iron, manganese remained consistently elevated in surface waters across the entire main transect section with values ranging from $1.93-4.04 \mathrm{nmol} \mathrm{L}^{-1}$. While this surface maximum is typical of manganese profiles from tropical and subtropical regions, its uniformity across the region is perhaps surprising. Aeolian deposition is often invoked as a primary source of manganese to the surface ocean (Statham et al. 1998). 
Given the low dust deposition predicted for this region (Fig. 4a), these observations suggest that there is likely an additional mechanism working to maintain the uniform surface maxima, such as redox cycling processes that act to retain dissolved manganese at the surface. Manganese-oxidizing bacteria are photoinhibited in the mixed layer, but below that, they are known to oxidize manganese, creating small neutrally buoyant manganese oxide particles that may be re-entrained into the mixed layer and photoreduced (Tebo et al. 2004; Sunda and Huntsman 1988). The combination of aeolian input from above and entrainment and recycling of manganese oxides from below could act to create and maintain the elevated dissolved manganese surface water inventory we observe across the gyre, similar to processes that concentrate dissolved metals at distinct strata in sedimentary environments (Heggie and Lewis 1984; Stumm and Morgan 1996).

Elevated concentrations of all three metals were found along the coast of Namibia and extended far into the South Atlantic basin in the main transect. For example, in the upper $60 \mathrm{~m}$ of Sta. 18 and 19, concentrations reached as high as $200 \mathrm{pmol} \mathrm{L}^{-1} \mathrm{Co}, 4.04$ nmol L $\mathrm{Ln}^{-1}$ and $3.03 \mathrm{nmol} \mathrm{L}{ }^{-1} \mathrm{Fe}$ with a second, spatially pronounced, westward propagating feature for all three metals observed around $400 \mathrm{~m}\left(163 \mathrm{pmol} \mathrm{L}^{-1} \mathrm{Co}, 3.1 \mathrm{nmol}\right.$ $\mathrm{L}^{-1} \mathrm{Fe}, 1.1 \mathrm{nmol} \mathrm{L}{ }^{-1} \mathrm{Mn}$; Figs. 1, 3, 5). Of the three metals, the subsurface cobalt extended furthest into the basin, for a distance of approximately $2500 \mathrm{~km}$ from the Namibian coast. This cobalt plume spatially made up $37 \%$ of the upper $1000 \mathrm{~m}$ of the zonal section, but disproportionately contributed more than $50 \%$ of the total cobalt therein (Fig. 5; see Methods for calculations). There are two potential causes of these plumes. First, these metals could be released from coastal sedimentary sources and advected westward. Second, 
the plumes could be formed by the remineralization of exported particulate organic matter from the euphotic zone. Both of these mechanisms are consistent with regional circulation and high productivity, and hence a combination of these two processes likely contributes to the plumes, as discussed in later sections.

Within the Angola Gyre, the edge of the Angola Dome is identified by a high temperature signature at Sta. 17 and 18 between 0 and $50 \mathrm{~m}$ depth (Lass and Mohrholz 2008, Fig. 6c). Cobalt, iron, and manganese concentrations at $400 \mathrm{~m}$ are 2.2, 3.8, and 3.8 times higher in the Angola Gyre (Sta. 15-19), respectively, than at comparable depths in the center of the oligotrophic subtropical gyre (Sta. 3-5), and 2.6, 2.5, and 1.9 times higher than at comparable depths off the coast in the southern transect (Sta. 25-27). The elevated metals generally co-occur with the oxygen depletion that occurs as a result of organic matter remineralization and poor ventilation in the shadow zone near the African coast (Figs. 2, 5, 6a Lass and Mohrholz 2008; Mohrholz et al. 2008). South Atlantic Central Water (100-500 m) carries this low oxygen signature south and eventually westward upon convergence with Eastern South Atlantic Central Water (Mohrholz et al. 2008). Additional oxygen consumption is driven by the Benguela Upwelling to the south, which also injects nutrients into surface waters.

In contrast to the high abundances observed offshore in the main transect through the Angola Gyre (Fig. 5), the iron, manganese, and cobalt concentrations were lower in the offshore southern zonal transect (Fig. 1). Metal concentrations were high along the coast at Sta. 23, reaching $203 \mathrm{pmol} \mathrm{L}^{-1} \mathrm{Co}, 7.9 \mathrm{nmol} \mathrm{L}{ }^{-1} \mathrm{Fe}$, and $2.8 \mathrm{nmol} \mathrm{L}^{-1} \mathrm{Mn}$, and dissolved oxygen was low (as low as $11 \mu \mathrm{mol} \mathrm{kg}^{-1}$ ), but this signal did not extend westward at this 
latitude. This likely reflects the differences in physical processes where the main transect encounters surface and subsurface westward advection resulting from the convergence of the southward flowing Angola Current and the northward flowing Benguela Coastal Current (at the ABFZ) and from the northwestward flowing Benguela Ocean Current that is driven by the strong trade winds (Peterson and Stramma 1991; Mohrholz et al. 2008). In contrast, the southern zonal transect crosses the northward flowing Benguela Coastal Current when it likely still follows the coastline north, and water mass movement westward is more restricted (Peterson and Stramma 1991). In order to compare cobalt, iron, and manganese biogeochemical cycles in the South Atlantic Basin, the sources and sinks acting on this system are described based on our current understanding. This includes estimates of relative differences in removal timescales for these metals, a discussion of the potential role of sediments and dust as sources, and consideration of lateral and vertical mechanisms for delivery of metals to the basin.

\subsubsection{Cobalt, iron, and manganese plumes across the South Atlantic basin - One}

of the striking observations in this dataset is the differing extent to which cobalt, iron, and manganese penetrate into the subsurface OMZ of the South Atlantic Gyre (Fig. 5). While cobalt extends furthest and shows an inverse linear correlation with oxygen (Fig. 7), iron and manganese show exponential removal relative to both distance from the coast and oxygen. Their differing reaches into the basin could be explained by differences in each metal's scavenging rates upon the horizontally advected and vertical remineralized flux, and/or by differences in supply from remineralized vertical flux, for example by changes in the Co:Mn:Fe stoichiometry of exported material. For horizontal advection, mixing and 
scavenging control the dissipation of the coastal signal as water flows westward with time, and since mixing should affect these metals identically, scavenging likely controls the observed differences in the subsurface distributions. Iron and manganese show exponential removal when compared to distance from coastal source waters and oxygen (Fig. 8e-h). Concentrations along the 27.0-27.1 density layer (400 m depth) approach 0.55 $\mathrm{nmol} \mathrm{L}{ }^{-1} \mathrm{Fe}\left(0.2 \mathrm{nmol} \mathrm{L}^{-1}\right.$ for $\left.\mathrm{Mn}\right)$ at the western end of the basin where oxygen concentrations also plateau, which may represent some fraction of the dissolved pool that is protected from biotic or abiotic scavenging by binding to strong organic ligands over time (Johnson et al. 1997). As a result, the aggregate scavenging behavior $\left(\mathrm{k}_{\text {scav }}\right)$ can be described by fitting a curve to the data using a simple parameterization representing biotic or abiotic processes:

$$
[\mathrm{M}]_{\mathrm{x}}=[\mathrm{M}]_{\mathrm{x}-1}-\left([\mathrm{M}]_{\mathrm{x}-1}-[\mathrm{M}]_{\mathrm{L}}\right) \mathrm{k}_{\mathrm{scav}} \mathrm{d}_{\mathrm{x}}
$$

In this equation, $[\mathrm{M}]_{\mathrm{x}}$ is the metal concentration in $\mathrm{mol} \mathrm{L}^{-1}$ at a distance $\mathrm{x},[\mathrm{M}]_{\mathrm{x}-1}$ is the concentration of metal at an "upstream" sample location at the same depth, and $[\mathrm{M}]_{\mathrm{L}}$ is the concentration of metal that is presumed to be protected from scavenging by binding to organic ligands. The longitudinal distance between one data point $\left([\mathrm{M}]_{\mathrm{x}-1}\right)$ and the next $\left([M]_{x}\right)$ is denoted by $d_{x}$. This simple equation assumes that metals are being scavenged along a westward path, and does not account for additional vertical input. Empirically estimating $[\mathrm{M}]_{\mathrm{L}}$ to be the asymptotic concentration observed in the western end of the basin, and defining $[\mathrm{M}]_{\mathrm{x}=0}$ to be the observed concentration in the eastern end of the basin, the relative removal rates for each metal were estimated. A non-linear fit to the iron concentrations at $400 \mathrm{~m}$ suggests a $0.0025 \mathrm{~km}^{-1}$ scavenging rate. If oxygen concentrations 
reached extreme lows, they may influence the pseudo first-order rate constant by reducing the re-oxidation rate. It appears, however, that the oxygen concentration range we observe here is likely still too high to decrease either iron or manganese scavenging significantly. Estimating manganese scavenging by the same approach, and using $0.20 \mathrm{nmol} \mathrm{L}^{-1}$ for $[\mathrm{M}]_{\mathrm{L}}$, we calculate a scavenging rate of $0.001 \mathrm{~km}^{-1}$, which is 2.5 times slower than the rate observed for iron.

In comparison to iron and manganese, cobalt appears to be insensitive to scavenging within the oxygen minimum zone, indicated by an inverse correlation with oxygen $\left(r^{2}=0.73\right)$, as observed previously in the Angola Dome (Pohl et al. 2011), and also by a correlation with $\mathrm{N}_{2} \mathrm{O}\left(r^{2}=0.80\right)($ Fig. $7 \mathrm{a}, \mathrm{b})$. If higher oxygen concentrations were increasing cobalt oxidation kinetics and scavenging, one would expect to see a relationship similar to that observed for iron and manganese. Because this is not observed, it suggests that cobalt scavenging rates were slower than physical mixing processes or that cobalt concentrations are at steady state, where scavenging rates were slow enough to be equally matched by lateral and vertical input (Fig. 8c, d). The cause of the Co: $\mathrm{N}_{2} \mathrm{O}$ relationship is unknown, and while the source mechanisms of these species may not be directly connected, their general coastal source locations likely are. The advection and persistence of these two chemical species with similarly slow removal timescales could lead to the observed correlation. There could also be specific mechanistic, enzymatic, or chemical explanations for the correlation. For example, $\mathrm{NO}$ and $\mathrm{N}_{2} \mathrm{O}$ are often produced through the same processes (Yoshida, 1988) and NO levels can be elevated in low-oxygen waters (Ward and Zafiriou, 1988). Cobalt-containing cobalamin and cobinamide molecules are known to 
have high affinities for NO (Sharma et al. 2003), and it may be possible that elevated NO concentrations stabilize labile cobalt and protect it from scavenging.

Using Equation (1), the relative removal of cobalt was estimated to be $0.0003 \mathrm{~km}^{-1}$ at $400 \mathrm{~m}$. This low value likely primarily reflects mixing processes since there is no mixing term in Eq. 1 and exponential removal was not observed. $[\mathrm{M}]_{\mathrm{L}}$ was calculated by taking the average difference between the total and labile cobalt concentrations at 400,600, and $800 \mathrm{~m}$ in the western end of the basin (Sta. 1-9), and was determined to be quite uniform with concentrations of $68 \pm 15.9 \mathrm{pmol} \mathrm{L}^{-1}(n=8)$ at $400 \mathrm{~m}, 60 \pm 11.0 \mathrm{pmol} \mathrm{L}^{-1}(n$ $=7)$ at $600 \mathrm{~m}$, and $60 \pm 8.9 \mathrm{pmol} \mathrm{L}^{-1}(n=8)$ at $800 \mathrm{~m}$. The decrease in standard deviation with depth may be reflective of more uniform concentrations of cobalt at depth and less biological and chemical variability.

The observation that cobalt persists much further westward than manganese in the $\mathrm{OMZ}$ is intriguing since previous process studies have demonstrated that these metals are linked by a common scavenging mechanism: the oxidation by manganese-oxidizing bacteria (Tebo et al. 1984; Moffett and Ho 1996). The cobalt and manganese concentrations in the coastal end-member are enriched $\left(0.200 \mathrm{nmol} \mathrm{L}^{-1}\right.$ and $2.0 \mathrm{nmol} \mathrm{L}{ }^{-1}$ respectively) relative to their oceanic deep-water concentrations $\left(0.045 \mathrm{nmol} \mathrm{L}^{-1}\right.$ and 0.20 nmol L ${ }^{-1}$ respectively), yet unlike cobalt, these enhanced coastal manganese abundances do not result in a significant plume. There are several possible explanations for this difference. First, cobalt is present in much lower concentrations than manganese $\left(0.006-0.200 \mathrm{nmol} \mathrm{L}^{-}\right.$ ${ }^{1}$ for Co vs. $0.10-4.03 \mathrm{nmol} \mathrm{L}^{-1}$ for $\mathrm{Mn}$ ), thus the elevated cobalt concentrations in the plume may be below concentrations at which a quantitatively significant coupling between 
manganese and cobalt scavenging might be observed. Second, manganese scavenging may be more apparent due to its preferential oxidation relative to cobalt (Moffett and Ho 1996). This notion is supported by the differences in the dissolved to particulate trace metal ratios for cobalt $(\mathrm{dCo} / \mathrm{pCo})$ and manganese $(\mathrm{dMn} / \mathrm{pMn})$ at Sta. 13 (Fig. 9), where $\mathrm{dCo} / \mathrm{pCo}$ was 5 to 20 times higher than $\mathrm{dMn} / \mathrm{pMn}$ below $\sim 150 \mathrm{~m}$. Third, oxygen concentrations may be low enough that microbially mediated oxidation of cobalt is kinetically unfavorable; we are unaware of studies examining the relative rates of oxidation of cobalt and manganese in low oxygen environments. Fourth, the degree of cobalt and manganese complexation likely influences the relative scavenging/oxidation processes. $\mathrm{Co}(\mathrm{III}), \mathrm{Co}(\mathrm{II})$, and $\mathrm{Mn}$ (III) have been shown to form strong complexes with natural organic molecules and siderophores, respectively (Saito et al. 2005; Homann et al. 2009). The cobalt in the plume generally had more than $75 \%$ of the total cobalt present in strongly complexed form (Figs. 1, 5), leaving less than a quarter of the labile cobalt available for co-oxidation, assuming complexed cobalt is not bioavailable to manganese-oxidizing bacteria. It is likely that the observed phenomenon is the result of a contribution from all four factors. There may be protection from scavenging by complexation for manganese as well, though little is known about the susceptibility of ligand-bound $\mathrm{Mn}$ (III) to bacterial manganese oxidation (Parker et al. 2007; Homann et al. 2009).

\subsubsection{Implications for hybrid-type metals with ocean deoxygenation - A recent} study by Stramma et al. (2008) found that the distribution of OMZs globally has increased significantly during the past 50 years. Based on our observations within the South Atlantic OMZ, it seems possible that a coincident increase in ocean inventories of certain trace 
metals may occur with deoxygenation, particularly for the hybrid-type elements that have small oceanic inventories due to the confluence of nutrient uptake and intermediate depth scavenging processes. The OMZ in the South Atlantic is relatively weak compared to the OMZs of the Pacific and Arabian Sea, where higher concentrations of dissolved iron and manganese have been observed $\left(10 \mathrm{nmol} \mathrm{L}^{-1} \mathrm{Mn}\right.$ and $5 \mathrm{nmol} \mathrm{L}{ }^{-1} \mathrm{Fe}$, Pacific, Martin et al. 1989; $2 \mathrm{nmol} \mathrm{L}^{-1} \mathrm{Fe}$, Arabian Sea, Moffett et al. 2007), consistent with this notion of a connection between low oxygen and hybrid-type metal flux. It is difficult to extrapolate the influence of intensified oxygen depletion on metal concentrations in the South Atlantic because of the complexities and uncertainties associated with estimating changes in the coastal sedimentary flux due to an expansion of regional low bottom water oxygen (see sedimentary metal source section). As a first approximation, we can use the linear relationship observed between cobalt and oxygen (Fig. 7), and a rate of oxygen depletion in the South Atlantic OMZ region of $-0.17 \mu \mathrm{mol} \mathrm{O}_{2} \mathrm{~kg}^{-1} \mathrm{yr}^{-1}$ over the past 50 years observed by Stramma et al. (2008). Using this deoxygenation rate, the cobalt inventory in the upper $1000 \mathrm{~m}$ of the South Atlantic Basin could increase $\sim 10 \%$ by the year 2100 . An important potential effect of increased oceanic cobalt, iron, and manganese inventories is a stimulation of marine primary productivity and nitrogen fixation and/or changes in marine ecology driven by species with distinct trace element preferences. In particular, the nitrogen limiting conditions of the South Atlantic could be alleviated by increases in nitrogen fixation activity with an enhanced OMZ iron supply (Saito et al. 2011). If primary productivity and export were to increase, the resultant intensified remineralization would consume more $\mathrm{O}_{2}$, creating a positive feedback mechanism for further expansion of OMZs. 
The extent of this potential feedback mechanism may be limited by the phosphate inventory as the limiting factor for microbial nitrogen fixation. The South Atlantic has a higher photic zone phosphate inventory than the North Atlantic (Fig. 2, Wu et al., 2000), however; suggesting that an increased coastal iron flux could contribute to this feedback mechanism prior to the onset of phosphate limitation. Future studies should investigate where the oxygen thresholds are with respect to reductions in metal scavenging rates, as well as the influence of expanded OMZs on coastal and sedimentary metal fluxes.

3.3.4 A sedimentary metal source - Cobalt, iron, and manganese concentrations increased with proximity to the Angolan and Namibian coasts, particularly between 200 and $1000 \mathrm{~m}$ depth. The source of these elevated metals is likely derived from the coastal sediments rather than aeolian deposition or riverine discharge. Coastal sediments can be a source of metals via dissolved fluxes from anoxic sediments and/or particulate fluxes through resuspension of sedimentary material and subsequent desorption or dissolution in the water column. This source could reach the basin through both lateral (advection of coastal waters westward at depth) and vertical (dissolution/desorption in the euphotic zone and subsequent remineralization of sinking biogenic material) vectors. The relative importance of the processes that create these plumes likely varies for each metal, and the magnitude of dissolved and particulate fluxes from sediments can be influenced by advection, bottom water oxygen concentration, and continental shelf width (Chase et al. 2005; Bruland et al. 2005, Lohan and Bruland 2008). Below, we discuss the potential sedimentary source mechanisms for each metal based on oxygen concentrations, dissolved 
and particulate trace metal abundances, and comparative transects over a narrow (northern) and broad (southern) shelf area in the Angola/Benguela African region.

Anoxic bottom water conditions can generate metal fluxes from sediments by the reductive dissolution of metal-oxides and diffusion out of those sediments (Heggie and Lewis 1984; Johnson et al. 1988; Lohan and Bruland 2008). Critical to this process is the prevention of reoxidation by oxygen depletion at the sediment water interface. The Namibian coastal shelf waters experience seasonal anoxia during the austral summer, coincident with detection of $\mathrm{H}_{2} \mathrm{~S}$ in bottom water due to sulfate reduction and occasional episodes of detectable methane (Bruchert et al. 2003; Emeis et al. 2004; Mohrholz et al. 2008). Conditions can even become reducing enough to allow for water column microbial sulfide oxidation, and a loss of nitrate due to denitrification and annamox (Lavik et al. 2009, Kuypers et al. 2005). Based on comparison of thermodynamic energy yields of redox couples, the presence of $\mathrm{H}_{2} \mathrm{~S}$ in the water column implies that more energetically favorable terminal electron acceptors, including manganese, iron, and cobalt oxide minerals, would have already been reduced and their soluble products released into pore waters. While precipitation of these reduced metals as metal sulfides might occur, aqueous sulfide complexes can form, and reduced metal maxima are often observed at the $\mathrm{H}_{2} \mathrm{~S}-\mathrm{O}_{2}$ interface, as has previously been observed in the anoxic Baltic Sea (Dyrssen and Kremling 1990). While reduced iron is sensitive to oxidative re-precipitation (Elrod et al. 2004), reduced manganese is less sensitive and reduced cobalt has been observed to diffuse the furthest of the three toward or past the sediment-water interface before oxidative reprecipitation (Klinkhammer 1980; Kremling 1983; Heggie and Lewis 1984). This 
reductive flux mechanism is likely occurring along the African shelf, and the highly reducing sediments here are likely instrumental in generating the cobalt plume observed in the zonal section (Figs. 1, 2, 5), as we have previously invoked to explain the high abundances in the north and south Eastern Subtropical Pacific (Saito et al. 2004, 2005). While the OMZ of the South Atlantic is generally considered to be much weaker than that of the Tropical Pacific, the observation of a plume here suggests that localized coastal anoxia may be more important than eventual OMZ oxygen levels in generating metal fluxes.

In recent years, the importance of advected particulate iron as a source to the oceanic iron inventory has been demonstrated (Lam et al. 2006; Lohan and Bruland 2008; Moore and Braucher 2008). The influence of advected particulate material on the dissolved inventory of other bioactive metals has been less studied (Johnson et al. 1992; Chase et al. 2005), but may also be significant (Lamborg et al. 2008). Turbidity and particulate metal concentration measurements from the CoFeMUG cruise suggest that this release mechanism may be important for the inventories of these metals, with potential implications for the dissolved iron concentrations, though less so for dissolved manganese and least of all for dissolved cobalt. A significant increase in dissolved iron concentration corresponds with an increase in particulate iron at Sta. 19 that is 7 times higher than the dissolved iron (Fig. 9). Cobalt dissolved and particulate concentrations also increase at the bottom sampled depths (Fig. 9f), but the particulate cobalt concentration is only a small fraction $\left(1 / 20^{\text {th }}\right)$ of the dissolved cobalt concentration and a similar trend is observed for manganese (pMn $\sim 1 / 10^{\text {th }}$ of the $\mathrm{dMn}$ ) (Fig. $9 \mathrm{~g}$ ). These differences in phase partitioning 
suggest that while the dissolved flux from the sediments may be more important than the particulate flux for cobalt and manganese, this may not be the case for iron. Cycling between the dissolved and particulate phase at the sediment-water interface can support a flux of iron to the water column if a fraction of the dissolved pool is bound by ligands before re-precipitation, as has been observed in the shelf waters along the coast of Oregon and Washington State (Lohan and Bruland 2008). While similar processes may exist for cobalt and manganese cycling, the higher water column dissolved concentrations do not support this as a dominant cobalt or manganese source here. Further south, sampling at coastal Sta. 21, 22 and 23 approached or penetrated the benthic bottom layer where an increase in total dissolved cobalt and iron concentrations, and to a lesser extent, manganese were observed (Fig. 10). The beam attenuation coefficient, a proxy for sediment and/or particle resuspension (Lam and Bishop 2008), also increased with depth near the bottom of the water column, and oxygen concentrations plummeted (Fig. 10). Given the nearby observation of high particulate iron and low particulate cobalt and manganese at Sta. 19 (Fig. 9), the elevated dissolved iron here was likely derived from a combination of reductive dissolution and particulate fluxes, but the cobalt concentrations were likely primarily derived from reductive dissolution. The smaller manganese trend may be a function of the high concentrations of manganese observed in surface waters (see above) masking a sedimentary signal in these shallow waters.

Broad continental shelf environments can enhance metal fluxes by providing more sedimentary surface area from which reduced metals and resuspended particles may be released (Bruland et al. 2005). Consistent with this, the concentration of dissolved iron at 
depth along the narrow shelf abutting the main transect was high $(\sim 10 \mathrm{~km}$ shelf width, 3 $\mathrm{nmol} \mathrm{L}{ }^{-1} \mathrm{Fe}$ ), but the highest concentration of iron was found at depth further south along the broad shelf (Sta. $23 \sim 150 \mathrm{~km}$ shelf width, $8 \mathrm{nmol} \mathrm{L}^{-1}$ ). Yet, the high iron concentrations found at this southern coastal station decreased immediately to the west (Fig. 1), likely reflecting the differences in physical processes between regions (Fig. 6a, b, c, g, h, i, Table 3). Consistent with this, there is a strong oxygen gradient heading westward from Sta. 23 (Figs. 2, 6g). To the north, oxygen is depleted (as low as $18 \mu \mathrm{mol} \mathrm{kg}^{-1}$ at Sta. $17300 \mathrm{~m}$ ), and the gradual oxygen gradient observed is consistent with offshore advection (Figs. 2, 6a). Our results follow the trend of wide shelves being important iron sources (Table 2); however, they also suggest that low oxygen concentrations and physical processes also contribute to the effective transport of these dissolved metals to the open ocean.

\subsubsection{Consideration of aeolian and riverine sources - Both modeled dust} deposition studies (Fig. 4a, Mahowald et al. 2005), and low surface dissolved aluminum concentrations are consistent with relatively low aeolian input to this region. Aluminum is a scavenged-type trace element with a surface water residence time of $\sim 5$ yrs (Jickells et al., 1994). The open ocean surface water concentrations have been used to estimate aeolian deposition using the MADCOW model yielding values that represent a $\sim 5 \mathrm{yr}$ running mean of dust deposition and that agree reasonably well with independent estimates over a large range of dust depositions (Measures and Vink, 2000). Previous studies of surface aluminum concentrations in the Atlantic show a significant range of concentrations that were higher near the Brazilian coast, lower in the Benguela Upwelling, and highest off the Sahara Desert (Measures 1995; Measures et al. 2008). In the eastern Atlantic, close to our 
Sta. 13, Measures (1995) showed that there is a very strong N-S gradient in aluminum concentrations from $\sim 12 \mathrm{nmol} \mathrm{L}^{-1}$ at $15^{\circ} \mathrm{S}$ to $<2 \mathrm{nmol} \mathrm{L}^{-1}$ at $16^{\circ} \mathrm{S}$ which was attributed to the convergence of the Angola Current and Benguela Current in the Angola Benguela Frontal Zone. These observations all suggest that while aeolian input is high in the Western Atlantic and the North East Atlantic, dust inputs are generally low in the South East Atlantic.

Surface samples of dissolved aluminum from CoFeMUG are consistent with previous observations (Fig. 4b). Aluminum concentrations imply dust depositions that are at a maximum of $9.4 \mathrm{mg} \mathrm{m}^{-2} \mathrm{~d}^{-1}$ at Sta. 11 and range from 5.7 to $7.67 \mathrm{mg} \mathrm{m}^{-2} \mathrm{~d}^{-1}$ to the west. East of Sta. 11, dust deposition values drop rapidly to $1-3 \mathrm{mg} \mathrm{m}^{-2} \mathrm{~d}^{-1}$ with the lowest values $<1 \mathrm{mg} \mathrm{m}^{-2} \mathrm{~d}^{-1}$ observed along the coast and the southern transect. It should be noted however that in upwelling regions the assumptions of a steady state surface water layer are incorrect and thus the MADCOW model will underpredict aeolian deposition (Kaupp et al, 2011). Aluminum concentrations were highest at Sta. 11, and concentrations ranged from 26 to $35 \mathrm{nmol} \mathrm{L}^{-1}$ to the west. East of Sta. 11, aluminum concentrations decreased, with the lowest concentrations observed along the coast and in the southern transect $\left(0.7 \mathrm{nmol} \mathrm{L}{ }^{-1}\right.$ to $7 \mathrm{nmol} \mathrm{L}{ }^{-1}$; Fig 4b). In contrast, cobalt surface concentrations increased from 24 pmol $\mathrm{L}^{-1}$ at Sta. 11 to $184 \mathrm{pmol} \mathrm{L}^{-1}$ at Sta. 19. These opposite trends provide evidence that dust was not a primary contributor to the cobalt signals observed in the main transect. While aluminum concentrations are historically high just north of our sampled stations, there was little aeolian contribution during our cruise and at these latitudes. These observations are consistent with results from the Atlantic Meridional Transect (AMT) expeditions which 
also found elevated iron and cobalt but decreased aluminum within the Benguela Upwelling relative to the gyre (Bowie et al. 2002).

We can derive an estimate of potential cobalt input from dust in this region from dissolved aluminum concentrations and dust deposition models. If the composition of dust entering the South Atlantic is similar to the composition of the upper crust $(\mathrm{Co}: \mathrm{Mn}: \mathrm{Fe}=$ 1:60:3500 from Taylor and McLennan 1985), the input of cobalt and manganese would be small relative to iron. Additionally, recent results suggest that the solubility of cobalt in dust may be quite low $(0.14 \%$ for natural dust and $0.78 \%$ for anthropogenic dust; Thuroczy et al. 2010), though some evidence shows that surface cobalt may display evidence of episodic aeolian input, for instance in the Sargasso Sea (Shelley et al. 2010). Using this solubility range, a dust input of $0.5 \mathrm{mg} \mathrm{m}^{-2} \mathrm{~d}^{-1}$ (from Fig. 4a, Mahowald et al. 2005, and from deposition estimates using our surface aluminum data Fig. 4b), and a mixed layer depth of 20 m estimated from temperature gradients (Fig. 6c), aeolian input would contribute only $0.002-0.012 \mathrm{pmol} \mathrm{Co} \mathrm{L}^{-1} \mathrm{y}^{-1}$ to the eastern end of the basin. This amount would be less than $1 \%$ of the total cobalt observed, with the conservative assumption of a cobalt surface water concentration of $10 \mathrm{pmol} \mathrm{L}^{-1}$, and in comparison to an estimated surface ocean residence time of $<7.6$ years (Saito and Moffett 2002).

The Congo River empties to the eastern South Atlantic Basin at $6^{\circ} \mathrm{S}, 12^{\circ} \mathrm{E}$. Inland from the Congo River, the African Copper Belt $\left(12^{\circ} \mathrm{S}, 26^{\circ} \mathrm{E}\right)$ previously produced $40 \%$ of the world's cobalt through mining (Prasad 1989). If high cobalt-containing dust reached the Congo River and was carried to the coast, this could contribute to the elevated cobalt observed relative to other metals, although we are unaware of any published Congo River 
cobalt data. Riverine removal rates of manganese and iron due to flocculation have been observed to be 5-10 times faster than that of cobalt (Sholkovitz 1976), which might enhance cobalt abundances relative to iron and manganese. Salinity and temperature signatures are good indications of surface water source and intrusion of waters from the Angolan Current are characterized by temperatures above $21^{\circ} \mathrm{C}$ and salinities above 35.9 (John et al. 2004). Based on these criteria, some intrusion of the Angolan Current is observed at Sta. 17 and 18 within the Angola Dome, but the highest surface cobalt concentrations are found at Sta. 19, which has temperature and salinity signatures of the Benguela Coastal Current. This suggests that upwelling along the coast may be a stronger source of cobalt than riverine input. Given the available evidence, aeolian and riverine sources do not appear to be major contributors to the subsurface metal plumes observed in this study, relative to the sedimentary sources discussed above.

\subsubsection{Vertical and lateral metal fluxes to the South Atlantic Basin - Metals from}

the coastal sediments can make their way into the open ocean by both lateral and vertical input vectors. The vertical introduction of these metals occurs primarily via remineralization of sinking organic and inorganic particulate material. Combining dissolved metals data with sediment trap and particulate metal data is useful in considering the influence of vertical and lateral processes on the metal distributions, and a similar collection of data proved useful in understanding the biogeochemistries of manganese and iron in the pacific (Landing and Bruland 1987). Sediment trap data provide an estimate of the vertical fluxes of exported material delivered to intermediate depths, albeit with some potential bias (Buesseler et al. 2007). Comparison of the particulate metal to dissolved 
metal concentrations can be informative of potential for transformations between phases, and we observe two overriding trends. First, dissolved and particulate cobalt, iron, and manganese showed parallel trends with depth at the coast (Sta. 19, Fig. 9f-h), suggesting that their sources are related and/or that there is interaction between phases. Second, in all cases but that of iron, the relative concentrations of the particulate material were orders of magnitude lower than that of the dissolved counterpart $\left(\mathrm{pP}: \mathrm{dPO}_{4}{ }^{3-} \approx 1: 780, \mathrm{pCo}: \mathrm{dCo} \approx\right.$ $1: 25, \mathrm{pMn}: \mathrm{dMn} \approx 1: 6$ along the coast and $\mathrm{pP}: \mathrm{dPO}_{4}{ }^{3-} \approx 1: 4260, \mathrm{pCo}: \mathrm{dCo} \approx 1: 180$, $\mathrm{pMn}: \mathrm{dMn} \approx 1: 14$ within the gyre, $\mathrm{p}=$ particulate and $\mathrm{d}=$ dissolved, Fig. 9). These large differences indicate that if the existing inventory of $\mathrm{pP}, \mathrm{pCo}$, and $\mathrm{pMn}$ were dissolved or remineralized, they would not contribute significantly to the inventory of $\mathrm{dPO}_{4}{ }^{3-}, \mathrm{dCo}$, and $\mathrm{dMn}$ in the water column. This highlights the likely shorter residence times of these particulate metals relative to their dissolved phases. In the following paragraphs, we compare the dissolved, particulate, and sediment trap metal data from a coastal station (Sta. 19) to an open ocean station (Sta. 13).

At the open ocean Sta. 13, convex vertical profile structure was observed for dissolved cobalt, iron, and phosphorus, consistent with input via remineralization and the diffusion of metals from below (Sta. 13, Fig. 9a, b, d). Particulate profiles at this station exhibit surface particle maxima for cobalt and phosphorus, consistent with uptake and remineralization; however, this trend was not observed in the particulate iron and manganese profiles. Sediment trap data provide an estimate of remineralization rates, showing a decrease in flux with depth between the $60 \mathrm{~m}$ and $150 \mathrm{~m}$ traps (Fig. 11b-e). The integrated input from remineralization was calculated by taking the difference between the 
fluxes and dividing it by the depth between the two traps. By this estimate, remineralized metal fluxes of 0.013 pmol Co L ${ }^{-1}$ day $^{-1}, 8.5 \mathrm{pmol} \mathrm{Fe} \mathrm{L}^{-1}$ day $^{-1}, 0.11 \mathrm{pmol} \mathrm{Mn} \mathrm{L}^{-1}$ day $^{-1}$, and 11.2 nmol $\mathrm{P} \mathrm{L}^{-1}$ day $^{-1}$ were delivered to the water column between these sediment trap depths (Sta. 13). Enhanced primary productivity was observed over the region with the subsurface metal plumes (http://las.pfeg.noaa.gov/; Fig. 4c). In addition to these observations, there is a strong linear relationship between cobalt and phosphate along the

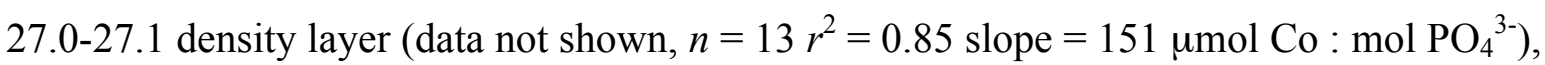
suggesting that timescales of removal of cobalt are slow, and reflect an integrated biological remineralization signal similar to that of $\mathrm{PO}_{4}{ }^{3-}$. These three lines of evidence support the input of metals via remineralization. However, this creates a conundrum since, as discussed earlier, the surface waters were depleted in cobalt and iron inventory and do not show a significant source from aeolian flux. As a result, it seems likely that both lateral fluxes and vertical fluxes contribute to the formation of the subsurface metal plumes, with supply to phytoplankton from the plume itself by upward diffusion/advection and lateral advection in surface waters also contributing to the propagation of the plume into the South Atlantic gyre.

Along the coast (Sta. 19, Fig. 9), the processes that control the dissolved and particulate trace metal distributions are more complex, and both surface and bottom water maxima are observed in the particulate profiles of manganese, phosphorus, and cobalt (Fig. 9f-h). Consistent with the degradation of sinking particulate organic matter, shallow maxima are observed in the dissolved cobalt, iron, manganese, and phosphorus profiles, coincident with a local oxygen minimum (Fig. 6a, 9f-h). The $60 \mathrm{~m}$ flux along the coast is 
indistinguishable from the open ocean flux within the error of the measurement. The sediment trap data do not detect remineralization for iron, manganese, and phosphorus at the coastal station (Sta. 19, Fig. 11b, d, e), likely due to other particulate cycling processes such as the preservation of sinking particulate matter due to high productivity and low oxygen and/or lateral advection of resuspended particulate matter. Total mass flux at 150 $\mathrm{m}$ is larger than that at $60 \mathrm{~m}$, with no noticeable change in the iron, manganese, and phosphorus fluxes (Fig. 11a, b), which supports this possible influence of particulate cycling processes other than remineralization. Table 3 shows some values of interest at Sta. 13, Sta. 19, and Sta. 25, off the coast in the southern transect. While average Chl a concentrations and primary production estimated from satellite data (http://oceancolor.gsfc.nasa.gov/, http://las.pfeg.noaa.gov/) are quite similar at Sta. 17 and 25 , their respective subsurface metal and oxygen signals are different. Additionally, the iron and manganese concentrations at Sta. 13 are quite similar to that of Sta. 25, even though primary productivity and $C h l a$ concentrations are twice as high. This again highlights the different physical processes influencing the northern and southern transects and suggests that the subsurface metal plumes are likely generated by the confluence of vertical and lateral processes that are not easily separated.

The particulate trace metal profiles for iron and manganese at the open ocean Sta. 13 also show steady increases in concentration from the surface to $600 \mathrm{~m}$ (Fig. 9c, d), and the particulate concentrations were respectively 140 and 6 fold less than the respective coastal particulate concentrations, consistent with a loss of particulate iron and manganese with water movement westward (Lam and Ohnemus unpubl., Fig. 9c, d, g, h). The changes 
in concentration of particulate and dissolved metals can also be compared between the open ocean and coastal region, assuming lateral advection contributes to transporting a fraction of both phases westward within the OMZ from Sta. 19 to 13. Calculated as the average decrease in concentration for each species between 300 and $400 \mathrm{~m}$ depth from Sta. 19 to Sta. 13, particulate iron decreased 28 times faster than dissolved iron (Fig. 9d, h), particulate cobalt decreased 7 times faster than dissolved cobalt (Fig. 9b, f), and particulate manganese decreased 2 times faster than dissolved manganese (Fig. 9c, f, g). This could also be evidence of decreased productivity and/or export. Low particulate cobalt concentrations at intermediate depths suggest that the scavenging of the laterally advected dissolved cobalt occurs slowly enough that it does not imprint a signal on the vertical profile of particulate cobalt (Figs. 9b, 11c), and this is supported by the observed persistence of enhanced dissolved concentrations into the center of the South Atlantic basin and the linear relationships with dissolved oxygen and nitrous oxide (Figs. 5, 7). While these simultaneous dissolved, particulate and sediment trap data provide an intriguing picture of the complex coastal and open ocean environments, future process studies are needed to better characterize the interactions between phases and processes.

3.3.7 Shallow coastal cobalt plume - The high surface concentrations of cobalt $\left(151-200\right.$ pmol L ${ }^{-1}$ in the upper $60 \mathrm{~m}$, Fig. 5) observed near the coast in the main transect are likely associated with the upwelling of waters high in reduced metals. Oxygen concentrations drop below $100 \mu \mathrm{mol} \mathrm{kg}{ }^{-1}$ at $15 \mathrm{~m}$ depth and reach as low as $29 \mu \mathrm{mol} \mathrm{kg}{ }^{-1}$ in the upper $60 \mathrm{~m}$. This high cobalt signal is coincident with strong beam attenuation likely due to high biological productivity (data not shown) and high nitrogen fixation 
activity (Sohn et al. in press.). Thermocline nitrate $\delta^{15} \mathrm{~N}$ values also decrease to the east and correlate with cobalt (data not shown, $r^{2}=0.70$ ), which would be consistent with an input of low $\delta^{15} \mathrm{~N}$ material from nitrogen fixation (Casciotti et al. 2008), although other processes may contribute to this pattern. While cobalt is known to be a required micronutrient in the marine cyanobacteria (Saito et al. 2005), cobalt requirements in nitrogen fixing marine diazotrophs have not been well characterized, and it is possible that the abundant cobalt and iron contribute to the nitrogen fixation measured in this region. Surface concentrations of iron are also elevated along the coast, though not to the same extent, which might be due to increased biological uptake. Surface concentrations of dissolved manganese were not significantly elevated relative to westward stations, though a source in this region could be masked by the pervasively high surface concentrations observed across the basin.

This manuscript highlights the advantages of a sectional approach to trace metal distribution studies, where the influence of important processes are more readily apparent in a section relative to vertical profiles. Specifically, we observed the eastern African boundary of the Southern Atlantic to be a strong source of cobalt. Iron and manganese were also elevated near the coast, but their concentrations rapidly decreased with distance from shore, likely due to faster oxidation/scavenging kinetics of these metals in this environment. The coastal source identified here is important because cobalt, iron, and manganese have relatively small oceanic inventories, and perturbations in their concentrations can create large gradients, significantly affecting their cycling and subsequent roles as important micronutrients to oceanic phytoplankton. Relationships 
between metals, low oxygen, and high beam attenuation in bottom waters near the coast suggest that reductive dissolution and particle resuspension were the major sources of the elevated metal concentrations observed. Dissolved oxygen concentration and shelf size influenced the magnitude of the coastal metal source fluxes, while the delivery of these metals to the ocean interior was dependent upon the physical processes influencing the system (wind-driven upwelling, advection, and slow ventilation), the extent to which each element was susceptible to scavenging, and the biological processes that deliver metals exported from the surface through uptake and remineralization. With the observed and predicted expansion of low oxygen regions in oceanic and coastal marine environments globally (Stramma et al. 2008), the fluxes of iron, cobalt, and manganese will also likely increase in magnitude in OMZs with potentially important implications for ocean primary productivity and nitrogen fixation. 


\section{References For Chapter 3:}

Atlas, E. L., S. W. Hager, L. I. Gordon and P. K. Park. 1971. A practical manual for use of the Technicon Autoanalyzer TM in seawater nutrient analyses; revised. Technical Report 215. Oregon State University, Dept of Oceanography, Ref. No. 71-22.

Bernhardt, H. and A. Wilhelms. 1967. The continuous determination of low level iron, soluble phosphate and total phosphate with the AutoAnalyzer TM. Technicon Symp., 1967, Vol. I, p. 386.

Bertrand, E. M., M.A. Saito, J. M. Rose, C.R. Riesselman, M.C. Lohan, A.E. Noble, P.A. Lee, and G.R. DiTullio. 2007. Vitamin $B_{12}$ and iron co-limitation of phytoplankton growth in the Ross Sea. Limnol. Oceanogr. 52: 1079-1093.

Bruchert, V., B. B. Jorgensen, K. Neumann, D. Riechmann, M. Schlosser, and H. Schulz. 2003. Regulation of bacterial sulfate reduction and hydrogen sulfide fluxes in the central Namibian coastal upwelling zone. Geochim. Cosmochim. Acta 67: 45054518.

Bruland, K., K. Orians, and J. Cowen. 1994. Reactive trace metals in the stratified central North Pacific. Geochim. Cosmochim. Acta 58: 3171-3182.

Bruland, K. W., and M. C. Lohan. 2003. Controls of trace metals in seawater, p. 23-47, Vol. 6 In H.D. Holland and K.K. Turekian [eds.], Treatise on Geochemistry. Elsevier.

Bruland, K. W., E. L. Rue, G. J. Smith and G. R. Ditullio. 2005. Iron, macronutrients and diatom blooms in the Peru Upwelling regime: Brown and blue waters of Peru. Mar. Chem. 93: 81-103.

Bowie, A. R., D. J. Whitworth, E. P. Achterberg, R.Fauzi, C. Mantoura, and P. J. Worsfold. 2002. Biogeochemistry of Fe and other trace elements (Al, Co, Ni) in the upper Atlantic Ocean. Deep Sea Res. I 49: 605-636.

Buesseler, K. O., A. N. Antia, M.Chen, S. W. Fowler, W. D. Gardner, O. Gustafsson, K. Harada, A. F. Michaels, M. R. van der Loeff, M. Sarin, D. K. Steinberg, and T. Trull. 2007. An assessment of the use of sediment traps for estimating upper ocean particle fluxes. J. Mar. Systems 65: 345-416.

Bruland, K. W.: GEOTRACES Co Intercalibration Results, http://www.obsvlfr.fr/GEOTRACES/libraries/documents/Intercalibration/SAFe Co.pdf, 2010.

Casciotti, K. L., T. W. Trull, D. M. Glover, and D. Davies. 2008. Constraints on nitrogen cycling at the subtropical North Pacific Station ALOHA from isotopic measurements of nitrate and particulate nitrogen. Deep-Sea Res. II 55: 1661-1672.

Chase, Z., K.S. Johnson, V. A. Elrod, J. N. Plant, S. E. Fitzwater, L. Pickell, and C. M. Sakamoto. 2005. Manganese and iron distributions off central California influenced by upwelling and shelf width. Mar. Chem. 95: 235-254.

Dyrssen, D., and K. Kremling. 1990. Increasing hydrogen sulfide concentration and trace metal behavior in the anoxic Baltic waters. Marine Chem. 30 : 193-204.

Elrod, V. A., W. M. Berelson, K. H. Coale, and K. Johnson. 2004. The flux of iron from continental shelf sediments: A missing source for global budgets. Geophys. Res. Lett. 31: L12307, doi:10.1029/2004GL020216

Emeis, K.-C., V. Bruchert, B. Currie, R. Endlier, T. Ferdelman, A. Kiessling, T. Leipe, K. 
Noli-Peard, U. Struck, and T. Vogt. Shallow gas in shelf sediments of the Namibian coastal upwelling ecosystem. Continental Shelf Res. 24: 627-642.

Gordon, L. I., J. C. Jennings, Jr., A. A Ross, and J. M. Krest. 1994. A suggested protocol for continuous flow analysis of seawater nutrients (phosphate, nitrate, nitrite, and silicic acid) in the WOCE Hydrographic Program and the Joint Global Ocean Fluxes Study. WHP Office Report 91-1. Revision 1, Nov 1994.

Heggie, D., and T. Lewis. 1984. Cobalt in pore waters of marine sediments. Nature 311 : 453-455.

Homann, V. V., M. Sandy, J. A. Tincu, A. S. Templeton, B. M. Tebo, and A. Butler. 2009. Loihichelins A-F, a suite of amphiphilic siderophores produced by the marine bacterium Halomonas LOB-5. J. Natural Prod. 72 : 884-888.

Jickells, T., T. Church, A. Veron, and R. Arimoto. 1994. Atmospheric inputs of manganese and aluminium to the Sargasso Sea and their relation to surface water concentrations. Mar. Chem. 46: 283-292.

John, H. -Ch., V. Mohrholz, J. R. E. Lutjeharms, S. Weeks, R. Cloete, A. Kreiner, and D. da Silva Neto. 2004. Oceanographic and faunistic structures across an Angola Current intrusion into northern Namibian waters. J. Mar. Systems. 46: 1-22.

Johnson, K. S., P. M. Stout, W. M. Berelson, and C. M. Sakamoto-Arnold. 1988. Cobalt and copper distributions in the waters of Santa Monica Basin, California. Nature 332: $527-530$.

Johnson, K. S., W.M. Berelson, K.H. Coale, T.L. Coley, V.A. Elrod, W.R. Fairey, H.D. Iams, T.E. Kilgore, and J.L. Nowicki. 1992. Manganese Flux from Continental Margin Sediments in a Transect Through the Oxygen Minimum. Science 257: 1242-1245.

Johnson, K. S., R. M. Gordon, and K. H. Coale. 1997. What controls dissolved iron in the world ocean? Mar. Chem 57: 137-161.

Johnson, K. S., E. A. Boyle, K. Bruland, K Coale, C. Measures, J. Moffett, A.Aguilar-Islas, K. Barbeau, B. Bergquist, A. Bowie, K. Buck, Y. Cai, Z., Chase, J. Cullen, T. Doi, V. Elrod, S. Fitzwater, M. Gordon, A. King, P. Laan, L. Laglera-Baquer, W. Landing, M. Lohan, J. Mendez, A. Milne, H. Obata, L.Ossiander, J. Plant, G. Sarthou, P. Sedwick, G.J. Smith, B. Sohst, S. Tanner, S. Van den Berg, and J. Wu. 2007. Developing Iron Standards for Seawater. EOS Transactions 88: No. 11, P. 131-132, doi:10.1029/2007EO110003

Kaupp, L., Measures, C.I., Selph., K.E., Mackenzie, F.T., The Distribution of Dissolved $\mathrm{Fe}$ and $\mathrm{Al}$ in the upper waters of the Eastern Equatorial Pacific, Deep Sea Research II, 58, 296, 2011.

Klinkhammer, G. P. 1980. Observations of the distribution of manganese over the East Pacific Rise. Chem. Geol. 29: 211-226.

Kremling, K. 1983. The Behavior of $\mathrm{Zn}, \mathrm{Cd}, \mathrm{Cu}, \mathrm{Ni}, \mathrm{Co}, \mathrm{Fe}$, and $\mathrm{Mn} \mathrm{In}$ anoxic Baltic waters. Mar. Chem. 13: 87-108.

Kuypers, M. M. M., G. Lavik, D. Woebken, M. Schmid, B. M. Fuchs, R. Amann, B. B. Jorgensen, M. S. M. Jetten, and J. M. Hayes. 2005. Massive nitrogen loss from the Benguela Upwelling system through anaerobic ammonium oxidation. Proc. Natl. Acad. Sci. 102: 6478-6483. 
Kostianoy, A. G., and J. R. E. Lutjeharms, 1999: Atmospheric effects in the AngolaBenguela frontal zone. J. Geophys. Res., 104:20962-20970.

Lam, P. J., J. K. B. Bishop, C. C. Henning, M. A. Marcus, G. A. Waychunas, and I. Y. Fung. 2006. Wintertime phytoplankton bloom in the subarctic Pacific supported by continental margin iron. Global Biogeochem. Cycles 20: GB1006, doi: $10.1029 / 2005 \mathrm{gb} 002557$.

Lam, P. J., and J. K. B. Bishop. 2008. The continental margin is a key source of iron to the HNLC North Pacific Ocean. Geophys. Res. Lett. 35: L07608 doi: $10.1029 / 2008 \mathrm{~g} 1033294$

Lamborg, C. H., K. O. Buesseler, and P. J. Lam. 2008. Sinking fluxes of minor and trace elements in the North Pacific Ocean measured during the VERTIGO program. Deep-Sea Res. II 55: 1564-1577.

Lass, H. U., and V. Mohrholz. 2008. On the interaction between the subtropical gyre and the Subtropical Cell on the shelf of the SE Atlantic. J. Marine Systems 74: 1-43.

Lavik, G., T. Stuhrmann, V. Bruchert, A. Van der Plas, V. Mohrholz, P. Lam, M. MuBmann, B. M. Fuchs, R. Amann, U. Lass, and M.M.M. Kuypers. 2009. Detoxification of suphidic African shelf waters by blooming chemolithotrophs. Nature 457: 581-585, doi:10.1038/nature07588

Lohan, M. C., and K. W. Bruland. 2008. Elevated Fe(II) and Dissolved Fe in Hypoxic Shelf Waters off Oregon and Washington: An Enhanced Source of Iron to Coastal Upwelling Regimes. Environ. Sci. Technol. 42: 6462-6468.

Mahowald, N. M., A. R. Baker, G. Bergametti, N. Brooks, R. A. Duce, T. D. Jickells, N. Kubilay, J. M. Prospero, and I. Tegen. 2005. Atmospheric global dust cycle and iron inputs to the ocean. Glob. Biogeochem. Cycle GB4025, doi: $10.1029 / 2004 \mathrm{gb} 002402$

Martin, J. H., R. M. Gordon, S. Fitzwater, and W. W. Broenkow. 1989. VERTEX: phytoplankton/iron studies in the Gulf of Alaska. Deep-Sea Res. 36: 649-680.

McIlvin, M. R., and K. L. Casciotti. 2010. Fully automated system for stable isotopic analyses of dissolved nitrous oxide at natural abundance levels. Limnol. Oceanogr. : Meth. 8: 54-66.

McIlvin, M. R., and K. L. Casciotti. in press. Technical updates to the bacterial method for nitrate isotopic analyses. Anal. Chem.

Measures, C. I. and J. M. Edmond. 1990. Aluminium in the South Atlantic: steady state distribution of a short residence time element. J. Geophys. Res. 95: 5331-5340.

Measures, C. I.. 1995. The distributions of Al in the IOC stations of the eastern Atlantic between $30^{\circ} \mathrm{S}$ and $34^{\circ} \mathrm{N}$. Mar. Chem 49: 267-281.

Measures, C.I., and S. Vink. 2000. On the use of dissolved aluminium in surface waters to estimate dust deposition to the ocean, Global Biogeochemical Cycles, 14, 317-327.

Measures, C. I., W. M. Landing, M. T. Brown, and C. S. Buck. 2008. High-resolution Al and Fe data from the Atlantic Ocean CLIVAR-CO2 Repeat Hydrography A16N transect: Extensive linkages between atmospheric dust and upper ocean geochemistry. Global Biogeochem. Cycles 22: GB1005, doi: $10.1029 / 2007 \mathrm{gb} 003042$

Moffett, J. W., and J. Ho. 1996. Oxidation of cobalt and manganese in seawater via a 
common microbially catalyzed pathway. Geochim. Cosmochim. Acta 60 : 34153424.

Moffett, J. W., T. J. Goepfert, and S. W. A. Naqvi. 2007. Reduced iron associated with secondary nitrite maxima in the Arabian Sea. Deep-Sea Res. I 54: 1341-1349.

Mohrholz, V., C. H. Bartholomae, A. K. Van Der Plas, and H. U. Lass. 2008. The seasonal variability of the northern Benguela undercurrent and its relation to the oxygen budget on the shelf. Continental Shelf Res. 28: 424-441.

Moore, C. M., M. M. Mills, E. P. Achterberg, R. J. Geider, J. LaRoche, M. I. Lucas, E. L. McDonagh, X. Pan, A. J. Poulton, M. J. A. Rijkenberg, D. J. Suggett, S. J. Ussher, and E. M. S. Woodward. 2009. Large-scale distribution of Atlantic nitrogen fixation controlled by iron availability. Nature Geosci. 2: 867-871.

Moore, J. K., S. C. Doney, and K. Lindsay. 2004. Upper ocean ecosystem dynamics and iron cycling in a global three-dimensional model. Global Biogeochem. Cycles 18: GB4028. doi:4010.1029/2004GB002220

Moore, J. K., and O. Braucher. 2008. Sedimentary and mineral dust sources of dissolved iron to the World Ocean. Biogeosciences 4: 1279-1327.

Morel, F. M. M., A. J. Milligan, M. A. Saito, D. H. Heinrich, and K. T. Karl. 2003. Marine Bioinorganic Chemistry: The Role of Trace Metals in the Oceanic Cycles of Major Nutrients, p. 113-143. Vol. 6 In H.D. Holland and K.K. Turekian [eds.], Treatise on Geochemistry. Elsevier.

Noble, A. E., M. A. Saito, K. Maiti, and C. Benitez-Nelson. 2008. Cobalt, manganese, and iron near the Hawaiian Islands: A potential concentrating mechanism for cobalt within a cyclonic eddy and implications for the hybrid-type trace metals. Deep-Sea Res. II 55: 1473-1490.

Orians, K. J., and K. W. Bruland. 1986. The biogeochemistry of aluminum in the Pacific Ocean. Earth and Planetary Sci. Lett. 78: 397-410, doi: 10.1016/0012821X(86)90006-3

Panzeca, C., A. J. Beck, K. Leblanc, G. T. Taylor, D. A. Hutchins, and S. A. SanudoWilhelmy. 2008. Potential cobalt limitation of vitamin $B_{12}$ synthesis in the North Atlantic Ocean. Global Biogeochem. Cycles 22: GB2029. doi:10.1029/2007GB003124

Parker, D. L., T. Morita, M. L. Mozafarzadeh, R. Verity, J. K. McCarthy, and B. M. Tebo. 2007. Inter-relationships of $\mathrm{MnO}_{2}$ precipitation, siderophore-Mn(III) complex formation, siderophore degradation, and iron limitation in $\mathrm{Mn}(\mathrm{II})$-oxidizing bacterial cultures. Geochim. Cosmochim. Acta 71: 5672-5683.

Patton, C. J. 1983. Design, characterization and applications of a miniature continuous flow analysis system. Ph.D. thesis, Mich. State U.

Peterson, R. G., and L. Stramma. 1991. Upper-level circulation in the South Atlantic Ocean. Prog. Oceanogr. 26: 1-73.

Pohl, C., P. L. Croot, U. Hennings, T. Daberkow, G. Budeus, and M. R. V. D. Loeff. 2011. Synoptic transects on the distribution of trace elements $(\mathrm{Hg}, \mathrm{Pb}, \mathrm{Cd}, \mathrm{Cu}, \mathrm{Ni}, \mathrm{Zn}, \mathrm{Co}$, $\mathrm{Mn}, \mathrm{Fe}$, and Al) in surface waters of the Northern- and Southern East Atlantic. J. Marine Systems 84: 28-41.

Prasad, M. S. 1989. Production of copper and cobalt at Gecamines, Zaire. Minerals 
Engineering 2: 521-541.

Roberts, S., T. Lane, and F. M. M. Morel. 1997. Carbonic anhydrase in the marine diatom Thalassiosira weissflogii (Bacillariophyceae). J. Phycol. 33 : 845-850.

Saito, M. A., and J. W. Moffett. 2001. Complexation of cobalt by natural organic ligands in the Sargasso Sea as determined by a new high-sensitivity electrochemical cobalt speciation method suitable for open ocean work. Mar. Chem. 75: 49-68.

Saito, M. A., and J. W. Moffett. 2002. Temporal and spatial variability of cobalt in the Atlantic Ocean. Geochim. Cosmochim. Acta 66: 1943-1953.

Saito, M. A., J. W. Moffett, S. W. Chisholm, and J. B. Waterbury. 2002. Cobalt limitation and uptake in Prochlorococcus. Limnol. Oceanogr. 47: 1629-1636.

Saito, M. A., J. W. Moffett, and G. Ditullio. 2004. Cobalt and Nickel in the Peru Upwelling Region: a Major Flux of Cobalt Utilized as a Micronutrient. Global Biogeochem. Cycles 18: GB4030, doi:10.1029/2003GB002216

Saito, M. A., G. Rocap, and J. W. Moffett. 2005. Production of cobalt binding ligands in a Synechococcus feature at the Costa Rica Upwelling Dome. Limnol. Oceanogr. 50: 279-290.

Saito, M. A., and D. L. Schneider. 2006. Examination of precipitation chemistry and improvements in precision using the $\mathrm{Mg}(\mathrm{OH})_{2}$ preconcentration ICP-MS method for high-throughput analysis of open-ocean Fe and Mn in seawater. Anal. Chim. Acta 565: 222-233.

Saito, M. A., and T. J. Goepfert. 2008. Zinc-cobalt colimitation in Phaeocystis antarctica. Limnol. Oceanogr. 53: 266-275.

Saito, M. A., T. J. Goepfert, and J. T. Ritt. 2008. Some thoughts on the concept of colimitation: three definitions and the importance of bioavailability. Limnol. Oceanogr. 53: 276-290.

Saito, M. A., E. M. Bertrand, V. Bulygin, D. Moran, S. Dutkiewicz, F. Monteiro, M.J. Follows, F. W. Valois, and J. B. Waterbury. 2011. Iron conservation by reduction of metalloenzyme inventories in the marine diazotroph Crocosphaera watsonii. Proc. Natl. Acad. Sci. 6: 2184-2189, doi: 10.1073/pnas.1006943108

Schlitzer, R., Ocean Data View, http://odv.awi.de, 2009.

Sharma, V. S., R. B. Pilz, G. R. Boss, and D. Magde. 2003. Reactions of Nitric Oxide with Vitamin $B_{12}$ and Its Precursor, Cobinamide. Biochemistry 42: 8900-8908.

Shelley, R. U., B. Zachhuber, P. Sedwick, P. J. Worsfold, and M. C. Lohan. 2010. Determination of total dissolved cobalt in UV-irradiated seawater using flow injection with chemiluminescence detection. Limnol. Oceanogr. Methods. 8: 352362.

Sholkovitz, E. R. 1976. Flocculation of dissolved organic and inorganic matter during the mixing of river water and seawater. Geochim. Cosmochim. Acta 40: 831-845.

Statham, P. J., P. A. Yeats, and W. M. Landing. 1998. Manganese in the eastern Atlantic Ocean: processes influencing deep and surface water distributions. Mar. Chem. 61 : 55-68.

Steinberg, D. K., C. A. Carlson, N. R. Bates, R. J. Johnson, A. F. Michaels, and A. H. Knap. 2001. Overview of the US JGOFS Bermuda Atlantic Time-series Study 
(BATS): a decade-scale look at ocean biology and biogeochemistry. Deep-Sea Res. II 48: 1405-1448.

Stramma, L., G. C. Johnson, J. Sprintall, and V. Mohrholz. 2008. Expanding OxygenMinimum Zones in the Tropical Oceans. Science 32 0: 655-658.

Stumm, W., and J. J. Morgan. 1996. Aquatic Chemistry: Chemical Equilibria and Rates in Natural Waters, third edition. John Wiley \& Sons.

Sunda, W. G., and S. A. Huntsman. 1988. Effect of sunlight on redox cycles of manganese in the southwestern Sargasso Sea. Deep-Sea Res. 35: 1297-1317.

Sunda, W., and S. A. Huntsman. 1995. Cobalt and zinc interreplacement in marine phytoplankton: Biological and geochemical implications. Limnol. Oceanogr. 40: 1404-1417.

Tebo, B., K. Nealson, S. Emerson, and L. Jacobs. 1984. Microbial meditation of Mn(II) and $\mathrm{Co}(\mathrm{II})$ precipitation at the $\mathrm{O}_{2} / \mathrm{H}_{2}$ interfaces in two anoxic fjords. Limnol. Oceanogr. 29: 1247-1258.

Tebo, B. M., J.R. Bargar, B.G. Clement, G. J. Dick, K. J. Murray, D. Parker, R. Verity, and S. M. Webb. 2004. Biogenic manganese oxides: properties and mechanisms of formation. Annu. Rev. Earth Planet. Sci. 32 : 287-328.

Taylor, S. R., and S. M. McLennan. 1985. The Continental Crust: its composition and evolution. Blackwell Scientific Publications.

Thuroczy, C.-E., M. Boye, and R. Losno. 2010. Dissolution of cobalt and zinc from natural and anthropogenic dusts in seawater. Biogeosciences. 7: 1927-1936.

Vink, S. and C. Measures. 2001. The role of dust deposition in determining surface water distributions of Al and Fe in the South West Atlantic. Deep-Sea Res. II 48: 27872809.

Ward, B.B. and O.C. Zafiriou. 1988. Nitrification and nitric oxide in the oxygen minimum of the eastern tropical North Pacific. Deep-Sea Res. 35:1127-1142.

Weeks, S. J., and F. A. Shillington. 1994. Interannual scales of variation of pigment concentrations from coastal zone color scanner data in the Benguela Upwelling system and the Subtropical Convergence zone south of Africa, J. Geophys. Res., 99: 7385-7399.

Wu, J., W. Sunda, E. A. Boyle, and D. M. Karl. 2000. Phosphate depletion in the Western North Atlantic Ocean. Science 289: p. 759-762.

Yoshida, N. 1988. ${ }^{15} \mathrm{~N}$-depleted $\mathrm{N}_{2} \mathrm{O}$ as product of nitrification. Nature 355: p. 528-529. 


\section{Tables and Figures for Chapter 3:}

Table 1. SAFe and GEOTRACES standard seawater cobalt analysis results for UV digested samples. All values are in $\mathrm{pmol} \mathrm{L}^{-1}$.

\begin{tabular}{ccccc} 
Sample & Dec 2008 & Aug 2010 & Oct 2010 & Current consensus \\
\hline S1 & $7 \pm 1.4(n=5)$ & $4 \pm 2.9(n=4)$ & na & $4.2 \pm 1.9$ \\
D2 & $49 \pm 5.8(n=5)$ & $45 \pm 2.9(n=2)$ & na & $43.1 \pm 3.2$ \\
GS & $31 \pm 1.7(n=4)$ & $25 \pm 1.2(n=4)$ & $31 \pm 2.9(n=7)$ & na \\
GD & $70 \pm 5.3(n=4)$ & $59 \pm 5.2(n=6)$ & $70 \pm 5.2(n=9)$ & na
\end{tabular}

Table 2. Comparison of shelf width and iron concentration in upwelling regions.

\begin{tabular}{|c|c|c|c|c|c|c|}
\hline Study & Location & Shelf width & lowest $\left[\mathrm{O}_{2}\right]$ reported & Fe species & {$[\mathrm{Fe}] \mathrm{nmol} \mathrm{L}^{-1}$} & Depth range \\
\hline Chase et al. 2005 & California coast & $2.5 \mathrm{~km}$ & $50-100 \mu \mathrm{mol} \mathrm{L}^{-1}$ & $<20 \mu \mathrm{m} \mathrm{Fe}(\mathrm{III})$ & $\begin{array}{l}0.6 \\
\text { nd }\end{array}$ & $\begin{array}{l}\text { upwelled waters } \\
\text { bottom }\end{array}$ \\
\hline Chase et al. 2005 & California coast & $10 \mathrm{~km}$ & $50-100 \mu \mathrm{mol} \mathrm{L}^{-1}$ & $<20 \mu \mathrm{m} \mathrm{Fe}(\mathrm{III})$ & $\begin{array}{c}12-20 \\
\text { nd }\end{array}$ & $\begin{array}{l}\text { upwelled waters } \\
\text { bottom }\end{array}$ \\
\hline Bruland et al. 2005 & Peruvian coast & $10 \mathrm{~km}$ & $<5 \mu \mathrm{mol} \mathrm{L}^{-1}$ & $<0.4 \mu \mathrm{m} \mathrm{FeTd} *$ & $\begin{array}{l}0.1-0.2 \\
1.4-4.3\end{array}$ & $\begin{array}{l}\text { upwelled waters } \\
\text { bottom }\end{array}$ \\
\hline Bruland et al. 2005 & Peruvian coast & $150 \mathrm{~km}$ & $<5 \mu \mathrm{mol} \mathrm{L}^{-1}$ & $<0.4 \mu \mathrm{m} \mathrm{FeTd}$ & $\begin{array}{l}3.5-16 \\
48-51\end{array}$ & $\begin{array}{l}\text { upwelled waters } \\
\text { bottom }\end{array}$ \\
\hline This study & SE Atlantic Sta. 19 & $10 \mathrm{~km}$ & $35 \mu \mathrm{mol} \mathrm{L}^{-1}$ & $<0.4 \mu \mathrm{m} \mathrm{FeTd}$ & $\begin{array}{l}1 \\
3\end{array}$ & $\begin{array}{c}10 \mathrm{~m} \\
\text { bottom }\end{array}$ \\
\hline This study & SE Atlantic Sta. 23 & $150 \mathrm{~km}$ & $11 \mu \mathrm{mol} \mathrm{L}^{-1}$ & $<0.4 \mu \mathrm{m} \mathrm{FeTd}$ & $\begin{array}{c}1.6 \\
8\end{array}$ & $\begin{array}{c}10 \mathrm{~m} \\
\text { bottom }\end{array}$ \\
\hline
\end{tabular}


Table 3. Comparison of chemical properties at $400 \mathrm{~m}$ depth to surface productivity for two stations from the main transect and one station from the southern transect. Differences in MODIS Chl $a$ concentrations do not mirror differences in trace metals, suggesting that vertical and lateral inputs likely work together to create the observed plumes. Chl $a$ concentrations are estimated from an image of MODIS satellite data for a $4 \mathrm{~km}$ resolution average for November 2007 (http://oceancolor.gsfc.nasa.gov/) and average primary productivity numbers are from Seawifs December 2007 averaged data http://oceanwatch.pfeg.noaa.gov/.

\begin{tabular}{cccccccc} 
Sta. & $\begin{array}{c}\text { Surf. Chl } a \\
\left(\mathrm{mg} \mathrm{m}^{-3}\right)\end{array}$ & $\begin{array}{c}\text { average PP } \\
\left(\mathrm{mg} \mathrm{m}^{-2} \mathrm{~d}^{-1}\right)\end{array}$ & $\begin{array}{c}\text { dist. to land } \\
(\mathrm{km})\end{array}$ & $\begin{array}{c}\mathrm{O}_{2} \\
\left(\mu \mathrm{mol} \mathrm{kg}^{-1}\right)\end{array}$ & $\begin{array}{c}\mathrm{Co} \\
\left(\mathrm{pmol} \mathrm{L}^{-1}\right)\end{array}$ & $\begin{array}{c}\mathrm{Fe} \\
\left(\mathrm{nmol} \mathrm{L}^{-1}\right)\end{array}$ & $\begin{array}{c}\mathrm{Mn} \\
\left(\mathrm{nmol} \mathrm{L}^{-1}\right)\end{array}$ \\
\hline 13 & $0.2-0.5$ & 424 & 1300 & 42 & 130 & 0.71 & 0.43 \\
17 & $0.8-1.00$ & 831 & 275 & 21 & 146 & 0.96 & 0.78 \\
25 & $0.8-1.00$ & 860 & 290 & 163 & 42 & 0.68 & 0.41
\end{tabular}


Figure 1) Cruise track with station locations and full depth ocean sections of total dissolved iron, manganese, cobalt, and labile cobalt for the CoFeMUG expedition across the Southern Atlantic Ocean. A large plume of cobalt, with corresponding but smaller plumes of iron and manganese, originates at the Southwest African coast and extends to the center of the basin. Coastal sources are likely due to a combination of reductive dissolution and resuspension of particulate matter in the sediments along the shelf. A plume of iron and manganese also extends westward from the Mid-Atlantic Ridge, with no corresponding elevated concentrations for cobalt (Saito et al. unpubl.). Note that the main transect (E-W), the meridional transect along the Namibian coast (N-S), and the southern zonal transect (W-E) are connected to form a continuous wrap-around section for each analyte, by cruise trace distance from Sta. 1 . 


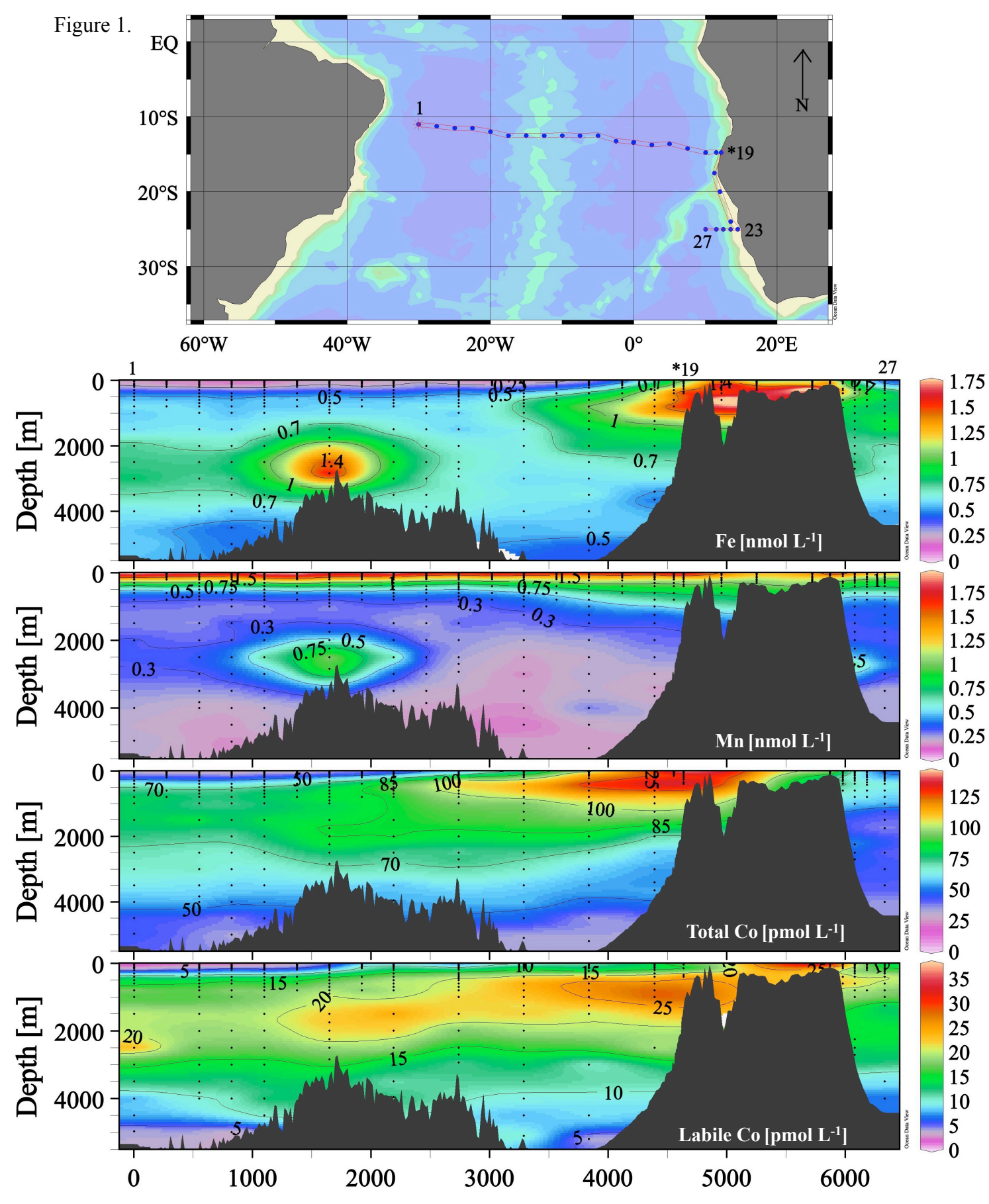

Distance [km] 


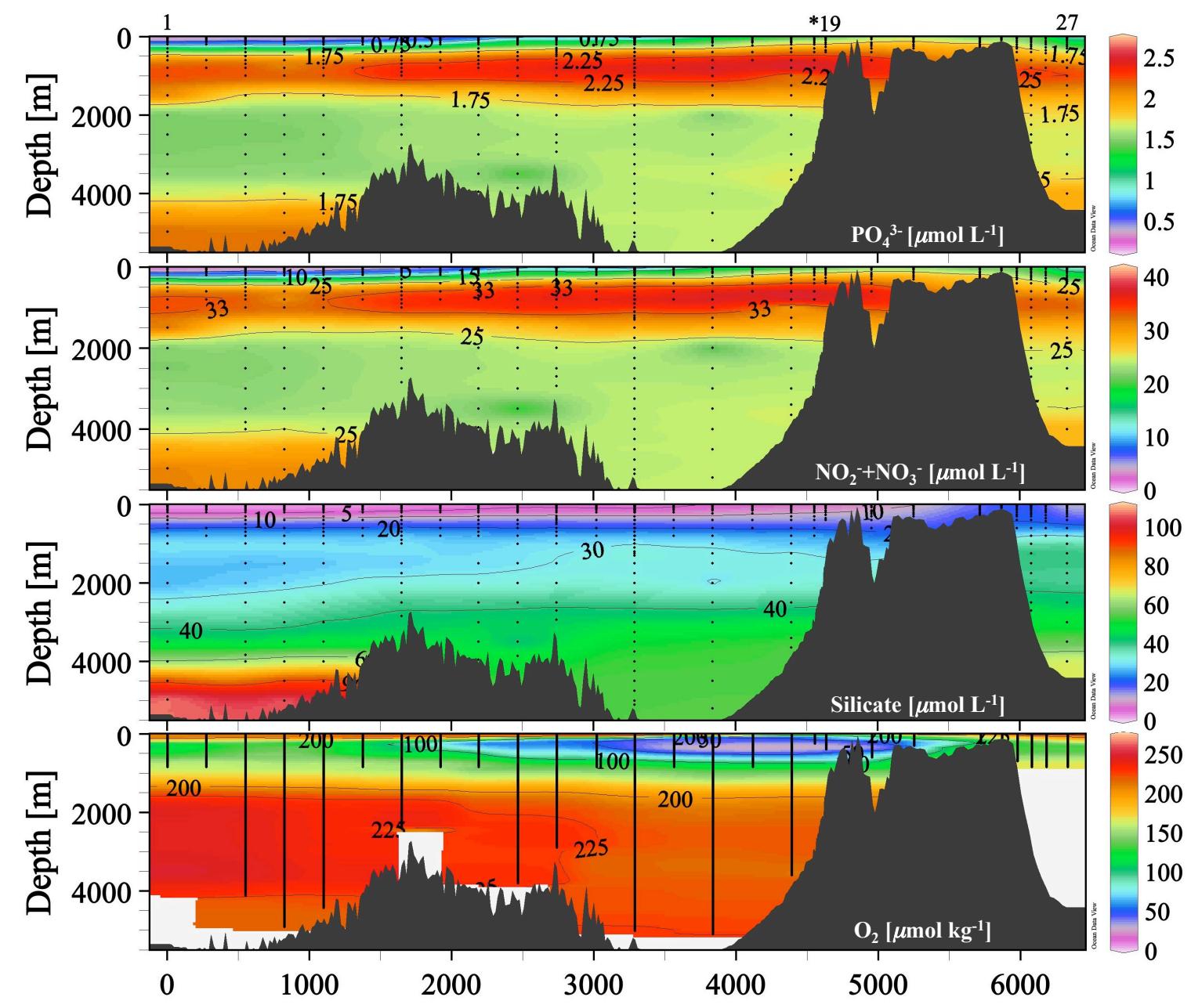

\section{Distance $[\mathrm{km}]$}

Figure 2) Full depth ocean sections of macronutrients, and oxygen for the CoFeMUG expedition. There is an oxygen minimum zone in the eastern end of the basin due to high productivity and subsequent organic matter decomposition and the limited water mass ventilation. 

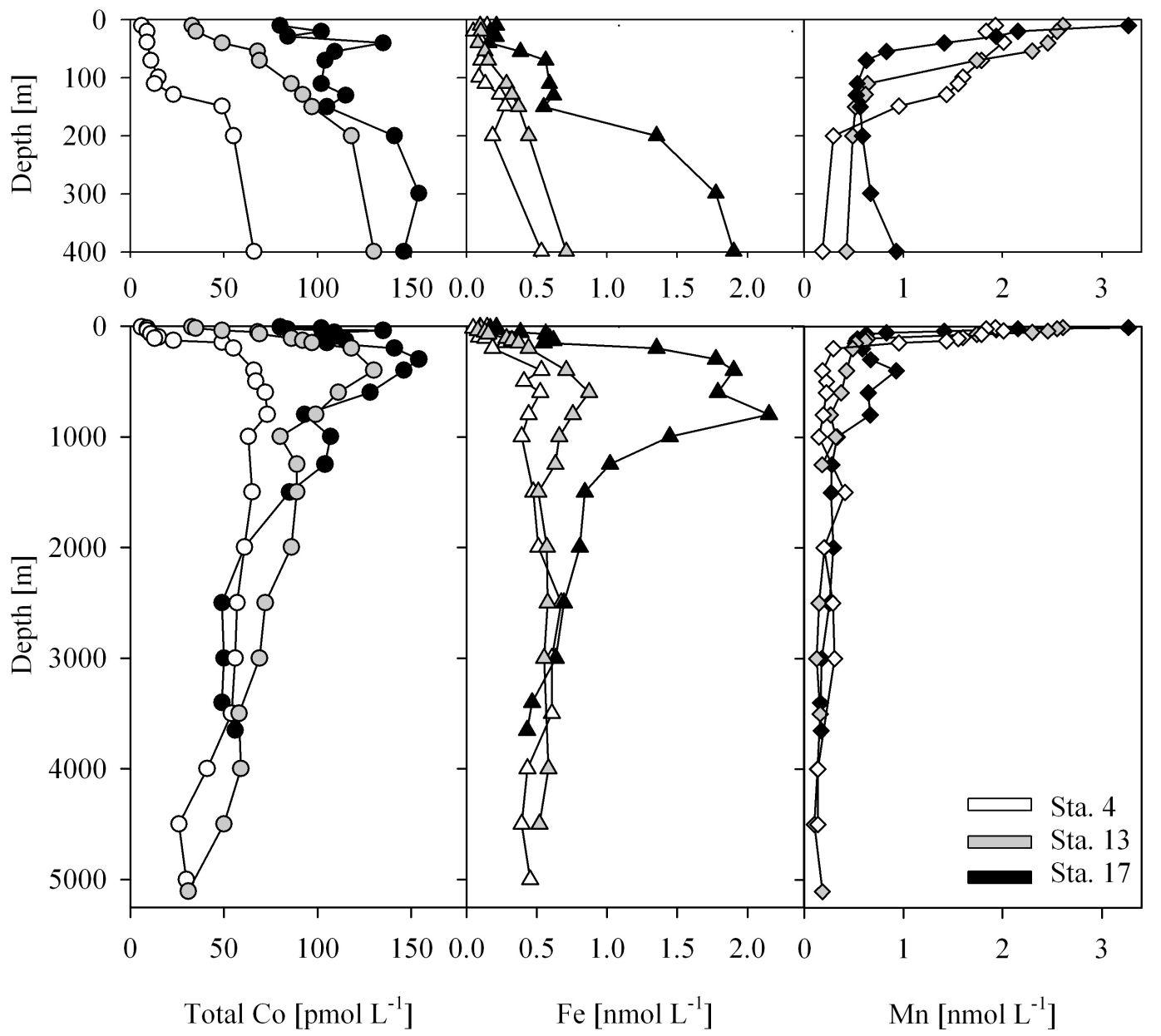

Figure 3) Representative vertical profiles from the CoFeMUG dataset, showing the subsurface maxima for cobalt, iron, and manganese with proximity to the coast. 

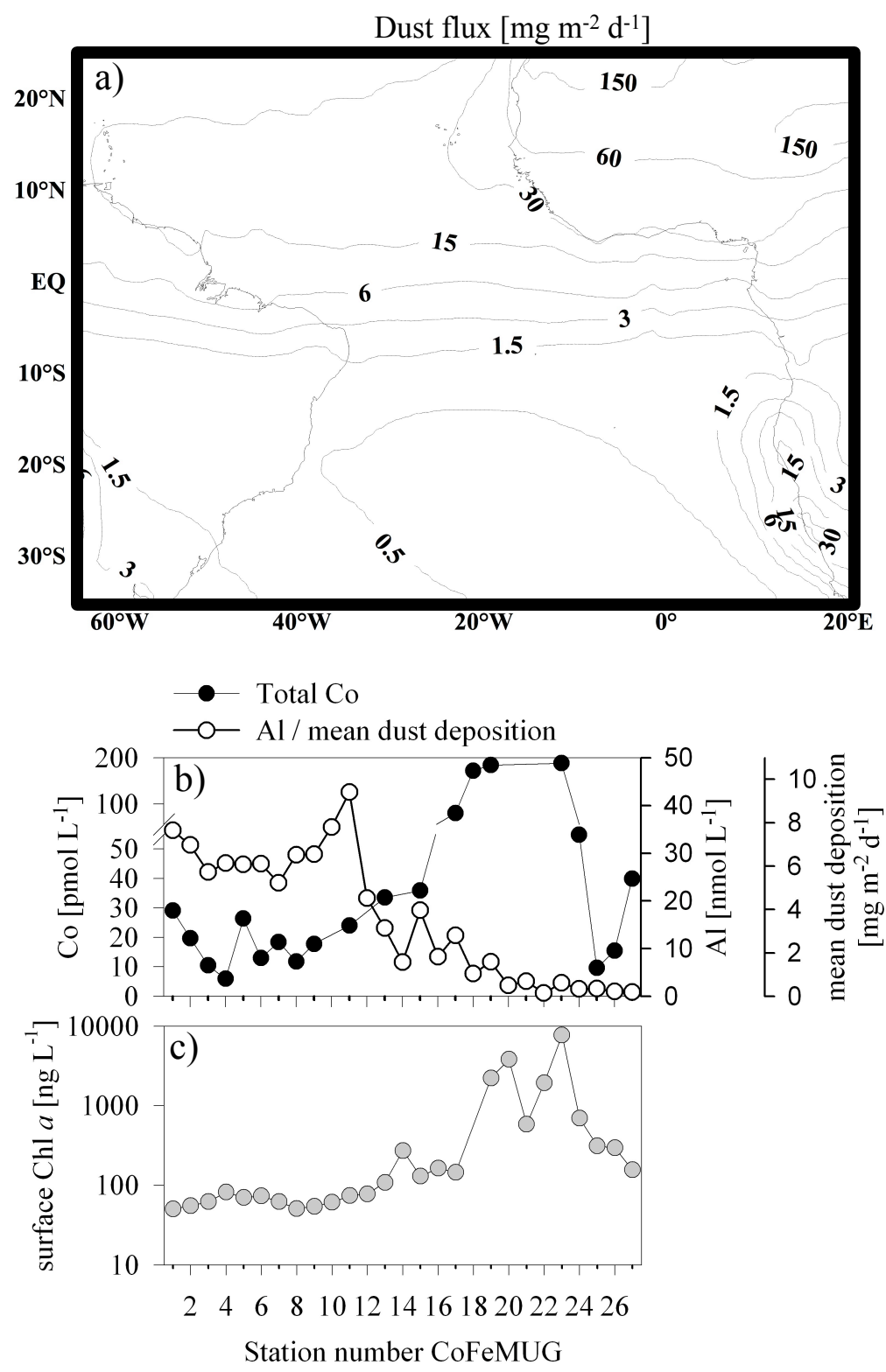

Figure 4) a) Estimates of dust input to the South Atlantic region, generated using data and a model generously provided by N. Mahowald (Mahowald et al. 2005). Inputs of dust are estimated to be much higher in the North Atlantic than the South Atlantic. b) surface transect of total dissolved cobalt and dissolved aluminum showing an opposite relationship, suggesting that the high dissolved cobalt near the coast is not derived from dust. c) measured surface $\mathrm{Chl} a$ concentrations. 


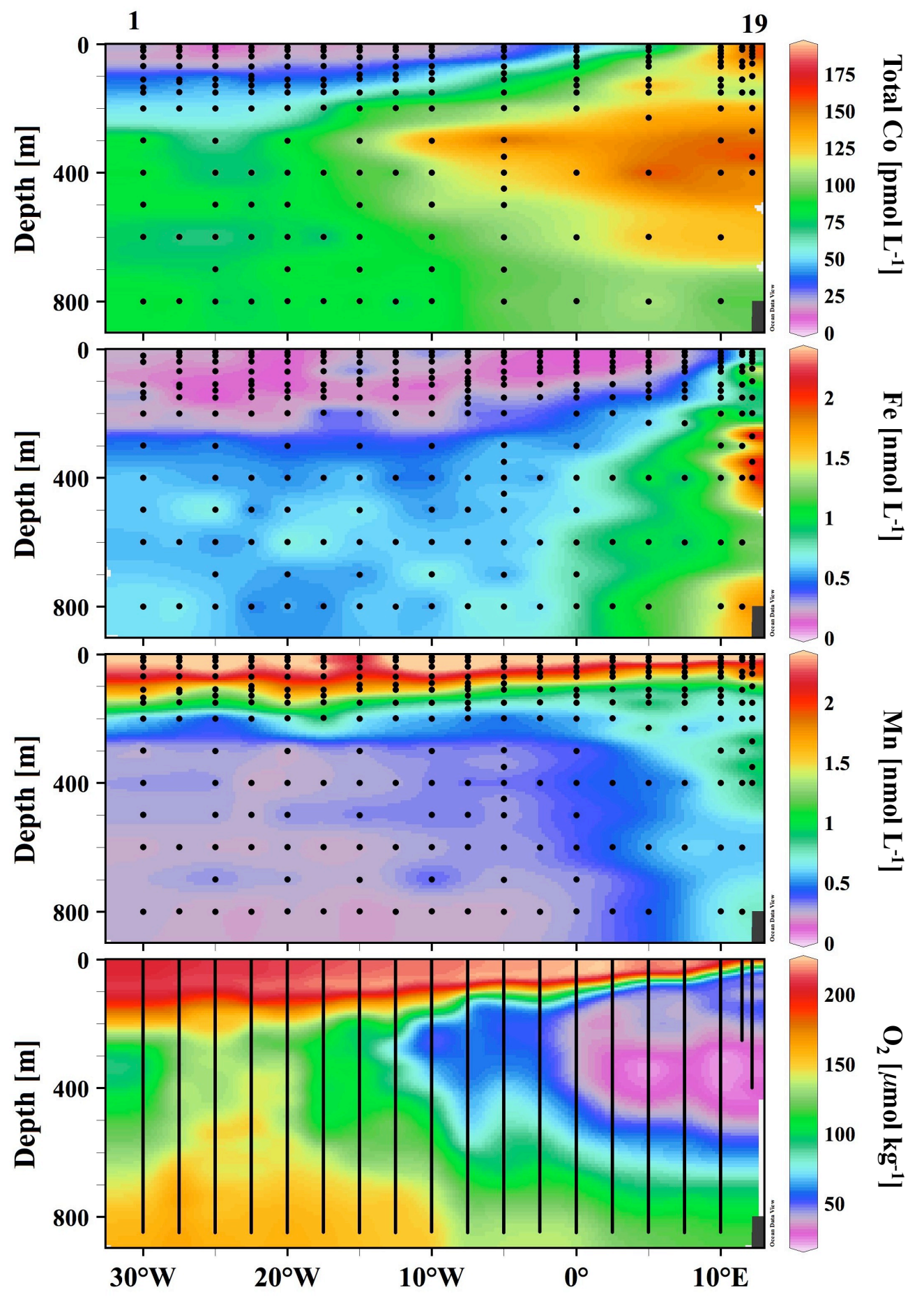

Figure 5) Expanded upper water column sections for the northern transect. Metal concentrations decrease with distance from the coast, and the coincidence of the oxygen minimum zone with iron, manganese, and particularly cobalt, is evident. 


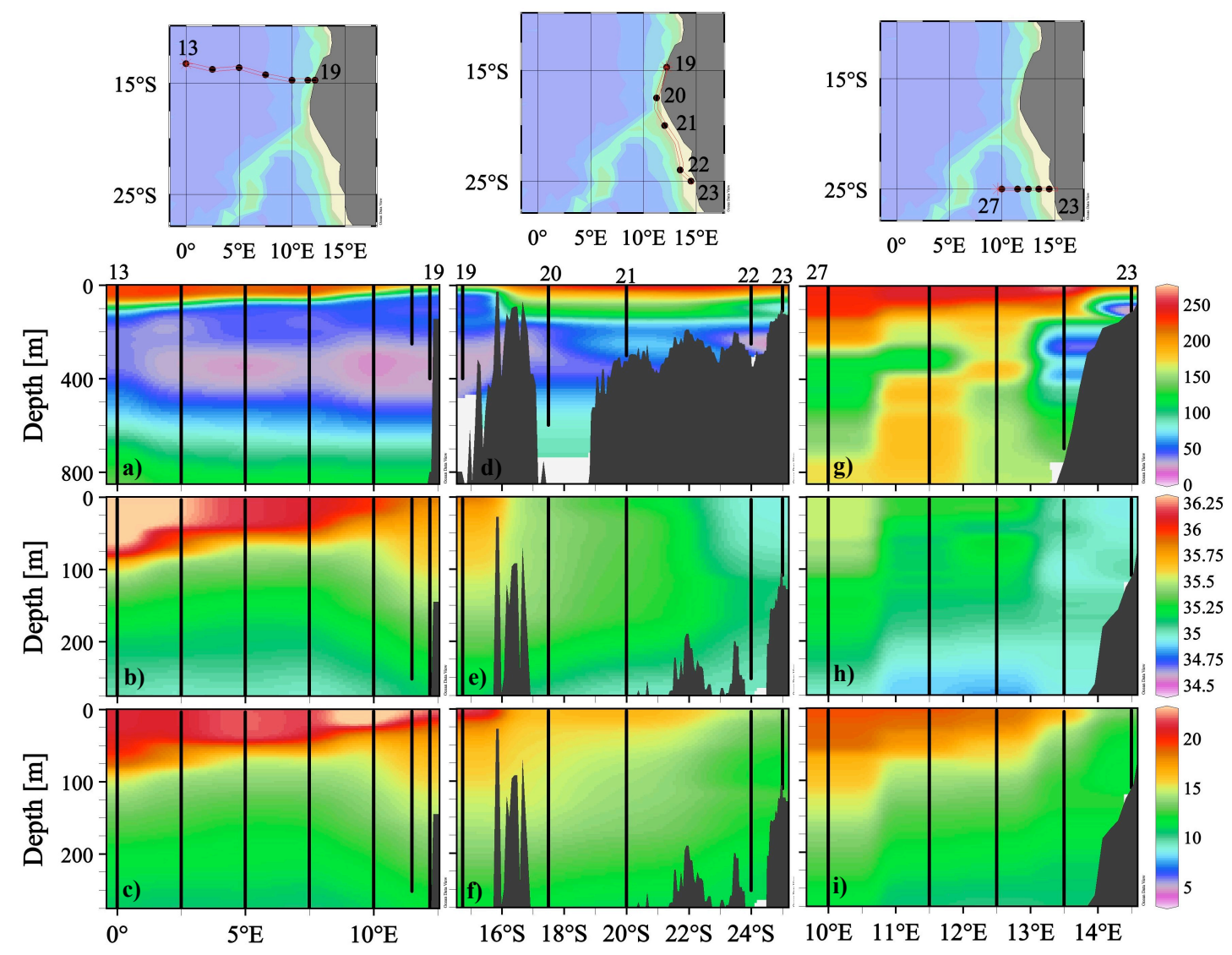

Figure 6) Dissolved oxygen ( $\mu \mathrm{mol} \mathrm{kg}{ }^{-1}$ ) in the upper $800 \mathrm{~m}$ near the Southwest African coast for the (a) northern, (d) coastal, and (g) southern transects, salinity $(\%$ o $)$ in the upper $300 \mathrm{~m}$ near the Southwest African coast for the (b) northern, (e) coastal, and (h) southern transects, and potential temperature $\left({ }^{\circ} \mathrm{C}\right.$ ) for the (c) northern, (f) coastal, and (i) southern transects. Oxygen concentrations are much higher in the southern transect than the northern and coastal transects, and low oxygen waters along the coast do not appear to be advected westward in the south as they are in the north. 


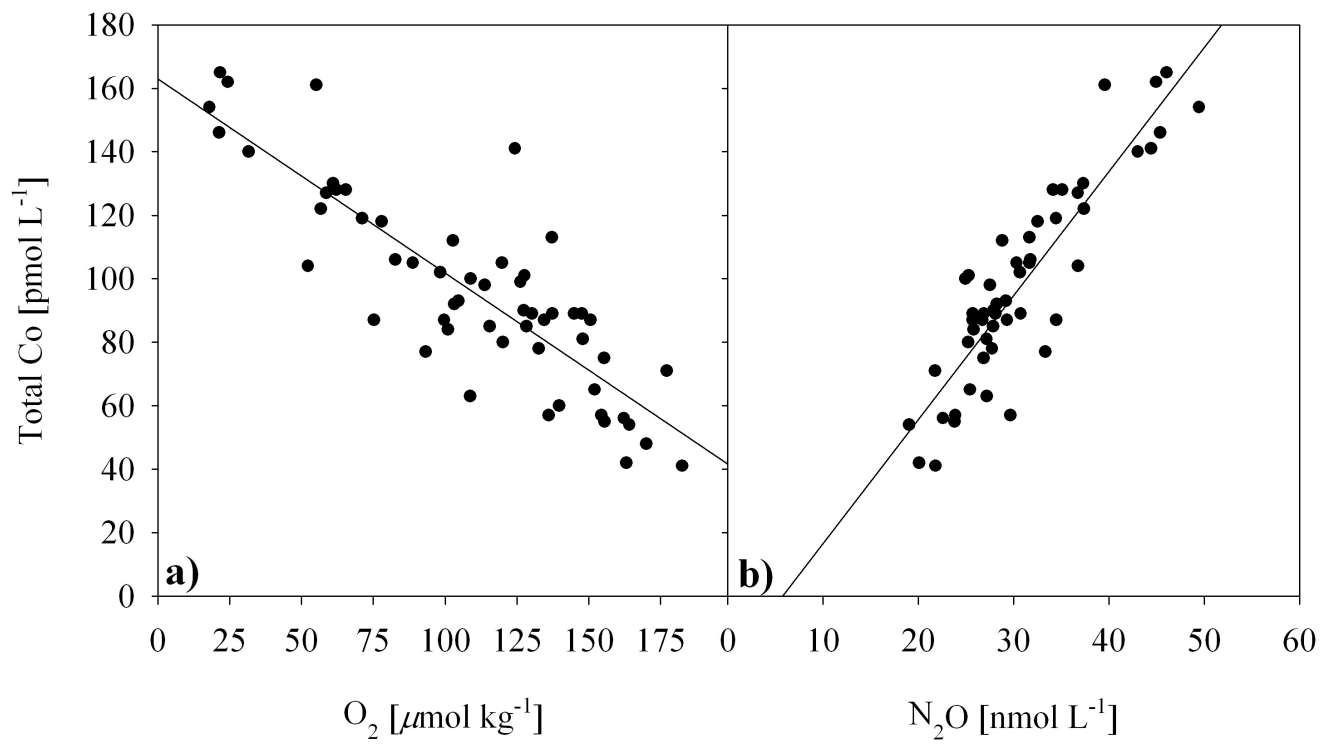

Figure 7) Relationships between (a) oxygen and total dissolved cobalt and (b) $\mathrm{N}_{2} \mathrm{O}$ and total dissolved cobalt for stations $5-27$ between 300 and $800 \mathrm{~m}$ depth. The $300-800 \mathrm{~m}$ analyses showed a linear correlation between $\mathrm{O}_{2}$ and $\mathrm{Co}\left(r^{2}=0.73\right) . \mathrm{N}_{2} \mathrm{O}$ also shows a linear correlation with cobalt $\left(r^{2}=0.80\right)$, suggesting that the two chemical species have similar coastal source locations. 
Figure 8) Distributions of dissolved cobalt, iron, and manganese along isopycnal surfaces that correspond to 400, 600, and $800 \mathrm{~m}$ depths from the main transect. Potential density with respect to (a) oxygen and (b) distance are plotted at the three depths. Scavenging rates are estimated using Sta. 19 as the starting point and data to the west increasing in distance. Linear correlations between (c) total dissolved cobalt and oxygen, and (d) cobalt and distance are indicative of advection for both chemical species. The relationships between (e) iron and oxygen, (f) iron and distance, (g) manganese and oxygen, and (h) manganese and distance are all non-linear. When plotted as a function of distance, the data points fall along the same curve (f, h). Error bars here represent a standard deviation of two analyses, where in most cases, the first analysis was conducted in batches by profile over a number of discrete ICP-MS runs (10 days of analyses total), while the second data point verified precision by analysis of all samples along each isopycnal on a single ICP-MS run. This approach verified that the day-to-day variability on the ICP-MS was not systematically affecting the isopycnal analysis. 


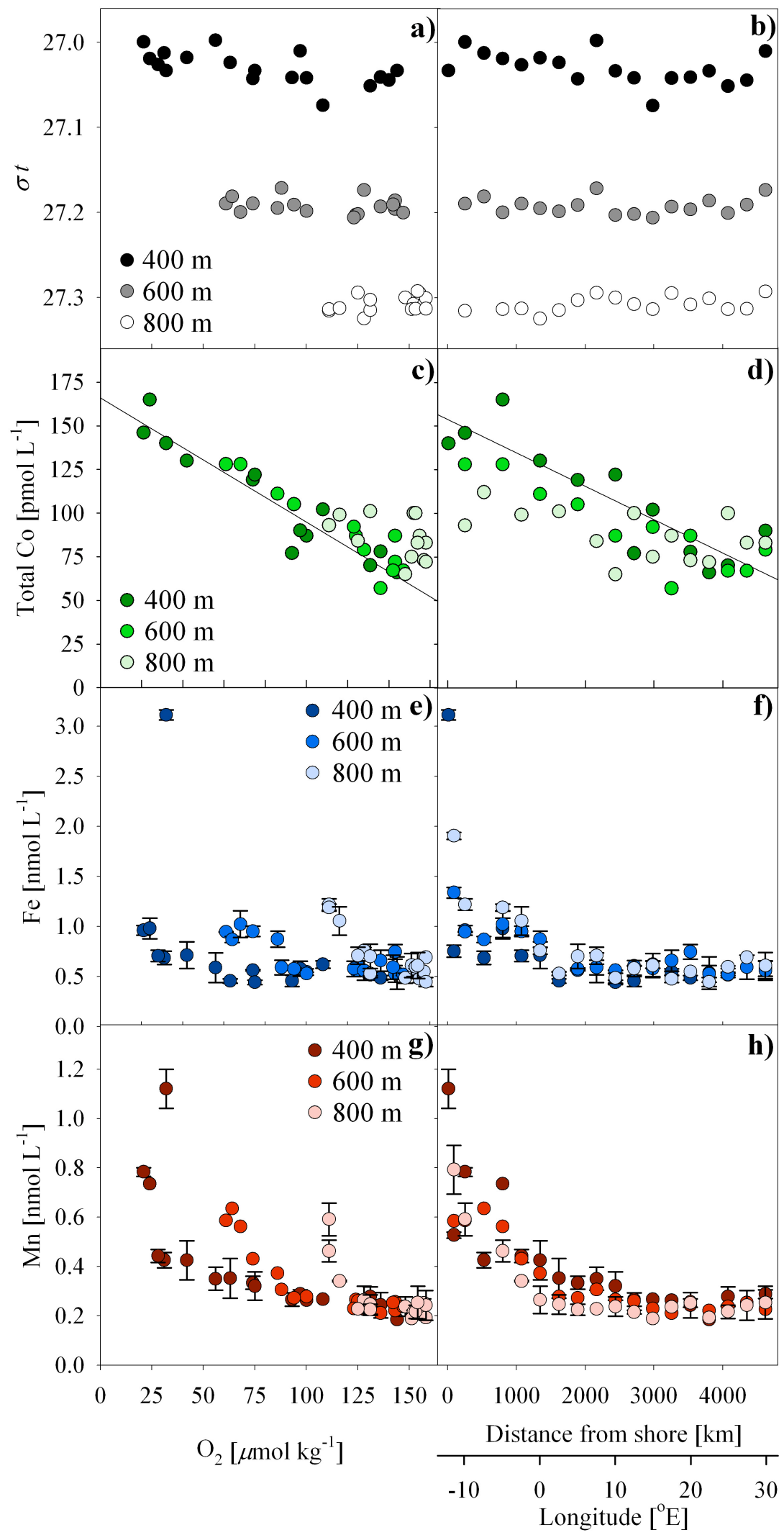


Figure 9) Trace metal particulate (open symbols) and dissolved (filled symbols) profiles for the upper $800 \mathrm{~m}$ from Sta. 13 (top panel) and 19 (bottom panel). Dissolved concentrations of phosphorus, cobalt, and manganese far surpass that of their respective particulate concentrations, however the particulate concentrations of iron are approximately 5 times higher than that of the dissolved phase. A brief schematic is presented beneath the profiles to summarize the observations for each metal and facilitate comparison. 

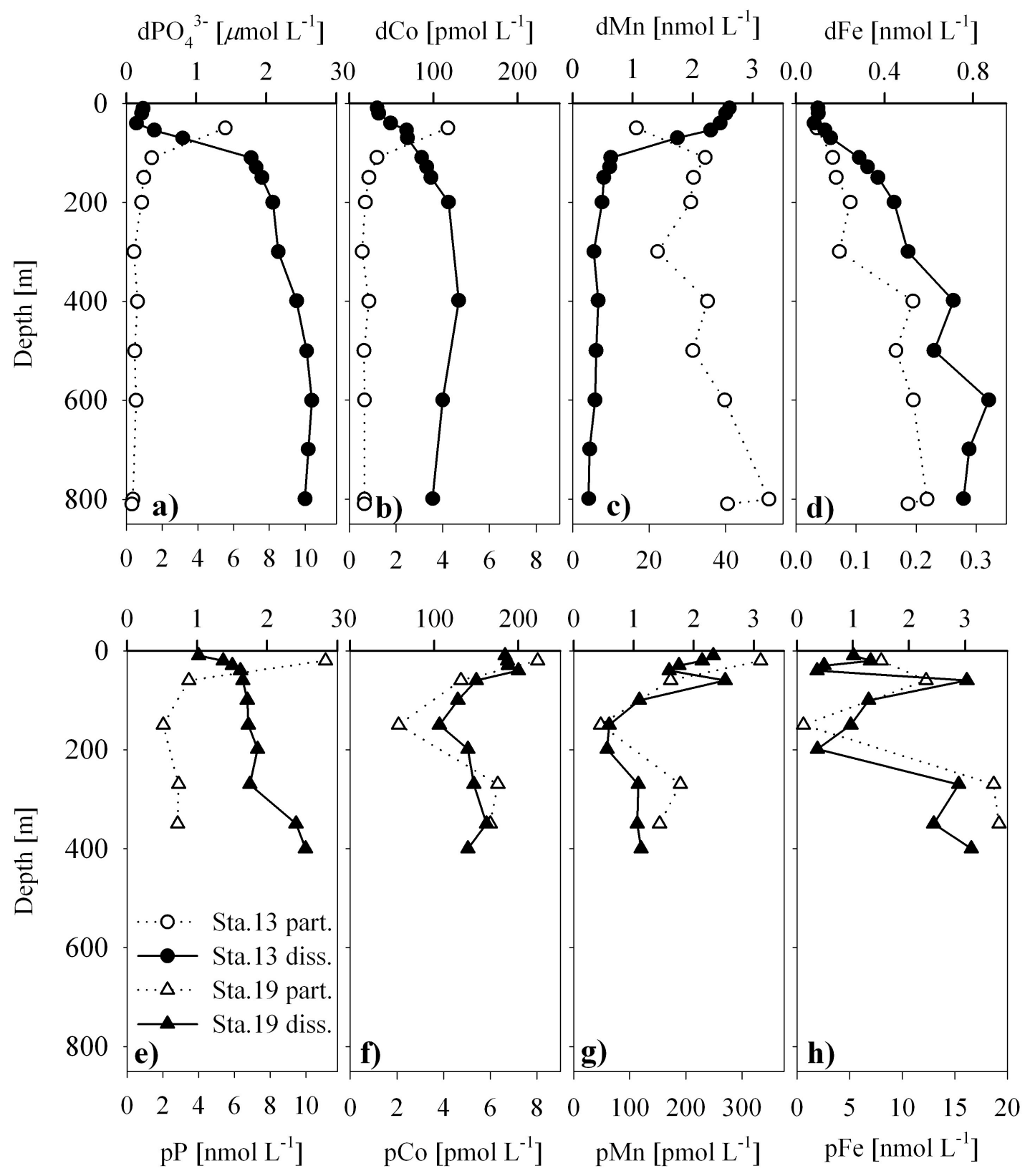

\begin{tabular}{cccc} 
& dissolved & particulate & spatial observations \\
\hline $\mathrm{Co}$ & $\begin{array}{c}\text { low } \\
\text { scavenging }\end{array}$ & coastal $>$ open ocean & $\begin{array}{l}\mathrm{dCo}_{\text {coast }}>>\mathrm{pCo}_{\text {coast }} \\
\mathrm{dCo}_{\text {basin }}>>\mathrm{pCo}_{\text {basin }}\end{array}$ \\
\hline \multirow{2}{*}{$\mathrm{Fe}$} & high & coastal $>>$ open ocean & $\mathrm{dFe}_{\text {coast }}<\mathrm{pFe}_{\text {coast }}$ \\
& scavenging & & $\mathrm{dFe}_{\text {basin }} \approx \mathrm{pFe}_{\text {basin }}$ \\
\hline $\mathrm{Mn}$ & $\begin{array}{c}\text { moderate } \\
\text { scavenging }\end{array}$ & coastal $>$ open ocean & $\begin{array}{l}\mathrm{dMn}_{\text {coast }}>\mathrm{pMn}_{\text {coast }} \\
\mathrm{dMn}_{\text {basin }}>\mathrm{pMn}_{\text {basin }}\end{array}$ \\
\hline
\end{tabular}




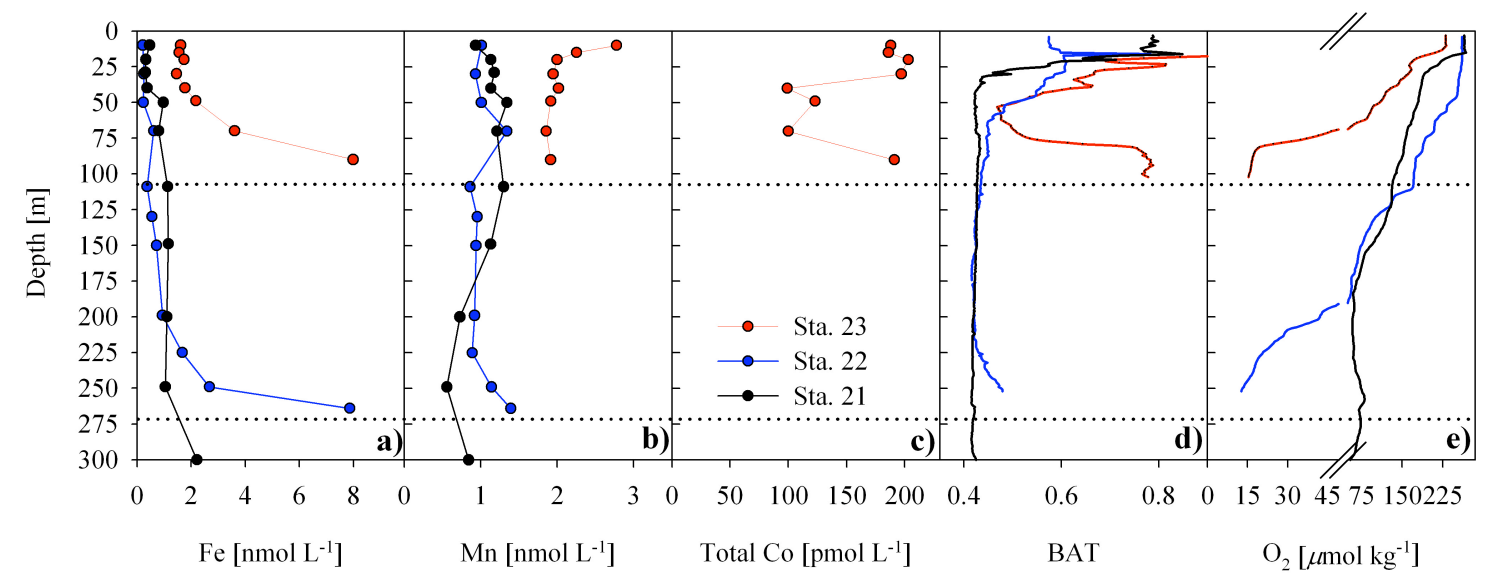

Figure 10) Vertical profiles of three stations sampled along the Namibian coast. Beam attenuation coefficient (BAT), a proxy for particulate material, increased near the deepest depths sampled for metals. Horizontal dotted lines indicate the bottom depth for the corresponding stations. The lowest oxygen concentration was observed coincident with the high beam attenuation and a marked increase in iron and cobalt concentrations, supporting the notion of a sedimentary source for the metals. (Note, only Sta. 23 is available for total dissolved cobalt due to methodological interferences in these productive coastal waters). 


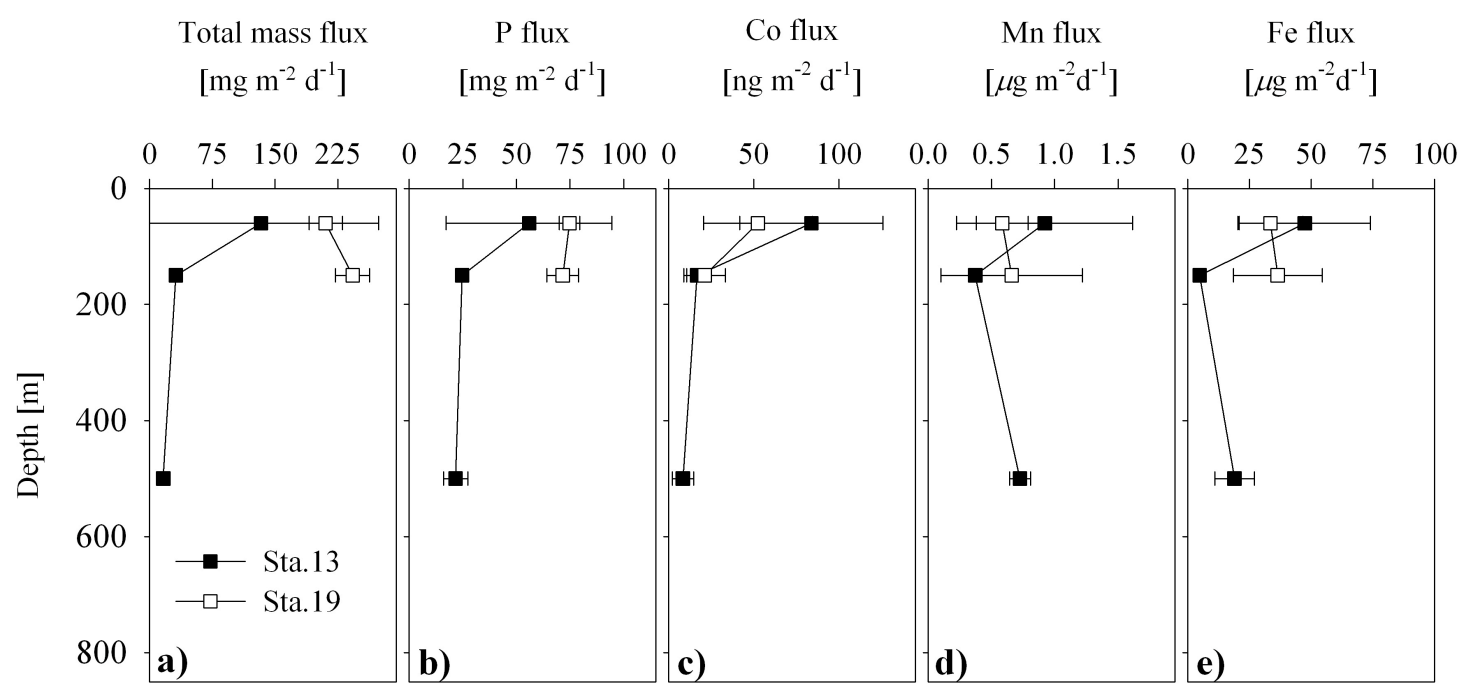

Figure 11) Sediment traps were deployed at Sta. 13 (black squares) and 19 (white squares) for 3 and 4 days respectively. As expected, the productive coast shows a higher total mass flux and total P flux, (a proxy for biologically generated particulate matter) than the oligotrophic gyre. In addition, the Sta. 19 total mass flux at $150 \mathrm{~m}$ is greater than the flux at $70 \mathrm{~m}$, suggesting that some horizontal particle movement may be occurring. Error bars were calculated as the standard deviation of triplicate measurements. 


\title{
Chapter 4: Dissolved and particulate cobalt, iron, and manganese under the McMurdo Sound seasonal sea ice
}

\begin{abstract}
This study presents dissolved and particulate hybrid-type metal data from the water column beneath the McMurdo Sound seasonal sea ice in the Ross Sea of Antarctica. Concentrations of total dissolved and labile cobalt were observed to be quite uniform with depth beneath the sea ice, suggesting that the water column was well mixed and that mixing was occurring on timescales faster than removal via co-oxidation by manganeseoxidizing bacteria, particle scavenging, and photosynthesis. Previously, we observed nutrient-like profiles of cobalt in the water column within the polynyas of the Ross Sea, and hypothesized that scavenging by manganese-oxidizing bacteria could be quite slow due to reduced populations or activities of these bacteria in the cold Antarctic waters, or that mixing processes were occurring on timescales fast enough to smooth out this influence on the vertical distributions of dissolved cobalt (Saito et al. 2010). In the current study, the likely presence of manganese oxides from manganese-oxidizing bacteria was observable in the particulate profiles, but not the dissolved, suggesting that the latter hypothesis was true. The dissolved iron and manganese profiles showed more variability than that of cobalt, but also did not show evidence of scavenging with depth. Comparison of particulate analyses to published sediment composition showed sediment-like Fe:Al ratios throughout much of the water column, and the sediments in this region appeared to be biogenically influenced, with Fe/Al ratios in excess of the crustal value. Excluding the depths where biological signals were strong, a linear correlation was observed for all permutations of particulate $\mathrm{Al}, \mathrm{Fe}, \mathrm{Mn}$, and Co, suggesting that the three metals had similar particulate cycles here during the winter/early spring months. The elevated particulate metals observed at these stations that were located in proximity to Ross Island and the Delbridge Islands support a likely particulate contribution from island effects and sediment resuspension. At one station, dissolved and particulate metal concentrations were significantly elevated in the shallowest depth, coincident with high biological activity on the underside of the sea ice, elevated particulate phosphorus, and slight drawdown of dissolved soluble reactive phosphorus. We suggest that this algal community concentrates metals there and helps to fuel productivity in the Ross Sea during the spring melt. Lastly, a comparison between different fractions of the iron concentration suggests that long term, weak acid leaching of unfiltered seawater accompanied by determination by inductivelycoupled plasma mass spectrometry detects more of the total particulate load than a short term, heated nitric acid digestion of polycarbonate filters, consistent with previous observations (Boyle et al. 2005).
\end{abstract}




\subsection{Introduction}

The HNLC region of the Ross Sea is an important sink for atmospheric $\mathrm{CO}_{2}$, with high pulses of primary productivity and subsequent export occurring during the spring and summer blooms (Arrigo et al. 2008). The waters of the Ross Sea also influence the chemical composition of the deep ocean when they eventually sink and contribute to Antarctic Bottom Water (AABW) formation (Orsi et al. 2009). There are a number of factors that are thought to impact biological productivity in these waters including light, mixed layer depth, seeding from sea ice, and iron (Sedwick et al. 2000; Smith et al. 1996; Arrigo et al. 1999; Sedwick and DiTullio 1997; Leventer and Dunbar 1996). Sedwick et al. (2000) suggest that the availability of iron exerts one of the major controls on phytoplankton growth in the Ross Sea. While iron concentrations have been observed to vary dramatically both seasonally and annually, dissolved iron concentrations are often very low during summer blooms (Sedwick et al. 2000). Recent results have also demonstrated that iron can be drawn down quickly to very low concentrations during the spring bloom as well (Sedwick et al. in review). This observation leads to questions regarding the sources of iron and respective mechanisms of input, and the origin of iron supply needed to support the summer bloom.

Evidence of particulate iron and manganese in the Ross Sea suggest that sediment resuspension can be a significant source of these elements to the water column (Corami et al. 2005), and sea ice melt water has also been observed to contribute significantly to particulate iron in surface waters near the receding ice edge during the austral summer (Fitzwater et al. 2000). In this coastal sea, terrestrial material may influence metal 
distributions. Previous work has suggested that elevated concentrations of metals accumulate with deep convective mixing during the winter, potentially mobilizing sediment when deep water intrudes onto the shelf. This "winter reserve" has been implicated as a likely source of micronutrients for phytoplankton in the spring, but sampling these waters during traditional shipboard fieldwork is difficult. Sampling the remnants of winter coastal waters was a goal of this field study to test the winter reserve hypothesis.

In addition to these questions regarding the sources of metals to the Ross Sea, the distributions of cobalt, iron, and manganese have all been observed to display nutrient-like profiles in this shallow sea (Sedwick et al. 2000, Saito et al. 2010). This is perhaps surprising because cobalt, iron, and manganese are hybrid-type metals, and in addition to being affected by biological utilization, their distributions are also affected by scavenging. This removal process tends to impose a decreasing concentration trend on the dissolved distributions below the upper water column uptake and remineralization signals. We have previously suggested that this may be due to shallow, fast mixing across the shelf at a pace that is faster than scavenging and/or biotic oxidation (Saito et al. 2010).

While particulate and dissolved iron have been studied in the Ross Sea previously, this study provides a novel dataset of dissolved and particulate metals sampled under the seasonal sea ice in McMurdo Sound as well as an examination of the potential contribution of Antarctic terrestrial sources to the Ross Sea. In this manner, this dataset provides a useful temporal and geographic point study of sources and processes influencing micronutrients in the Ross Sea. 


\subsection{Methods}

4.2.1 Sampling Techniques - Fieldwork took place on the seasonal sea ice of McMurdo Sound during three short expeditions between 11/17 and 11/23, 2009 (Chief Scientists, Mak Saito and Andy Allen). Three stations were occupied during the field season, Sta. $1\left(166.424^{\circ} \mathrm{E}, 77.650^{\circ} \mathrm{S}\right)$, Sta. $2\left(166.168^{\circ} \mathrm{E}, 77.659^{\circ} \mathrm{S}\right)$ and Sta. $3(165.750$ ${ }^{\circ} \mathrm{E}, 77.673^{\circ} \mathrm{S}$; Fig. 1), and all stations were sampled to near bottom with the exception of Sta. 3, which was sampled to $600 \mathrm{~m}$ and exhausted the maximum length of the sampling line. The locations of Sta. 1, 2, and 3 are located to the west of Mt. Erebus and north of the Erebus Glacier Ice Tongue (Fig. 1), and pass between two of the Delbridge Islands (Tent Is. and Inaccessible Is.). An aluminum tripod was used to suspend a block above each station sampling hole, with a meter read-out connected to it to determine the sampled depths, assuming no wire angle. A generator-powered winch was used to lower the $8 \mathrm{~L} \mathrm{X-Niskin}$ sampling bottles (Ocean Test Equipment, Inc., Fort Lauderdale, FL) on a Kevlar line through the ice for water sampling. The holes for each station were drilled and melted out. Bottles were tripped using teflon messengers, and then removed from the line upon retrieval and transferred to the trace metal clean sled hut where they were racked under a positive pressure class-100 hood. Bottles were immediately pressurized with $99.999 \% \mathrm{~N}_{2}$ at $\sim 5 \mathrm{psi}$, and seawater was either collected unfiltered for total dissolvable metals, or filtered through teflon tubing and a $142 \mathrm{~mm}, 0.4 \mu \mathrm{m}$ polycarbonate filter within a plastic sandwich filter holder (Geotech Environmental Equipment Inc.). All tubing and filters were acid-washed prior to use. Samples intended for iron and manganese analyses were acidified to $\mathrm{pH} 1.7$ with high purity $\mathrm{HCl}$ (Seastar Inc.) within 4 months of sampling, and 
stored acidified at room temperature for at least 3 months prior to analysis. Samples intended for all cobalt analyses were filled to the top of the bottle, not acidified, and were kept at $4^{\circ} \mathrm{C}$ in darkness until analysis.

For each X-Niskin bottle sampled, a separate filter was used. The filters were collected with trace metal clean plastic forceps into cleaned polyethylene bottles and frozen for later particulate analyses.

Low-density polyethylene sample storage bottles were soaked overnight in the acidic detergent, Citranox, rinsed thoroughly with Milli-Q water (Millipore), filled with $10 \% \mathrm{HCl}$ to soak for 10 days, rinsed thoroughly with Milli-Q water adjusted to $\mathrm{pH} 2$, and double-bagged until use.

4.2.2 Cobalt Analyses - Concentrations of total dissolved and labile cobalt were determined in the lab 2 months after sampling, using a previously described adsorptive cathodic stripping voltammetry (ACSV) method (Saito and Moffett 2001, Saito et al. 2004). Measurements were made using the Eco-Chemie $\mu$ AutolabIII systems connected to Metrohm 663 VA Stands equipped with hanging mercury drop electrodes and Teflon sampling cups. Standard additions were carried out with Metrohm 765 Dosimats using a programmed dosing procedure (Noble and Saito et al. 2008).

For total dissolved cobalt analyses, samples were UV-irradiated for $1 \mathrm{~h}$ prior to analysis using a Metrohm 705 UV digester to degrade the organic ligands that bind cobalt and allow binding by the added electroactive cobalt ligand, dimethylglyoxime. Samples were analyzed in $8.5 \mathrm{~mL}$ aliquots with the addition of $30 \mu \mathrm{L}$ recrystalized dimethylglyoxime (DMG, $0.1 \mathrm{~mol} \mathrm{~L}^{-1}$ in methanol), $1.5 \mathrm{~mL}$ purified sodium nitrite $\left(1.5 \mathrm{~mol} \mathrm{~L}^{-1}\right.$ in Milli-Q 
water), and $50 \mu \mathrm{L}$ purified N-(2-hydroxyethyl)piperazine-N-(3-propanesulfonic acid) (EPPS) buffer $\left(0.5 \mathrm{~mol} \mathrm{~L}^{-1}\right.$ in Milli-Q water). Reagent purification protocols were identical to those previously published (Saito and Moffett 2001). Analysis began with a 180 s purge with $99.999 \% \mathrm{~N}_{2}$. Each sample was conditioned at $-0.6 \mathrm{~V}$ for $90 \mathrm{~s}$ at a stir-rate of $2500 \mathrm{rpm}$ followed by a $10 \mathrm{~s}$ equilibration step and a linear sweep from $-0.6 \mathrm{~V}$ to $-1.4 \mathrm{~V}$ at a rate of $10 \mathrm{~V} \mathrm{~s}^{-1}$. Cobalt concentrations were determined by the standard additions technique, with initial concentrations measured in triplicate followed by four $25 \mathrm{pmol} \mathrm{L}^{-1}$ cobalt additions. The analytical blank was determined by analyzing seawater that had been UV-irradiated for $1 \mathrm{~h}$, equilibrated overnight with prepared Chelex 100 resin beads (BioRad), and UV-irradiated a second time to degrade any leached synthetic ligands. Reagent blanks (nitrite, DMG, EPPS) were subtracted from the initial sample concentration, and blank analyses were made continually throughout the analysis of the sample set. The averaged blank concentration was 3 pmol L ${ }^{-1} \pm 0.5 \operatorname{pmol~L}^{-1}(n=7)$.

For labile cobalt analyses, $8.5 \mathrm{~mL}$ of sample were pipetted into acid-washed teflon vials that were preconditioned with a small aliquot of sample water. $30 \mu \mathrm{L}$ of DMG were added to each vial and allowed to equilibrate overnight in the dark prior to analysis (Saito et al. 2004). Analyses were then performed as described for total concentrations with the addition of the remaining two reagents and use of the standard addition technique.

Previously, we determined that natural cobalt ligands in seawater have a conditional stability constant of $>10^{16.8}$ (Saito et al. 2005). This suggests that the cobalt is bound very tightly to the cobalt ligands. Thus, we define labile cobalt as the fraction of total dissolved cobalt that is either bound to weak organic and inorganic ligands in seawater or present as 
free $\mathrm{Co}^{2+}$, and is then exchangeable with the complexing agent (DMG) used for analysis (Saito et al. 2004, Saito et al. 2005). The difference between the total dissolved cobalt and the labile cobalt can then be used as an estimation of the strong cobalt ligand concentration.

\subsubsection{Total dissolved and total dissolvable iron and manganese analyses - Total}

dissolved and total dissolvable iron and manganese were measured using inductively coupled plasma mass spectrometry (ICP-MS), as described in detail by Saito and Schneider (2006). Total dissolved samples were filtered as described above, and total dissolvable samples were unfiltered. Briefly, $13.0 \mathrm{~mL}$ aliquots of acidified seawater were weighed into acid-leached polypropylene centrifuge tubes, to which an ${ }^{57} \mathrm{Fe}$ solution was added for isotope dilution analysis and allowed to equilibrate overnight. Following equilibration, concentrated ammonium hydroxide (Seastar) was added to induce $\mathrm{Mg}(\mathrm{OH})_{2}$ and trace metal co-precipitation into a pellet. This was accomplished by allowing a precipitate to form for 3 minutes, followed by centrifugation for 3 minutes at $3000 \mathrm{rpm}$ (1460 x g) using an Eppendorf Centrifuge 5810R. The supernatant was decanted, the sample was centrifuged a second time to further remove residual seawater, and the pellet was re-dissolved in 5\% nitric acid (Seastar) made with $1 \mathrm{ppb}$ indium. The 5\% nitric acid resuspension solution was used to estimate the blank, and signal suppression due to matrix effects was accounted for by using a ratio of the indium in the blank to the indium in the resuspended sample solution.

4.2.4 Particulate metal analyses - For particulate metal analysis, filters were kept frozen at $-20{ }^{\circ} \mathrm{C}$ until analysis. Each filter was transferred to its own teflon digestion vial to which $4 \mathrm{~mL}$ of $50 \%$ nitric acid with $1 \mathrm{ppb}$ indium was added as an internal standard. 
The filters and acid were then refluxed at $100-110{ }^{\circ} \mathrm{C}$ for $6 \mathrm{~h}$. Following digestion, the tubes were allowed to cool, and the remaining filter was removed from the digestion vials with trace metal clean plastic forceps. The samples were then diluted 1:10 with Milli-Q water such that the nitric acid was diluted to $5 \%$ concentration, and analyzed by ICP-MS. A 1-4 ppb metal reference standard curve (1, 2, 3, 4 ppb) was used to determine the instrument sensitivity. Two processed filter blanks were run as well, where a cleaned, unused filter was digested following the above protocol. The metal values from this filter blank were subtracted from the sample blanks, corrected by the indium in each. Sample recovery and volume corrections post-indium addition were also corrected for by multiplying the metal counts per second (cps) by the ratio of indium cps estimated in the digest solution to the indium in the sample. The estimated indium cps in the $50 \%$ nitric digest solution ( $\left.\operatorname{In}_{\text {digestion }}\right)$ was calculated by analyzing a 1:10 dilution of the $50 \%$ nitric digest solution to achieve a 5\% nitric acid solution matrix match, and multiplying that value by 10 . The equation used for calculations described above is:

$$
M_{\text {particulate }}=\left[\frac{M_{\text {sample }}}{I_{\text {sample }}}-\frac{M_{\text {blank }}}{I n_{\text {blank }}}\right] \times \frac{\text { In digestion }}{M_{\text {slope }}} \times \frac{V_{\text {digested }}}{V_{\text {filtered }}}
$$

where the $V_{\text {filtered }}$ was the volume estimated to have passed through the filter $(6 \mathrm{~L}), \mathrm{V}_{\text {digested }}$ was the volume used to digest the sample $(4.0 \mathrm{~mL}), \mathrm{M}_{\text {slope }}$ is the slope of the metal of interest in cps $\mathrm{ppb}^{-1}, \mathrm{In}_{\text {sample }}$ is the indium measured in the sample in cps, $\operatorname{In}_{\text {blank }}$ is the indium measured in the blank in cps, and the concentration of the metal of interest $\left(\mathrm{M}_{\text {particulate }}\right)$, is in $\mathrm{ppb}\left(\mu \mathrm{g} \mathrm{L}{ }^{-1}\right)$. The filter blank for these analyses was sometimes quite high. The filter blank associated with particulate phosphorus was sometimes as much as $50 \%$ of the sample signal, reducing confidence in the quantitative values of particulate 
phosphorus, though it is unclear why the filter blank for phosphorus was high. All other analyses had filter blanks that were less than $6 \%$ of the signal, with the exception of cobalt, which occasionally was as high as $30 \%$. This may be expected for cobalt, however, as particulate cobalt concentrations are generally quite low $[<10 \mathrm{pM}]$.

4.2.5 Nutrient analyses - Nutrient analyses for these samples were processed at Oregon State University, using identical methods to those described in Noble et al. in review (see Chapter 3).

\subsection{Results and Discussion}

4.3.1 Oceanographic context - The Ross Sea is relatively well mixed as a result of being a shallow sea with an average $500 \mathrm{~m}$ depth and gyre-like water movement that drives across the shelf (Smith et la. 2007, Orsi et al. 2009). McMurdo Sound connects to the Ross Sea, and hydrographic data has revealed homogeneity throughout the water column near the sea ice edge during the spring, implying that the shallow coastal waters are well mixed down to the bottom during the winter (Smith et al. 2007, Dinniman et al. 2003, Fig. 1). The Ross Sea is generally covered in ice throughout the winter months, with a polynya forming in the springtime. McMurdo Sound is characterized by an annual sea ice sheet that forms in the winter and melts in the late spring / summer and feeds into the Ross Sea. During this field season (November, 2009), the sea ice sheet was $\sim 2 \mathrm{~m}$ thick, and the data from this study provide a description of early season shelf water composition beneath the seasonal sea ice. These results provide evidence of two mechanisms of metal input to the Ross Sea from the Sound: one due to mobilization of the largely biogenic sediment when waters mix across the shelf and one due to processes involving the breakup and melting of 
the seasonal sea ice. In order to put this dataset in a broader context, we compare it to recent observations of dissolved metals in the Ross Sea from two seasonal expeditions in 2005 and 2006 that comprised the Controls on Ross Sea Algal Community Structure (CORSACS) program (Sedwick et al. in review; Saito et al. 2010). CORSACS-I took place during the austral summer of 2005-2006 (December 272005 - January 22 2006), and CORSACS-II took place during the austral spring of 2006 (November 8 - December 3 2006).

4.3.2 Total dissolved and labile cobalt distributions - The concentrations of total dissolved and labile cobalt were uniform with depth with the exception of one surface sample at Sta. 2 (Fig. 1, 2). The concentration range of total dissolved cobalt observed here $(50 \pm 3.5 \mathrm{pM}, \mathrm{n}=32)$ was similar to those previously observed below the euphotic zone during the summer in the Ross Sea $(53 \pm 4.5 \mathrm{pM} \mathrm{n}=44$, CORSACS-I study Saito et al. 2010, Fig. 3). Biological drawdown in the summer decreases the surface concentrations of cobalt relative to those observed during the spring beneath the McMurdo Sound sea ice (Saito et al. 2010). In contrast, the spring CORSACS cobalt concentrations were higher than those observed under the sea ice (Fig. 3). Perhaps the timing of the spring cruise coincided with an input of dissolved cobalt due to physical break up of the sea ice, and that the waters beneath the sea ice were somewhat less perturbed than the recently ice-free stations sampled during the spring CORSACS expedition. Interestingly, the labile concentrations observed during both CORSACS expeditions were higher than those observed during this study (Fig. 3). During both CORSACS studies, total dissolved cobalt correlated strongly with soluble reactive phosphate throughout the water column, implying 
that biological uptake and remineralization are the primary influences on cobalt distributions during the spring and summer (Saito et al. 2010, Fig. 4). The small range of water column concentrations of both cobalt and phosphate observed during this study did not appear to have a strong linear relationship, however; when compared to our data from CORSACS, the $\mathrm{Co}: \mathrm{PO}_{4}{ }^{3-}$ ratio range for the under sea ice data fell along the previously observed relationship, consistent with early season input and winter mixing homogenization of dissolved distributions (Fig. 4). The coherence of the McMurdo sound data with the upper values of the CORSACS cobalt data suggests that while biological processes did not create gradients in these species during winter months, a biologically driven $\mathrm{Co}: \mathrm{PO}_{4}{ }^{3-}$ ratio persisted in the subsurface waters (Fig. 4). The total cobalt in the surface sample from Sta. 2, which fell above the Co: $\mathrm{PO}_{4}{ }^{3-}$ correlation (87 pM), was likely elevated due to sources from the sea ice and is discussed in a later section.

In addition to these trends, we observed two overriding features in the cobalt dataset that were consistent with our previous observations in the Ross Sea: 1) nonsaturating concentrations of cobalt-binding ligand throughout the water column, and 2) no evidence of cobalt scavenging with depth, which is typically seen for this hybrid-type element in other oceanic regimes (Saito et al. 2010, Fig. 2). As we have previously argued, the presence of labile cobalt throughout the water column may be attributable to the absence of marine cyanobacteria, which possess the biosynthetic pathway for synthesizing $\mathrm{B}_{12}$, an important cobalt-containing vitamin and potential source of strong cobalt binding ligands (Saito et al, 2010). The absence of these organisms likely leads to less complexation of cobalt in these cold waters (Saito et al. 2010). Although biochemical 
studies have discovered the presence of native heterotrophic bacteria that can synthesize $\mathrm{B}_{12}$ (Bertrand et al. 2011), their $\mathrm{B}_{12}$ production rates are likely low, as demonstrated by observations of $\mathrm{B}_{12}$ and iron colimitation in the Ross Sea and Southern Ocean (Bertrand et al. 2007, Panzeca et al. 2006). The lack of a scavenged profile for cobalt with depth implies that either there is a reduction in the activity of manganese-oxidizing bacteria, that these bacteria do not exist in Antarctic waters, or that deep seasonal mixing across the shelf essentially erases the evidence of this removal process on the dissolved distributions. We have previously suggested that some combination of these processes might be occurring (Saito et al. 2010), although particulate metals were not measured during that study to look for evidence of manganese oxide particles. The combination of dissolved and particulate cobalt and manganese measurements from this study reveals that manganese-oxidizing bacteria are likely present (see section 4.3 .6 below). Additionally, it may be possible that abiotic scavenging of cobalt could be associated with a shift in the composition of suspended particles in the water column from organic to lithogenic dominated particles. Perhaps the Ross Sea is too shallow to observe this phenomenon if the water column particles are generally dominated by organic material. In the North East Atlantic, the apparent onset of cobalt scavenging was coincident with a notable decrease in both the absolute and relative amount of particulate organic material relative to the lithogenic fraction (P. Lam personal communication, see Chapter 5).

4.3.3 Total dissolved manganese and iron distributions - Total dissolved manganese concentrations varied between 0.83 and $1.33 \mathrm{nM}$ with the exception of the shallowest sample at Sta. 2 (3.88 nM, Fig. 2). This range of concentrations is generally 
higher than deep water concentrations in the open ocean, which have been observed to be between 0.1-0.3 nM (Landing and Bruland 1987; Martin et al. 1985) and higher than surface and deep waters found in the Atlantic sector of the Southern Ocean with the exception of a hydrothermal signal observed along the Mid-Atlantic Ridge ( $\sim 0.1-0.5 \mathrm{nM}$ Middag et al. in press, Saito et al. in prep). The range is also lower than many surface manganese concentrations in the tropical / subtropical ocean, which often display surface maxima due to aeolian input and photoreduction (Sunda and Huntsman 1988).

Total dissolved iron distributions were similar to those of manganese, though lower in concentration with the exception of a few shallow samples. Iron concentrations ranged between 0.4 and $0.9 \mathrm{nM}$ with the exception of 3 shallow samples (Sta. 1, $25 \mathrm{~m}, 1.2 \mathrm{nM}$; Sta. 2, 4 m, 11.9 nM; Sta. 2, 8 m, 1.5 nM; Fig. 2). These concentrations were generally elevated above previously observed iron concentrations in the Ross Sea during both CORSACS expeditions (Sedwick et al. in press), but were consistent with previous studies in the Ross Sea during early (October-November 1996; Coale et al. 2005) and late spring (November - December 1994; Sedwick et al 2000). During the spring CORSACS expedition, dissolved iron concentrations were significantly depleted to sometimes below $0.1 \mathrm{nM}$ in surface waters due to biological uptake during the spring bloom (Fig. 3), but some deeper waters were sampled that are consistent with the concept of a "winter-reserve" hypothesized by that study (Sedwick et al. in review). The upper water column average dissolved iron concentrations for both seasons of the CORSACS study are lower than those observed beneath the McMurdo Sound seasonal sea ice (Fig. 3). This trend is the opposite of what was observed for cobalt, where the spring CORSACS concentrations were found 
to be higher than those observed beneath the McMurdo Sound seasonal sea ice. Perhaps this was due in part to a higher biological demand for iron relative to cobalt. If the waters sampled during this expedition were somewhat representative of winter distributions beneath the seasonal sea ice across the coastal regions of the Ross Sea, these finding are consistent with the hypothesis that well-mixed "winter-reserve" shelf water could supply iron to surface waters during the spring bloom. Additionally, satellite data showed evidence of a stronger bloom occurring later in the season following CORSACS-II. Given the low detected iron concentrations in the water column during the early spring, a midseason source is required to support the late bloom. Sedwick et al. (in review) suggested that a late-season ice melt may have been responsible for this source. Our results support this hypothesis and possible mechanisms of metal accumulation within the sea ice are discussed below in section 4.3.7.

\subsubsection{Particulate metal distributions and comparison of acid leachable particulate}

iron to particulate iron - The particulate concentrations of all three metals had very similar distributions and showed much more structure in their vertical profiles than observed for the dissolved phase (Fig. 5). When the particulate concentrations for the entire field season were compared in paired scatter plots, strong linear relationships were evident between all pairings of metals, suggesting that their particulate distributions beneath the sea ice sheet were related by source or process (Fig. 6). Samples that fell outside the general trend are shown in red, and represent samples from the upper $10 \mathrm{~m}$ of the water column where phytoplankton biological processes were present. These data 
points appeared to show a linear relationship as well, though the relationship was driven by only three data points.

In addition to filtered analyses and particulate analyses, unfiltered samples were also collected and acidified for iron and manganese analyses. The interpretation of the unfiltered fraction is still debated because different analytical techniques may detect different fractions of the total metal (Bowie et al. 2004). Previous studies have used analyses from unfiltered samples in conjunction with dissolved analyses (filtered samples) to infer the acid leachable particulate concentration by taking the difference between the two (Sedwick et al. in review, Chever et al. 2010). These studies have generally employed flow injection analysis techniques, which likely do not detect refractory iron that may pass through the instrument. Additionally, a comparison study between different flow injection analysis techniques have observed slight differences likely due to the detection of different physiochemical fractions of iron (Bowie et al. 2004). Lastly, other work has suggested that analysis of the unfiltered fraction using ICP-MS techniques is likely to be a closer estimate of the total iron than the acid leachable particulate iron, as particles are may be precipitated during the co-precipitation preconcentrating step, and then subsequently ionized if taken up into the instrument during analysis (Boyle et al. 2005). Investigating these differences was beyond the scope of this study, and while the unfiltered samples discussed in this study may have measured a fraction closer to the total iron due to analysis via ICP-MS, the following paragraphs refer to the unfiltered samples as total dissolvable. These samples were treated similarly to those in Sedwick et al. (in review) and were used to calculate the acid leachable particulate iron as described above. 
The total dissolvable samples were analyzed after storage and leaching under acidic conditions for at least 3 months using the same analytical protocol described for the dissolved analyses (see methods section). Fig. 7 shows the results of the total dissolvable and dissolved analyses for both iron (top panel) and manganese (bottom panel). A large difference between the acid leachable and dissolved iron indicates that the acid leachable particulate metal should be resolvable for iron. In contrast, the difference between the total dissolvable manganese and dissolved manganese was small, suggesting that the acid leachable particulate manganese was a small fraction of the total, consistent with the measured particulate manganese being approximately 20 times less than the measured dissolved manganese (Fig. 5).

When the acid leachable particulate Fe was calculated (ALP Fe hereon) and compared to that of the measured particulate $\mathrm{Fe}$, a couple of observations could be made (Fig. 8). First, the broad trends observed in the particulate Fe profiles were also observed in the ALP Fe profiles, and the ALP Fe and particulate Fe below the surface at Sta. 2 agreed well. However, in general, the measured particulate Fe concentrations were lower than the ALP Fe. This seems odd given that the particulate analyses were a result of a total filter digestion performed with a strong acid $\left(50 \% \mathrm{HNO}_{3}\right)$ at high temperature, but the ALP Fe values were derived from analyses of samples that were weakly acidified $(\mathrm{pH} 1.7$ with $\mathrm{HCl})$ at room temperature. Both the dissolved and total dissolvable metal samples were acidified with the same batch of acid, the bottles were treated identically during acid washing, and were identically treated and analyzed by ICP-MS, so contamination seems unlikely. The use of a combined acid mixture that includes HF is typically used to digest 
all recalcitrant lithogenic material (Chever et al. 2010, Lannuzel et al. 2010, D. Ohnemus and P. Lam personal communication). Given the digestion treatment used in this study, the particulate metal measured should be considered to consist of metals that were associated with organic material and/or present in a relatively labile particulate phase (Lannuzel et al. 2010). It is interesting that the long-term, but less acidic dissolution and room temperature leaching step produced ALP Fe concentrations that were up to $40 \%$ higher than the heated,

particulate digestion. Additionally, a few of the total dissolvable samples were re-analyzed a month later, and the concentrations measured were slightly higher, suggesting that iron continued to be released or desorbed from particulate matter in the sample bottles. Lastly, it is possible that the higher values derived from the unfiltered samples were due in part to the ionization of refractory iron that may have precipitated during the $\mathrm{Mg}(\mathrm{OH})_{2}$ coprecipitation preconcentrating step and been carried into the instrument during analysis as was suggested by Boyle et al. (2005).

\subsubsection{Consideration of island effects, terrestrial input and/or sedimentary}

influences - The influence of terrestrial inputs is important to consider given the proximity of the stations to many islands (Ross Island and the Delbridge Islands, Fig. 1). If particulate material from these islands was a source to the surrounding waters of McMurdo Sound and the Ross Sea, stations located further from the Island sources would be expected to have lower particulate concentrations. Some evidence of sources from this region could be seen when the ALP Fe distributions from this study were compared to the ALP Fe distributions from both CORSACS studies (Fig. 9, Sedwick et al. in review). The average concentrations of 6 stations from the spring (Stas. NX12-15, 17, 20) and summer 
(Stas. NX1-4, 7, 10) CORSACS cruises are shown relative to each of the stations from this study. The CORSACS ALP Fe values were lower in the upper $200 \mathrm{~m}$ than observed during our field season, and did not vary considerably from spring to summer. The much higher concentrations of ALP Fe observed at Sta. 1, located between the Delbridge Islands and Ross Island (Fig. 1), suggest that there may be a considerable amount of particulate matter coming from the sediments in proximity to these volcanic islands. This may also be reflective of the difference in water column depth. Some stations from the CORSACS expeditions showed significant evidence of sediment mobilization by elevated ALP Fe concentrations up to $68 \mathrm{nM}$ at depth, but those stations had bottom depths as deep at 700 and are not shown here (Sedwick et al. in review). The particulate iron maximum at Sta. 1 was 4.5-6.0 nM ALP Fe, 3.0-4.3 nM particulate Fe, but was considerably smaller than the high concentration observed in the surface sample at Sta. 2 (128 nM ALP Fe, $80 \mathrm{nM}$ particulate $\mathrm{Fe})$.

The potential for terrestrial/continental sources can be inferred by comparing ratios of each metal with aluminum in the particulate phase to nearby sediment and to crustal values. Using data from a sediment core from the Ross Sea for iron and manganese (Ianni et al. 2010) and from the Western Antarctic Peninsula for cobalt (Ravanelli et al. 1997), Fig. 10 shows the relationships between the metal/Al ratios in the suspended particulate material, the crustal ratios (Taylor and McLennan 1985), and that of the local sediment. The Fe/Al ratio found at most depths for all stations was close to that found in the sediment. The particulate manganese concentrations, however, far exceeded that expected to be found in the sediments and this is discussed in a later section (see the following section 
4.3.6, Fig. 10). Interestingly, there was no large bottom depth point source observed for either the particulate iron or ALP iron profiles. This may have been a result of winter mixing across much of the sound and/or the digestion used for these analyses. For total particulate digestions, hydrofluoric acid (HF) is often used. No HF was used for the particulate analyses in this study, and it is likely that strong lithogenic sources may be missed. This is interesting given the similarity observed between the Fe/Al ratio in most of the suspended particulate analyses and the $\mathrm{Fe} / \mathrm{Al}$ ratio of the sediment core from the Ross Sea (Ianni et al. 2010). The Ianni study values that were used for the Fe/Al and Mn/Al sediment composition comparison were complete digestions processed with HF (Ianni et al. 2010). The similarity between their ratios and ours suggests that these sediments were largely comprised of labile and/or biogenic material. When compared to crustal values, the suspended particulate and sediment core metal/Al ratios were both higher. This may be slightly skewed for the cobalt sediment core $\mathrm{Co} / \mathrm{Al}$ ratio as no data were available for a complete digestion processed with $\mathrm{HF}$, though this influence is likely small as cobalt is not typically considered to be a silicate forming metal. The $\mathrm{Co} / \mathrm{Al}$ sediment core ratio was taken from a sediment core study that processed their samples using a cold, weak acid leach (0.3 N HCl at room temperature for 2 h, Ravanelli et al. 1997).

\subsubsection{Particulate and dissolved cobalt and manganese distributions: evidence of} manganese-oxidizing bacteria and fast mixing - Previously, we observed nutrient-like profiles of cobalt in the water column in the Ross Sea, and hypothesized that either scavenging by manganese-oxidizing bacteria could be quite slow, due to reduced populations or activities of these bacteria in the cold Antarctic waters, or that mixing 
processes were occurring on timescales fast enough to smooth out this influence on the vertical distributions of dissolved cobalt (Saito et al. 2010). The dissolved manganese and particulate cobalt and manganese data help resolve the question of which mechanism is more important in the Ross Sea. In the particulate data, maxima of manganese and cobalt were suggestive of manganese oxidation and co-oxidation of cobalt by manganeseoxidizing bacteria either in the water column or in resuspended sedimentary material (Fig. 5). At Sta. 2, an increase in particulate manganese and a concomitant but smaller increase in particulate cobalt were observed between 75 and $175 \mathrm{~m}$ depth (Fig. 5). There was also a maximum of particulate manganese at $300 \mathrm{~m}$ in Sta. 3 (Fig. 5). In the particulate metal scatter plots discussed earlier, cobalt and manganese correlated exceptionally well with only one exception in the surface sample from Sta. 2 (Fig. 6). This also supports the proposed coupling of the particulate phases of these metals through the bacterial manganese oxidation pathway (Tebo et al. 1984). It is important to note that our study can examine for the presence of manganese particles, but does not measure oxidation rates directly. Hence, we cannot discern if any manganese particles present were formed in the water column or advected from sedimentary material, however; the particulate $\mathrm{Mn} / \mathrm{Al}$ ratios observed in the water column were elevated relative to both the sediment core study and crustal values, suggesting that the excess particulate manganese is a result of manganese oxide production occurring in the water column. While manganese oxides from manganese-oxidizing bacteria appeared to be present in this polar environment, the evidence was observable in the particulate profiles, but not the dissolved, in contrast to observations in the Equatorial Pacific (Saito unpubl.). This suggests that water column 
mixing was faster than the influence of biotic oxidation of dissolved cobalt and manganese by manganese-oxidizing bacteria.

4.3.7 Sea ice sources - A striking feature in this dataset was the elevated dissolved and particulate concentrations found in the shallowest sample of Sta. 2 (Fig. 1, 5). This observation implies that the sea ice was a source of these metals to the water column, though the mechanism is not immediately clear. Seasonal sea ice has previously been suggested to accumulate metals via aeolian deposition and / or from entrainment of organic and inorganic terrestrial and near shore sedimentary material (Sedwick et al. in review). The metals contained in the ice are then introduced to the water column via both physical breakup of the seasonal sea ice and melting. An additional mechanism for metal accumulation in the sea ice was apparent in our studies of McMurdo Sound sea ice. Intense biological production was observed in the bottom 2-3 inches of the cores of all three McMurdo Sound studies, which were thick with brown biomass. In a companion sea ice proteomic study, particulate iron determined in an ice core bottom was extremely high (Saito et al. in prep). When these values were compared to the biomass in that core bottom estimated from chlorophyll $a$ analyses, the Fe:C ratios were within the range of the lower cellular iron demand limit required for diatoms (Sunda and Huntsman 1995). This suggested that these analyses were not contaminated for iron, and that the organisms occupying the ice core bottom may have been iron limited, consistent with the proteomic results. Given this presence of high biogenic particulate iron, these algal communities were likely gradually concentrating metals at the sea ice-seawater interface as the wellmixed "winter reserve" waters came in contact with the ice. These high concentrations 
found during melting of the seasonal sea ice both in the upper water column and in the sea ice (Saito et al. in prep.) support hypotheses that metals are delivered to the Ross Sea during the spring melt. In support of this biochemical concentrating mechanism at the sea ice-seawater interface, the shallow sample at Sta. 2 was also the only observation of any noticeable drawdown of water column $\mathrm{PO}_{4}{ }^{3-}$ (Fig. 2). When disturbed (either by sloughing off of the interface layer during spring melting or physical breakup of sea ice), the organisms that populate the bottom of the seasonal sea ice and their iron content could be an important flux of iron. It is possible that our sampling efforts also disturbed these communities, potentially sloughing some biomass off the bottom of the sea ice. Elevated particulate metals were also observed at $10 \mathrm{~m}$ at Sta. 3, with no elevated signal above it. This could perhaps be due to an advected signal from biomass sloughed off the ice sheet at some upstream location.

4.4 Conclusions - This study presents dissolved and particulate cobalt, iron, and manganese distributions in the water column beneath the seasonal sea ice, and demonstrates that winter concentrations of these important metal micronutrients were relatively high. Contrary to other environments where cobalt, iron, and manganese have been observed to behave quite differently from each other, it seems that in McMurdo Sound, biological activity under sea ice is slow relative to the physical processes controlling hybrid-type metal distributions. The likely presence of manganese oxides from manganese-oxidizing bacteria was observable in the particulate profiles, but not the dissolved, suggesting that water column mixing was faster than the influence of biotic oxidation of dissolved cobalt and manganese by manganese-oxidizing bacteria. This study 
provides an explanation of conditions beneath the sea ice, and supports the previous hypothesis that elevated "winter-reserve" concentrations of metals exist there.

Additionally, the bioconcentration of metals in biota populating the underside of the sea ice sheet may constitute a previously undocumented but important source of metals to the highly productive Ross Sea. 


\section{References for Chapter 4:}

Arrigo, K. R. and others 1999. Phytoplankton Community Structure and the Drawdown of Nutrients and $\mathrm{CO}_{2}$ in the Southern Ocean. Science 283 : 365-367.

Arrigo, K. R., G. V. Dijken, and M. Long. 2008. Coastal Southern Ocean: A strong anthropogenic CO2 sink. Geophys. Res. Lett. 35: L21602, doi:21610.21029/22008GL035624.

Bertrand, E. M. and others 2007. Vitamin $\mathrm{B}_{12}$ and iron co-limitation of phytoplankton growth in the Ross Sea. Limnol. Oceanogr. 52: 1079-1093.

Bertrand, E. M., Saito, M. A., Jeon, Y. J. and Neilan, B. A. (2011), Vitamin B12 biosynthesis gene diversity in the Ross Sea: the identification of a new group of putative polar $\mathrm{B}_{12}$ biosynthesizers. Environmental Microbiology, 13: 1285-1298. doi: 10.1111/j.1462-2920.2011.02428.x

Bowie, A. R., P. N. Sedwick, P. J. Worsfold. 2004. Analytical intercomparison between

flow injection-chemiluminescence and flow injection-spectrophotometry for the determination of picomolar concentrations of iron in seawater. Limnol. Oceanogr. Methods. 2 42-54.

Boyle, E.A., Berquist, B.A., Kayser, R.A., Mahowald, N., 2005. Iron, manganese, and lead at Hawaii Ocean Time-series station ALOHA: Temporal variability and an intermediate water hydrothermal plume. Geochimica et Cosmochimica Acta. 69 (4), 933-952.

Chever, F., G. Sarthou, E. Bucciarelli, S. Blain, and A. R. Bowie. 2010. An iron budget during the natural iron fertilisation experiment KEOPS (Kerguelen Islands, Southern Ocean). Biogeosciences 7: 455-468.

Coale, K. H., R. Michael Gordon, and X. Wang. 2005. The distribution and behavior of dissolved and particulate iron and zinc in the Ross Sea and Antarctic circumpolar current along $170^{\circ}$ W. Deep Sea Res. I 52: 295-318.

Corami, F., G. Capodaglio, C. Turetta, F. Soggia, E. Magi, and M. Grotti. 2005. Summer distribution of trace metals in the western sector of the Ross Sea, Antarctica. J. Environ. Monit. 7: 1256-1264.

Dinniman, M. S., J. M. Klinck, and W. O. Smith. 2003. Cross-shelf exchange in a model of the Ross Sea circulation and biogeochemistry. Deep Sea Research Part II: Topical Studies in Oceanography 50: 3103-3120.

Fitzwater, S. E., K. S. Johnson, R. M. Gordon, K. H. Coale, and W. O. Smith. 2000. Trace metal concentrations in the Ross Sea and their relationship with nutrients and phytoplankton growth. Deep-Sea Res. II 47: 3159-3179.

Ianni, C., E. Magi, F. Soggia, P. Rivaro, and R. Frache. 2010. Trace metal speciation in coastal and off-shore sediments from Ross Sea (Antarctica). Microchemical Journal 96: 203-212.

Landing, W. M., and K. W. Bruland. 1987. The contrasting biogeochemistry of iron and manganese in the Pacific Ocean. Geochimica et Cosmochimica Acta 51 : 29-43.

Lannuzel, D. and others. 2010. Distribution of dissolved iron in Antarctic sea ice: Spatial, seasonal, and inter-annual variability. J. Geophys. Res.-Biogeosci. 115: 13.

Leventer, A., and R. B. Dunbar. 1996. Factors influencing the distribution of diatoms and other algae in the Ross Sea. J. Geophys. Res. 101: 18489-18500. 
Martin, J. H., and G. A. Knauer. 1985. Lateral transport of Mn in the north-east Pacific Gyre oxygen minimum. Nature 314: 524-526.

Middag, R., H. J. W. De Baar, P. Laan, P. H. Cai, and J. C. Van Ooijen. 2011. Dissolved manganese in the Atlantic sector of the Southern ocean. Deep Sea Research Part II: Topical Studies in Oceanography In press.

Noble, A. E., M. A. Saito, K. Maiti, and C. Benitez-Nelson. 2008. Cobalt, manganese, and iron near the Hawaiian Islands: A potential concentrating mechanism for cobalt within a cyclonic eddy and implications for the hybrid-type trace metals. Deep Sea Res II 55: 1473-1490.

Orsi, A. H., and C. L. Weiderwohl. 2009. A recount of Ross Sea waters. Deep-Sea Res. II 56: $778-795$.

Panzeca, C. and others 2006. B Vitamins as Regulators of Phytoplankton Dynamics. EOS 87: 593.

Ravanelli, M., O. Tubertini, S. Valcher, and W. Martinotti. 1997. Heavy metal distribution in sediment cores from Western Ross Sea (Antartica). Water, Air, \&amp; Soil Pollution 99: 697-704.

Saito, M. A., G. Rocap, and J. W. Moffett. 2005. Production of cobalt binding ligands in a Synechococcus feature at the Costa Rica Upwelling Dome. Limnol. Oceanogr. 50: 279-290.

Saito, M. A., and D. L. Schneider. 2006. Examination of precipitation chemistry and improvements in precision using the $\mathrm{Mg}(\mathrm{OH})_{2}$ preconcentration ICP-MS method for high-throughput analysis of open-ocean Fe and Mn in seawater. Anal. Chim. Acta 565: 222-233.

Saito, M. A., and J. W. Moffett. 2001. Complexation of cobalt by natural organic ligands in the Sargasso Sea as determined by a new high-sensitivity electrochemical cobalt speciation method suitable for open ocean work. Mar. Chem. 75: 49-68.

Saito, M. A., J. W. Moffett, and G. Ditullio. 2004. Cobalt and Nickel in the Peru Upwelling Region: a Major Flux of Cobalt Utilized as a Micronutrient. Global Biogeochem. Cycles 18: doi:10.1029/2003GB002216

Saito, M. A., T. J. Goepfert, A. E. Noble, E. M. Bertrand, P. N. Sedwick, and G. R. Ditullio. 2010. A seasonal study of dissolved cobalt in the Ross Sea, Antarctica: micronutrient behavior, absence of scavenging, and relationships with $\mathrm{Zn}, \mathrm{Cd}$, and P. Biogeosciences 7: 4059-4082.

Schlitzer, R., Ocean Data View, http://odv.awi.de, 2009.

Sedwick, P. N., and G. R. Ditullio. 1997. Regulation of algal blooms in Antarctic Shelf Waters by the release of iron from melting sea ice. Geophys. Res. Lett. 24: 25152518.

Sedwick, P. N., G. R. Ditullio, and D. J. Mackey. 2000. Iron and manganese in the Ross Sea, Antarctica: Seasonal iron limitation in Antarctic shelf waters. J. Geophys. Res 105: 11321-11336.

Smith, W. O., Jr., D. M. Nelson, G. R. Ditullio, and A. R. Leventer. 1996. Temporal and spatial patterns in the Ross Sea: Phytoplankton biomass, elemental composition, productivity and growth rates. J. Geophys. Res. 101: 18455-18465. 
Smith, W.O., D.G. Ainley, R. Cattaneo-Vietti. 2007. Trophic interactions within the Ross Sea continental shelf ecosystem. Phil. Trans. R. Soc. B. 362, 95-111.

Sunda, W. G., and S. A. Huntsman. 1988. Effect of sunlight on redox cycles of manganese in the southwestern Sargasso Sea. Deep-Sea Research 35: 1297-1317.

Sunda, W. G., and S. A. Huntsman. 1995. Cobalt and Zinc interreplacement in marine phytoplankton: biological and geochemical implications. Limnol. Oceanogr. 40 : 1404-1417.

Tebo, B., K. Nealson, S. Emerson, and L. Jacobs. 1984. Microbial meditation of Mn(II) and $\mathrm{Co}(\mathrm{II})$ precipitation at the $\mathrm{O}_{2} / \mathrm{H}_{2}$ interfaces in two anoxic fjords. Limnol. Oceanogr. 29: 1247-1258.

Taylor, S. R., and S. M. Mclennan. 1985. The Continental Crust: its Composition and Evolution. Blackwell Scientific Publications. 


\section{Figures for Chapter 4:}

Figure 1) Maps of the study area. The red boxes indicate the approximate study area from the continental scale (top left), to the Ross Sea, which abuts the large permanent Ross Ice Shelf (top right), to McMurdo Sound (middle) with an aerial photo of the sea ice at the time of the expedition (bottom). The approximate sampling stations are indicated in red, and the approximate location of where the bottom photograph was taken is indicated by a blue triangle in the map of McMurdo Sound. The photograph shows the location of the Delbridge islands, the receding sea ice edge, and Mt. Erebus relative to the approximate locations of the stations. Maps were taken from

http://tiger.gsfc.nasa.gov/images/RossArea.jpg, http://nauticallog.blogspot.com (2010), and generated by Ocean Data View (Schlitzer 2009). 


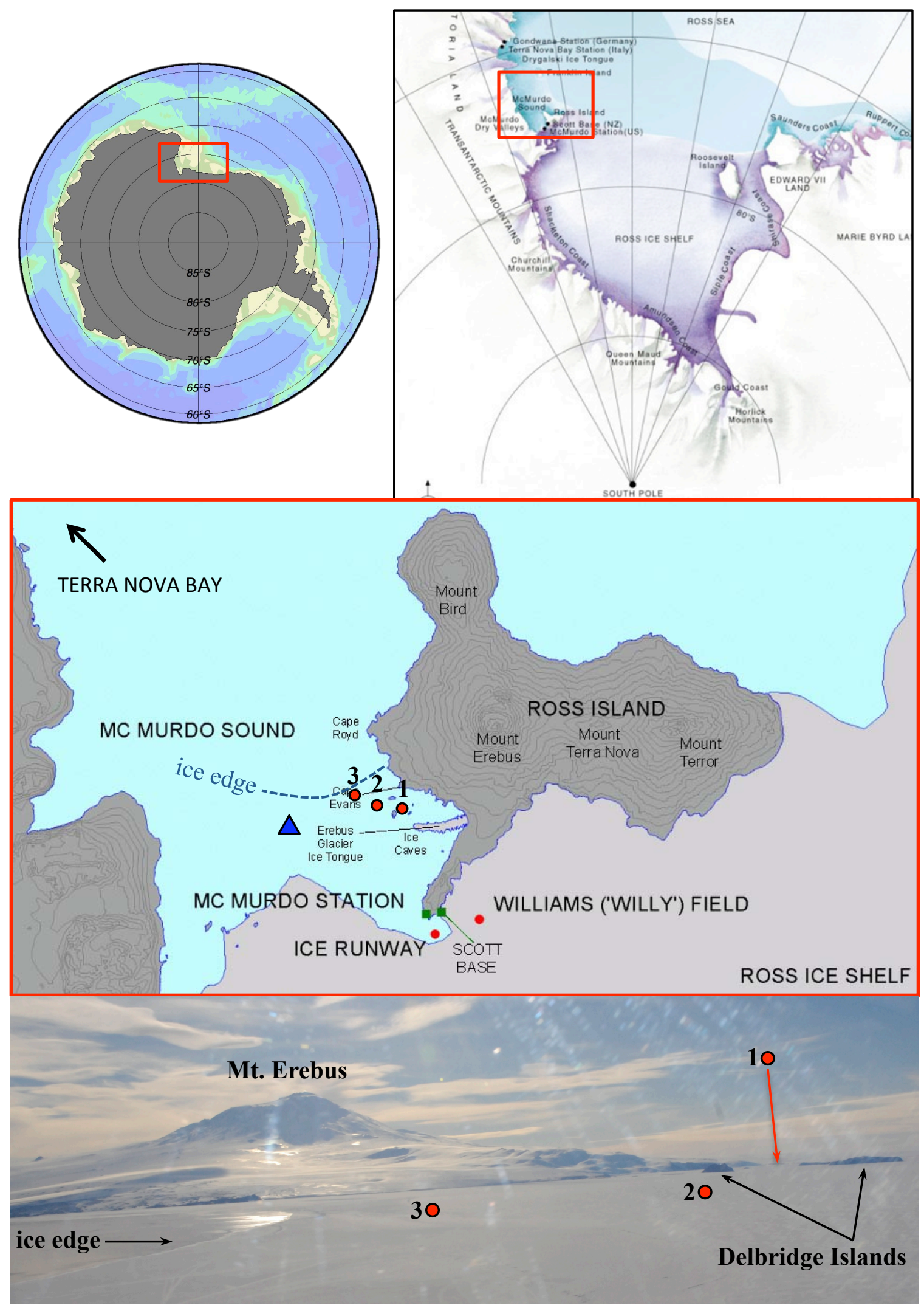


Figure 2. Dissolved distributions of metals at Sta. 1, 2, and 3. Concentrations of total dissolved cobalt, labile cobalt, and phosphate were relatively uniform with the exception of the $4 \mathrm{~m}$ sample at Sta. 2 that showed elevated metals and slightly depleted dissolved phosphate. Iron and manganese trends were similar and varied within a relatively small range of concentrations with the exception of the surface sample at Sta. 2. 

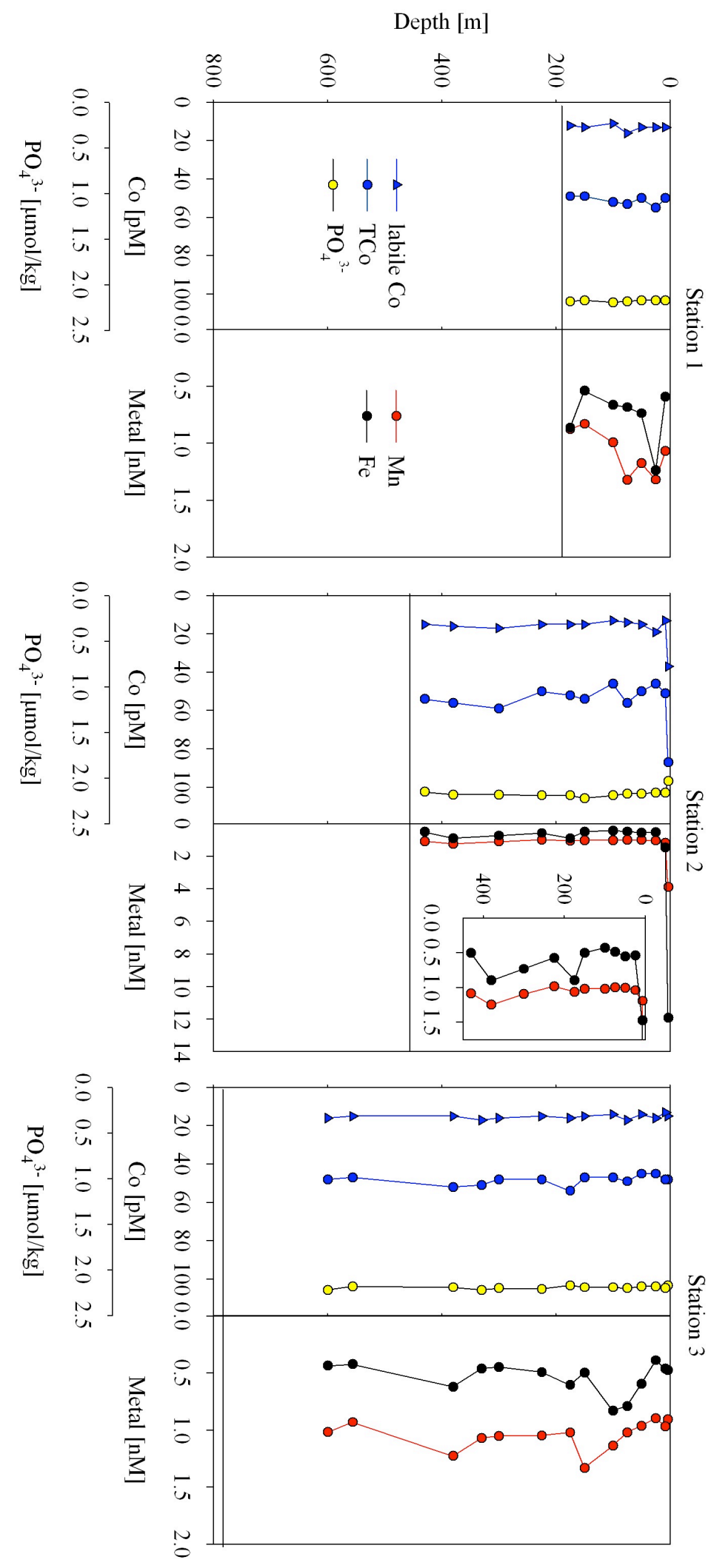
Figure 3. Averaged profiles of dissolved iron, dissolved cobalt, and labile cobalt from this study (all panels, Stas. 1-3), the spring CORSACS study (top panels Stas. NX12-15, NX17, NX20; data from Saito et al. 2010, Sedwick in review), and the summer CORSACS study (bottom panels, Stas. NX1-4, NX7, NX10; data from Saito et al. 2010, Sedwick in review). Stations from the CORSACS study were chosen based on proximity of station location to this study. A similarity between the intermediate depth concentrations of total dissolved cobalt during this study and the summer CORSACS study was apparent. While total cobalt was drawn down in surface waters over the season, labile cobalt was higher in than under the McMurdo sea ice, perhaps due to the introduction to deeper waters via remineralization in the spring and summer. The biotic drawdown of iron appeared to penetrate to deeper depths than observed for cobalt. 


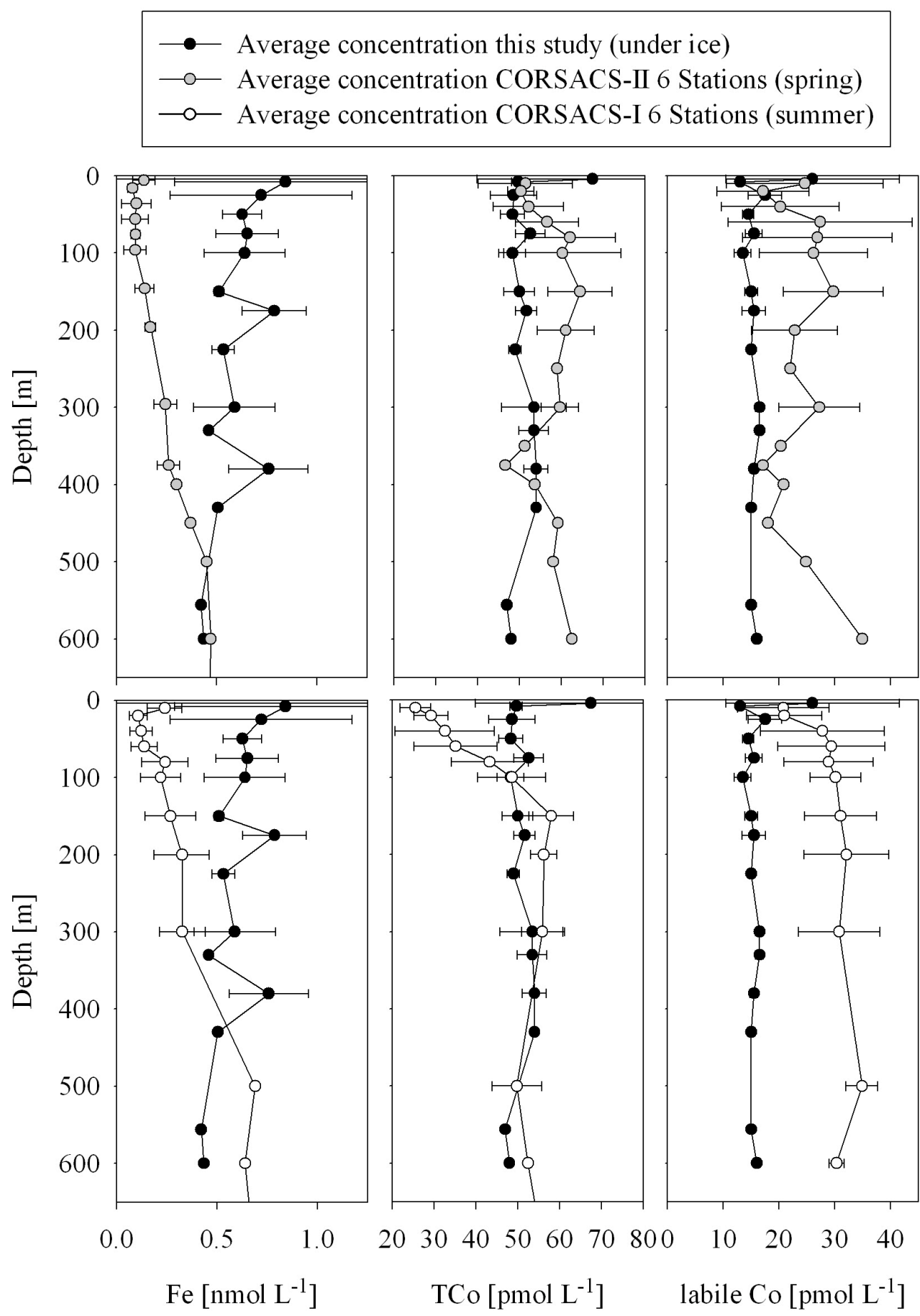




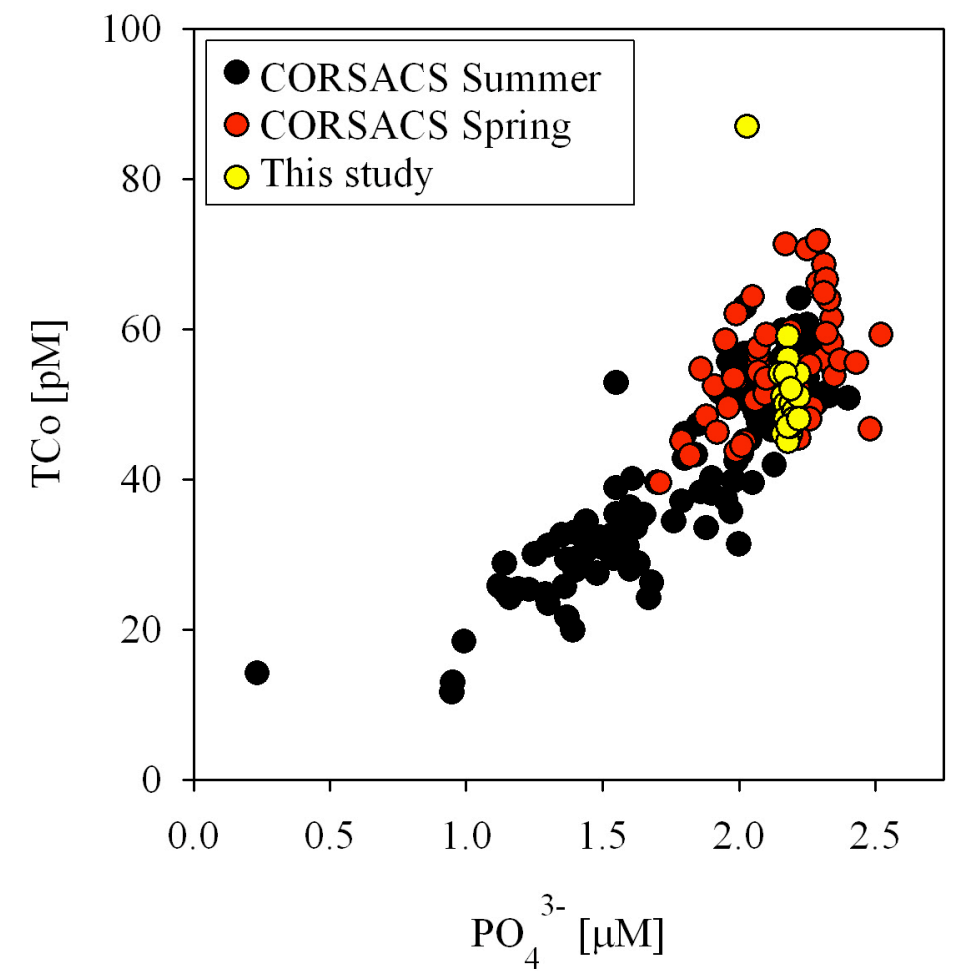

Figure 4. A comparison of cobalt and phosphate relationships under the sea ice (this study) and in the Ross Sea Polynya (CORSACS spring and summer, Saito et al. 2010). The data from this study are consistent with our previous $\mathrm{Co}: \mathrm{PO}_{4}{ }^{3-}$ observations. 
Figure 5. Particulate iron, cobalt, manganese, aluminum, and phosphorus distributions at Stas. 1, 2, and 3 .

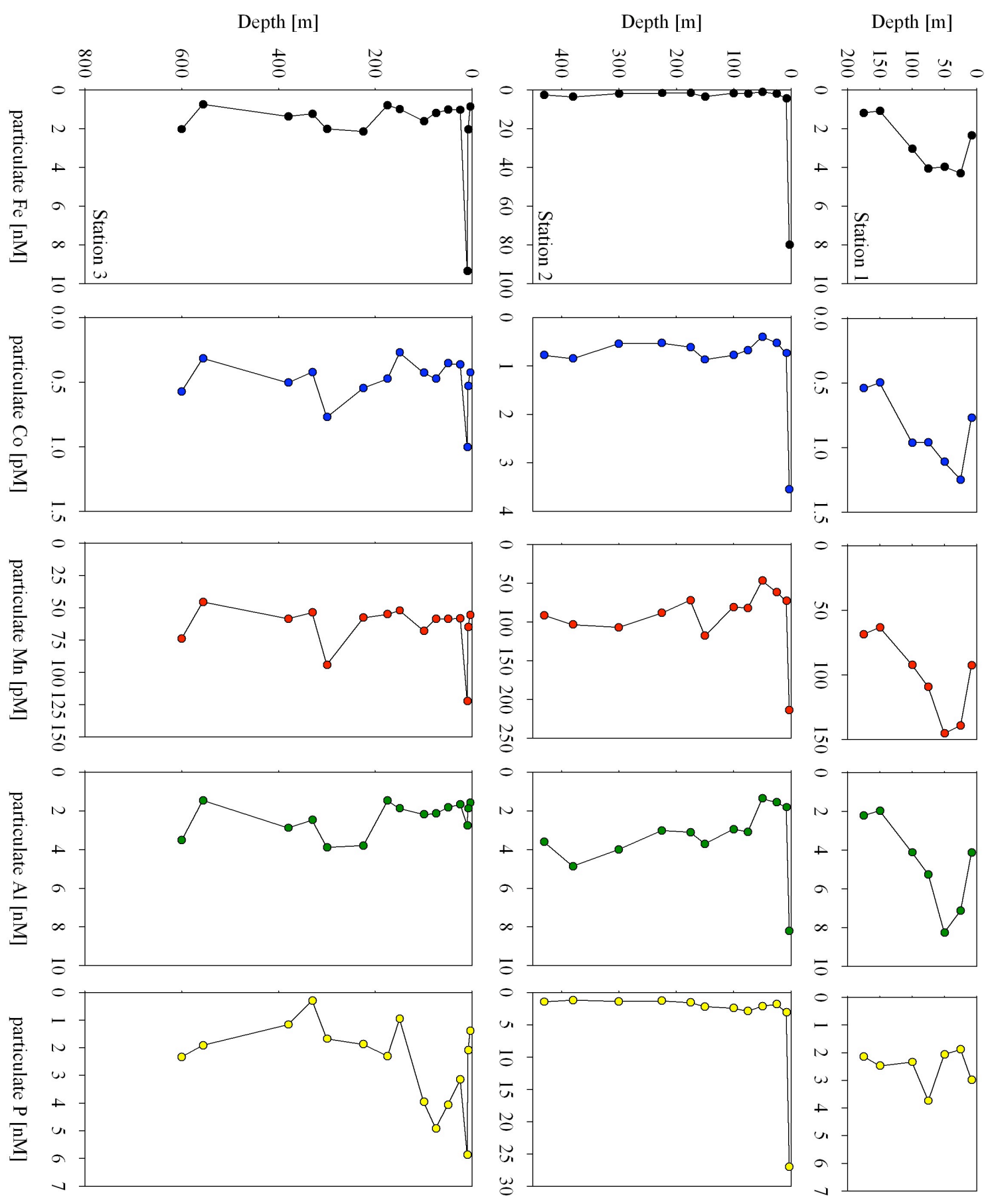


Figure 6. Correlations between particulate bioactive trace metals for all three stations sampled. Surface samples where biological activity was evidently high are shown in red (Sta. 2 3m, Sta. 2 8m, Sta. $310 \mathrm{~m}$ ).

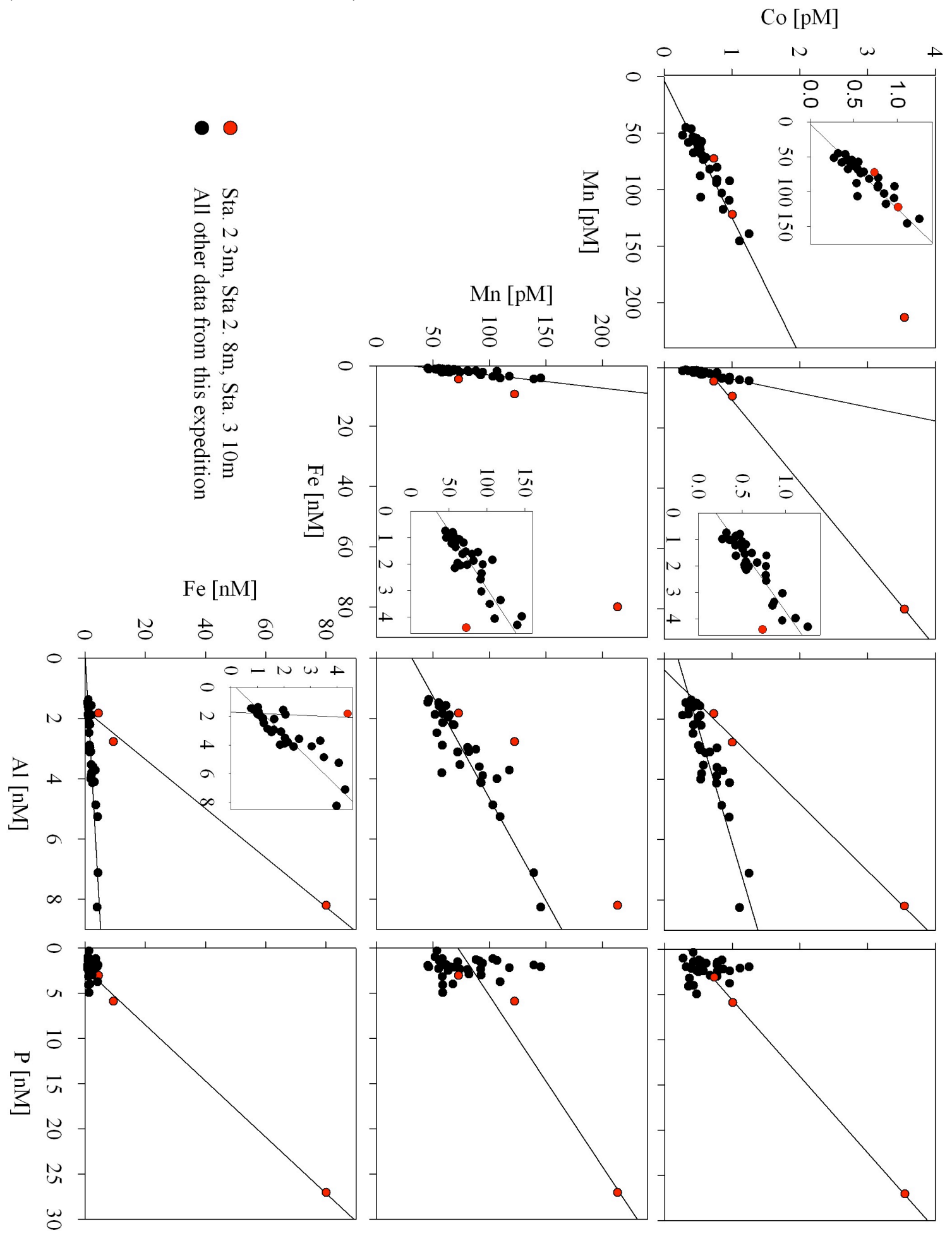



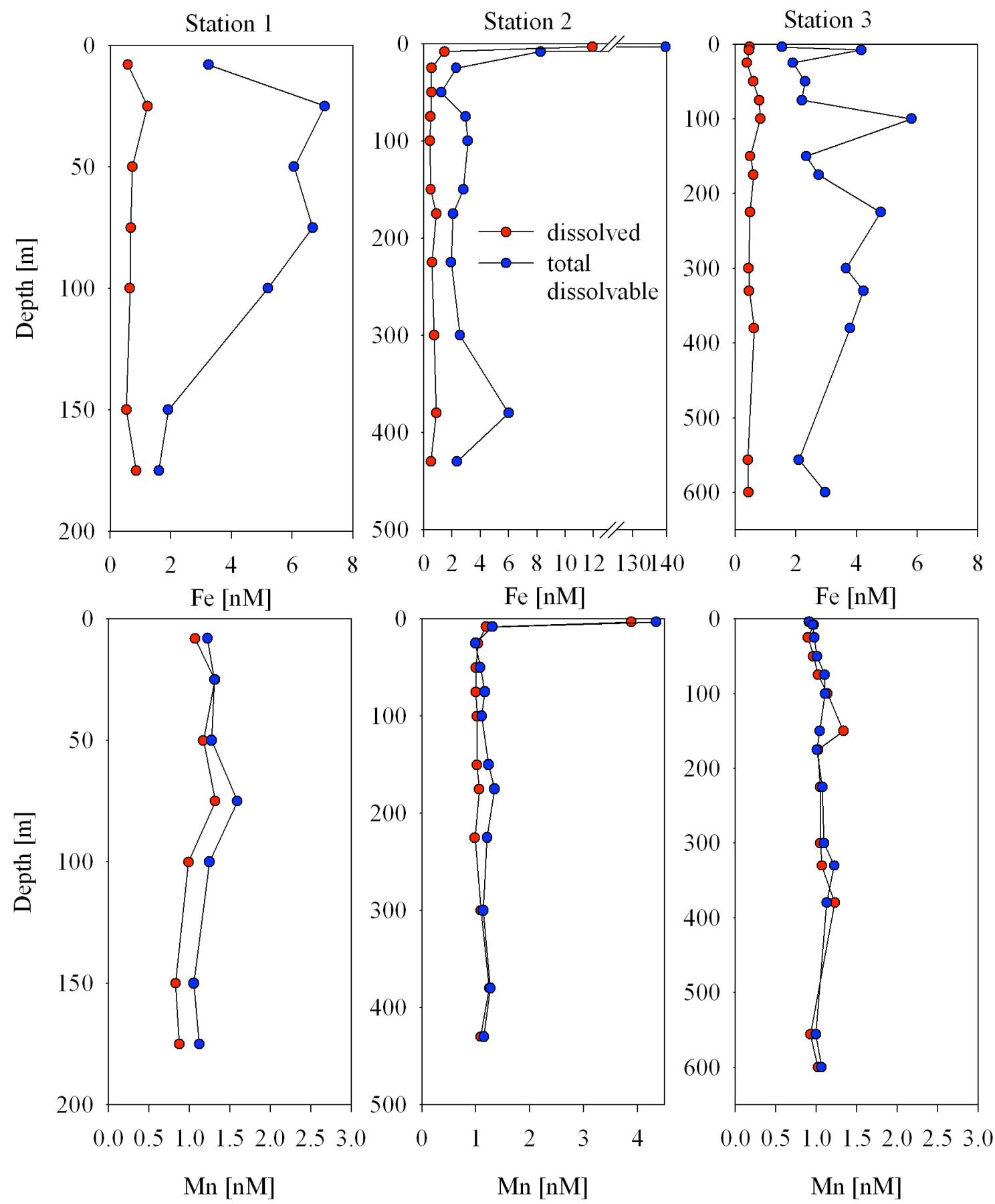

Figure 7. Depth profiles of total dissolvable and dissolved iron and manganese. The acid leachable particulate concentration is determined by the difference between these two concentrations. This demonstrates that the acid leachable particulate iron should be resolvable using this method, and that the acid leachable particulate manganese fraction appears to be very small. 

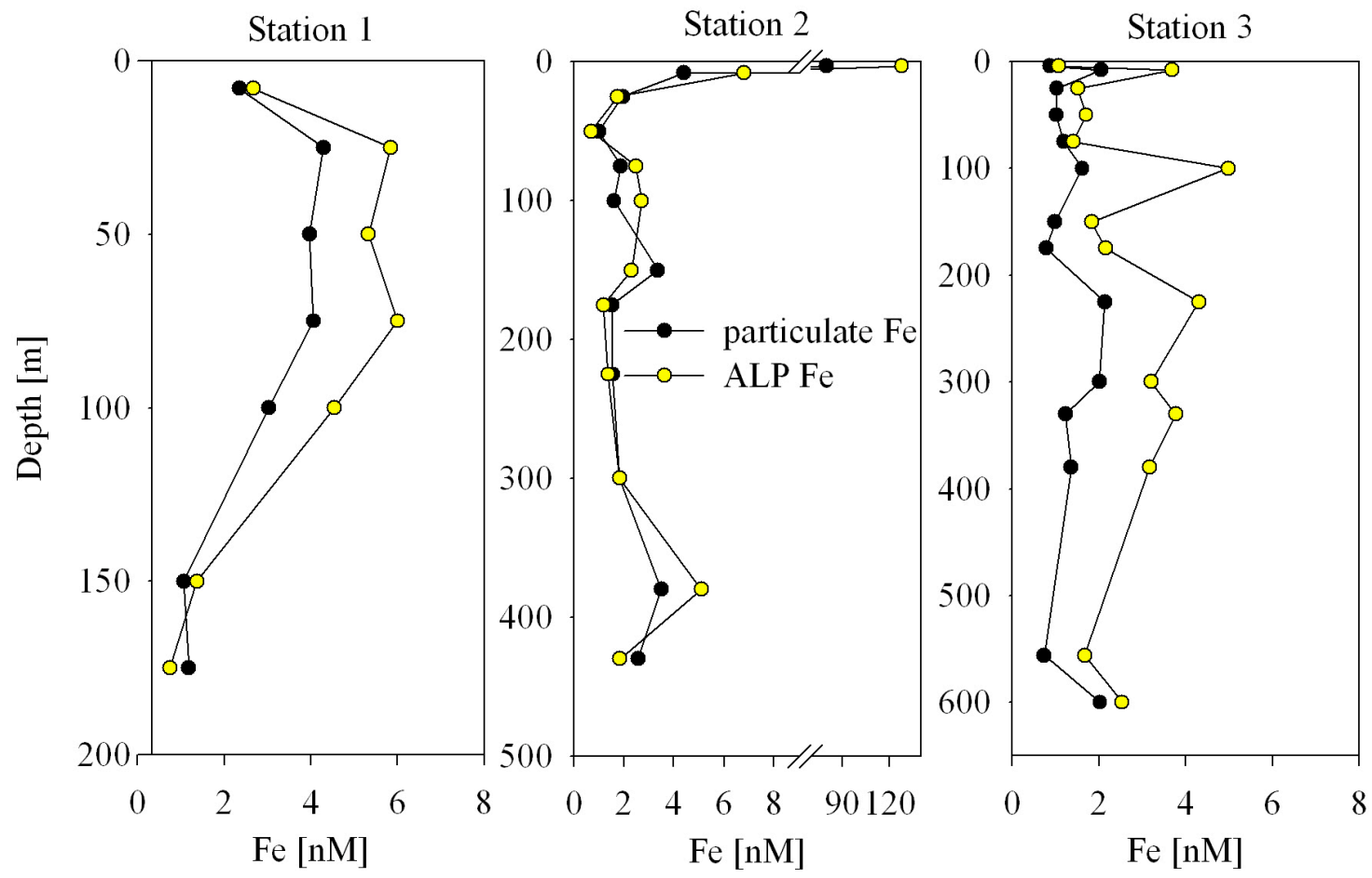

Figure 8. Depth profiles of calculated acid leachable particulate iron (ALP Fe) compared to particulate iron. 
Figure 9. A comparison of acid leachable particulate iron from the CORSACS expeditions (Sedwick et al. in review) to acid leachable particulate iron profiles from this study. Depth $[\mathrm{m}]$
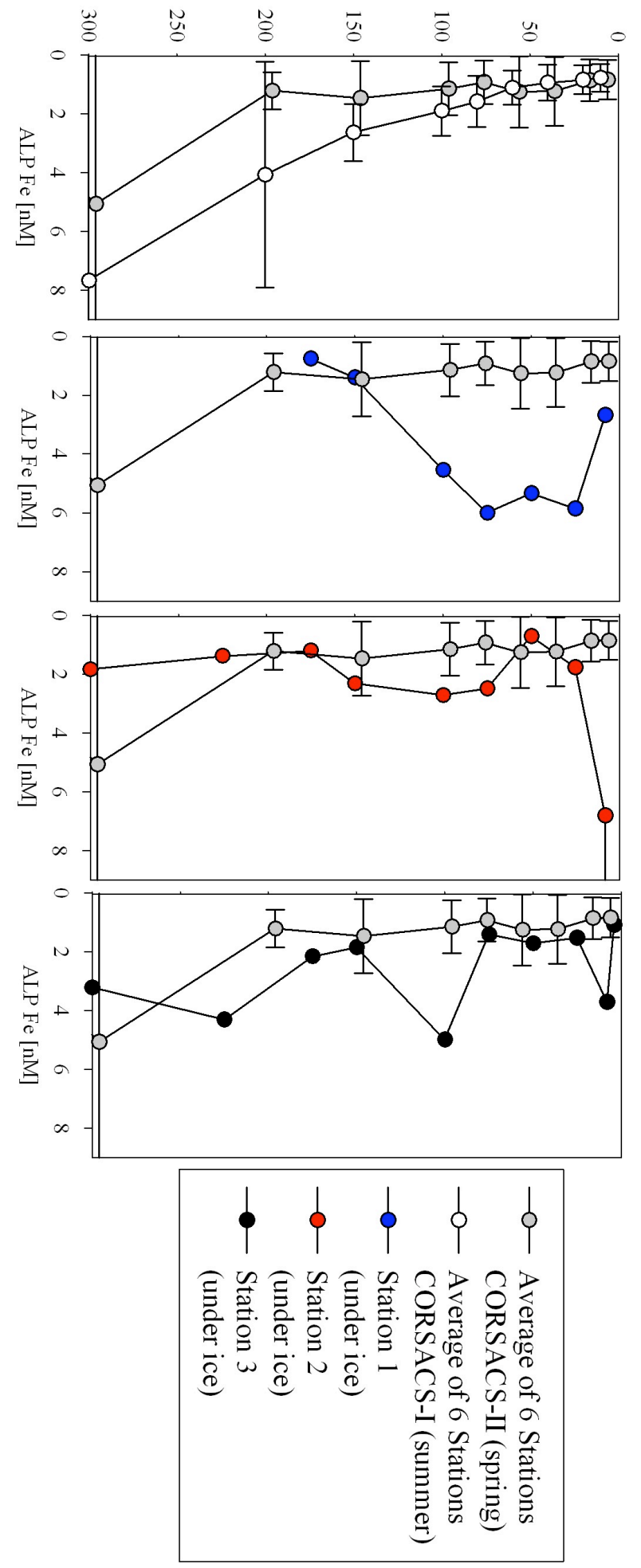

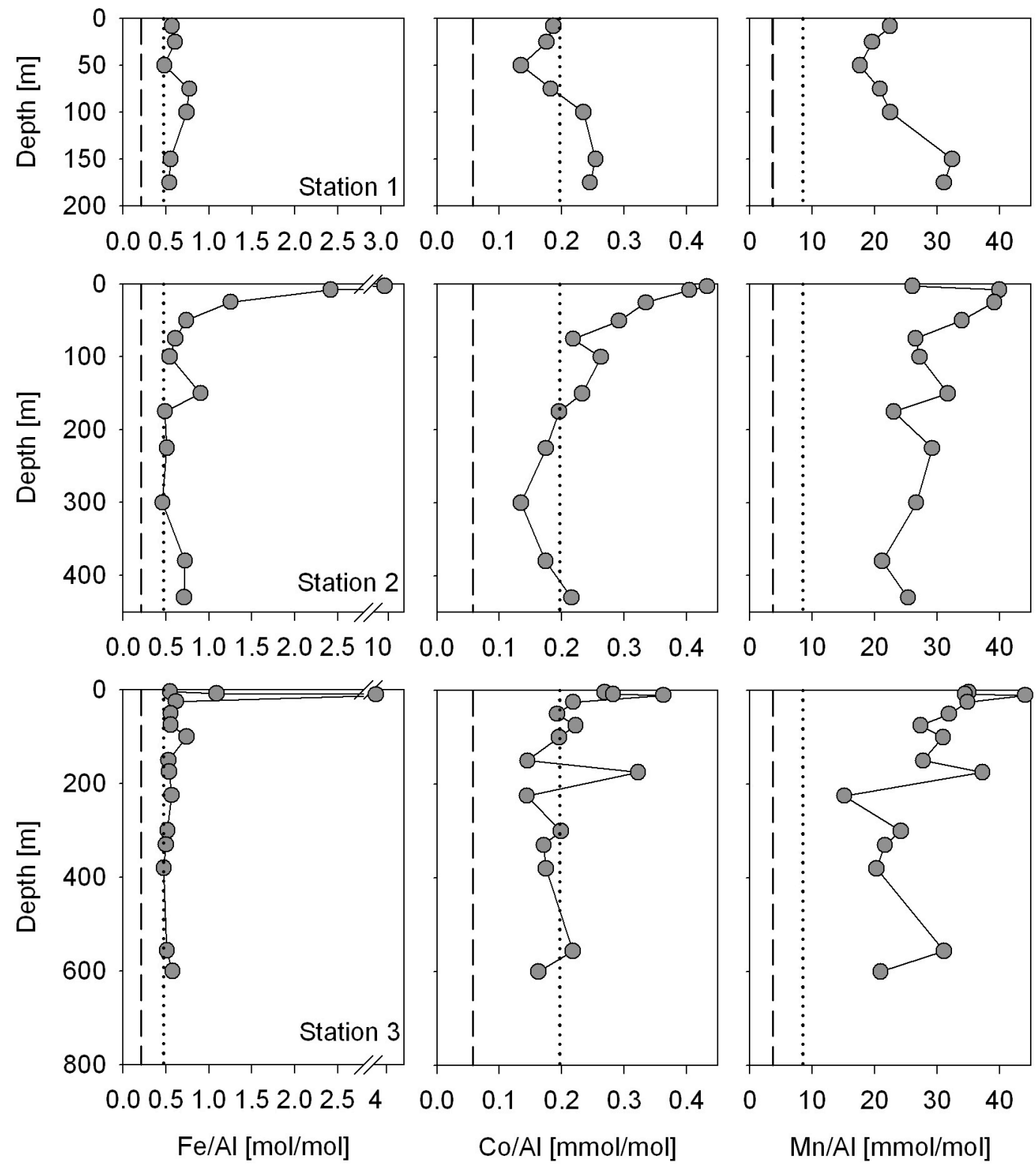

Figure 10. Aluminum normalized metal profiles. The Fe/Al ratios are similar the sediment composition value for two Ross Sea sediment core study sites (dotted lines, Ianni et al. 2010, Ravanelli et al. 1997), but both the sediment and water column ratios are higher than crustal ratios (dashed lines, Taylor and McLennan 1985), suggesting that the particulate influences observed here are more biogenic than lithogenic. 


\title{
Chapter 5: Distributions of dissolved cobalt from the US North Atlantic GEOTRACES cruise: relationships with phosphate and oxygen, and a comparison to the South Atlantic Ocean
}

\begin{abstract}
Cobalt is an important micronutrient to many phytoplankton in the open ocean. It is also a hybrid-type metal with low oceanic concentrations due to the low solubility of the oxidized ion in seawater. This study examined the $\mathrm{Co}: \mathrm{PO}_{4}{ }^{3-}$ and $\mathrm{Co}: \mathrm{O}_{2}$ relationships in the North and South Atlantic Ocean. The data for these comparisons were derived from two expeditions: the CoFeMUG (Cobalt Iron and Microorganisms from the Upwelling to the Gyre) and the US-NAGZT (United States North Atlantic GEOTRACES Zonal Transect) research cruises. From these data, a number of trends were observed. First, linear covariance between total cobalt and phosphate, and between labile cobalt and phosphate suggest a strong biological influence was exerted on the surface distributions of cobalt. Second, a broad comparison between the North and South Atlantic basins revealed similar $\mathrm{Co}: \mathrm{PO}_{4}{ }^{3-}$ ratios, but the different surface phosphate inventories and average dust deposition rates in these basins caused their respective $\mathrm{Co}: \mathrm{PO}_{4}{ }^{3-}$ correlation lines to be offset from each other. Third, a range of $\mathrm{Co}: \mathrm{PO}_{4}{ }^{3-}$ ratios were observed when the regions were examined more closely: the lowest $\mathrm{Co}: \mathrm{PO}_{4}{ }^{3-}$ ratios were found in the oligotrophic South Atlantic Subtropical Gyre and European North East Atlantic, and the highest were found in the West African North East Atlantic and the Angola Gyre. These observations build upon the growing global dataset of linear $\mathrm{Co}: \mathrm{PO}_{4}{ }^{3-}$ relationships, which have been observed to range over an order of magnitude, and demonstrate the real oceanic variability of this important micronutrient. Strong linear relationships were also observed for total and labile cobalt when compared to $\mathrm{O}_{2}$ within the oxygen minimum zones of these basins. Lastly, when cobalt and phosphate were compared at depth, removal of cobalt relative to phosphate was observed in a "curl" that began at variable depths in $\mathrm{Co}: \mathrm{PO}_{4}{ }^{3-}$ space for each of the regions investigated.
\end{abstract}


5.1 Introduction - Cobalt is a hybrid-type micronutrient that is present at very low concentrations in the ocean. The aggregate nutritive utilization of trace metal micronutrients has been examined by comparing the dissolved trace metal concentration to macronutrients like soluble reactive phosphate or nitrate (Sunda and Huntsman 1995, Cullen 2006, Sunda and Huntsman 2000, Saito et al. 2004, Noble and Saito et al. 2008, Saito et al. 2010). Correlations between metal and phosphate concentrations in the upper water column can be indicative of the biological processes of uptake and remineralization dominating the dissolved distribution of the metal. The slope of these correlations has been termed the "ecological stoichiometry" of the metal because it reflects the integrated uptake and remineralization of that metal by the aggregate community (Sterner and Elser 2002; Saito et al. 2010). Abiotic processes, such as aeolian deposition, particle scavenging, or sedimentary supply tend to decouple cobalt and phosphate because they tend to exert a greater influence on cobalt distributions than phosphate (Noble and Saito et al. 2008; Saito et al. 2010). For these reasons, the ecological stoichiometries of cobalt are often obscured in regions that are dominated by abiotic processes and/or by non-nutritive biotic utilization such as co-oxidation of cobalt by manganese oxidizing bacteria (Tebo et a. 1984).

Dynamic changes in $\mathrm{Co}: \mathrm{PO}_{4}{ }^{3-}$ relationships have previously been observed. In the Peru Upwelling system where a large flux of cobalt correlated with phosphate, the correlation was not observed further from shore as cobalt became highly complexed and phosphate became depleted (Saito et al. 2004). Photic zone coupling of cobalt and phosphate has also been observed in the North Pacific, Subtropical North Pacific, and Ross 
Sea (Martin et al. 1989; Noble and Saito et al. 2008; Saito et al. 2010). Co:PO ${ }^{3-}$ ratios derived from linear regressions of dissolved cobalt and soluble reactive phosphate vary by more than an order of magnitude in the oceans. This observation suggests that real variations in biological utilization of this micronutrient are linked to other processes of ecological and biogeochemical significance, such as differences in supply, lability, and the presence of competing micronutrients. This study sought to compare $\mathrm{Co}: \mathrm{PO}_{4}{ }^{3-}$

relationships across distinct oceanic regimes in the Atlantic, and build upon the results of previous studies from other geographic regions.

These linear relationships between cobalt and phosphate are often observed in the upper water column only, as scavenging of cobalt occurs with depth, preferentially removing cobalt relative to phosphate, and resulting in a downward "curl" in Co: $\mathrm{PO}_{4}{ }^{3-}$ space. If the relationship between cobalt and phosphate are compared through the entire water column, the depth at which cobalt removal becomes apparent can be observed visually and determined mathematically by deterioration of the linear relationship. This study also sought to examine the variability in the apparent depth of cobalt scavenging in different geographic regions, although the implications of this variability are still unclear.

In addition to the previous observations of linear relationships between cobalt and phosphate, recent work has revealed an inverse linear relationship between cobalt and oxygen within the oxygen minimum zone of the South Atlantic (Noble et al. in review). In an effort to expand on those observations, this study presents a comparison of the South Atlantic $\mathrm{Co}: \mathrm{O}_{2}$ relationship to the trends observed within the low oxygen regions of the 
North Atlantic. With this high-resolution dataset of labile cobalt, comparisons between labile cobalt and oxygen could also be made.

\subsection{Methods}

5.2.1 NAGZT Cruise Sampling Techniques - The first leg of the NAGZT (North Atlantic Geotraces Zonal Transect) cruise took place aboard the $R / V$ Knorr $(10 / 14-11 / 3,2010$, Chief Scientists: William Jenkins and Ed Boyle) and employed a trace metal sampling program using the ODU GEOTRACES Carousel. The cruise began near Lisbon, Portugal, at $9.66^{\circ} \mathrm{W}, 38.32^{\circ} \mathrm{N}$ (Sta. 1) and headed south, sampling Mediterranean outflow water (Fig. 1, Sta. 1-6), and then continued south to perform a short zonal transect from the Mauritanian coast along $17.35^{\circ} \mathrm{N}$ (Fig. 1, Sta. 9-12). The carousel was used to collect 24 samples from surface to near bottom waters, and an additional sample was collected from a surface towfish at each station. Stas. 2, 6, and 8 were sampled to $1200 \mathrm{~m}$ and consisted of 12 carousel samples plus one towfish sample. Pre-conditioned, teflon-coated Go-Flo sampling bottles (General Oceanics, Miami, FL) of $12 \mathrm{~L}$ capacity were deployed on a polyurethane powder-coated aluminum rosette with titanium pilings and pressure housings (Sea-Bird Electronics, Inc., Bellevue, WA) attached to a Kevlar, non-metallic conducting cable. For more information regarding carousel deployment, please refer to the GEOTRACES Program Cruise and Methods Manual, located on the GEOTRACES Program website (www.GEOTRACES.org). Following the retrieval of the carousel, GoFlo bottles were moved to the GEOTRACES Program class-100 trace metal clean van, and pressurized with $99.999 \% \mathrm{~N}_{2}$ at $<7 \mathrm{psi}$ for sampling. Surface towfish samples were 
collected by suspending the towfish off the starboard side with a boom, and sampled water at approximately $2 \mathrm{~m}$ depth using a Teflon diaphragm pump following the GEOTRACES Program Cruise and Methods Manual sampling recommendations. All cobalt samples described here were collected through $0.2 \mu \mathrm{m}$ Acropak filters, which had been cleaned and conditioned according to the GEOTRACES Program Cruise and Methods Manual.

Sample storage bottles were soaked overnight in the acidic detergent, Citranox, rinsed thoroughly with Milli-Q water (Millipore), filled with $10 \% \mathrm{HCl}$ to soak for 10 days, rinsed thoroughly with Milli-Q water adjusted to $\mathrm{pH}$ 2, and double-bagged. The sample storage bottles were made of low-density polyethylene. Once filled, they were kept at $4^{\circ} \mathrm{C}$ in darkness until analysis. All analyses reported here were made within a week of sampling.

5.2.2 CoFeMUG Cruise Sampling Techniques -The CoFeMUG (Cobalt, iron and microorganisms from the upwelling to the gyre) cruise aboard the R/V Knorr (16 November - 13 December 2007, Chief Scientist, Saito) employed a trace metal sampling program for three transects in the South Atlantic Ocean. Further details of this expedition and sampling can be found in Noble et al. (in review).

5.2.3 Cobalt Analyses - Concentrations of total dissolved and labile cobalt during the NAGZT cruise were determined ship-board using a previously described cathodic stripping voltammetry (CSV) method (Saito and Moffett 2001, Saito et al. 2004). Measurements were made using the Eco-Chemie $\mu$ AutolabIII systems connected to Metrohm 663 VA Stands equipped with hanging mercury drop electrodes and Teflon sampling cups. Standard additions were carried out with Metrohm 765 Dosimats using a 
programmed dosing procedure (Noble and Saito et al. 2008). Identical methods to those described here were employed for the CoFeMUG cruise as well, and are described in detail in Noble et al. in review.

For total dissolved cobalt analyses, samples were UV-irradiated for $1 \mathrm{~h}$ prior to analysis using a Metrohm 705 UV digester to degrade the organic ligands that bind cobalt and allow binding by the added electroactive cobalt ligand, dimethylglyoxime. Samples were analyzed in $8.5 \mathrm{~mL}$ aliquots with the addition of $30 \mu \mathrm{L}$ recrystalized dimethylglyoxime (DMG, $0.1 \mathrm{~mol} \mathrm{~L}^{-1}$ in methanol), $1.5 \mathrm{~mL}$ purified sodium nitrite $\left(1.5 \mathrm{~mol} \mathrm{~L}^{-1}\right.$ in Milli-Q water), and $50 \mu \mathrm{L}$ purified N-(2-hydroxyethyl)piperazine-N-(3-propanesulfonic acid) (EPPS) buffer $\left(0.5 \mathrm{~mol} \mathrm{~L}^{-1}\right.$ in Milli-Q water). Reagent purification protocols were identical to those previously published (Saito and Moffett 2001). Analysis began with a 180 s purge with $99.999 \% \mathrm{~N}_{2}$. Each sample was conditioned at $-0.6 \mathrm{~V}$ for $90 \mathrm{~s}$ at a stir-rate of $2500 \mathrm{rpm}$ followed by a $10 \mathrm{~s}$ equilibration step and a linear sweep from $-0.6 \mathrm{~V}$ to $-1.4 \mathrm{~V}$ at a rate of $10 \mathrm{~V} \mathrm{~s}^{-1}$. Cobalt concentrations were determined by the standard additions technique, with initial concentrations measured in triplicate followed by four $25 \mathrm{pmol} \mathrm{L}^{-1}$ cobalt additions. The analytical blank was determined by analyzing seawater that had been UV-irradiated for $1 \mathrm{~h}$, equilibrated overnight with prepared Chelex 100 resin beads (BioRad), and UV-irradiated a second time to degrade any leached synthetic ligands. Blanks for each reagents batch (nitrite, DMG, EPPS) were subtracted from the initial sample concentration. During the cruise, five batches of reagents were used. Blank analyses for each reagent batch were made at the beginning and end of use to confirm that the blank 
remained constant during analyses. The averaged blank concentrations for the five reagent batches used in this dataset are listed below in Table 1.

For labile cobalt analyses, $8.5 \mathrm{~mL}$ of sample was pipetted into acid washed Teflon vials that were preconditioned with a small aliquot of sample water. $30 \mu \mathrm{L}$ of DMG was added to each vial and allowed to equilibrate overnight in the dark prior to analysis (Saito et al. 2004). Analyses were then performed as described for total concentrations with the addition of the remaining two reagents and use of the standard addition technique. Previously, we determined that natural cobalt ligands in seawater have a conditional stability constant of $>10^{16.8}$ (Saito et al. 2005). This suggests that the cobalt is very strongly bound to ligands. Thus, we define labile cobalt as the fraction of total dissolved cobalt that is either bound to weak organic and inorganic ligands in seawater or present as free $\mathrm{Co}(\mathrm{II})$, and is then exchangeable with the complexing agent (DMG) used for analysis (Saito et al. 2004, Saito et al. 2005). The difference between the total dissolved cobalt and the labile cobalt can then be used as an estimation of the strong cobalt ligand concentration. Two full electrochemical systems were utilized during the cruise. One electrode was dedicated to total analyses and the other to labile analyses. GEOTRACES standard seawater and internal standard lab seawater were analyzed over the course of the cruise to ensure that the two electrodes were intercalibrated, and functioning properly. The results of these analyses are reported below in Table 2. GEOTRACES standard seawater was UV irradiated and neutralized using Optima ammonium hydroxide to bring the $\mathrm{pH}$ up to 7.5. Standard seawater internal to our lab was UV irradiated as well, but was not previously acidified so received no further treatment prior to analysis. These results demonstrate that 
the methodologies employed to produce this dataset detect concentrations within the standard deviation of current consensus values for UV irradiated samples, which can be found on the International GEOTRACES Program website (www.geotraces.org).

5.2.4 Nutrient analyses - Nutrient analyses for CoFeMUG were processed at Oregon State University and the methods described in Noble et al. in review (see Chapter 3). Nutrient analyses for NAGZT were processed shipboard by Susan Becker from the University of California, San Diego.

\subsection{Results and Discussion}

Relationships between cobalt and phosphate can help characterize the influences on oceanic cobalt distributions and this study expands upon previous observations to include $\mathrm{Co}: \mathrm{PO}_{4}{ }^{3-}$ linear relationships from the North and South Atlantic. In order to examine the changes in these ratios, two types of comparisons are presented here: 1) a broad North and South Atlantic basin comparison, and 2) a subset of this comparison where the data generated from the NAGZT and CoFeMUG cruises were separated into 4 regions. The first approach focused on trends that reflected the large-scale basin differences and similarities between the North and South Atlantic. These differences are summarized in Table 3, and one of the most notable similarities between the two basins was the observation of a strong linear relationship between cobalt and oxygen observed within the low oxygen regions of both basins (Fig. 3). The second comparison approach, for which the four regions are described below, focused on more subtle differences in the $\mathrm{Co}: \mathrm{PO}_{4}{ }^{3-}$ relationships and inferences derived from chemical speciation analyses. The last section 
focused on the differences in scavenging behavior of cobalt across a number of geographic regions.

5.3.1 Description of the oceanographic regions studied - The first of the four regions is the European N. E. Atlantic, which is comprised of Stas. 1, 2, 3, 5, and 6 from the NAGZT cruise (Figs. 1, 2, 4). These stations showed extreme surface depletion of macronutrients (Fig. 2). Sta. 3 and 5 were also characterized by intense surface depletion of total and labile cobalt. Some aeolian input may have been observed at Sta. 6, and elevated surface concentrations at Sta. 1 and 2 were likely due to anthropogenic aerosol sources from Europe (A. Aguilar-Islas and R. Shelley personal communication). The data points that may be skewed by anthropogenic influence were excluded from the $\mathrm{Co}: \mathrm{PO}_{4}{ }^{3-}$ relationship study (4 data points from Sta. 1 (2, 24, 34, and $40 \mathrm{~m}$ ) and 2 from Sta. 2 (2 and $49 \mathrm{~m}$ ).

The second region is the W. African N. E. Atlantic, which is comprised of Stas. 712 from the NAGZT cruise (Figs. 1, 3, 4). This region was distinct from the European N. E. Atlantic due to notable differences in oxygen minimum zone depth and chemical character. In Stas. 1-6, the oxygen minimum was located at $\sim 800 \mathrm{~m}$ and concentrations remained above $140 \mu \mathrm{mol} \mathrm{kg}$. . This contrasted with Stas. 7-12 which had shallower oxygen minima ( $400 \mathrm{~m}$, Fig. 3,4$)$ and concentrations ranging from $40-110 \mu \mathrm{mol} \mathrm{kg}^{-1}$. These differences result from different water masses being present in each region, and perhaps the influence of different remineralization rates, which could be driven in part by the upwelling that occurs near the W. African coast, and the resultant higher productivity.

The third region is the S. E. Atlantic Angola Gyre/Benguela Front, which is comprised of Stas. 8-17 from the CoFeMUG cruise (Fig. 1; Noble et al. in review). Stas. 
18-27 have been omitted here to focus on the open ocean basin and specifically on the Angola Gyre. This region was characterized by low oxygen concentrations due to winddriven upwelling and remineralization, intense biological productivity, and slow ventilation near the African coast. Oxygen concentrations reached as low as $18 \mu \mathrm{mol} \mathrm{\textrm {kg } ^ { - 1 }}$ in coincidence with a large plume of cobalt, which we previously suggested was fueled by reductive dissolution in the coastal sediments during seasonal anoxia (Fig. 2; Noble et al. in review).

The fourth region is the South Atlantic Subtropical Gyre, which consists of data from Stas. 1-7 of the CoFeMUG cruise, east of the Mid-Atlantic Ridge (Fig. 1; Noble et al. in review). Surface waters were depleted in cobalt and macronutrients, and the chlorophyll maximum was found at depths $>100 \mathrm{~m}$. In general, the concentrations of cobalt observed in this region were lower than the other three regions.

5.3.2 Sources of cobalt to the N. E. Atlantic-There appeared to be three sources of cobalt to the N. E. Atlantic. First, dust from the Sahara Desert deposits significant amounts of iron and other metals to the N. E. Atlantic (Measures 1995; Measures and Vink 2000; Measures et al. 2008). However, cobalt is much less abundant than iron in average crustal material (1:2600, Taylor and McLennan 1985) so dust is often not considered to be as important a source of cobalt as it is for iron to the open ocean. The NAGZT cruise and its proximity to the Sahara Desert examined the potential for aeolian contribution: a dust event occurred during Sta. 9, where visibility was reduced. This was reflected in increasing surface cobalt concentrations with proximity to the desert source (Fig. 5). Dissolved aluminum, a tracer of natural dust deposition, showed a significant linear 
relationship with total cobalt in surface waters between Sta. 8 and $12\left(r^{2}=0.96\right.$, Fig. 5). This Co:Al relationship in surface waters was not observed in the South Atlantic where dust input is known to be much lower (Noble et al. in review). A deviation from this trend was seen near the coast of Portugal where total cobalt in surface waters is high (Sta. 1, Fig. $4,5)$ and the corresponding aluminum concentrations are low (data not shown). This decoupling is consistent with an increasing influence of anthropogenic aerosols at these stations, though anthropogenic contamination in natural dust plumes from the Sahara has also been observed (A. Aguilar-Islas and R. Shelley, personal communication; Erel et al. 2006, 2007).

Upwelling occurs in the W. African N. E. Atlantic as well, which leads to higher productivity and an oxygen minimum zone that extends from the coast (Fig. 2). Closer to the coast, a shoaling of the mixed layer depth and chlorophyll maximum are observed (data not shown). Oxygen concentrations below the euphotic zone are negatively correlated to cobalt, similar to our observations in the Benguela and Angola Upwelling systems (Fig. 2, Noble et al. in review). Reductive dissolution of cobalt associated with manganese oxides in sediments along the coast may contribute to this cobalt plume, as we have previously suggested for the Benguela and Angola system. However, as aeolian influences were significant, it is likely that a fraction of this subsurface plume was due to remineralization as a result of surface productivity fueled by aeolian input. While oxygen concentrations here are not as low as those observed in the South Atlantic, particulate $\mathrm{FeS}_{2}$ has been observed in both the sediments and suspended particulate matter near our sampling sites (Lam et al. in review), which suggests that there may be sufficiently low oxygen 
concentrations along the shelf to allow the escape of reduced cobalt from the sediments without reprecipitation as oxides.

5.3.3 $\mathrm{Co}_{\mathrm{PO}}{ }_{4}^{3-}$ relationships in the Atlantic - In the upper water column of both the North and South Atlantic datasets, linear relationships were observed between total dissolved cobalt and phosphate (Figs. 6,7,8). These relationships, referred to as the ecological stoichiometries, are represented by the slope in cobalt versus phosphate plots, and are listed in Table 4, along with the currently published values for other geographic regions. A number of similarities were apparent in the upper $300 \mathrm{~m}$ of the North and South Atlantic basins (Figs. 6 and 7). Biological processes appeared to dominate the distributions of cobalt in the South Atlantic between $\sim 50$ and $300 \mathrm{~m}$, as demonstrated by the significant linear correlation of total dissolved cobalt with phosphate $\left(r^{2}=0.83\right)$. This slope provided an estimated $\mathrm{Co}: \mathrm{PO}_{4}{ }^{3-}$ ecological stoichiometry of $63 \pm 3.3 \mu \mathrm{mol} \mathrm{mol}^{-1}$ for this system, indicative of the aggregate confluence of biological uptake and remineralization processes. Biological processes also influenced the $\sim 50-300 \mathrm{~m}$ cobalt distributions in the North Atlantic basin, and the corresponding relationship observed there was similar $\left(67 \pm 3.0 \mu \mathrm{mol} \mathrm{mol}{ }^{-1}, r^{2}=0.89\right)$. Upon close examination, another much higher $\mathrm{Co}: \mathrm{PO}_{4}{ }^{3-}$ ratio appeared to be present within the shallowest waters of both basins, and this "kink" is described later in this section.

Despite the similarity in the patterns of $\mathrm{Co}: \mathrm{PO}_{4}{ }^{3-}$ relationships between the two basins, there was a notable offset indicated by two arrows in Fig. 6b. Along the phosphate axis, there is a shift to the left between the South and North Atlantic. This was likely due to the much lower surface phosphate inventory observed in the North Atlantic (Wu et al. 
2000). This was clearly illustrated when cobalt was compared to nitrate (NAGZT) and nitrate plus nitrite (CoFeMUG) in Fig. 6c, where the horizontal offset is no longer observed. There is also a vertical offset, likely due to higher cobalt concentrations in the North Atlantic than in the South Atlantic. This could be due to additional sources of cobalt to the North Atlantic. As discussed earlier, one likely source was dust deposition. This could have increased the cobalt concentrations relative to phosphate between the two regions because the North Atlantic experiences significant input from the Saharan Desert compared to much lower dust inputs to the South Atlantic, and aeolian deposition is not considered to be a source of phosphorus. This offset can also be seen in the $\mathrm{Co}: \mathrm{O}_{2}$ plots as a vertical shift (Fig. 3), perhaps similarly caused by the higher dust contribution in the North Atlantic.

If the two basins are separated into the four defined regions described above (section 5.3.1), further spatial differences emerge (Fig. 8). In the shallowest waters, much steeper slopes were observed in three of the four regions, imposing a kink on the overall correlation between cobalt and phosphate (Fig. 8a-d). The slopes were too steep to calculate a meaningful slope due to the small dynamic range of phosphate in the data used to determine these correlations and the severe drawdown of nitrate and nitrite to below detection (Fig. 6c). All slopes reported here were determined by a 2-way linear regression. The average mixed layer depth and the depths at which the most coherent 2-way linear regression was observed in deeper waters were used to determine the different depths of the observed kinks. The steep slopes to the left of the kink in $\mathrm{Co}: \mathrm{PO}_{4}{ }^{3-}$ space were likely the result of a combination of three factors: the abundances of other metals, a shift in 
biological community structure and the associated cellular macro- and micronutrient requirements, and a change in bioavailability of cobalt and other metals (Sunda and Huntsman 1995; Saito et al. 2004; Noble and Saito et al. 2008). In particular, the depletion of zinc and cadmium that occurs in the upper water column would likely increase cobalt utilization by biota. The high Co: $\mathrm{PO}_{4}{ }^{3-}$ ratio found in the shallowest waters of the South Atlantic Subtropical Gyre is consistent with the dominance of zeaxanthin cyanobacterial pigment found in this region (Noble et al. in review.) since marine cyanobacteria have an absolute requirement for cobalt and a low zinc requirement (Saito et al. 2002, 2005). This change in $\mathrm{Co}: \mathrm{PO}_{4}{ }^{3-}$ also coincides with a kink in the $\mathrm{NO}_{3}{ }^{-}+\mathrm{NO}_{2}: \mathrm{PO}_{4}{ }^{3-}$ ratio in the South Atlantic dataset as nitrate becomes depleted (Fig. 6d), which is characteristic of oligotrophic conditions. In comparison to other regions, $\mathrm{Co}: \mathrm{PO}_{4}{ }^{3-}$ correlations have been observed in high nutrient low chlorophyll waters (HNLC, Sunda and Huntsman 1995; Saito et al. 2010), such a relationship was not found in the Sargasso Sea (Saito and Moffett 2002), but a relationship was observed during mesoscale eddies near the Hawaiian Islands (Noble and Saito et al. 2008).

Below these surface waters that display high $\mathrm{Co}: \mathrm{PO}_{4}{ }^{3-}$ ratios, slightly different stoichiometries are observed in each of the four regions. Between $90-900 \mathrm{~m}$ depth in the European N. E. Atlantic, a significant Co: $\mathrm{PO}_{4}{ }^{3-}$ relationship of $44 \pm 2.0 \mu \mathrm{mol} \mathrm{mol}{ }^{-1}\left(r^{2}=\right.$ 0.92) is observed, similar to that observed in the oligotrophic South Atlantic Subtropical Gyre $\left(44 \pm 5.4 \mu \mathrm{mol} \mathrm{mol}^{-1}, r^{2}=0.71\right)$. The two upwelling regions showed linear relationships that displayed steeper slopes (Fig. 8b, d). While the W. African N. E. Atlantic showed evidence of a kink in the $\mathrm{Co}: \mathrm{PO}_{4}{ }^{3-}$ relationship with a deeper $\mathrm{Co}: \mathrm{PO}_{4}{ }^{3-}$ 
relationship of $57 \pm 3.9 \mu \mathrm{mol} \mathrm{mol}^{-1}\left(49-421 \mathrm{~m}, \mathrm{r}^{2}=0.83\right)$, the Angola Gyre was characterized by a more diffuse linear relationship with no observable kink and an aggregate $\mathrm{Co}: \mathrm{PO}_{4}{ }^{3-}$ ratio of $60 \pm 3.5 \mu \mathrm{mol} \mathrm{mol}^{-1}\left(0-400 \mathrm{~m}, \mathrm{r}^{2}=0.84\right.$, Fig. 8d). Upwelling is known to be a source of macronutrients, but is not generally considered to be a strong source of hybrid-type metals, as they have depleted inventories at depth relative to macronutrients. However, near the coast, the confluence of upwelling and mixing along the shelf can allow reduced metals to escape the sediments and be brought to the surface and/or advected offshore (Noble et al. in review). The decoupled sources of cobalt and phosphate obscured the influence of biological processes in the Angola Gyre where upwelling is very strong, but biological influences still appeared to control surface cobalt distributions in the W. African N. E. Atlantic where the upwelling tends to be weaker. These regions compare interestingly to $\mathrm{Co}: \mathrm{PO}_{4}{ }^{3-}$ from two HNLC regions (Fig. 9). Despite the similar cobalt concentrations observed in the Ross Sea, N. E. Pacific, and South Atlantic Subtropical Gyre area, the differences in the phosphate concentrations set each dataset apart. Samples from the Ross Sea fall the furthest to the right due to the high phosphate concentrations found there. There is also no kink observed for either the Ross Sea or the N. E. Pacific. We have previously suggested that this lack of a kink was due to an inadequate supply of cobalt beneath the euphotic zone to allow for excess uptake which would presumably increase the slope of the $\mathrm{Co}: \mathrm{PO}_{4}{ }^{3-}$ relationship (Saito et al. 2010). In this case, there also may be a relationship with nitrate as the kink observed here appears to be related to extreme depletion of nitrate, where this is not typically observed in the Ross Sea. 
5.3.4 Complexation and Speciation - In addition to the correlation between total cobalt and phosphate, some stations also showed a linear relationship between labile cobalt and phosphate (Fig. 7, Table 4). Labile cobalt is defined as the fraction of the total cobalt that is either present as the free ion in seawater or is bound to weak ligands (Saito et al. 2004). The labile Co: $\mathrm{PO}_{4}{ }^{3-}$ slopes for the South and North Atlantic basins were much shallower than that of the total cobalt (both $27 \mu \mathrm{mol} \mathrm{mol}^{-1}$, Table 4), suggesting that either a large percentage of remineralized cobalt is released in a strongly complexed form, or that it is organically bound rapidly after remineralization. Recent studies have demonstrated that bacterial remineralization can release ligand bound iron, and this may also be the case for cobalt (Boyd et al. 2010). We have previously suggested that the organic ligands that bind cobalt in seawater are a partially degraded form of vitamin $B_{12}$ (Saito et al. 2010). $B_{12}$ is an important vitamin that contains cobalt at its metal center and is required by many organisms, but only produced by some bacteria and archaea. Although $\mathrm{B}_{12}$ concentrations in seawater are in the femtomolar range (Bertrand et al. 2007, Panzeca et al. 2006, Menzel 1962), photodegraded $B_{12}$ has been observed to bind cobalt at conditional stability constants in excess of $10^{16.8}$ in seawater, even after 20 hours of exposure to sunlight (see Appendix III). Previous work has demonstrated that cyanobacteria are capable of producing and taking up the ligand-bound fraction of cobalt (Saito et al. 2004, 2005). This implicates cyanobacteria as a link to understanding the speciation of cobalt in the oceans.

Chemical speciation also appeared to be related to the observation of a kink in the $\mathrm{Co}: \mathrm{PO}_{4}{ }^{3-}$ relationship. This is best illustrated in some example profiles for upper $200 \mathrm{~m}$ of the NAGZT cruise (Fig. 10). There was a noticeable offset in the depth of the 
"phosphocline" and the "cobalt-cline" at some stations. At Sta. 3, the phosphocline is coincident with the bottom of the chlorophyll maximum (99 m), whereas the cobalt-cline coincides with the mixed layer depth (77 m, Fig. 10). This initially suggests that cobalt concentrations were regulated by physical mixing. However, when the phosphate profiles were compared to the labile profiles, it appeared that mixing was not the sole regulating process. Similarly to phosphate, labile cobalt was drawn down below the mixed layer to the bottom of the chlorophyll maximum and biological uptake and complexation appeared to control cobalt speciation on a timescale faster than mixing and diffusion. It has recently been discovered that cyanobacteria are capable of operating under non-Redfieldian N:P stoichiometries by partially substituting sulfolipids for phospholipids, thereby lowering their phosphate requirement (Van Mooy et al. 2006). A reduced phosphate requirement could potentially contribute to the observations of higher $\mathrm{Co}: \mathrm{PO}_{4}{ }^{3-}$ relationships, and efficient recycling in the upper water column might concentrate cobalt in the surface mixed layer. While these relationships are tantalizing, careful biochemical studies are required to truly constrain the processes that influence these distributions in a meaningful way.

\subsubsection{Scavenging and deepwater regional trends - In all 4 regions, removal of} cobalt relative to phosphate was observed in deeper waters (Fig. 11). The depth at which scavenging becomes apparent was different among the regions sampled, beginning at 200 $\mathrm{m}$ in the South Atlantic Subtropical Gyre, $400 \mathrm{~m}$ in the Angola Gyre, $421 \mathrm{~m}$ in the W. African N. E. Atlantic, and $900 \mathrm{~m}$ in the European N. E. Atlantic (Fig. 11). The shape of the scavenging behavior is referred to as a cobalt "curl" in the $\mathrm{Co}: \mathrm{PO}_{4}{ }^{3-}$ plots where cobalt concentrations change significantly without a simultaneous change in phosphate due to 
scavenging. The depth at which the 2-way linear regression relationship between cobalt and phosphate began to deteriorate was taken as the depth that defined the onset of cobalt scavenging. The shapes of the cobalt curls were similar within the two low-oxygen, zonal upwelling regions (Fig. 12b). Scavenging in the Pacific has also been observed, and was apparent at very shallow depths (80-100 m, Martin et al. 1989), with a curl that was similar in shape to that of the European N. E. Atlantic (Fig. 12a). It is possible that changes in productivity may influence scavenging rates between the northern and southern stations of the North Atlantic. In general, the North Atlantic is characterized by nitrogen and phosphorus limitation, but a clear increase in productivity can be seen in satellite images on the Ocean Color website of the Mauritanian coast (http://oceancolor.gsfc.nasa.gov). In a global circulation model for iron cycling in the ocean, the scavenging rates were increased around the Saharan dust plume in order to match the observed trends (Moore et al. 2004). This suggests that an increase in sinking particulate material could increase the scavenging of iron. It is possible that this may also affect cobalt scavenging and could be used to explain the large difference in the apparent depth of scavenging between the European N. E. Atlantic and the W. African N. E. Atlantic, however; it may not be solely due to the total particulate load, but rather a combination of both higher biogenic and lithogenic fractions. In the North East Atlantic, the apparent onset of cobalt scavenging was coincident with a notable decrease in both the absolute and relative amount of particulate organic material relative to the lithogenic fraction (P. Lam personal communication). Increased productivity likely increases the amount of cobalt released with remineralization. Below the depth of remineralization, where the organic fraction 
decreases, if there are high concentrations of lithogenic particles remaining, this may have a strong influence on the irreversible scavenging of that remineralized cobalt. This suggests that it may not be the total particulate load that determines the depth at which cobalt scavenging occurs, but rather the confluence of the magnitude of the particle load with a shift in the composition of particles from organic to lithogenic-dominated particles. In the W. African N. E. Atlantic, lithogenic particles could be derived from aeolian input but also from advection from the coast.

Other potential influences on scavenging are oxygen and mixing. In addition to the difference in dust input between the European and W. African N. E. Atlantic, clear differences in subsurface water masses present were observed in a temperature salinity plot (Fig. 13a). Antarctic Intermediate Water (AAIW) at $\sim 800 \mathrm{~m}$ and Mediterranean Outflow Water (MOW) at 650-1200 m were well-defined, and below these water masses, two distinct deep water scavenging "curls" were observed in $\mathrm{Co}: \mathrm{PO}_{4}{ }^{3-}$ space. AAIW carries high concentrations of phosphate due to its origin in HNLC waters of the Antarctic, while MOW carries much lower concentrations of phosphate due to the hyper-oligotrophic conditions in the Mediterranean. The Co: $\mathrm{PO}_{4}{ }^{3-}$ ratios of AAIW were very different from the shallower North Atlantic Central Water (NACW) Co: $\mathrm{PO}_{4}{ }^{3-}$, which may indicate limited interaction between AAIW and the waters above, which is consistent with water traveling along, instead of across isopycnals. Perhaps the much tighter relationship between the $\mathrm{Co}: \mathrm{PO}_{4}{ }^{3-}$ character of MOW and NACW reflects stronger mixing due to the shallower intrusion and larger depth range that MOW occupies relative to AAIW when it enters the Atlantic basin. It could also be related to the proximity of the sources of these two water 
masses since AAIW originates in a very different nutrient regime than either NACW or MOW. At depth, all stations converged upon a similar concentration of phosphate, perhaps unsurprisingly given the timescale of North Atlantic Deep Water (NADW) circulation relative to the proximity of these stations to each other. Cobalt concentrations also converged upon a uniform concentration at depth, and the influence of scavenging can clearly be seen in the consistency of the phosphate concentrations with continual removal of cobalt with depth. In a comparison of the deepwater in the upwelling regions of the North and South Atlantic, very slight evidence of phosphate accumulation with thermohaline circulation was evident (Fig. 12b).

With respect to oxygen, strong linear relationships were observed for both total dissolved and labile cobalt when compared to oxygen (Fig. 2) and the slopes of these relationships are reported in Table 5. It is interesting that while the oxygen concentrations appeared to be lowest in the South Atlantic, the cobalt concentrations were higher in the North Atlantic. As mentioned earlier, this was likely due to the additional input from aeolian sources in the North Atlantic.

5.4 Conclusion - The differences in observed $\mathrm{Co}: \mathrm{PO}_{4}{ }^{3-}$ relationships between these regions implies that a wide variety of cobalt utilization regimes exist. The processes of uptake and remineralization exerted control on cobalt in the oligotrophic surface waters of the South Atlantic Subtropical Gyre and the European N. E. Atlantic. In the South Atlantic Subtropical Gyre and both regions in the N. E. Atlantic, cobalt displayed two distinct $\mathrm{Co}: \mathrm{PO}_{4}{ }^{3-}$ ratios in the upper water column. This was demonstrated by tight correlations with phosphate observed in subsurface waters there, and the strong drawdown of 
phosphate, nitrate, and total dissolved and labile cobalt. When geochemical metal inputs and physical processes imposed a strong influence, these tight correlations became more diffuse, blurring the biological processes that operate to couple these species. These processes obscured the biological influences on cobalt cycling in the Angola Gyre, but were matched by strong biological demand in the W. African N.E. Atlantic. As more studies collect dissolved cobalt data from around the world during the GEOTRACES program, the table of stoichiometries will continue to grow. Combining these datasets with future biochemical studies will improve our understanding of the influence of cobalt biogeochemical cycling and its interaction with ocean marine ecology. 


\section{References for Chapter 5:}

Bertrand, E. M. and others 2007. Vitamin $\mathrm{B}_{12}$ and iron co-limitation of phytoplankton growth in the Ross Sea. Limnol. Oceanogr. 52: 1079-1093.

Bown, J., M. Boye, A. Baker, E. Duvieilbourg, F. Lacan, F. Le Moigne, F. Planchon, S. Speich, D. M. Nelson. The biogeochemical cycle of dissolved cobalt in the Atlantic and Southern Ocean south off the coast of Africa. Marine Chemistry. In press.

Boyd, P.W., E. Ibisanmi, S.G. Sander, K. A. Hunter, G. A. Jackson, 2010. Remineralization of upper ocean particles: Implications for iron biogeochemistry. Limnol. and Oceanogr., 55 (3), 1271-1288.

Cullen, J. T. 2006. On the Nonlinear Relationship between Dissolved Cadmium and Phosphate in the Modern Global Ocean: Could Chronic Iron Limitation of Phytoplankton Growth Cause the Kink? Limnol. Oceanogr. 51: 1369-1380.

Erel, Y, U. Dayan, R. Rabi, Y. Rudich, M. Stein. 2006. Trans Boundary Transport of Pollutants by Atmospheric Mineral Dust. Environ. Sci. Technol. 40 2996-3005.

Erel, Y, B. Kalderon-Asael, U. Dayan, A. Sandler. 2007. European Atmospheric Pollution Imported by Cooler Air Masses to the Eastern Mediterranean during the Summer. Environ. Sci. Technol. 41, 5198-5203.

Martin, J. H., R. M. Gordon, S. Fitzwater, and W. W. Broenkow. 1989. VERTEX: phytoplankton/iron studies in the Gulf of Alaska. Deep-Sea Res. 36: 649-680.

Measures, C. I.. 1995. The distributions of Al in the IOC stations of the eastern Atlantic between $30^{\circ} \mathrm{S}$ and $34^{\circ} \mathrm{N}$. Mar. Chem 49: 267-281.

Measures, C.I., and S. Vink. 2000. On the use of dissolved aluminium in surface waters to estimate dust deposition to the ocean, Global Biogeochemical Cycles, 14, 317-327.

Measures, C. I., W. M. Landing, M. T. Brown, and C. S. Buck. 2008. High-resolution Al and Fe data from the Atlantic Ocean CLIVAR-CO2 Repeat Hydrography A16N transect: Extensive linkages between atmospheric dust and upper ocean geochemistry. Global Biogeochem. Cycles 22: GB1005, doi: $10.1029 / 2007 \mathrm{gb} 003042$

Menzel, D. W., and J. P. Spaeth. 1962. Occurrence of vitamin $B_{12}$ in the Sargasso Sea. Limnol. Oceanogr. 7: 151-154.

Moore, J. K., S. C. Doney, and K. Lindsay. 2004. Upper ocean ecosystem dynamics and iron cycling in a global three-dimensional model. Global Biogeochem. Cycles 18: GB4028. doi:4010.1029/2004GB002220. .

Noble, A. E., M. A. Saito, K. Maiti, and C. Benitez-Nelson. 2008. Cobalt, manganese, and iron near the Hawaiian Islands: A potential concentrating mechanism for cobalt within a cyclonic eddy and implications for the hybrid-type trace metals. Deep-Sea Res. II 55: 1473-1490.

Panzeca, C., A. J. Beck, K. Leblanc, G. T. Taylor, D. A. Hutchins, and S. A. SanudoWilhelmy. 2006. Potential cobalt limitation of vitamin B12 synthesis in the North Atlantic Ocean. Global Biogeochem. Cycles 22.

Saito, M. A., and J. W. Moffett. 2001. Complexation of cobalt by natural organic ligands in the Sargasso Sea as determined by a new high-sensitivity electrochemical cobalt speciation method suitable for open ocean work. Mar. Chem. 75: 49-68. 
Saito, M. A., and J. W. Moffett. 2002. Temporal and spatial variability of cobalt in the Atlantic Ocean. Geochim. Cosmochim. Acta 66: 1943-1953.

Saito, M. A., J. W. Moffett, S. W. Chisholm, and J. B. Waterbury. 2002. Cobalt limitation and uptake in Prochlorococcus. Limnol. Oceanogr. 47: 1629-1636.

Saito, M. A., J. W. Moffett, and G. Ditullio. 2004. Cobalt and Nickel in the Peru Upwelling Region: a Major Flux of Cobalt Utilized as a Micronutrient. Global Biogeochem. Cycles 18: GB4030, doi:10.1029/2003GB002216

Saito, M. A., G. Rocap, and J. W. Moffett. 2005. Production of cobalt binding ligands in a Synechococcus feature at the Costa Rica Upwelling Dome. Limnol. Oceanogr. 50: 279-290.

Saito, M. A., T. J. Goepfert, A. E. Noble, E. M. Bertrand, P. N. Sedwick, and G. R. Ditullio. 2010. A seasonal study of dissolved cobalt in the Ross Sea, Antarctica: micronutrient behavior, absence of scavenging, and relationships with $\mathrm{Zn}, \mathrm{Cd}$, and P. Biogeosciences 7: 4059-4082.

Schlitzer, R., Ocean Data View, http://odv.awi.de, 2009.

Sterner, R. W., and J. J. Elser. 2002. Ecological Stoichiometry: The Biology of Elements from Molecules to the Biosphere. Princeton University Press.

Sunda, W., and S. A. Huntsman. 1995. Cobalt and zinc interreplacement in marine phytoplankton: Biological and geochemical implications. Limnol. Oceanogr. 40: 1404-1417.

Sunda, W. G., and S. A. Huntsman. 2000. Effect of Zn, Mn, and Fe on Cd accumulation in phytoplankton: Implications for oceanic Cd cycling. Limnol. Oceanogr. 45: 15011516.

Taylor, S. R., and S. M. McLennan. 1985. The Continental Crust: its Composition and Evolution. Blackwell Scientific Publications.

Wu, J., W. Sunda, E. A. Boyle, D. M. Karl. 2000. Phosphate depletion in the Western North Atlantic Ocean. Science. Vol 289, 759-762.

Van Mooy, B. A. S., G. Rocap, H. F. Fredricks, C. T. Evans, and A. H. Devol. 2006. Sulfolipids dramatically decrease phosphorus demand by picocyanobacteria in oligotrophic marine environments. Proceedings of the National Academy of Sciences 103: 8607-8612. 
172 


\section{Tables and Figures for Chapter 5:}

Table 1. Blanks determined and applied for each electrode during the NAGZT expedition.

Total dissolved cobalt electrode

\begin{tabular}{cccc}
\hline $\begin{array}{c}\text { Analysis } \\
\text { Date }\end{array}$ & $\begin{array}{c}\text { Reagent } \\
\text { batch }\end{array}$ & Blank [pM] & $\begin{array}{c}\text { Blank } \\
\text { applied }\end{array}$ \\
\hline 10.18 .10 & A & 4.3 & 3.3 \\
10.18 .10 & A & 3.5 & \\
10.23 .10 & A & 2.2 & \\
10.21 .10 & B & 2 & 2.4 \\
10.21 .10 & B & 1.6 & \\
10.27 .10 & B & 3.5 & 3.1 \\
10.25 .10 & C & 3.5 & \\
10.27 .10 & $\mathrm{C}$ & 2.7 & 2.4 \\
11.4 .10 & D & 3.2 & \\
10.27 .10 & D & 2.1 & \\
10.29 .10 & D & 1.8 & \\
10.31 .10 & E & 1.9 & 2.4 \\
11.4 .10 & E & 2.9 & \\
\hline \multicolumn{5}{c}{ Average $=$} & 2.7 \\
Stdev $=$ & 0.84 \\
n $=$ & 13
\end{tabular}

Labile cobalt electrode

\begin{tabular}{cccc}
\hline $\begin{array}{c}\text { Analysis } \\
\text { Date }\end{array}$ & $\begin{array}{c}\text { Reagent } \\
\text { Batch }\end{array}$ & Blank [pM] & $\begin{array}{c}\text { Blank } \\
\text { applied }\end{array}$ \\
\hline 10.18 .10 & A & 3.5 & 3.5 \\
10.18 .10 & A & 3.3 & \\
10.23 .10 & A & 3.6 & 5.9 \\
10.21 .10 & B & 5.9 & \\
10.21 .10 & B & 5.7 & \\
10.27 .10 & B & 6.1 & 4.2 \\
10.25 .10 & C & 4.2 & \\
10.27 .10 & C & 4.2 & 3.5 \\
11.4 .10 & D & 3.9 & \\
10.27 .10 & D & 3.6 & \\
10.29 .10 & D & 3.1 & \\
10.31 .10 & E & 2 & \\
11.4 .10 & E & 2.5 & \\
\hline \multicolumn{5}{r}{ Average $=$} & 4.0 \\
Stdev $=$ & 1.26 \\
n $=$ & 13
\end{tabular}


Table 2. Standards run during the NAGZT expedition. CSW is our internal lab standard seawater which has been UV irradiated and is kept in the dark. GD is the deep GEOTRACES standard, GS is the shallow standard. "-s" denotes a labile measurement in the sense that the samples were equilibrated overnight with DMG and not UV-irradiated. As the GS sample was acidified and not refrigerated, this measurement was for investigative / intercalibration purposes only and does not represent a true labile value. 
Total dissolved cobalt electrode

\begin{tabular}{|c|c|c|c|}
\hline Sample ID & $\begin{array}{c}\text { Analaysis } \\
\text { Date }\end{array}$ & $\begin{array}{l}\text { Reagent } \\
\text { Batch }\end{array}$ & Co $[\mathrm{pM}]$ \\
\hline \multirow[t]{15}{*}{ CSW } & 10.17 .10 & $\mathrm{~A}$ & 51 \\
\hline & 10.17 .10 & A & 53 \\
\hline & 10.18 .10 & A & 53 \\
\hline & 10.18 .10 & A & 54 \\
\hline & 10.18 .10 & A & 49 \\
\hline & 10.21 .10 & B & 51 \\
\hline & 10.25 .10 & $\mathrm{C}$ & 50 \\
\hline & 10.25 .10 & $\mathrm{C}$ & 49 \\
\hline & 10.25 .10 & $\mathrm{C}$ & 53 \\
\hline & 10.26 .10 & $\mathrm{C}$ & 51 \\
\hline & 10.26 .10 & $\mathrm{C}$ & 46 \\
\hline & 10.31 .10 & $\mathrm{D}$ & 45 \\
\hline & 11.4 .10 & $\mathrm{E}$ & 50 \\
\hline & & Average $=$ & 50 \\
\hline & & Stdev $=$ & 2.7 \\
\hline \multirow[t]{8}{*}{ GD-20 } & 10.17 & A & 74 \\
\hline & 10.18 & A & 73 \\
\hline & 10.25 & $\mathrm{C}$ & 72 \\
\hline & 10.25 & $\mathrm{C}$ & 68 \\
\hline & 10.31 & $\mathrm{D}$ & 69 \\
\hline & 11.4 & $\mathrm{D}$ & 60 \\
\hline & & Average $=$ & 69 \\
\hline & & Stdev $=$ & 5.1 \\
\hline \multirow[t]{8}{*}{ GS-28 } & 10.17 & $\mathrm{~A}$ & 32 \\
\hline & 10.18 & A & 30 \\
\hline & 10.25 & $\mathrm{C}$ & 35 \\
\hline & 10.25 & $\mathrm{C}$ & 30 \\
\hline & 10.31 & $\mathrm{D}$ & 28 \\
\hline & 11.4 & $\mathrm{D}$ & 26 \\
\hline & & Average $=$ & 30 \\
\hline & & Stdev $=$ & 3.1 \\
\hline
\end{tabular}

*These results were done during efforts to trouble shoot. They have been included to highlight the value of an internal standard in helping identify instrumental issues and address them quickly.
Labile cobalt electrode

\begin{tabular}{cccc}
\hline Sample ID & $\begin{array}{c}\text { Analaysis } \\
\text { Date }\end{array}$ & $\begin{array}{c}\text { Reagent } \\
\text { Batch }\end{array}$ & Co [pM] \\
\hline CSW & 10.18 .10 & A & 58 \\
& 10.18 .10 & A & 55 \\
10.18 .10 & A & 55 \\
10.18 .10 & A & 53 \\
10.21 .10 & B & $81^{*}$ \\
10.21 .10 & B & $74^{*}$ \\
10.21 .10 & B & 49 \\
10.25 .10 & C & 49 \\
& 11.4 .10 & D & 46 \\
\cline { 2 - 4 } & \multicolumn{3}{c}{ Average $=$} \\
& Stdev $=$ & 52 \\
& &
\end{tabular}

\begin{tabular}{|c|c|c|c|}
\hline \multirow[t]{6}{*}{ GD-20 } & 10.18 .10 & $\mathrm{~A}$ & 77 \\
\hline & 10.18 .10 & A & 68 \\
\hline & 10.26 .10 & $\mathrm{D}$ & 68 \\
\hline & 10.26 .10 & D & 65 \\
\hline & & Average $=$ & 70 \\
\hline & & Stdev $=$ & 5.2 \\
\hline \multirow[t]{4}{*}{ GS-28 } & 10.18 .20 & $\mathrm{~A}$ & 35 \\
\hline & 10.18 .20 & $\mathrm{~A}$ & 33 \\
\hline & & Average $=$ & 34 \\
\hline & & Stdev $=$ & 1.4 \\
\hline \multirow[t]{6}{*}{ CRUD-s } & 10.18 .20 & $\bar{A}$ & 8 \\
\hline & 10.19 .20 & A & 10 \\
\hline & 10.25 .20 & $\mathrm{C}$ & 7 \\
\hline & 11.4 .20 & $\mathrm{D}$ & 7 \\
\hline & & Average $=$ & 8 \\
\hline & & Stdev $=$ & 1.4 \\
\hline \multirow[t]{5}{*}{ GS-s } & 10.25 .20 & $\bar{C}$ & 19 \\
\hline & 11.1.20 & $\mathrm{E}$ & 13 \\
\hline & 11.4 .20 & D & 17 \\
\hline & & Average $=$ & 16 \\
\hline & & Stdev $=$ & 3.1 \\
\hline \multirow[t]{5}{*}{ RS-s } & 10.25 .20 & $\mathrm{C}$ & 15 \\
\hline & 11.4 .20 & $\mathrm{D}$ & 14 \\
\hline & 10.18 .20 & $\mathrm{~A}$ & 19 \\
\hline & & Average $=$ & 16 \\
\hline & & Stdev $=$ & 2.6 \\
\hline
\end{tabular}


Table 3. Comparison of the character of the basins sampled by the CoFeMUG (South Atlantic basin) and NAGZT (N. E. Atlantic basin) expeditions.

South Atlantic basin

N. E. Atlantic Basin

\begin{tabular}{cc}
\hline Strong wind-driven upwelling & Weaker wind-driven upwelling \\
Slow ventilation rates & Faster ventilation rates \\
Oxygen minimum zone & Oxygen deficient zone \\
Low rates of aeolian deposition & High rates of aeolian deposition \\
Large cobalt plume observed & Large cobalt plume observed
\end{tabular}


Table 4. Compilation of $\mathrm{Co}: \mathrm{PO}_{4}{ }^{3-}$ and $\mathrm{Co}: \mathrm{N}$ ratios from the literature and from this study. Ecological stoichiometries range over an order of magnitude, suggesting real variability in cobalt utilization in the ocean. All data are total cobalt unless otherwise noted. The depth ranges reported for this study refer to the exact depth ranges based on sample bottle depths, and the values reported for this study were determined by applying a two-way linear regression least squares fit in MATLAB using the script lsqfitma.m. 


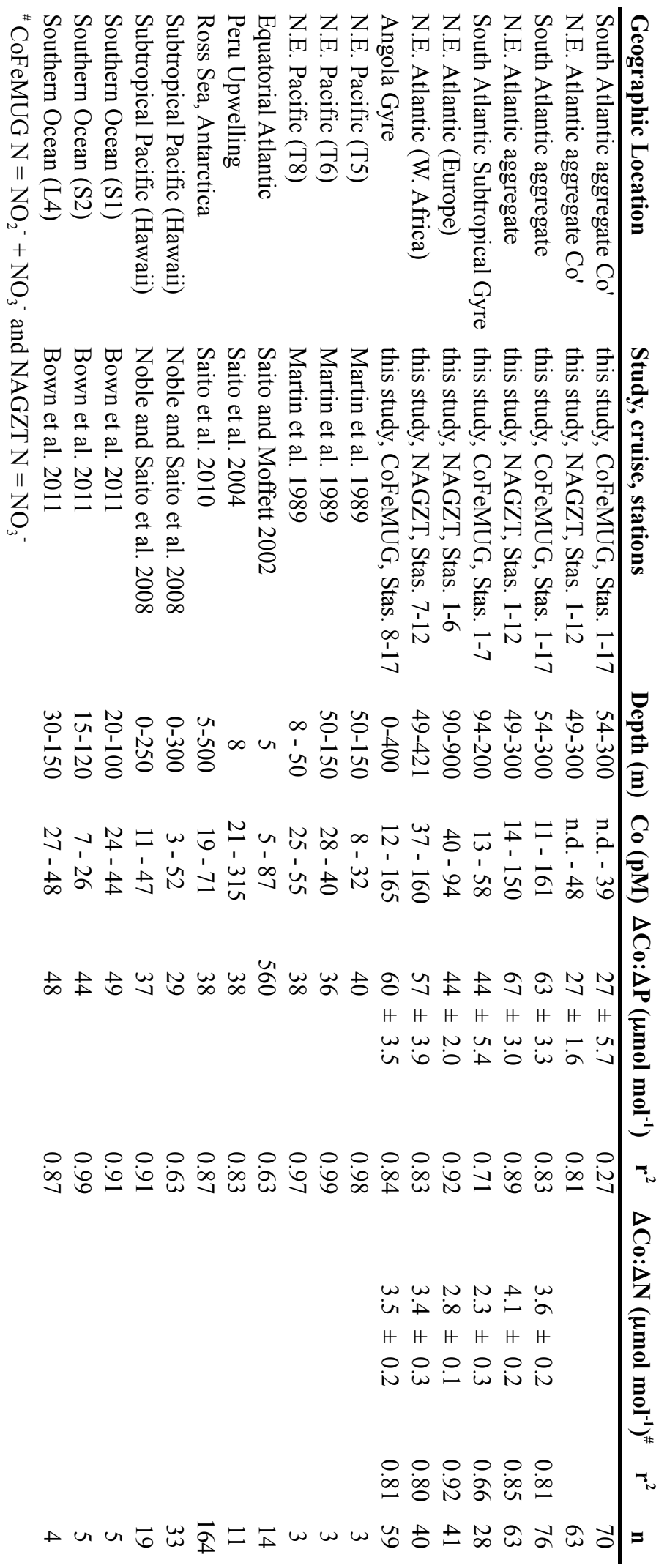


Table 5. Regional slopes for the relationship between cobalt and oxygen, which shows covariance between 50 and $800 \mathrm{~m}$ for both total dissolved and labile cobalt in the N. and $\mathrm{S}$. Atlantic basins.

\begin{tabular}{lcc} 
Geographic Location & $\boldsymbol{\Delta} \mathbf{C o :} \Delta \mathbf{O}_{\mathbf{2}}\left(\boldsymbol{\mu} \mathbf{m o l ~} \mathbf{~ m o l}^{-\mathbf{1}}\right)$ & $\mathbf{r}^{\mathbf{2}}$ \\
\hline N.E. Atlantic TCo & -0.51 & 0.87 \\
N.E. Atlantic LCo & -0.17 & 0.70 \\
South Atlantic TCo & -0.58 & 0.82 \\
South Atlantic LCo & -0.09 & 0.24
\end{tabular}




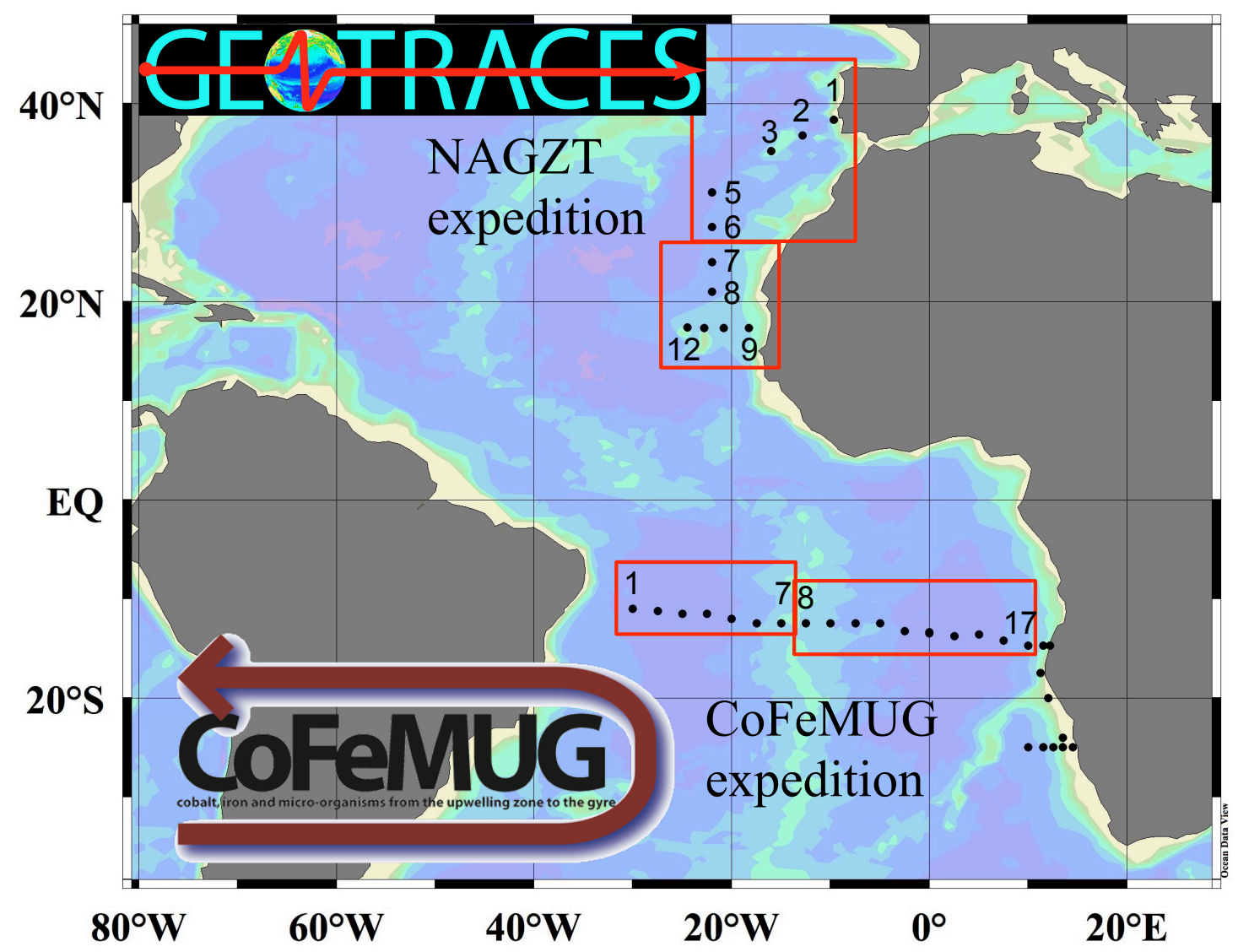

Figure 1) Map of cruise tracks for the CoFeMUG (cobalt iron and microorganisms from the upwelling to the gyre) and NAGZT (North Atlantic GEOTRACES zonal transect) expeditions. Station numbers are labeled with red boxes outlining the different regions discussed in the text. 

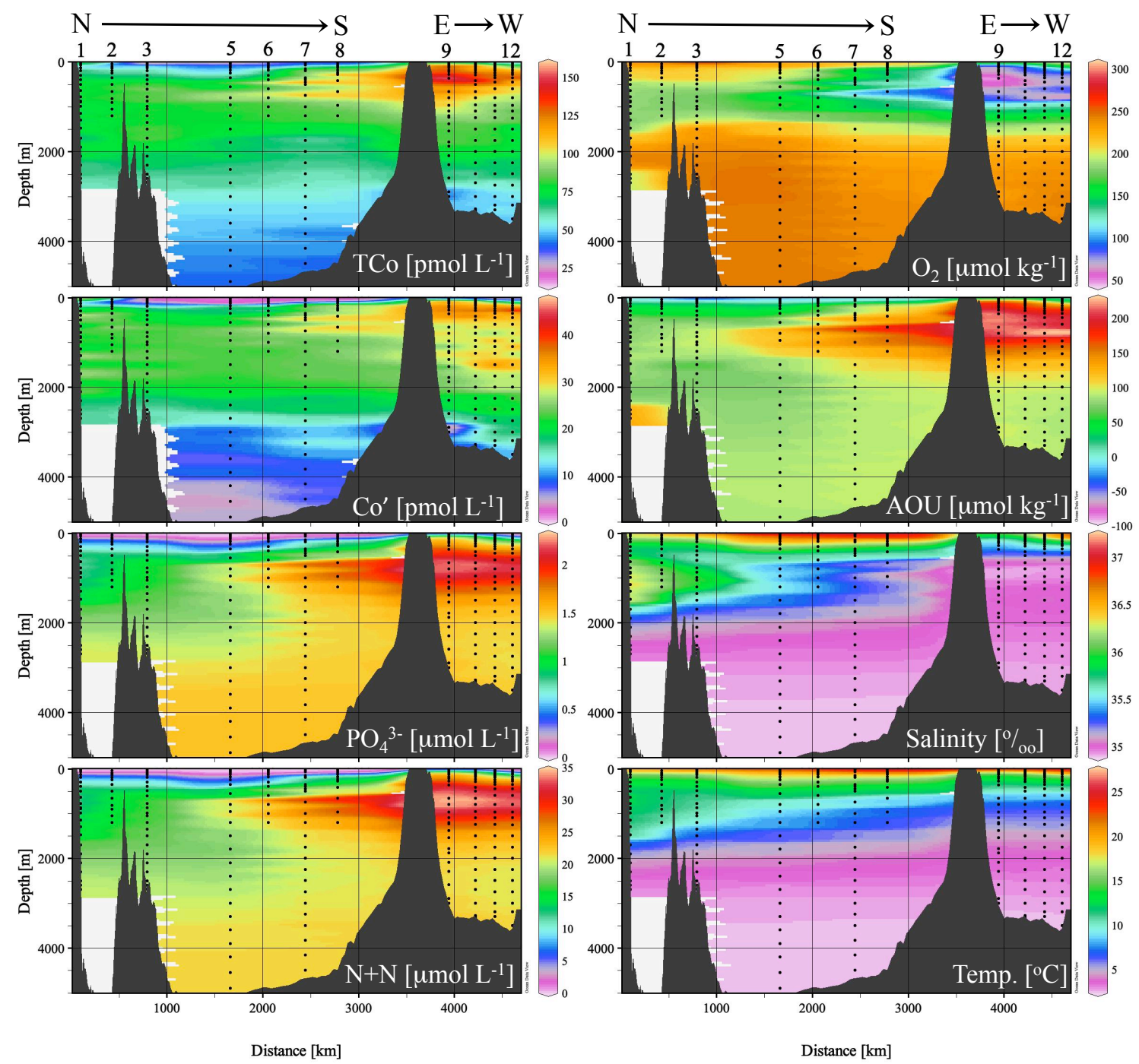

Figure 2) Ocean sections of cobalt, macronutrients, and hydrographic data. A large plume of cobalt emanates from the West African coast, coincident with low oxygen concentrations and high macronutrients. Mediterranean outflow water is characterized in Sta. 1 by salinities less than $36 \%$ between 500-1200 m. Note the directional arrows at the top of the figure to orient with the cruise map. Figures were created using Ocean Data View (Schlitzer 2009). 
Figure 3) Zoom in on the upper $1500 \mathrm{~m}$ of both the NAGZT and CoFeMUG cruises to highlight the negative correlation between oxygen concentration and total cobalt concentration. The scatter plots below demonstrate the vertical shift in the linear relationship, suggesting that there may be an additional input to the North Atlantic that is not experienced in the South Atlantic (i.e. aeolian deposition). The linear regression data are presented in Table 5. 

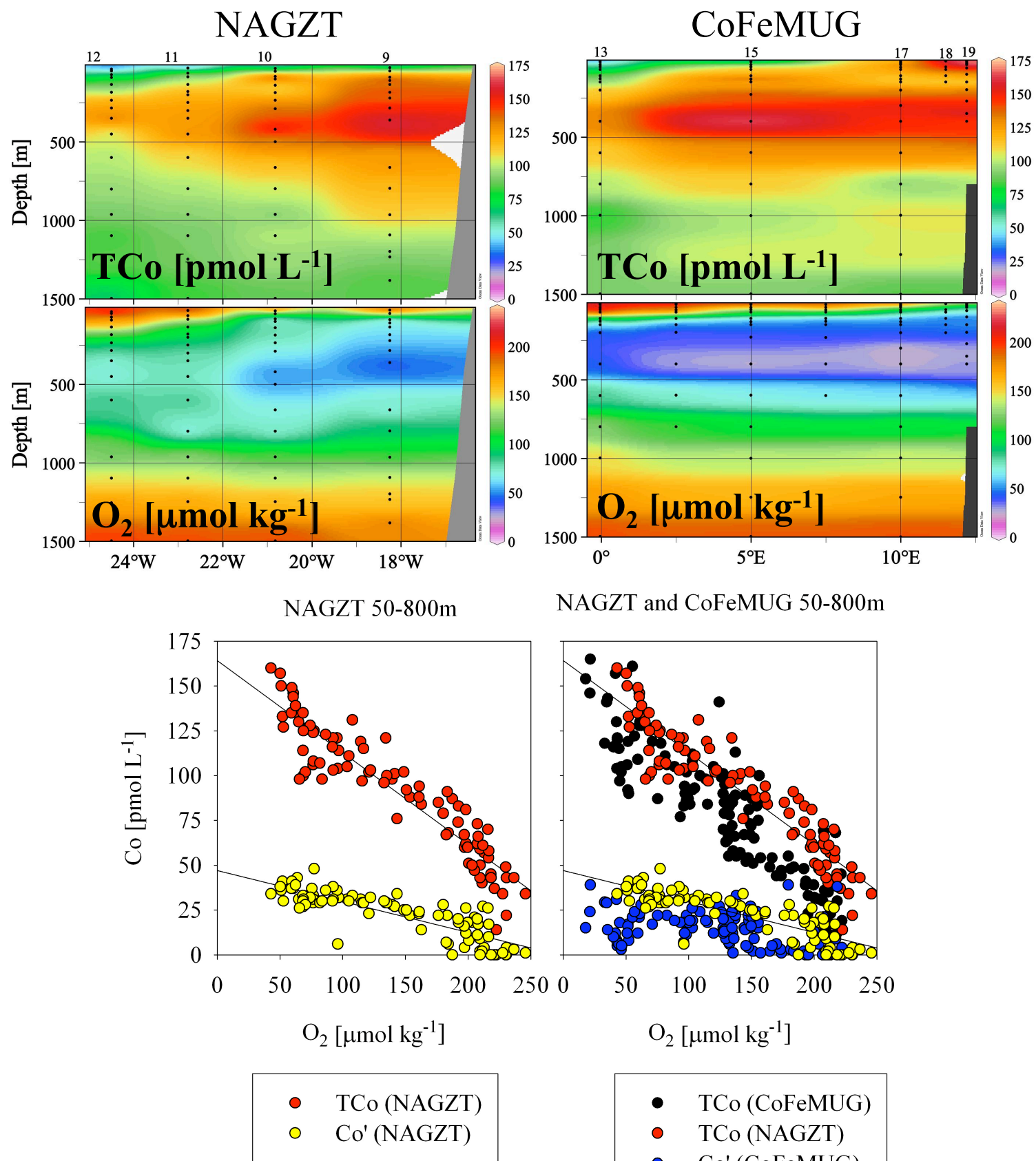

- $\mathrm{TCo}$ (CoFeMUG)

- TCo (NAGZT)

- $\mathrm{Co}^{\prime}(\mathrm{CoFeMUG})$

- Co' (NAGZT) 
Figure 4) Profiles of total and labile cobalt for the NAGZT cruise. The surface waters are characterized by depletion due to biological uptake, with a subsurface maximum due to remineralization and inputs from advection of reduced cobalt from the coast. The top row of stations comprises the European N. E. Atlantic region, while the bottom row of stations makes up the data for the West African N. E. Atlantic region. The low values for both total and labile cobalt in the deepest samples at Sta. 9 coincide with low $230^{\mathrm{Th}}$ concentrations, indicative of sampling within the nepheloid layer (R. Anderson, E. Boyle personal communication). This suggests that these low values may be due to scavenging of cobalt onto particles. 


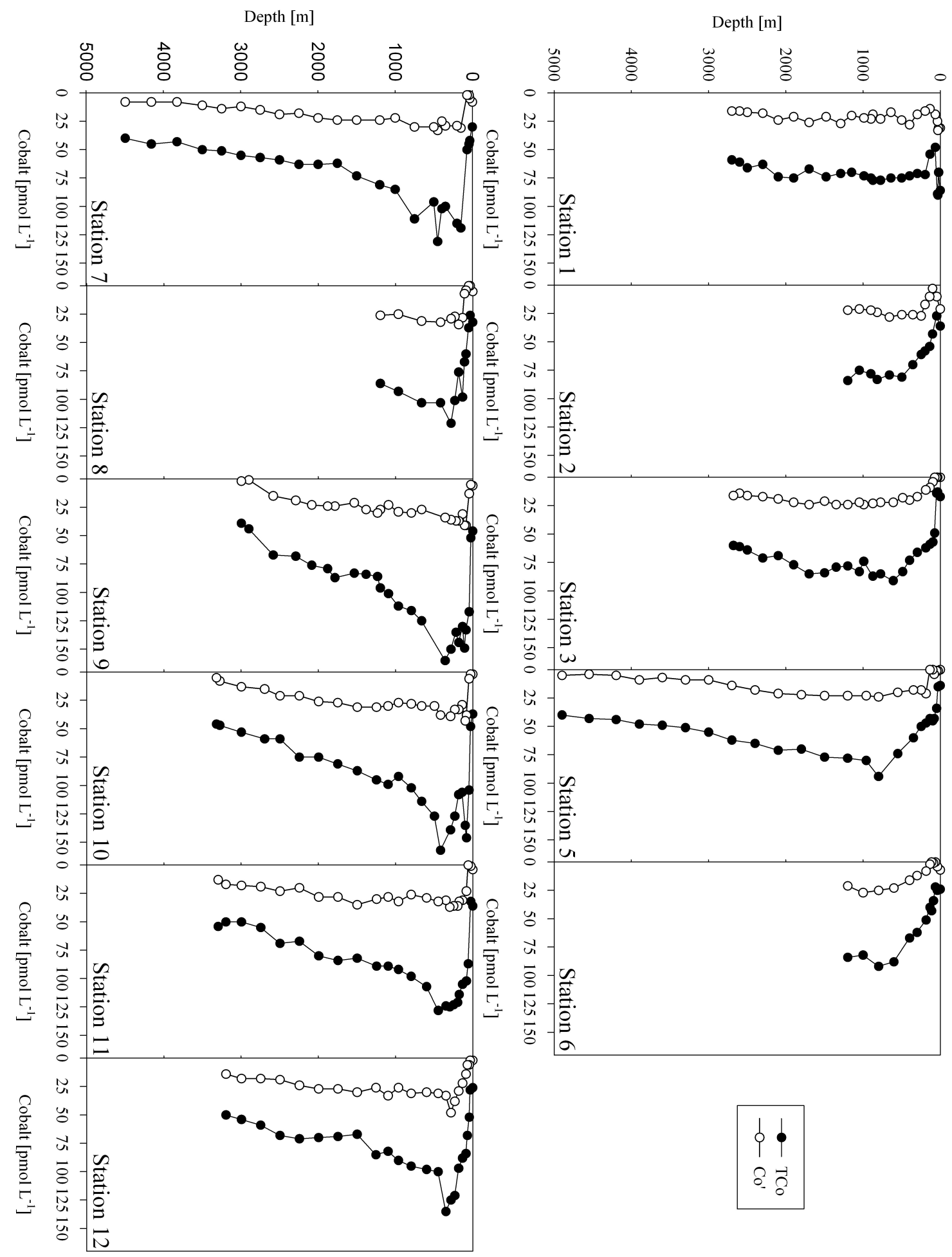



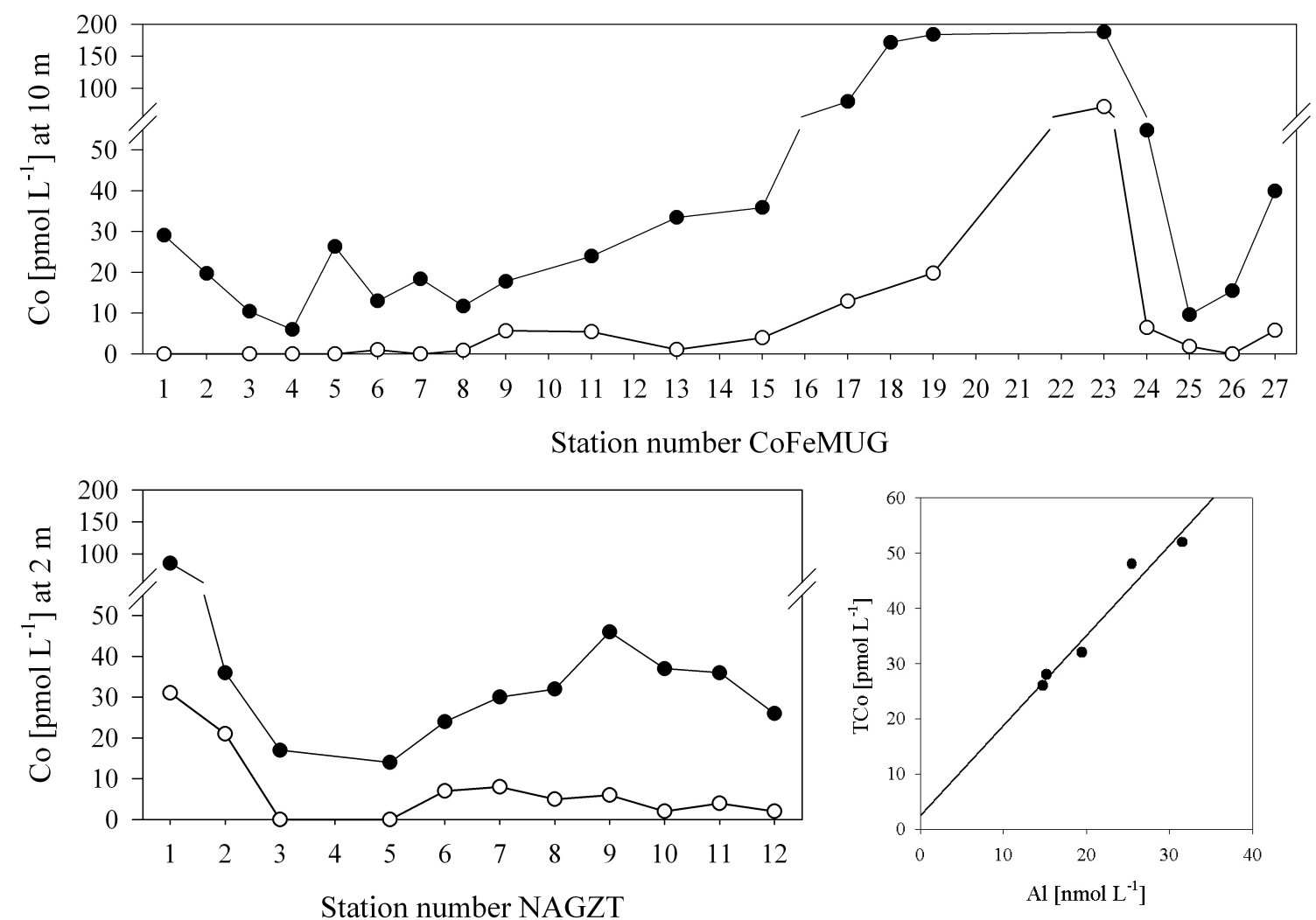

Figure 5) Surface transects of total (filled circles) and labile (open circles) cobalt from the CoFeMUG and NAGZT expeditions. The scatter plot to the right displays a linear relationship between dissolved aluminum and total dissolved cobalt in surface waters between Sta. 8-12 of the NAGZT cruise. This suggests that there is an aeolian component to the sources of cobalt to the North Atlantic. North of these stations, the relationship was not observed, perhaps due to biological drawdown at Stas. 3-7, and a stronger influence of anthropogenic aerosols at Stas. 1 and 2 (A. Aguilar-Islas and R. Shelley personal communication). 
Figure 6) Aggregate basin ecological stoichiometries of the North (red) and South (black) Atlantic. The slopes of the linear regressions are reported in Table 4 and reflect the cumulative uptake and remineralization of the resident biota. Interestingly, there appear to be two general slopes, indicated in panel b), and in the surface waters (filled circles), the basin differences in phosphate inventory are apparent (horizontal arrow) which is more easily observed when cobalt and nitrate are compared and the offset is not observed in panel c). Aeolian input may also be detectable by the slight vertical shift in the North Atlantic dataset relative to the South Atlantic dataset (vertical arrow). 


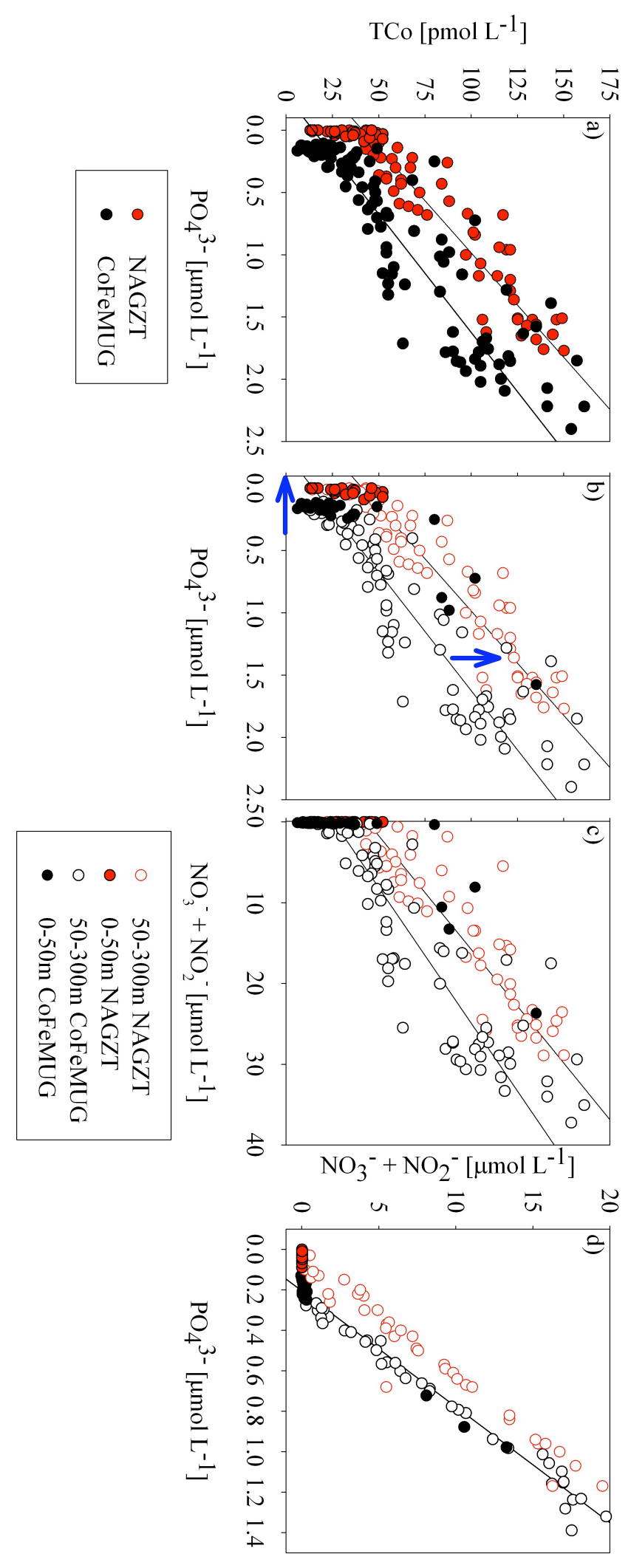



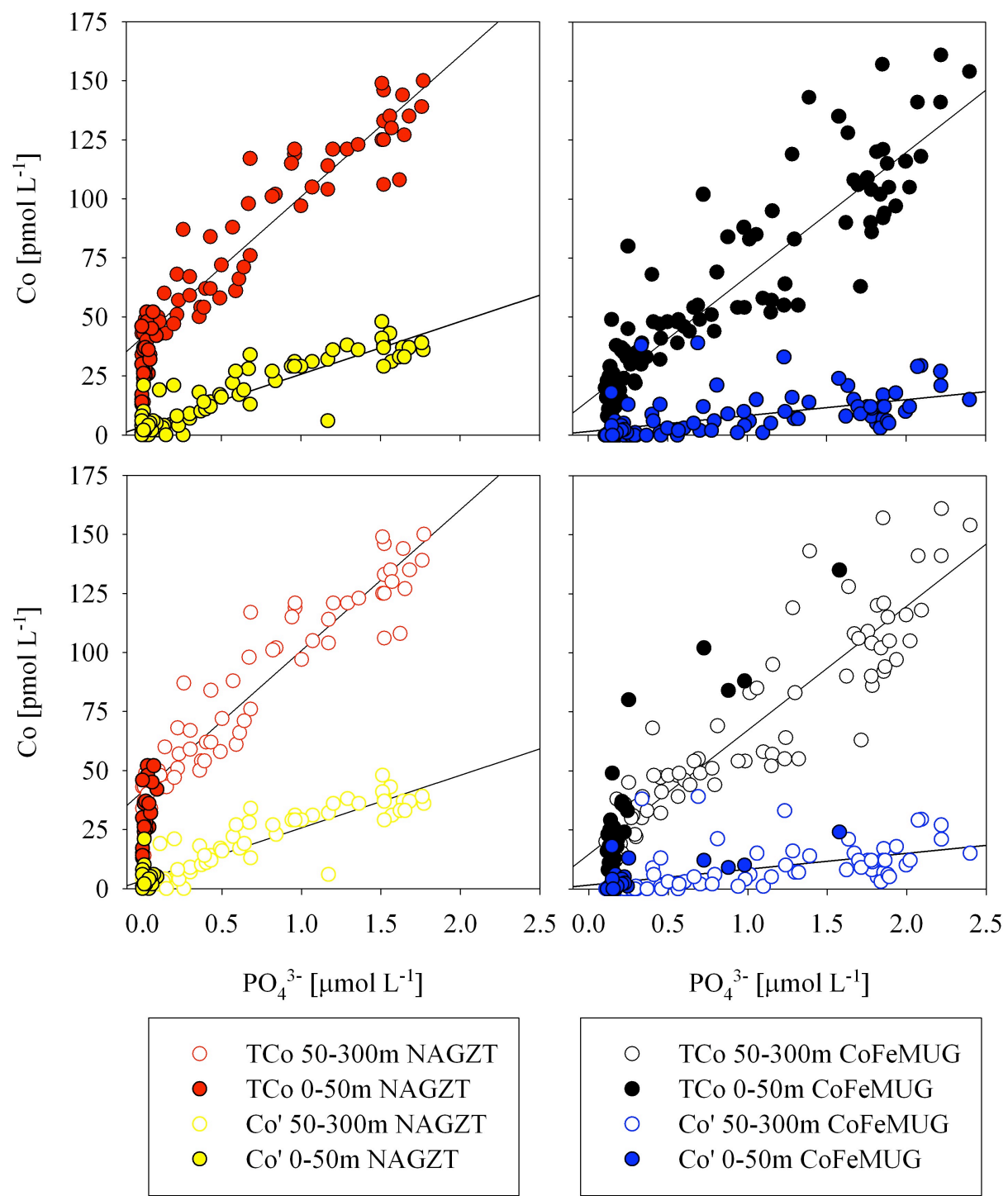

Figure 7) Linear relationship observed between total cobalt and phosphate. Labile cobalt also appears to have a linear relationship. There was a relationship between the degree of complexation and the onset of the observed kink in the $\mathrm{TCo}: \mathrm{PO}_{4}{ }^{3-}$ correlation, where close to $100 \%$ complexation (filled circles, bottom panels) generally co-occurs with phosphate depletion and a higher TCo: $\mathrm{PO}_{4}{ }^{3-}$ slope. 
Figure 8) When the stations are examined closely, each cruise can generally be separated into two regions that display different stoichiometries. The linear relationships are supported to different depths with the European stations (1-6) displaying a linear relationship down to $900 \mathrm{~m} \mathrm{(a),} \mathrm{compared} \mathrm{to} \mathrm{the} \mathrm{W.} \mathrm{African} \mathrm{N.} \mathrm{E.} \mathrm{Atlantic} \mathrm{(b),} \mathrm{and} \mathrm{the}$ South Atlantic Subtropical Gyre (c). Within the Angola Gyre, strong upwelling obscures the relationship between cobalt and phosphate $(\mathrm{d})$. 


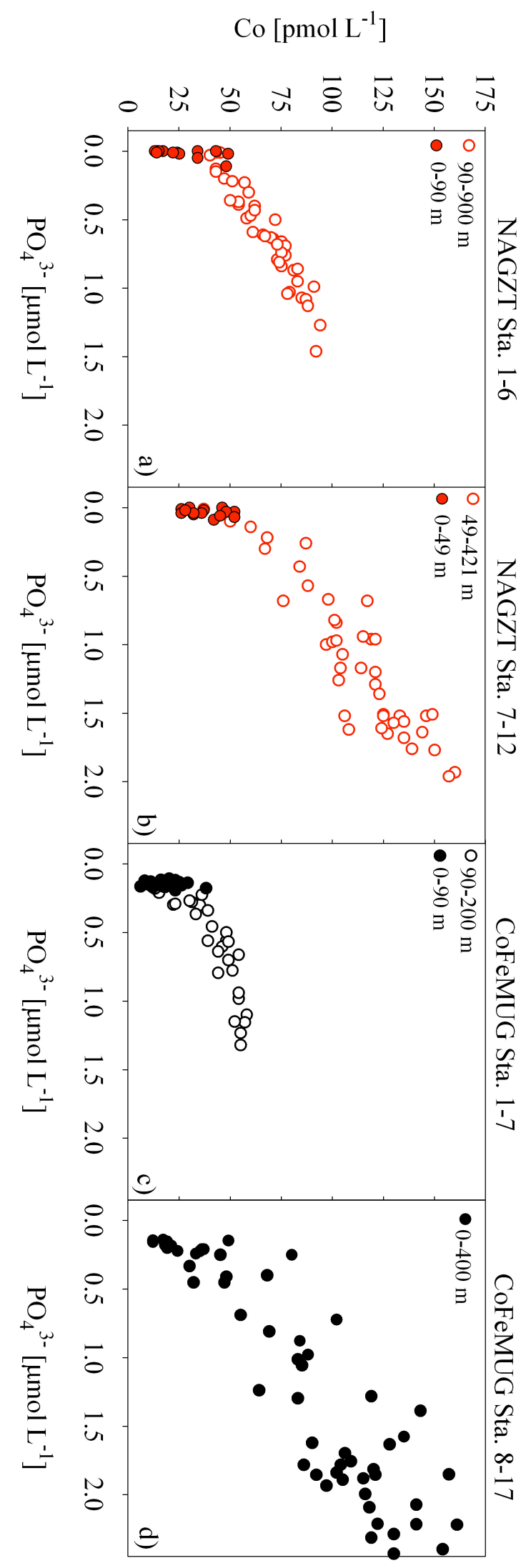




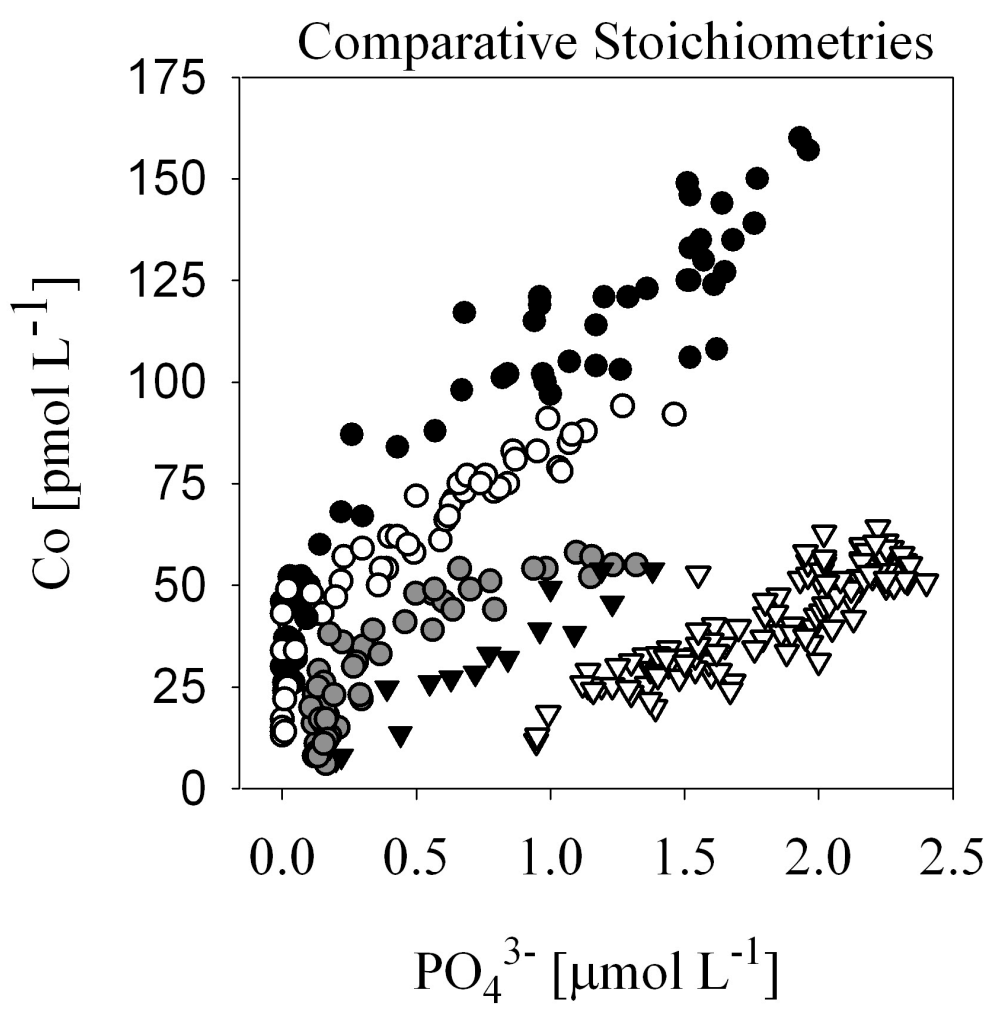

\begin{tabular}{|ll|}
\hline$\bullet$ & NAGZT Sta. $7-120-425 \mathrm{~m}$ \\
$\circ$ & NAGZT Sta. 1-6 0-900m \\
$\circ$ & CoFeMUG Sta. 1-7 0-200m \\
$\nabla$ & N.E. Pacific (T5,T6,T8) 0-150m \\
$\nabla$ & Ross Sea 0-500m \\
\hline
\end{tabular}

Figure 9) Regional differences are highlighted when cobalt and phosphate relationships are compared from this and other studies. It is interesting that while many of the ecological stoichiometries reported in Table 4 are similar, the data plot in different regions of $\mathrm{Co}: \mathrm{PO}_{4}{ }^{3-}$ space, primarily due to the large differences in macronutrient surface water inventory. It is interesting to note these similarities despite the large differences in macronutrients, which might be expected to have a significant effect on the biological species composition. 

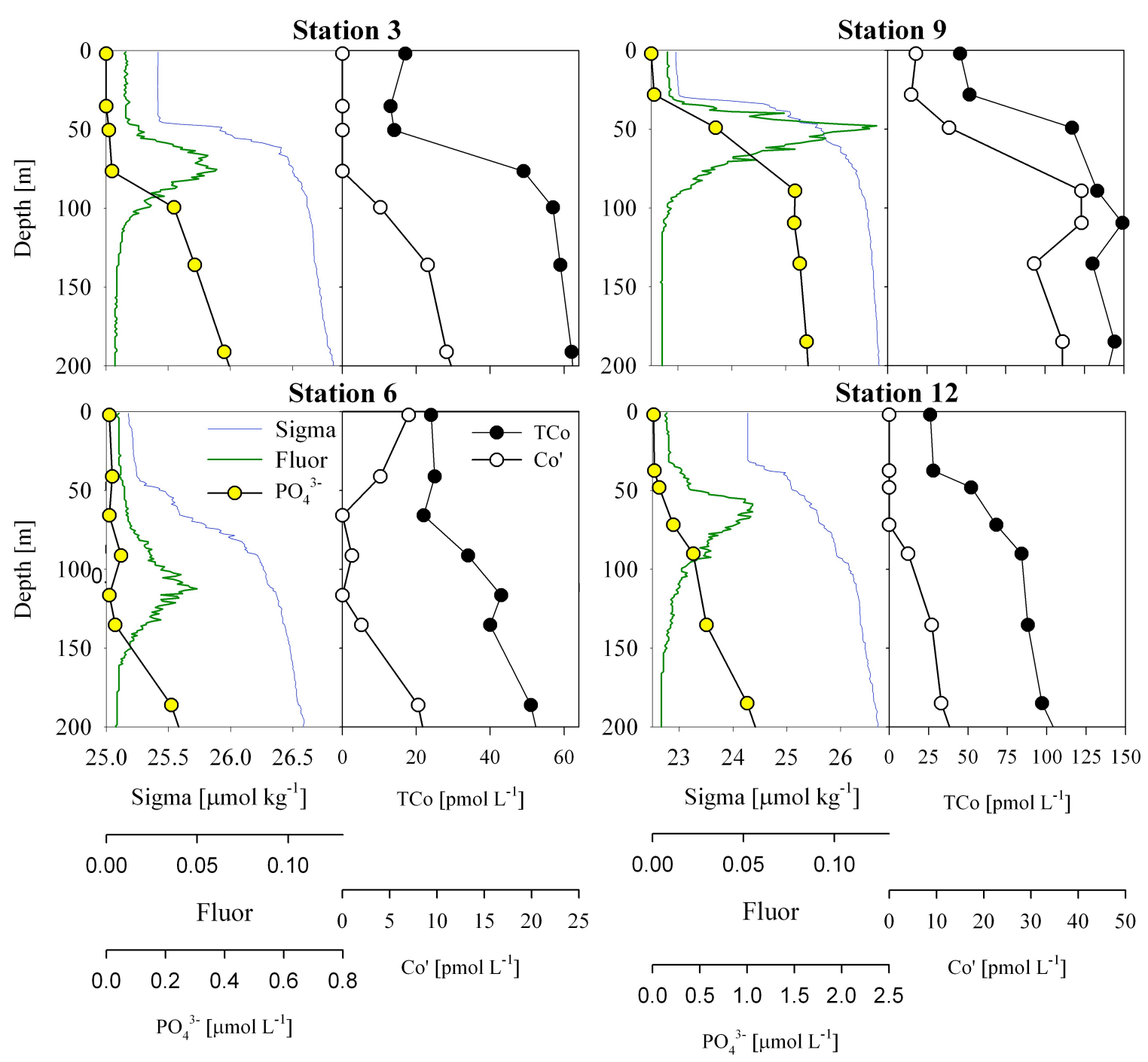

Figure 10) Upper water column profiles of fluorescence, sigma-theta, and phosphate (left plots) and labile and total cobalt (right plots) for four stations from the NAGZT cruise. Sta. 3 and 12 highlight the depth offsets in the labile and total "cobalt-clines." It is interesting to note that the labile cobalt-cline is coincident with the phosphocline, and the total cobaltcline is coincident with the mixed layer depth. At Sta. 9, the phosphocline and mixed layer depth align, likely due to the upwelling that occurs along the coast, and cobalt distributions follow this. At Sta. 6, the cobalt distributions are similar to that of Sta. 3 and 12 with the exception of the labile cobalt in surface waters, which display a slight maxima, potentially due to some aeolian input. 
Figure 11) Full depth scatter of TCo: $\mathrm{PO}_{4}{ }^{3-}$ relationships reveal differences in both the depth at which scavenging of cobalt becomes apparent, and the pattern observed in the scavenged "curl." Little is known about the mechanisms that might influence the depth at which cobalt scavenging becomes apparent, although this depth is observed to be coincident with a change in the dominant type of particle load toward a more lithogenic fraction in the African N.E. Atlantic (P. Lam personal communication). It may also be be related to oxygen concentrations and chemical speciation. 


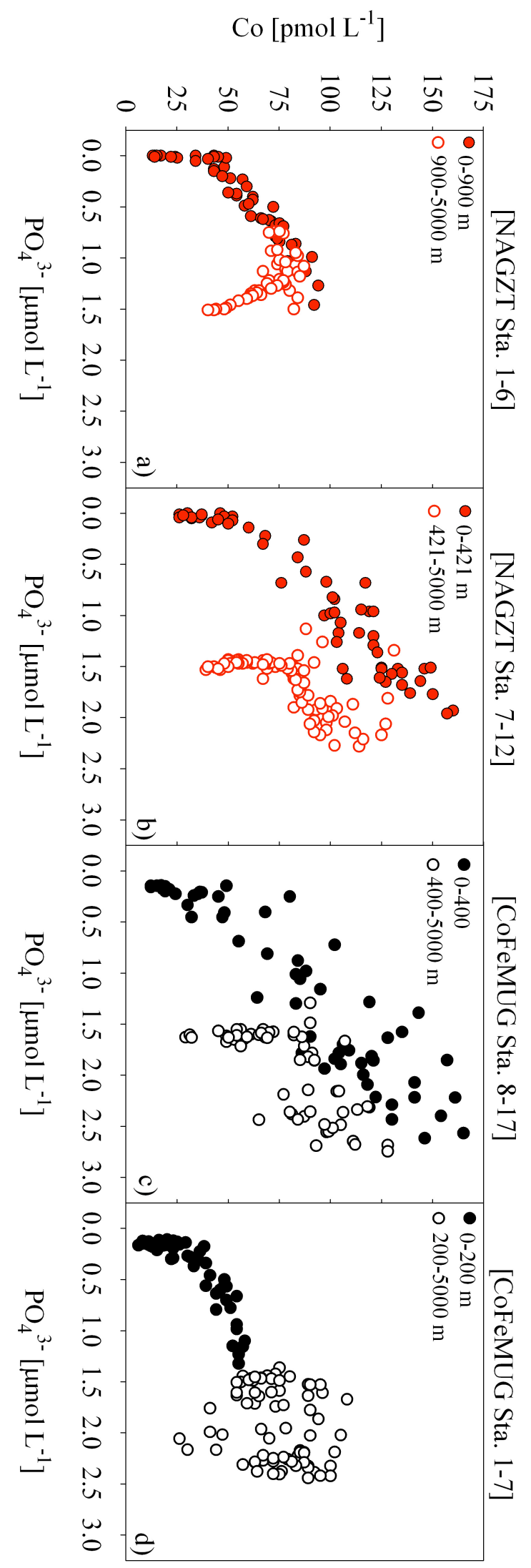




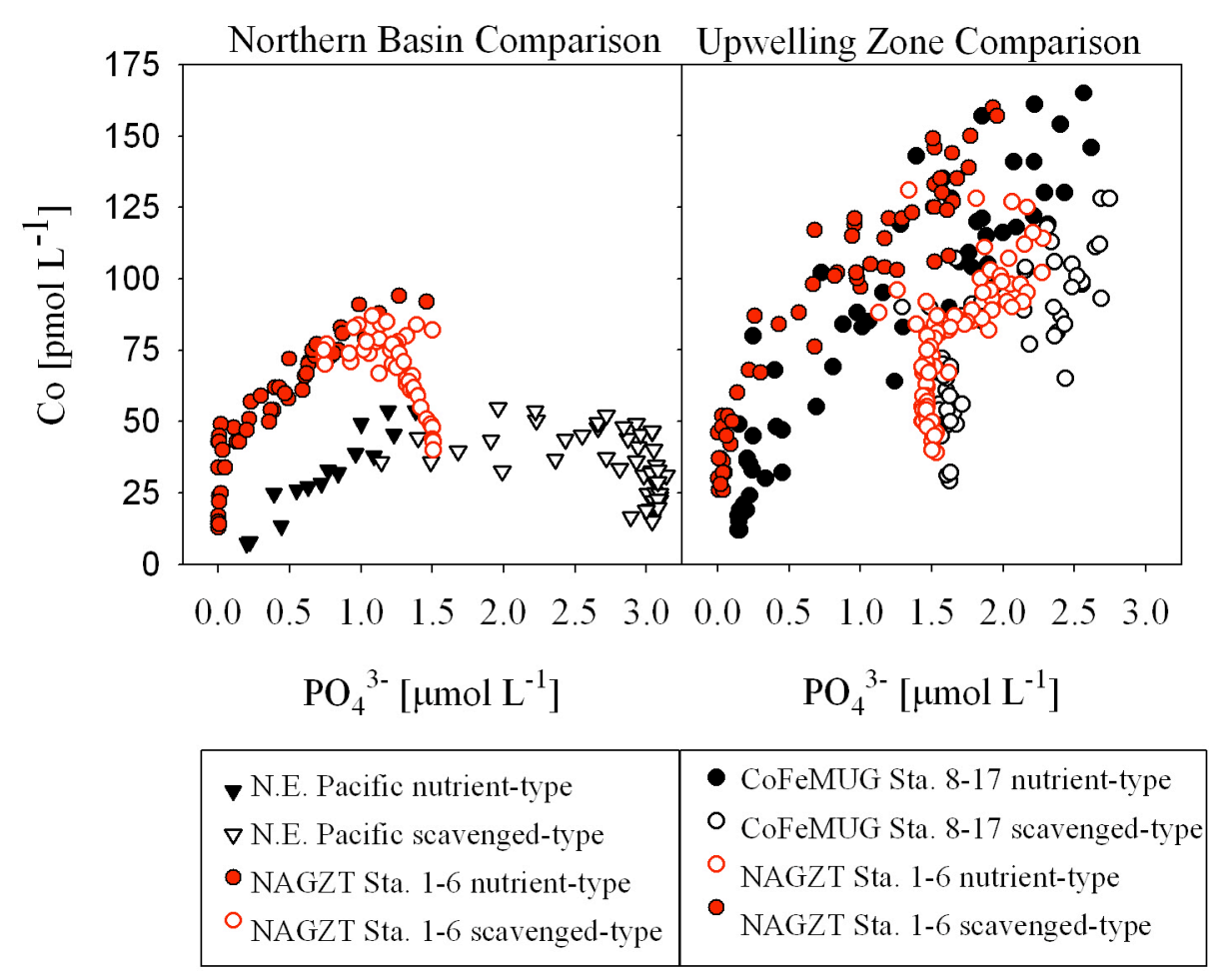

Figure 12) Shapes of cobalt "curls" for four regions: a) The European E.N. Atlantic and the E.N. Pacific (Martin et al. 1989), and b) The upwelling regions of the North and South Atlantic. The upwelling regions show similar trends, perhaps due to the OMZ pulses of both cobalt and phosphate, and similar water mass influences associated with intrusion of AAIW at $800 \mathrm{~m}$ depth. The E.N. Atlantic and E.N Pacific also show similar scavenging patterns and the differences in the deepwater inventories of phosphate due to accumulation with thermohaline circulation are apparent. 

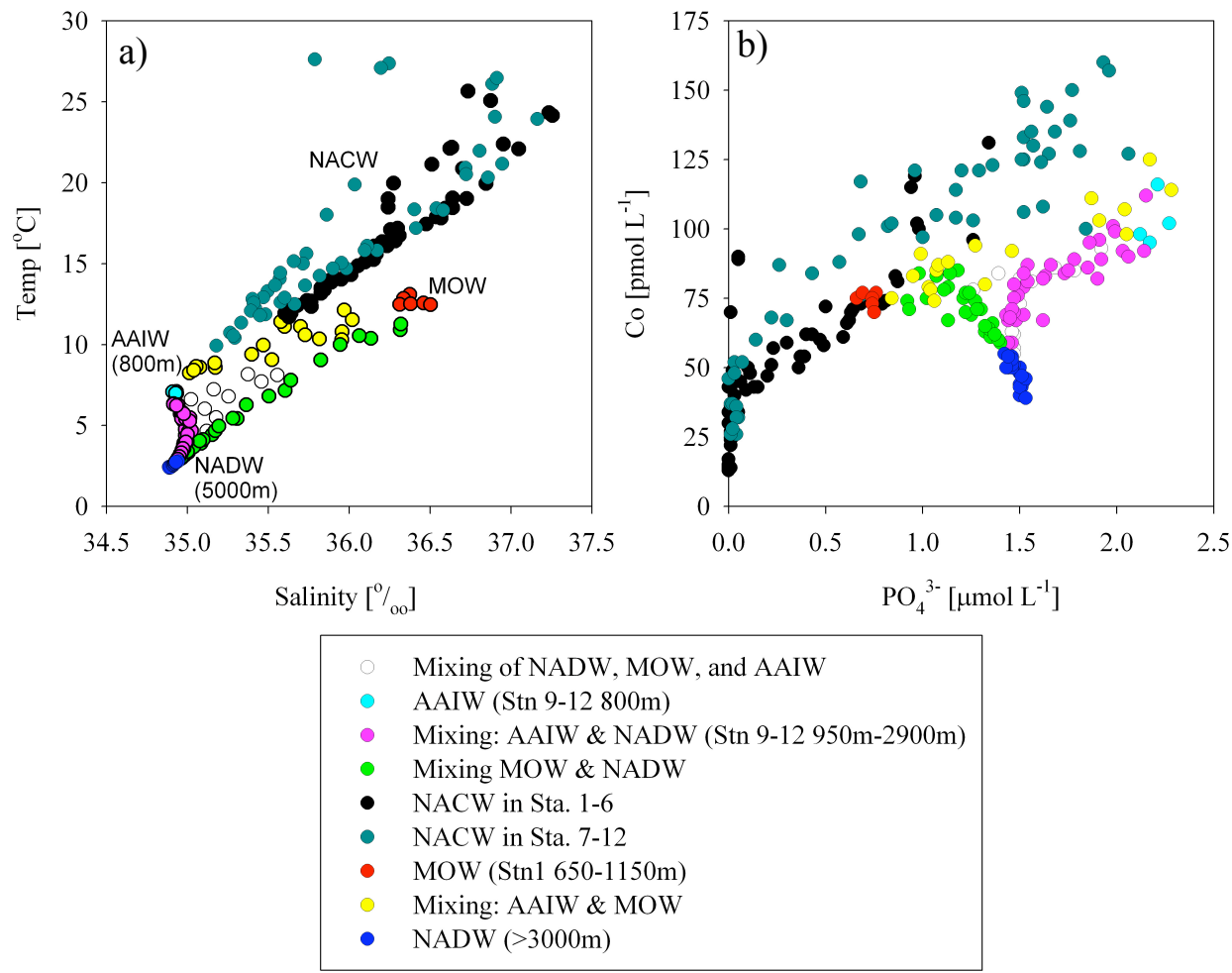

Figure 13) Water masses and mixing identified by color from the NAGZT dataset in a) temperature-salinity relationships and b) $\mathrm{TCo}_{\mathrm{PO}}{ }_{4}^{3-}$ space. It is interesting to note the different water masses, and where those data fall in $\mathrm{TCo}_{\mathrm{PO}}{ }_{4}{ }^{3-}$ space. AAIW carries high phosphate concentrations due to its origin in the HNLC waters of the Antarctic, while MOW carries a lower phosphate concentrations, and the mixing of these waters creates an interesting pattern. 


\title{
Chapter 6: Identification of a novel redox sensitive strong cobalt ligand class in oxygen minimum zones and a simple total dissolved cobalt preservation technique by the application of gas absorbing satchels
}

\author{
Abigail E. Noble ${ }^{1,2}$ and Mak A. Saito ${ }^{1 *}$
}

1) Marine Chemistry and Geochemistry Department, Woods Hole Oceanographic Institution, Woods Hole, Massachusetts 02543

2) MIT-WHOI Joint Program in Chemical Oceanography Massachusetts Institute of

Technology, 77 Massachusetts Ave, Cambridge, Massachusetts 02139

* Corresponding Author: msaito@whoi.edu 
Acknowledgements: We thank Ed Boyle and Bill Jenkins, the chief scientists of the GEOTRACES cruise, and we thank Jess Fitzsimmons, Peter Morton, and Geo Smith for their sampling and filtering efforts during the cruise. We are particularly indebted to the Captain and Crew of the R/V Knorr from the CoFeMUG cruise. This research was supported by US National Science Foundation Chemical Oceanography (OCE-0928414). 
Abstract: The measurement of picomolar total cobalt concentrations in seawater is challenging and in recent years has been accomplished primarily by adsorptive cathodic stripping voltammetry (ACSV) electrochemical techniques (Vega and Van den Berg 1997; Ellwood and Van den Berg 2001; Saito and Moffett 2001). Because this method operates at ambient seawater $\mathrm{pH}$, analysis of refrigerated, filtered seawater is ideal for minimizing handling and reagent blanks associated with acidification of samples for storage, yet, little work has focused on demonstrating successful methods of sample preservation for cobalt analyses. We present evidence that preservation of samples with headspace at $4^{\circ} \mathrm{C}$ in the dark is not sufficient for samples from all geographic regions on timescales of months to years. While samples from the Ross Sea showed good agreement between analyses made at-sea and analyses made after 17 months of storage, samples from the South Atlantic Gyre and the Eastern North Atlantic showed variable loss of total cobalt after 4-5 months of storage likely due to exposure to oxygen and the presence of colloidal material from aeolian and hydrothermal inputs. Results of speciation analyses suggest that this artifact was caused by a loss of cobalt that was not bound to organic ligands. We developed protocols for sample preservation, where successful storage for at least 4 months was documented with the use of metal-free gas absorbing satchels and heat-sealed, gasimpermeable storage bags. Excellent agreement was attained from this storage technique when compared to at-sea analyses for a 25-point profile from the North Atlantic (average recovery of $97 \pm 3.6 \%, \mathrm{n}=25$ ), demonstrating that this method is a successful alternative to immediate analysis at sea. Speciation studies on these preserved samples suggest that a novel class of strong redox sensitive ligands exist that bind cobalt in oxygen minimum zones. This use of gas absorber satchels provides a new avenue through which to probe metal speciation and ligand chemistry in seawater. 


\subsection{Introduction}

Cobalt is a metal of increasing interest as an important micronutrient to phytoplankton in the surface ocean. It is categorized as a hybrid-type metal, where both biological utilization and scavenging influence its distributions with depth (Bruland and Lohan 2003, Noble and Saito et al. 2008). The known biological uses of cobalt in the marine environment are as a metal co-factor in both the $\delta$ carbonic anhydrase and within vitamin $B_{12}$ (Roberts et al. 1997; Lane and Morel 2000). While some phytoplankton have an absolute requirement for cobalt, other organisms are capable of partially or fully satisfying their metal requirements with zinc or cadmium (Saito et al. 2004, Saito et al. 2002, Sunda and Huntsman 1995, Lane et al. 2005). B $_{12}$ is required by many organisms but is synthesized by only certain bacteria and archaea, including the marine cyanobacteria Prochlorococcus, which are responsible for the majority of surface ocean primary production (Partensky et al. 1999). Accurate methods to measure dissolved cobalt concentrations in seawater are therefore important to understanding the biogeochemistry and biochemistry of cobalt nutrition in the ocean.

The analysis of total cobalt concentrations in seawater is analytically challenging because cobalt is present in even lower concentrations than iron and is strongly bound by organic ligands. The seawater chemistry of cobalt is believed to be influenced by redox and complexation, both of which have the potential to complicate measurements of its total dissolved abundances. The use of a UV-oxidation step has proven successful for both the

reduction of cobalt species and the destruction of organic ligands in the samples (Saito and Moffett 2001). The total dissolved cobalt can then be determined in a sample without 
interference of organic matter or redox processes. Earlier methods relied on the use of reductants to assure that $\mathrm{Co}^{3+}$ was reduced to $\mathrm{Co}^{2+}$ (Donat and Bruland 1988), though this step has since been found to be unnecessary (Vega and Van den Berg 1997), perhaps due to the reduction of cobalt species during UV oxidation by superoxide. Following this UV oxidation step, cobalt samples can retain their integrity for long periods of time, and adsorption to the sides of the sample bottles appears to be minimal (Noble and Saito unpubl.).

In addition to total analyses, the speciation of cobalt has been probed using competitive ligand exchange adsorptive cathodic stripping voltammetry (CLE-ACSV, Saito and Moffett 2001, Saito et al. 2005). Competitive ligand exchange has been used both for ACSV and anodic stripping voltammetry (ASV) to determine the conditional stability constants of naturally occurring ligands in seawater for a number of transition metals including copper, zinc, nickel, cobalt, and iron (Jakuba et al. 2008; Saito et al. 2002, 2005; Buck and Bruland 2005, 2007; Buck et al. 2010; Donat et al. 1994) and even to detect different oxidation states of dissolved species in seawater such as $\mathrm{Mn}(\mathrm{III})$ (Trouwborst et al. 2006). The conditional stability constants of many naturally occurring transition metal ligands have been observed to be high, and incubation studies suggest that many are organic and biologically produced. For example, shipboard incubation studies of the cyanobacteria Synechococcus have revealed that these organisms can produce large quantities of strong cobalt binding ligands, and that Prochlorococcus can access ligandbound cobalt more readily than labile cobalt (Saito et al. 2002, 2005). While the structure of these molecules is largely unknown, the conditional stability constants of cobalt binding 
ligands in seawater give some indication of the oxidation state of the ligand-bound metal. For example, $\mathrm{Co}^{3+}$ is essentially insoluble in oxygenated seawater, but the exceptionally high stability constants of cobalt binding ligands (on the order of $10^{16.8}$ or greater) suggest that this ligand-bound cobalt is likely present in the +III oxidation state (Saito and Moffett 2001, Saito et al. 2005). If labile cobalt in seawater is present as $\mathrm{Co}^{2+}$, oxidation of cobalt may occur during or following complexation. This chemistry complicates its behavior in the water column and the considerations involved in measuring concentrations accurately. While the potential for loss of cobalt by oxidation and precipitation at ambient $\mathrm{pH}$ has not been previously observed in seawater analyses, as it has for iron, it has also not been carefully investigated. From an analytical perspective, the complex redox reactions that are possible for cobalt in the environment suggest that changes in cobalt speciation and redox have the potential to induce artifacts during storage.

High-throughput techniques for the determination of trace metal concentrations in seawater are in demand due to the international GEOTRACES program that measures global distributions of trace elements and isotopes. Electrochemical methods like ACSV provide an efficient avenue through which to study both the total concentrations and the speciation of cobalt using a single analytical technique that involves no significant perturbation of the sample state. For example, the precision of the ACSV method for samples at ambient $\mathrm{pH}$ is excellent (e.g. mean and standard deviation of triplicate analyses of a surface water sample $=32 \pm 0.7 \mathrm{pmol} \mathrm{L}^{-1}$, Noble et al. in review), but the challenges involved in the neutralization of acidified samples decrease the precision associated with the measurement (Noble and Saito unpubl.). Recent intercalibration efforts have also 
discovered that these organic ligands are so strong that they do not dissociate at $\mathrm{pH} 1.7$, which then requires that inductively coupled plasma mass spectrometry (ICP-MS) and flow injection methods incorporate a UV oxidation step prior to analysis (Bruland 2010; Shelley et al. 2010). Additionally, studies have shown that the $\mathrm{MgOH}_{2}$ co-precipitation method has a low scavenging efficiency, requiring very large precipitates in order to attain good cobalt recovery (Saito and Schneider 2006, Appendix I).

The analysis of cobalt concentrations at ambient $\mathrm{pH}$ by ACSV has many advantages. First, the method is highly sensitive due to the use of the electroactive ligand dimethylglyoxime, which involves a 10 electron reduction reaction of the bis complex $\mathrm{CoHDMG}_{2}$ from the hanging mercury drop (Bobrowski 1989, Baxter et al. 1998). Second, the method is relatively fast; the $90 \mathrm{~s}$ deposition time allows for a 7 -scan automated titration to be completed in less than 20 minutes. Third, analyses are easily conducted at sea. This methodology can also measure chemical speciation. Because ambient cobalt binding ligands have high stability constants $\left(>10^{16.8}\right)$ and are often found in non-saturating concentrations beneath the euphotic zone, rapid analyses of labile cobalt are also attainable (Saito et al. 2005). This also allows for an estimation of the total cobalt ligand concentration in waters where labile is detected because this value can be estimated by the difference between the total and labile concentrations. These advances in cobalt electrochemical analysis have made it possible to include the analysis of total and labile cobalt in many of the initial GEOTRACES expeditions.

While samples can be processed relatively quickly, there are often logistical advantages to being able to store samples for analysis in the laboratory at a later date. This 
study investigated the sample integrity over time of samples taken from three different regions, and presents results for a successful and simple preservation technique. Lastly, the work presented here suggests that the preservation of samples for shore-based analysis may be affected by a loss of signal in oxygen minimum zones and/or in high dust regions.

\subsection{Methods}

6.2.1 Sampling Techniques - All samples were taken using pre-conditioned, teflon-coated sampling bottles, either from X-Niskin (Ocean Test Equipment) or Go-Flo sampling bottles (General Oceanics) attached to a Kevlar, non-metallic conducting cable. Sampling bottles were moved to a class-100 trace metal clean van, and pressurized with $99.999 \% \mathrm{~N}_{2}$ at $<7 \mathrm{psi}$ for sampling. Samples were collected through either a $0.2 \mu \mathrm{m}$ Acropak filters, or a $142 \mathrm{~mm} 0.4 \mu \mathrm{m}$ polycarbonate filter (Osmonics), both of which were cleaned and preconditioned using trace metal clean techniques. Sample storage bottles were soaked overnight in the acidic detergent, Citranox, rinsed thoroughly with Milli-Q water (Millipore), filled with $10 \% \mathrm{HCl}$ to soak for 10 days, rinsed thoroughly with Milli-Q water adjusted to $\mathrm{pH} 2$, and double-bagged. After sample collection, bottles were double bagged and kept in the dark at $4^{\circ} \mathrm{C}$ until analysis.

6.2.2 Cobalt Analyses - Concentrations of total dissolved and labile cobalt were determined using a previously described cathodic stripping voltammetry (CSV) method (Saito and Moffett 2001, Saito et al. 2004). Measurements were made using the EcoChemie $\mu$ AutolabIII systems connected to Metrohm 663 VA Stands equipped with hanging mercury drop electrodes and Teflon sampling cups. Standard additions were 
carried out with Metrohm 765 Dosimats using a programmed dosing procedure (Noble and Saito et al. 2008).

For total dissolved cobalt analyses, samples were UV-irradiated for $1 \mathrm{~h}$ prior to analysis using a Metrohm 705 UV digester to degrade the organic ligands that bind cobalt and allow binding by the added electroactive cobalt ligand, dimethylglyoxime. Samples were analyzed in $8.5 \mathrm{~mL}$ aliquots with the addition of $30 \mu \mathrm{L}$ recrystalized dimethylglyoxime (DMG, $0.1 \mathrm{~mol} \mathrm{~L}^{-1}$ in methanol), $1.5 \mathrm{~mL}$ purified sodium nitrite $(1.5$ mol L ${ }^{-1}$ in Milli-Q water), and $50 \mu \mathrm{L}$ purified N-(2-hydroxyethyl)piperazine-N-(3propanesulfonic acid) (EPPS) buffer $\left(0.5 \mathrm{~mol} \mathrm{~L}^{-1}\right.$ in Milli-Q water). Reagent purification protocols were identical to those previously published (Saito and Moffett 2001). Analysis began with a 180 s purge with $99.999 \% \mathrm{~N}_{2}$. Each sample was conditioned at $-0.6 \mathrm{~V}$ for 90 $\mathrm{s}$ at a stir-rate of $2500 \mathrm{rpm}$ followed by a $10 \mathrm{~s}$ equilibration step and a linear sweep from $0.6 \mathrm{~V}$ to $-1.4 \mathrm{~V}$ at a rate of $10 \mathrm{~V} \mathrm{~s}^{-1}$. Cobalt concentrations were determined by the standard additions technique, with initial concentrations measured in triplicate followed by

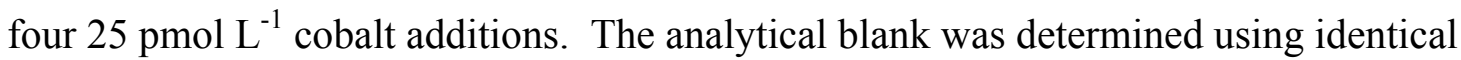
techniques to those previously described (Saito and Moffett 2001), and blanks for each reagents batch (nitrite, DMG, EPPS) were subtracted from the initial sample concentration. The blanks for the analyses presented here were all less than $6 \mathrm{pmol} \mathrm{L}^{-1}$.

For labile cobalt analyses, $8.5 \mathrm{~mL}$ of sample were pipetted into acid washed Teflon vials that were preconditioned with a small aliquot of sample water. $30 \mu \mathrm{L}$ of DMG were added to each vial and allowed to equilibrate overnight in the dark prior to analysis (Saito et al. 2004). Analyses were then performed as described for total concentrations with the 
addition of the remaining two reagents and use of the standard addition technique.

Previously, we determined that natural cobalt ligands in seawater have conditional stability constants $>10^{16.8}$ (Saito et al. 2005). This suggests that the cobalt is bound very tightly to the cobalt ligands. Thus, we define labile cobalt as the fraction of total dissolved cobalt that is either bound to weak organic and inorganic ligands in seawater or present as free $\mathrm{Co}(\mathrm{II})$, and is then exchangeable with the complexing agent (DMG) used for analysis (Saito et al. 2004, Saito et al. 2005). The difference between the total dissolved cobalt and the labile cobalt can then be used as an estimation of the strong cobalt ligand concentration. 6.2.3 Preservation technique - Samples were collected into $60 \mathrm{~mL}$ LDPE bottles and filled to the top. Following collection, 6-7 samples were places in a 2-mil plastic bag, partially sealed, and then placed in a PTS bag (Mitsubishi Gas Chemical Company Inc., Tokyo, Japan) along with 5 PR System ${ }^{\circledast}$ oxygen absorber satchels (model 56-PR3K-25 Mitsubishi Gas Chemical Company Inc., Tokyo, Japan). The PTS bags were then heat sealed using an 11 in. pouch sealer (Ampac Flexibles, Cincinnati, OH). The sealed bags were stored at $4^{\circ} \mathrm{C}$ in the dark until analysis with no further treatment. Each oxygen absorber satchel is rated to absorb $60 \mathrm{~mL}$ of oxygen and $10 \mathrm{ppm} \mathrm{H}_{2} \mathrm{~S}$ per $300 \mathrm{~mL}$ of air, and is iron-free (http://www.mgc-a.com/AGELESS/). Many oxygen absorber satchels that are commercially available and inexpensive use an iron-based powder as the absorption agent. The PTS bags have a high gas barrier with an oxygen transmission rate of $0.5 \mathrm{cc} \mathrm{m}^{-}$ $2 \mathrm{~d}^{-1} \mathrm{~atm}^{-1}$. 


\subsection{Results}

To examine the influence of storage on unacidified samples stored for dissolved cobalt analysis by adsorptive cathodic stripping voltammetry (ACSV), samples were reanalyzed after varying lengths of time from collection and compared to at-sea analyses. Two types of cobalt analyses are reported here: total dissolved cobalt (TCo), and labile cobalt $\left(\mathrm{Co}^{\prime}\right)$, where $\mathrm{Co}^{\prime}$ refers to the fraction of the TCo that is present either as the free ion, or is bound to weak ligands in seawater. The shipboard analyses occurred within days of collection, and are referred to in this manuscript as $\mathrm{TCo}_{a t-s e a}$ and $\mathrm{Co}_{\text {at-sea. }}$. Results from analyses performed on samples stored in the dark at $4^{\circ} \mathrm{C}$ with some headspace for 4 - 17 months depending on the sample set (Table 1) are referred to as $\mathrm{TCo}_{\text {stored }}$ and $\mathrm{Co}_{\text {stored. }}$ In order to determine if the stability of a sample over time might be influenced by the particular chemistry of a geographic region, $\mathrm{TCo}_{\text {stored }}$ and $\mathrm{Co}^{\prime}$ stored were compared to their respective $\mathrm{TCo}_{\text {at-sea }}$ and $\mathrm{Co}^{\prime}$ at-sea concentrations in three distinct oceanic regions (the Ross Sea, the South Atlantic Gyre, and the North East Atlantic). 6 full-depth profiles are presented in Fig. 1 and described in Table 1 with an inventory of the types of analyses performed. On the US North Atlantic GEOTRACES cruise, a duplicate set of samples was preserved in heat sealed, gas-impermeable bags containing gas absorber satchels, and stored in the dark at $4^{\circ} \mathrm{C}$ for at least 4 months. These samples had little-to-no headspace and are referred to as $\mathrm{TCo}_{\text {preserved }}$ and $\mathrm{Co}_{\text {preserved }}^{\prime}$ and we present preserved results from one of the stations sampled in duplicate (Sta. 9).

6.3.1 Comparison of at-sea to stored analyses - The South Atlantic Gyre Stas. 7, 9, and 11 showed two noticeable trends after 4 months of storage. First, there was noticeable loss of 
TCo within the oxygen minimum zone in all three stations, implying that samples taken within oxygen minimum zones have shorter shelf lives (Fig. 1a-c). This might be due to oxygen invasion into the sample bottles, followed by eventual oxidation and precipitation within the sample bottle. Second, loss of TCo was also observed between 1500 and 2500 m depth at Sta. 7 (Fig. 1a). This station was located above the Mid-Atlantic Ridge. We hypothesize that elevated colloidal material from hydrothermal activity was present in the filtered samples and scavenged cobalt in the sample bottle over time. While we do not have colloidal data from this station, a large plume of dissolved iron and manganese concentrations was detected above the ridge at these depths (Saito et al. unpubl.; Mullaugh and Luther 2011; Yucel et al. 2011).

In contrast to these selected South Atlantic stations, samples from the Ross Sea showed very good agreement between $\mathrm{TCo}_{\mathrm{at}-\text { sea }}$ and $\mathrm{TCo}_{\text {stored }}$ with the exception of one datapoint at $300 \mathrm{~m}$ (average recovery of $101 \pm 10 \%, \mathrm{n}=8$, Fig. 1f). These $\mathrm{TCo}_{\text {stored }}$ analyses were made 17 months after collection and suggest that issues with sample preservation are not uniform for all compositions of seawater. Repeat analyses of one sample from this region also demonstrated exceptional stability of $\mathrm{Co}^{\prime}$, suggesting that labile cobalt can be stable in some environments for long periods of time as well (Table 2).

In the Eastern North Atlantic, analyses from Sta. 5 and 9 showed 4 major features (Fig. 1d, e). First, good agreement between $\mathrm{TCo}_{\text {at-sea }}$ and $\mathrm{TCo}_{\text {stored }}$ (stored 5 months) was observed in the upper $50 \mathrm{~m}$, which may be related to processes in the euphotic zone, such as biological production of organic ligands and complexation of cobalt. Second, somewhat consistent loss of TCo during storage was observed for most depths below the euphotic 
zone in both Sta. 9 and Sta. 5. Third, there was a large difference in TCo loss between Sta. 5 and Sta. $9 . \mathrm{TCo}_{\text {stored }}$ at Sta. 5 was $\sim 15 \%$ less than $\mathrm{TCo}_{\text {at-sea }}$ (approximately 9 pmol $\mathrm{L}^{-1} \operatorname{loss}$ per sample), while $\mathrm{TCo}_{\text {stored }}$ at Sta. 9 was $\sim 40 \%$ less than $\mathrm{TCo}_{\text {at-sea }}$ (approximately 37 pmol $\mathrm{L}^{-1}$ loss per sample). Lastly, $\mathrm{Co}_{\text {stored }}$ analyses at Sta. 9 showed an almost $100 \%$ loss relative to $\mathrm{Co}_{\text {at-sea }}^{\prime}$ after 5 months of storage.

6.3.2 Preserved analyses: Analysis of the preserved samples for Sta. 9 from the North Atlantic displayed excellent reproducibility throughout the water column compared with the at-sea analyses (Fig. 2). For a 25-point vertical profile, the average reproducibility was $97+/-3.6 \%(n=25)$. Agreement between the $\mathrm{TCo}_{\text {at-sea }}$ and $\mathrm{TCo}_{\text {preserved }}$ profiles is evident in Fig. 2a, and when compared in a scatter plot, data fall along the 1:1 line (Fig. 2b). These results also imply that the shelf-life of the reagents used for these analyses is long. The nitrite solution used for these analyses was dissolved and purified 2 years prior, compared to the nitrite used for at-sea analyses, which was fresh (1-2 months old). Additionally, while purified DMG crystals were freshly dissolved for each analysis batch, the crystals had been recrystallized two years prior.

\subsection{Discussion}

The accurate and precise determination of dissolved cobalt in seawater is a challenge given the low oceanic concentrations, and the results of this study suggest that additional complications can arise when samples are not analyzed or preserved soon after collection for samples from certain geographic locations. The trends in sample integrity over time were not uniform across all stored cobalt samples: while $\mathrm{TCo}_{\text {stored }}$ from the Ross Sea showed little to no loss of TCo, stations from other geographic regions showed loss at 
specific depths, and still other stations showed uniform loss over large depth ranges. The following sections focus on the merits of sample preservation using gas absorber satchels and a discussion of three potential influences on cobalt sample integrity: 1) dissolved oxygen, 2) particle load, and 3) chemical speciation. Additionally, in the application of this new total cobalt preservation technique, novel insights into the nature of cobalt speciation were obtained, and they are presented here as well.

\subsubsection{A method of preservation for total cobalt analyses by ACSV - The TCo $\mathrm{o}_{\mathrm{preserved}}$} concentrations agreed well with the $\mathrm{TCo}_{\text {at-sea }}$ concentrations (Fig. 2), demonstrating the viability of preservation with gas absorbers as an alternative to performing TCo analyses at sea. While these results are presented for only one station, this station demonstrated the highest loss of TCo among all regions (up to 53\%) for the $\mathrm{TCo}_{\text {stored }}$ analyses (Fig. 1e, k). Among the environmental factors that may affect dissolved cobalt solubility and stability, low oxygen concentrations have been observed to have an inverse correlation with elevated cobalt concentrations (Saito et al. 2004, Noble et al. in review). If samples experience a change in oxygen concentration during storage by either equilibration with headspace in sample bottles or invasion of oxygen into the sample bottles over time, this may alter the cobalt concentration. Dissolved oxygen at Sta. 9 dropped to $40 \mu \mathrm{mol} \mathrm{kg}^{-1}$ between 350 and $400 \mathrm{~m}$ and agreement between $\mathrm{TCo}_{\text {preserved }}$ and $\mathrm{TCo}_{\text {at-sea }}$ was still excellent (Fig. 1e, 2a, b). Additionally, this station was located near the Sahara Desert, and a significant amount of dust was deposited during occupation of this station, which has been observed to correlate with higher concentrations of colloidal material (Aguilar-Islas et al. 2010). Higher concentrations of colloidal material in filtered samples may increase the potential for 
removal of cobalt within the sample by aggregation, adsorption, and precipitation. The demonstrated success of this preservation method at a highly dynamic geographic location with both low oxygen concentrations and high dust input (i.e. a worst-case scenario) provides confidence in this new preservation approach.

6.4.2 Influence of oxygen - Dissolved oxygen concentrations likely affect the susceptibility of a sample to loss of cobalt over time, though the mechanisms are unclear. Low oxygen waters have previously been observed to support higher concentrations of cobalt, likely due to differences in solubilities of cobalt redox states (Pohl et al. 2010, Noble et al. in review, Saito et al. 2004, Saito et al., 2005). If oxygen concentration were to change significantly between sample collection and analysis, the redox state of the sample may also change, which could induce oxidation and subsequent precipitation of cobalt(III) oxides. The oxygen concentration in a sample could change as a result of equilibration with headspace in sample bottles or with oxygen invasion into the gaspermeable bottles with time. This is supported by our observations from the CoFeMUG stations. As mentioned above, Sta. 7, 9, and 11 from the CoFeMUG cruise showed higher loss of TCo at depths within the oxygen minimum zone than above and below (Fig. 1a-c, g-i). If these originally low oxygen samples experienced significant changes in the oxidizing capacity via increases in oxygen concentration during storage, they might be expected to experience the most loss of TCo through oxidation of reduced chemical species. In contrast, in the Ross Sea, oxygen concentrations were initially above $252 \mu \mathrm{mol}$ $\mathrm{kg}^{-1}$, and good consistency was observed between $\mathrm{TCo}_{\mathrm{at}-\text { sea }}$ and $\mathrm{TCo}_{\text {stored }}$ (Fig. 1f, 1). With the seawater oxygen concentrations being high at the time of sampling, equilibration with 
the atmosphere would not appreciably change the oxygen concentration in the sample, and thus would not significantly affect the redox character of the sample during storage.

The loss of TCo with sample storage does not appear to be solely explained by changes in oxygen concentration, however, and two lines of evidence support this. First, both Sta. 9 from the North Atlantic and Sta. 11 from the South Atlantic Gyre sampled waters that were characterized by similarly low $\mathrm{O}_{2}$ concentrations, and similar cobalt profiles and concentration ranges (Fig. 1c, e). These stations showed distinct trends in sample integrity with $\mathrm{TCo}_{\text {stored }}$ analyses: while the North Atlantic station (Sta. 9) showed a $\sim 40 \%$ loss of TCo through much of the water column, the South Atlantic station (Sta. 11) showed an average of $14 \%$ loss of TCo except in the oxygen minimum where an $\sim 22 \%$ loss of TCo was observed. Because the oxygen distributions and $\mathrm{TCo}_{\mathrm{at}-\mathrm{sea}}$ concentrations were similar at each location, but the loss observed was different, oxygen is likely not the sole factor controlling sample integrity. It is interesting to note, however, that the South Atlantic samples were collected in $250 \mathrm{~mL}$ bottles, while the North Atlantic samples were collected in $60 \mathrm{~mL}$ bottles. While both bottles were initially filled to the top, $\mathrm{TCo}_{\text {at-sea }}$ analyses created $\sim 20 \mathrm{~mL}$ of headspace in both sets of bottles that were stored for $\mathrm{TCo}_{\text {stored }}$ analyses. It is possible that the higher headspace to volume ratio in the $60 \mathrm{~mL}$ bottles caused the larger loss of TCo due to the greater abundance of oxygen in the headspace. As a simple preventative measure, bottles should be filled to the top during collection.

The second line of evidence that TCo loss was not solely controlled by oxygen was observed in the comparison of the two North Atlantic stations. The oxygen concentrations dropped to $\sim 40 \mu \mathrm{mol} \mathrm{kg}{ }^{-1}$ in the oxygen minimum zone of Sta. 9 and oxygen 
concentrations did not drop below $150 \mu \mathrm{mol} \mathrm{kg}{ }^{-1}$ at Sta. 5 (Fig. 1d, e). While the TCo loss observed in the oxygen minimum zone of Sta. 9 (40\%) was much higher than that observed in the oxygen minimum zone of Sta. 5 (24\%), and the loss was somewhat uniform throughout the water column below the euphotic zone, despite a large range of oxygen concentrations (40-225 $\mu \mathrm{mol} \mathrm{kg}{ }^{-1}$, Fig. 3a). This contrasts with the observations in the South Atlantic, where the major loss of TCo was restricted to the oxygen minimum zone, with the exception of the Mid-Atlantic Ridge samples. The high $\mathrm{O}_{2}$ samples within the Mid-Atlantic Ridge iron and manganese plumes likely have high concentrations of colloidal material (Yucel et al. 2011), and suggest that another mechanism may be responsible for the cobalt loss observed in those samples (see following section below). It is important to note, however, that these observations do not discount the possibility that oxygen invasion into low oxygen samples over time could cause oxidation and precipitation of cobalt.

6.4.3 Potential influence of particle load - Another factor to consider is the presence of colloidal material in the filtered samples. Here, colloids are defined as particulate matter that is less than either $0.4 \mu \mathrm{m}$ (CoFeMUG samples) or $0.2 \mu \mathrm{m}$ (NAGZT samples) in diameter, but larger than $0.02 \mu \mathrm{m}$ (Bergquist et al. 2007). Fieldwork in the Atlantic Ocean has demonstrated that a large percentage of the variability observed in iron concentrations is found in this colloidal fraction (Bergquist et al. 2007). In regions that have an increased particulate load the concentration of colloidal material may be higher, and it has recently been demonstrated that the majority of iron delivered from aerosols is colloidal (AguilarIslas et al. 2010). The presence of colloids in filtered samples may provide reaction sites 
for adsorption and/or accelerated rates of aggregation and precipitation. Numerical models suggest that scavenging rates may be faster for iron in areas with higher particle loads: in a global circulation model of dissolved iron, accelerated scavenging rates were required to reproduce the observed distributions of iron off the North West African coast (Moore et al. 2004). Without this constraint on the system, predicted iron concentrations would be much too high. Similarly, it is possible that cobalt scavenging rates may also be influenced by particle load. One difference between Sta. 9 in the North Atlantic and Sta. 11 in the South Atlantic is the average dust input. The South Atlantic generally experiences much lower rates of dust deposition than the high dust input from the Saharan Desert in the North Atlantic (Measures 1995, Measures and Vink 2000, Measures and Edmond 1990). The potential impact of colloids and particle deposition on the loss of total cobalt with storage raises questions regarding the role of the gas absorber satchels in preventing this loss. While the mechanisms are still unclear, if cobalt is only removed by the particulate phase as it is oxidized, the presence of the satchels could be crucial in preventing the sample from becoming more oxidizing than its initial sampled state.

\subsubsection{The role of complexation and a new avenue for the exploration of cobalt speciation}

- In addition to particle load, the chemical speciation of cobalt is an important factor to consider. Oceanic cobalt complexation is largely driven by a strong organic ligand class,

which binds cobalt with conditional stability constants in excess of $10^{16.8}$ (Saito et al. 2005). These ligands likely increase the solubility of cobalt in seawater, and protect it from scavenging to some degree. Interestingly, our laboratory has found that these strong ligands are not typically observed to be present in excess of the total cobalt, and picomolar 
concentrations of labile cobalt are frequently detected below the euphotic zone (Saito et al. 2004, 2005, Noble and Saito et al. 2008) and sometimes throughout the entire water column (Saito et al. 2010).

Speciation measurements that estimated the labile cobalt $\left(\mathrm{Co}^{\prime}\right)$ were performed on Sta. 5 and Sta. 9 samples at sea ( $\left.\mathrm{Co}^{\prime}{ }_{\text {at-sea }}\right)$ and after 5 months of storage ( $\mathrm{Co}^{\prime}$ stored), from the NAGZT expedition (Fig. 4), and on one sample from the Ross Sea (Table 2). At Sta. 9 Co' was detectable in all samples at sea, with only 4 depths where concentrations fell below 10 pmol L ${ }^{-1}$ (Fig. 4a). In the lab, however; $\mathrm{Co}^{\prime}$ stored was below detection for most samples after 5 months of storage. Some loss of $\mathrm{Co}^{\prime}$ was also observed in a comparison of $\mathrm{Co}^{\prime}$ at-sea to $\mathrm{Co}^{\prime}$ stored for Sta. 5 (Fig. 4b). These results were in contrast to the repeat analyses from the Ross Sea, which showed no loss of Co' over 17 months of storage (Table 2). Our lab has also found that Co' in UV-digested seawater is quite stable even at room temperature, and can be used successfully as an internal lab standard $(51 \pm 3.2 \mathrm{pM}, n=21$ over 2 months, Chapter 5). Wall loss has also not been observed to be a problem for cobalt as it has for other metals like zinc (Jakuba et al. 2008, Saito unpubl.). These observations suggest that the loss of Co' observed for the N. E. Atlantic Sta. 5 and 9 may be somewhat unique to seawater from low-oxygen and high deposition environments.

In addition to $\mathrm{Co}^{\prime}{ }_{\text {at-sea }}$ and $\mathrm{Co}^{\prime}$ stored analyses, $\mathrm{Co}^{\prime}$ preserved analyses were also performed for Sta. 9. Interestingly, $\mathrm{Co}^{\prime}$ preserved tended to be higher than $\mathrm{Co}^{\prime}{ }_{\text {at-sea }}$ (Fig. 5b). This increased lability was most pronounced within the oxygen minimum zone (stippled blue area in Fig. 5b), and was not observed in the surface waters. This suggests that there may be a second ligand class that exists predominantly within oxygen minimum zones and 
was subject to removal by the gas absorber satchels ( $\mathrm{CoL}_{\mathrm{OMZ}}$ hereon). As mentioned earlier, we have previously demonstrated that the biologically derived, organic ligands that bind cobalt in seawater have extremely high conditional stability constants and are inert to exchange with the added synthetic ligand (Saito et al. 2005). Defining this ligand class as $\mathrm{CoL}_{\text {organic }}$, the total ligand-bound cobalt concentration $(\mathrm{CoL})$ may be described as:

$\mathrm{CoL}=\mathrm{CoL}_{\text {organic }}+\mathrm{CoL}_{\mathrm{OMZ}}$

and $\mathrm{TCo}_{\text {at-sea }}$ can be described as:

$\mathrm{TCo}_{\text {at-sea }}=\mathrm{Co}^{\prime}{ }_{\text {at-sea }}+\mathrm{CoL}=\mathrm{Co}^{\prime}{ }_{\text {at-sea }}+\mathrm{CoL}_{\text {organic }}+\mathrm{CoL}_{\mathrm{OMZ}}$

In addition to the removal of $\mathrm{O}_{2}$, the absorber satchels are also known to remove $\mathrm{H}_{2} \mathrm{~S}$ and likely other gases present as well (http://www.mgca.com/AGELESS/RPSystemFeatures.html). The observation that the presence of the absorber satchels converted $\mathrm{CoL}_{\mathrm{OMZ}}$ to $\mathrm{Co}^{\prime}$, suggests that a gas phase sensitive functional group such as sulfide, nitric oxide, or nitrous oxide may be part of the $\mathrm{CoL}_{\mathrm{OMz}}$ molecular structure, and that equilibration within the sealed bags could have caused the dissociation of the reactive functional group/s with the removal of any residual gaseous $\mathrm{H}_{2} \mathrm{~S}, \mathrm{NO}$, or $\mathrm{N}_{2} \mathrm{O}$. If the cobalt in $\mathrm{CoL}_{\mathrm{OMZ}}$ is present in the $\mathrm{Co}^{2+}$ state, it would remain in solution after dissociating from the ligand, thus increasing $\mathrm{Co}^{\prime}$ preserved relative to $\mathrm{Co}^{\prime}$ at-sea. This mechanism is consistent with our observations in Fig. $5 b$ (see schematic of mechanism in Fig. 6), and the increase in $\mathrm{Co}^{\prime}$ in the preserved analyses can then be attributed to the conversion of $\mathrm{CoL}_{\mathrm{OMZ}}$ to $\mathrm{Co}^{\prime}$ caused by the satchel preservation:

$\mathrm{Co}^{\prime}{ }_{\text {preserved }}=\mathrm{Co}^{\prime}{ }_{\text {at-sea }}+\mathrm{CoL}_{\mathrm{OMZ}}$ 
In contrast to the increase in $\mathrm{Co}^{\prime}$ caused by the satchel preservation, storage with headspace caused a loss of both the $\mathrm{Co}^{\prime}$ at-sea and $\mathrm{CoL}_{\mathrm{OMz}}$ fractions of $\mathrm{TCo}_{\text {at-sea }}$ (Fig. $5 \mathrm{~b}$, open triangles). At Sta. 9 in the North Atlantic, both $\mathrm{Co}^{\prime}{ }_{\text {at-sea }}$ and $\mathrm{CoL}_{\mathrm{OMz}}$ were not stable over 5 months. By comparison with TCo, these fractions appeared to be equivalent to the TCo lost during storage ( $\mathrm{TCO}_{\text {lost }}$ Fig. 5a blue area):

$$
\mathrm{TCo}_{\text {lost }}=\mathrm{Co}_{\text {at-sea }}^{\prime}+\mathrm{CoL}_{\mathrm{OMZ}}
$$

We also observed the complimentary relationship between the remaining total cobalt after storage $\left(\mathrm{TCo}_{\text {stored }}\right)$ and organically complexed cobalt $\left(\mathrm{CoL}_{\text {organic }}\right)$ :

$\mathrm{TCo}_{\text {stored }}=\mathrm{CoL}_{\text {organic }}=\mathrm{TCo}_{\text {at-sea }}-\mathrm{TCo}_{\text {lost }}$

These relationships are apparent in scatter plots of $\mathrm{CoL}_{\text {organic }} \mathrm{vs} . \mathrm{TCo}_{\text {stored }}$ (Fig. 5c) and $\mathrm{TCo}_{\text {lost }}$ vs. [Co' ${ }^{\prime}$ at-sea $\left.+\mathrm{CoL}_{\mathrm{OMz}}\right]$ (Fig. 5d). The relationship observed was linear, with a slope that was close to 1 , suggesting that the observed loss of $\mathrm{Co}^{\prime}{ }_{\text {at-sea }}$ and $\mathrm{CoL}_{\mathrm{OMz}}$ could account for $\mathrm{TCO}_{\text {lost. }}$. These observations have important implications for our understanding of the chemical structure of cobalt ligands and the subsequent biogeochemical reactions of these molecules in the environment. The agreement between $\mathrm{CoL}_{\text {organic }}$ and $\mathrm{TCo}_{\text {stored }}$ also emphasizes the importance of these strong cobalt-binding ligands as a mechanism for protecting cobalt from scavenging in the water column, even after dramatic changes in $\mathrm{O}_{2}$.

If the cobalt within $\mathrm{CoL}_{\mathrm{OMZ}}$ is present as $\mathrm{Co}(\mathrm{II})$, the influence of increased oxygen in the sample over time may cause the oxidation of $\mathrm{Co}^{2+}$ to $\mathrm{Co}^{3+}$, which would be quickly precipitated due to the exceedingly low solubility of $\mathrm{Co}^{3+}$ in seawater (Morel and Herring 1994). This would suggest that the oxidation of $\mathrm{Co}^{\prime}$ in these solutions occurs on timescales of a couple of months, however; the evidence from the Ross Sea Co' analyses (Table 2) 
and repeat analyses of UV-oxidized seawater suggest that the inorganic oxidation of $\mathrm{Co}^{\prime}$ is a slow process, at least in well oxygenated waters. Given these observations, a mechanism is required to explain the accelerated oxidation and precipitation loss of $\mathrm{Co}^{\prime}$ in low oxygen high deposition waters relative to samples from well oxygenated regions. Perhaps the processes controlling the stability of $\mathrm{Co}^{\prime}$ in low oxygen regions are more directly related to other reactive species that exist in low oxygen environments rather than to the oxygen concentration itself. The study of $\mathrm{B}_{12}$ as a scavenger of potentially harmful NO molecules in biological systems has demonstrated that NO has a strong affinity for the cobalt atom in $\mathrm{B}_{12}$ and can be effectively reduced (Cheng and $\mathrm{Su}$ 1994; Broderick et al. 2005). Porphyrin-based cobalt-containing molecules have been demonstrated to reduce nitrite, nitrate, and nitrous oxide in lab experiments (Banks et al. 1968; Kudrik et al. 2003) and have also been shown to be reduced by nitric oxide on an electrode surface under anoxic conditions (Zheng et al. 2002). Given that these species are typically found under low oxygen conditions, it is possible that their presence and affinity for the cobalt atom in cobalt-containing molecules could catalyze the oxidation of cobalt such that it would be precipitated and removed from the water column in the presence of oxygen. The observation that nitrite can be a good catalyst for cobalt redox reactions has also been demonstrated in electrochemical studies (Bobrowski 1989). If Co' in OMZ waters has an NO group bound to it, this may catalyze the oxidation of that $\mathrm{Co}^{2+}$ to $\mathrm{Co}^{3+}$, when exposed to $\mathrm{O}_{2}$. In the highly oxygenated waters of the Ross Sea, there is likely no NO present and thus could explain the stability of Co' in those waters, and the UV-oxidation step might also remove these species. 
While these results suggest that complications may arise with cobalt analyses, the implications for existing findings in the literature should be small, since the timescales of storage until analysis for most studies have been quite short (days to weeks) and there has not been much detailed work on cobalt in OMZs and high dust environments to our knowledge. The extent to which this loss of TCo documented in this study may also occur in acidified samples is currently unknown, but the finding that even acidified samples require a UV-oxidation step for all currently employed methods of analysis (electrochemical, Saito and Moffett 2001; ICP-MS, Bruland 2010; flow injection, Shelley et al. 2010) suggest that further examination is needed. Development and examination of preservation methods for cobalt speciation studies should be investigated in future studies, since the preservation method documented here is complicated for speciation analysis.

\subsection{Conclusion}

This study documents a novel preservation method of seawater samples for total cobalt analyses to resolve an observed artifact associated with sample storage within OMZ and high dust deposition environments. Benefits of this preservation method are that the technique is simple and easy to carry out, and samples can be analyzed with minimal perturbation, such as freezing or acidification and neutralization that can introduce reagent blanks, and influence precision and recovery. This preservation approach is applicable to the GEOTRACES cruises, which are generating more samples than can be processed shipboard. This preservation method for TCo appears to also serve as a novel tool for the investigation of the chemical speciation and redox properties of cobalt. Addition of gas absorbing satchels causes the removal of strong ligands with a gas phase chemistry, 
implying the importance of such species in OMZ environments. New variables for cobalt analyses are being uncovered, and this emphasizes the importance of continued investigations of the influences on sample integrity, precision, and accuracy for all currently employed analytical methods. 


\section{References for Chapter 6:}

Aguilar-Islas, A. M., J. Wu, R. Rember, A. M. Johansen, and L. M. Shank. Dissolution of aerosol-derived iron in seawater: Leach solution chemistry, aerosol type, and colloidal iron fraction. Marine Chemistry 120: 25-33.

Banks, R. G. S., R. J. Henderson, and J. M. Pratt. 1968. Reactions of gases in solution. Part III. Some reactions of nitrous oxide with transition-metal complexes. Journal of the Chemical Society A: Inorganic, Physical, Theoretical: 2886-2889.

Baxter, L. and others 1998. Electrochemical and Spectroscopic Investigation of the Reduction of Dimethylglyoxime at Mercury Electrodes in the Presence of Cobalt and Nickel. Analytical Chemistry 70: 1312-1323.

Bergquist, B.A., J. Wu, E.A. Boyle. 2007. Variability in oceanic dissolved iron is dominated by the colloidal fraction. Geochim. et. Cosmochim. Acta. 71: 29602974.

Bertrand, E. M. and others 2007. Vitamin $\mathrm{B}_{12}$ and iron co-limitation of phytoplankton growth in the Ross Sea. Limnol. Oceanogr. 52 : 1079-1093.

Bobrowski, A. 1989. Polarographic Methods for Ultratrace Cobalt Determination Based on Adsorption-Catalytic Effects in Cobalt(II)-Dioxime-Nitrite Systems. Analytical Chemistry 61: 2178-2184.

Broderick, K. E. and others 2005. Nitric Oxide Scavenging by the Cobalamin Precursor Cobinamide. Journal of Biological Chemistry 280: 8678-8685.

Bruland, K. W. 2010. GEOTRACES Co Intercalibration Results. http://www.geotraces.org/documents/SAFeReferenceSample-Co.pdf.

Bruland, K. W., M. C. Lohan, 2003. Controls of Trace Metals in Seawater, p. 23-47. In Treatise on Geochemistry. Elsevier. Eds. D. H. Heinrich, and K. T. Karl.

Buck, K. N., and K. W. Bruland. 2005. Copper speciation in San Francisco Bay: A novel approach using multiple analytical windows. Marine Chemistry 96: 185-198.

Buck, K. N., and K. W. Bruland. 2007. The physicochemical speciation of dissolved iron in the Bering Sea, Alaska. Limnol. Oceanogr. (2) 5: 1800-1808.

Buck, K. N., K. E. Selph, and K. A. Barbeau. Iron-binding ligand production and copper speciation in an incubation experiment of Antarctic Peninsula shelf waters from the Bransfield Strait, Southern Ocean. Marine Chemistry 122: 148-159.

Cheng, S.-H., and Y. O. Su. 1994. Electrocatalysis of Nitric Oxide Reduction by WaterSoluble Cobalt Porphyrin. Spectral and Electrochemical Studies. Inorganic Chemistry 33: 5847-5854.

Donat, J. R., and K. W. Bruland. 1988. Direct Determination of Dissolved Cobalt and Nickel in Seawater by differential Pulse Cathodic Stripping Voltammetry Preceded by Adsorptive Collection of Cyclohexane-1,2,-dione Dioxime Complexes. Anal. Chem. 60: 240-244.

Donat, J. R., K. A. Lao, and K. W. Bruland. 1994. Speciation of dissolved copper and nickel in South San Francisco Bay: a multi-method approach. Anal. Chim. Acta 284: 547-571.

Ellwood, M. J., and C. M. G. Van Den Berg. 2001. Determination of organic complexation of cobalt in seawater by cathodic stripping voltammetry. Mar. Chem. 75: 33-47. 
Jakuba, R. W., J. W. Moffett, and S. T. Dyhrman. 2008. Evidence for the linked biogeochemical cycling of zinc, cobalt, and phosphorus in the western North Atlantic Ocean. Global Biogeochem. Cycles 22.

Kudrik, E. V., S. V. Makarov, A. Zahl, and R. Van Eldik. 2002. Kinetics and Mechanism of the Cobalt Phthalocyanine Catalyzed Reduction of Nitrite and Nitrate by Dithionite in Aqueous Solution. Inorganic Chemistry 42: 618-624.

Lane, T.W. and F.M.M. Morel. 2000. Regulation of Carbonic Anhydrase Expression by Zinc, Cobalt, and Carbon Dioxide in the Marine Diatom Thalassiosira weissflogii. Plant. Physiology. doi: 10.1104/pp.123.1.345 123 (1) 345-352.

Lane, T. W., M. A. Saito, G. N. George, I. J. Pickering, R. C. Prince, and F. M. M. Morel. 2005. A cadmium enzyme from a marine diatom. Nature 435: 42.

Measures, C. I. and J. M. Edmond. 1990. Aluminium in the South Atlantic: steady state distribution of a short residence time element. J. Geophys. Res. 95: 5331-5340.

Measures, C. I.. 1995. The distributions of Al in the IOC stations of the eastern Atlantic between $30^{\circ} \mathrm{S}$ and $34^{\circ} \mathrm{N}$. Mar. Chem 49: 267-281.

Measures, C.I., and S. Vink. 2000. On the use of dissolved aluminium in surface waters to estimate dust deposition to the ocean, Global Biogeochemical Cycles, 14, 317-327.

Moore, J. K., S. C. Doney, and K. Lindsay. 2004. Upper ocean ecosystem dynamics and iron cycling in a global three-dimensional model. Global Biogeochem. Cycles 18: GB4028. doi:4010.1029/2004GB002220. .

Morel, F. M. M., and J. G. Hering. 1993. Principles and Applications of Aquatic Chemistry. John Wiley \& Sons Inc.

Mullaugh K. M., and G. W. Luther III. 2011. Growth kinetics and long-term stability of $\mathrm{CdS}$ nanoparticles in aqueous solution under ambient conditions. J. Nanopart. Res. doi: 10.1007/s11051-010-0045-9

Noble, A. E., M. A. Saito, K. Maiti, and C. Benitez-Nelson. 2008. Cobalt, manganese, and iron near the Hawaiian Islands: A potential concentrating mechanism for cobalt within a cyclonic eddy and implications for the hybrid-type trace metals. Deep Sea Res II 55: 1473-1490.

Partensky, F., W. R. Hess, and D. Vaulot. 1999. Prochlorococcus, a Marine Photosynthetic Prokaryote of Global Significance. Microbiology and Molecular Biology Reviews 63: 106-127.

Pohl, C., P. L. Croot, U. Hennings, T. Daberkow, G. Budeus, and M. R. V. D. Loeff. 2011. Synoptic transects on the distribution of trace elements $(\mathrm{Hg}, \mathrm{Pb}, \mathrm{Cd}, \mathrm{Cu}, \mathrm{Ni}, \mathrm{Zn}, \mathrm{Co}$, $\mathrm{Mn}, \mathrm{Fe}$, and $\mathrm{Al}$ ) in surface waters of the Northern- and Southern East Atlantic. Journal of Marine Systems 84: 28-41.

Roberts, S., T. Lane, and F. M. M. Morel. 1997. Carbonic anhydrase in the marine diatom Thalassiosira weissflogii (Bacillariophyceae). J. Phycol. 33 : 845-850.

Saito, M. A., and J. W. Moffett. 2001. Complexation of cobalt by natural organic ligands in the Sargasso Sea as determined by a new high-sensitivity electrochemical cobalt speciation method suitable for open ocean work. Mar. Chem. 75: 49-68.

Saito, M. A., G. Rocap, and J. W. Moffett. 2005. Production of cobalt binding ligands in a Synechococcus feature at the Costa Rica Upwelling Dome. Limnol. Oceanogr. 50: 279-290. 
Saito, M. A., J. W. Moffett, and G. Ditullio. 2004. Cobalt and Nickel in the Peru Upwelling Region: a Major Flux of Cobalt Utilized as a Micronutrient. Global Biogeochem. Cycles 18: doi:10.1029/2003GB002216

Saito, M. A., J. W. Moffett, S. W. Chisholm, and J. B. Waterbury. 2002. Cobalt limitation and uptake in Prochlorococcus. Limnol. Oceanogr. 47: 1629-1636.

Saito, M. A., and D. L. Schneider. 2006. Examination of precipitation chemistry and improvements in precision using the $\mathrm{Mg}(\mathrm{OH})_{2}$ preconcentration ICP-MS method for high-throughput analysis of open-ocean Fe and Mn in seawater. Anal. Chim. Acta 565: 222-233.

Shelley, R. U., B. Zachhuber, P. N. Sedwick, P. J. Worsfold, and M. C. Lohan. 2010. Determination of total dissolved cobalt in UV-irradiated seawater using flow injection with chemiluminescence detection. Limnol. Oceanogr. Meth. 8: 352-362.

Sunda, W. G., and S. A. Huntsman. 1995. Cobalt and Zinc interreplacement in marine phytoplankton: biological and geochemical implications. Limnol. Oceanogr. 40 : 1404-1417.

Vega, M., and C. M. G. Van Den Berg. 1997. Determination of Cobalt in Seawater by Catalytic Adsorptive Cathodic Stripping Voltammetry. Anal. Chem. 69: 874-881.

Yucel, M., A. Gartman, C. S. Chan, and G. W. Luther. Hydrothermal vents as a kinetically stable source of iron-sulphide-bearing nanoparticles to the ocean. Nature Geosci 4: In press.

Zheng, D., L. Yan, and R. L. Birke. 2002. Electrochemical and Spectral Studies of the Reactions of Aquocobalamin with Nitric Oxide and Nitrite Ion. Inorganic Chemistry 41: 2548-2555. 


\section{Tables and Figures for Chapter 6:}

Table 1. Samples presented in this manuscript

\begin{tabular}{|c|c|c|c|c|c|c|c|c|}
\hline Cruise & $\begin{array}{c}\text { Research } \\
\text { Vessel } \\
\end{array}$ & Sample Dates & Lat. $[\mathrm{N}]$ & Long. [E] & Region & Sta. & $\begin{array}{c}\text { Storage time } \\
\text { prior to analysis }\end{array}$ & $\begin{array}{c}\text { Storage } \\
\text { conditions }\end{array}$ \\
\hline CORSACS $^{a}$ & $\begin{array}{l}\text { R/V N.B. } \\
\text { Palmer }\end{array}$ & 1.7 .2006 & -76.00 & -176.62 & Ross Sea & NX6 & 17 months & $4^{\circ} \mathrm{C}$ \\
\hline \multirow[t]{3}{*}{$\mathrm{CoFeMUG}^{\mathrm{b}}$} & R/V/ Knorr & 11.24 .2007 & -12.50 & 345.00 & S. Atlantic Gyre & 7 & 4 months & $4^{\circ} \mathrm{C}$ \\
\hline & & 11.25 .2007 & -12.50 & 350.00 & S. Atlantic Gyre & 9 & 4 months & $4^{\circ} \mathrm{C}$ \\
\hline & & 11.27 .2007 & -12.50 & 355.00 & S. Atlantic Gyre & 11 & 4 months & $4^{\circ} \mathrm{C}$ \\
\hline \multirow[t]{3}{*}{ NAGZT-1 $^{\mathrm{c}}$} & R/V Knorr & 10.22 .2010 & 31.00 & -22.00 & $\begin{array}{l}\text { N.E. Atlantic } \\
\text { (Europe) }\end{array}$ & 5 & 5 months & $4^{\circ} \mathrm{C}$ \\
\hline & & 10.27 .2010 & 17.35 & -18.25 & $\begin{array}{l}\text { N.E. Atlantic } \\
\text { (W. Africa) }\end{array}$ & 9 & 5 months & $4^{\circ} \mathrm{C}$ \\
\hline & & 10.27 .2010 & 17.35 & -18.25 & $\begin{array}{l}\text { N.E. Atlantic } \\
\text { (W. Africa) }\end{array}$ & 9 & 5 months & $\begin{array}{c}4^{\circ} \mathrm{C} \text { and } \\
\mathrm{O}_{2} \text {-sealed }\end{array}$ \\
\hline
\end{tabular}

${ }^{\mathrm{a} C o n t r o l s ~ o n ~ R o s s ~ S e a ~ A l g a l ~ C o m m u n i t y ~ S t r u c t u r e ~}$

${ }^{\mathrm{b} C o b a l t}$ iron and microorganisms from the upwelling to the gyre

cNorth Atlantic Geotraces Zonal Transect Leg 1

Table 2. Speciation analyses of Ross Sea sample NX3-3

\begin{tabular}{cc}
\hline Analaysis Date & $\mathrm{Co}^{\prime}[\mathrm{pM}]$ \\
\hline 1.8 .06 & 17 \\
10.25 .10 & 15 \\
11.4 .10 & 14 \\
10.18 .10 & 19 \\
\hline Average $=$ & 16 \\
Stdev $=$ & 2.6
\end{tabular}


Figure 1) Comparison of $\mathrm{TCo}_{\text {at-sea }}$ (black circles) to $\mathrm{TCo}_{\text {stored }}$ (white circles) for 6 stations from three cruises. The dissolved oxygen is plotted as a grey line with a separate $\mathrm{x}$ axis, and the vertical black line between 2000-3000 m denotes the depth range where evidence of a hydrothermal is observed in the dissolved iron and manganese data (Saito et al. in review). The CoFeMUG stations are from the South Atlantic Gyre, The NAGZT stations are from the North East Atlantic, and the CORSACS station is from the Ross Sea. The top panel shows a zoom in of the upper $300 \mathrm{~m}$ with the full profile displayed beneath. The bottom panel shows a scatter plot comparison of $\mathrm{TCo}_{\text {at-sea }}$ to $\mathrm{TCo}_{\text {stored }}$ for each corresponding station (grey circles). The datapoints that fall along the 1:1 line (dashed black line) showed good reproducibility during the analysis of stored samples. 


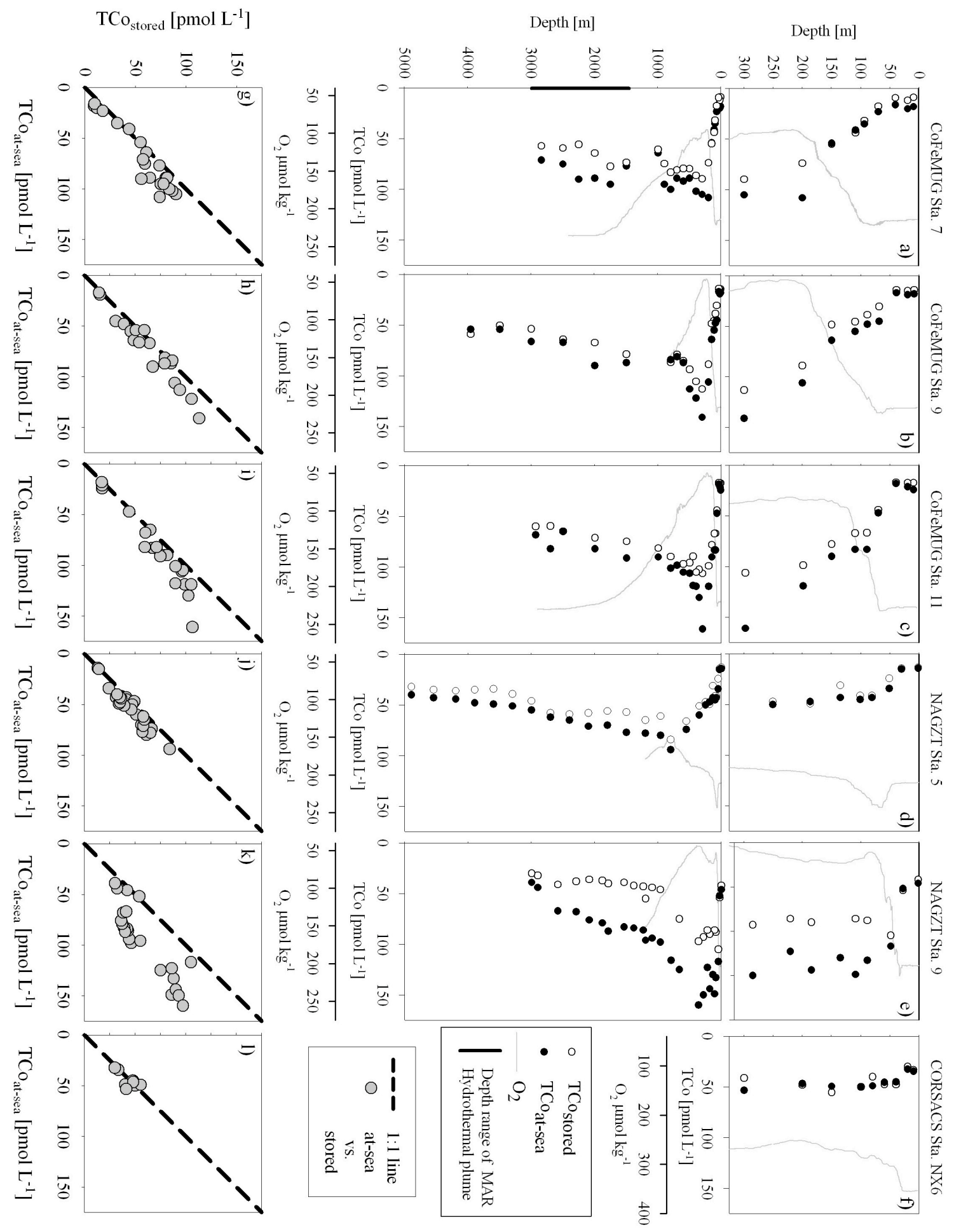



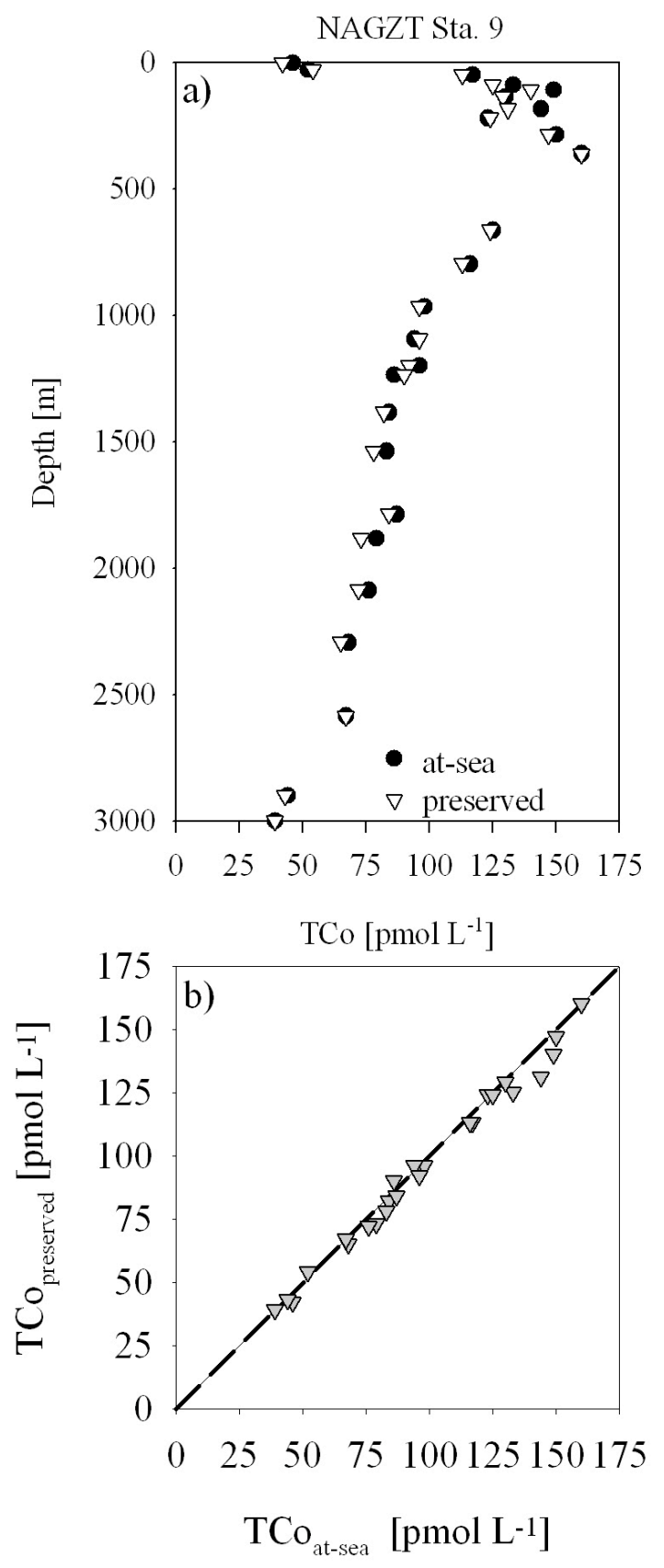

Figure 2) Comparison of $\mathrm{TCO}_{\text {at-sea }}$ (black circles) to $\mathrm{TCo}_{\text {preserved }}$ (white triangles) for Sta. 9 from the Eastern North Atlantic. Excellent reproducibility is observed using this preservation method. The data are presented in a) profile format, and b) scatter plot format, showing that most data fall along the 1:1 line. 

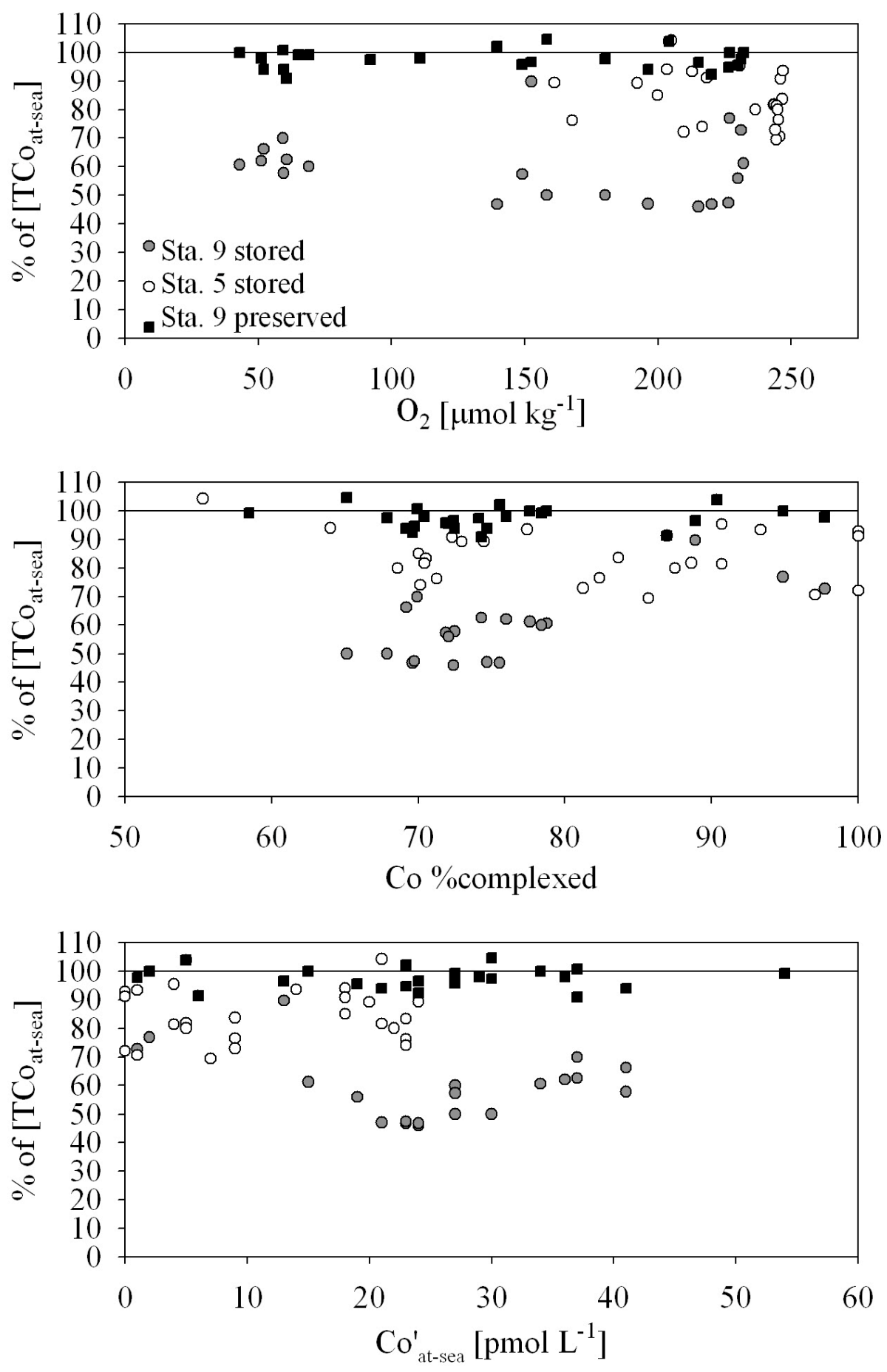

Figure 3) Percent of cobalt lost compared to at-sea analysis versus a) oxygen concentration b) percent of total cobalt that is complexed to strong organic ligands, and c) labile cobalt for NAGZT dataset. The preserved samples show good agreement with $\mathrm{TCo}_{\text {at-sea }}$ across a large range of oxygen concentrations, and the relationship between $\mathrm{TCo}_{\text {at-sea }}$ and $\mathrm{TCo}_{\text {stored }}$ analyses does not appear to be consistently related to dissolved oxygen, in the seawater. 

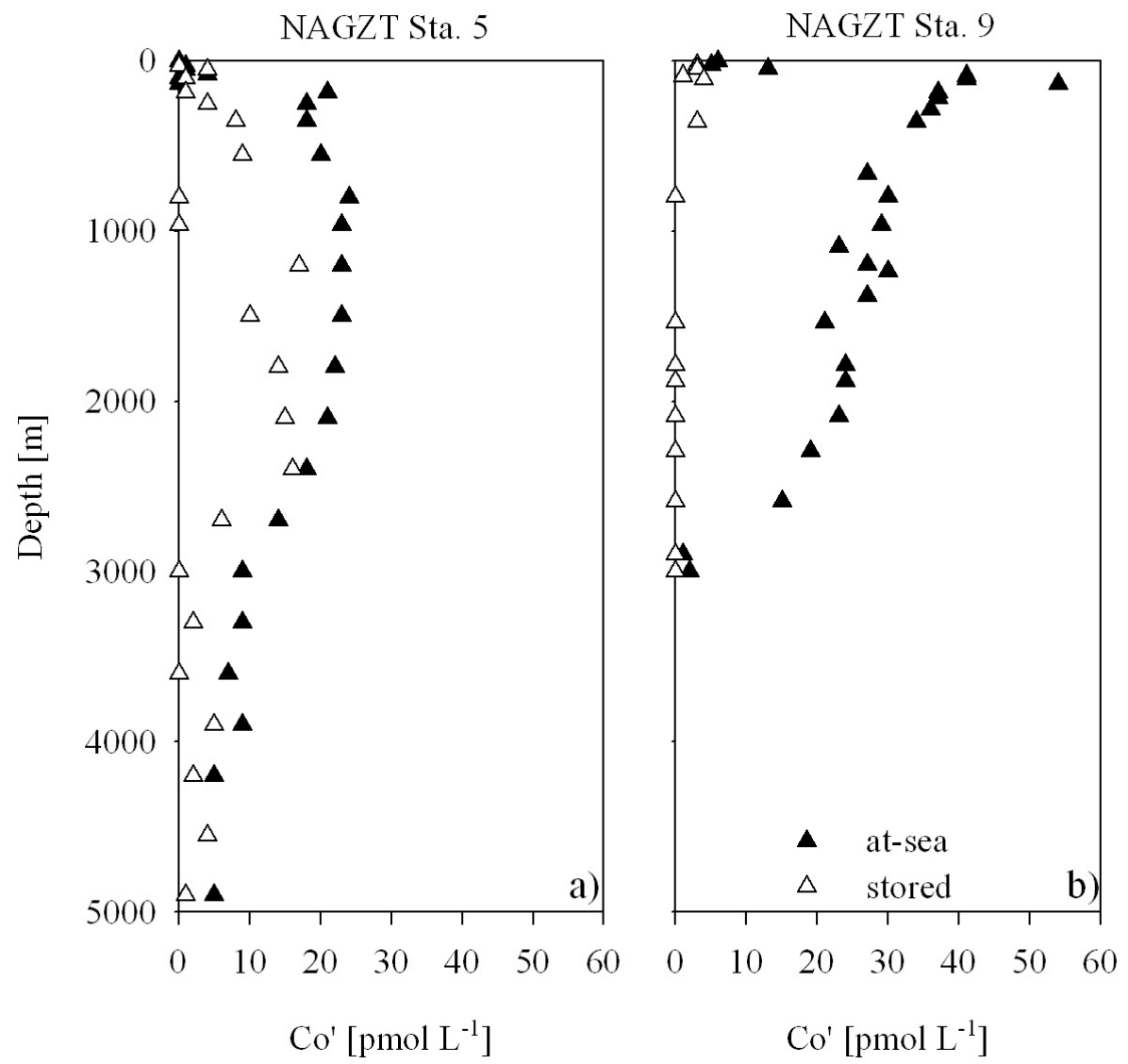

Figure 4) Comparison of $\mathrm{Co}^{\prime}$ at-sea (black triangles) to $\mathrm{Co}^{\prime}$ stored (white triangles) analyses for Sta. 5 and 9 from the NAGZT expedition in profile format (a), and in scatter plot format (b). These results suggest that either the chemical speciation of cobalt has changed considerably, or cobalt has been lost from the sample. 

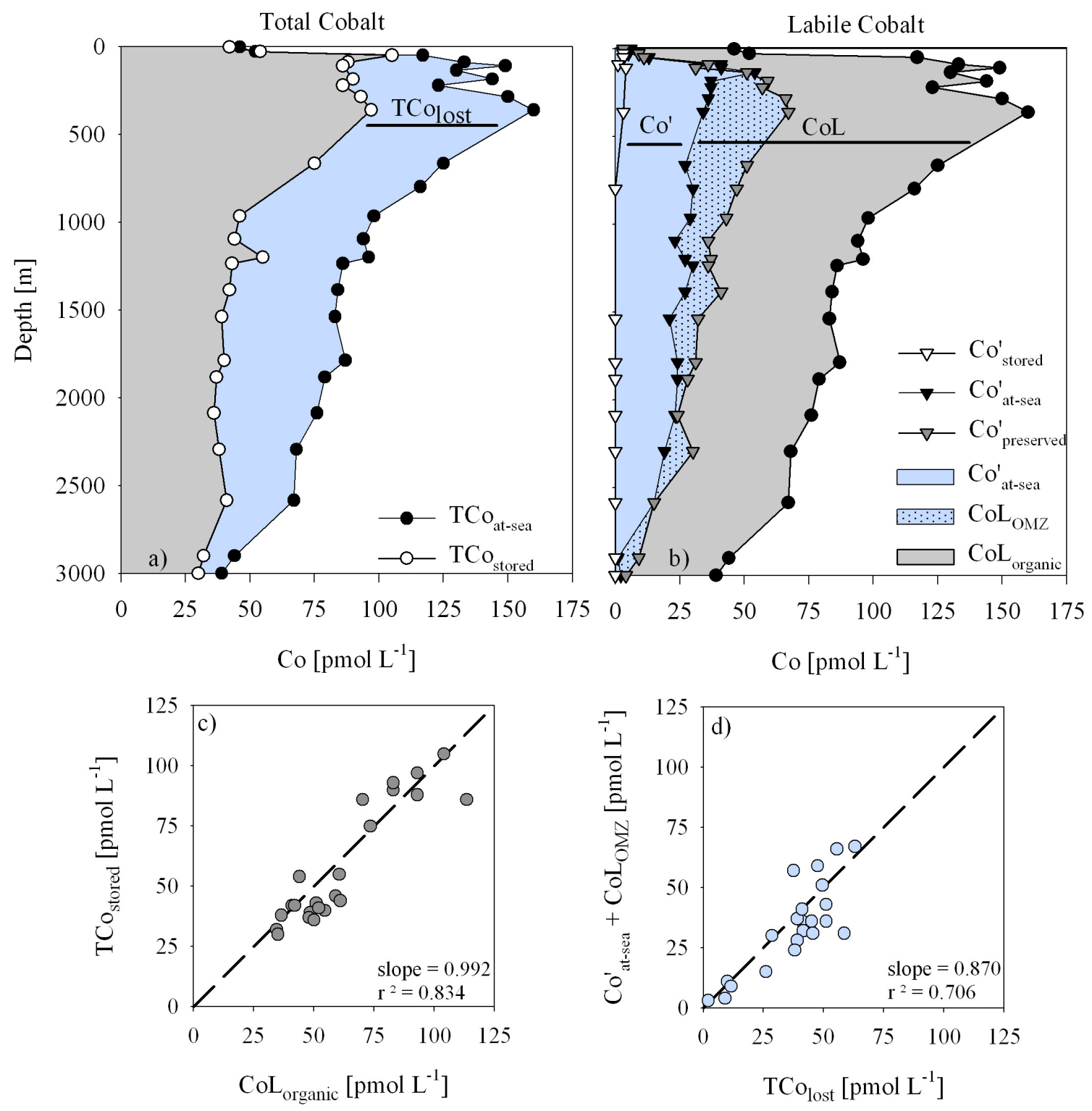

Figure 5) a) Profiles of $\mathrm{TCo}_{\text {at-sea }}$ and $\mathrm{TCo}_{\text {stored }}$ at Sta. 9 compared to b) profiles of the labile and complexed fractions of cobalt. The blue shaded areas and grey shaded areas correlate well when plotted against each other in c) and d), demonstrating how speciation can influence the storage of these samples with time. 


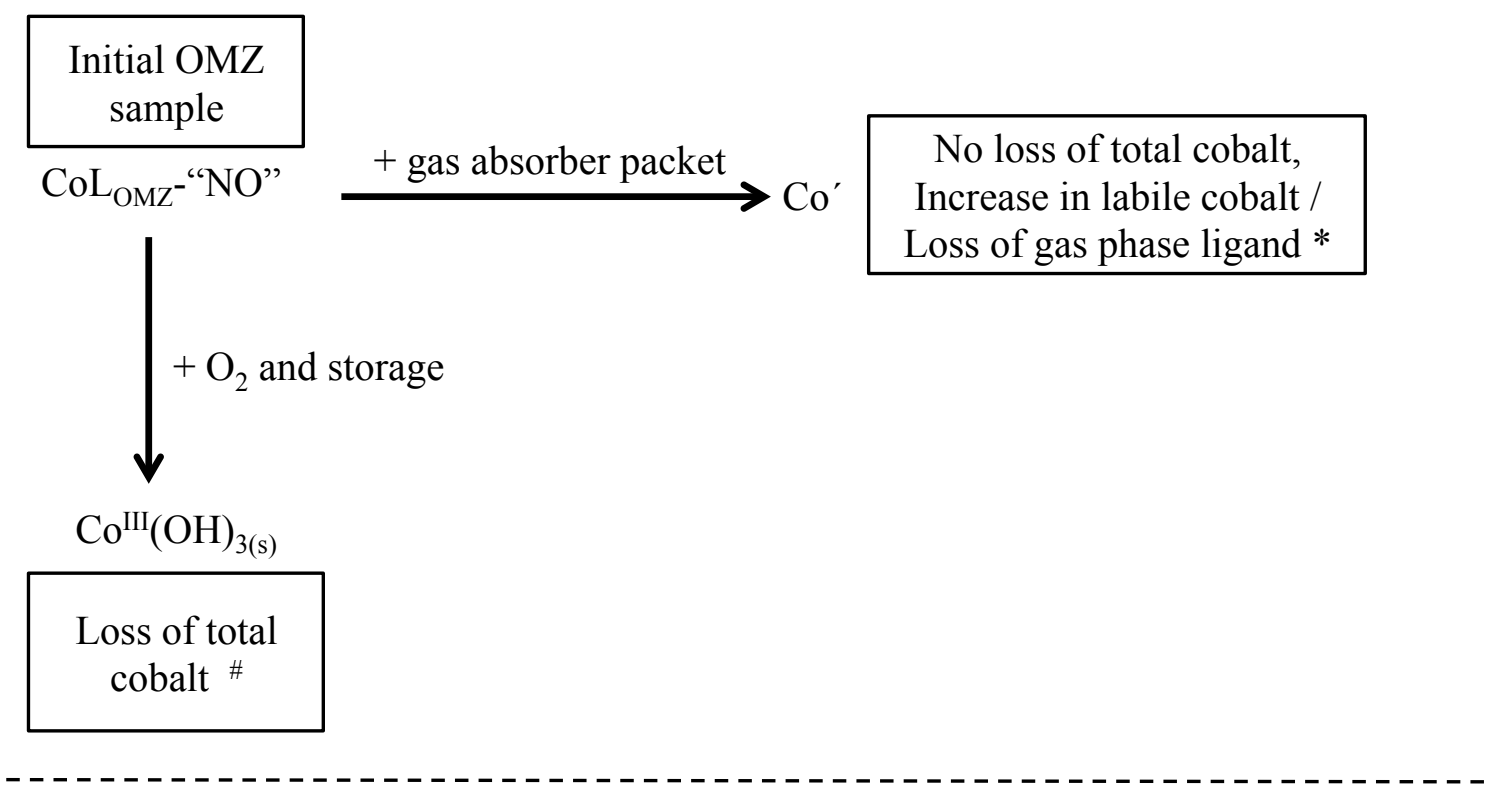

*possible reactions

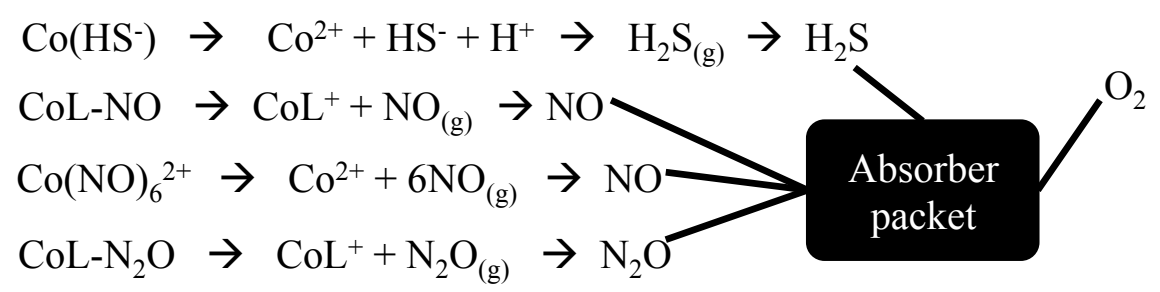

\# likely effect of increased $\mathrm{O}_{2}$ on fractions of TCo

$$
\begin{aligned}
& \mathrm{Co}^{\mathrm{II}}+\mathrm{O}_{2} \rightarrow \mathrm{Co}^{\mathrm{III}}(\mathrm{OH})_{3(\mathrm{~s})} \\
& \mathrm{Co}^{\mathrm{II}} \mathrm{L}_{\mathrm{OMZ}}+\mathrm{O}_{2} \rightarrow \mathrm{Co}^{\mathrm{III}}(\mathrm{OH})_{3(\mathrm{~s})} \\
& \mathrm{Co}^{\mathrm{III}} \mathrm{L}_{\text {organic }}+\mathrm{O}_{2} \rightarrow \text { no reaction, no loss of this fraction } \\
& \mathrm{K}_{\mathrm{sp}\left(25^{\circ} \mathrm{C} \text { in water }\right)}=[\mathrm{Co}][\mathrm{OH}]^{3} / \mathrm{Co}(\mathrm{OH})_{3(\mathrm{~s})}=10^{-44}
\end{aligned}
$$

Figure 6. Schematic for possible mechanisms of cobalt loss and behavior of $\mathrm{CoL}_{\mathrm{OMZ}}$ 


\title{
Appendix I: Prospects for a multi-element method to measure cobalt, iron, and manganese concentrations in seawater via inductively-coupled plasma mass spectrometry
}

\begin{abstract}
The importance of trace metals in global biogeochemical cycling as micronutrients, toxins, and process tracers motivates the development of methods to measure them accurately and efficiently. This study focuses on the potential for inductively-coupled plasma mass spectrometry (ICP-MS) to facilitate a multi-element analytical method that would include cobalt. With streamlined analytical methods, the need for greater resolution and oceanographic coverage of trace metals can be achieved (e.g. as proposed in the international GEOTRACES program). The low concentrations in seawater, often laborious sample workup, and high potential for sample contamination, however, tend to limit sample throughput, and make method development particularly challenging. Like iron, cobalt is a "hybrid-type" element, and is affected by both biological and abiotic processes such as uptake by phytoplankton and particle scavenging (Bruland and Lohan 2003, Noble and Saito et al. in press) and should be considered in multi-trace-element analytical methods. Here, a previously described method to measure total iron and manganese concentrations in seawater (Wu and Boyle 1998, Saito and Schneider 2006) has been modified for the analysis of cobalt at the picomolar level. Three major findings from this study are discussed. First, low detection limits for measuring iron have been previously achieved by isotope dilution and preconcentration by coprecipitation, but isotope dilution is not an option for elements with only one stable isotope, like manganese and cobalt. For manganese, the ${ }^{57} \mathrm{Fe}$ isotope spike can be used as an internal standard because the sample preparation recovery is reproducible and near complete. This use of ${ }^{57} \mathrm{Fe}$ as an internal standard has not been possible for cobalt due to low sample recovery (Saito and Schneider 2006). The work presented here demonstrates that with a greater than or equal to $8 \mathrm{mg}$ precipitate and the addition of at least $250 \mu \mathrm{L}$ ammonia, cobalt recovery is increased to acceptable analytical levels $(88 \%)$. Secondly, the large precipitate requires additional steps to remove salt matrices that interfere with sample introduction and analysis on the ICP-MS. The investigation of binding capacities and capabilities of four commercially available resins (Chelex100, NTA-Histidine, NTA-Superflow, and Toyopearl) suggested that Toyopearl resin was superior for the objectives of this study. The use of a chelating resin successfully removes interfering matrices and produces high precision in standard additions of $0.1 \mathrm{nM}\left(\mathrm{r}^{2}=0.998\right)$ with excellent reproducibility $( \pm 3 \mathrm{pM}, \mathrm{n}=5)$. Thirdly, previous ICP-MS methods rely on acidification to dissociate all organic metal-ligand complexes in stored samples. A comparison of UV-oxidized, acidified seawater to acidified seawater shows a $16 \mathrm{pM}$ systematic offset, suggesting that acidification may not sufficiently dissociate all cobalt-ligand complexes. This is not unreasonable as the conditional stability constant of cobalt-binding ligands is high (on the order of $10^{16.3}$ or higher, Saito and Moffett 2001). While accuracy and blanks are still works in progress, the relative merits of this method are discussed, specifically with regards to electrochemical methods and the importance of cobalt as a trace element of interest to biogeochemical studies.
\end{abstract}




\section{Introduction}

With the successful marriage of biochemical and trace metal oceanographic research, the importance of bioactive trace metals to marine phytoplankton ecology is now evident (Martin et al. 1989, Price and Morel 1990, Price and Morel 1991, Morel et al. 1994, Sunda and Huntsman 1995, Fitzwater et al. 1996, Saito and Moffett 2001, Saito et al. 2002a, Morel et al. 2003, Morel and Price 2003, Saito et al. 2004, Lane et al. 2005). Many trace metals are known to follow biological processes as micronutrients, particle reactive elements, or tracers (GEOTRACES science plan 2006). As the poster-child for the importance of trace metal biogeochemical cycling, iron is accepted as the limiting nutrient in a large part of the ocean (Moore et al. 2004). It is required for photosynthesis and respiration in phytoplankton, and in many aspects of the biological nitrogen cycle as demonstrated by lab studies (Morel et al. 2003, and references therein). Field studies have also observed iron to limit phytoplankton growth in macronutrient rich waters due to its requirements in these enzymatic processes (Martin et al. 1989, Martin et al. 1991, Fitzwater et al 1996). These biological demands, coupled with its extremely low seawater solubility, make iron a difficult element to ignore in ocean biological cycles. In recent years, many other trace elements have joined the ranks as micronutrients (though most to a lesser degree than iron), including zinc, nickel, cobalt, cadmium, manganese and copper (Price and Morel 1990, Morel et al. 1994, Moffett 1995, Moffett and Brand 1996, Morel et al. 2003). Cobalt can serve as a micronutrient by occupying the active site of carbonic anhydrase in marine phytoplankton (Price and Morel 1990, Sunda and Huntsman 1995, Saito et al. 2002a). While the cyanobacterial species Prochlorococcus and Synechococcus have an absolute requirement for cobalt, the diatoms Thalassiosira pseudonana and Thalassiosira oceanica have the ability to use both cobalt and zinc to fulfill some biochemical metal requirements (Sunda and Huntsman 1995, Saito et al. 2002).

In addition to their biological functions, both iron and cobalt are considered hybridtype elements, also affected by scavenging and other abiotic processes (Bruland and Lohan 2003, Noble and Saito et al. in press). In contrast to micronutrients like zinc and cadmium, present at nanomolar concentrations and tending to have nutrient-like depth profiles, iron and cobalt are present at picomolar concentrations in open ocean profiles with iron reaching maxima between 1-2nM and cobalt reaching maxima $\sim 200 \mathrm{pM}$ (Morel et al. 2004, Boyle et al. 2005, Noble and Saito et al. in press). Due to these low concentrations and scavenging processes, small perturbations can be seen in depth profiles, greatly complicating the simpler explanations of uptake and remineralization used to describe typical zinc and cadmium profiles. A number of studies demonstrate the variety of influences on cobalt and iron: eddy processes have been observed to concentrate cobalt below the euphotic zone in oligotrophic waters off Hawaii, and also to draw iron off the coast and stimulate blooms in high nutrient, low chlorophyll (HNLC) waters in the Gulf of Alaska (Johnson et al. 2005, Noble and Saito et al. in press). Groundwater or potential input from shelf sediments has also been observed for cobalt at $\sim 400-600 \mathrm{~m}$ depth off Hawaii, and the nearby Loihi Seamount has been traced as a source for iron to deepwater at Station ALOHA (Noble and Saito et al. in press, Boyle et al. 2005). Microbial cooxidation of cobalt by manganese-oxidizing bacteria has been observed, lateral delivery of 
particulate iron has been traced to continental margin sources $900 \mathrm{~km}$ away, and these are just a handful of examples (Tebo et al. 1984, Moffett and Ho 1996, Lam et al. 2006).

With such a variety of inputs and removal mechanisms affecting these micronutrients, there has been an increasing demand for high resolution global coverage of the oceans for bioactive trace metals. Culture studies, bottle incubations, and discrete depth profile analyses have identified the importance of trace metals to ocean biogeochemistry, but a sophisticated understanding of the larger cycles of these elements must be attained through global scale sampling. It is important to know where these elements limit growth, how they behave throughout the water column, and how these behaviors change in different chemical environments across the world oceans. Attempts have been made to model the world oceans for iron; however, it is clear that as a scientific community, we are still "data poor," and the well-crafted models are only as good as the quantity and quality of the data with which they are supplied (Johnson et al. 1997, Fung et al. 2000, Archer and Johnson 2000, Moore et al. 2004, Parekh et al. 2004, Weber et al. 2007).

Addressing this question of quantity and quality is an overarching goal of the recently launched GEOTRACES program, an international initiative to map the world oceans for trace elements. Similar to the World Ocean Circulation Experiment (WOCE) and the Joint Global Ocean Flux Study (JGOFS), this program will divide the world oceans into sections, sampling across ocean basins for many trace elements and isotopes (GEOTRACES

Science Plan 2006). Samples and analyses from this program will supply data to flesh out existing models for trace element cycling. For this program to be successful, it is imperative that analytical techniques be intercalibrated, efficient, and streamlined.

Trace metal analysis is difficult due to extremely low seawater concentrations and the high potential for contamination. While many elements can be simultaneously measured in coastal samples via ICP-MS (Field et al. 1999), the extremely low concentrations in open ocean samples prohibit the measurement of many trace metals without preconcentration. Current methods generally include electrochemical techniques such as anodic or cathodic stripping voltammetry (ASV or CSV) and inductively-coupled plasma mass spectrometry (ICP-MS). Electrochemical methods have been quite successful, especially for the determination of labile metals (Cd, Bruland et al. 1985; Fe, Rue and Bruland 1995; Cu, Moffett 1995; Co and Ni, Saito and Moffett 2001; Zn, Ellwood 2004), but these methods are time consuming because they use standard addition and many require a preconcentrating conditioning step. ICP-MS methods can be more efficient, although dissolved salts in seawater affect instrument performance, and salts must be removed by additional processing steps which increase potential for contamination.

A variety of ICP-MS methods for measuring different trace elements in seawater have emerged in recent years, demonstrating the great potential for a multi-trace-element analytical method using this approach. These methods have used resin preconcentration coupled with flow injection (Wells and Bruland 1998, Lohan et al. 2005, Rahmi et al. 2007), coprecipitation with magnesium hydroxide (Wu and Boyle 1997, Wu and Boyle 1998, Saito and Schneider 2006, Wisniewski 2006), and coprecipitation with gallium (Akagi 1985). Focusing on that potential, this study discusses the optimization of a method to measure cobalt in seawater with the possibility of expanding the method to include $\mathrm{Zn}, \mathrm{Fe}, \mathrm{Cd}, \mathrm{Mn}, \mathrm{Cu}$, and $\mathrm{Ni}$. The GEOTRACES program will generate thousands 
of samples that need to be analyzed for many metals, so the development of a method that could achieve simultaneous measurement of many metals is desirable. Sample volume, preservation, and efficiency of analysis are issues of concern that that are explored here. The elements included here are chosen based on their low concentrations in seawater (pM$\mathrm{nM}$ ), and their aforementioned importance in ocean biogeochemical cycles. This study demonstrates that measurement of cobalt by ICP-MS may be possible by coupling coprecipitation and resin techniques.

\section{Materials and Methods}

2.1. Trace metal clean sampling and preservation - Seawater used for experiments was collected with acid-cleaned, teflon-coated 10L Go-Flo bottles (General Oceanics Inc.) deployed on Kevlar wire. The Go-Flo bottles were tripped with epoxy-coated lead messengers. Immediately following collection, Go-Flo bottles were pressurized with $99.999 \% \mathrm{~N}_{2}$ at $\sim 5 \mathrm{psi}$ and the seawater was filtered through $0.4 \mu \mathrm{m}, 142 \mathrm{~mm}$, polycarbonate filters into acid-washed polyethylene or polycarbonate carboys in a positive pressure class 100 clean environment. Samples were acidified to $\mathrm{pH} 1.7$ using Seastar grade HCl. Additional samples were preserved unacidified and refrigerated for electrochemical measurements. Acidified seawater was used for all experiments unless otherwise stated. All tubing and filters were vigorously acid-washed prior to use. Sampling bottles and carboys had been soaked overnight in acidic detergent, Citranox, rinsed thoroughly with Milli-Q water (Millipore), filled with $10 \% \mathrm{HCl}$ to soak for 10 days, and rinsed with Milli$\mathrm{Q}$ water adjusted to $\mathrm{pH} 2$ before used to store seawater.

2.2. Magnesium-hydroxide preconcentration - The concentration of metals onto a pellet by co-precipitation with magnesium-hydroxide has been previously described (Wu and Boyle 1997, Wu and Boyle 1998, Saito and Schneider 2006). Briefly, 13.0mL of acidified seawater were measured gravimetrically into acid-washed polypropylene centrifuge tubes to avoid pipette contamination, spiked with $100 \mu \mathrm{L}$ of an ${ }^{57} \mathrm{Fe}$ solution, and allowed to equilibrate overnight. Seastar grade concentrated ammonia was added to induce $\mathrm{Mg}(\mathrm{OH})_{2}$ and trace metal co-precipitation into a pellet. This was accomplished by allowing the precipitate to form for 90s undisturbed followed by inverting the sample 6 times to mix the precipitate throughout, letting it precipitate undisturbed for another $90 \mathrm{~s}$, and centrifuging for 3 minutes at 3000rpm (Brinkmann 5810 swinging bucket). The supernatant was decanted, the sample was centrifuged a second time to further remove residual seawater, and the pellet was re-dissolved in $500 \mu \mathrm{L}$ of $5 \%$ Seastar grade nitric acid spiked with trace amounts of indium. The 5\% Seastar grade nitric acid resuspension solution was used as a blank, and signal suppression due to matrix effects was accounted for by using a ratio of the indium signals for the blank and sample to calibrate the effective blank of each isotope for each sample. For all the radioactive ${ }^{57} \mathrm{Co}$ experiments, this method was modified, eliminating the ${ }^{57} \mathrm{Fe}$ spike, and samples were measured using counting techniques (see below) as opposed to measuring by ICP-MS. All other samples were treated as described above unless otherwise stated.

2.3. Inductively coupled plasma mass spectrometry - A Thermo-Finnigan Element 2 inductively coupled plasma mass spectrometer (ICP-MS) equipped with X skimmer cones was used for all mass spectrometry measurements. An Aridus desolvating sample introduction system (Cetac Technologies) was coupled with a low-flow PFA-Teflon 
nebulizer (Elemental Scientific Inc.) and an ASX-100 auto sampler (Cetac Technologies) to perform all ICP-MS measurements.

2.4. Calculation of radioactive Co concentrations / counting techniques - The half-life of ${ }^{57} \mathrm{Co}$ is 271.8 days. A $1.2 \mathrm{mCi} / \mathrm{L}\left(3 \mathrm{nM}{ }^{57} \mathrm{Co}\right)$ stock dilution was made in Milli-Q water from a ${ }^{57} \mathrm{CoCl}_{2}$ in $0.1 \mathrm{M} \mathrm{HCl}$ stock (Isotope Products Laboratories). ${ }^{57} \mathrm{Co}$ counting was performed at $122 \mathrm{keV}$ on a Canberra Germanium Gamma Detector. Aliquots of acidified seawater $(10 \mathrm{~mL})$ were spiked with $5-20 \mathrm{pM}{ }^{57} \mathrm{Co}$ by the addition of $20-100 \mu \mathrm{L}$ of the stock. Scavenging efficiency was determined by comparing the activity of ${ }^{57} \mathrm{Co}$ in the seawater prior to precipitation to that of the resuspended coprecipitate. Percent recovery for resin studies was determined by comparing the activity of ${ }^{57} \mathrm{Co}$ in resuspended coprecipitate with that of the final processed sample. Because results are reported in percent and samples were processed from start to finish within 24 hours of preparation, decay of ${ }^{57} \mathrm{Co}$ was considered negligible and was not taken into account when calculating recovery because the processing time was significantly less than the half-life of the radioisotope. The activity of the stock was frequently recalculated during the study period to ensure that the concentration range examined remained within $5-20 \mathrm{pM}{ }^{57} \mathrm{Co}$.

2.5. Electrochemical determination of total cobalt - A previously described method to measure cobalt via cathodic stripping voltammetry (CSV) (Saito and Moffett 2001) was used to measure field samples for intercalibration and comparison with the method described here. Briefly, unacidified, refrigerated seawater was UV oxidized to destroy any natural ligands prior to measurement with a hanging mercury drop electrode. An electroactive ligand, dimethylglyoxime, was added to bind the cobalt and adsorb to the mercury drop upon applying a negative potential to the cell. The complex was reduced off the drop by ramping the cell towards a more negative potential and concentration was determined by sequential addition of a known standard.

\subsection{Experimental Designs}

2.6.1 Study of ammonia addition - A batch of acidified seawater was spiked with ${ }^{57} \mathrm{Co}$, and $10 \mathrm{~mL}$ aliquots were each precipitated in duplicate with increasing amounts of Seastar grade ammonia to examine the effect of precipitate size on cobalt scavenging efficiency. Precipitations were performed as described above (Section 2.2) and pellets were resuspended and assessed for ${ }^{57} \mathrm{Co}$ scavenging efficiency by gamma detection.

2.6.2 Multi-variable experiment - The range between the additions of $250 \mu \mathrm{L}-400 \mu \mathrm{L}$ ammonia was investigated, as well as manipulating a number of variables to identify potential changes that could further optimize scavenging efficiency. Variables included UV oxidation for $1 \mathrm{hr}$ (705 UV digester, Metrohm), equilibration of ${ }^{57} \mathrm{Co}$ with seawater, and drying the pellet prior to resuspension. Scavenging efficiency was assessed as described in the previous section.

\subsubsection{Comparison of four commercially available resins and volume of resin - Four} commercially available chelating resins were examined for binding capacity and efficiency. Sample resuspensions were prepared by the addition of ${ }^{57} \mathrm{Co}$ to acidified seawater, precipitation with $350 \mu \mathrm{L}$ ammonia, and resuspension with $2 \mathrm{~mL}$ dilute acetate buffer to maintain a $\mathrm{pH}$ between 4 and 7 . Resins were acid-washed and restored to neutral $\mathrm{pH}$ with the addition of dilute trace metal grade $\mathrm{NaOH}$. Resins were added to each resuspension in 
differing quantities and were allowed to equilibrate overnight. Aliquots of each equilibrated solution were subsampled to assess binding to the resin.

2.6.4 Resin retention and $\mathrm{pH}$ sensitivity - The use of a synthetic chelator bound to resin, Chelex-100 (BioRad) was tested to determine the ability to remove salt matrices. Sample precipitates were prepared by addition of ${ }^{57} \mathrm{Co}$ to acidified seawater and precipitation with $350 \mu \mathrm{L}$ ammonia. Precipitates were resuspended to differing $\mathrm{pH}$ values using a $2 \%$ solution of $\mathrm{HCl}$. Chelex-100 was added to the solutions and allowed to equilibrate overnight. The resin was then washed a number of times, and metals were finally leached from the resin with 5\% nitric acid (Seastar). Washes, resuspension, leachate, and resin were all counted for ${ }^{57} \mathrm{Co}$ activity to follow the retention and loss of ${ }^{57} \mathrm{Co}$ at each stage of the process.

2.6.5 Limitations on resin binding rates: kinetic and equilibrium experiments - The investigation of equilibration time versus available binding sites due to cation exchange was studied using two resins. Sample resuspensions were prepared as described above in Section 2.6.3. To assess equilibration time, resin was added to the resuspension solution, and solution was subsampled over time to determine percent ${ }^{57} \mathrm{Co}$ bound to resin until no further changes were observed. To assess the observable rates of binding, resuspension solution and Milli-Q water were alternately and repeatedly run through resin columns, and resuspension solution was subsampled following each load. Milli-Q washes were intended to remove excess magnesium due to the high concentration in seawater and subsequent precipitation.

2.6.6 Precision and accuracy when applied to ICP-MS - Viability of the method was tested on the ICP-MS by performing a standard addition on acidified seawater collected from the Costa Rica Upwelling Dome. Seawater was weighed into $15 \mathrm{~mL}$ trace metal clean centrifuge tubes. A Co standard (Fisher Scientific) was added in 100pM increments and an ${ }^{57} \mathrm{Fe}$ isotope spike was added to each sample to be used as an internal standard. $\mathrm{Mg}(\mathrm{OH})_{2}$ precipitates were formed using $350 \mu \mathrm{L}$ of concentrated Seastar ammonia. EPPS buffer $(0.25 \mathrm{M})$ or acetic acid (1\% in Milli-Q water) was used to resuspend the pellets.

Resuspensions were transferred to resin columns to which a chelating resin was added and allowed to equilibrate. Following equilibration, resins were washed thoroughly with MilliQ water and eluted with 5\% nitric acid (SeaStar) into acid-washed autosampler tubes to be measured for total metals. A field sample was also measured in quintuplicate for precision and reproducibility.

2.6.7 Vertical profile comparison to an electrochemical method - A depth profile from the Ross Sea was measured on acidified seawater using the method described in Section 2.6.6 without the addition of cobalt. A duplicate profile was measured with the exception that samples were UV-oxidized for $1 \mathrm{hr}$ prior to precipitation (705 UV Digester, Metrohm). A third replicate profile was measured on unacidified, refrigerated samples using a previously described CSV method (Saito and Moffett 2001). These profiles were compared for accuracy with the profile that was measured at the time of sampling by CSV (Saito et al. 2010, Saito and Moffett 2001).

\section{Results and Discussion}

As proposed by Saito and Schneider (2006), there is potential (and perhaps even a demand) to develop a method for measuring cobalt in seawater via ICP-MS. This potential 
is complicated by the determination that cobalt was poorly scavenged during $\mathrm{Mg}(\mathrm{OH})_{2}$ coprecipitation, a necessary preconcentrating step in the analysis of Fe and $\mathrm{Mn}$ in previously described methods (Saito and Schneider 2006). The primary goals of the study described here were to 1) optimize cobalt scavenging efficiency and 2) determine the feasibility of an ICP-MS method to measure total dissolved cobalt in acidified seawater samples. Optimization of scavenging efficiency was investigated by determining the effects of complex formation, precipitation time, and precipitate size on scavenging. After optimization was achieved, feasibility studies of an ICP-MS method focused on the ability to generate a concentrated sample that was compatible with the mass spectrometer. Trace metal samples introduced to the ICP-MS are required to be in an acidic solution and have a low dissolved salt load (Dave Schneider, personal communication). The results described here demonstrate the feasibility and precision of the method. Accuracy is still a work in progress.

3.1 Optimization Studies - Because ICP-MS instrument time is expensive and method development tends to require much iteration, this study took advantage of the radioisotope ${ }^{57} \mathrm{Co}$. Exhibiting the same chemical properties as stable ${ }^{59} \mathrm{Co},{ }^{57} \mathrm{Co}$ has the advantages of easy measurement at low concentrations by gamma detection, a long halflife (271.8 days) relative to the timescale of the studies ( $\sim 24$ hours), and no significant health risks due to low energy and small quantities used. By spiking seawater samples with a known activity of ${ }^{57} \mathrm{Co}$, each step of the method can be tracked for loss and recovery of the radioisotope by comparing the activity of subsampled aliquots to the measured initial activity. This approach was efficient and cost effective for method development.

The first experiments examined the effect of larger precipitates on cobalt recovery. The working hypothesis was that if the chemical properties of cobalt prevent it from scavenging as efficiently as iron and manganese, a larger induced precipitate may allow for increased cobalt scavenging. This was tested both by allowing the precipitate to form for a longer period of time (data not shown, see Appendix 1b), and by adding more ammonia (Figure 1). The largest precipitates were generally achieved with larger additions of ammonia, and the increase in precipitate size significantly increased the scavenging efficiency of cobalt into a pellet. The addition of between 250 and $400 \mu \mathrm{L}$ fresh ammonia resulted in a plateau of $\sim 90 \%$ recovery of cobalt onto the pellet. This implies that optimal scavenging is achieved at precipitate masses greater than or equal to $8 \mathrm{mg}$, ammonia additions greater than $200 \mu \mathrm{L}$, and a supernatant $\mathrm{pH}$ above 10.3 . While the relative strength of the ammonia solutions cannot be directly compared, these results are consistent with previous observations, which considered precipitates formed with the addition of $160 \mu \mathrm{L}$ ammonia and resulted in $\sim 20 \%$ cobalt recovery (Saito and Schneider 2006).

Several hypotheses arose from the observed plateau in scavenging efficiency, and an experiment was designed to investigate the nuances of the precipitation step in which three variables were tested. The first variable, drying the pellet prior to resuspension, was tested with the hypothesis that residual liquid associated with the pellet might be introducing dilution volume error. This would lead to lower apparent efficiencies due to underestimation of dilution when back calculating the activity of the resuspension. The second variable examined whether the ${ }^{57} \mathrm{Co}$ added at the beginning of the sampling process might complex with strong ligands in the seawater, potentially preventing complexed 
cobalt from precipitating. For this experiment, spiked seawater samples were allowed to equilibrate overnight before precipitation, while others were processed immediately after spiking. The third variable also addressed potential ligand effects. Samples were UV oxidized to destroy all complexing ligands in case any particularly strong ligands might still form complexes at the $\mathrm{pH}$ of the acidified seawater ( $\mathrm{pH} 1.7)$. In retrospect, examination of this variable would be best tested solely during ICP-MS studies because UV oxidation would release stable cobalt and would likely have no effect on the detectable

${ }^{57} \mathrm{Co}$. The results demonstrated that these variables were not significant (Table 1). The reproducibility within the range of $250-400 \mu \mathrm{L}$ of ammonia added is excellent, however, and scavenging efficiency is greatly improved from $20 \%$ in earlier studies to $88 \%+/-2 \%$ with $n=19$ (Saito and Schneider 2006).

Scavenging did not increase significantly above $90 \%$, suggesting that this is the maximum possible cobalt precipitated by this method, or that other factors should be examined. It is also important to recognize that these experiments were done at the lowend of the range of cobalt concentrations observed in the field. This experiment tested the method at $13 \mathrm{pM}{ }^{57} \mathrm{Co}$. Total cobalt concentrations reported in the open ocean are generally 5-200pM (Martin et al. 1989, Martin et al. 1993, Saito and Moffett 2002). It is possible that a higher scavenging efficiency might be achieved with higher concentrations. For example, an absolute loss of 1.6pM during precipitation would account for the $88 \%$ scavenged on a $12 \mathrm{pM}$ sample. For a concentration of $40 \mathrm{pM}$, however, an absolute loss of $1.6 \mathrm{pM}$ would result in $96 \%$ scavenged. Future experiments should test recoveries across the relevant concentration range. For our purposes, these results were sufficient to justify intercomparison studies with accepted methods to determine the feasibility of this method. 3.2 Feasibility Studies - Assuming $88 \%$ scavenging efficiency is sufficient to accurately measure total cobalt on the ICP-MS, the success of this method then depends on the ability to make the concentrated sample compatible with the mass spectrometer. Dissolved solids are known to clog the ICP-MS by creating buildup of oxides on the skimmer cones (Dave Schneider, personal communication). This decreases instrument sensitivity and requires frequent cleaning and replacement of the skimmer cones. The concentrated sample proposed here would have a high content of dissolved solids and would clog the ICP-MS, thus the concentrated sample must be treated to remove dissolved salts while retaining the metals.

It has been previously demonstrated that synthetic chelating resins can be used to concentrate and isolate metals from a solution (Bruland et al. 1985, Wells and Bruland 1998, Lohan et al. 2005). Difficulties with the use of resins include sensitivity to $\mathrm{pH}$, assurance of complete dissociation of all ligand-metal complexes prior to loading, and pretreatment of the resins prior to use for trace metal work. Samples collected for total iron and manganese analysis are generally acidified to $\mathrm{pH} 1.7$ upon collection (Wu and Boyle 1998, Saito and Schneider 2006). It is generally assumed that at this $\mathrm{pH}$, all metal-ligand complexes dissociate and wall-loss effects by adsorption to the inside of the bottle are prevented. At this $\mathrm{pH}$, however, many resins release metals rather than bind them, and $\mathrm{pH}$ adjustment prior to loading on the column is often necessary, which may alter the availability of the metals in the seawater matrix. Resuspending the coprecipitated pellet in a buffer, as proposed here, may be successful as much of the seawater matrix is removed 
prior to loading on the column. Alternatively, samples could be stored unacidified, but shelf-life would then be called into question.

Four commercially available resins were investigated for this study: Toyopearl, NTA Superflow, Chelex-100, and NTA Histidine. All of these resins have been used with some success for trace metal analyses (Bruland et al. 1985, Lohan et al. 2005, Maeve Lohan personal communication). Chelex-100 has the advantage of a history of use, being used to remove metals from unacidified seawater (to be used as analytical blanks), from stock reagents (to reduce reagent blanks), and to concentrate metals from unacidified seawater (Bruland et al. 1985, Price et al. 1989, Saito and Moffett 2001). NTA Superflow has the advantage of use at low $\mathrm{pH}$ ranges for some metals ( $\mathrm{Fe}$ and $\mathrm{Cu}$ ) and has been determined to be clean for work with iron (Lohan et al. 2005). Toyopearl is more amenable to a suite of metals than the NTA resins and is easier to work with (Maeve Lohan, personal communication). All studies done here were performed with gravity columns, but future work should include the use of a vacuum or peristaltic pump due to extremely slow elution times and to improve throughput efficiency. Initial resin experiments used Chelex-100 resin because it has been the primary resin used in our lab, and the bead size was amenable to gravity column treatment.

Initial tests of the Chelex-100 resin determined that metals bound most completely at a resuspended pellet $\mathrm{pH}$ of between 4 and 7 . After running the resuspended solution through the column, it was determined that metals were not lost during successive washes with Milli-Q water. This step is important to remove dissolved solids and seawater matrix prior to eluting. One concern that arose was that $4 \%$ percent of the metal remained in the resuspended buffer solution, and that an additional $4.7 \%$ percent remained on the resin even after eluting with nitric acid (Figure 2). These findings were addressed in later experiments, and loss was likely due to insufficient amount of resin added to the resuspension solution and the short time of resin exposure to nitric acid.

Following this study, the four resins were tested in varying volumes to determine their relative capacity to bind cobalt. For this experiment, pellets were resuspended with a Chelex-treated EPPS buffer to control $\mathrm{pH}$ (Seth John, personal communication). All resuspension solutions were allowed to equilibrate with the resin overnight before leaching. The trend can be seen between the amount of resin added to the column and the percentage bound (Figure 3). The resuspension solution was measured for ${ }^{57} \mathrm{Co}$ activity by removing an aliquot from the solution in the resin column. It should also be noted that the percentages reported for these and the following studies are the percentage recovered from the buffered resuspension solution (i.e. the percentage of the $88 \%$ of the total added during the first the sample processing step).

Given the apparent differences in binding of these resins, two were chosen to determine if kinetic barriers prevent the complete binding of cobalt to the resin in the timescale studied here. One experiment was designed to examine equilibration time with the beads, and a second experiment involved repetitively loading the sample solution through the column seven times, with Milli-Q washes of the resin between each load. (Figures $4 \mathrm{a}$ and $4 \mathrm{~b}$ ). The hypothesis was that equilibrium would be reached quickly if ${ }^{57} \mathrm{Co}$ in solution did not change after loading onto the resin. Approaching equilibrium would be limiting if ${ }^{57} \mathrm{Co}$ in solution decreased slowly over the subsampling period before 
reaching a steady state concentration (Figure 4a), assuming that a decrease in ${ }^{57} \mathrm{Co}$ of the buffered solution indicated increased binding to the resin.

Three conclusions were drawn from this study. First, it appears that greater than $300 \mu \mathrm{L}$ of Chelex resin is required to completely bind cobalt because equilibrium appeared to be reached after $\sim 30$ hours and $20 \%$ of the cobalt remained in solution (Figure $4 \mathrm{a}$ ). Second, the increase in resin volume causes the equilibration time to decreases noticeably from $\sim 30$ hours with $300 \mu \mathrm{L}$ Chelex, to $\sim 30$ minutes for $600 \mu \mathrm{L}$ Chelex (Figure 4a). Lastly, it appears that equilibration may not be required given a sufficient volume of resin (Figure 4b.) In summary, short equilibration times efficiently concentrate metals given a sufficient number of available binding sites. For both Toyopearl resin additions, near complete elution was observed for the equilibration experiment $(200 \mu \mathrm{L}, 92.7 \% ; 400 \mu \mathrm{L}, 90 \%)$.

These results may be understood from a theoretical perspective with a brief consideration of the kinetics and thermodynamics of this system. The concentrations of the dominant cations in seawater (mM) and the trace metals of interest to this study (pM-nM) are different by orders of magnitude. From a kinetic perspective, if all elements have similar binding rates, and second order kinetics are assumed, calcium and magnesium, the dominant cations, will control the observed rate of resin binding site saturation. This may, in turn, reduce the observed binding rates of trace elements. With respect to Figure 4a, the dissociation rate of $\mathrm{Mg}$-Chelex may dominate cobalt binding at $300 \mathrm{~mL}$ resin volume, but this may become irrelevant at higher volumes of resin where equilibrium appears to be reached quickly. From an equilibrium perspective, relative cation concentration differences are also important because cations compete for available resin binding sites. The dominant cations will control the concentration of free Chelex if the equilibrium constants of the considered cations are of the same order of magnitude. To examine this possibility, Chelex was modeled as a free ligand. Chelex has iminodiacetic acid functional groups to bind metals (Rengan 1997). Stability constants for N-Methyliminodiacetic acid (MIDA) were used to model the concentration of free binding sites relative to percent cobalt bound. This demonstrated that there should be a threshold of resin concentration above which almost complete binding of cobalt should be observed (Figure 5). For further discussion of theory, equations, and model details, see Appendix 2.

3.3 Preliminary ICP-MS results - With successful removal of dissolved solids, it was possible to test the precision and accuracy of this method. A standard addition was prepared and results are shown below in Figure 6. Chelex was used to bind metals for ICP-MS work because these tests were run prior to the resin comparison experiment. Standard additions were made by adding stable cobalt to a batch of acidified seawater collected from the Costa Rica Upwelling Dome. Nitric acid was in contact with the resin for 30 minutes prior to extracting for sampling. The zero-addition and 400pM addition samples were run in triplicate to examine the precision within the range of interest. While this demonstrated great precision, it also revealed problems with accuracy. The slope of the standard addition should ideally be 1, which would demonstrate that the known amount added corresponded to the calculated amount measured. The slope of 0.89 implies that a fraction of the added cobalt is systematically removed during sample processing. It is possible that all the metals were leached and that the loss here is directly correlated to the $88 \%$ determined to be scavenged during the precipitation step. 
To investigate $\mathrm{pH}$ effects on binding and chemical properties of buffers, EPPS and acetate buffers were compared. Identical standard additions were run using the EPPS and acetate buffers in conjunction with the acid-leached Chelex-100 (Figure 7). These results raised questions regarding buffer effects and leaching techniques because the slopes were lower than the previous standard addition and the $\mathrm{r}^{2}$ values were not as high (EPPS slope $0.67, \mathrm{R}^{2} 0.966$; acetate slope $0.88, \mathrm{R}^{2} 0.993$ ). While both zero-addition samples gave concentrations very close to one another (122pM for EPPS, $125 \mathrm{pM}$ for acetate), there appears to be a difference in the interaction of the added cobalt with the buffer for the two standard additions. This could be due to slight differences in $\mathrm{pH}$. The $\mathrm{pH}$ of the supernatant was checked initially, but was not monitored for all samples. Differences in $\mathrm{pH}$ could reflect insufficient resuspension of the pellet or less successful binding of metals in one buffer relative to the other. These standard additions were performed using $100 \mu \mathrm{L}$ Chelex-100 to concentrate metals, prior to the decision that more Chelex was needed to achieve binding potential. Ultimately, however, the slope differences should only reflect scavenging loss because normalizing to ${ }^{57} \mathrm{Fe}$ should correct for any other processing inefficiencies (Table 2). Replicates were performed on a field sample to confirm precision, which was $65 \mathrm{pM}+/-3 \mathrm{pM}(\mathrm{n}=5)$, equivalent to that of the CSV method (Saito and Moffett 2001).

To determine accuracy of the ICP-MS method on actual field samples, a depth profile from the Ross Sea was analyzed and compared to concentrations determined at sea using our routine CSV method (Saito and Moffett 2001, Noble and Saito et al. in press, Saito and Noble unpublished data). Four profiles were analyzed: 1) by CSV on board shortly after sampling, 2) by CSV in lab 1.5years after sampling, 3) by ICP-MS, this method, and 4) by ICP-MS, this method with UV oxidation of samples for $1 \mathrm{hr}$ prior to precipitation. CSV measurements were made on unacidified samples stored at $4^{\circ} \mathrm{C}$, while ICP-MS measurements were made on samples acidified to $\mathrm{pH} 1.7$. For the CSV method, it is preferred that samples be processed immediately after collection and at $\mathrm{pH} 8$, however acidified samples have been run successfully with $\mathrm{pH}$ adjustment (Saito and Moffett 2002b).

When compared, these profiles raise a number of questions. First, there is a clear difference between the samples that were UV irradiated and those that were not. This may suggest that either the UV oxidation is in fact destroying cobalt binding ligands that prevent scavenging, or there is a contamination problem during this step. These differences may be difficult to deconvolve. Secondly, by comparison to the CSV shipboard results, there appears to be a $\sim 16 \mathrm{pM}$ systematic offset in accuracy with this ICP-MS approach (Figure $8 \mathrm{~b}$ ). The results of efforts to determine the blank were unclear, but if this offset reflects the actual blank, it implies that true concentrations may be underestimated without the UV oxidation step. This profile correlation motivates further work to gain confidence in blank concentrations.

Lastly, there is a noticeable artifact at $75 \mathrm{~m}$ depth (Figure 8a). Both the ICP-MS nonUV oxidized sample as well as the sample processed by CSV 1.5 years after collection show a decrease in concentration. The CSV method has been established for samples processed relatively shortly after sampling and the shelf-life of the refrigerated samples analyzed by this method has not been scrutinized. For the refrigerated sample, it is 
possible that cobalt may have adsorbed to the inside of the sample bottle over time. For the ICP-MS sample, acidification may have prevented bottle effects, but it is possible that there may be a higher concentration of strong ligands at this depth due to remineralization of organic matter, and that these ligands may bind cobalt over time and alter the apparent total concentration. UV oxidation may resolve this by destroying those ligands. These issues are difficult to resolve without further investigation, which should eventually include analysis of certified reference seawater such as CASS-3, NASS-5, and SAFE once blanks are low and predictable.

Recent results validate the question of refrigerated sample shelf-life, specifically in low oxygen regions (Saito and Noble unpublished data, Figure 8c and Appendix 3). Data processed from a cruise transecting the Southern Atlantic in November 2007 show a decrease in detected cobalt at $300 \mathrm{~m}$ across the basin when comparing samples measured at sea to samples measured 3 months after collection. The electrochemical method is currently the primary method for measuring total cobalt, and if these samples must be processed immediately, the method may not be reasonable for use in conjunction with GEOTRACES, especially in low oxygen regions.

3.4 Future Applications and Global Significance - If the accuracy of this method can be resolved, and the blank determined and lowered, it could be used as a substitute in measuring total concentrations of cobalt during large process cruises like those planned for GEOTRACES. An advantage would be identical sample preservation to that currently used for total iron and manganese analysis, and the samples would presumably have longer shelf-lives than those of the refrigerated samples. This will be particularly valuable because the number of samples generated by these process cruises will greatly surpass the capability of processing at sea.

Cobalt is present in the lowest concentration range of the bioactive trace metals, which would provide a standard for detection limits should a cobalt ICP-MS method be used as a template to expand for use with other metals. In addition, it is vital that cobalt be considered on a GEOTRACES scale because its complex chemistry is affected by so many processes that are not easily generalized or detected by a few vertical profiles (scavenging, biological uptake, remineralization, redox chemistry, aeolian deposition, and groundwater discharge among others). For example, samples analyzed from a transect across the Southern Atlantic demonstrate that lateral advection and upwelling may play a role in delivering cobalt to the euphotic zone through the Benguela upwelling region (Saito and Noble, unpublished data, see Appendix 3). In the lee of the Hawaiian Islands, regularly forming eddies potentially concentrate cobalt in the upper $100 \mathrm{~m}$ due to upwelling of deepwater, biological uptake, and remineralization below the euphotic zone (Noble and Saito et al., in press). This same study suggested that groundwater discharge or sheer with nearby island shelves may also be introducing cobalt to the water column at mid-depths of 400-600m (Noble and Saito et al., in press). A reliable method to measure cobalt on acidified samples is necessary in order to uncover other potential nuances of cobalt biogeochemical cycling.

4. Conclusion - The intricacies of cobalt biogeochemical cycling make it a difficult metal to measure. This study demonstrates that an ICP-MS method to measure cobalt may be feasible in the near future, and that this may be imperative given that recent studies 
suggest that the shelf-life may be short for some samples preserved for electrochemical methods, particularly in low oxygen regions (Saito and Noble, unpublished data). The increase in pellet size by coprecipitation with magnesium hydroxide greatly improves scavenging relative to previous studies. There appears to be a threshold volume of resin required for complete binding in order to retain metals and remove salt matrix, but issues remain with regards to blank determination. Precision is quite good however, and comparison to an accepted method on field samples demonstrates potential for this method to succeed. Future studies should include systematic blank determination and comparison with certified seawater standards. 


\section{Tables and Figures for Appendix I:}

Table 1. Multi-variable experiment. All samples were spiked to ${ }^{12} \mathrm{pM}{ }^{57} \mathrm{Co}$. Variables included UV oxidation for $1 \mathrm{hr}$ (UV), drying precipitates in the oven (Dry), and overnight equilibration with the ${ }^{57} \mathrm{Co}$ spike (Equil). Experiments were repeated at differing additions of ammonia as noted. It appears that within the additions of $250 \mu \mathrm{L}-400 \mu \mathrm{L}$ ammonia, these variables have no significant effect on scavenging.

\begin{tabular}{|c|c|c|c|c|}
\hline \multirow[t]{2}{*}{$\mathrm{NH}_{4}{ }^{+}$added (uL) } & \multicolumn{3}{|c|}{ Sample Treatment } & \multirow[t]{2}{*}{$\%$ scavenged } \\
\hline & Equil & Dry & UV & \\
\hline 250 & \multicolumn{3}{|c|}{ CONTROL } & 89.4 \\
\hline 250 & $\mathbf{x}$ & & & 87.9 \\
\hline 250 & \multicolumn{3}{|c|}{$\mathbf{x}$} & 89.7 \\
\hline 300 & \multicolumn{3}{|c|}{ CONTROL } & 82.8 \\
\hline 300 & $\mathbf{x}$ & & & 87.0 \\
\hline 300 & \multicolumn{3}{|c|}{$\mathbf{x}$} & 91.2 \\
\hline $350(n=2)$ & \multicolumn{3}{|c|}{ CONTROL } & $93.3,88.4$ \\
\hline $350(n=2)$ & $\mathbf{x}$ & & & $88.2,90.5$ \\
\hline $350(n=2)$ & \multicolumn{3}{|c|}{$\mathbf{x}$} & $86.8,90.1$ \\
\hline $350(n=2)$ & $\mathbf{x}$ & & $\mathbf{x}$ & $93.0,89.8$ \\
\hline $350(n=2)$ & & & $\mathbf{x}$ & $90.3,88.3$ \\
\hline 400 & \multicolumn{3}{|c|}{ CONTROL } & 89.1 \\
\hline 400 & $\mathbf{x}$ & & & 89.6 \\
\hline 400 & \multicolumn{3}{|c|}{$\mathrm{x}$} & 89.2 \\
\hline Aver & $7 \mathrm{Co}$ & & & 88.3 \\
\hline & \multicolumn{3}{|c|}{ Standard deviation = } & 2.2 \\
\hline
\end{tabular}

Table 2. Recoveries for cobalt at each processing step. Assuming that $\mathrm{Fe}$ and $\mathrm{Co}$ behave similarly with respect to binding and eluting, all inefficiencies in recovery can be corrected for using the ${ }^{57} \mathrm{Fe}$ internal standard except for the initial coprecipitation. Actual efficiencies may be closer to $100 \%$ due to loss through pipette error or other error associated with the ${ }^{57} \mathrm{Co}$ counting process.

\begin{tabular}{lcc} 
& $\begin{array}{c}\text { Recovery / } \\
\text { Efficiency }\end{array}$ & $\begin{array}{c}\text { Corrected for w/ } \\
57 \text { Fe internal std }\end{array}$ \\
\hline Processing step & $85-90 \%$ & No \\
precipitation/scavenging & $95 \%$ & ${ }^{*}$ \\
resin binding & $90-93 \%$ & Yes $^{*}$
\end{tabular}

${ }^{*}$ It is assumed that these metals have similar binding and eluting efficiencies 

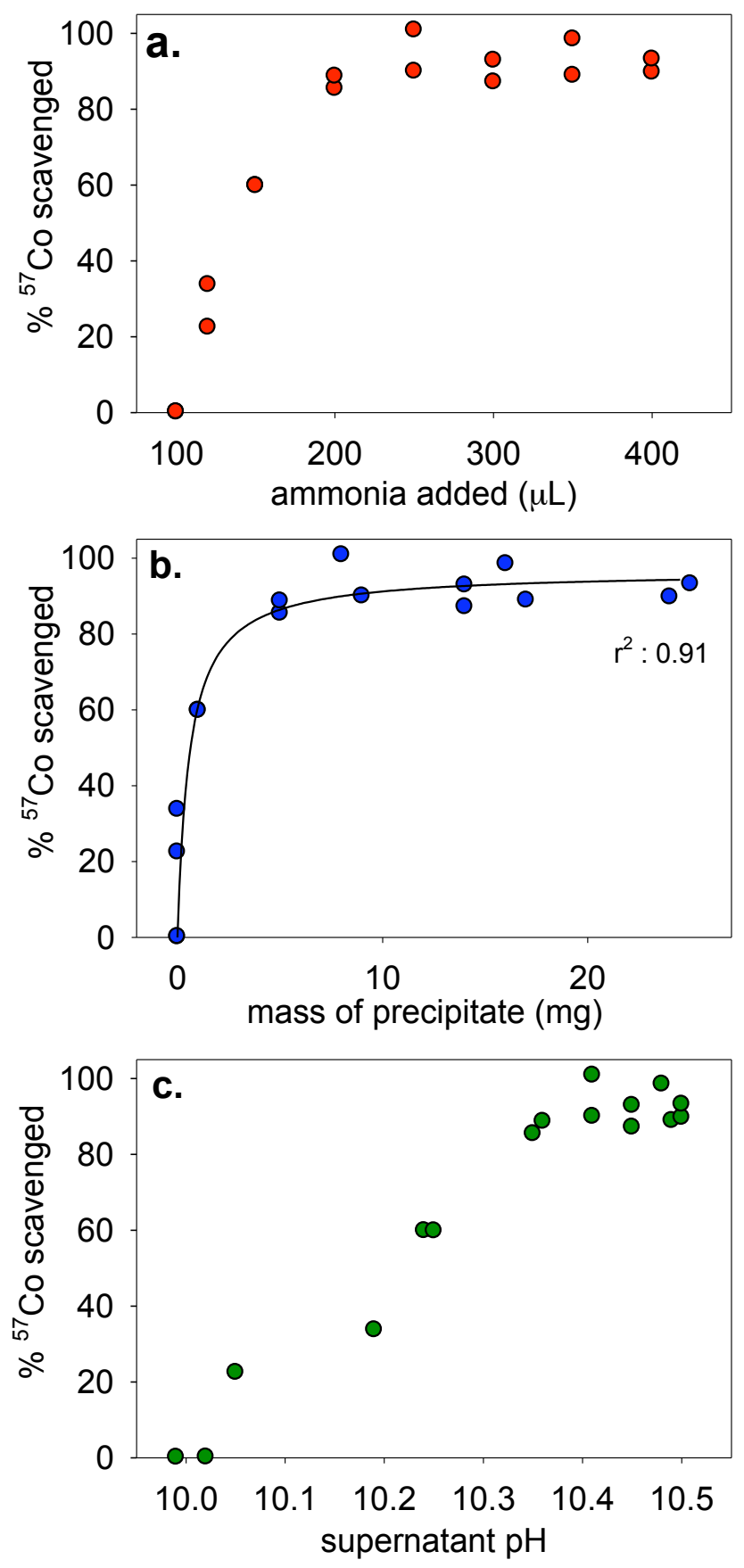

Figure 1. The effect of ammonia addition on 57Co scavenging assessed by amount of ammonia added (a), precipitate mass (b), and supernatant $\mathrm{pH}$ (c). Optimal scavenging is reached at ammonia additions of at least $250 \mathrm{~mL}$, which induces precipitates equal to or greater than $8 \mathrm{mg}$ with $\mathrm{pH}$ values above 10.3 . 


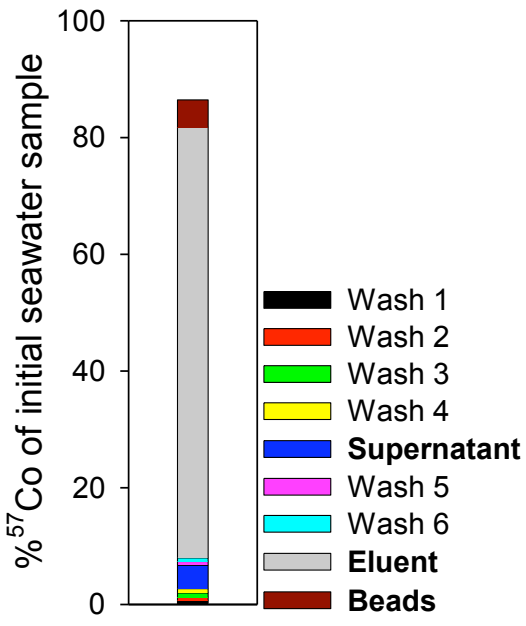

Figure 2. Wash loss and pH sensitivity experiment. The precipitate was resuspended in $2 \mathrm{~mL}$ to $\mathrm{pH} 7$ using a dilute solution of HCl. Subsamples were taken from each processing step and compared to the total.

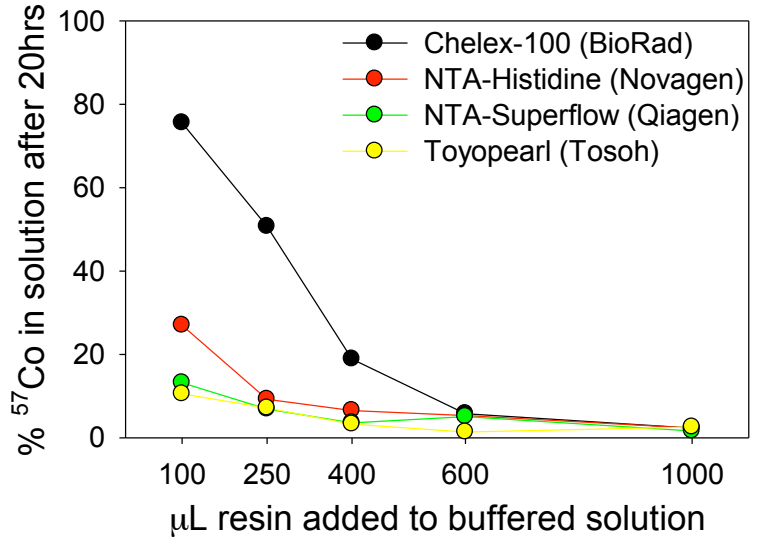

Figure 3. Comparison of available resins for binding ${ }^{57} \mathrm{Co}$. Percent was determined by removing an aliquot for cobalt activity measurements, and correcting for dilution.

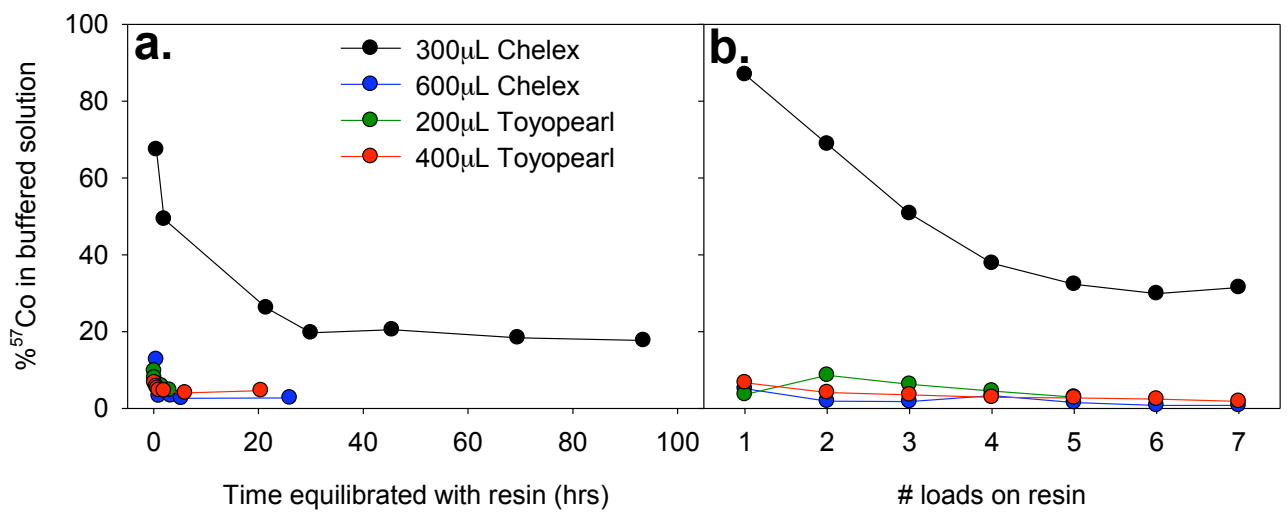

Figure 4. Kinetics and equilibrium experiments. a.) Study of time required to reach equilibrium with different volumes of two resins. b.) Examination of rates affecting binding. Note clear difference in $300 \mu \mathrm{L}$ Chelex-100 resin capacity relative to that of higher resin volumes and different resins. Rate effects appear to be amplified when insufficient resin is added. Sufficient resin volumes increase binding of cobalt and reduce time required to reach equilibrium. 


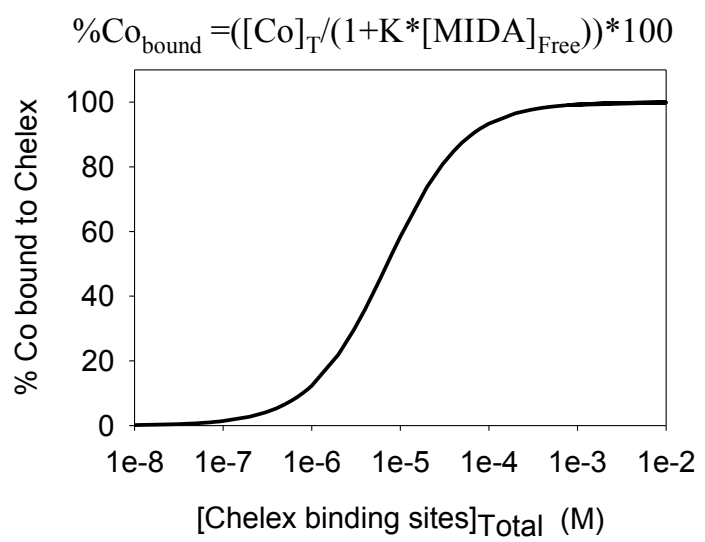

Figure 5. Theoretical speciation of bound cobalt, modeling Chelex as the free ligand $\mathrm{N}$-Methyliminodiacetic acid (MIDA) and assuming a total concentration of cobalt in seawater of 50pM. For further discussion of aquatic chemical theory and model details, see Appendix 2.

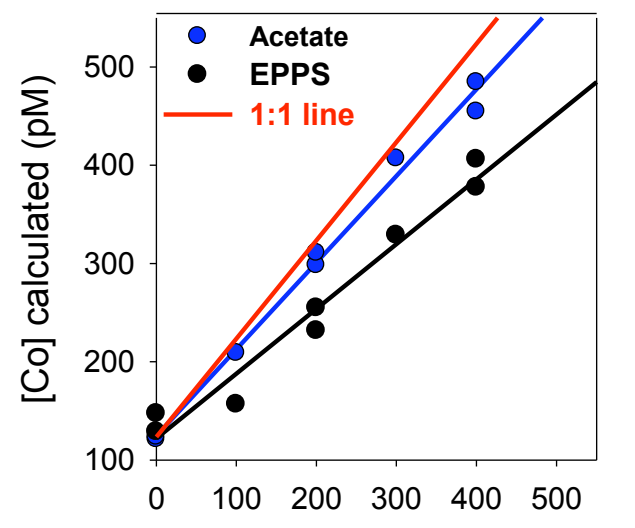

[Co] added to $13 \mathrm{~mL}$ seawater $(\mathrm{pM})$

Figure 7) Different buffers and potential effects on binding to resin. Both buffers completely dissolved the pellet, however slight differences in pH may affect the ability of all the cobalt to bind. This should be corrected for with the ${ }^{57} \mathrm{Fe}$ normalization. Deviation from the 1:1 line may reflect either the difference in scavenging efficiency between iron and cobalt, or differences in the binding affinities of iron and cobalt.

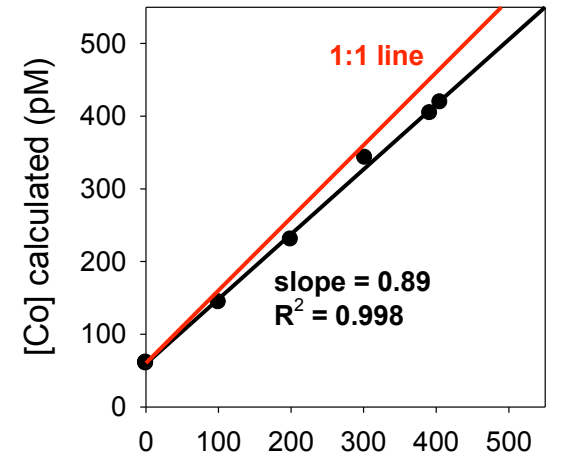

[Co] added to $13 \mathrm{ml}$ seawater (pM)

Figure 6. Standard addition by ICP-MS. EPPS buffer, no UV oxidation, 30 minute leach per sample. All calculated concentrations are normalized to ${ }^{57} \mathrm{Fe}$. 1:1 line plotted in red. See discussion for detail. 

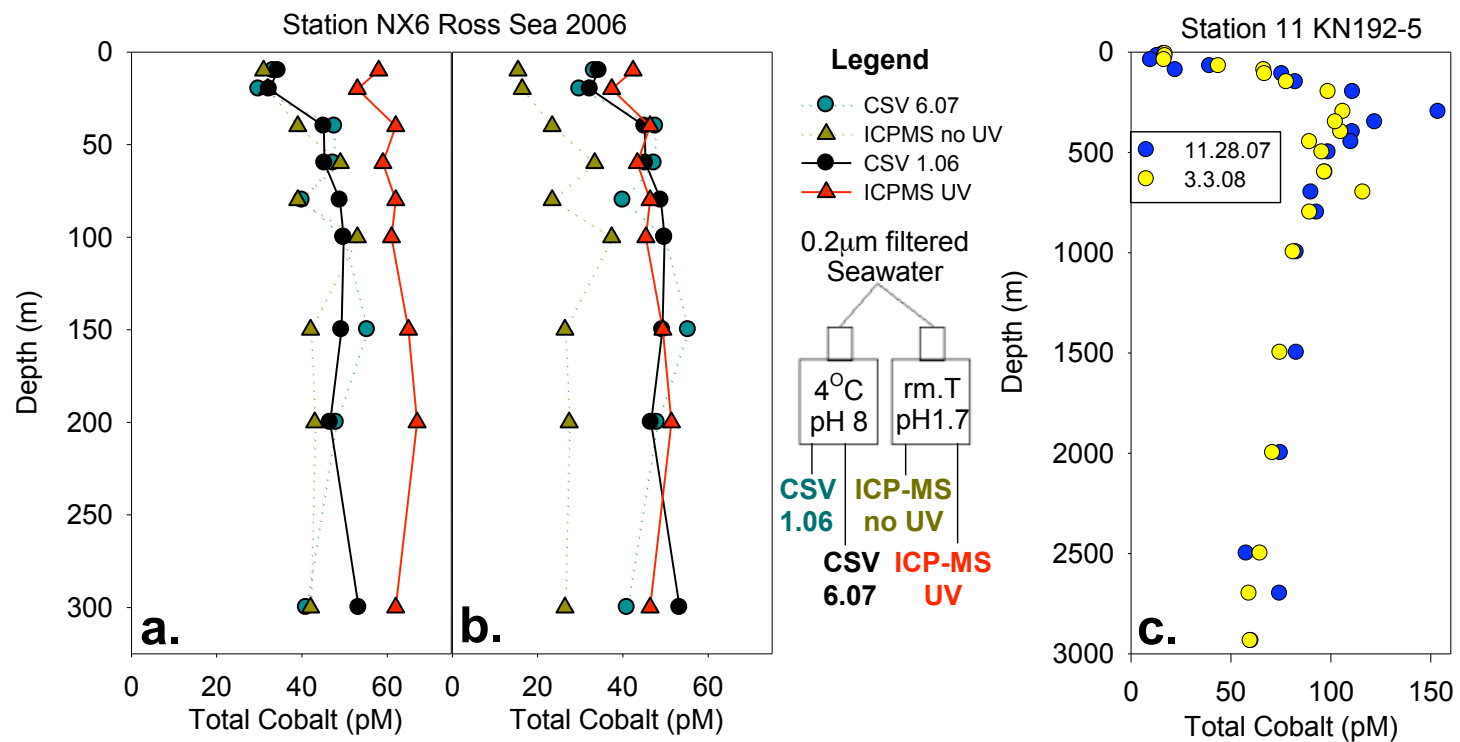

Figure 8. a. and b. ) Ross Sea profile. 1.06 and 6.07 refer to analysis date. Samples were collected in January, 2006. ICP-MS samples were processed with $100 \mu \mathrm{L}$ Chelex resin to concentrate metals, an amount later determined to be too little to achieve complete binding. Acetate buffer was used to resuspend the pellet. a.) ICP-MS profiles are not blank adjusted. Precision of this method is excellent, but the accuracy is still a work in progress due to difficulty determining the blank. b.) ICP-MS profiles moved to likely $16 \mathrm{pM}$ blank offset to allow comparison of UV irradiated to CSV accepted profile (see text for discussion). c.) South Atlantic profile. Samples were collected on 11.27.2007 and are plotted by the date they were analyzed. Note excellent agreement except for dampening of the peak at $400 \mathrm{~m}$ depth observed for samples processed 3 months following sampling.

\section{Additional experiments}

Figure 9 Probe for existence of weak $\mathrm{CoCO}_{3}$ complexes - A bulk batch of acidified seawater was spiked to 300pM Co and divided into two aliquots. One aliquot was purged with $99.999 \% \mathrm{~N}_{2}$ for 90 minutes with acid-washed Teflon tubing while the other was not. Both aliquots were measured in quintuplicate for Fe, Mn, and Co following the previously described magnesium-hydroxide coprecipitation method as described in Section 2.2.

Figure 10 Relative importance of precipitation time versus size $-{ }^{57} \mathrm{Co}$ counting techniques were used to investigate if the size of the $\mathrm{Mg}(\mathrm{OH})_{2}$ precipitate induced and/or the time that precipitation was allowed to occur affected the scavenging efficiency. Increasing ammonia additions and precipitation time were varied and the scavenging efficiency was measured by comparing the activity of the precipitates formed to that of the initial sample. Samples were analyzed at 3, 12, and 30 minutes precipitation time with 120,350 and $600 \mu \mathrm{L}$ ammonia added. 


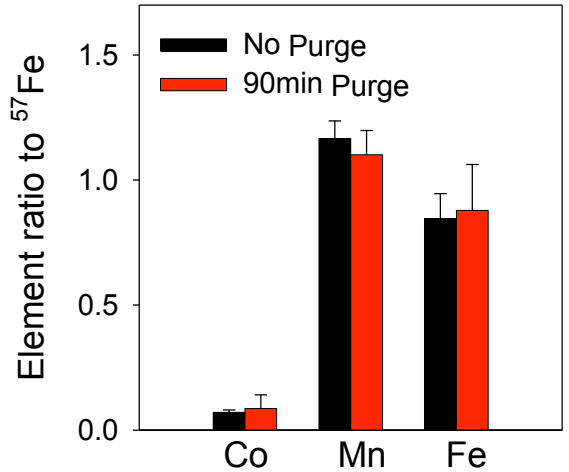

Appendix 1a) Effect of DIC degassing. An unlikely but easily solvable problem with scavenging would be the possible existence of unknown weak $\mathrm{Co}-\mathrm{CO}_{3}$ complexes which might prevent cobalt from precipitating. Seawater was purged with $99.999 \% \mathrm{~N}_{2}$ to degas DIC from solution and cause dissociation of potential $\mathrm{CoCO}_{3}$ complexes, making cobalt available for precipitation assuming equilibrium is reached. No significant increase in Co was observed, suggesting that $\mathrm{CoCO}_{3}$ complexes do not exist or there is so little cobalt bound to these complexes that their dissociation has no detectable effect on scavenging efficiency. Also note relative counts normalized to ${ }^{57} \mathrm{Fe}$, which reflect relative concentrations in seawater. For error bars, $\mathbf{n}=\mathbf{5}$.

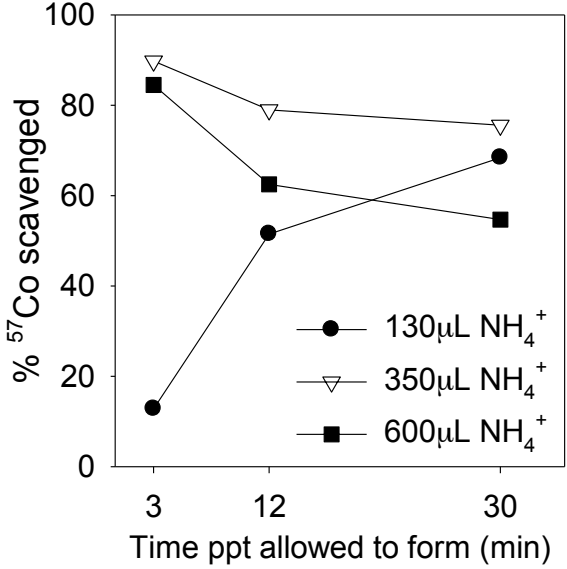

Appendix 1b) Time and ammonia addition experiment. It appears that 3 minutes and $350 \mu \mathrm{L}$ ammonia is optimal. Unclear results are found with longer precipitation times and a decrease is found for larger additions of ammonia combined with longer precipitation times. It is possible that loss with longer precipitation times may be due to re-dissolution of the pellet. These results are consistent with previous observations (Saito and Schneider 2006). 


\section{Rates, equilibrium equations, and conditions used for Chelex model}

Taking only magnesium and cobalt into consideration and assuming second order kinetics, the respective rate equations governing the system would be:

$$
\begin{aligned}
\frac{d \text { Chelex }}{d t} & =-\mathrm{k}_{\mathrm{fMg}}\left[\mathrm{Mg}^{2+}\right][\text { Chelex }]_{\text {Free }}+\mathrm{k}_{\mathrm{bMg}}[\text { MgChelex }]+\mathrm{k}_{\mathrm{bCo}}[\text { CoChelex }]-\mathrm{k}_{\mathrm{fCo}}\left[\mathrm{Co}^{2+}\right][\text { Chelex }] \\
\frac{d \text { MgChelex }}{d t} & =\mathrm{k}_{\mathrm{fMg}}\left[\mathrm{Mg}^{2+}\right][\text { Chelex }]_{\mathrm{Free}}-\mathrm{k}_{\mathrm{bMg}}[\text { MgChelex }] \\
\frac{d \text { CoChelex }}{d t} & =\mathrm{k}_{\mathrm{fCo}}\left[\mathrm{Co}^{2+}\right][\text { Chelex }]_{\mathrm{Free}}-\mathrm{k}_{\mathrm{bCo}}[\text { CoChelex }] \\
\frac{d \mathrm{Co}}{d t} & =-\mathrm{k}_{\mathrm{fCo}}\left[\mathrm{Co}^{2+}\right][\text { Chelex }]_{\mathrm{Free}}+\mathrm{k}_{\mathrm{bCo}}[\text { CoChelex }]
\end{aligned}
$$

We assumed that dissociation between cobalt and Chelex did not happen, thus the term $\mathrm{k}_{\mathrm{bCo}}$ would drop out of these equations and cobalt concentrations would be solely dependent upon the rate that cobalt binds Chelex, adjusted by the concentrations of free cobalt and free Chelex. Because the concentration of magnesium is orders of magnitude more than that of cobalt, magnesium rates will control the observed time rate of change in free Chelex. This would, in turn, affect the observed rate of cobalt binding to the resin. This could be avoided if the Chelex were in great excess of all other species so that the rate would no longer depend on Chelex availability, and the rate of cobalt binding could be considered pseudo first order.

If one assumed instantaneous establishment of thermodynamic equilibrium, rates may be ignored and the speciation of Chelex and cobalt may be expressed:

$$
\begin{aligned}
& {[\text { Chelex }]_{\mathrm{T}}=[\text { Chelex }]_{\text {free }}+[\text { MgChelex }]+[\text { CaChelex }]+[\text { FeChelex }]+[\text { MnChelex }]+[\text { CoChelex }]} \\
& {[\text { Chelex }]_{\mathrm{T}}=[\text { Chelex }]_{\text {free }}\left(1+\mathrm{K}_{\mathrm{dMg}}\left[\mathrm{Mg}^{2+}\right]+\mathrm{K}_{\mathrm{dCa}}\left[\mathrm{Ca}^{2+}\right]+\mathrm{K}_{\mathrm{dFe}}\left[\mathrm{Fe}^{3+}\right]+\mathrm{K}_{\mathrm{dMn}}\left[\mathrm{Mn}^{2+}\right]+\mathrm{K}_{\mathrm{dCo}}\left[\mathrm{Co}^{2+}\right]\right)} \\
& {\left[\mathrm{Co}^{2+}\right]_{\mathrm{T}}=\left[\mathrm{Co}^{2+}\right]_{\text {free }}+[\text { CoChelex }]}
\end{aligned}
$$

The distribution coefficients, $\mathrm{K}_{\mathrm{d}}$, describe the distribution of the compound of interest between the solid-associated phase and the dissolved phase, assuming equilibrium. If the concentrations of magnesium and calcium are in great excess of the available binding sites on the Chelex, and similar $\mathrm{K}_{d} \mathrm{~S}$ are observed for all species, there may not be sufficient free binding sites to completely bind the trace metals. $\mathrm{K}_{\mathrm{d}}$ values were not available in the literature, so the system was modeled as the free ligand N-Methyliminodiacetic acid (MIDA). Appendix 2 shows the conditions used for the model, and it appears from Figure 4 , that there is a tipping point after which the concentrations of magnesium and calcium no longer prevent the Chelex from being in sufficient excess of free cobalt to bind it completely. This model is not directly comparable to the system under consideration in this paper though. Corrections for the ionic strength of the buffer would need to be made, but the same general trend should be expected. 


\begin{tabular}{|c|c|c|c|}
\hline \\
\hline \multicolumn{4}{|c|}{$\begin{array}{l}\text { Appendix 2) Values used for theoretical } \\
\text { Chelex speciation calculations. Average } \\
\text { concentrations of Ca and Mg in seawater } \\
\text { were taken from Morel and Hering (1993) } \\
\text { and metal estimates were based on } \\
\text { previous observations (Noble and Saito et } \\
\text { al. in press). Buffer concentrations are } \\
\text { calculated assuming a 10mg precipitate } \\
\text { (see Figure 1) dissolved in } 2 \mathrm{~mL} \text { of buffer. } \\
\text { K constants for Mg, Ca, and Co with the } \\
\text { free ligand were acquired from the NIST } \\
\text { database at an ionic strength of } 0.1 \text { as this } \\
\text { was the only data available. Speciation of } \\
\text { free Chelex was calculated for the } \\
\text { buffered solution assuming the precipitate } \\
\text { was comprised of } 95 \% \mathrm{Mg}(\mathrm{OH})_{2} \text { and } 5 \% \\
\text { Ca(OH) })_{2} \text { by mass, and that trace metal } \\
\text { precipitates contributed negligibly to the } \\
\text { total mass. } \\
\text { Metal }\left[M 1 \text { in SW } \text {.Ml in buffer } \mathrm{K}_{M-M D A}\right.\end{array}$} \\
\hline \multicolumn{2}{|c|}{$0532 \mathrm{M} \quad 0.081 \mathrm{M} \quad 10^{3.51}$} & & \\
\hline $\mathrm{Ca}$ & $0.0102 \mathrm{M}$ & $0.0034 \mathrm{M}$ & $10^{3.8}$ \\
\hline Co & $0.05 \mathrm{nM}$ & $0.325 \mathrm{nM}$ & $10^{1.0}$ \\
\hline $\mathrm{Fe}$ & $0.5 \mathrm{nM}$ & $3.25 \mathrm{nM}$ & 10 \\
\hline $\mathrm{Mn}$ & $0.5 \mathrm{nM}$ & & 10 \\
\hline
\end{tabular}




\section{References for Appendix I:}

Akagi, T., Fuwa, K., Haraguchi, H., 1985. Simultaneous multi-element determination of trace metals in sea water by inductively-coupled plasma atomic emission spectrometry after coprecipitation with gallium. Analytica Chimica Acta, 177, 139-151.

Archer, D.E., Johnson, K., 2000. A Model of the iron cycle in the ocean. Global Biogeochemical Cycles, 14 (1), 269-279.

Boyle, E.A., Berquist, B.A., Kayser, R.A., Mahowald, N., 2005. Iron, manganese, and lead at Hawaii Ocean Time-series station ALOHA: Temporal variability and an intermediate water hydrothermal plume. Geochimica et Cosmochimica Acta. 69 (4), 933-952.

Bruland, K.W., Coale, K.H., Mart, L., 1985. Analysis of seawater for dissolved cadmium, copper, and lead: an intercomparison of voltammetric and atomic absorption methods. Marine Chemistry, 17, 285-300.

Bruland, K.W., Lohan, M.C. 2003. Controls of Trace Metals in Seawater. Treatise on Geochemistry Volume 6 The Oceans and Marine Geochemistry. Elderfield, H., Holland, H.D., Turekian, K.K., 6.02, 23-47.

Ellwood, M.J., 2004. Zinc and cadmium speciation in subantarctic waters east of New Zealand. Marine Chemistry, 87, 37-58.

Field, M.P., Cullen, J.T., Sherrell, R.M., 1999. Direct determination of 10 trace metals in $50 \mu \mathrm{L}$ samples of coastal seawater using desolvating micronebulization sector field ICP-MS. Journal of Analytical Atomic Spectrometry. 14, 1425-1431.

Fitzwater, S.E., Coale, K.H., Gordon, M.R., Johnson, K.S., Ondrusek, M.E., 1996. Iron deficiency and phytoplankton growth in the equatorial Pacific. Deep-Sea Research II, 43 (4-6) 995-1015.

Fung, I.Y., Meyn, S.K., Tegen, I., Doney, S.C., John, J.G., Bishop, J.K.B., 2000. Iron supply and demand in the upper ocean. Global Biogeochemical Cycles, 14 (1), 281-295.

GEOTRACES Planning Group, 2006. GEOTRACES Science Plan. Baltimore, Maryland: Scientific Committee on Oceanic Research.

John, Seth. Postdoctoral Scholar in Geochemistry, California Institute of Technology, Pasadena, California. Personal communication, 2007.

Johnson, K.S., Gordon, R.M., Coale, K.H., 1997. What controls dissolved iron concentrations in the world ocean? Marine Chemistry, 57, 137-161.

Johnson, W.K., Miller, L.A., Sutherland, N.E., Wong, C.S., 2005. Iron transport by mesoscale Haida eddies in the Gulf of Alaska. Deep-Sea Research Part II, 52, 933-953.

Lam, P.J., Bishop, J.K., Henning, C.C., Marcus, M.A., Waychunas, G.A., Fung, I.Y., 2006. Wintertime phytoplankton bloom in the subarctic Pacific supported by continental margin iron. Global Biogeochemical Cycles, doi:10.1029/2005GB002557.

Lane, T.W., Saito, M.A., George, G.N., Pickering, I.J., Prince, R.C., Morel, F.M.M., 2005. A cadmium enzyme from a marine diatom. Nature, 435, 42.

Lohan, M.C., Aguilar-Islas, A.M., Franks, R.P., Bruland, K.W., 2005. Determination of iron and copper in seawater at $\mathrm{pH} 1.7$ with a new commercially available chelating resin, NTA Superflow. Analytica Chimica Acta. 530, 121-129. 
Lohan, Maeve. Lecturer in Biogeochemistry and Environmental Analytical Chemistry, School of Earth, Ocean \& Environmental Sciences, University of Plymouth, United Kingdom. Personal communication, 2006.

Martin, J.H., Gordon, R.M., Fitzwater, S., Broenkow, W.W., 1989. VERTEX: phytoplankton/iron studies in the Gulf of Alaska. Deep-Sea Research I, 36 (5), 649-680.

Martin, J.H., Gordon, R.M., Fitzwater, S.E., 1991. The Case for Iron. Limnology and Oceanography, 36 (8), 1793-1802.

Martin, J.H., Fitzwater, S.E., Gordon, R.M., Hunter, C.N., Tanner, S.J., 1993. Iron, primary production and carbon-nitrogen flux studies during the JGOFS North Atlantic Bloom Experiment. Deep-Sea Research II 40 (1/2), 115-134.

Moffett, J.W., 1995. Temporal and spatial variability of copper complexation by strong chelators in the Sargasso Sea. Deep-Sea Research I, 42 (8), 1273-1295.

Moffett, J.W., Brand, L.E., 1996. Production of strong, extracellular Cu chelators by marine cyanobacteria in response to $\mathrm{Cu}$ stress. Limnology Oceanography, 41 (3) 388395.

Moffett, J.W., Ho, J., 1996. Oxidation of cobalt and manganese in seawater via a common microbially catalyzed pathway. Geochimica et Cosmochimica Acta, 60 (18), 34153424.

Moore, J.K., Doney, S.C., Lindsay, K., 2004. Upper ocean ecosystem dynamics and iron cycling in a global three-dimensional model. Global Biogeochemical Cycles. 18 doi:10.1029/2004GB002220.

Morel, F.M.M., Hering, J.G., 1993. Principles and Applications of Aquatic Chemistry. John Wiley and Sons, New York, NY.

Morel, F.M.M., Reinfelder, J.R., Roberts, S.B., Chamberlain, C.P., Lee, J.G., Yee, D., 1994. Zinc and carbon co-limitation of marine phytoplankton. Nature, 369, 740-742.

Morel, F.M.M., Price, N.M., 2003. The Biogeochemical Cycles of Trace Metals in the Oceans. Science, 300, 944-947.

Morel, F.M.M., Milligan, A.J., Saito, M.A., 2003. Marine Bioinorganic Chemistry: The Role of Trace Metals in the Oceanic Cycles of Major Nutrients. Treatise on Geochemistry Volume 6, The Oceans and Marine Geochemistry. Elderfield, H., Holland, H.D., Turekian, K.K., 6.05, 113-143.

Noble, A.E., Saito, M.A., Maiti, K., Benitez-Nelson, C., 2008. Cobalt, manganese, and iron near the Hawaiian Islands: A potential concentrating mechanism for cobalt within a cyclonic eddy and implications for the hybrid-type trace metals. Deep-Sea Research Part II, in press.

Parekh, P., Follows, M.J., Boyle, E., 2004. Modeling the global ocean iron cycle. Global Biogeochemical Cycles. 18 doi:10.1029/2003GB002061.

Price, N.M, Harrison, G.I., Hering, J.G., Hudson, R.J., Nirel, P.M., Palenik, B., Morel, F.M.M., 1989. Preparation and Chemistry of the Artificial Algal Culture Medium Aquil. Biological Oceanography, 6, 443-461.

Price, N.M., Morel, F.M.M., 1990. Cadmium and cobalt substitution for zinc in a marine diatom. Nature, 344, 658-660.

Price, N.M., Morel, F.M.M., 1991. Colimitation of phytoplankton growth by nickel and nitrogen. Limnology and Oceanography, 36 (6), 1071-1077. 
Rahmi, D., Zhu, Y., Fujimori, E., Umemura, T., Haraguchi, H., 2007. Multielement determination of trace metals in seawater by ICP-MS with aid of down-sized chelating resin-packed minicolumn for preconcentration. Talanta 72, 600-606.

Rengan, K., 1997. Chelating resins: Sorption characteristics in chloride media. Journal of Radioanalytical and Nuclear Chemistry, 219 (2), 211-215.

Rue, E.L., Bruland, K.W., 1995. Complexation of iron(III) by natural organic ligands in the Central North Pacific as determined by a new competitive ligand equilibration/adsorptive Cathodic stripping voltammetric method. Marine Chemistry 50, 117-138.

Saito, M.A., Moffett, J.W., 2001. Complexation of cobalt by natural organic ligands in the Sargasso Sea as determined by a new high-sensitivity electrochemical cobalt speciation method suitable for open ocean work. Marine Chemistry, 75, 49-68.

Saito, M.A., Moffett, J.W., Chisholm, S.W., Waterbury, J.B., 2002a. Cobalt limitation and uptake in Prochlorococcus. Limnology and Oceanography, 47(6), 1629-1636.

Saito, M.A., Moffett, J.W., 2002b. Temporal and spatial variability of cobalt in the Atlantic Ocean. Geochimica et Cosmochimica Acta. 66 (11), 1943-1953.

Saito, M.A., Moffett, J.W., DiTullio, G.R., 2004. Cobalt and nickel in the Peru upwelling region: A major flux of labile cobalt utilized as a micronutrient. Global Biogeochemical Cycles, 18, dio:10.1029/2003GB002216.

Saito, M.A., Schneider D.L., 2006. Examination of precipitation chemistry and improvements in precision using the $\mathrm{Mg}(\mathrm{OH}) 2$ preconcentration inductively coupled plasma mass spectrometry (ICP-MS) method for high-throughput analysis of openocean Fe and Mn in seawater. Analytical Chimica Acta, 565, 222-233.

Saito, M.A., Noble, A.E. Unpublished data from Research Cruise in the Ross Sea. R/V N.B. Palmer, cruise NBP0601. 12.12.2005-1.31.2006.

Saito. M.A., Noble, A.E. unpublished data from Research Cruise transecting the South Atlantic Ocean. R/V/ Knorr, cruise KN192-5. 11.13.2007-12.13.2007.

Schneider, Dave. Research Associate III, Department of Marine Chemistry and Geochemistry, Woods Hole Oceanographic Institution. Woods Hole, MA. Personal communication, 2006.

Sunda, W.G., Huntsman, S.A., 1995. Cobalt and Zinc Intereplacement in Marine Phytoplankton: Biological and Geochemical Implications. Limnology and Oceanography, 40 (8) 1404-1417.

Tebo, B.M., Nealson, K.H., Emerson, S., Jacobs, L., 1985. Microbial Mediation of Mn(II) and $\mathrm{Co}(\mathrm{II})$ Precipitation at the O2/H2S Interfaces in Two Anoxic Fjords. Limnology and Oceanography, 29 (6), 1247-1258.

Weber, L., Volker, C., Oschlies, A., Burchard, H., 2007. Iron profiles and speciation of the upper water column at the Bermuda Atlantic Time-series Study site: a model based sensitivity study. Biogeosciences. 4, 689-706.

Wells, M.L., Bruland, K.W., 1998. An improved method for rapid preconcentration and determination of bioactive trace metals in seawater using solid phase extraction and high resolution inductively coupled plasma mass spectrometry. Marine Chemistry 63, 145-153. 
Wisniewski, R.J., 2006. Relating the Biogeochemistries of Zinc, Cobalt, and Phosphorus to Phytoplankton Activities in the Sea. Thesis for Doctorate of Philosophy in Chemical Oceanography at Massachusetts Institute of Technology and Woods Hole Oceanographic Institution.

Wu, J., Boyle, E.A., 1997. Low Blank Preconcentration Technique for the Determination of Leah, Copper, and Cadmium in Small-Volume Seawater Samples by Isotope Dilution ICPMS. Analytical Chemistry. 69 (13) 2464-2470.

$\mathrm{Wu}$, J., Boyle, E.A., 1998. Determination of iron in seawater by high-resolution isotope dilution inductively coupled plasma mass spectrometry after $\mathrm{Mg}(\mathrm{OH})_{2}$ coprecipitation. Analytica Chimica Acta. 367, 183-191. 


\section{Appendix II: Experiments investigating the lability of cobalt in naturally photodegraded Vitamin $B_{12}$}

\section{Experimental method:}

$500 \mathrm{~mL}$ of trace metal clean seawater was UV-irradiated and chelexed to be used for all experiments. Each experiment was conduced in a quartz tube to which $15 \mathrm{~mL}$ of seawater was added, along with an aliquot of $\mathrm{B}_{12}$ to yield an approximate initial concentration of 2.5 $\mu \mathrm{M}$. The tube was then either exposed to natural sunlight, or to UV light and subsamples were taken over time, both for $\mathrm{B}_{12}$ concentration determination by UV-Vis spectroscopy and labile cobalt concentration by ACSV.

For $\mathrm{B}_{12}$ determination, $2 \mathrm{~mL}$ of the subsample was transferred to a $2 \mathrm{~mL}$ Epindorf tube and then pushed through a Sep Pak column with a $1 \mathrm{~mL}$ syringe. $5 \mathrm{~mL}$ of Milli-Q water were pushed through to remove salts and then eluted with $2 \mathrm{~mL}$ of $\mathrm{MeOH}$, and divided among 4 $1 \mathrm{~mL}$ Epindorf tubes. The aliquots were concentrated, and then combined in $100 \mu \mathrm{L}$ of Milli-Q water. For UV-Vis analyses, 1-2 $\mu \mathrm{L}$ were placed on a Nanodrop and the absorbance at $361 \mathrm{~nm}$ was used to determine the concentration of $\mathrm{B}_{12}$. This solution was also used for Mass Spectrometry analyses.

For labile cobalt analyses, $100 \mu \mathrm{L}$ of the solution were subsampled and diluted into $10 \mathrm{~mL}$ of Milli-Q water. From this sample $102 \mu \mathrm{L}$ were added to an $8.5 \mathrm{~mL}$ sample of UVirradiated seawater and assessed for labile cobalt by ACSV, as described in previous chapters.

\section{Results:}

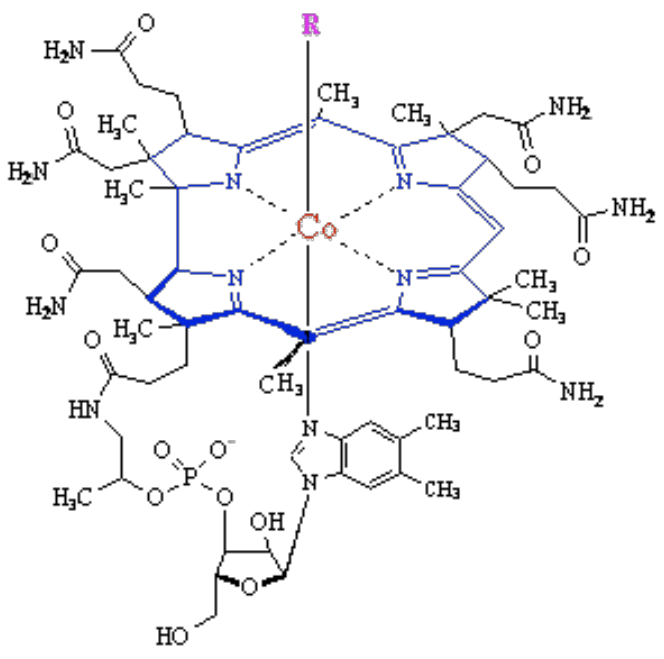

Molecular structure of $B_{12}$, where $R$ is either an adenosyl functional group (coenzyme $B_{12}$ ) or a $\mathrm{CN}$ - functional group (cyanocobalamin) 


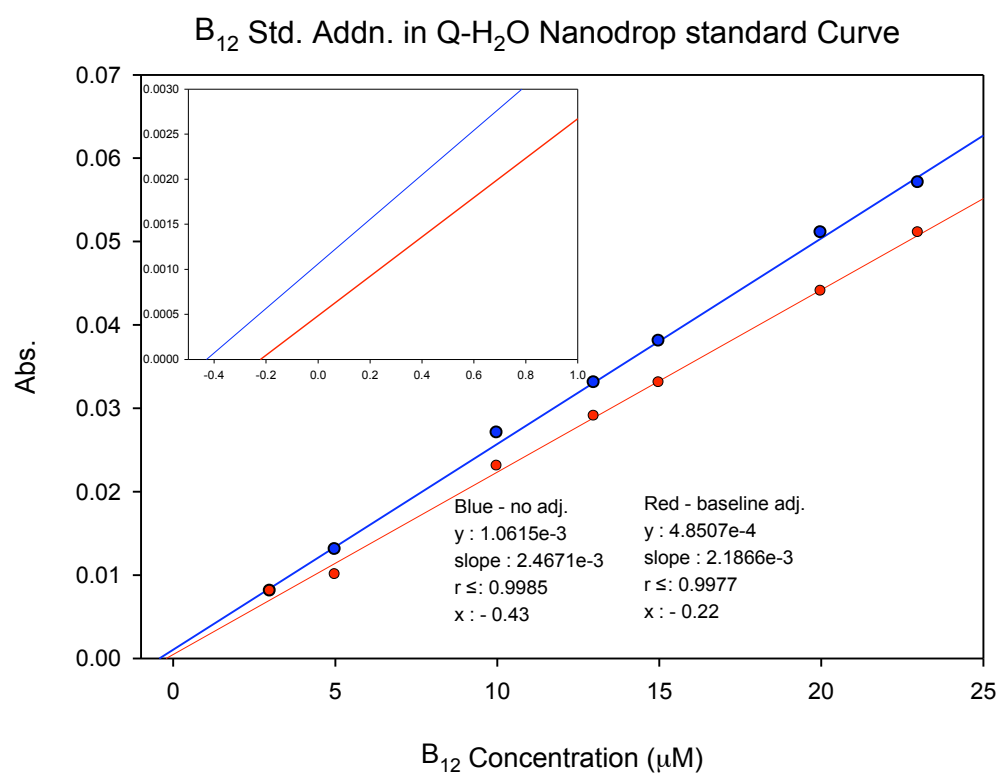

Fig. 1 Standard curve for $\mathrm{B}_{12}$ in Q-water. Red line is a standard curve with the noise subtracted out, and the blue line is the raw data.

Table 1. Measured $\mathrm{B}_{12}$ and labile cobalt for a natural sunlight exposure experiment.

\begin{tabular}{ccc} 
Hours & $\mathrm{B}_{12}[\mu \mathrm{M}]$ & $\mathrm{Co}^{\prime}[\mathrm{pM}]$ \\
\hline 0 & 52 & 16 \\
0.5 & 48 & 14 \\
2 & 45 & 15 \\
4.5 & 33 & 15 \\
6.5 & 27 & 18
\end{tabular}


Cobalt and B12 concentration with increasing UV exposure of 300pM B12 UV Chelexed SW 12/06/04

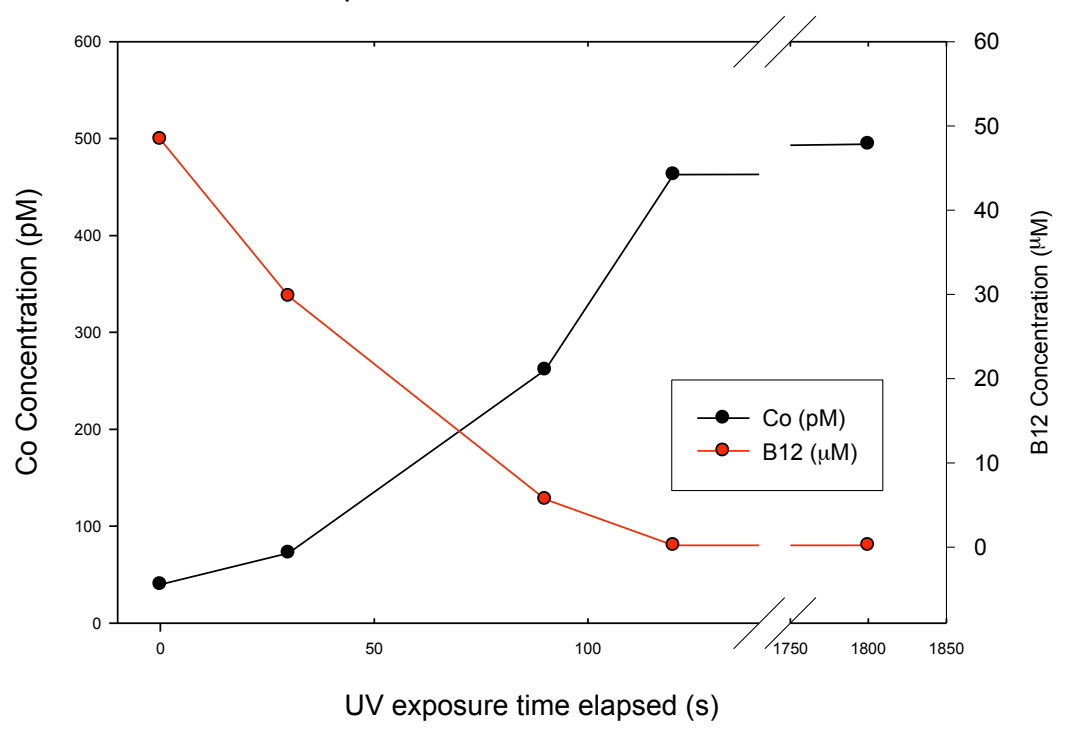

Degradation of B12 over time with exposure to UV light 12/6/04

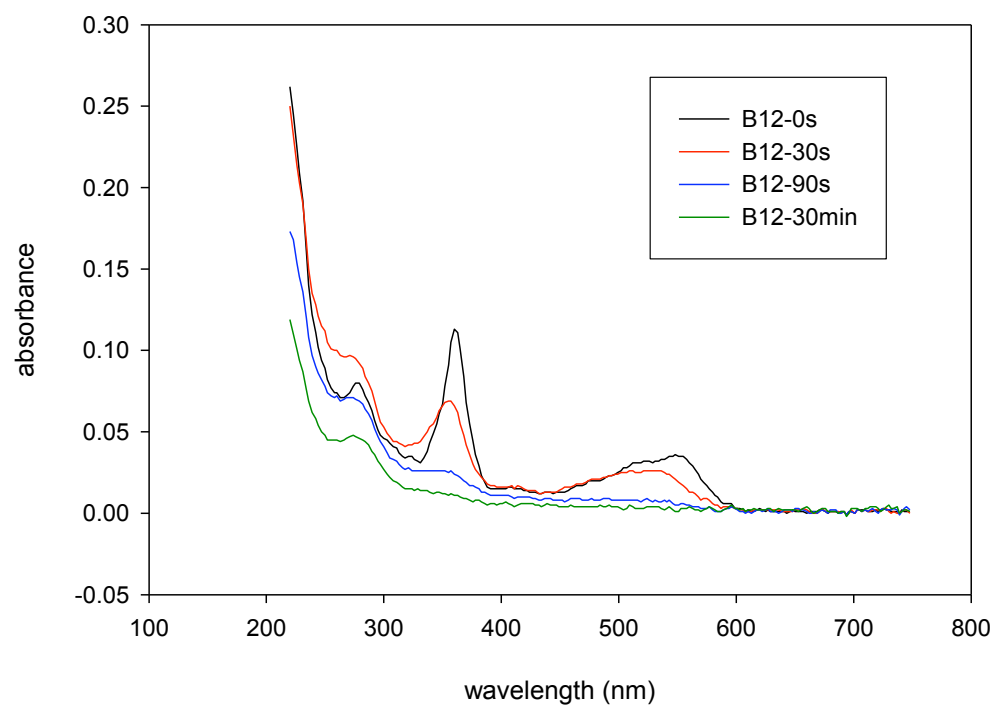

Fig. 2 Initial experiment conducted on a sample with an initial $\mathrm{B}_{12}$ concentration of $45 \mu \mathrm{M}$. The sample was exposed to UV light and subsampled over time. As $\mathrm{B}_{12}$ degraded under UV-light, labile cobalt was released from the metal center of the $\mathrm{B}_{12}$ molecule. 
The degradation of B12 with exposure to natural sunlight $12 / 15 / 04$
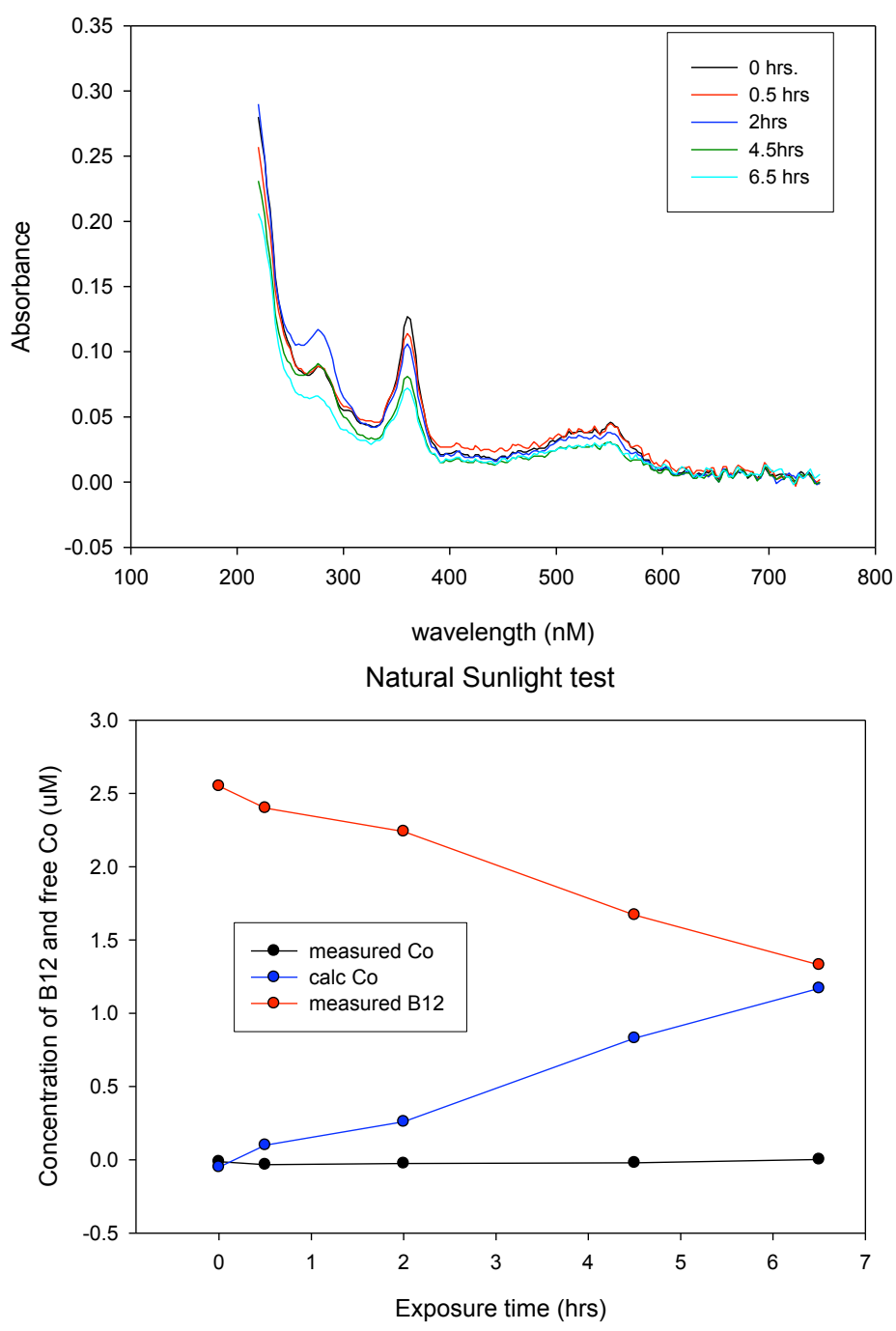

Fig. 3 Identical experiment conducted with exposure to natural sunlight. While the results of the UV-Vis indicate $\mathrm{B}_{12}$ degradation, the ACSV results did not show any release of Co over the exposure to sunlight, suggesting that the degraded $\mathrm{B}_{12}$ maintained conditional stability constants high enough to compete with the electroactive ligand dimethylglyoxime. These figures do not show the $30 \mathrm{hr}$ and $72 \mathrm{hr}$ results because they don't appear to correlate with the other data, and don't accurately describe the number of hours of sunlight that the sample was exposed to as these included many hours of darkness. 


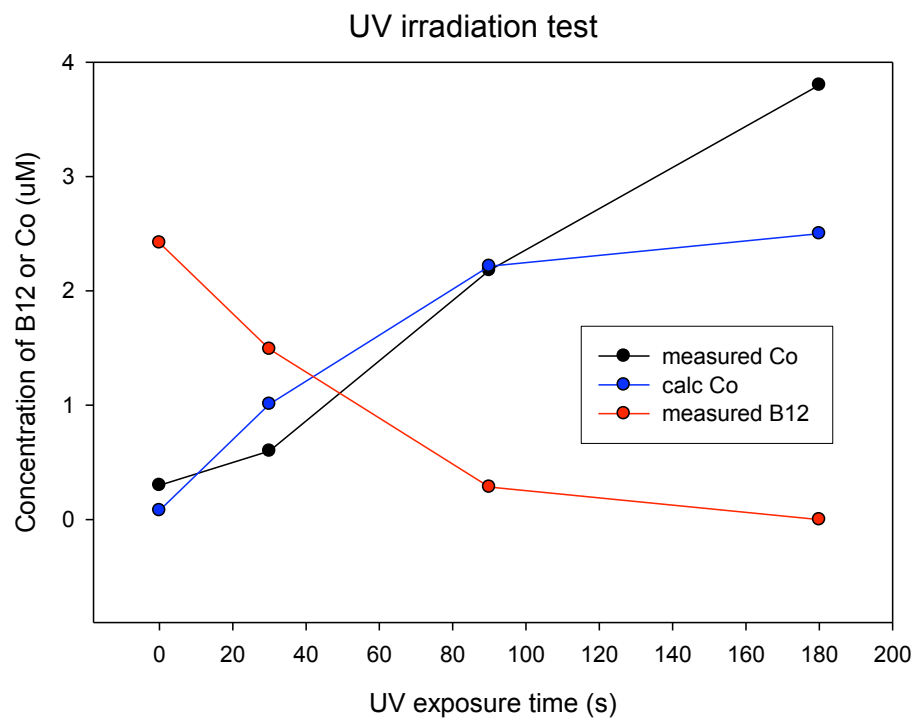

Degradation of B12 with exposure to Natural Sunlight in Miami
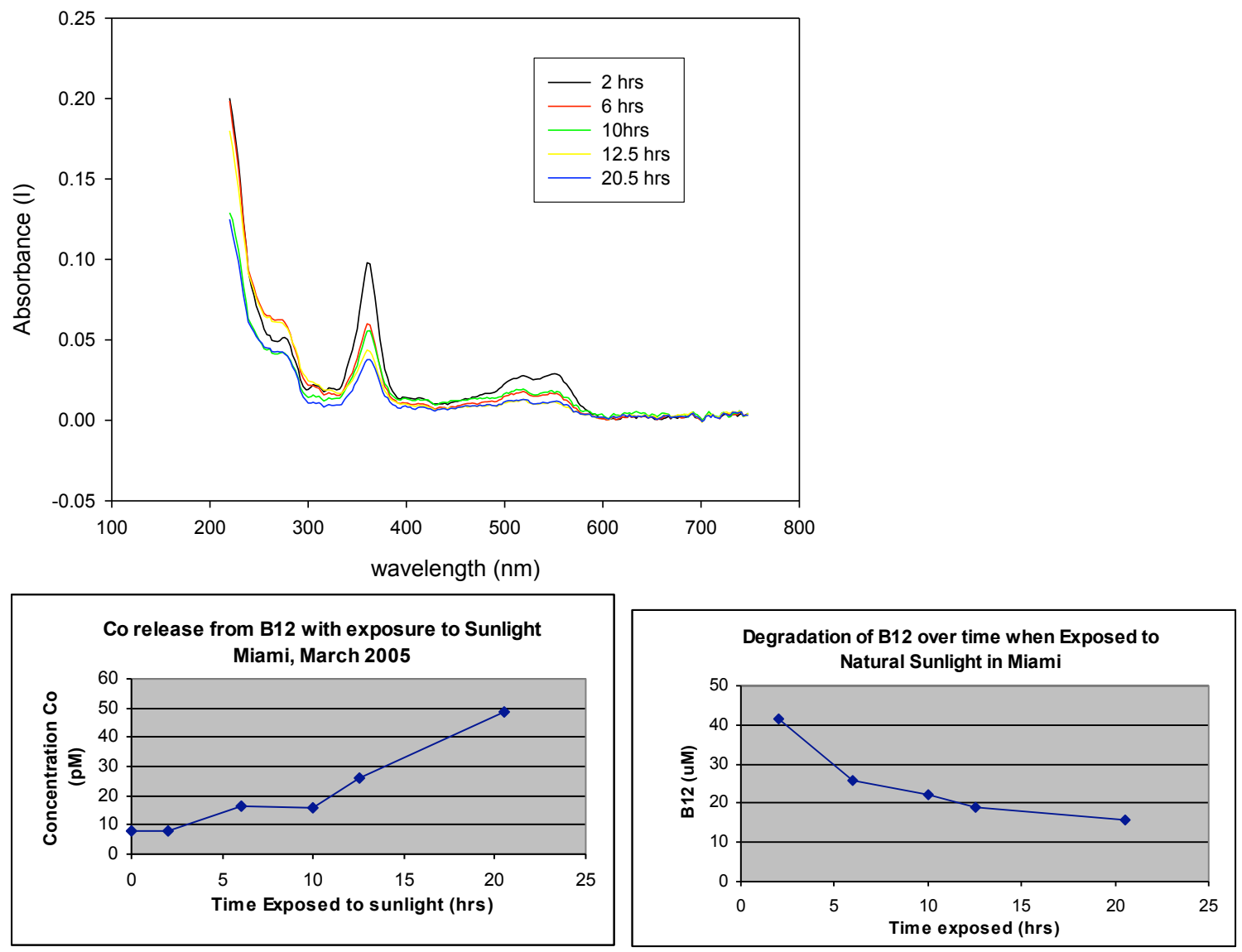
Later tests included parallel experiments with CoenzymeB $_{12}$, which has an adenosyl functional group instead of $-\mathrm{CN}$ in the hopes that parallel mass spectrometry analyses would be fruitful. The timepoints for the below experiment are listed below.

$\begin{array}{ccc}\text { Timepoint } & \text { Time } & \text { Date } \\ \text { to } & 12: 00 & 4.12 .2005 \\ \text { t1 } & 17: 00 & 4.12 .2005 \\ \text { t2 } & 12: 30 & 4.12 .2005 \\ \text { t3 } & 16: 00 & 2.14 .2005 \\ \text { t4 } & 8: 30 & 2.18 .2005\end{array}$
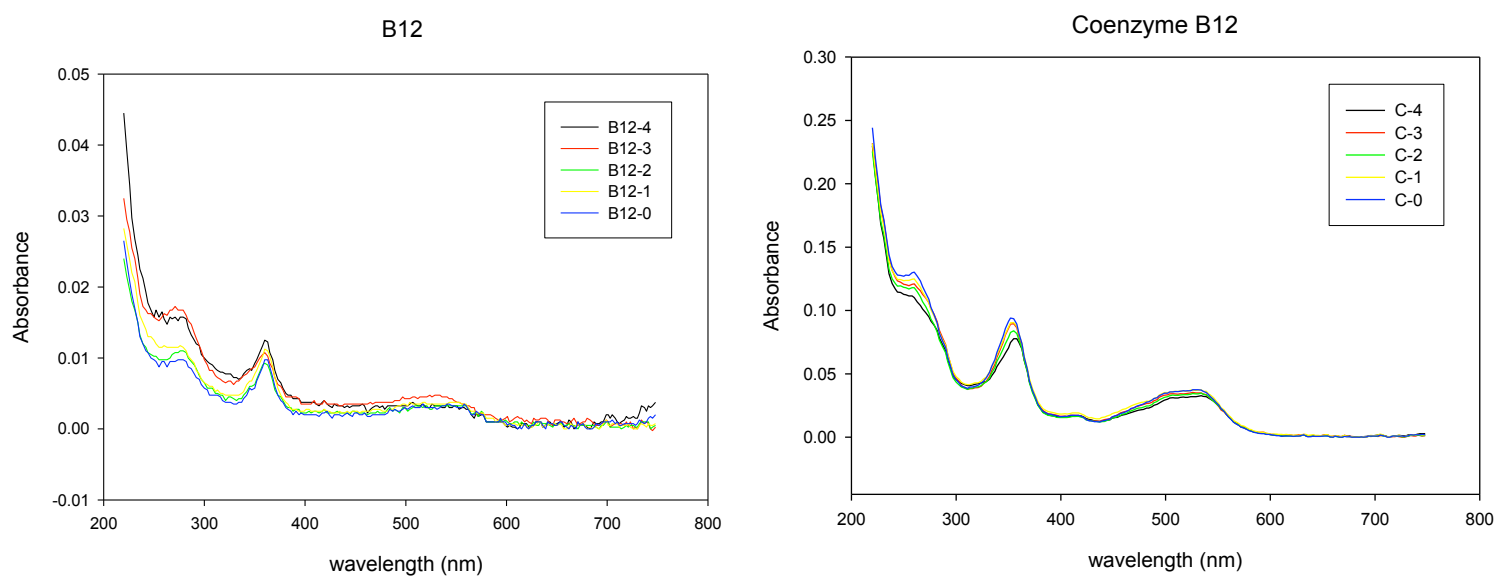


\section{Appendix III: Dissolved cobalt in the Beaufort Sea from the Canadian Arctic GEOTRACES}

The Canadian Arctic GEOTRACES section took place from 8.27.2009-9.13.2009 (Chief Scientist, Roger Francois) and employed a trace metal sampling program in Arctic Ocean.

Sample storage bottles were cleaned identically to those described in previous chapters. Analyses were performed approximately 1 year following collection. Samples were unfiltered.

Cobalt analyses were performed identically to those described in Chapter 5. One set of reagents was used, with a blank of $3 \pm 0.6 \mathrm{pM}(n=6)$.

Figures:

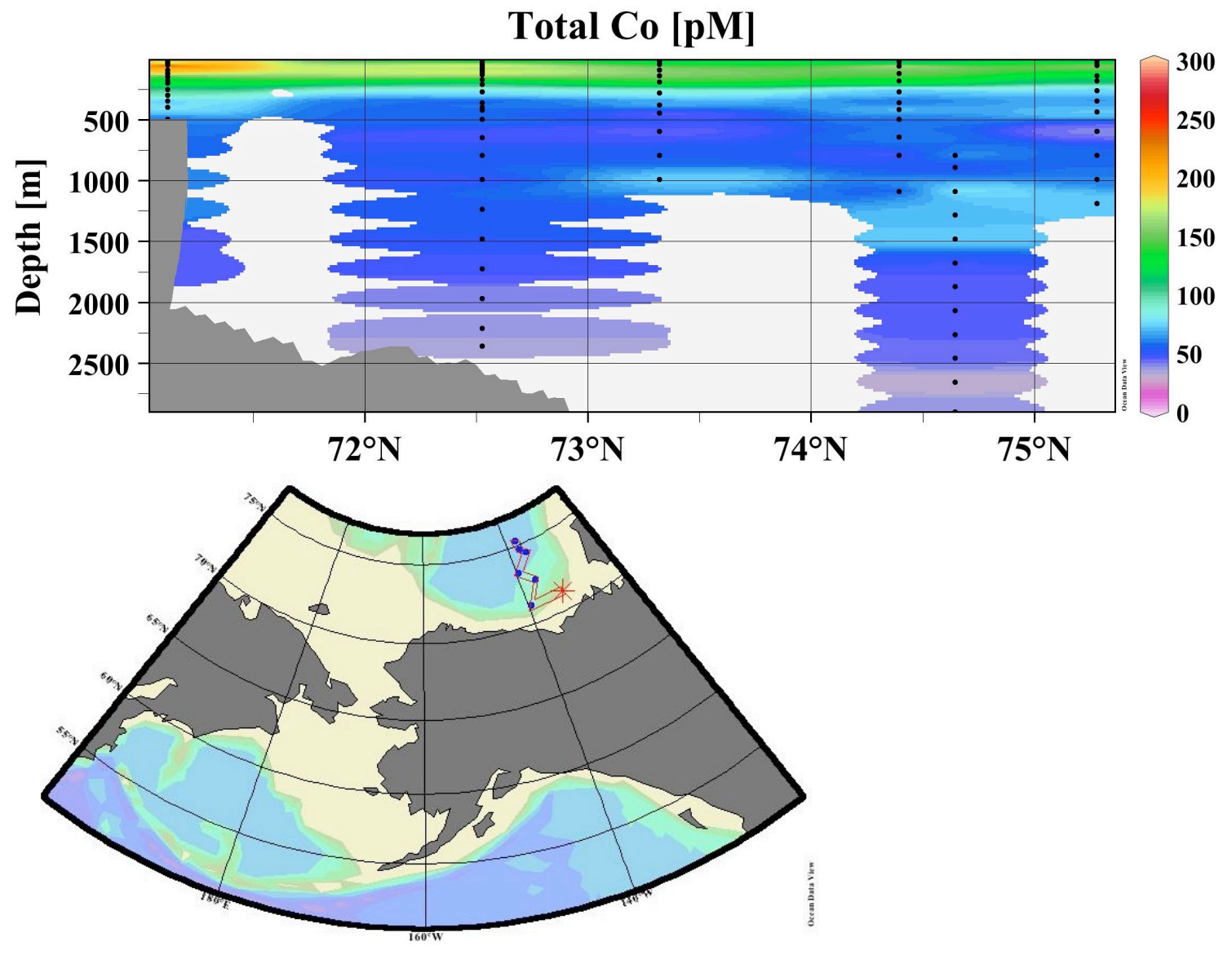

Figure 1. Ocean Data View rendering of the data from the cruise and plot of cruise track. 

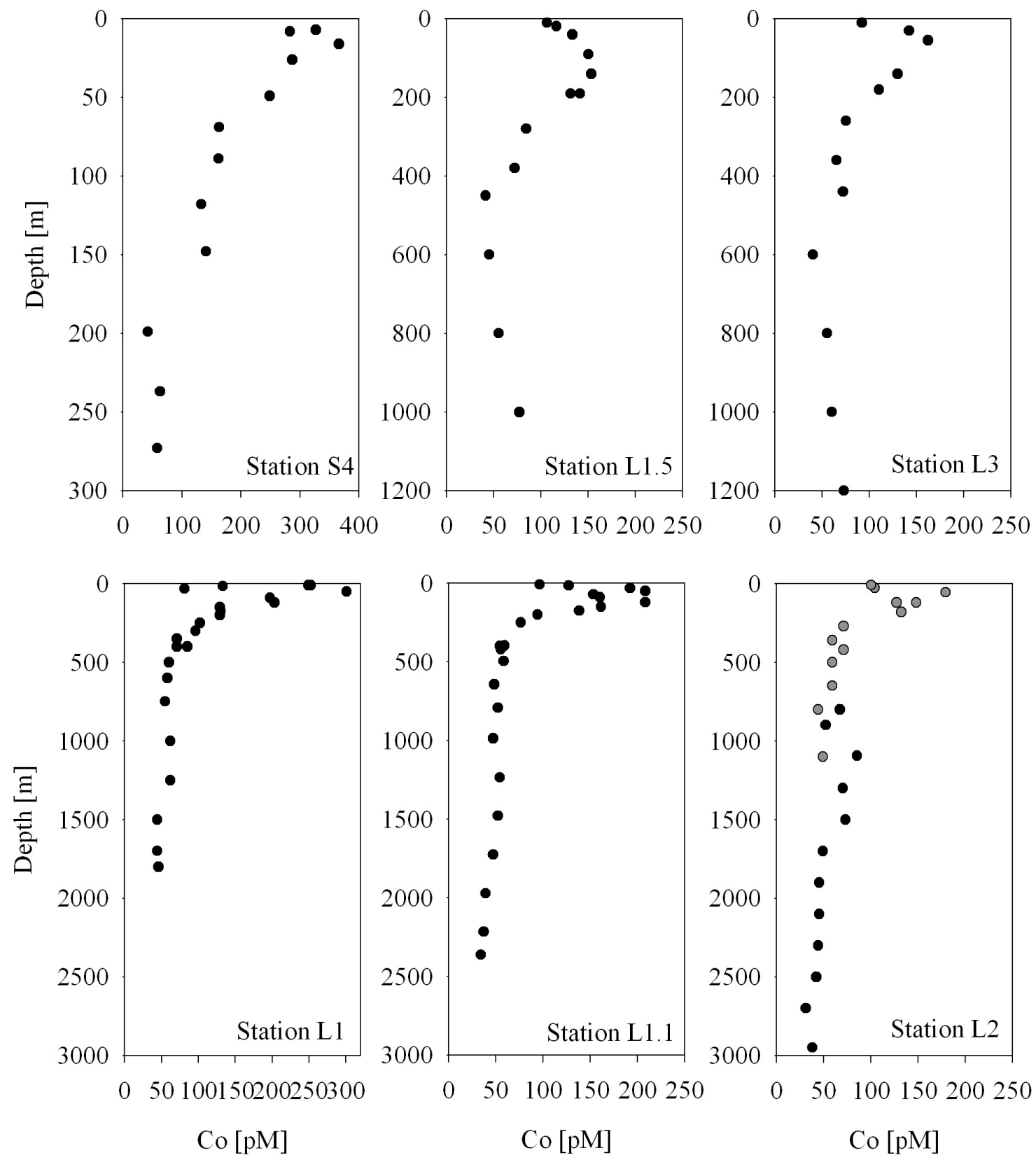

Figure 2. Total cobalt profiles for all stations from the Canadian GEOTRACES Arctic expedition. 

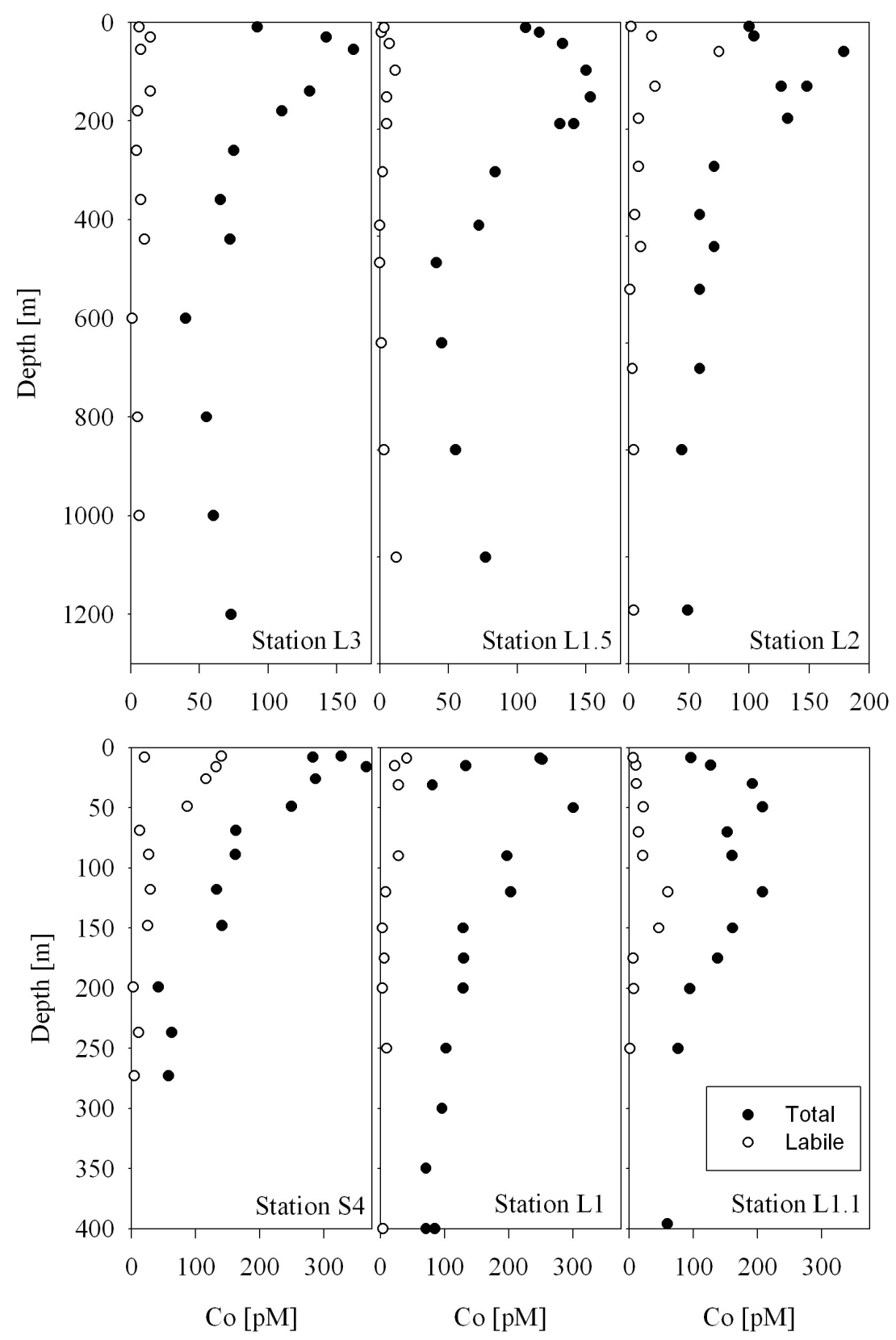

Figure 3. Upper $1200 \mathrm{~m}$ and $300 \mathrm{~m}$ for all Stations showing both total (black) and labile (white) cobalt. The strong surface signal of total cobalt is not reflected in the labile, which is consistent with a strong riverine input which may contain high concentrations of humic substances or other organic molecules capable of strongly complexing cobalt. 


\section{Appendix IV: Data tables}

For all tables, solid black indicates no bottle for a given depth, and an asterisk indicates an oceanographically inconsistent measured sample concentration. For labile cobalt analyses "n.d." denotes that the concentration was not detectable because the there was no measurable peak in the initial scans due to near complete complexation of cobalt by strong ligands. 
Table 1. Data from Chapter 3

\begin{tabular}{|c|c|c|c|c|c|c|c|c|c|}
\hline $\begin{array}{c}\text { Station } \\
{[\#]}\end{array}$ & $\begin{array}{c}\text { Longitude } \\
\text { [deg. E] }\end{array}$ & $\begin{array}{l}\text { Latitude } \\
\text { [deg. N] }\end{array}$ & $\begin{array}{c}\text { Depth } \\
\text { [m] }\end{array}$ & $\begin{array}{l}\mathbf{N}+\mathbf{N} \\
{[\mathbf{u M}]}\end{array}$ & $\begin{array}{l}\mathrm{PO}_{4}{ }^{3-} \\
{[\mathrm{uM}]}\end{array}$ & $\begin{array}{c}\text { TCo } \\
{[\mathrm{pM}]}\end{array}$ & $\begin{array}{c}\mathrm{Co}^{\prime} \\
{[\mathbf{p M}]}\end{array}$ & $\begin{array}{l}\text { Td Fe } \\
{[\mathrm{nM}]}\end{array}$ & $\begin{array}{c}\text { Mn } \\
{[\mathrm{nM}]}\end{array}$ \\
\hline 1 & 330.00 & -11.00 & 10 & 0.14 & 0.137 & 29 & n.d. & $0.64^{*}$ & 2.42 \\
\hline 1 & 330.00 & -11.00 & 20 & 0.08 & 0.119 & 23 & n.d. & 0.23 & 2.45 \\
\hline 1 & 330.00 & -11.00 & 40 & 0.07 & 0.115 & 16 & n.d. & 0.24 & 2.34 \\
\hline 1 & 330.00 & -11.00 & 69 & 0.18 & 0.107 & 20 & n.d. & $0.97^{*}$ & 2.30 \\
\hline 1 & 330.00 & -11.00 & 110 & 0.17 & 0.224 & 36 & n.d. & 0.16 & 1.96 \\
\hline 1 & 330.00 & -11.00 & 135 & 1.35 & 0.366 & 33 & n.d. & 0.35 & 1.75 \\
\hline 1 & 330.00 & -11.00 & 150 & 5.17 & 0.567 & 49 & 2 & 0.31 & 1.10 \\
\hline 1 & 330.00 & -11.00 & 200 & 12.38 & 0.939 & 54 & 1 & 0.19 & 0.46 \\
\hline 1 & 330.00 & -11.00 & 299 & 29.66 & 1.864 & 94 & 12 & 0.49 & 0.24 \\
\hline 1 & 330.00 & -11.00 & 400 & 33.00 & 2.025 & 90 & 18 & 0.58 & 0.29 \\
\hline 1 & 330.00 & -11.00 & 499 & 34.67 & 2.222 & 84 & 18 & 0.57 & 0.27 \\
\hline 1 & 330.00 & -11.00 & 599 & 35.42 & 2.259 & 79 & 13 & 0.56 & 0.23 \\
\hline 1 & 330.00 & -11.00 & 800 & 35.58 & 2.321 & 83 & 24 & 0.61 & 0.25 \\
\hline 1 & 330.00 & -11.00 & 999 & 34.27 & 2.281 & 81 & 14 & 0.49 & 0.24 \\
\hline 1 & 330.00 & -11.00 & 1499 & 34.77 & 2.237 & 77 & 21 & 0.55 & 0.25 \\
\hline 1 & 330.00 & -11.00 & 2000 & 20.45 & 1.362 & 75 & 20 & 0.76 & 0.33 \\
\hline 1 & 330.00 & -11.00 & 2499 & 21.23 & 1.442 & 69 & 30 & 0.75 & $0.10^{*}$ \\
\hline 1 & 330.00 & -11.00 & 3000 & 22.41 & 1.468 & 66 & 16 & 0.82 & 0.31 \\
\hline 1 & 330.00 & -11.00 & 3499 & 22.40 & 1.486 & 62 & 12 & 0.71 & 0.25 \\
\hline 1 & 330.00 & -11.00 & 4000 & 24.08 & 1.632 & 54 & 5 & 0.57 & 0.22 \\
\hline 1 & 330.00 & -11.00 & 4499 & 29.32 & 1.990 & 41 & 8 & & \\
\hline 1 & 330.00 & -11.00 & 4968 & 31.45 & 2.166 & 44 & 3 & 0.56 & 0.21 \\
\hline 2 & 332.50 & -11.25 & 10 & 0.08 & 0.158 & 20 & & 0.33 & 2.29 \\
\hline 2 & 332.50 & -11.25 & 20 & 0.08 & 0.132 & 18 & & 0.16 & 2.39 \\
\hline 2 & 332.50 & -11.25 & 39 & 0.01 & 0.128 & 11 & & 0.09 & 2.13 \\
\hline 2 & 332.50 & -11.25 & 69 & 0.12 & 0.142 & 17 & & 0.17 & 2.02 \\
\hline 2 & 332.50 & -11.25 & 110 & 0.27 & 0.277 & 31 & & 0.10 & 1.80 \\
\hline 2 & 332.50 & -11.25 & 118 & 1.25 & 0.338 & 39 & & 0.13 & 1.57 \\
\hline 2 & 332.50 & -11.25 & 149 & 6.72 & 0.637 & 44 & & 0.12 & 0.81 \\
\hline 2 & 332.50 & -11.25 & 200 & 16.95 & 1.154 & 57 & & 0.39 & 0.31 \\
\hline 2 & 332.50 & -11.25 & 399 & & & & & & \\
\hline 2 & 332.50 & -11.25 & 599 & 34.80 & 2.269 & 67 & & 0.59 & 0.25 \\
\hline 2 & 332.50 & -11.25 & 799 & 35.23 & 2.324 & 100 & & 0.69 & 0.24 \\
\hline 3 & 335.00 & -11.50 & 9 & 0.26 & 0.136 & 10 & n.d. & 0.15 & 2.86 \\
\hline 3 & 335.00 & -11.50 & 20 & 0.08 & 0.121 & 8 & n.d. & 0.30 & 2.56 \\
\hline 3 & 335.00 & -11.50 & 39 & 0.13 & 0.128 & 18 & n.d. & 0.19 & 2.50 \\
\hline 3 & 335.00 & -11.50 & 69 & 0.29 & 0.164 & 17 & n.d. & 0.16 & 2.40 \\
\hline 3 & 335.00 & -11.50 & 109 & 5.51 & 0.559 & 48 & 2 & 0.14 & 1.24 \\
\hline 3 & 335.00 & -11.50 & 129 & 6.38 & 0.602 & 46 & 3 & 0.12 & 1.22 \\
\hline 3 & 335.00 & -11.50 & 150 & 9.73 & 0.776 & 51 & 2 & 0.09 & 0.82 \\
\hline 3 & 335.00 & -11.50 & 200 & 16.99 & 1.149 & 52 & 5 & 0.23 & 0.31 \\
\hline 3 & 335.00 & -11.50 & 300 & 25.48 & 1.713 & 63 & 9 & 0.65 & 0.26 \\
\hline 3 & 335.00 & -11.50 & 399 & 31.56 & 2.054 & 70 & 15 & 0.51 & 0.28 \\
\hline 3 & 335.00 & -11.50 & 499 & 33.45 & 2.172 & 85 & 12 & 0.71 & 0.28 \\
\hline 3 & 335.00 & -11.50 & 599 & 34.74 & 2.220 & 67 & 15 & 0.52 & 0.24 \\
\hline 3 & 335.00 & -11.50 & 699 & 35.75 & 2.375 & 76 & 15 & 0.57 & 0.31 \\
\hline 3 & 335.00 & -11.50 & 800 & 35.72 & 2.401 & 72 & 16 & 0.59 & 0.22 \\
\hline 3 & 335.00 & -11.50 & 999 & 33.48 & 2.288 & 63 & 13 & 0.70 & 0.24 \\
\hline 3 & 335.00 & -11.50 & 1499 & 24.06 & 1.607 & 96 & 20 & 0.62 & 0.17 \\
\hline
\end{tabular}




\begin{tabular}{|c|c|c|c|c|c|c|c|c|c|}
\hline $\begin{array}{c}\text { Station } \\
{[\#]}\end{array}$ & $\begin{array}{l}\text { Longitude } \\
\text { [deg. E] }\end{array}$ & $\begin{array}{l}\text { Latitude } \\
\text { [deg. N] }\end{array}$ & $\begin{array}{c}\text { Depth } \\
{[\mathrm{m}]}\end{array}$ & $\begin{array}{l}\mathbf{N}+\mathbf{N} \\
{[\mathbf{u M}]}\end{array}$ & $\begin{array}{l}\mathrm{PO}_{4}{ }^{3-} \\
{[\mathrm{uM}]}\end{array}$ & $\begin{array}{r}\text { TCo } \\
{[\mathbf{p M}]}\end{array}$ & $\begin{array}{l}\mathrm{Co}^{\prime} \\
{[\mathrm{pM}]}\end{array}$ & $\begin{array}{l}\text { Td Fe } \\
{[\mathrm{nM}]}\end{array}$ & $\begin{array}{c}\text { Mn } \\
{[n M]}\end{array}$ \\
\hline 3 & 335.00 & -11.50 & 2000 & 20.92 & 1.422 & 73 & 17 & 0.70 & 0.24 \\
\hline 3 & 335.00 & -11.50 & 2499 & 21.62 & 1.462 & 66 & 14 & 0.72 & 0.29 \\
\hline 3 & 335.00 & -11.50 & 3000 & 21.67 & 1.487 & 75 & 12 & 0.72 & 0.25 \\
\hline 3 & 335.00 & -11.50 & 3500 & 21.27 & 1.451 & 63 & 14 & 0.69 & 0.24 \\
\hline 3 & 335.00 & -11.50 & 3832 & 23.51 & 1.606 & 63 & 11 & 0.66 & 0.20 \\
\hline 3 & 335.00 & -11.50 & 3999 & 25.06 & 1.711 & 59 & 9 & 0.64 & 0.17 \\
\hline 4 & 337.50 & -11.50 & 10 & 0.10 & 0.164 & 6 & n.d. & 0.15 & 1.93 \\
\hline 4 & 337.50 & -11.50 & 20 & 0.14 & 0.146 & 9 & n.d. & 0.05 & 1.83 \\
\hline 4 & 337.50 & -11.50 & 39 & -0.04 & 0.131 & 9 & 1 & 0.11 & 2.01 \\
\hline 4 & 337.50 & -11.50 & 70 & 0.12 & 0.156 & 11 & n.d. & 0.10 & 1.79 \\
\hline 4 & 337.50 & -11.50 & 99 & 0.11 & 0.209 & 15 & 1 & 0.09 & 1.60 \\
\hline 4 & 337.50 & -11.50 & 110 & 0.16 & 0.178 & 13 & 1 & 0.13 & 1.55 \\
\hline 4 & 337.50 & -11.50 & 129 & 1.29 & 0.290 & 23 & n.d. & 0.23 & 1.44 \\
\hline 4 & 337.50 & -11.50 & 149 & 8.29 & 0.701 & 49 & 2 & 0.28 & 0.95 \\
\hline 4 & 337.50 & -11.50 & 200 & 18.12 & 1.232 & 55 & 33 & 0.19 & 0.29 \\
\hline 4 & 337.50 & -11.50 & 399 & 30.66 & 1.962 & 66 & 20 & 0.53 & 0.18 \\
\hline 4 & 337.50 & -11.50 & 499 & & & 67 & 19 & 0.41 & 0.22 \\
\hline 4 & 337.50 & -11.50 & 599 & 34.55 & 2.280 & 72 & 24 & 0.52 & 0.22 \\
\hline 4 & 337.50 & -11.50 & 800 & 21.60 & 1.741 & 73 & 27 & 0.44 & 0.19 \\
\hline 4 & 337.50 & -11.50 & 999 & 33.54 & 2.283 & 63 & 15 & 0.39 & 0.15 \\
\hline 4 & 337.50 & -11.50 & 1499 & 24.62 & 1.640 & 65 & & 0.47 & 0.41 \\
\hline 4 & 337.50 & -11.50 & 1999 & 21.78 & 1.466 & 61 & 19 & 0.51 & 0.20 \\
\hline 4 & 337.50 & -11.50 & 2499 & 21.87 & 1.444 & 57 & 19 & 0.68 & 0.29 \\
\hline 4 & 337.50 & -11.50 & 3000 & 22.07 & 1.502 & 56 & 14 & 0.61 & 0.31 \\
\hline 4 & 337.50 & -11.50 & 3499 & 22.43 & 1.506 & 54 & 9 & 0.61 & 0.22 \\
\hline 4 & 337.50 & -11.50 & 4000 & 25.89 & 1.759 & 41 & 8 & 0.43 & 0.14 \\
\hline 4 & 337.50 & -11.50 & 4500 & 29.93 & 2.055 & 26 & & 0.39 & 0.14 \\
\hline 4 & 337.50 & -11.50 & 5002 & 31.16 & 2.164 & 30 & 2 & 0.46 & 0.15 \\
\hline 5 & 340.00 & -12.00 & 10 & 0.26 & 0.155 & 26 & n.d. & 0.11 & 2.67 \\
\hline 5 & 340.00 & -12.00 & 19 & 0.14 & 0.141 & 23 & n.d. & 0.16 & 2.62 \\
\hline 5 & 340.00 & -12.00 & 39 & 0.24 & 0.133 & 25 & n.d. & 0.07 & 2.40 \\
\hline 5 & 340.00 & -12.00 & 70 & 0.23 & 0.176 & 38 & n.d. & 0.09 & 2.24 \\
\hline 5 & 340.00 & -12.00 & 110 & 0.94 & 0.267 & 30 & n.d. & 0.10 & 2.17 \\
\hline 5 & 340.00 & -12.00 & 130 & 4.83 & 0.499 & 48 & 3 & 0.05 & 1.55 \\
\hline 5 & 340.00 & -12.00 & 150 & 7.78 & 0.662 & 54 & 5 & 0.29 & 1.52 \\
\hline 5 & 340.00 & -12.00 & 199 & 16.87 & 1.098 & 58 & 1 & 0.18 & 0.52 \\
\hline 5 & 340.00 & -12.00 & 300 & 27.20 & 1.776 & 90 & 12 & 0.42 & 0.20 \\
\hline 5 & 340.00 & -12.00 & 399 & 30.63 & 1.954 & 78 & 15 & 0.48 & 0.24 \\
\hline 5 & 340.00 & -12.00 & 499 & 34.21 & 2.191 & 85 & 14 & 0.63 & 0.33 \\
\hline 5 & 340.00 & -12.00 & 599 & 34.51 & 2.198 & 87 & 15 & 0.74 & 0.26 \\
\hline 5 & 340.00 & -12.00 & 699 & 35.41 & 2.340 & 89 & 14 & 0.51 & 0.28 \\
\hline 5 & 340.00 & -12.00 & 799 & 35.49 & 2.288 & 87 & 21 & 0.55 & 0.25 \\
\hline 5 & 340.00 & -12.00 & 999 & 33.86 & 2.249 & 72 & 16 & 0.69 & 0.22 \\
\hline 5 & 340.00 & -12.00 & 1500 & 24.92 & 1.635 & 89 & 19 & 0.64 & 0.25 \\
\hline 5 & 340.00 & -12.00 & 1999 & 22.11 & 1.449 & 80 & 18 & 0.79 & 0.35 \\
\hline 5 & 340.00 & -12.00 & 2499 & 22.54 & 1.471 & 71 & 16 & 1.05 & 0.92 \\
\hline 5 & 340.00 & -12.00 & 2999 & 22.97 & 1.479 & 62 & 12 & 0.99 & 0.41 \\
\hline 5 & 340.00 & -12.00 & 3499 & 22.85 & 1.482 & 60 & 15 & 0.87 & 0.29 \\
\hline 5 & 340.00 & -12.00 & 4000 & 23.28 & 1.606 & 54 & 16 & 0.55 & 0.20 \\
\hline 5 & 340.00 & -12.00 & 4502 & 30.07 & 2.017 & 47 & 11 & 0.48 & 0.23 \\
\hline
\end{tabular}




\begin{tabular}{|c|c|c|c|c|c|c|c|c|c|}
\hline $\begin{array}{c}\text { Station } \\
{[\#]}\end{array}$ & $\begin{array}{c}\text { Longitude } \\
\text { [deg. E] }\end{array}$ & $\begin{array}{l}\text { Latitude } \\
\text { [deg. N] }\end{array}$ & $\begin{array}{c}\text { Depth } \\
\text { [m] }\end{array}$ & $\begin{array}{l}\mathbf{N}+\mathbf{N} \\
{[\mathbf{u M}]}\end{array}$ & $\begin{array}{l}\mathrm{PO}_{4}{ }^{3-} \\
{[\mathrm{uM}]}\end{array}$ & $\begin{array}{l}\text { TCo } \\
{[\mathrm{pM}]}\end{array}$ & $\begin{array}{c}\mathrm{Co}^{\prime} \\
{[\mathrm{pM}]}\end{array}$ & $\begin{array}{l}\text { Td Fe } \\
{[\mathrm{nM}]}\end{array}$ & $\begin{array}{c}\text { Mn } \\
{[\mathrm{nM}]}\end{array}$ \\
\hline 6 & 342.50 & -12.50 & 10 & 0.12 & 0.160 & 13 & 1 & 0.14 & 2.41 \\
\hline 6 & 342.50 & -12.50 & 20 & 0.06 & 0.138 & 9 & 1 & 0.18 & 1.97 \\
\hline 6 & 342.50 & -12.50 & 39 & 0.11 & 0.134 & 8 & n.d. & 0.20 & 2.97 \\
\hline 6 & 342.50 & -12.50 & 69 & 0.16 & 0.170 & 12 & n.d. & 0.35 & 2.71 \\
\hline 6 & 342.50 & -12.50 & 110 & 1.42 & 0.296 & 22 & 1 & 0.15 & 2.12 \\
\hline 6 & 342.50 & -12.50 & 129 & 6.06 & 0.561 & 39 & n.d. & 0.23 & 1.52 \\
\hline 6 & 342.50 & -12.50 & 149 & 10.16 & 0.793 & 44 & 6 & 0.13 & 0.97 \\
\hline 6 & 342.50 & -12.50 & 198 & 19.73 & 1.320 & 55 & 7 & 0.50 & 1.06 \\
\hline 6 & 342.50 & -12.50 & 400 & 34.00 & 2.191 & 87 & 18 & 0.54 & 0.26 \\
\hline 6 & 342.50 & -12.50 & 599 & 35.15 & 2.311 & 57 & 9 & 0.66 & 0.21 \\
\hline 6 & 342.50 & -12.50 & 798 & 35.47 & 2.405 & 75 & 18 & 0.47 & 0.24 \\
\hline 7 & 345.00 & -12.50 & 10 & 0.08 & 0.168 & 18 & n.d. & 0.25 & 1.95 \\
\hline 7 & 345.00 & -12.50 & 20 & 0.08 & 0.160 & 20 & n.d. & 0.26 & 2.12 \\
\hline 7 & 345.00 & -12.50 & 41 & 0.12 & 0.153 & 16 & n.d. & 0.27 & 2.16 \\
\hline 7 & 345.00 & -12.50 & 70 & 0.17 & 0.192 & 23 & n.d. & 0.47 & 1.92 \\
\hline 7 & 345.00 & -12.50 & 94 & 1.03 & 0.301 & 35 & n.d. & 0.19 & 1.85 \\
\hline 7 & 345.00 & -12.50 & 109 & 4.12 & 0.457 & 41 & n.d. & 0.17 & 1.64 \\
\hline 7 & 345.00 & -12.50 & 150 & 13.39 & 0.983 & 54 & 4 & 0.16 & 0.74 \\
\hline 7 & 345.00 & -12.50 & 200 & 25.49 & 1.670 & 108 & 15 & 0.38 & 0.52 \\
\hline 7 & 345.00 & -12.50 & 300 & 30.72 & 2.021 & 105 & 12 & 0.43 & 0.26 \\
\hline 7 & 345.00 & -12.50 & 399 & 33.67 & 2.186 & 102 & 22 & 0.62 & 0.27 \\
\hline 7 & 345.00 & -12.50 & 500 & 35.54 & 2.323 & 89 & 22 & 0.65 & 0.32 \\
\hline 7 & 345.00 & -12.50 & 599 & 36.59 & 2.385 & 92 & 19 & 0.58 & 0.23 \\
\hline 7 & 345.00 & -12.50 & 700 & 36.34 & 2.443 & 89 & 22 & 0.50 & 0.24 \\
\hline 7 & 345.00 & -12.50 & 799 & 35.88 & 2.422 & 100 & 24 & 0.61 & 0.19 \\
\hline 7 & 345.00 & -12.50 & 899 & 35.36 & 2.420 & 95 & 21 & 0.65 & 0.23 \\
\hline 7 & 345.00 & -12.50 & 999 & 34.48 & 2.377 & 64 & 25 & 0.53 & 0.21 \\
\hline 7 & 345.00 & -12.50 & 1499 & 25.09 & 1.729 & 77 & 23 & 0.92 & 0.27 \\
\hline 7 & 345.00 & -12.50 & 1749 & 23.02 & 1.529 & 95 & 24 & 0.60 & 0.24 \\
\hline 7 & 345.00 & -12.50 & 1999 & 22.36 & 1.525 & 89 & 22 & 1.42 & 0.48 \\
\hline 7 & 345.00 & -12.50 & 2249 & 22.84 & 1.528 & 90 & 23 & 1.38 & 0.94 \\
\hline 7 & 345.00 & -12.50 & 2500 & 23.11 & 1.586 & 75 & 18 & 1.79 & 1.38 \\
\hline 7 & 345.00 & -12.50 & 2841 & 23.26 & 1.600 & 71 & 17 & 1.89 & 1.13 \\
\hline 8 & 347.50 & -12.50 & 10 & 0.15 & 0.157 & 12 & 1 & 0.16 & 2.40 \\
\hline 8 & 347.50 & -12.50 & 19 & 0.01 & 0.146 & 15 & & 0.15 & 2.37 \\
\hline 8 & 347.50 & -12.50 & 40 & 0.03 & 0.145 & 12 & 18 & 0.10 & 2.29 \\
\hline 8 & 347.50 & -12.50 & 70 & 0.11 & 0.199 & 19 & 4 & 0.23 & 1.95 \\
\hline 8 & 347.50 & -12.50 & 94 & 1.73 & 0.333 & 30 & 38 & 0.22 & 1.71 \\
\hline 8 & 347.50 & -12.50 & 110 & 5.12 & 0.452 & 32 & 13 & 0.09 & 1.56 \\
\hline 8 & 347.50 & -12.50 & 150 & 16.04 & 1.057 & 85 & 15 & 0.13 & 0.51 \\
\hline 8 & 347.50 & -12.50 & 199 & 16.23 & 1.158 & 95 & & 0.25 & 0.47 \\
\hline 8 & 347.50 & -12.50 & 400 & 35.75 & 2.186 & 77 & 18 & 0.46 & 0.27 \\
\hline 8 & 347.50 & -12.50 & 599 & 37.05 & 2.360 & & 24 & 0.61 & 0.26 \\
\hline 8 & 347.50 & -12.50 & 800 & 37.46 & 2.435 & 65 & 9 & 0.58 & 0.22 \\
\hline 9 & 350.00 & -12.50 & 9 & 0.08 & 0.167 & 18 & 6 & 0.34 & 2.88 \\
\hline 9 & 350.00 & -12.50 & 20 & 0.02 & 0.152 & 19 & 3 & 0.36 & 3.01 \\
\hline 9 & 350.00 & -12.50 & 39 & 0.13 & 0.141 & 17 & 1 & 0.20 & 2.48 \\
\hline 9 & 350.00 & -12.50 & 69 & 0.24 & 0.250 & 45 & n.d. & 0.26 & 2.51 \\
\hline 9 & 350.00 & -12.50 & 89 & 3.19 & 0.409 & 48 & 6 & 0.19 & 2.01 \\
\hline 9 & 350.00 & -12.50 & 110 & 8.31 & 0.689 & 55 & $39 *$ & 0.07 & 1.48 \\
\hline
\end{tabular}




\begin{tabular}{|c|c|c|c|c|c|c|c|c|c|}
\hline $\begin{array}{c}\text { Station } \\
{[\#]}\end{array}$ & $\begin{array}{c}\text { Longitude } \\
\text { [deg. E] }\end{array}$ & $\begin{array}{l}\text { Latitude } \\
\text { [deg. N] }\end{array}$ & $\begin{array}{l}\text { Depth } \\
{[\mathbf{m}]}\end{array}$ & $\begin{array}{l}\mathbf{N}+\mathbf{N} \\
{[\mathbf{u M}]}\end{array}$ & $\begin{array}{l}\mathrm{PO}_{4}{ }^{3-} \\
{[\mathrm{uM}]}\end{array}$ & $\begin{array}{r}\text { TCo } \\
\text { [pM] }\end{array}$ & $\begin{array}{c}\mathrm{Co}^{\prime} \\
{[\mathrm{pM}]}\end{array}$ & $\begin{array}{l}\text { Td Fe } \\
{[\mathrm{nM}]}\end{array}$ & $\begin{array}{c}\text { Mn } \\
{[n M]}\end{array}$ \\
\hline 9 & 350.00 & -12.50 & 150 & 17.58 & 1.238 & 64 & 10 & 0.12 & 0.67 \\
\hline 9 & 350.00 & -12.50 & 200 & 26.63 & 1.697 & 106 & 12 & 0.20 & 0.52 \\
\hline 9 & 350.00 & -12.50 & 300 & 34.01 & 2.217 & 141 & 27 & 0.42 & 0.30 \\
\hline 9 & 350.00 & -12.50 & 399 & 33.58 & 2.213 & 122 & 24 & 0.44 & 0.32 \\
\hline 9 & 350.00 & -12.50 & 499 & 35.72 & 2.337 & 113 & 24 & 0.50 & 0.34 \\
\hline 9 & 350.00 & -12.50 & 599 & 35.85 & 2.403 & 87 & 22 & 0.56 & 0.27 \\
\hline 9 & 350.00 & -12.50 & 699 & 35.77 & 2.380 & 81 & 19 & 0.71 & 0.38 \\
\hline 9 & 350.00 & -12.50 & 799 & 35.50 & 2.432 & 84 & 19 & 0.48 & 0.24 \\
\hline 9 & 350.00 & -12.50 & 899 & & & & & & \\
\hline 9 & 350.00 & -12.50 & 998 & & & & & & \\
\hline 9 & 350.00 & -12.50 & 1499 & 24.60 & 1.714 & 87 & 28 & 0.61 & 0.21 \\
\hline 9 & 350.00 & -12.50 & 1999 & 22.03 & 1.487 & 90 & 24 & 0.84 & 0.32 \\
\hline 9 & 350.00 & -12.50 & 2499 & 22.76 & 1.549 & 67 & 21 & 0.93 & 0.95 \\
\hline 9 & 350.00 & -12.50 & 3000 & 23.27 & 1.585 & 66 & 11 & 0.86 & 0.32 \\
\hline 9 & 350.00 & -12.50 & 3499 & 22.75 & 1.552 & 54 & 10 & 0.67 & 0.20 \\
\hline 9 & 350.00 & -12.50 & 3959 & 23.43 & 1.610 & 54 & 12 & 0.61 & 0.17 \\
\hline 10 & 352.50 & -12.50 & 9 & 0.21 & 0.176 & & & 0.28 & 2.42 \\
\hline 10 & 352.50 & -12.50 & 20 & 0.15 & 0.164 & & & 0.18 & 2.49 \\
\hline 10 & 352.50 & -12.50 & 39 & 0.19 & 0.218 & & & 0.13 & 2.82 \\
\hline 10 & 352.50 & -12.50 & 70 & 0.85 & 0.282 & & & 0.17 & 2.27 \\
\hline 10 & 352.50 & -12.50 & 89 & 6.98 & 0.592 & & & 0.10 & 1.96 \\
\hline 10 & 352.50 & -12.50 & 100 & 25.09 & 1.639 & & & 0.25 & 0.87 \\
\hline 10 & 352.50 & -12.50 & 109 & 18.32 & 1.173 & & & 0.32 & 1.07 \\
\hline 10 & 352.50 & -12.50 & 129 & 30.05 & 1.903 & & & 0.41 & 0.78 \\
\hline 10 & 352.50 & -12.50 & 150 & 30.20 & 1.863 & & & 0.49 & 0.65 \\
\hline 10 & 352.50 & -12.50 & 169 & 31.31 & 2.011 & & & 0.41 & 0.40 \\
\hline 10 & 352.50 & -12.50 & 199 & 30.46 & 1.877 & & & 0.45 & 0.38 \\
\hline 10 & 352.50 & -12.50 & 399 & 36.81 & 2.278 & & & 0.59 & 0.35 \\
\hline 10 & 352.50 & -12.50 & 499 & 39.69 & 2.542 & & & 0.55 & 0.33 \\
\hline 10 & 352.50 & -12.50 & 599 & 39.23 & 2.545 & & & 0.59 & 0.31 \\
\hline 10 & 352.50 & -12.50 & 799 & 36.50 & 2.465 & & & 0.71 & 0.23 \\
\hline 10 & 352.50 & -12.50 & 1000 & 35.36 & 2.390 & & & 0.59 & 0.19 \\
\hline 10 & 352.50 & -12.50 & 1499 & 26.40 & 1.744 & & & 0.58 & 0.14 \\
\hline 10 & 352.50 & -12.50 & 1999 & 23.34 & 1.588 & & & 0.62 & 0.16 \\
\hline 10 & 352.50 & -12.50 & 2500 & 23.33 & 1.544 & & & 0.72 & 0.17 \\
\hline 10 & 352.50 & -12.50 & 2999 & 23.81 & 1.583 & & & 0.66 & 0.17 \\
\hline 10 & 352.50 & -12.50 & 3499 & 15.51 & 1.172 & & & 0.64 & 0.16 \\
\hline 10 & 352.50 & -12.50 & 3859 & 23.89 & 1.578 & & & 0.62 & 0.17 \\
\hline 11 & 355.00 & -12.50 & 10 & 0.04 & 0.223 & 24 & 5 & 0.22 & 2.40 \\
\hline 11 & 355.00 & -12.50 & 20 & 0.10 & 0.182 & 21 & 2 & 0.13 & 2.20 \\
\hline 11 & 355.00 & -12.50 & 40 & 0.15 & 0.178 & 18 & 1 & 0.13 & 2.38 \\
\hline 11 & 355.00 & -12.50 & 70 & 4.23 & 0.451 & 47 & 1 & 0.12 & 2.08 \\
\hline 11 & 355.00 & -12.50 & 90 & 15.62 & 1.013 & 83 & 6 & 0.14 & 1.16 \\
\hline 11 & 355.00 & -12.50 & 110 & 20.04 & 1.297 & 83 & 7 & 0.14 & 0.77 \\
\hline 11 & 355.00 & -12.50 & 150 & 27.35 & 1.621 & 90 & 8 & 0.26 & 0.58 \\
\hline 11 & 355.00 & -12.50 & 199 & 17.09 & 1.282 & 119 & 16 & 0.35 & 0.39 \\
\hline 11 & 355.00 & -12.50 & 298 & 35.08 & 2.219 & 161 & 21 & 0.58 & 0.29 \\
\hline 11 & 355.00 & -12.50 & 350 & 36.22 & 2.288 & 130 & 23 & 0.58 & 0.27 \\
\hline 11 & 355.00 & -12.50 & 399 & 36.26 & 2.314 & 119 & 17 & 0.56 & 0.33 \\
\hline 11 & 355.00 & -12.50 & 449 & 37.71 & 2.306 & 118 & 29 & 0.62 & 0.27 \\
\hline 11 & 355.00 & -12.50 & 500 & 38.56 & 2.361 & 106 & 22 & 0.57 & 0.28 \\
\hline
\end{tabular}




\begin{tabular}{|c|c|c|c|c|c|c|c|c|c|}
\hline $\begin{array}{c}\text { Station } \\
{[\#]}\end{array}$ & $\begin{array}{c}\text { Longitude } \\
\text { [deg. E] }\end{array}$ & $\begin{array}{l}\text { Latitude } \\
\text { [deg. N] }\end{array}$ & $\begin{array}{c}\text { Depth } \\
{[\mathrm{m}]}\end{array}$ & $\begin{array}{l}\mathbf{N}+\mathbf{N} \\
{[\mathbf{u M}]}\end{array}$ & $\begin{array}{l}\mathrm{PO}_{4}{ }^{3-} \\
{[\mathrm{uM}]}\end{array}$ & $\begin{array}{r}\text { TCo } \\
{[\mathbf{p M}]}\end{array}$ & $\begin{array}{l}\mathrm{Co}^{\prime} \\
{[\mathrm{pM}]}\end{array}$ & $\begin{array}{l}\text { Td Fe } \\
{[\mathrm{nM}]}\end{array}$ & $\begin{array}{c}\text { Mn } \\
{[n M]}\end{array}$ \\
\hline 11 & 355.00 & -12.50 & 600 & 39.10 & 2.485 & 105 & 19 & 0.57 & 0.27 \\
\hline 11 & 355.00 & -12.50 & 700 & 38.57 & 2.554 & 98 & 19 & 0.50 & 0.26 \\
\hline 11 & 355.00 & -12.50 & 800 & 37.63 & 2.515 & 101 & 20 & 0.70 & 0.23 \\
\hline 11 & 355.00 & -12.50 & 998 & 35.40 & 2.357 & 90 & 23 & 0.42 & 0.13 \\
\hline 11 & 355.00 & -12.50 & 1499 & 26.54 & 1.783 & 91 & 21 & 0.50 & 0.37 \\
\hline 11 & 355.00 & -12.50 & 1999 & 23.76 & 1.608 & 82 & 15 & 0.60 & 0.24 \\
\hline 11 & 355.00 & -12.50 & 2500 & 23.42 & 1.604 & 65 & 14 & 0.61 & 0.19 \\
\hline 11 & 355.00 & -12.50 & 2700 & 23.60 & 1.578 & 82 & 12 & 0.64 & 0.18 \\
\hline 11 & 355.00 & -12.50 & 2936 & 23.77 & 1.629 & 68 & 9 & 0.56 & 0.17 \\
\hline 12 & 357.50 & -13.25 & 9 & -0.01 & 0.170 & & & 0.14 & 2.73 \\
\hline 12 & 357.50 & -13.25 & 20 & 0.04 & 0.182 & & & 0.06 & 2.68 \\
\hline 12 & 357.50 & -13.25 & 40 & 0.04 & 0.207 & & & 0.15 & 2.71 \\
\hline 12 & 357.50 & -13.25 & 57 & 1.48 & 0.339 & & & 0.17 & 1.94 \\
\hline 12 & 357.50 & -13.25 & 70 & 3.83 & 0.488 & & & 0.04 & 1.83 \\
\hline 12 & 357.50 & -13.25 & 109 & 16.70 & 1.234 & & & 0.11 & 0.91 \\
\hline 12 & 357.50 & -13.25 & 149 & 26.07 & 1.724 & & & 0.28 & 0.71 \\
\hline 12 & 357.50 & -13.25 & 199 & 30.20 & 1.983 & & & $4.66^{*}$ & 0.50 \\
\hline 12 & 357.50 & -13.25 & 399 & 36.63 & 2.386 & & & 0.45 & 0.35 \\
\hline 12 & 357.50 & -13.25 & 600 & 37.83 & 2.463 & & & 0.53 & 0.28 \\
\hline 12 & 357.50 & -13.25 & 800 & 37.07 & 2.532 & & & 0.53 & 0.25 \\
\hline 13 & 359.96 & -13.48 & 10 & 0.16 & 0.242 & 33 & 1 & 0.10 & 2.61 \\
\hline 13 & 359.96 & -13.48 & 20 & 0.26 & 0.224 & 35 & 2 & 0.10 & 2.55 \\
\hline 13 & 359.96 & -13.48 & 40 & 0.20 & 0.147 & 49 & 4 & 0.08 & 2.46 \\
\hline 13 & 359.96 & -13.48 & 54 & 2.78 & 0.401 & 68 & 9 & 0.13 & 2.30 \\
\hline 13 & 359.96 & -13.48 & 70 & 10.64 & 0.809 & 69 & 21 & 0.16 & 1.74 \\
\hline 13 & 359.96 & -13.48 & 110 & 28.11 & 1.783 & 86 & 11 & 0.29 & 0.64 \\
\hline 13 & 359.96 & -13.48 & 129 & 29.45 & 1.855 & 92 & 17 & 0.32 & 0.62 \\
\hline 13 & 359.96 & -13.48 & 150 & 30.62 & 1.935 & 97 & 18 & 0.37 & 0.51 \\
\hline 13 & 359.96 & -13.48 & 200 & 33.31 & 2.092 & 118 & 29 & 0.44 & 0.49 \\
\hline 13 & 359.96 & -13.45 & 300 & 34.68 & 2.169 & & & 0.51 & 0.35 \\
\hline 13 & 359.96 & -13.48 & 399 & 38.59 & 2.431 & 130 & 31 & 0.71 & 0.43 \\
\hline 13 & 359.96 & -13.45 & 500 & 39.74 & 2.574 & & & 0.62 & 0.39 \\
\hline 13 & 359.96 & -13.48 & 600 & 41.01 & 2.646 & 111 & 30 & 0.87 & 0.37 \\
\hline 13 & 359.96 & -13.45 & 699 & 37.65 & 2.599 & & & 0.78 & 0.28 \\
\hline 13 & 359.96 & -13.48 & 799 & 37.93 & 2.551 & 99 & 24 & 0.76 & 0.26 \\
\hline 13 & 359.96 & -13.48 & 999 & 35.17 & 2.360 & 80 & 22 & 0.66 & 0.32 \\
\hline 13 & 359.96 & -13.48 & 1249 & 31.06 & 2.145 & 89 & 19 & 0.63 & 0.18 \\
\hline 13 & 359.96 & -13.48 & 1499 & 26.74 & 1.819 & 89 & 24 & 0.51 & $0.08 *$ \\
\hline 13 & 359.96 & -13.48 & 1999 & 23.60 & 1.625 & 86 & 20 & 0.57 & 0.20 \\
\hline 13 & 359.96 & -13.48 & 2499 & 23.49 & 1.574 & 72 & 17 & 0.58 & 0.15 \\
\hline 13 & 359.96 & -13.48 & 3000 & 23.96 & 1.632 & 69 & 14 & 0.55 & 0.12 \\
\hline 13 & 359.96 & -13.48 & 3500 & 24.12 & 1.639 & 58 & 11 & $0.39 *$ & 0.16 \\
\hline 13 & 359.96 & -13.48 & 3999 & 24.00 & 1.623 & 59 & 9 & 0.58 & 0.13 \\
\hline 13 & 359.96 & -13.48 & 4499 & 24.10 & 1.630 & 50 & 7 & 0.52 & 0.10 \\
\hline 13 & 359.96 & -13.48 & 5106 & 23.99 & 1.604 & 31 & 11 & $2.98^{*}$ & 0.18 \\
\hline 14 & 2.50 & -13.75 & 10 & 0.35 & 0.265 & & & 0.08 & 2.19 \\
\hline 14 & 2.50 & -13.75 & 20 & 0.61 & 0.257 & & & 0.11 & 2.41 \\
\hline 14 & 2.50 & -13.75 & 40 & 2.01 & 0.358 & & & 0.07 & 2.10 \\
\hline 14 & 2.50 & -13.75 & 55 & 7.79 & 0.709 & & & 0.07 & 1.81 \\
\hline 14 & 2.50 & -13.75 & 70 & 9.34 & 0.738 & & & 0.08 & 1.72 \\
\hline
\end{tabular}




\begin{tabular}{|c|c|c|c|c|c|c|c|c|c|}
\hline $\begin{array}{c}\text { Station } \\
{[\#]}\end{array}$ & $\begin{array}{c}\text { Longitude } \\
\text { [deg. E] }\end{array}$ & $\begin{array}{l}\text { Latitude } \\
\text { [deg. N] }\end{array}$ & $\begin{array}{c}\text { Depth } \\
{[\mathrm{m}]}\end{array}$ & $\begin{array}{l}\mathbf{N}+\mathbf{N} \\
{[\mathbf{u M}]}\end{array}$ & $\begin{array}{l}\mathrm{PO}_{4}{ }^{3-} \\
{[\mathrm{uM}]}\end{array}$ & $\begin{array}{r}\text { TCo } \\
\text { [pM] }\end{array}$ & $\begin{array}{r}\mathrm{Co}^{\prime} \\
{[\mathrm{pM}]}\end{array}$ & $\begin{array}{l}\text { Td Fe } \\
{[\mathrm{nM}]}\end{array}$ & $\begin{array}{c}\text { Mn } \\
{[\mathbf{n M}]}\end{array}$ \\
\hline 14 & 2.50 & -13.75 & 109 & 28.59 & 1.758 & & & 0.27 & 0.72 \\
\hline 14 & 2.50 & -13.75 & 149 & 32.14 & 1.932 & & & 0.54 & 0.70 \\
\hline 14 & 2.50 & -13.75 & 199 & 33.58 & 1.993 & & & 0.61 & 0.50 \\
\hline 14 & 2.50 & -13.75 & 400 & 40.32 & 2.500 & & & 0.70 & 0.44 \\
\hline 14 & 2.50 & -13.75 & 599 & 41.11 & 2.667 & & & 0.95 & 0.43 \\
\hline 14 & 2.50 & -13.75 & 799 & 39.25 & 2.565 & & & 1.06 & 0.34 \\
\hline 15 & 5.00 & -13.60 & 10 & 0.05 & 0.208 & 36 & 4 & 0.18 & 3.13 \\
\hline 15 & 5.00 & -13.60 & 20 & 0.32 & 0.209 & 37 & 2 & 0.12 & 2.73 \\
\hline 15 & 5.00 & -13.60 & 40 & 13.27 & 0.979 & 88 & 10 & 0.08 & 1.87 \\
\hline 15 & 5.00 & -13.60 & 54 & 25.22 & 1.633 & 128 & 21 & 0.16 & 1.35 \\
\hline 15 & 5.00 & -13.60 & 70 & 28.54 & 1.814 & 120 & 5 & 0.30 & 1.07 \\
\hline 15 & 5.00 & -13.60 & 110 & 29.97 & 1.854 & 121 & 7 & 0.21 & 0.70 \\
\hline 15 & 5.00 & -13.60 & 130 & 29.40 & 1.851 & 157 & 12 & 0.18 & 0.58 \\
\hline 15 & 5.00 & -13.60 & 150 & 31.61 & 1.996 & 116 & 10 & 0.55 & 1.14 \\
\hline 15 & 5.00 & -13.60 & 229 & 17.51 & 1.389 & 143 & 14 & 0.54 & 0.93 \\
\hline 15 & 5.00 & -13.60 & 399 & 40.40 & 2.567 & 165 & 39 & 0.98 & 0.74 \\
\hline 15 & 5.00 & -13.60 & 599 & 41.21 & 2.683 & 128 & 31 & 1.02 & 0.56 \\
\hline 15 & 5.00 & -13.60 & 800 & 39.76 & 2.676 & 112 & 26 & 1.19 & 0.46 \\
\hline 15 & 5.00 & -13.60 & 1000 & 35.92 & 2.482 & 97 & 27 & 0.85 & 0.35 \\
\hline 15 & 5.00 & -13.60 & 1249 & 31.37 & 2.157 & 103 & 22 & 0.99 & 0.33 \\
\hline 15 & 5.00 & -13.60 & 1499 & 26.70 & 1.853 & 92 & 17 & 0.77 & 0.32 \\
\hline 15 & 5.00 & -13.60 & 1999 & 16.35 & 1.290 & 90 & & 0.89 & 0.22 \\
\hline 15 & 5.00 & -13.60 & 2500 & 23.59 & 1.579 & 70 & & 0.62 & 0.18 \\
\hline 15 & 5.00 & -13.60 & 2999 & 23.30 & 1.550 & 56 & 8 & 0.58 & 0.23 \\
\hline 15 & 5.00 & -13.60 & 3499 & 24.50 & 1.652 & 53 & & 0.65 & 0.20 \\
\hline 15 & 5.00 & -13.60 & 3999 & 23.94 & 1.566 & 45 & & 0.56 & 0.31 \\
\hline 15 & 5.00 & -13.60 & 4499 & 24.10 & 1.627 & 29 & 9 & 0.55 & 0.18 \\
\hline 15 & 5.00 & -13.60 & 5196 & 24.31 & 1.631 & 32 & 2 & 0.40 & 0.17 \\
\hline 16 & 7.50 & -14.25 & 9 & 0.24 & 0.259 & & & 0.23 & 3.47 \\
\hline 16 & 7.50 & -14.25 & 20 & 0.28 & 0.248 & & & 0.20 & 2.77 \\
\hline 16 & 7.50 & -14.25 & 40 & 4.62 & 0.552 & & & 0.10 & 2.37 \\
\hline 16 & 7.50 & -14.25 & 55 & 15.49 & 1.131 & & & 0.11 & 1.84 \\
\hline 16 & 7.50 & -14.25 & 70 & 26.96 & 1.743 & & & 0.19 & 1.06 \\
\hline 16 & 7.50 & -14.25 & 109 & 30.58 & 1.963 & & & 0.46 & 1.25 \\
\hline 16 & 7.50 & -14.25 & 129 & 31.72 & 1.996 & & & 0.53 & 0.70 \\
\hline 16 & 7.50 & -14.25 & 150 & 32.26 & 2.072 & & & 0.44 & 0.80 \\
\hline 16 & 7.50 & -14.25 & 230 & 34.06 & 2.144 & & & 0.63 & 0.45 \\
\hline 16 & 7.50 & -14.25 & 400 & 39.70 & 2.517 & & & 0.68 & 0.43 \\
\hline 16 & 7.50 & -14.25 & 600 & 41.62 & 2.716 & & & 0.87 & 0.64 \\
\hline 17 & 10.00 & -14.75 & 10 & 0.31 & 0.250 & 80 & 13 & 0.21 & 3.27 \\
\hline 17 & 10.00 & -14.75 & 20 & 8.08 & 0.723 & 102 & 12 & 0.17 & 2.15 \\
\hline 17 & 10.00 & -14.75 & 29 & 10.53 & 0.878 & 84 & 9 & 0.21 & 1.94 \\
\hline 17 & 10.00 & -14.75 & 40 & 23.68 & 1.576 & 135 & 24 & 0.16 & 1.41 \\
\hline 17 & 10.00 & -14.75 & 55 & 27.28 & 1.756 & 109 & 12 & 0.38 & 0.83 \\
\hline 17 & 10.00 & -14.75 & 70 & 27.54 & 1.781 & 104 & 8 & 0.56 & 0.62 \\
\hline 17 & 10.00 & -14.75 & 110 & 28.15 & 1.838 & 102 & 3 & 0.59 & 0.53 \\
\hline 17 & 10.00 & -14.75 & 130 & 28.92 & 1.881 & 115 & 6 & 0.62 & 0.52 \\
\hline 17 & 10.00 & -14.75 & 150 & 29.07 & 1.891 & 105 & 5 & 0.55 & 0.56 \\
\hline 17 & 10.00 & -14.75 & 200 & 32.14 & 2.072 & 141 & 29 & 1.35 & 0.58 \\
\hline 17 & 10.00 & -14.75 & 299 & 37.25 & 2.399 & 154 & 15 & 0.86 & 0.68 \\
\hline
\end{tabular}




\begin{tabular}{|c|c|c|c|c|c|c|c|c|c|}
\hline $\begin{array}{c}\text { Station } \\
{[\#]}\end{array}$ & $\begin{array}{c}\text { Longitude } \\
\text { [deg. E] }\end{array}$ & $\begin{array}{l}\text { Latitude } \\
\text { [deg. N] }\end{array}$ & $\begin{array}{c}\text { Depth } \\
{[\mathrm{m}]}\end{array}$ & $\begin{array}{l}\mathbf{N}+\mathbf{N} \\
{[\mathbf{u M}]}\end{array}$ & $\begin{array}{l}\mathrm{PO}_{4}{ }^{3-} \\
{[\mathrm{uM}]}\end{array}$ & $\begin{array}{l}\text { TCo } \\
{[\mathrm{pM}]}\end{array}$ & $\begin{array}{c}\mathrm{Co}^{\prime} \\
{[\mathrm{pM}]}\end{array}$ & $\begin{array}{l}\text { Td Fe } \\
{[\mathrm{nM}]}\end{array}$ & $\begin{array}{c}\text { Mn } \\
{[n M]}\end{array}$ \\
\hline 17 & 10.00 & -14.75 & 399 & 40.33 & 2.618 & 146 & 24 & 0.96 & 0.78 \\
\hline 17 & 10.00 & -14.75 & 600 & 41.50 & 2.745 & 128 & 28 & 0.94 & 0.59 \\
\hline 17 & 10.00 & -14.75 & 799 & 41.06 & 2.689 & 93 & 31 & 1.22 & 0.59 \\
\hline 17 & 10.00 & -14.75 & 999 & 19.01 & 1.666 & 107 & 31 & 1.45 & 0.33 \\
\hline 17 & 10.00 & -14.75 & 1249 & 31.31 & 2.155 & 104 & & 1.02 & 0.28 \\
\hline 17 & 10.00 & -14.75 & 1500 & 26.70 & 1.849 & 85 & & 0.84 & 0.27 \\
\hline 17 & 10.00 & -14.75 & 1999 & 23.29 & 1.598 & 61 & & 0.80 & 0.29 \\
\hline 17 & 10.00 & -14.75 & 2500 & 23.55 & 1.616 & 49 & 20 & 0.69 & 0.25 \\
\hline 17 & 10.00 & -14.75 & 3000 & 23.81 & 1.626 & 50 & & 0.64 & 0.18 \\
\hline 17 & 10.00 & -14.75 & 3400 & 24.24 & 1.672 & 49 & & 0.47 & 0.16 \\
\hline 17 & 10.00 & -14.75 & 3650 & 24.56 & 1.715 & 56 & & 0.43 & 0.17 \\
\hline 18 & 11.50 & -14.75 & 10 & 5.53 & 0.605 & 172 & & 0.46 & 4.03 \\
\hline 18 & 11.50 & -14.75 & 17 & 6.91 & 0.702 & 175 & & 0.70 & 3.49 \\
\hline 18 & 11.50 & -14.75 & 54 & 19.96 & 1.434 & 158 & & 1.38 & 2.33 \\
\hline 18 & 11.50 & -14.75 & 70 & 22.26 & 1.571 & 141 & & 1.26 & 2.33 \\
\hline 18 & 11.50 & -14.75 & 110 & 27.69 & 1.730 & 113 & & 1.16 & 1.12 \\
\hline 18 & 11.50 & -14.75 & 150 & 28.46 & 1.816 & 101 & & 0.88 & 1.01 \\
\hline 18 & 11.50 & -14.75 & 200 & 29.61 & 1.855 & & & 1.11 & 0.78 \\
\hline 18 & 11.50 & -14.75 & 300 & 34.49 & 2.141 & & & 0.93 & 0.70 \\
\hline 18 & 11.50 & -14.75 & 399 & 36.77 & 2.322 & & & 0.75 & 0.53 \\
\hline 18 & 11.50 & -14.75 & 599 & 41.71 & 2.683 & & & 1.34 & 0.58 \\
\hline 18 & 11.50 & -14.75 & 799 & 38.89 & 2.606 & & & 1.90 & 0.79 \\
\hline 19 & 12.20 & -14.75 & 10 & 12.20 & 1.022 & 184 & 20 & 1.01 & 2.33 \\
\hline 19 & 12.20 & -14.75 & 20 & 19.10 & 1.373 & 187 & 23 & 1.31 & 2.14 \\
\hline 19 & 12.20 & -14.75 & 30 & 21.63 & 1.501 & 188 & 24 & 0.49 & 1.75 \\
\hline 19 & 12.20 & -14.75 & 40 & 24.38 & 1.618 & 200 & 22 & 0.36 & 1.59 \\
\hline 19 & 12.20 & -14.75 & 60 & 25.77 & 1.652 & 150 & & 3.03 & 2.52 \\
\hline 19 & 12.20 & -14.75 & 100 & 27.62 & 1.714 & 128 & & 1.28 & 1.09 \\
\hline 19 & 12.20 & -14.75 & 150 & 27.25 & 1.729 & 106 & 4 & 0.96 & 0.59 \\
\hline 19 & 12.20 & -14.75 & 199 & 30.30 & 1.861 & 140 & & 0.36 & 0.56 \\
\hline 19 & 12.20 & -14.75 & 270 & 26.00 & 1.753 & 147 & & 2.88 & 1.08 \\
\hline 19 & 12.20 & -14.75 & 350 & 38.01 & 2.411 & 162 & 32 & 2.44 & 1.06 \\
\hline 19 & 12.20 & -14.75 & 400 & 39.92 & 2.546 & 140 & 39 & 3.11 & 1.12 \\
\hline 20 & 11.25 & -17.50 & 11 & 21.90 & 1.431 & & & 2.28 & 1.60 \\
\hline 20 & 11.25 & -17.50 & 29 & 22.92 & 1.579 & & & 2.41 & 1.54 \\
\hline 20 & 11.25 & -17.50 & 70 & 25.32 & 1.723 & & & 2.62 & 1.55 \\
\hline 20 & 11.25 & -17.50 & 150 & 29.09 & 2.056 & & & 5.18 & 1.55 \\
\hline 20 & 11.25 & -17.50 & 190 & 30.60 & 1.958 & & & 3.77 & 0.82 \\
\hline 20 & 11.25 & -17.50 & 239 & 32.94 & 2.095 & & & 0.95 & 0.53 \\
\hline 20 & 11.25 & -17.50 & 299 & 36.13 & 2.258 & & & 1.28 & 0.56 \\
\hline 20 & 11.25 & -17.50 & 400 & 39.26 & 2.525 & & & 0.88 & 0.50 \\
\hline 20 & 11.25 & -17.50 & 600 & 39.75 & 2.623 & & & 3.30 & 0.51 \\
\hline 21 & 12.00 & -20.00 & 10 & 13.31 & 1.124 & & & 0.46 & 0.93 \\
\hline 21 & 12.00 & -20.00 & 20 & 16.86 & 1.313 & & & 0.32 & 1.13 \\
\hline 21 & 12.00 & -20.00 & 29 & 20.57 & 1.578 & & & 0.30 & 1.18 \\
\hline 21 & 12.00 & -20.00 & 40 & 21.96 & 1.658 & & & 0.37 & 1.13 \\
\hline 21 & 12.00 & -20.00 & 50 & 23.90 & 1.716 & & & 0.97 & 1.34 \\
\hline 21 & 12.00 & -20.00 & 70 & 26.64 & 1.908 & & & 0.79 & 1.21 \\
\hline 21 & 12.00 & -20.00 & 109 & 27.55 & 1.988 & & & 1.13 & 1.30 \\
\hline 21 & 12.00 & -20.00 & 149 & 30.34 & 2.072 & & & 1.16 & 1.13 \\
\hline
\end{tabular}




\begin{tabular}{|c|c|c|c|c|c|c|c|c|c|}
\hline $\begin{array}{c}\text { Station } \\
{[\#]}\end{array}$ & $\begin{array}{c}\text { Longitude } \\
\text { [deg. E] }\end{array}$ & $\begin{array}{l}\text { Latitude } \\
\text { [deg. N] }\end{array}$ & $\begin{array}{c}\text { Depth } \\
{[\mathrm{m}]}\end{array}$ & $\begin{array}{l}\mathbf{N}+\mathbf{N} \\
{[\mathbf{u M}]}\end{array}$ & $\begin{array}{l}\mathrm{PO}_{4}{ }^{3-} \\
{[\mathrm{uM}]}\end{array}$ & $\begin{array}{r}\text { TCo } \\
{[\mathbf{p M}]}\end{array}$ & $\begin{array}{c}\mathrm{Co}^{\prime} \\
{[\mathrm{pM}]}\end{array}$ & $\begin{array}{l}\text { Td Fe } \\
{[\mathrm{nM}]}\end{array}$ & $\begin{array}{c}\text { Mn } \\
{[\mathbf{n M}]}\end{array}$ \\
\hline 21 & 12.00 & -20.00 & 200 & 29.74 & 1.980 & & & 1.11 & 0.73 \\
\hline 21 & 12.00 & -20.00 & 249 & 29.30 & 1.958 & & & 1.04 & 0.56 \\
\hline 21 & 12.00 & -20.00 & 300 & 33.23 & 2.249 & & & 2.21 & 0.84 \\
\hline 22 & 13.50 & -24.00 & 10 & 6.02 & 0.558 & & & 0.21 & 1.01 \\
\hline 22 & 13.50 & -24.00 & 30 & 12.88 & 0.916 & & & 0.25 & 0.93 \\
\hline 22 & 13.50 & -24.00 & 50 & 13.34 & 0.984 & & & 0.24 & 1.01 \\
\hline 22 & 13.50 & -24.00 & 70 & 20.75 & 1.411 & & & 0.62 & 1.34 \\
\hline 22 & 13.50 & -24.00 & 109 & 19.35 & 1.331 & & & 0.37 & 0.86 \\
\hline 22 & 13.50 & -24.00 & 130 & 25.38 & 1.682 & & & 0.55 & 0.96 \\
\hline 22 & 13.50 & -24.00 & 150 & 28.99 & 1.881 & & & 0.72 & 0.94 \\
\hline 22 & 13.50 & -24.00 & 199 & 32.27 & 2.076 & & & 0.94 & 0.92 \\
\hline 22 & 13.50 & -24.00 & 225 & 33.68 & 2.311 & & & 1.67 & 0.89 \\
\hline 22 & 13.50 & -24.00 & 249 & 33.10 & 2.448 & & & 2.68 & 1.14 \\
\hline 22 & 13.50 & -24.00 & 264 & 33.74 & 2.602 & & & 7.86 & 1.39 \\
\hline 23 & 14.50 & -25.00 & 10 & 25.92 & 1.792 & 188 & 71 & 1.61 & 2.78 \\
\hline 23 & 14.50 & -25.00 & 15 & 25.14 & 1.824 & 186 & 72 & 1.55 & 2.25 \\
\hline 23 & 14.50 & -25.00 & 20 & 26.35 & 1.853 & 203 & 88 & 1.74 & 2.00 \\
\hline 23 & 14.50 & -25.00 & 30 & 27.15 & 1.899 & 197 & 101 & 1.46 & 1.95 \\
\hline 23 & 14.50 & -25.00 & 40 & 12.05 & 1.355 & 99 & & 1.77 & 2.02 \\
\hline 23 & 14.50 & -25.00 & 49 & 28.49 & 1.971 & 123 & & 2.18 & 1.92 \\
\hline 23 & 14.50 & -25.00 & 70 & 30.25 & 1.981 & 100 & & 3.61 & 1.86 \\
\hline 23 & 14.50 & -25.00 & 90 & 28.87 & 1.702 & 191 & 60 & 7.99 & 1.91 \\
\hline 24 & 13.50 & -25.00 & 10 & 8.18 & 0.851 & 55 & 6 & 0.49 & 2.39 \\
\hline 24 & 13.50 & -25.00 & 20 & 10.60 & 0.963 & 78 & 20 & 0.38 & 2.19 \\
\hline 24 & 13.50 & -25.00 & 30 & 12.09 & 0.989 & 69 & 26 & 0.39 & 2.82 \\
\hline 24 & 13.50 & -25.00 & 50 & 13.35 & 1.054 & 78 & 25 & 0.31 & 1.73 \\
\hline 24 & 13.50 & -25.00 & 100 & 19.07 & 1.376 & 100 & 35 & 0.59 & 1.29 \\
\hline 24 & 13.50 & -25.00 & 149 & 24.46 & 1.673 & 61 & 16 & & \\
\hline 24 & 13.50 & -25.00 & 200 & 27.20 & 1.887 & 71 & & & \\
\hline 24 & 13.50 & -25.00 & 260 & 33.84 & 2.253 & 86 & & 1.36 & 0.77 \\
\hline 24 & 13.50 & -25.00 & 299 & 33.72 & 2.358 & 104 & & 2.58 & 0.85 \\
\hline 24 & 13.50 & -25.00 & 399 & 36.07 & 2.449 & 127 & 40 & 1.82 & 0.68 \\
\hline 24 & 13.50 & -25.00 & 600 & 36.16 & 2.424 & 89 & 31 & 1.04 & 0.36 \\
\hline 25 & 12.50 & -25.00 & 10 & 27.27 & 1.861 & 10 & 2 & 0.13 & 1.18 \\
\hline 25 & 12.50 & -25.00 & 11 & 29.92 & 2.082 & & & & \\
\hline 25 & 12.50 & -25.00 & 20 & 36.00 & 2.459 & 12 & 4 & 0.12 & 1.21 \\
\hline 25 & 12.50 & -25.00 & 39 & 34.30 & 2.383 & 36 & 6 & 0.15 & 1.51 \\
\hline 25 & 12.50 & -25.00 & 41 & 31.69 & 2.183 & & & & \\
\hline 25 & 12.50 & -25.00 & 70 & 28.26 & 1.950 & 36 & 10 & 0.17 & 0.81 \\
\hline 25 & 12.50 & -25.00 & 109 & 25.86 & 1.790 & 30 & 9 & 0.20 & 0.47 \\
\hline 25 & 12.50 & -25.00 & 149 & 24.93 & 1.703 & 40 & 8 & 0.25 & 0.62 \\
\hline 25 & 12.50 & -25.00 & 200 & 24.16 & 1.684 & 27 & 6 & 0.38 & 0.51 \\
\hline 25 & 12.50 & -25.00 & 250 & 24.10 & 1.688 & 49 & 10 & 0.49 & 0.48 \\
\hline 25 & 12.50 & -25.00 & 300 & 23.98 & 1.684 & 54 & 21 & 0.47 & 0.53 \\
\hline 25 & 12.50 & -25.00 & 400 & 27.27 & 1.861 & 42 & 6 & 0.68 & 0.41 \\
\hline 25 & 12.50 & -25.00 & 600 & 29.92 & 2.082 & 55 & 20 & 0.65 & 0.32 \\
\hline 25 & 12.50 & -25.00 & 800 & 36.00 & 2.459 & 57 & 12 & 0.71 & 0.31 \\
\hline 25 & 12.50 & -25.00 & 1000 & 34.30 & 2.383 & 55 & 20 & 0.79 & 0.29 \\
\hline 25 & 12.50 & -25.00 & 1250 & 31.69 & 2.183 & & & 0.77 & 0.28 \\
\hline 25 & 12.50 & -25.00 & 1500 & 28.26 & 1.950 & & & 0.72 & 0.30 \\
\hline
\end{tabular}




\begin{tabular}{|c|c|c|c|c|c|c|c|c|c|}
\hline $\begin{array}{c}\text { Station } \\
{[\#]}\end{array}$ & $\begin{array}{c}\text { Longitude } \\
\text { [deg. E] }\end{array}$ & $\begin{array}{l}\text { Latitude } \\
\text { [deg. N] }\end{array}$ & $\begin{array}{c}\text { Depth } \\
\text { [m] }\end{array}$ & $\begin{array}{l}\mathbf{N}+\mathbf{N} \\
{[\mathbf{u M}]}\end{array}$ & $\begin{array}{l}\mathbf{P O}_{4}{ }^{3-} \\
{[\mathbf{u M}]}\end{array}$ & $\begin{array}{r}\text { TCo } \\
\text { [pM] }\end{array}$ & $\begin{array}{c}\mathrm{Co}^{\prime} \\
{[\mathbf{p M}]}\end{array}$ & $\begin{array}{l}\text { Td Fe } \\
{[\mathrm{nM}]}\end{array}$ & $\begin{array}{c}\text { Mn } \\
{[\mathrm{nM}]}\end{array}$ \\
\hline 25 & 12.50 & -25.00 & 1750 & 25.86 & 1.790 & & & 0.71 & 0.29 \\
\hline 25 & 12.50 & -25.00 & 1999 & 24.93 & 1.703 & & & 0.69 & 0.26 \\
\hline 25 & 12.50 & -25.00 & 2249 & 24.16 & 1.684 & 57 & 16 & 0.74 & 0.44 \\
\hline 25 & 12.50 & -25.00 & 2499 & 24.10 & 1.688 & 52 & 13 & 0.86 & 0.39 \\
\hline 25 & 12.50 & -25.00 & 2706 & 23.98 & 1.684 & 45 & 12 & 0.80 & 1.02 \\
\hline 26 & 11.50 & -25.00 & 10 & 2.18 & 0.431 & 15 & n.d. & 0.16 & 1.32 \\
\hline 26 & 11.50 & -25.00 & 30 & 5.14 & 0.586 & 19 & 2 & 0.13 & 1.53 \\
\hline 26 & 11.50 & -25.00 & 50 & 3.74 & 0.466 & 29 & 5 & 0.10 & 1.38 \\
\hline 26 & 11.50 & -25.00 & 109 & 12.38 & 0.997 & 53 & 17 & 0.16 & 0.97 \\
\hline 26 & 11.50 & -25.00 & 150 & 17.18 & 1.232 & 57 & 29 & 0.29 & 0.73 \\
\hline 26 & 11.50 & -25.00 & 200 & 19.83 & 1.434 & 43 & 14 & 0.44 & 0.65 \\
\hline 26 & 11.50 & -25.00 & 275 & 23.93 & 1.787 & 58 & & 0.74 & 0.78 \\
\hline 26 & 11.50 & -25.00 & 350 & 28.29 & 2.018 & 63 & & 0.81 & 0.75 \\
\hline 26 & 11.50 & -25.00 & 424 & 29.36 & 1.964 & 56 & & 0.74 & 0.44 \\
\hline 26 & 11.50 & -25.00 & 600 & 32.33 & 2.216 & 41 & & 0.72 & 0.63 \\
\hline 26 & 11.50 & -25.00 & 800 & 33.85 & 2.343 & 71 & 26 & 0.79 & 0.30 \\
\hline 27 & 10.00 & -25.00 & 10 & 0.29 & 0.231 & 40 & 6 & 0.12 & 1.21 \\
\hline 27 & 10.00 & -25.00 & 20 & 0.32 & 0.161 & 14 & 2 & 0.15 & 1.38 \\
\hline 27 & 10.00 & -25.00 & 39 & 0.39 & 0.222 & 18 & 6 & 0.14 & 1.39 \\
\hline 27 & 10.00 & -25.00 & 60 & 0.91 & 0.265 & 23 & 6 & 0.11 & 1.40 \\
\hline 27 & 10.00 & -25.00 & 70 & 2.80 & 0.377 & 19 & 5 & 0.13 & 1.24 \\
\hline 27 & 10.00 & -25.00 & 110 & 7.15 & 0.576 & 36 & 9 & 0.22 & 1.52 \\
\hline 27 & 10.00 & -25.00 & 150 & 9.97 & 0.728 & 33 & 4 & 0.20 & 0.79 \\
\hline 27 & 10.00 & -25.00 & 200 & 12.67 & 0.880 & 29 & 11 & 0.24 & 0.33 \\
\hline 27 & 10.00 & -25.00 & 250 & 17.40 & 1.152 & 31 & 12 & 0.42 & 0.48 \\
\hline 27 & 10.00 & -25.00 & 299 & 9.96 & 0.995 & 80 & 29 & 0.82 & 0.85 \\
\hline 27 & 10.00 & -25.00 & 400 & 29.35 & 1.891 & 85 & 30 & 0.77 & 0.71 \\
\hline 27 & 10.00 & -25.00 & 600 & 23.24 & 1.676 & 60 & 16 & 0.71 & 0.47 \\
\hline 27 & 10.00 & -25.00 & 800 & 35.09 & 2.317 & 48 & 12 & 0.69 & 0.32 \\
\hline 27 & 10.00 & -25.00 & 1000 & 34.08 & 2.338 & 57 & 17 & 0.84 & 0.28 \\
\hline 27 & 10.00 & -25.00 & 1250 & 31.00 & 2.154 & 29 & 8 & 0.94 & 0.32 \\
\hline 27 & 10.00 & -25.00 & 1500 & 28.04 & 1.933 & 38 & & 0.98 & 0.47 \\
\hline 27 & 10.00 & -25.00 & 1750 & 25.33 & 1.781 & 32 & & 0.77 & 0.25 \\
\hline 27 & 10.00 & -25.00 & 2000 & 24.26 & 1.661 & & & 0.60 & 0.27 \\
\hline 27 & 10.00 & -25.00 & 2500 & 23.69 & 1.639 & 29 & & 0.51 & 0.24 \\
\hline 27 & 10.00 & -25.00 & 3000 & 24.64 & 1.650 & 42 & & 0.59 & 0.20 \\
\hline 27 & 10.00 & -25.00 & 3500 & 24.82 & 1.704 & 41 & 9 & 0.61 & 0.19 \\
\hline 27 & 10.00 & -25.00 & 4000 & 28.23 & 1.945 & 34 & 6 & 0.51 & 0.22 \\
\hline
\end{tabular}




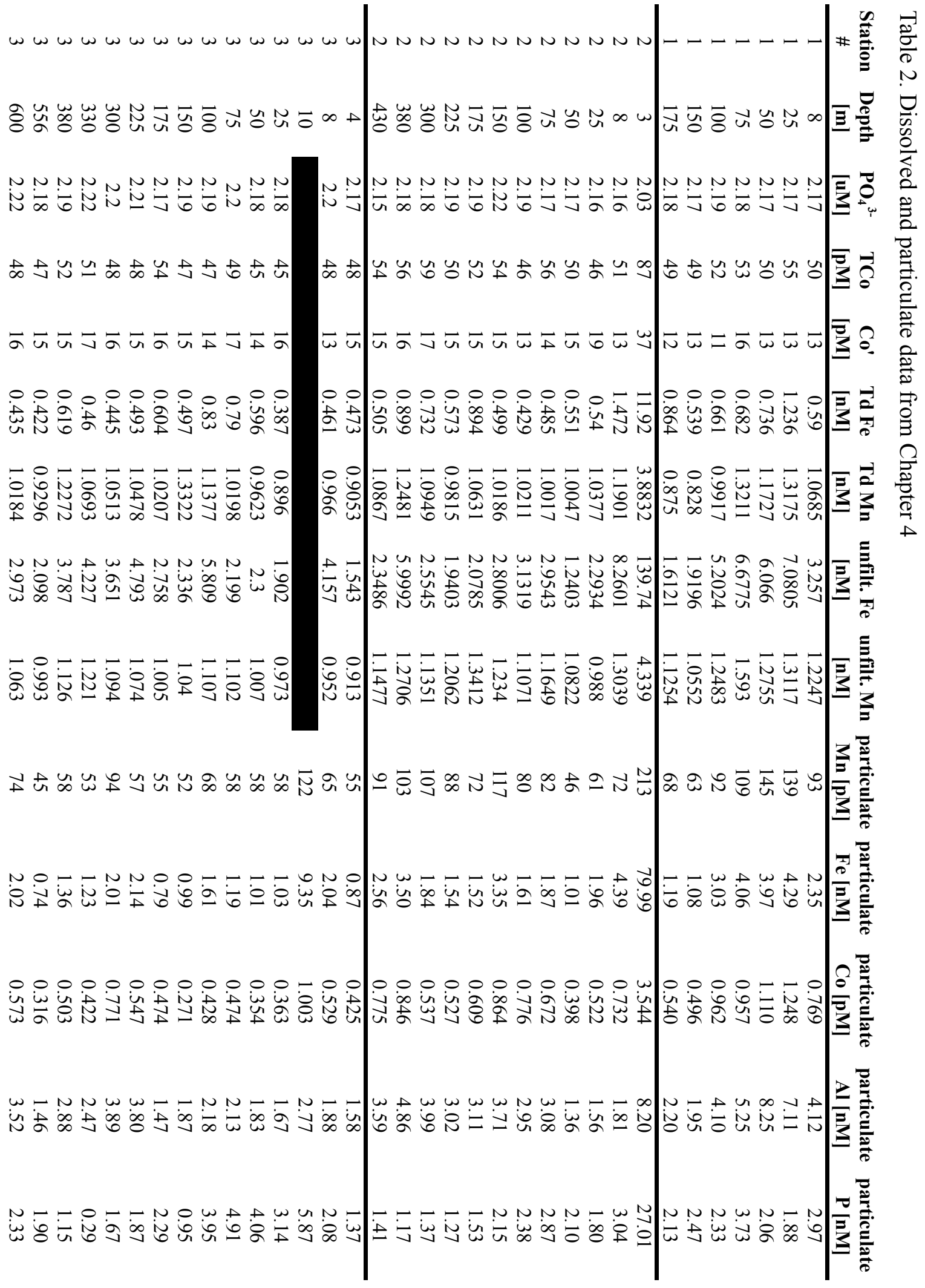


Table 3. Data from Chapter 5

\begin{tabular}{|c|c|c|c|c|c|c|c|c|c|c|}
\hline $\begin{array}{c}\text { Station } \\
{[\#]}\end{array}$ & $\begin{array}{c}\text { Longitude } \\
\text { [deg. E] }\end{array}$ & $\begin{array}{l}\text { Latitude } \\
\text { [deg. N] }\end{array}$ & $\begin{array}{c}\text { Depth } \\
\text { [m] }\end{array}$ & $\begin{array}{c}\text { CTDPRS } \\
\text { [dbars] }\end{array}$ & $\begin{array}{c}\text { Nitrate } \\
{[\mathbf{u M}]}\end{array}$ & $\begin{array}{c}\text { Nitrite } \\
{[\mathrm{uM}]}\end{array}$ & $\begin{array}{l}\mathrm{PO}_{4}{ }^{3-} \\
{[\mathrm{uM}]}\end{array}$ & $\begin{array}{r}\text { TCo } \\
{[\mathbf{p M}]}\end{array}$ & $\begin{array}{c}\mathbf{C o}^{\prime} \\
{[\mathbf{p M}]}\end{array}$ & $\begin{array}{l}\text { GEOTR } \\
\text { SMPL \# }\end{array}$ \\
\hline 1 & -9.66 & 38.32 & 24 & 24 & 0 & 0 & 0.01 & 70 & 31 & 5052 \\
\hline 1 & -9.66 & 38.32 & 35 & 35 & 0.04 & 0.02 & 0.05 & 90 & 33 & 5054 \\
\hline 1 & -9.66 & 38.32 & 40 & 40 & 0.10 & 0.05 & 0.05 & 89 & 25 & 5056 \\
\hline 1 & -9.66 & 38.32 & 68 & 69 & 0.64 & 0.09 & 0.11 & 48 & 19 & 5058 \\
\hline 1 & -9.66 & 38.32 & 136 & 136 & 5.44 & 0.01 & 0.39 & 54 & 14 & 5060 \\
\hline 1 & -9.66 & 38.32 & 199 & 201 & 7.54 & 0 & 0.50 & 72 & 16 & 5062 \\
\hline 1 & -9.66 & 38.32 & 300 & 302 & 10.08 & 0 & 0.64 & 71 & 19 & 5064 \\
\hline 1 & -9.66 & 38.32 & 401 & 404 & 12.40 & 0 & 0.79 & 73 & 28 & 5066 \\
\hline 1 & -9.66 & 38.32 & 502 & 506 & 13.28 & 0 & 0.84 & 75 & 24 & 5068 \\
\hline 1 & -9.66 & 38.32 & 642 & 648 & 10.66 & 0 & 0.66 & 75 & 17 & 5070 \\
\hline 1 & -9.66 & 38.32 & 777 & 784 & 11.21 & 0 & 0.69 & 77 & 23 & 5072 \\
\hline 1 & -9.66 & 38.32 & 876 & 884 & 12.15 & 0 & 0.76 & 77 & 19 & 5074 \\
\hline 1 & -9.66 & 38.33 & 900 & 909 & 12.21 & 0 & 0.74 & 75 & 23 & 5088 \\
\hline 1 & -9.66 & 38.33 & 990 & 1001 & 12.08 & 0 & 0.74 & 73 & 22 & 5090 \\
\hline 1 & -9.66 & 38.33 & 1150 & 1163 & 12.33 & 0 & 0.75 & 70 & 20 & 5092 \\
\hline 1 & -9.66 & 38.33 & 1293 & 1308 & 14.59 & 0 & 0.93 & 71 & 27 & 5094 \\
\hline 1 & -9.66 & 38.33 & 1480 & 1497 & 14.58 & 0 & 0.92 & 74 & 21 & 5096 \\
\hline 1 & -9.66 & 38.33 & 1701 & 1721 & 17.11 & 0 & 1.13 & 67 & 26 & 5098 \\
\hline 1 & -9.66 & 38.33 & 1901 & 1924 & 17.99 & 0 & 1.21 & 75 & 21 & 5100 \\
\hline 1 & -9.66 & 38.33 & 2101 & 2128 & 18.59 & 0 & 1.27 & 74 & 24 & 5102 \\
\hline 1 & -9.66 & 38.33 & 2301 & 2331 & 19.17 & 0 & 1.32 & 63 & 18 & 5104 \\
\hline 1 & -9.66 & 38.33 & 2501 & 2536 & 19.70 & 0 & 1.36 & 66 & 17 & 5106 \\
\hline 1 & -9.66 & 38.33 & 2601 & 2638 & 19.93 & 0 & 1.37 & 61 & 16 & 5108 \\
\hline 1 & -9.66 & 38.33 & 2701 & 2740 & 20.14 & 0 & 1.40 & 59 & 16 & 5110 \\
\hline 2 & -12.83 & 36.76 & 49 & 49 & 0 & 0 & 0.01 & 27 & 10 & 5174 \\
\hline 2 & -12.83 & 36.76 & 102 & 103 & 1.10 & 0.13 & 0.13 & 43 & 3 & 5176 \\
\hline 2 & -12.83 & 36.76 & 141 & 142 & 5.51 & 0.01 & 0.37 & 54 & 10 & 5178 \\
\hline 2 & -12.83 & 36.76 & 201 & 202 & 7.44 & 0.01 & 0.49 & 58 & 17 & 5180 \\
\hline 2 & -12.83 & 36.76 & 251 & 253 & 9.31 & 0 & 0.59 & 61 & 27 & 5182 \\
\hline 2 & -12.83 & 36.76 & 359 & 362 & 9.88 & 0 & 0.63 & 70 & 26 & 5184 \\
\hline 2 & -12.83 & 36.76 & 501 & 505 & 13.76 & 0 & 0.87 & 81 & 26 & 5186 \\
\hline 2 & -12.83 & 36.76 & 662 & 667 & 16.32 & 0 & 1.03 & 79 & 28 & 5188 \\
\hline 2 & -12.83 & 36.76 & 821 & 828 & 14.88 & 0 & 0.95 & 83 & 24 & 5190 \\
\hline 2 & -12.83 & 36.76 & 902 & 911 & 16.41 & 0 & 1.04 & 78 & 22 & 5192 \\
\hline 2 & -12.83 & 36.76 & 1051 & 1061 & 16.11 & 0 & 1.02 & 75 & 21 & 5194 \\
\hline 2 & -12.83 & 36.76 & 1201 & 1213 & 15.50 & 0 & 0.98 & 84 & 22 & 5196 \\
\hline 3 & -16.00 & 35.20 & 35 & 36 & 0 & 0 & 0 & 13 & n.d & 5212 \\
\hline 3 & -16.00 & 35.20 & 51 & 51 & 0 & 0 & 0.01 & 14 & n.d & 5214 \\
\hline 3 & -16.00 & 35.20 & 77 & 77 & 0.11 & 0.02 & 0.02 & 49 & n.d & 5216 \\
\hline 3 & -16.00 & 35.20 & 100 & 100 & 4.01 & 0.04 & 0.23 & 57 & 4 & 5218 \\
\hline 3 & -16.00 & 35.20 & 136 & 137 & 4.92 & 0.01 & 0.30 & 59 & 9 & 5220 \\
\hline 3 & -16.00 & 35.20 & 191 & 193 & 6.42 & 0 & 0.40 & 62 & 11 & 5222 \\
\hline 3 & -16.00 & 35.20 & 301 & 303 & 9.82 & 0 & 0.61 & 66 & 17 & 5224 \\
\hline 3 & -16.00 & 35.20 & 401 & 404 & 10.95 & 0 & 0.68 & 73 & 20 & 5226 \\
\hline 3 & -16.00 & 35.20 & 490 & 494 & 13.72 & 0 & 0.86 & 83 & 18 & 5228 \\
\hline 3 & -16.00 & 35.20 & 611 & 616 & 15.91 & 0 & 0.99 & 91 & 22 & 5230 \\
\hline 3 & -16.00 & 35.20 & 777 & 783 & 16.98 & 0 & 1.07 & 85 & 22 & 5232 \\
\hline
\end{tabular}




\begin{tabular}{|c|c|c|c|c|c|c|c|c|c|c|}
\hline $\begin{array}{c}\text { Station } \\
{[\#]}\end{array}$ & $\begin{array}{c}\text { Longitude } \\
\text { [deg. E] }\end{array}$ & $\begin{array}{l}\text { Latitude } \\
\text { [deg. N] }\end{array}$ & $\begin{array}{c}\text { Depth } \\
\text { [m] }\end{array}$ & $\begin{array}{c}\text { CTDPRS } \\
\text { [dbars] }\end{array}$ & $\begin{array}{c}\text { Nitrate } \\
{[\mathrm{uM}]}\end{array}$ & $\begin{array}{c}\text { Nitrite } \\
{[\mathrm{uM}]}\end{array}$ & $\begin{array}{l}\mathrm{PO}_{4}{ }^{3-} \\
{[\mathrm{uM}]}\end{array}$ & $\begin{array}{r}\text { TCo } \\
{[\mathrm{pM}]}\end{array}$ & $\begin{array}{c}\mathrm{Co}^{\prime} \\
{[\mathbf{p M}]}\end{array}$ & $\begin{array}{l}\text { GEOTR } \\
\text { SMPL \# }\end{array}$ \\
\hline 3 & -16.00 & 35.20 & 876 & 884 & 16.81 & 0 & 1.08 & 87 & 23 & 5234 \\
\hline 3 & -16.00 & 35.20 & 994 & 1004 & 16.67 & 0 & 1.06 & 74 & 24 & 5257 \\
\hline 3 & -16.00 & 35.20 & 1051 & 1062 & 16.81 & 0 & 1.07 & 83 & 22 & 5259 \\
\hline 3 & -16.00 & 35.20 & 1201 & 1213 & 17.16 & 0 & 1.11 & 78 & 24 & 5261 \\
\hline 3 & -16.00 & 35.20 & 1351 & 1365 & 17.36 & 0 & 1.13 & 79 & 24 & 5263 \\
\hline 3 & -16.00 & 35.20 & 1501 & 1518 & 17.34 & 0 & 1.14 & 84 & 21 & 5265 \\
\hline 3 & -16.00 & 35.20 & 1701 & 1721 & 17.66 & 0 & 1.18 & 85 & 24 & 5267 \\
\hline 3 & -16.00 & 35.20 & 1901 & 1924 & 18.09 & 0 & 1.22 & 77 & 22 & 5269 \\
\hline 3 & -16.00 & 35.20 & 2101 & 2128 & 18.59 & 0 & 1.25 & 69 & 19 & 5271 \\
\hline 3 & -16.00 & 35.20 & 2300 & 2331 & 18.98 & 0 & 1.30 & 71 & 17 & 5273 \\
\hline 3 & -16.00 & 35.20 & 2501 & 2536 & 19.47 & 0 & 1.34 & 64 & 16 & 5275 \\
\hline 3 & -16.00 & 35.20 & 2601 & 2637 & 19.71 & 0 & 1.35 & 61 & 14 & 5277 \\
\hline 3 & -16.00 & 35.20 & 2681 & 2719 & 19.89 & 0 & 1.39 & 60 & 16 & 5279 \\
\hline 5 & -22.00 & 31.00 & 31 & 31 & 0 & 0 & 0 & 15 & 1 & 5332 \\
\hline 5 & -22.00 & 31.00 & 52 & 52 & 0 & 0 & 0 & 34 & 1 & 5334 \\
\hline 5 & -22.00 & 31.00 & 81 & 81 & 0 & 0 & 0 & 43 & 4 & 5336 \\
\hline 5 & -22.00 & 31.00 & 101 & 102 & 0.07 & 0.07 & 0.01 & 45 & n.d & 5338 \\
\hline 5 & -22.00 & 31.00 & 136 & 137 & 2.74 & 0.02 & 0.15 & 43 & n.d & 5340 \\
\hline 5 & -22.00 & 31.00 & 187 & 188 & 3.78 & 0.01 & 0.20 & 47 & 21 & 5342 \\
\hline 5 & -22.00 & 31.00 & 251 & 253 & 5.66 & 0.01 & 0.36 & 50 & 18 & 5344 \\
\hline 5 & -22.00 & 31.00 & 351 & 353 & 7.63 & 0 & 0.47 & 60 & 18 & 5346 \\
\hline 5 & -22.00 & 31.00 & 553 & 557 & 12.97 & 0 & 0.81 & 74 & 20 & 5348 \\
\hline 5 & -22.00 & 31.00 & 802 & 809 & 19.98 & 0 & 1.27 & 94 & 24 & 5350 \\
\hline 5 & -22.00 & 31.00 & 963 & 971 & 20.15 & 0 & 1.32 & 80 & 23 & 5352 \\
\hline 5 & -22.00 & 31.00 & 1202 & 1213 & 19.26 & 0 & 1.26 & 78 & 23 & 5384 \\
\hline 5 & -22.00 & 31.00 & 1501 & 1518 & 18.54 & 0 & 1.24 & 77 & 23 & 5399 \\
\hline 5 & -22.00 & 31.00 & 1800 & 1822 & 17.99 & 0 & 1.22 & 70 & 22 & 5401 \\
\hline 5 & -22.00 & 31.00 & 2100 & 2127 & 18.62 & 0 & 1.28 & 71 & 21 & 5403 \\
\hline 5 & -22.00 & 31.00 & 2401 & 2433 & 18.99 & 0 & 1.32 & 65 & 18 & 5405 \\
\hline 5 & -22.00 & 31.00 & 2701 & 2739 & 19.68 & 0 & 1.37 & 62 & 14 & 5407 \\
\hline 5 & -22.00 & 31.00 & 3001 & 3046 & 20.38 & 0 & 1.42 & 55 & 9 & 5409 \\
\hline 5 & -22.00 & 31.00 & 3301 & 3353 & 20.88 & 0 & 1.46 & 51 & 9 & 5411 \\
\hline 5 & -22.00 & 31.00 & 3600 & 3659 & 21.22 & 0 & 1.49 & 49 & 7 & 5413 \\
\hline 5 & -22.00 & 31.00 & 3899 & 3966 & 21.44 & 0 & 1.50 & 48 & 9 & 5415 \\
\hline 5 & -22.00 & 31.00 & 4199 & 4275 & 21.53 & 0 & 1.50 & 44 & 5 & 5417 \\
\hline 5 & -22.00 & 31.00 & 4549 & 4634 & 21.53 & 0 & 1.51 & 43 & 4 & 5419 \\
\hline 5 & -22.00 & 31.00 & 4899 & 4995 & 21.56 & 0 & 1.51 & 40 & 5 & 5421 \\
\hline 6 & -22.00 & 27.52 & 41 & 41 & 0.01 & 0 & 0.02 & 25 & 4 & 5427 \\
\hline 6 & -22.00 & 27.52 & 66 & 66 & 0 & 0 & 0.01 & 22 & n.d & 5429 \\
\hline 6 & -22.00 & 27.52 & 91 & 92 & 0 & 0 & 0.05 & 34 & 1 & 5431 \\
\hline 6 & -22.00 & 27.52 & 116 & 117 & 0 & 0.06 & 0.01 & 43 & n.d & 5433 \\
\hline 6 & -22.00 & 27.52 & 135 & 136 & 0.52 & 0.03 & 0.03 & 40 & 2 & 5435 \\
\hline 6 & -22.00 & 27.52 & 186 & 187 & 3.64 & 0.01 & 0.22 & 51 & 8 & 5437 \\
\hline 6 & -22.00 & 27.52 & 302 & 304 & 7.19 & 0 & 0.43 & 62 & 12 & 5439 \\
\hline 6 & -22.00 & 27.52 & 401 & 404 & 10.21 & 0 & 0.62 & 67 & 16 & 5441 \\
\hline 6 & -22.00 & 27.52 & 602 & 607 & 18.12 & 0 & 1.13 & 88 & 23 & 5443 \\
\hline 6 & -22.00 & 27.52 & 801 & 808 & 22.85 & 0 & 1.46 & 92 & 25 & 5445 \\
\hline 6 & -22.00 & 27.52 & 1003 & 1012 & 22.85 & 0 & 1.50 & 82 & 27 & 5447 \\
\hline
\end{tabular}




\begin{tabular}{|c|c|c|c|c|c|c|c|c|c|c|}
\hline $\begin{array}{c}\text { Station } \\
{[\#]}\end{array}$ & $\begin{array}{c}\text { Longitude } \\
\text { [deg. E] }\end{array}$ & $\begin{array}{l}\text { Latitude } \\
\text { [deg. N] }\end{array}$ & $\begin{array}{c}\text { Depth } \\
\text { [m] }\end{array}$ & $\begin{array}{c}\text { CTDPRS } \\
\text { [dbars] }\end{array}$ & $\begin{array}{c}\text { Nitrate } \\
\text { [uM] }\end{array}$ & $\begin{array}{c}\text { Nitrite } \\
{[\mathbf{u M}]}\end{array}$ & $\begin{array}{l}\mathrm{PO}_{4}{ }^{3-} \\
{[\mathbf{u M}]}\end{array}$ & $\begin{array}{c}\text { TCo } \\
{[\mathbf{p M}]}\end{array}$ & $\begin{array}{c}\mathrm{Co}^{\prime} \\
{[\mathbf{p M}]}\end{array}$ & $\begin{array}{l}\text { GEOTR } \\
\text { SMPL \# }\end{array}$ \\
\hline 6 & -22.00 & 27.52 & 1202 & 1213 & 20.95 & 0 & 1.39 & 84 & 21 & 5449 \\
\hline 7 & -22.00 & 24.00 & 35 & 35 & 0 & 0 & 0.09 & 42 & 5 & 5467 \\
\hline 7 & -22.00 & 24.00 & 46 & 46 & 0 & 0 & 0.06 & 45 & 2 & 5469 \\
\hline 7 & -22.00 & 24.00 & 71 & 71 & 0.19 & 0.05 & 0.10 & 50 & 2 & 5471 \\
\hline 7 & -22.00 & 24.00 & 151 & 152 & 15.32 & 0.01 & 0.96 & 119 & 31 & 5473 \\
\hline 7 & -22.00 & 24.00 & 201 & 202 & 15.15 & 0.01 & 0.94 & 115 & 29 & 5475 \\
\hline 7 & -22.00 & 24.00 & 351 & 354 & 16.31 & 0.01 & 0.98 & 100 & 29 & 5477 \\
\hline 7 & -22.00 & 24.00 & 398 & 400 & 15.93 & 0.01 & 0.97 & 102 & 25 & 5479 \\
\hline 7 & -22.00 & 24.00 & 451 & 454 & 22.00 & 0.01 & 1.34 & 131 & 33 & 5481 \\
\hline 7 & -22.00 & 24.00 & 501 & 505 & 20.51 & 0.01 & 1.26 & 96 & 30 & 5483 \\
\hline 7 & -22.00 & 24.00 & 751 & 757 & 29.11 & 0 & 1.87 & 111 & 30 & 5485 \\
\hline 7 & -22.00 & 24.00 & 1001 & 1010 & 26.38 & 0 & 1.78 & 85 & 22 & 5487 \\
\hline 7 & -22.00 & 24.00 & 1201 & 1212 & 23.62 & 0 & 1.56 & 81 & 24 & 5489 \\
\hline 7 & -22.00 & 24.00 & 1499 & 1516 & 21.98 & 0 & 1.50 & 73 & 24 & 5544 \\
\hline 7 & -22.00 & 24.00 & 1750 & 1771 & 21.36 & 0 & 1.47 & 62 & 24 & 5546 \\
\hline 7 & -22.00 & 24.00 & 1999 & 2024 & 21.12 & 0 & 1.46 & 63 & 22 & 5548 \\
\hline 7 & -22.00 & 24.00 & 2248 & 2277 & 20.94 & 0 & 1.46 & 63 & 18 & 5550 \\
\hline 7 & -22.00 & 24.00 & 2499 & 2534 & 20.91 & 0 & 1.45 & 59 & 19 & 5552 \\
\hline 7 & -22.00 & 24.00 & 2749 & 2789 & 21.00 & 0 & 1.47 & 57 & 15 & 5554 \\
\hline 7 & -22.00 & 24.00 & 2998 & 3043 & 21.14 & 0 & 1.47 & 55 & 12 & 5556 \\
\hline 7 & -22.00 & 24.00 & 3248 & 3299 & 21.31 & 0 & 1.47 & 51 & 14 & 5558 \\
\hline 7 & -22.00 & 24.00 & 3498 & 3554 & 21.63 & 0.01 & 1.50 & 50 & 11 & 5560 \\
\hline 7 & -22.00 & 24.00 & 3827 & 3892 & 21.54 & 0.01 & 1.50 & 43 & 8 & 5562 \\
\hline 7 & -22.00 & 24.00 & 4157 & 4231 & 21.77 & 0 & 1.52 & 45 & 8 & 5564 \\
\hline 7 & -22.00 & 24.00 & 4496 & 4580 & 21.75 & 0 & 1.50 & 40 & 8 & 5566 \\
\hline 8 & -22.00 & 21.00 & 35 & 36 & 0.01 & 0 & 0.04 & 26 & n.d & 5583 \\
\hline 8 & -22.00 & 21.00 & 56 & 56 & 0 & 0 & 0.01 & 37 & n.d & 5585 \\
\hline 8 & -22.00 & 21.00 & 91 & 91 & 0.60 & 0.18 & 0.14 & 60 & 4 & 5587 \\
\hline 8 & -22.00 & 21.00 & 111 & 111 & 4.06 & 0.08 & 0.30 & 67 & 7 & 5589 \\
\hline 8 & -22.00 & 21.00 & 135 & 136 & 10.66 & 0.02 & 0.67 & 98 & 28 & 5591 \\
\hline 8 & -22.00 & 21.00 & 186 & 187 & 11.05 & 0.01 & 0.68 & 76 & 34 & 5593 \\
\hline 8 & -22.00 & 21.00 & 236 & 237 & 13.46 & 0.01 & 0.82 & 101 & 27 & 5595 \\
\hline 8 & -22.00 & 21.00 & 285 & 287 & 15.81 & 0.01 & 0.96 & 121 & 29 & 5597 \\
\hline 8 & -22.00 & 21.00 & 421 & 424 & 20.85 & 0 & 1.26 & 103 & 32 & 5599 \\
\hline 8 & -22.00 & 21.00 & 666 & 671 & 30.16 & 0 & 1.91 & 103 & 31 & 5601 \\
\hline 8 & -22.00 & 21.00 & 965 & 974 & 28.76 & 0 & 1.92 & 93 & 25 & 5603 \\
\hline 8 & -22.00 & 21.00 & 1200 & 1211 & 24.97 & 0 & 1.68 & 86 & 26 & 5605 \\
\hline 9 & -18.25 & 17.35 & 28 & 28 & 0 & 0 & 0.03 & 52 & 5 & 5637 \\
\hline 9 & -18.25 & 17.35 & 49 & 49 & 5.47 & 0.35 & 0.68 & 117 & 13 & 5639 \\
\hline 9 & -18.25 & 17.35 & 89 & 90 & 23.29 & 0.03 & 1.52 & 133 & 41 & 5641 \\
\hline 9 & -18.25 & 17.35 & 109 & 110 & 23.55 & 0.01 & 1.51 & 149 & 41 & 5643 \\
\hline 9 & -18.25 & 17.35 & 135 & 136 & 24.43 & 0 & 1.57 & 130 & 31 & 5645 \\
\hline 9 & -18.25 & 17.35 & 185 & 186 & 25.91 & 0 & 1.64 & 144 & 37 & 5647 \\
\hline 9 & -18.25 & 17.35 & 221 & 222 & 26.73 & 0 & 1.68 & 135 & 37 & 5649 \\
\hline 9 & -18.25 & 17.35 & 285 & 287 & 28.88 & 0 & 1.77 & 150 & 36 & 5651 \\
\hline 9 & -18.25 & 17.35 & 361 & 364 & 31.55 & 0 & 1.93 & 160 & 34 & 5653 \\
\hline 9 & -18.25 & 17.35 & 665 & 670 & 34.12 & 0 & 2.17 & 125 & 27 & 5655 \\
\hline 9 & -18.25 & 17.35 & 797 & 805 & 33.97 & 0 & 2.21 & 116 & 30 & 5706 \\
\hline
\end{tabular}




\begin{tabular}{|c|c|c|c|c|c|c|c|c|c|c|}
\hline $\begin{array}{c}\text { Station } \\
{[\#]}\end{array}$ & $\begin{array}{c}\text { Longitude } \\
\text { [deg. E] }\end{array}$ & $\begin{array}{l}\text { Latitude } \\
\text { [deg. N] }\end{array}$ & $\begin{array}{c}\text { Depth } \\
\text { [m] }\end{array}$ & $\begin{array}{c}\text { CTDPRS } \\
\text { [dbars] }\end{array}$ & $\begin{array}{c}\text { Nitrate } \\
{[\mathrm{uM}]}\end{array}$ & $\begin{array}{c}\text { Nitrite } \\
{[\mathrm{uM}]}\end{array}$ & $\begin{array}{l}\mathrm{PO}_{4}{ }^{3-} \\
{[\mathrm{uM}]}\end{array}$ & $\begin{array}{r}\text { TCo } \\
{[\mathbf{p M}]}\end{array}$ & $\begin{array}{c}\mathrm{Co}^{\prime} \\
{[\mathbf{p M}]}\end{array}$ & $\begin{array}{l}\text { GEOTR } \\
\text { SMPL \# }\end{array}$ \\
\hline 9 & -18.25 & 17.35 & 965 & 973 & 32.28 & 0 & 2.15 & 112 & 29 & 5657 \\
\hline 9 & -18.25 & 17.35 & 1095 & 1105 & 29.66 & 0 & 1.98 & 101 & 23 & 5708 \\
\hline 9 & -18.25 & 17.35 & 1200 & 1211 & 28.04 & 0 & 1.91 & 96 & 27 & 5659 \\
\hline 9 & -18.25 & 17.35 & 1236 & 1248 & 27.59 & 0 & 1.85 & 86 & 30 & 5710 \\
\hline 9 & -18.25 & 17.35 & 1385 & 1399 & 25.42 & 0 & 1.73 & 84 & 27 & 5712 \\
\hline 9 & -18.25 & 17.35 & 1538 & 1555 & 23.99 & 0 & 1.63 & 83 & 21 & 5714 \\
\hline 9 & -18.25 & 17.35 & 1787 & 1808 & 22.56 & 0 & 1.54 & 87 & 24 & 5716 \\
\hline 9 & -18.25 & 17.35 & 1883 & 1905 & 22.21 & 0 & 1.52 & 79 & 24 & 5718 \\
\hline 9 & -18.25 & 17.35 & 2088 & 2113 & 21.75 & 0 & 1.49 & 76 & 23 & 5720 \\
\hline 9 & -18.25 & 17.35 & 2294 & 2324 & 21.57 & 0 & 1.47 & 68 & 19 & 5722 \\
\hline 9 & -18.25 & 17.35 & 2585 & 2620 & 21.52 & 0 & 1.48 & 67 & 15 & 5724 \\
\hline 9 & -18.25 & 17.35 & 2900 & 2942 & 21.8 & 0 & 1.51 & 44 & 1 & 5726 \\
\hline 9 & -18.25 & 17.35 & 2999 & 3043 & 21.99 & 0 & 1.53 & 39 & 2 & 5728 \\
\hline 10 & -20.82 & 17.35 & 32 & 32 & 0.01 & 0 & 0.03 & 48 & 2 & 5779 \\
\hline 10 & -20.82 & 17.35 & 51 & 51 & 16.26 & 0.44 & 1.17 & 104 & 6 & 5781 \\
\hline 10 & -20.82 & 17.35 & 85 & 85 & 24.64 & 0.03 & 1.52 & 146 & 38 & 5783 \\
\hline 10 & -20.82 & 17.35 & 101 & 101 & 25.14 & 0.02 & 1.56 & 135 & 43 & 5785 \\
\hline 10 & -20.82 & 17.35 & 141 & 141 & 24.48 & 0.01 & 1.52 & 106 & 29 & 5787 \\
\hline 10 & -20.82 & 17.35 & 186 & 188 & 25.78 & 0.01 & 1.62 & 108 & 33 & 5789 \\
\hline 10 & -20.82 & 17.35 & 235 & 237 & 26.52 & 0.01 & 1.65 & 127 & 33 & 5791 \\
\hline 10 & -20.82 & 17.35 & 291 & 293 & 28.93 & 0.01 & 1.76 & 139 & 39 & 5793 \\
\hline 10 & -20.82 & 17.35 & 421 & 424 & 32.28 & 0 & 1.96 & 157 & 38 & 5795 \\
\hline 10 & -20.82 & 17.35 & 499 & 503 & 33.49 & 0 & 2.06 & 127 & 30 & 5797 \\
\hline 10 & -20.82 & 17.35 & 665 & 670 & 34.83 & 0 & 2.28 & 114 & 30 & 5799 \\
\hline 10 & -20.82 & 17.35 & 800 & 806 & 34.65 & 0 & 2.27 & 102 & 28 & 5801 \\
\hline 10 & -20.82 & 17.35 & 964 & 973 & 32.24 & 0 & 2.14 & 92 & 27 & 5812 \\
\hline 10 & -20.82 & 17.35 & 1100 & 1110 & 29.80 & 0 & 1.99 & 99 & 30 & 5814 \\
\hline 10 & -20.82 & 17.35 & 1249 & 1262 & 27.67 & 0 & 1.86 & 95 & 31 & 5816 \\
\hline 10 & -20.82 & 17.35 & 1498 & 1514 & 24.43 & 0 & 1.66 & 87 & 31 & 5818 \\
\hline 10 & -20.82 & 17.35 & 1750 & 1770 & 22.82 & 0 & 1.54 & 81 & 27 & 5820 \\
\hline 10 & -20.82 & 17.35 & 1999 & 2022 & 21.89 & 0 & 1.47 & 75 & 26 & 5822 \\
\hline 10 & -20.82 & 17.35 & 2249 & 2277 & 21.57 & 0 & 1.47 & 75 & 21 & 5824 \\
\hline 10 & -20.82 & 17.35 & 2499 & 2531 & 21.35 & 0 & 1.46 & 59 & 21 & 5826 \\
\hline 10 & -20.82 & 17.35 & 2698 & 2735 & 21.35 & 0 & 1.46 & 59 & 15 & 5828 \\
\hline 10 & -20.82 & 17.35 & 2999 & 3042 & 21.51 & 0 & 1.46 & 53 & 13 & 5830 \\
\hline 10 & -20.82 & 17.35 & 3278 & 3327 & 22.11 & 0 & 1.51 & 47 & 8 & 5832 \\
\hline 10 & -20.82 & 17.35 & 3321 & 3371 & 22.53 & 0.01 & 1.53 & 46 & 5 & 5834 \\
\hline 11 & -22.78 & 17.35 & 30 & 30 & 0 & 0 & 0.04 & 32 & 1 & 5873 \\
\hline 11 & -22.78 & 17.35 & 62 & 62 & 1.82 & 0.27 & 0.26 & 87 & n.d & 5875 \\
\hline 11 & -22.78 & 17.35 & 85 & 86 & 13.46 & 0.05 & 0.84 & 102 & 23 & 5877 \\
\hline 11 & -22.78 & 17.35 & 136 & 137 & 17.76 & 0 & 1.07 & 105 & 31 & 5879 \\
\hline 11 & -22.78 & 17.35 & 180 & 181 & 19.50 & 0 & 1.17 & 114 & 32 & 5881 \\
\hline 11 & -22.78 & 17.35 & 200 & 201 & 20.07 & 0 & 1.20 & 121 & 36 & 5883 \\
\hline 11 & -22.78 & 17.35 & 250 & 252 & 22.64 & 0 & 1.36 & 123 & 36 & 5885 \\
\hline 11 & -22.78 & 17.35 & 301 & 303 & 25.48 & 0 & 1.52 & 125 & 37 & 5887 \\
\hline 11 & -22.78 & 17.35 & 351 & 354 & 26.82 & 0 & 1.61 & 124 & 31 & 5889 \\
\hline 11 & -22.78 & 17.35 & 450 & 453 & 29.83 & 0 & 1.81 & 128 & 32 & 5891 \\
\hline 11 & -22.78 & 17.35 & 600 & 605 & 32.19 & 0 & 2.04 & 107 & 29 & 5893 \\
\hline
\end{tabular}




\begin{tabular}{|c|c|c|c|c|c|c|c|c|c|c|}
\hline $\begin{array}{c}\text { Station } \\
{[\#]}\end{array}$ & $\begin{array}{l}\text { Longitude } \\
\text { [deg. E] }\end{array}$ & $\begin{array}{l}\text { Latitude } \\
\text { [deg. N] }\end{array}$ & $\begin{array}{c}\text { Depth } \\
{[\mathrm{m}]}\end{array}$ & $\begin{array}{c}\text { CTDPRS } \\
\text { [dbars] }\end{array}$ & $\begin{array}{c}\text { Nitrate } \\
{[\mathrm{uM}]}\end{array}$ & $\begin{array}{l}\text { Nitrite } \\
{[\mathrm{uM}]}\end{array}$ & $\begin{array}{l}\mathrm{PO}_{4}{ }^{3-} \\
{[\mathrm{uM}]}\end{array}$ & $\begin{array}{l}\text { TCo } \\
{[\mathbf{p M}]}\end{array}$ & $\begin{array}{c}\mathrm{Co}^{\prime} \\
{[\mathbf{p M}]}\end{array}$ & $\begin{array}{l}\text { GEOTR } \\
\text { SMPL \# }\end{array}$ \\
\hline 11 & -22.78 & 17.35 & 801 & 807 & 32.67 & 0 & 2.12 & 98 & 26 & 5895 \\
\hline 11 & -22.78 & 17.35 & 964 & 973 & 30.67 & 0.01 & 2.03 & 92 & 32 & 5921 \\
\hline 11 & -22.78 & 17.35 & 1098 & 1109 & 28.74 & 0 & 1.92 & 89 & 28 & 5923 \\
\hline 11 & -22.78 & 17.35 & 1248 & 1260 & 26.41 & 0 & 1.78 & 89 & 30 & 5925 \\
\hline 11 & -22.78 & 17.35 & 1499 & 1514 & 23.94 & 0 & 1.62 & 82 & 35 & 5927 \\
\hline 11 & -22.78 & 17.35 & 1748 & 1768 & 22.29 & 0 & 1.52 & 84 & 28 & 5929 \\
\hline 11 & -22.78 & 17.35 & 1998 & 2021 & 21.50 & 0 & 1.47 & 80 & 28 & 5931 \\
\hline 11 & -22.78 & 17.35 & 2248 & 2275 & 21.04 & 0 & 1.44 & 67 & 20 & 5933 \\
\hline 11 & -22.78 & 17.35 & 2499 & 2531 & 20.92 & 0 & 1.43 & 69 & 23 & 5935 \\
\hline 11 & -22.78 & 17.35 & 2748 & 2785 & 20.95 & 0 & 1.43 & 55 & 19 & 5937 \\
\hline 11 & -22.78 & 17.35 & 2998 & 3040 & 21.07 & 0 & 1.43 & 50 & 18 & 5939 \\
\hline 11 & -22.78 & 17.35 & 3198 & 3245 & 21.28 & 0 & 1.44 & 50 & 17 & 5941 \\
\hline 11 & -22.78 & 17.35 & 3298 & 3347 & 21.56 & 0 & 1.46 & 54 & 13 & 5943 \\
\hline 12 & -24.50 & 17.40 & 38 & 38 & 0 & 0 & 0.02 & 28 & 2 & 5976 \\
\hline 12 & -24.50 & 17.40 & 48 & 48 & 0 & 0 & 0.07 & 52 & 6 & 5978 \\
\hline 12 & -24.50 & 17.40 & 72 & 72 & 1.71 & 0.20 & 0.22 & 68 & 6 & 5980 \\
\hline 12 & -24.50 & 17.40 & 90 & 91 & 6.00 & 0.08 & 0.43 & 84 & 14 & 5982 \\
\hline 12 & -24.50 & 17.40 & 135 & 136 & 9.23 & 0.02 & 0.57 & 88 & 22 & 5984 \\
\hline 12 & -24.50 & 17.40 & 185 & 186 & 16.73 & 0 & 1.00 & 97 & 29 & 5986 \\
\hline 12 & -24.50 & 17.40 & 235 & 237 & 21.35 & 0 & 1.29 & 121 & 38 & 5988 \\
\hline 12 & -24.50 & 17.40 & 286 & 287 & 25.18 & 0 & 1.51 & 125 & 48 & 5990 \\
\hline 12 & -24.50 & 17.40 & 351 & 353 & 27.89 & 0 & 1.68 & 135 & 33 & 5992 \\
\hline 12 & -24.50 & 17.40 & 451 & 454 & 30.30 & 0 & 1.84 & 100 & 31 & 5994 \\
\hline 12 & -24.50 & 17.40 & 601 & 606 & 32.38 & 0 & 2.05 & 98 & 30 & 5996 \\
\hline 12 & -24.50 & 17.40 & 801 & 807 & 33.31 & 0 & 2.17 & 95 & 31 & 5998 \\
\hline 12 & -24.50 & 17.40 & 964 & 973 & 31.21 & 0.01 & 2.06 & 90 & 26 & 6035 \\
\hline 12 & -24.50 & 17.40 & 1099 & 1110 & 28.42 & 0.01 & 1.90 & 82 & 33 & 6037 \\
\hline 12 & -24.50 & 17.40 & 1257 & 1269 & 25.98 & 0 & 1.75 & 85 & 26 & 6039 \\
\hline 12 & -24.50 & 17.40 & 1499 & 1514 & 23.82 & 0 & 1.62 & 67 & 30 & 6041 \\
\hline 12 & -24.50 & 17.40 & 1749 & 1768 & 22.35 & 0 & 1.52 & 69 & 27 & 6043 \\
\hline 12 & -24.50 & 17.40 & 1999 & 2022 & 21.58 & 0.01 & 1.47 & 70 & 27 & 6045 \\
\hline 12 & -24.50 & 17.40 & 2248 & 2275 & 21.22 & 0 & 1.46 & 71 & 24 & 6047 \\
\hline 12 & -24.50 & 17.40 & 2499 & 2531 & 21.14 & 0.01 & 1.45 & 68 & 19 & 6049 \\
\hline 12 & -24.50 & 17.40 & 2749 & 2785 & 21.05 & 0 & 1.44 & 59 & 18 & 6051 \\
\hline 12 & -24.50 & 17.40 & 2998 & 3040 & 21.10 & 0 & 1.44 & 54 & 18 & 6053 \\
\hline 12 & -24.50 & 17.40 & 3198 & 3245 & 21.37 & 0 & 1.46 & 50 & 14 & 6055 \\
\hline 12 & -24.50 & 17.40 & 3498 & 3551 & 21.76 & 0 & 1.47 & 48 & 11 & 6057 \\
\hline
\end{tabular}


Table 4. Data from Chapter 6

\begin{tabular}{|c|c|c|c|c|c|c|c|c|}
\hline Cruise & $\begin{array}{c}\text { Station } \\
\#\end{array}$ & $\begin{array}{c}\text { Depth } \\
{[\mathbf{m}]}\end{array}$ & $\begin{array}{c}\mathbf{T C o}_{\text {at-sea }} \\
{[\mathbf{p M}]}\end{array}$ & $\begin{array}{c}\mathbf{T C o}_{\text {stored }} \\
{[\mathbf{p M}]}\end{array}$ & $\begin{array}{c}\text { TCo } \text { preserved } \\
{[\text { [pM] }}\end{array}$ & $\begin{array}{c}\mathrm{Co}^{\prime}{ }_{\text {at-sea }} \\
{[\mathbf{p M}]}\end{array}$ & $\begin{array}{c}\mathbf{C o}^{\prime}{ }_{\text {stored }} \\
{[\mathbf{p M}]}\end{array}$ & $\begin{array}{c}\mathrm{Co}_{\text {preservec }}^{\prime} \\
{[\mathbf{p M}]}\end{array}$ \\
\hline CoFeMUG & 7 & 10 & 18 & 9 & & $\overline{\text { n.d }}$ & & \\
\hline CoFeMUG & 7 & 20 & 20 & 12 & & n.d & & \\
\hline CoFeMUG & 7 & 41 & 16 & 9 & & n.d & & \\
\hline CoFeMUG & 7 & 70 & 23 & 18 & & n.d & & \\
\hline CoFeMUG & 7 & 94 & 35 & 32 & & n.d & & \\
\hline CoFeMUG & 7 & 109 & 41 & 44 & & n.d & & \\
\hline CoFeMUG & 7 & 150 & 54 & 55 & & 4 & & \\
\hline CoFeMUG & 7 & 200 & 108 & 74 & & 15 & & \\
\hline CoFeMUG & 7 & 300 & 105 & 90 & & 12 & & \\
\hline CoFeMUG & 7 & 399 & 102 & 86 & & 22 & & \\
\hline CoFeMUG & 7 & 500 & 89 & 80 & & 22 & & \\
\hline CoFeMUG & 7 & 599 & 92 & 80 & & 19 & & \\
\hline CoFeMUG & 7 & 700 & 89 & 81 & & 22 & & \\
\hline CoFeMUG & 7 & 799 & 100 & 83 & & 24 & & \\
\hline CoFeMUG & 7 & 899 & 95 & 75 & & 21 & & \\
\hline CoFeMUG & 7 & 999 & 64 & 61 & & 25 & & \\
\hline CoFeMUG & 7 & 1499 & 77 & 73 & & 23 & & \\
\hline CoFeMUG & 7 & 1749 & 95 & 77 & & 24 & & \\
\hline CoFeMUG & 7 & 1999 & 89 & 64 & & 22 & & \\
\hline CoFeMUG & 7 & 2249 & 90 & 56 & & 23 & & \\
\hline CoFeMUG & 7 & 2500 & 75 & 59 & & 18 & & \\
\hline CoFeMUG & 7 & 2841 & 71 & 57 & & 17 & & \\
\hline CoFeMUG & 9 & 9 & 18 & 14 & & 6 & & \\
\hline CoFeMUG & 9 & 20 & 19 & 15 & & 3 & & \\
\hline CoFeMUG & 9 & 39 & 17 & 14 & & 1 & & \\
\hline CoFeMUG & 9 & 69 & 45 & 31 & & n.d & & \\
\hline CoFeMUG & 9 & 89 & 48 & 39 & & 6 & & \\
\hline CoFeMUG & 9 & 110 & 55 & 46 & & & & \\
\hline CoFeMUG & 9 & 150 & 64 & 48 & & 10 & & \\
\hline CoFeMUG & 9 & 200 & 106 & 89 & & 12 & & \\
\hline CoFeMUG & 9 & 300 & 141 & 113 & & 27 & & \\
\hline CoFeMUG & 9 & 399 & 122 & 106 & & 24 & & \\
\hline CoFeMUG & 9 & 499 & 113 & 94 & & 24 & & \\
\hline CoFeMUG & 9 & 599 & 87 & 85 & & 22 & & \\
\hline CoFeMUG & 9 & 699 & 81 & 79 & & 19 & & \\
\hline CoFeMUG & 9 & 799 & 84 & 87 & & 19 & & \\
\hline
\end{tabular}




\begin{tabular}{|c|c|c|c|c|c|c|c|c|}
\hline Cruise & $\begin{array}{c}\text { Station } \\
\#\end{array}$ & $\begin{array}{c}\text { Depth } \\
{[\mathrm{m}]}\end{array}$ & $\begin{array}{c}\mathbf{T C o}_{\text {at-sea }} \\
{[\mathbf{p M}]}\end{array}$ & $\begin{array}{c}\mathbf{T C} \mathbf{o}_{\text {stored }} \\
{[\mathbf{p M}]}\end{array}$ & $\begin{array}{c}\text { TCo } \\
\text { [preserved } \\
\text { [pM] }\end{array}$ & $\begin{array}{c}\mathbf{C o}^{\prime}{ }_{\text {at-sea }} \\
{[\mathbf{p M} \mathbf{M}]}\end{array}$ & $\begin{array}{c}\mathbf{C o}^{\prime}{ }_{\text {stored }} \\
{[\mathbf{p M} \mathbf{M}}\end{array}$ & $\begin{array}{c}\mathrm{Co}_{\text {preserved }}^{\prime} \\
{[\mathbf{p M}]}\end{array}$ \\
\hline CoFeMUG & 9 & 1499 & 87 & 79 & & 28 & & \\
\hline CoFeMUG & 9 & 1999 & 90 & 67 & & 24 & & \\
\hline CoFeMUG & 9 & 2499 & 67 & 64 & & 21 & & \\
\hline CoFeMUG & 9 & 3000 & 66 & 54 & & 11 & & \\
\hline CoFeMUG & 9 & 3499 & 54 & 50 & & 10 & & \\
\hline CoFeMUG & 9 & 3959 & 54 & 59 & & 12 & & \\
\hline CoFeMUG & 11 & 10 & 24 & 17 & & 5 & & \\
\hline CoFeMUG & 11 & 20 & 21 & 17 & & 2 & & \\
\hline CoFeMUG & 11 & 40 & 18 & 17 & & 1 & & \\
\hline CoFeMUG & 11 & 70 & 47 & 44 & & 1 & & \\
\hline CoFeMUG & 11 & 90 & 83 & 67 & & 6 & & \\
\hline CoFeMUG & 11 & 110 & 83 & 67 & & 7 & & \\
\hline CoFeMUG & 11 & 150 & 90 & 78 & & 8 & & \\
\hline CoFeMUG & 11 & 199 & 119 & 99 & & 16 & & \\
\hline CoFeMUG & 11 & 298 & 161 & 106 & & 21 & & \\
\hline CoFeMUG & 11 & 350 & 130 & 102 & & 23 & & \\
\hline CoFeMUG & 11 & 399 & 119 & 105 & & 17 & & \\
\hline CoFeMUG & 11 & 449 & 118 & 90 & & 29 & & \\
\hline CoFeMUG & 11 & 500 & 106 & 96 & & 22 & & \\
\hline CoFeMUG & 11 & 600 & 105 & 97 & & 19 & & \\
\hline CoFeMUG & 11 & 700 & 98 & & & 19 & & \\
\hline CoFeMUG & 11 & 800 & 101 & 90 & & 20 & & \\
\hline CoFeMUG & 11 & 998 & 90 & 81 & & 23 & & \\
\hline CoFeMUG & 11 & 1499 & 91 & 75 & & 21 & & \\
\hline CoFeMUG & 11 & 1999 & 82 & 71 & & 15 & & \\
\hline CoFeMUG & 11 & 2500 & 65 & 65 & & 14 & & \\
\hline CoFeMUG & 11 & 2700 & 82 & 59 & & 12 & & \\
\hline CoFeMUG & 11 & 2936 & 68 & 60 & & 9 & & \\
\hline CORSACS & NX6 & 10 & 34 & 33 & & 25 & & \\
\hline CORSACS & NX6 & 20 & 32 & 30 & & 25 & & \\
\hline CORSACS & NX6 & 40 & 45 & 48 & & 28 & & \\
\hline CORSACS & NX6 & 60 & 45 & 47 & & 28 & & \\
\hline CORSACS & NX6 & 80 & 49 & 40 & & 22 & & \\
\hline CORSACS & NX6 & 100 & 50 & 50 & & 30 & & \\
\hline CORSACS & NX6 & 150 & 49 & 55 & & 30 & & \\
\hline CORSACS & NX6 & 200 & 47 & 48 & & 25 & & \\
\hline CORSACS & NX6 & 300 & 53 & 41 & & 33 & & \\
\hline
\end{tabular}




\begin{tabular}{|c|c|c|c|c|c|c|c|c|}
\hline Cruise & $\begin{array}{c}\text { Station } \\
\#\end{array}$ & $\begin{array}{c}\text { Depth } \\
{[\mathbf{m}]}\end{array}$ & $\begin{array}{c}\mathbf{T C o}_{\text {at-sea }} \\
{[\mathbf{p M}]}\end{array}$ & $\begin{array}{c}\mathbf{T C o}_{\text {stored }} \\
{[\mathbf{p M}]}\end{array}$ & $\begin{array}{c}\text { TCo } \text { preserved } \\
{[\mathbf{p M}]}\end{array}$ & $\begin{array}{c}\mathbf{C o}^{\prime}{ }_{\text {at-sea }} \\
{[\mathbf{p M}]}\end{array}$ & $\begin{array}{c}\mathbf{C o}^{\prime}{ }_{\text {stored }} \\
{[\mathbf{p M}]}\end{array}$ & $\begin{array}{c}\mathbf{C o}_{\text {preserved }}^{\prime} \\
{[\mathbf{p M}]}\end{array}$ \\
\hline NAGZT & 9 & 2 & 46 & 42 & 42 & 6 & & 3 \\
\hline NAGZT & 9 & 28 & 52 & 54 & 54 & 5 & 3 & 9 \\
\hline NAGZT & 9 & 49 & 117 & 105 & 113 & 13 & 3 & 11 \\
\hline NAGZT & 9 & 89 & 133 & 88 & 125 & 41 & 1 & 36 \\
\hline NAGZT & 9 & 109 & 149 & 86 & 140 & 41 & 4 & 31 \\
\hline NAGZT & 9 & 135 & 130 & & 129 & 54 & & 51 \\
\hline NAGZT & 9 & 185 & 144 & 90 & 131 & 37 & & 59 \\
\hline NAGZT & 9 & 221 & 123 & 86 & 124 & 37 & & 57 \\
\hline NAGZT & 9 & 285 & 150 & 93 & 147 & 36 & & 66 \\
\hline NAGZT & 9 & 361 & 160 & 97 & 160 & 34 & 3 & 67 \\
\hline NAGZT & 9 & 665 & 125 & 75 & 124 & 27 & & 51 \\
\hline NAGZT & 9 & 797 & 116 & & 113 & 30 & n.d & 47 \\
\hline NAGZT & 9 & 965 & 98 & 46 & 96 & 29 & & 43 \\
\hline NAGZT & 9 & 1095 & 94 & 44 & 96 & 23 & & 36 \\
\hline NAGZT & 9 & 1200 & 96 & 55 & 92 & 27 & & 37 \\
\hline NAGZT & 9 & 1236 & 86 & 43 & 90 & 30 & & 36 \\
\hline NAGZT & 9 & 1385 & 84 & 42 & 82 & 27 & & 41 \\
\hline NAGZT & 9 & 1538 & 83 & 39 & 78 & 21 & n.d & 32 \\
\hline NAGZT & 9 & 1787 & 87 & 40 & 84 & 24 & n.d & 31 \\
\hline NAGZT & 9 & 1883 & 79 & 37 & 73 & 24 & n.d & 28 \\
\hline NAGZT & 9 & 2088 & 76 & 36 & 72 & 23 & n.d & 24 \\
\hline NAGZT & 9 & 2294 & 68 & 38 & 65 & 19 & n.d & 30 \\
\hline NAGZT & 9 & 2585 & 67 & 41 & 67 & 15 & n.d & 15 \\
\hline NAGZT & 9 & 2900 & 44 & 32 & 43 & 1 & n.d & 9 \\
\hline NAGZT & 9 & 2999 & 39 & 30 & 39 & 2 & n.d & 4 \\
\hline NAGZT & 5 & 2 & 14 & 13 & & n.d & & \\
\hline NAGZT & 5 & 31 & 15 & 14 & & 1 & n.d & \\
\hline NAGZT & 5 & 52 & 34 & 24 & & 1 & 4 & \\
\hline NAGZT & 5 & 81 & 43 & 41 & & 4 & & \\
\hline NAGZT & 5 & 101 & 45 & 41 & & n.d & 1 & \\
\hline NAGZT & 5 & 136 & 43 & 31 & & n.d & & \\
\hline NAGZT & 5 & 187 & 47 & 49 & & 21 & 1 & \\
\hline NAGZT & 5 & 251 & 50 & 47 & & 18 & 4 & \\
\hline NAGZT & 5 & 351 & 60 & 51 & & 18 & 8 & \\
\hline NAGZT & 5 & 553 & 74 & 66 & & 20 & 9 & \\
\hline NAGZT & 5 & 802 & 94 & 84 & & 24 & n.d & \\
\hline NAGZT & 5 & 963 & 80 & 61 & & 23 & n.d & \\
\hline
\end{tabular}




\begin{tabular}{|c|c|c|c|c|c|c|c|c|}
\hline Cruise & $\begin{array}{c}\text { Station } \\
\#\end{array}$ & $\begin{array}{c}\text { Depth } \\
{[\mathbf{m}]}\end{array}$ & $\begin{array}{c}\mathbf{T C o}_{\text {at-sea }} \\
{[\mathbf{p M}]}\end{array}$ & $\begin{array}{c}\mathbf{T C o}_{\text {stored }} \\
{[\mathbf{p M}]}\end{array}$ & $\begin{array}{c}\text { TCo } \mathbf{o}_{\text {preserved }} \\
\text { [pM] }\end{array}$ & $\begin{array}{c}\mathbf{C o}^{\prime}{ }_{\text {at-sea }} \\
{[\mathbf{p M}]}\end{array}$ & $\begin{array}{c}\mathbf{C o}^{\prime}{ }_{\text {stored }} \\
{[\mathbf{p M}]}\end{array}$ & $\begin{array}{c}\mathbf{C o}_{\text {preserved }}^{\prime} \\
{[\mathbf{p M ]}}\end{array}$ \\
\hline NAGZT & 5 & 1202 & 78 & 65 & & 23 & 17 & \\
\hline NAGZT & 5 & 1501 & 77 & 57 & & 23 & 10 & \\
\hline NAGZT & 5 & 1800 & 70 & 56 & & 22 & 14 & \\
\hline NAGZT & 5 & 2100 & 71 & 58 & & 21 & 15 & \\
\hline NAGZT & 5 & 2401 & 65 & 59 & & 18 & 16 & \\
\hline NAGZT & 5 & 2701 & 62 & 58 & & 14 & 6 & \\
\hline NAGZT & 5 & 3001 & 55 & 46 & & 9 & n.d & \\
\hline NAGZT & 5 & 3301 & 51 & 39 & & 9 & 2 & \\
\hline NAGZT & 5 & 3600 & 49 & 34 & & 7 & n.d & \\
\hline NAGZT & 5 & 3899 & 48 & 35 & & 9 & 5 & \\
\hline NAGZT & 5 & 4199 & 44 & 36 & & 5 & 2 & \\
\hline NAGZT & 5 & 4549 & 43 & 35 & & 4 & 4 & \\
\hline NAGZT & 5 & 4899 & 40 & 32 & & 5 & 1 & \\
\hline
\end{tabular}


Table 5. Data from Appendix III

Station Longitude Latitude Depth TCo Co'

\begin{tabular}{cccccc} 
[\#] & [deg. E] & [deg. N] & [m] & [pM] & [pM] \\
\hline L1-24 & -139.33 & 71.12 & 8 & 249 & 41 \\
L1-24 & -139.33 & 71.12 & 15 & 133 & 22 \\
L1-24 & -139.33 & 71.12 & 30 & 81 & 28 \\
L1-24 & -139.33 & 71.12 & 49 & 301 & \\
L1-24 & -139.33 & 71.12 & 70 & & \\
L1-24 & -139.33 & 71.12 & 89 & 197 & 28 \\
L1-24 & -139.33 & 71.12 & 119 & 203 & 8 \\
L1-24 & -139.33 & 71.12 & 148 & 129 & 3 \\
L1-24 & -139.33 & 71.12 & 173 & 130 & 6 \\
L1-24 & -139.33 & 71.12 & 198 & 129 & 3 \\
L1-24 & -139.33 & 71.12 & 248 & 102 & 10 \\
L1-24 & -139.33 & 71.12 & 396 & 85 & 4 \\
L1-32 & -139.33 & 71.12 & 9 & 252 & \\
L1-32 & -139.33 & 71.12 & 297 & 96 & \\
L1-32 & -139.33 & 71.12 & 346 & 71 & \\
L1-32 & -139.33 & 71.12 & 395 & 71 & \\
L1-32 & -139.33 & 71.12 & 494 & 60 & \\
L1-32 & -139.33 & 71.12 & 593 & 58 & \\
L1-32 & -139.33 & 71.12 & 740 & 55 & \\
L1-32 & -139.33 & 71.12 & 987 & 62 & \\
L1-32 & -139.33 & 71.12 & 1233 & 62 & \\
L1-32 & -139.33 & 71.12 & 1479 & 44 & \\
L1-32 & -139.33 & 71.12 & 1674 & 44 & \\
L1-32 & -139.33 & 71.12 & 1774 & 46 & \\
\hline L1.5 & -139.39 & 73.32 & 989 & 77 & 12 \\
L1.5 & -139.39 & 73.32 & 791 & 55 & 3 \\
L1.5 & -139.39 & 73.32 & 593 & 45 & 1 \\
L1.5 & -139.39 & 73.32 & 445 & 41 & n.d. \\
L1.5 & -139.39 & 73.32 & 376 & 72 & n.d. \\
L1.5 & -139.39 & 73.32 & 277 & 84 & 2 \\
L1.5 & -139.39 & 73.32 & 188 & 141 & 5 \\
L1.5 & -139.39 & 73.32 & 138 & 153 & 5 \\
L1.5 & -139.39 & 73.32 & 89 & 150 & 11 \\
L1.5 & -139.39 & 73.32 & 40 & 133 & 7 \\
L1.5 & -139.39 & 73.32 & 18 & 116 & 1 \\
L1.5 & -139.39 & 73.32 & 10 & 106 & 3 \\
\hline L2-35 & -137.34 & 74.64 & 2899 & 38 &
\end{tabular}




\begin{tabular}{|c|c|c|c|c|c|}
\hline $\begin{array}{c}\text { Station } \\
{[\#]}\end{array}$ & $\begin{array}{c}\text { Longitude } \\
\text { [deg. E] }\end{array}$ & $\begin{array}{r}\text { Latitude } \\
\text { [deg. N] }\end{array}$ & $\begin{array}{c}\text { Depth } \\
\text { [m] }\end{array}$ & $\begin{array}{r}\text { TCo } \\
{[\mathbf{p M}]}\end{array}$ & $\begin{array}{r}\mathrm{Co}^{\prime} \\
{[\mathrm{pM}]}\end{array}$ \\
\hline L2-35 & -137.34 & 74.64 & 2656 & 31 & \\
\hline L2-35 & -137.34 & 74.64 & 2460 & 42 & \\
\hline L2-35 & -137.34 & 74.64 & 2264 & 44 & \\
\hline L2-35 & -137.34 & 74.64 & 2068 & 45 & \\
\hline L2-35 & -137.34 & 74.64 & 1872 & 45 & \\
\hline L2-35 & -137.34 & 74.64 & 1676 & 49 & \\
\hline L2-35 & -137.34 & 74.64 & 1480 & 73 & \\
\hline L2-35 & -137.34 & 74.64 & 1283 & 70 & \\
\hline L2-35 & -137.34 & 74.64 & 1086 & 85 & \\
\hline L2-35 & -137.34 & 74.64 & 888 & 52 & \\
\hline L2-35 & -137.34 & 74.64 & 790 & 67 & \\
\hline $\mathrm{L} 2-51$ & -136.16 & 74.39 & 1086 & 49 & 4 \\
\hline L2-51 & -136.16 & 74.39 & 791 & 44 & 4 \\
\hline L2-51 & -136.16 & 74.39 & 641 & 59 & 3 \\
\hline L2-51 & -136.16 & 74.39 & 495 & 59 & 1 \\
\hline L2-51 & -136.16 & 74.39 & 416 & 71 & 10 \\
\hline L2-51 & -136.16 & 74.39 & 356 & 59 & 5 \\
\hline L2-51 & -136.16 & 74.39 & 268 & 71 & 8 \\
\hline L2-51 & -136.16 & 74.39 & 179 & 132 & 8 \\
\hline L2-51 & -136.16 & 74.39 & 119 & 148 & 22 \\
\hline L2-51 & -136.16 & 74.39 & 55 & 179 & 75 \\
\hline L2-51 & -136.16 & 74.39 & 25 & 104 & 19 \\
\hline L2-51 & -136.16 & 74.39 & 8 & 100 & 2 \\
\hline L3 & -137.56 & 75.28 & 1185 & 73 & \\
\hline L3 & -137.56 & 75.28 & 987 & 60 & 6 \\
\hline L3 & -137.56 & 75.28 & 791 & 55 & 5 \\
\hline L3 & -137.56 & 75.28 & 593 & 40 & 1 \\
\hline L3 & -137.56 & 75.28 & 435 & 72 & 10 \\
\hline L3 & -137.56 & 75.28 & 345 & 65 & 7 \\
\hline L3 & -137.56 & 75.28 & 257 & 75 & 4 \\
\hline L3 & -137.56 & 75.28 & 178 & 110 & 5 \\
\hline L3 & -137.56 & 75.28 & 138 & 130 & 14 \\
\hline L3 & -137.56 & 75.28 & 54 & 162 & 7 \\
\hline L3 & -137.56 & 75.28 & 31 & 142 & 14 \\
\hline L3 & -137.56 & 75.28 & 10 & 92 & 6 \\
\hline L1.1-59 & -136.59 & 72.51 & 357 & 54 & \\
\hline L1.1-59 & -136.59 & 72.51 & 267 & 76 & 1 \\
\hline L1.1-59 & -136.59 & 72.51 & 208 & 94 & 7 \\
\hline
\end{tabular}




\begin{tabular}{cccccc}
$\begin{array}{c}\text { Station } \\
\text { [\#] }\end{array}$ & $\begin{array}{c}\text { Longitude } \\
\text { [deg. E] }\end{array}$ & $\begin{array}{c}\text { Latitude } \\
\text { [deg. N] }\end{array}$ & $\begin{array}{c}\text { Depth } \\
\text { [m] }\end{array}$ & $\begin{array}{c}\text { TCo } \\
\text { [pM] }\end{array}$ & $\begin{array}{c}\text { Co' } \\
\text { [pM] }\end{array}$ \\
\hline L1.1-59 & -136.59 & 72.51 & 168 & 138 & 6 \\
L1.1-59 & -136.59 & 72.51 & 129 & 161 & 46 \\
L1.1-59 & -136.59 & 72.51 & 109 & 208 & 60 \\
L1.1-59 & -136.59 & 72.51 & 89 & 160 & 21 \\
L1.1-59 & -136.59 & 72.51 & 75 & 153 & 14 \\
L1.1-59 & -136.59 & 72.51 & 60 & 208 & 22 \\
L1.1-59 & -136.59 & 72.51 & 40 & 192 & 11 \\
L1.1-59 & -136.59 & 72.51 & 20 & 127 & 10 \\
L1.1-59 & -136.59 & 72.51 & 8 & 96 & 6 \\
\hline L1.1 & -136.94 & 72.54 & 2362 & 34 & \\
L1.1 & -136.94 & 72.54 & 2216 & 37 & \\
L1.1 & -136.94 & 72.54 & 1971 & 39 & \\
L1.1 & -136.94 & 72.54 & 1725 & 47 & \\
L1.1 & -136.94 & 72.54 & 1479 & 52 & \\
L1.1 & -136.94 & 72.54 & 1234 & 54 & \\
L1.1 & -136.94 & 72.54 & 987 & 47 & \\
L1.1 & -136.94 & 72.54 & 791 & 52 & \\
L1.1 & -136.94 & 72.54 & 643 & 48 & \\
L1.1 & -136.94 & 72.54 & 495 & 58 & \\
L1.1 & -136.94 & 72.54 & 420 & 55 & \\
L1.1 & -136.94 & 72.54 & 396 & 59 & \\
\hline S4 & -132.94 & 71.18 & 273 & 58 & 4 \\
S4 & -132.94 & 71.18 & 237 & 63 & 11 \\
S4 & -132.94 & 71.18 & 199 & 42 & 3 \\
S4 & -132.94 & 71.18 & 148 & 141 & 25 \\
S4 & -132.94 & 71.18 & 118 & 133 & 29 \\
S4 & -132.94 & 71.18 & 89 & 162 & 27 \\
S4 & -132.94 & 71.18 & 69 & 163 & 13 \\
S4 & -132.94 & 71.18 & 49 & 249 & 87 \\
S4 & -132.94 & 71.18 & 26 & 521 & 116 \\
S4 & -132.94 & 71.18 & 16 & 150 & 132 \\
S4 & -132.94 & 71.18 & 8 & 283 & 20 \\
S4 & -132.94 & 71.18 & 7 & 327 & 140 \\
\hline & & & & &
\end{tabular}

